\title{
Numerical and Experimental Optimization for Specific Fatigue Life Maximization of Additively Manufactured Ti- 6Al-4V Aerospace Components
}

\author{
by
}

Eric Paul Trudel

A thesis submitted to the Faculty of Graduate and Postdoctoral Affairs in partial fulfillment of the requirements for the degree of

\author{
Master of Applied Science \\ in \\ Mechanical and Aerospace Engineering \\ Carleton University \\ Ottawa, Ontario \\ (C) 2020, Eric Paul Trudel
}




\begin{abstract}
Computer Aided Design (CAD) in combination with structural optimization has allowed the rapid development of engineering prototypes. Topology and multi-scale design optimization techniques are capable of shaping highly efficient load paths. However, the resulting geometries are complex that they can only be manufactured with Additive Manufacturing (AM) technology. One of the main downfalls of AM parts is their poor fatigue life which hinders the potential merits of design optimization. This thesis investigates experimental and numerical strategies to extend the fatigue life of additively manufactured Ti-6Al-4V components manufactured by Direct Metal Laser Sintering (DMLS) technology. Three stages for the development of fatigue resistant AM parts are introduced. The first experimental stage is concerned with the application of Ultrasonic Impact Treatment (UIT) to enhance the fatigue life of AM parts and to derive the S-N curves for the fatigue behavior of the treated and the as built parts. The second numerical stage is to conduct design optimization employing multi-scale, multi-objective and topology optimization methods to maximize the specific fatigue life of mechanical components made of UIT treated Ti-6Al-4V. A third stage includes employing cellular material in the context of topology and multi-scale optimization to further minimize the weight and maximize the fatigue life in a multi-objective design optimization scheme. It is found that by applying UIT onto DMLS Ti-6Al-4V that the endurance limit increases by $25 \%$. When UIT is applied in the context of specific fatigue maximization, then a $51 \%$ mass reduction can be achieved while maintaining infinite life criterion.
\end{abstract}




\section{Acknowledgements}

I would like to acknowledge my supervisor professor Mostafa El Sayed for helping me with my thesis. I would also like to extend my appreciations to the machine shop employees, Steve Truttmann, Sinah Malz and Shaza Nosir for their help with the UIT experimental work. Additional gratitude is given to Denis Walsh and Leo Kok from Bombardier for funding and guidance for the experimental research. 


\section{Contributions}

The following papers have been published as a result of the research in this thesis:

Peer-Reviewed Journal Papers

- Walker, P.; Malz, S.; Trudel, E.; Nosir, S.; ElSayed, M.S.; Kok, L. Effects of Ultrasonic Impact Treatment on the Stress-Controlled Fatigue Performance of Additively Manufactured DMLS Ti-6Al-4V Alloy. Appl. Sci. 2019, 9, 4787.

\section{Conference Papers}

- Trudel, E. P., ElSayed, M.S.A, Kok, L., \& Provost, E. (2019). Multiscale Design Optimization of Additively Manufactured Aerospace Structures Employing Functionally Graded Lattice Materials. AIAA Scitech 2019 Forum. doi:10.2514/6.2019-0420

- Malz, S., Nosir, S., Trudel, E. P., ElSayed, M.S.A, Kok, L., \& Provost, E. (2019). Effect of Ultrasonic Impact Treatment on the Stress-Controlled Fatigue Performance of Additively Manufactured Ti-6Al-4V Alloy. AIAA Scitech 2019 Forum. doi:10.2514/6.2019-0414

\section{Technical Reports}

- Trudel, E, Topology Optimization Design Report, Bombardier (2020)

In addition, a list of research papers in the process of being published or currently in review is given below:

Papers in Review

- Trudel,E.P., Walker, P.,Nosir,S, ElSayed, M.S.A. (2020) Experimental Optimization for Fatigue Life Maximization of Additively Manufactured Ti-6Al4V Alloy Employing Ultrasonic Impact Treatment, Additive Manufacturing

- Trudel, E. P., ElSayed, M.S.A. (2020) Conformal Wireframe Lattice Nets, International Journal for Computational Methods in Engineering Science \& Mechanics

- Trudel, E. P., ElSayed, M.S.A. (2020) Procedural Global Optimization of Lattice Structures for Fatigue Enhancement, Journal of Multi-Disciplinary Engineering 
- Trudel, E. P., ElSayed, M.S.A. (2020) Comparison of Fatigue Gradients on Fatigue Minimization Topology Optimizations, International Journal of Engineering Technologies and Optimization 


\section{Table of Contents}

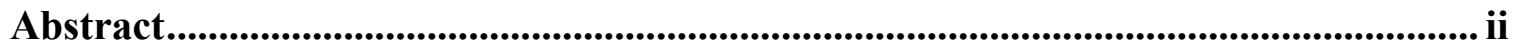

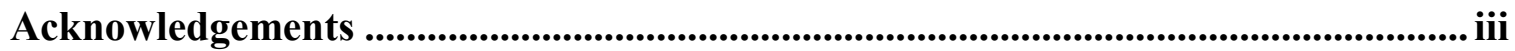

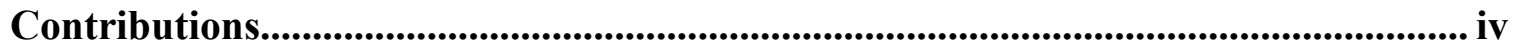

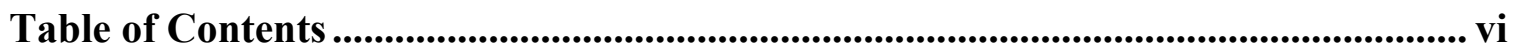

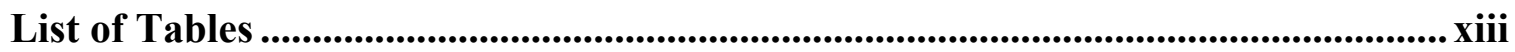

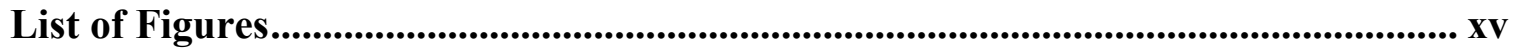

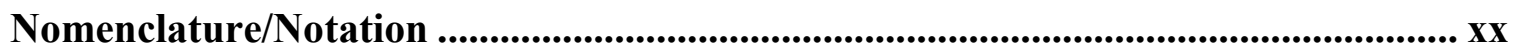

Chapter 1: Introduction and Motivations ........................................................................ 1

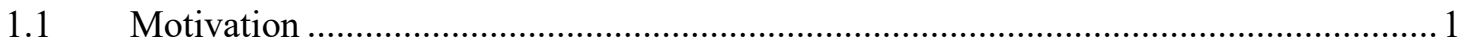

1.2 Proposed Multi -Scale and Multi-Objective Workflow ............................................ 4

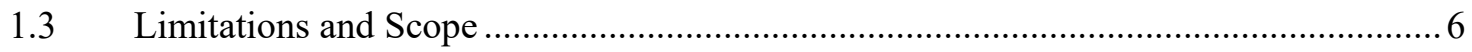

$1.4 \quad$ Thesis Outline

Chapter 2: Literature Review ..................................................................................................... 9

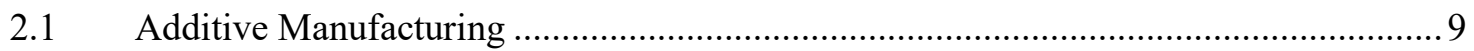

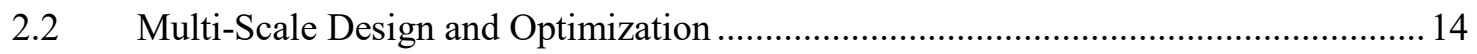

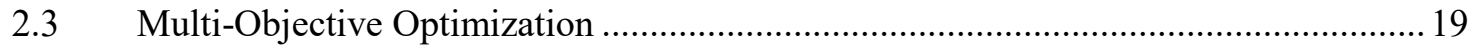

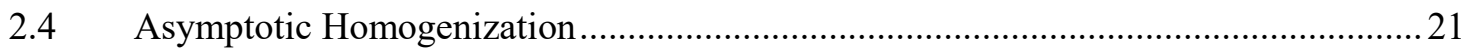

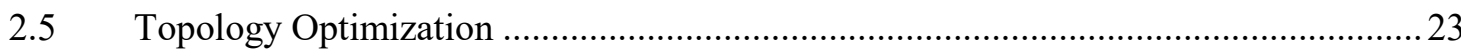

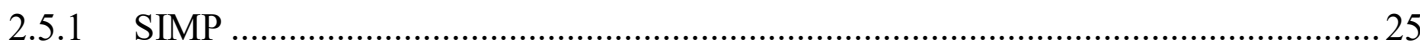

2.6 Lattice Optimization Literature Review ............................................................26

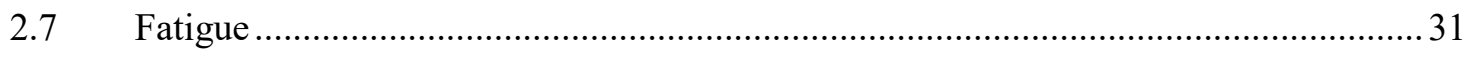

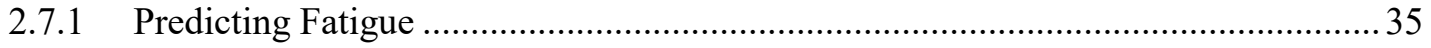

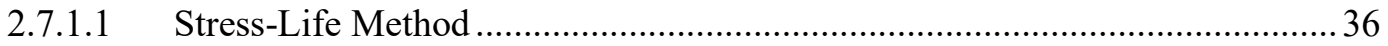




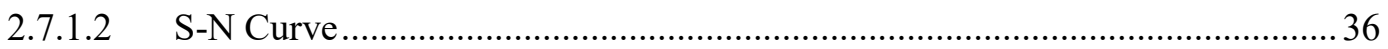

2.7.2 Consideration of Multi-Axial Loadings and Equivalent Stresses ........................... 38

2.7.3 Failure criteria and Mean Stress Correction from Fluctuating Loads ..................... 40

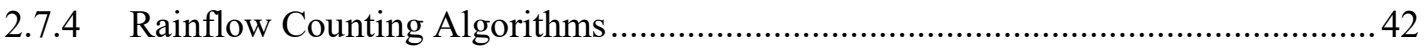

2.7.5 Palmgren Miner Cumulative Damage Hypotheses ............................................... 43

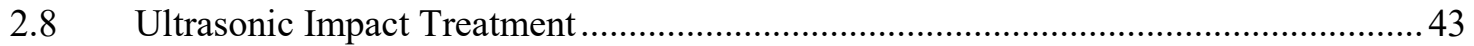

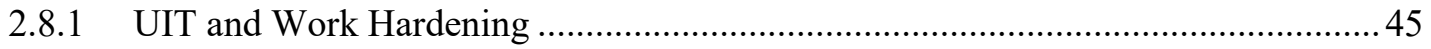

Chapter 3: DMLS Ti-6Al-4V Material Characterization ......................................... 48

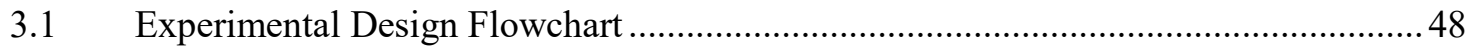

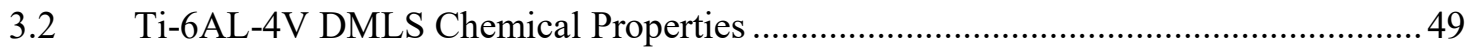

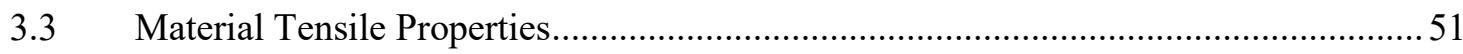

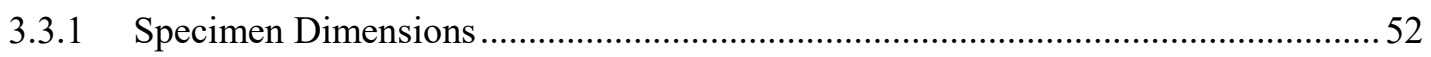

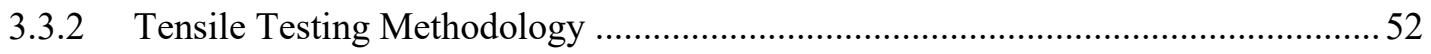

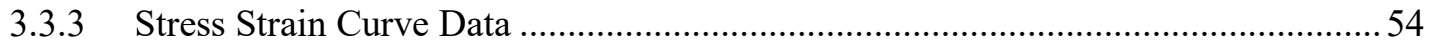

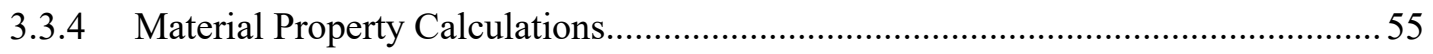

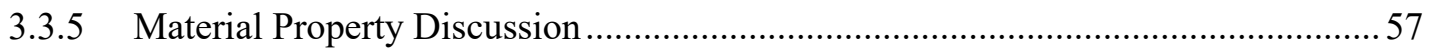

3.4 Untreated and UIT Treated Fatigue Behavior of DMLS Ti-6AL-4V .........................58

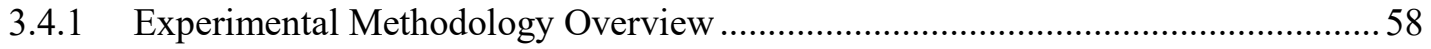

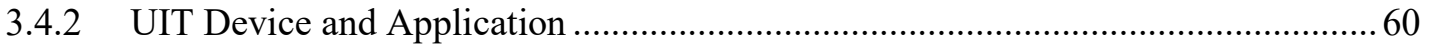

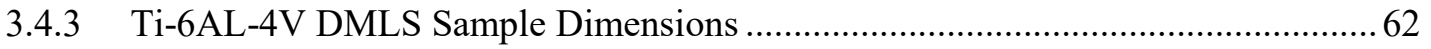

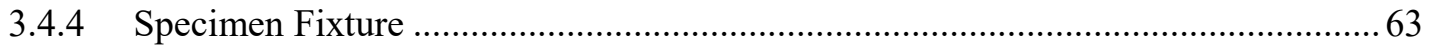

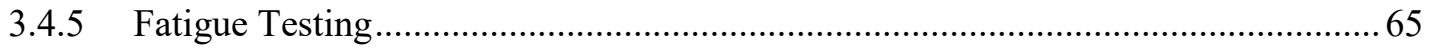

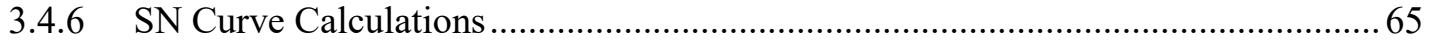

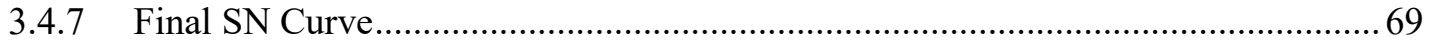

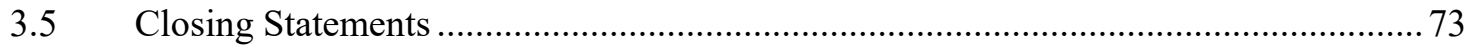


Chapter 4: Asymptotic Homogenization .............................................................................. 74

4.1 Numerical Asymptotic Homogenization ................................................................. 74

4.2 Homogenization Method for Elastic Materials........................................................... 75

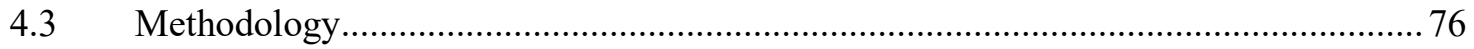

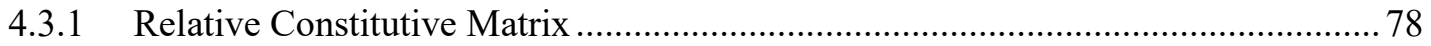

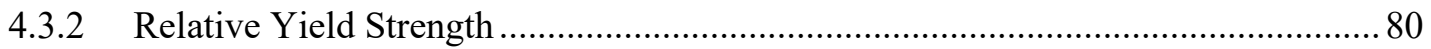

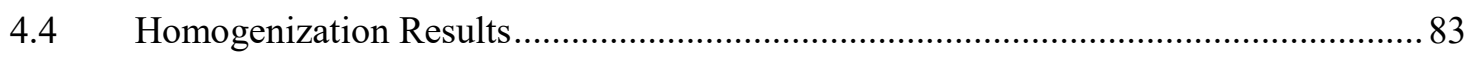

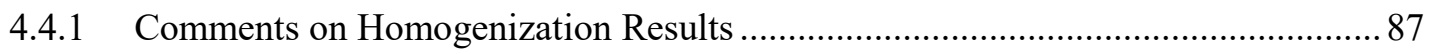

Chapter 5: Structural Optimization.................................................................. 89

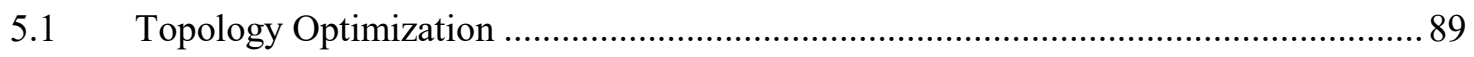

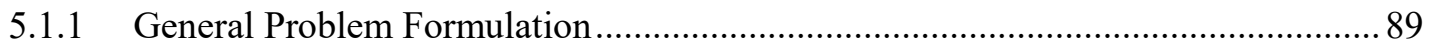

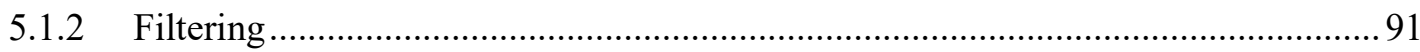

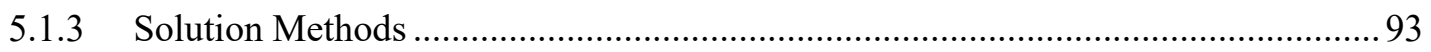

5.1.3.1 Method of Moving Asymptotes.................................................................. 94

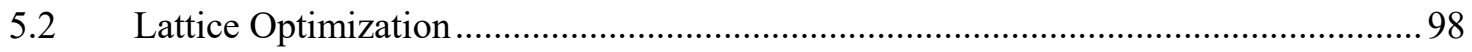

5.2.1 Sensitivity Analysis for Lattice SIMP Optimizations …..................................... 98

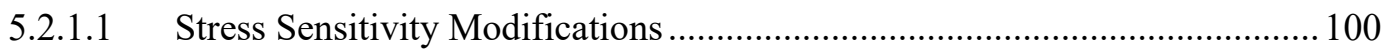

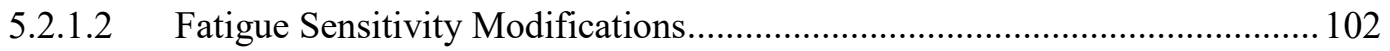

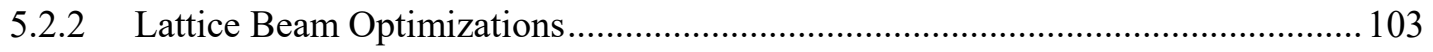

5.2.2.1 Problem Formulation for Lattice Beam Optimization................................. 104

5.2.2.2 Lattice Beam Sensitivity Analysis........................................................... 105

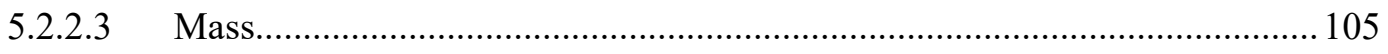

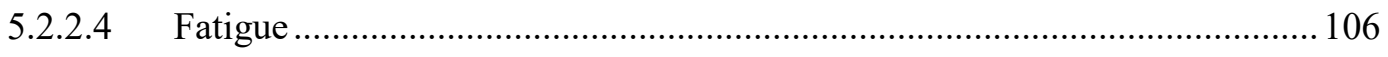

Chapter 6: Automated CAD from Topology Optimizations ........................................ 109

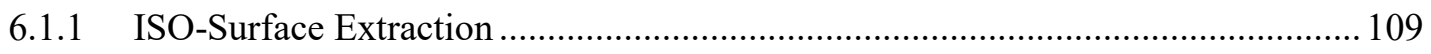


6.1.2 MATLAB Methodology for Lattice Embedded ISO-Surfaces .............................. 112

6.1.2.1 Lattice Topology Optimization Post-Processing ......................................... 113

6.1.2.2 Voxelization and Surface Reconstruction ................................................... 114

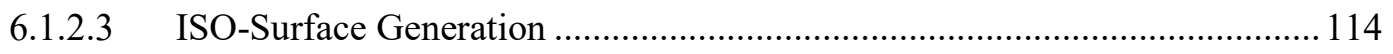

6.1.2.4 Lattice Embedded FEM Creation ........................................................... 116

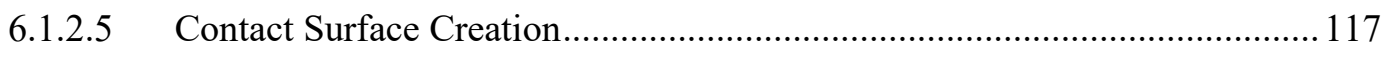

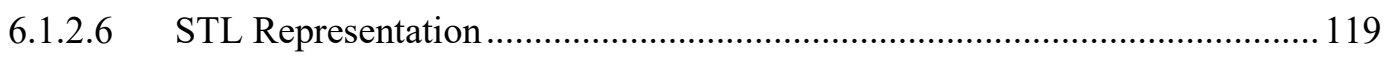

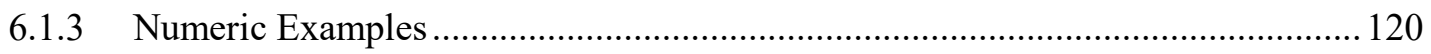

Chapter 7: Case Study and Industrial Multi-Scale Design ................................... 122

7.1 Multiple-Objective Gradient Based Optimization............................................... 122

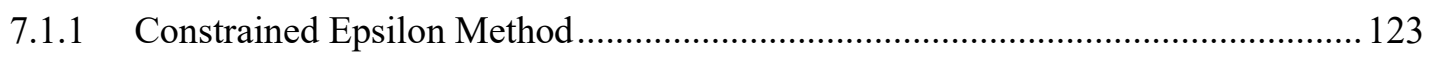

7.2 Multi-Objective Optimization Problem Definition and Sensitivities .......................... 124

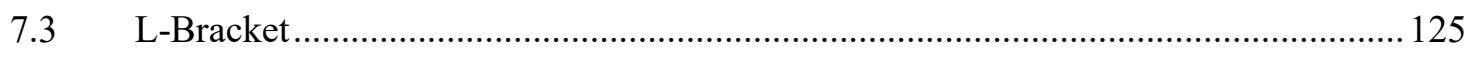

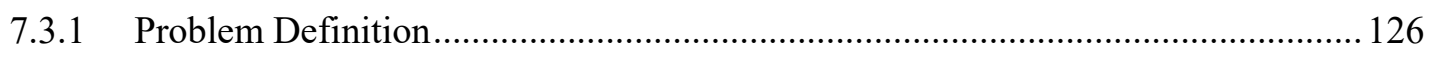

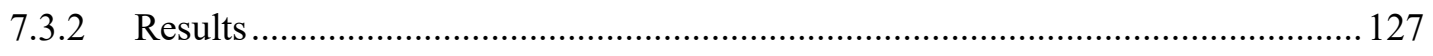

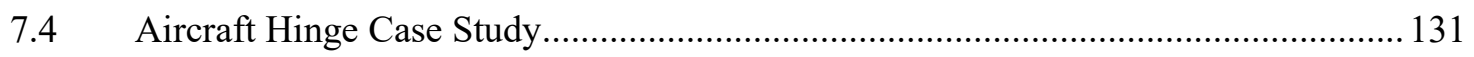

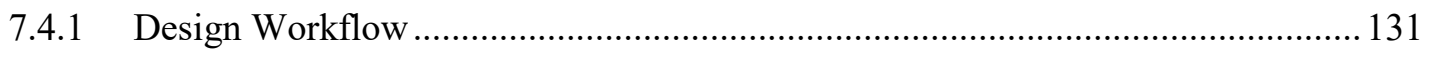

7.4.2 Surrogate Modeling and Global Optimization ................................................... 134

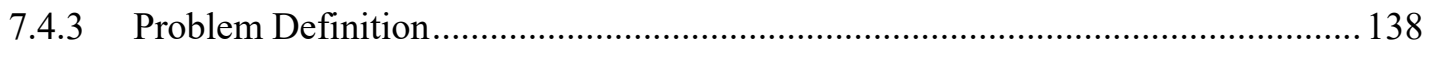

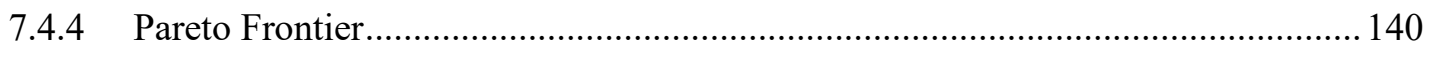

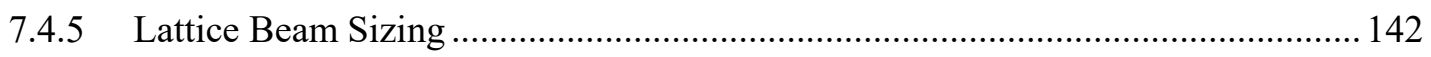

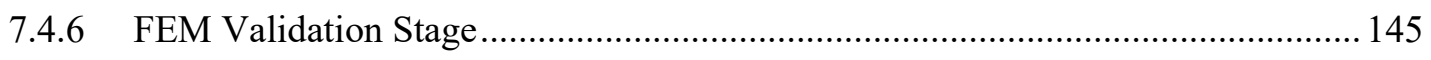

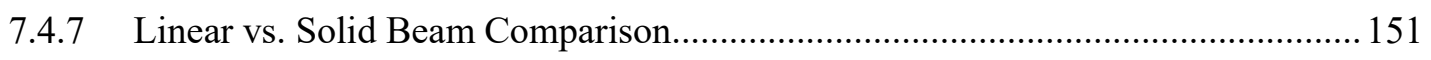

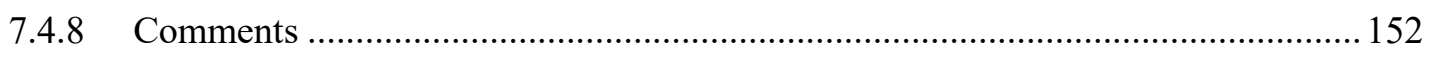

Chapter 8: Conclusion ......................................................................................................... 154 


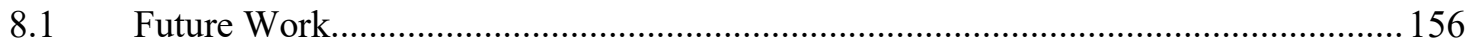

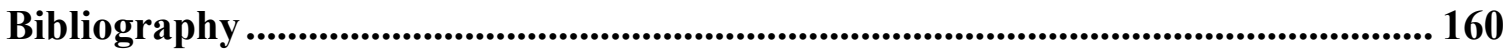

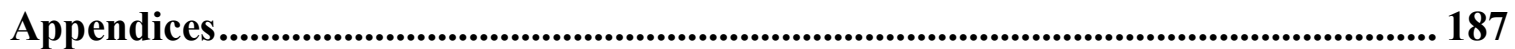

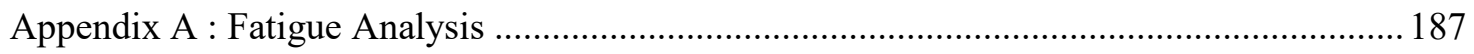

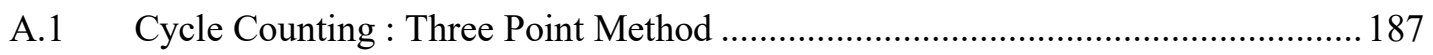

A.2 Fatigue Simulation Using Finite Element Method............................................ 187

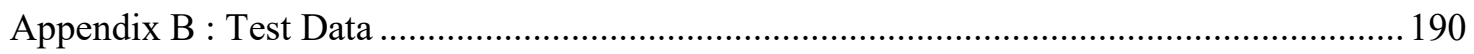

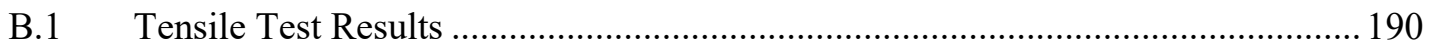

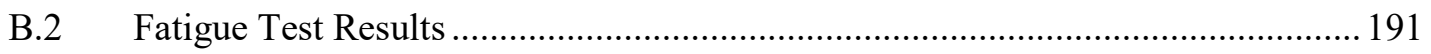

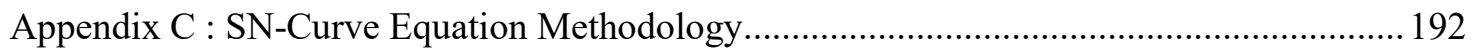

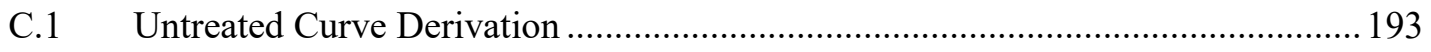

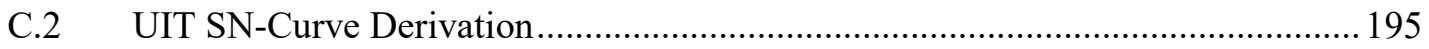

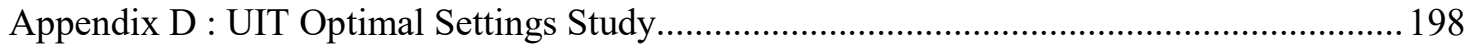

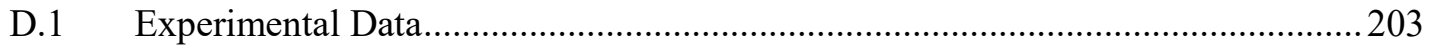

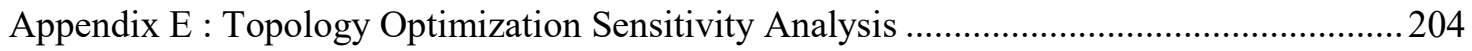

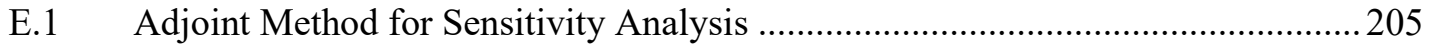

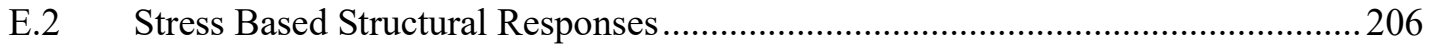

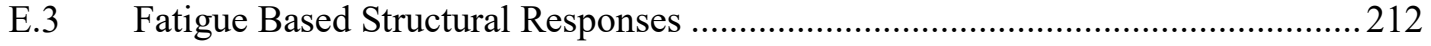

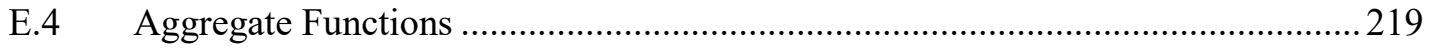

E.5 Local Stiffness and Constitutive Matrix.............................................................22

E.6 Finite Difference vs. Analytical Sensitivity Comparisons ...................................228

Appendix F : Numerical Homogenization Data and Interpolation Methods ........................... 232

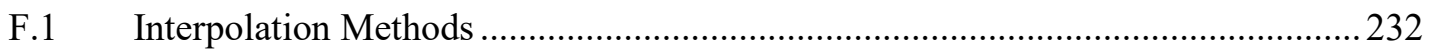

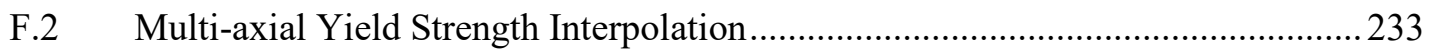

F.3 Effective Mechanical Properties ……........................................................... 235 


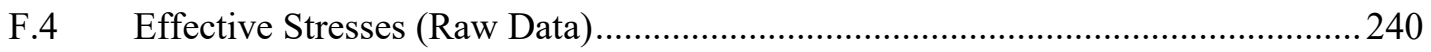

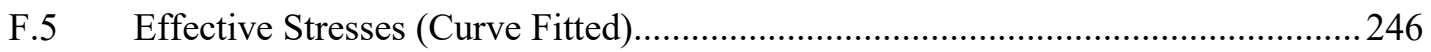

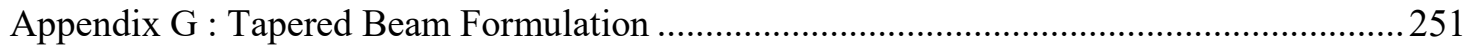

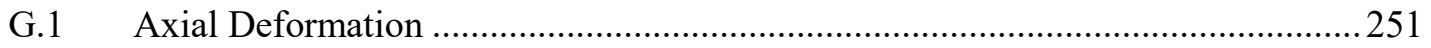

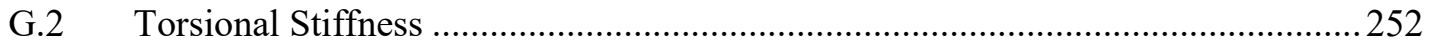

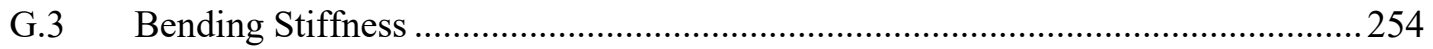

G.4 3D Tapered Frame Beam Element …….........................................................258

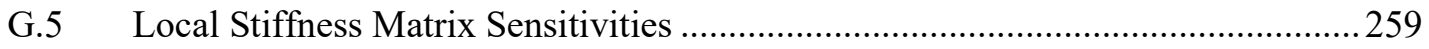

Appendix H : Trimmed Lattice and Conformal Wireframe Nets ..........................................2261

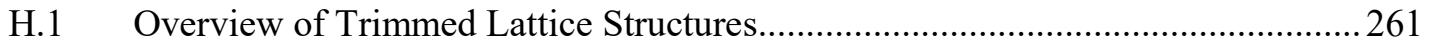

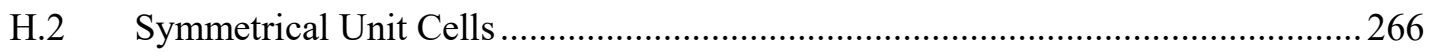

H.3 Algorithm for an Interior Trimmed Lattice .......................................................... 268

H.4 Algorithm for a Conformal Lattice Net for a Trimmed Symmetric Lattice............272

H.5 Additional Connections for the Conformal Lattice Net ........................................228

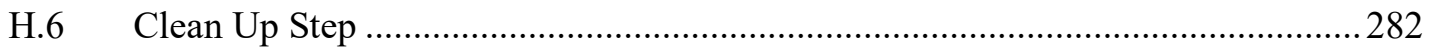

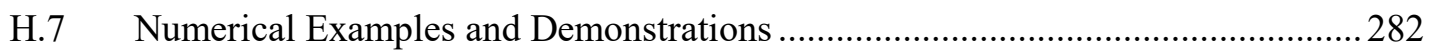

H.8 Advantages and Disadvantages of the Current Lattice Net Algorithm ..................285

H.9 Lattice Net Case Study Example for Engineering Applications ...........................2.286

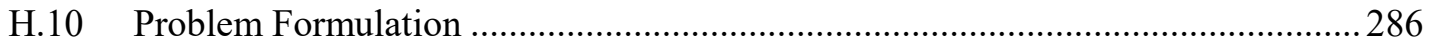

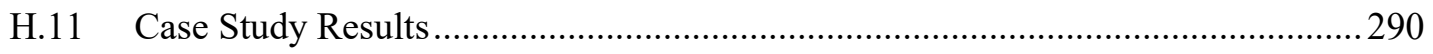

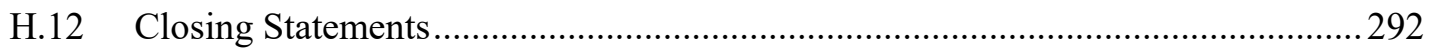

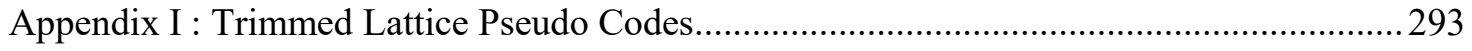

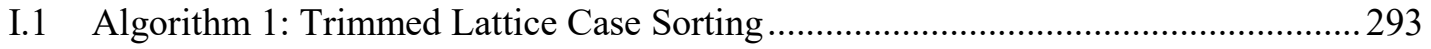

I.2 Algorithm 2: Trimmed Lattice Sub Algorithm..................................................... 294

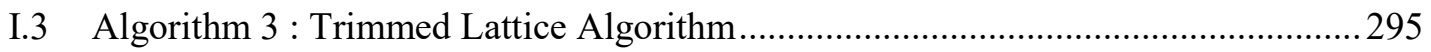




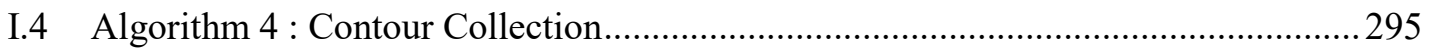

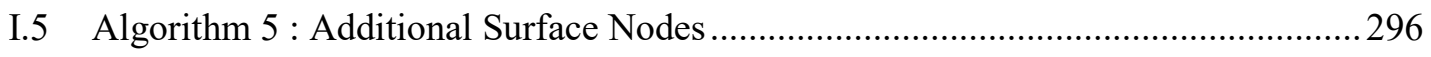

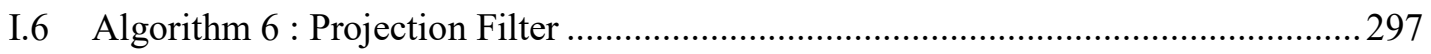

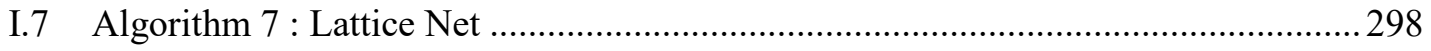

I.8 Algorithm 8 : Additional Connections Algorithm................................................298

I.9 Algorithm 9: in_polyhedron (Ray-Search Algorithm for interior and Exterior Points 299

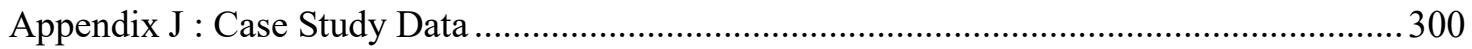

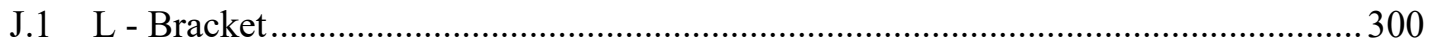

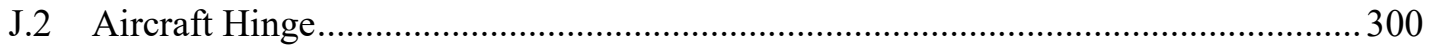




\section{List of Tables}

Table 1 : Chemical Composition of Ti-6Al-4V Grade 23 Power, AP\&C [137] ............. 50

Table 2: Company Rating for Material Properties [137] ............................................ 51

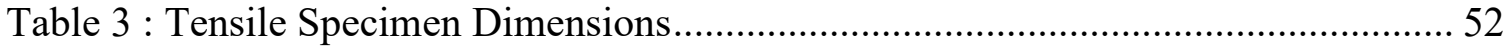

Table 4 : Material Properties Summary .............................................................................. 55

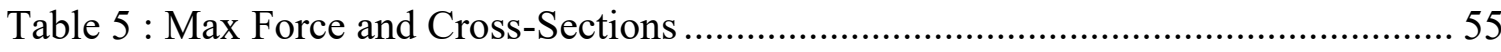

Table 6 : Young's Modulus Results from Raw MTS Strain Data.................................. 57

Table 7 : Yield Strength and Yield Elongation.......................................................... 57

Table 8: S-N Curve Logarithmic Regression Equations (99.99\% Survivability)............ 70

Table 9 : Equivalent Basquin Curve Equations (99.99\% Survivability) ......................... 70

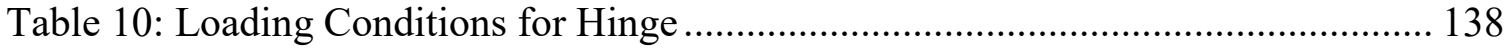

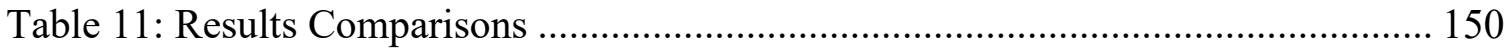

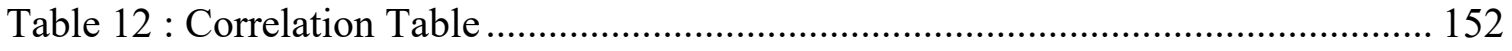

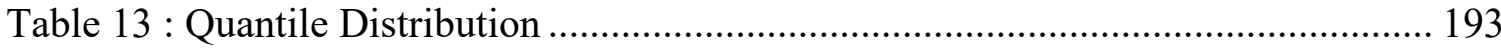

Table 14 : Tabular Data for Untreated SN-Curve Derivation A................................... 193

Table 15 : Tabular Data for Untreated SN-Curve Derivation B ................................. 194

Table 16 : Model Parameter Comparisons for Untreated SN Curve ............................. 195

Table 17 : Tabular Data for UIT SN-Curve Derivation A .......................................... 195

Table 18 : Tabular Data for UIT SN-Curve Derivation B ......................................... 196

Table 19 : Model Parameter Comparisons for UIT SN Curve .................................... 197

Table 20 : Average fatigue life at different UIT parameters .................................... 199

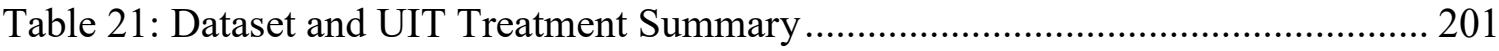


Table 22 : Lattice Net settings for Selected Topologies ......................................... 289

Table 23 : Lattice Net Case Study Results.................................................................. 290 


\section{List of Figures}

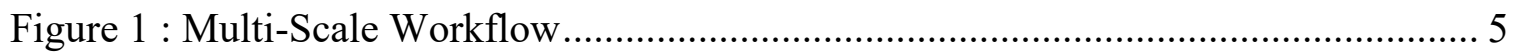

Figure 2 : Illustration of Homogenization - Microscale (Y) to Macroscale (X) [75] ....... 23

Figure 3 : SIMP Compliance Minimization Example with Mass Constraint (A) Before

(B) After SIMP (C) Equivalent SIMP with Cellular Material........................................ 25

Figure 4 : Example of Lattice Material [83] …………………………................... 27

Figure 5 : Appearance of the fatigue fracture surface [105] ............................................ 33

Figure 6 : (A) UIT Device (B) UIT Diagram [123] ....................................................... 44

Figure 7 : Block Diagram for Material Characterization of DMLS Ti-6AL-4V .............. 49

Figure 8 : Ti-6Al-4V Powder Size Distribution [137].................................................. 50

Figure 9: Tensile Specimen Dimension Standards [138] ............................................ 52

Figure 10 : Engineering Stress (MPa) - Strain (\%) Curve DMLS Ti-6AL-4V ................ 54

Figure 11 : Machining Fatigue Specimens (A) Pristine Sample Side Bottom (B) Pristine

Sample Side Top (C) Machined Sample .......................................................................... 59

Figure 12: Impact Needle Position in UIT Device ....................................................... 61

Figure 13: (a) Side View (b) Front View of UIT device (c) Schematic of treatment path - starting point, $\$ inner local spirals, $\$ outer global spiral, — end point (d) Pristine

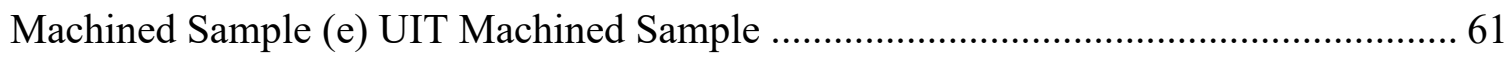

Figure 14 : (a) Schematic of flat sheet fatigue specimen with rectangular cross-section (b) Orientation of the specimens during DMLS manufacturing process................................ 63

Figure 15: (a) CAD of UIT Fixture Set-Up (b) CNC machine and Fixture Assembly (c)

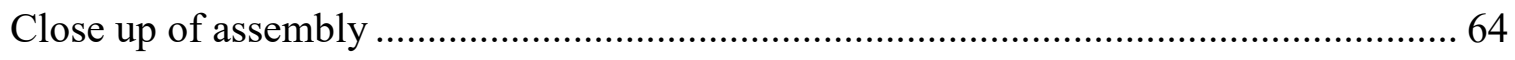


Figure 16: Logarithmic Regression SN Curve $(\mathrm{R}=0)$ for Treated and Untreated DMLS Ti$6 \mathrm{AL}-4 \mathrm{~V}$ A 71

Figure 17 : Logarithmic Regression SN Curve $(\mathrm{R}=0)$ for Treated and Untreated DMLS Ti-6AL-4V B 72

Figure 18 : Block Diagram for Asymptotic Homogenization Workflow ....................... 75

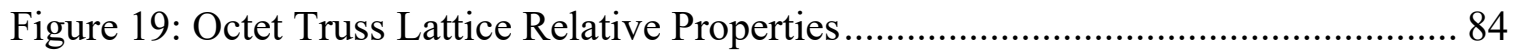

Figure 20: BCC Lattice Relative Properties ....................................................... 85

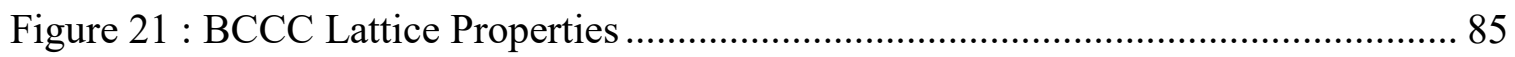

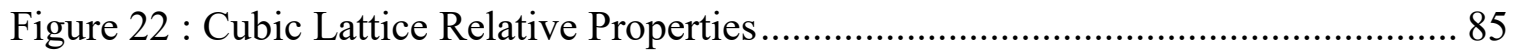

Figure 23 : Diamond Lattice Relative Properties .................................................. 86

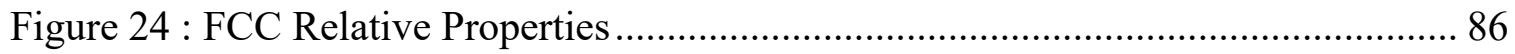

Figure 25 : Truncated Octahedron (Octa) Lattice Relative Properties ........................... 86

Figure 26 : Tesseract Lattice Relative Properties ...................................................... 87

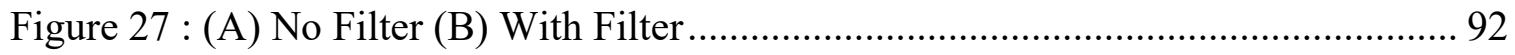

Figure 28 : Example of ISO-Surface Extraction Problems with OSSMooth (A) Iso-

Surface (B) Cross-Section of Meshed Areas (circled area shows meshing error) (C)

Zoomed Section displaying failed mesh is empty with no tetrahedrons ...................... 111

Figure 29 : Block Diagram Solid and Lattice Domain ISO-Surface Generation ........... 115

Figure 30 : Block Diagram for Embed Lattice Beams ......................................... 116

Figure 31 : Block Diagram for Contact Surface Creation with Lattice and Solid Skin.. 118

Figure 32: Lattice STL File Creation...................................................................... 119

Figure 33 : Lattice Embedded ISO-Surface Results (a) Lattice-Net (b) Lattice-Net Cross-

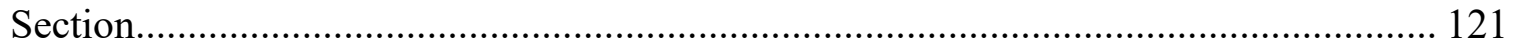




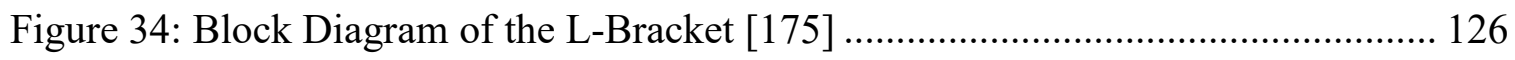

Figure 35 : Fatigue History for L-bracket (Force Multiplier).................................. 127

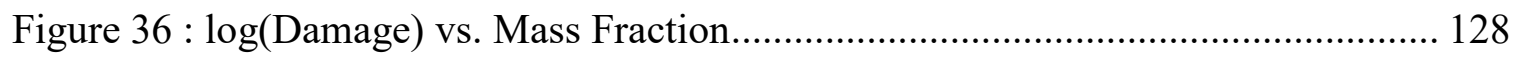

Figure 37 : L-Bracket Optimized Topologies at 43\% Mass Fraction (Dot $=$ Solid, ' $X$ ' =

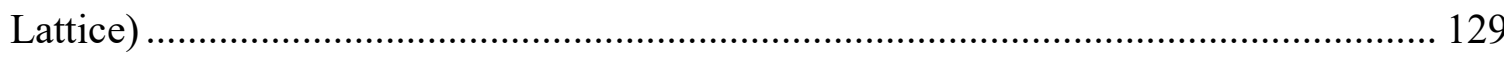

Figure 38 : Block Diagram for Multiple Objective Topology Optimizations with Lattice

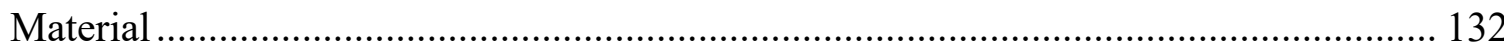

Figure 39 : Block Diagram for Global Optimization and Post-Processing Results........ 133

Figure 40 : Global vs. Local Design Variable Distinction.......................................... 136

Figure 41 : Aircraft Hinge (a) Original Design, (b) Expanded Design Space (Grey=

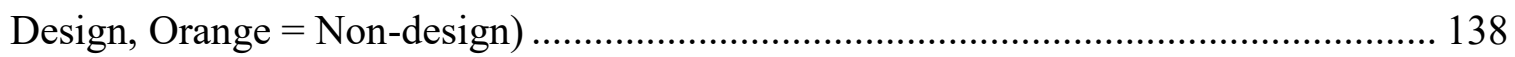

Figure 42 : Loading Conditions of (a) Newtons shown in Hypermesh (b) Schematic... 139

Figure 43 : Aircraft hinge Fatigue History (Force Multiplier) ................................. 139

Figure 44 : Pareto with Frontier for Aircraft Hinge............................................... 141

Figure 45 : Pareto Frontier with no Filter for Aircraft hinge ................................... 141

Figure 46 : Axis Alignment with Relation to Model for Lattice Rotation along X-Axis 144

Figure $47:$ Final design $($ Lattice $=$ Green $),($ Red $=$ Solid $)$, ISO $=0.95$, Rotation $=32^{\circ} .144$

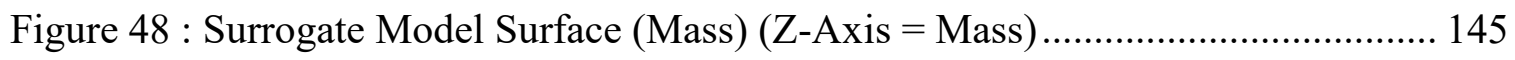

Figure 49 : STL Representation of the Aircraft Hinge ......................................... 147

Figure 50 : Fatigue life Contour of 3D Model ...................................................... 148

Figure 51 : Alternating Stress Von-Mises Contour ............................................. 149

Figure 52 : Alternating Stress for Beam Model (Tapered Beams) .............................. 149

Figure 53 : Alternating Stress for Beam Model (3D Elements) ................................ 149

Xvii 
Figure 54 : Fatigue Live Contour for Beam Model

Figure 55 : Maximum Von-Mises Comparison

Figure 56 : Finite Element Simulation for Multi-Axial Fatigue Prediction of Proportional

Loads 188

Figure 57 : Fatigue life at $400 \mathrm{MPa}$ for different UIT Parameters 199

Figure 58: Improvement Ratio (\%) vs. Static force (N) times Scanning Speed (mm/min) 201

Figure 59 : test Mesh for Sensitivity Analysis. 228

Figure 60 : Lattice Generation Methods a) Domain b) Swept C) Meshed d) Trimmed [194] 263

Figure 61: a) Trimmed Lattice Structure b) Lattice Structure with net-skin c) Net-Skin [194]. 264

Figure 62 : Bravais Cubic Lattices (a) Cubic/Primitive (b) Body-Centered Cubic (BCC)

(c) Face Centered Cubic (FCC) 266

Figure 63 : Miller Indices (n1, n2, n3) Planes for Cubic System (a) (100) (b) (010) (c) $(001)$ 267

Figure 64 : Interior Trimmed Lattice Workflow. 268

Figure 65 : Line Segment Classification within a Hollow Sphere (Red) Exterior Node (Blue) Interior Node (Green) Surface Node (Dotted Line) Interior Segment (Solid Line)

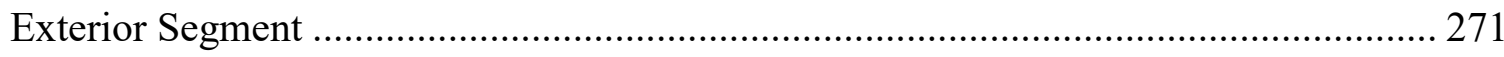

Figure 66 : Input Information for Lattice Net Generation ............................................ 273

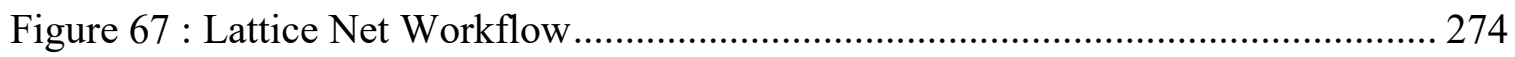

Figure 68: Axis Re-Alignment and Planar Elevations Calculations.............................. 275 
Figure 69: Surface Intersection (a) Orientation (b) Sphere Sliced by Triangle creates Contour at $Z \boldsymbol{i n}=\boldsymbol{d i}$ 276

Figure 70 : (a) Filtering of Lattice Net Segments for each Crystallographic Plane of a BCC unit cell (b) Input Mesh and (c) Final Construction 278

Figure 71: (a) Lattice net without preserved features and (b) Lattice net with preserved features 281

Figure 72 : FCC Net (a) No Filter (b) With filter applied (c,d) Cube with Lattice rotated $30^{\circ}$ with unit cell sizes 12 and 9 (e,f) Cylinder with unit cell sizes 12 and 9 283

Figure 73 : (a) Interior Diamond Lattice (b) Front view of Diamond Lattice (c) Diamond Lattice net using $\{110\}$ (d) Bunny interior of Octet Truss (e) Octet Truss Lattice net with $\{111\}$ (f) Octet Truss Lattice Net with no filter and planes (111), (111), (111) ....... 284 Figure 74 : MBB Initial Problem, Yellow = Design Space, Brown = Non-design Space 288

Figure 75: Optimized Results $($ Yellow $=$ Trimmed Interior Lattice, Green $=$ Lattice Net, Purple $=$ Single Unit Cell) (a) Cubic without net (b) Cubic with net (c) BCC without net (d) BCC with net (e) FCC without net (f) FCC with net (g) Octet truss without net (h) Octet truss with net 291 


\title{
Nomenclature/Notation
}

\author{
English Alphabet \\ Variable Description \\ A Cross sectional area of a tapered beam or regular beam element \\ a Amplitude stress for fatigue loading \\ B Strain Displacement Matrix \\ $b \quad$ Basquin Slope value \\ C Compliance \\ c Stress or Load scalar for Stress-Life Fatigue Analysis \\ D Constitutive Material Matrix \\ d Density \\ E Stiffness of element or young's modulus \\ $e \quad$ Index or subscript for element \\ F $\quad$ Force vector \\ $f \quad$ Arbitrary Function \\ G Slope of Goodman-Haigh Diagram \\ $g \quad$ Node or grid identifier in a structural response \\ $H \quad$ Accumulated damage from Miner's Rule \\ $h \quad$ Arbitrary Equality Constraint Function \\ I Iteration index for an optimization \\ $i \quad$ Summation Index number \\ $j \quad$ Index Number \\ K Global Stiffness Matrix \\ $k \quad$ Number of cycles in a load time history \\ $L \quad$ Length of a beam element \\ $l \quad$ MMA Lower Bound (Moving Asymptote) \\ $M \quad$ Mass \\ $m \quad$ Mean stress for fatigue loading \\ $N \quad$ Number of individual structural responses in an aggregate response
}




\begin{tabular}{ll}
$n$ & Number of cycles per load \\
$o$ & Origin (or Point Origin) \\
$P$ & Norm dimension factor \\
$p$ & SIMP penalization factor \\
$Q$ & Global vector which the constraints must satisfy (Multi-Point Constraints) \\
$q$ & Arbitrary exponent for stress structural response in SIMP optimizations \\
$R$ & Stress Ratio (fatigue) \\
$r$ & Radii for beam elements \\
$S$ & Factor of safety \\
$T$ & Temperature field \\
$t$ & Traction Vector \\
$U$ & Displacement Vector \\
$u$ & Utopia Point (Multi-objective optimization) \\
$V$ & Volume \\
$v$ & Element volume \\
$W$ & Function Weights for Multi-Objective Optimization \\
$w$ & Weighted Convolutions weighed factor for topology filtering \\
$z$ & Design Variable for Optimizations \\
$z$ & Local coordinate system of periodic medium \\
& Elevation or sequence of elevations \\
\hline &
\end{tabular}

\section{Greek Alphabet}

\section{Variable Description}

$\alpha \quad$ Adaptive Scaling factor for aggregate functions

$B \quad$ Mean stress multiplier

$\beta \quad$ Material parameter of mean stress influence (Sines Method)

$\Gamma \quad$ Function of relative modulus as a function of relative density

$\gamma \quad$ Lagrange Multiplier

$\Delta \quad$ Difference operator 


\begin{tabular}{|c|c|}
\hline$\delta$ & Kronecker Delta \\
\hline$\varepsilon$ & Strain \\
\hline$\zeta$ & Sign of the hydrostatic stress of an element \\
\hline$\eta$ & Damping ratio \\
\hline$\Theta$ & Numerical Tolerance \\
\hline$\vartheta$ & Relative Stress (or SN-Curve Intercept) function \\
\hline$\kappa$ & Mean Stress Correction Factor \\
\hline$\Lambda$ & Function of relative Poisson Ratio as a function of relative density \\
\hline$\lambda$ & Adjoint vector for the adjoint sensitivity analysis \\
\hline$\mu$ & The second Lame Parameter for the shear stress \\
\hline$v$ & Poisson Ration \\
\hline$\Xi$ & Thermal Conductivity \\
\hline$\xi$ & Move Limit \\
\hline$\Pi$ & Sum product \\
\hline$\pi$ & $\operatorname{Pi}(3.14 \ldots)$ \\
\hline$\varpi$ & The number of cycles to failure \\
\hline$\rho$ & Relative Density \\
\hline$\varrho$ & MMA variable \\
\hline$\Sigma$ & Summation \\
\hline$\sigma$ & Stress Measurement \\
\hline$\varsigma$ & Relative Damage Function \\
\hline$\tau$ & Mean stress Correction Function (Goodman, Soderberg) \\
\hline$\Upsilon$ & The first Lame Parameter \\
\hline$\Phi$ & Payoff Matrix for Normal Boundary Intersection \\
\hline$\varphi$ & Infeasibility Criterion \\
\hline$\phi$ & Convergence Criterion \\
\hline$\chi$ & Global coordinate system for a periodic medium \\
\hline , & Arbitrary Structural Response or function \\
\hline & Design Domain for topology optimization \\
\hline
\end{tabular}




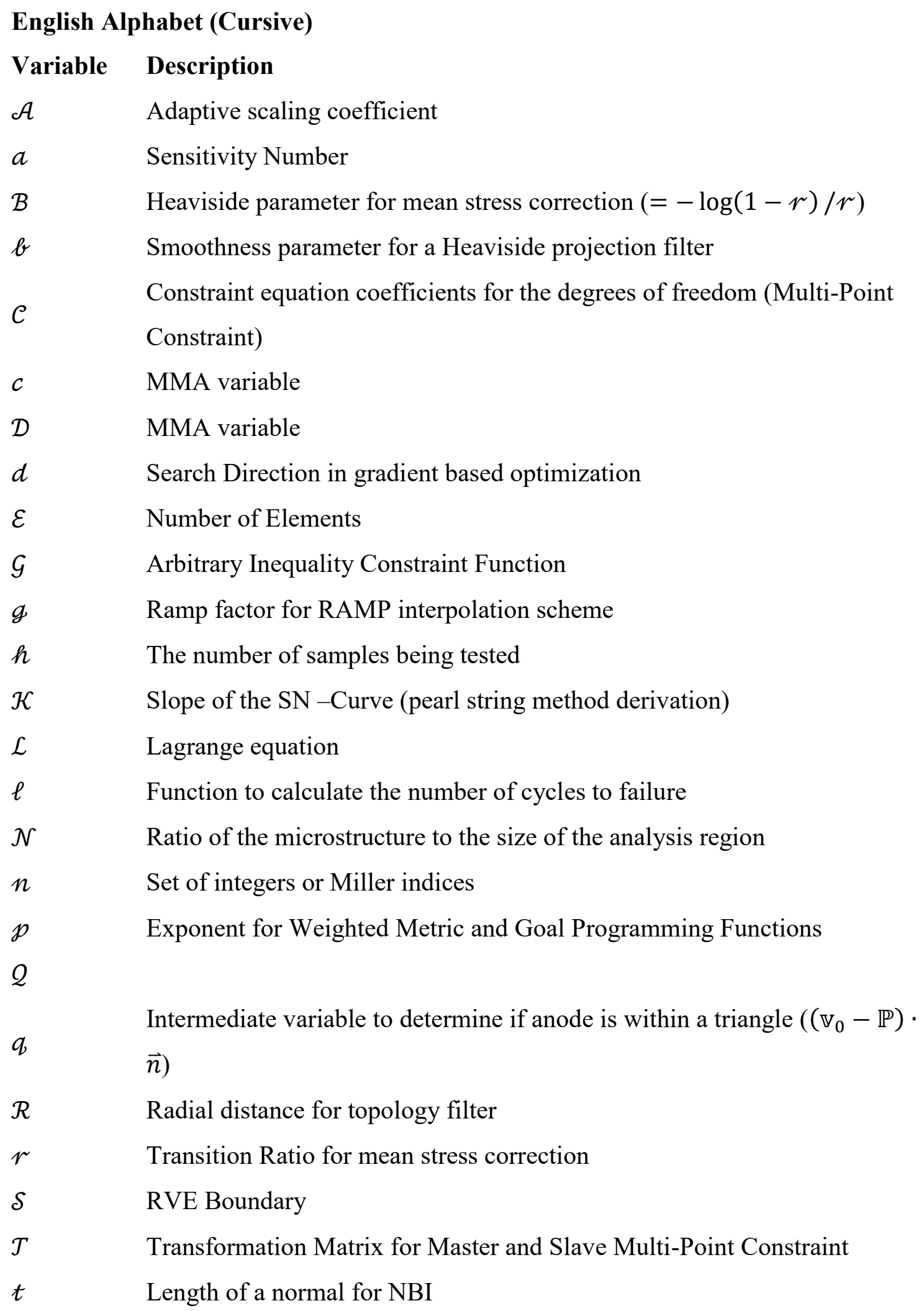




$\begin{array}{ll}u & \text { MMA Upper Bound (Moving Asymptote) } \\ u & \text { Non-Homogeneous Constraint Vector } \\ v, v_{\text {virtual }} & \text { Virtual Displacement field } \\ w & \text { Bary-centric Coordinates components } \\ y & \text { MMA variable } \\ y & \text { MMA variable } \\ z & \text { MMA variable }\end{array}$

\section{English Alphabet (Frakturs)}

\section{Variable Description}

$\mathfrak{A} \quad$ Linear weight at point $\mathrm{x}$ for Convexity definition

a Diagonal matrix with penalty terms

$\mathfrak{B} \quad$ Linear weight at point y for Convexity definition

c SN-Curve Constant for logarithmic regression formula (HCF)

c Center of Rotation

D Ray Direction

$\mathfrak{K} \quad$ Function to determine if the node lies on a surface

m Bravais Dimension Index

$\mathfrak{R} \quad$ Position of Bravais lattice points

$\mathfrak{s} \quad$ A small value $\approx 1 e^{-16}$ to avoid numerical instabilities

$\mathrm{t}$ Parametric Distance along a ray or line

\section{English Alphabet (Double Struck)}

\section{Variable Description}
al The periodicity of the unit cell
$\mathbb{B}$
Ratio between the objective functions rate of change and the change in
b Plane unit normal




$\begin{array}{ll}\mathbb{g} & \text { Projection of a Line } \\ \mathbb{k} & \text { Number of crystallographic planes } \\ \mathbb{I} & \text { Weighted Sum or Metric Multi-Objective Function } \\ \mathbb{M} & \text { Local Structure Tensor } \\ \mathbb{m} & \text { Number of Equality constraints } \\ \mathbb{m} & \text { Number of design variables } \\ \mathbb{P} & \text { A Point } \\ \mathbb{P} & \text { MMA variable } \\ \mathbb{Q} & \text { MMA sub-problem } \\ \mathbb{q} & \text { MMA variable } \\ \mathbb{R} & \text { Numbers in a given dimension space } \\ \mathbb{r} & \text { Number of inequality constraints } \\ \mathbb{S} & \text { Set of numbers containing the design variables } \\ \mathbb{T} & \text { Coordinate Transformation matrix (Beam Elements) } \\ \mathbb{U} & \text { Unit Normal Direction of the z-axis } \\ \mathbb{U} & \text { MMA number of differentiable functions } \\ \mathbb{V} & \text { Crystallographic plane normal } \\ \mathbb{V} & \text { Vertices of a triangle }\end{array}$

\section{Cyrillic Alphabet}

\section{Variable Description}

И Mass convolution matrix for Beam Sizing Optimizations

K Volume convolution matrix for Beam Sizing Optimizations

6 MMA variable (lower bound asymptote)

ж MMA variable (upper bound asymptote)

Д MMA variable

Ч MMA variable

Њ MMA variable 


\section{Chapter 1: Introduction and Motivations}

\subsection{Motivation}

Today, Computer Aided Design (CAD) is the norm and the line between structural optimization and mechanical design is evermore blurred. CAD has improved the productivity of engineers and designers to plan and optimize structures for high efficiency and performance [1]. Topology optimization techniques are commonly employed to determine optimal load paths using a limited amount of material [2]. Recent advances in modeling techniques have made it possible to quickly convert topology optimization results into draft-able designs [3]. Topology optimization results are often too complicated to be manufactured by conventional means. However, 3D-printing or additive manufacturing has made these complex designs realizable. Savings in both material costs and increased design productivity are possible by adopting these new design paradigms. Material savings by additive manufacturing can be further increased by including functionally graded lattice materials. However, applying additive manufacturing into fields such as aerospace or automotive industries are not without their issues.

Safety is a major concern in mechanical design. It is thus inherent to have mechanical components with long and reliable life cycles. These problems are exasperated when additive manufacturing is used to create new engineering components. Fatigue failure is a major concern in many industries ranging from aerospace, biomedical, automotive and transportation. Another aspect in mechanical engineering design which is highly sought 
after is the development of lighter weight components. Unfortunately, as the weight of mechanical parts decreases, the risk of failure due to fatigue tends to increase. This problem has led to the implementation of work hardening processes to help improve the fatigue properties by applying a layer of compressive residual stress at the surface. Many such techniques are currently available which include ultrasonic needle peening (UNP) or ultrasonic impact treatment (UIT) [4], shot peening [5], low plasticity ball burnishing [6] and deep rolling [7]. Each treatment has its own effectiveness in terms of improving the fatigue life of materials. Many metal alloys are available for AM such as aluminum and titanium and selecting the correct material for strength, cost and fatigue resistance is essential.

Titanium alloys are widely used in aerospace, in part due to their high strength to weight ratio, low density, and excellent corrosion resistance. In particular, Ti-6Al-4V's popularity in aerospace is also a result of its high strength at extreme temperatures $[8,9]$. Not surprisingly, several studies have been published examining the feasibility of fabricating Ti-based functionally graded materials (FGM) using AM methods [10]. The advantageous qualities of titanium make it an excellent candidate for high life cycle additively manufactured components

Ultrasonic impact treatment has been shown to substantially improve the fatigue life of hard metals; especially for titanium alloys [11]. The main mechanism for fatigue improvement is caused by applying a compressive residual stress on the surface of a metal. The presence of compressive residual stresses will cause the propagation and 
initiation of fatigue cracks to slow down [12]. According to fatigue theory, if a high enough compressive force is applied to the surface, fatigue cracks will cease to propagate [13]. Compressive forces are desired as they suppress the effects of tensile stresses on the surface which would accelerate the nucleation of fatigue cracks. UIT is capable of inducing large compressive residual stresses, making it an ideal surface treatment for improving the fatigue life of AM metals [11].

Combining the beneficial effects of UIT with topology optimization can create highly efficient structures with potentially very long lifetimes. Conflicts arise when designing lightweight engineering components with long life cycles because both objectives are in conflict with each other. As the fatigue life increases, the available mass must also increase and a trade-off must be chosen between the two performance metrics. This phenomenon is known as multi-objective optimization and commonly occurs in many engineering problems to which a solution will lie on a Pareto frontier [14]. Multiobjective optimization is an iterative procedure to determine all solutions along the Pareto frontier.

The motivation for writing this thesis is to give an outline or workflow of how an engineer would develop multiple designs through multi-objective optimization in the field of additive manufacturing. Material characterization of AM Ti-6Al-4V fatigue properties of both virgin and UIT treated material is discussed and is derived from scratch. Strategies of automated design are also presented to increase productivity time and to seamlessly integrate functionally graded materials into AM models. To maximize 
automation, the developed workflow is designed so that little intervention is needed between the engineer and the optimization process. The emphasis on automation is made so that the method can be applied in an industry setting to save manual labor costs.

\subsection{Proposed Multi-Scale and Multi-Objective Workflow}

There are six objectives in this thesis and they are:

1- To experimentally characterize the fatigue behavior of additively manufactured Ti-6AL-4V alloy with and without ultrasonic impact treatment.

2- $\quad$ To calculate relative mechanical properties for three dimensional lattice truss structures for FGM optimizations.

3- To develop a multi-scale workflow to optimize metal components with the numerically derived homogenized properties of the AM material. The multi-scale workflow uses homogenization theory to bridge the scales together between the micro scale properties and the macro scale effects. Dual objectives of minimum mass and maximum fatigue life are explored and fatigue performance to mass ratios is calculated. The solution closest to the utopia point is selected for further analysis in the proceeding steps.

4- A fourth goal of developing a global optimization scheme to further improve the fatigue performance is established and represents the functionally graded lattices are linear tapered Euler Bernoulli beams. Global effects such as the rotation of the lattice and lattice volume fraction are considered as "global design variables". 
5- The fifth goal is to compare and validate the optimum finite element model with linear beams to a finite element model meshed with three dimensional elements. The accuracy of the proposed optimization workflow is also discussed.

6- The final goal is to generate an STL file for 3D printing. However, physical printing for experimental validation is outside the scope of this thesis.

A global diagram outlining the major steps for the multi-scale and multi-objective optimization workflow is presented below in Figure 1.

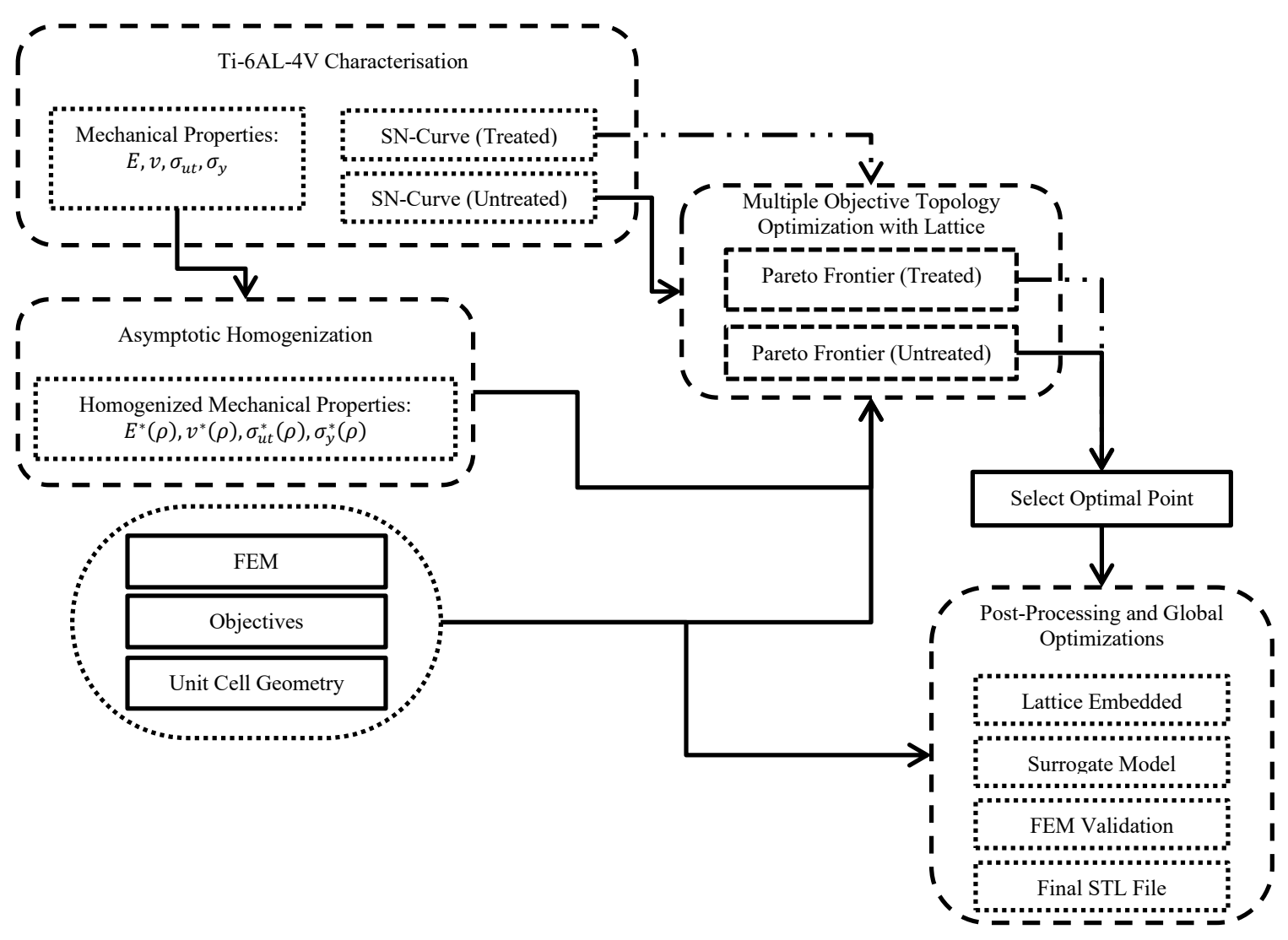

Figure 1 : Multi-Scale Workflow 


\subsection{Limitations and Scope}

Integration of $\mathrm{AM}$ components into aerospace structures requires consideration of its environment and monitoring for defects. Residual stress relaxation is seen to occur in shot peened components where the effectiveness of the surface treatment diminishes over time due to a build-up of tensile stresses [15]. This effect could also occur on the AM component after UIT, hence, additional maintenance such as non-destructive crack detection and additional UIT rounds may be needed. Aerospace components are also exposed to sudden temperature changes and other environmental corrosion. Titanium is highly resistance to corrosion and oxidation when compared to other widely used metal alloys [16]. Aerospace structures are also subjected to vibrations [17]. Effects other than cyclic loadings on the life of the AM component are not implemented in the multi-scale design workflow and are outside the scope of this thesis.

The 3D printing process contains many steps and checks to ensure both printability and defect-free components. Large thermal gradients are expected during the DMLS printing process and may create high internal residual stresses ensuing in cracks, warping or fracture [18]. Titanium alloys are known to have large residual stresses during the printing process which add complexity to the design to avoid pre-emptive material failure [18]. Support structures are required to allow the printing process to create complex structures [19]. The complex support system can be automatically generated using 3D printing software or by manual design from an engineer [20]. Issues arise with metals as sanding and additional machining is required to remove the complex supports. Post- 
process tooling will then add costs and tome to the design and in some cases supports may be too difficult to remove if the parts complexity is too high. To minimize the number of supports, the components are oriented in such a way to minimize the need to build them during printing. The pre-production stage of the 3D part is not included in this thesis and considerations of these steps are not included in the design stage and are outside the scope of this thesis.

The practical application of work hardening complex 3D printed shapes is not explored and is outside the scope of this thesis. Enhanced material properties are applied on the non-lattice material during optimizations. The micro stresses in the homogenized material are used to calculate the sensitivities for the fatigue life.

Finally, the research presented in this thesis is limited to the computational modeling of ISO-surfaces from multi-objective optimizations and the experimental fatigue life extension of such structures. It is also assumed that the lattice struts can be approximated as one-dimensional tapered beam elements. The effects of buckling on the lattice struts are also outside the scope of this manuscript. Metallurgical analysis of the AM material is also omitted and is outside the scope.

\subsection{Thesis Outline}

This thesis consists of 8 chapters which includes theoretical background on fatigue, nonlinear optimization and finite element meshing. The second chapter presents a literature review for important topics outlined in this thesis. Chapter 3 contains experimental work to characterize the mechanical and fatigue properties of the 3D printed titanium metal. 
Chapter 4 has information on asymptotic homogenization and is used to determine the average properties for lattice material in the multi-scale stages of the workflow. Chapter 5 is lattice optimization techniques and their roles in the MDO framework and differences when compared to regular topology optimizations. Chapter 6 describes a novel meshing algorithm for trimmed lattice structures and their use in lattice micro-strut optimizations. Chapter 6 also presents a novel ISO-surface reconstruction algorithm to post-process topology optimization results for both regular structural optimizations and lattice optimizations. Chapter 7 demonstrates the MDO workflow on the optimization of an aircraft hinge both with and without the UIT surface treatment for minimum weight and maximum fatigue life. Finally, Chapter 8 is the conclusion of the thesis and discusses the results of the proposed workflow and contributions to knowledge. 


\section{Chapter 2: Literature Review}

A background in both additive manufacturing and multi-scale modeling is required for the optimization workflow in this thesis. Additive manufacturing is a field which enables the fabrication of highly complex structures such as lattices or cellular material. The properties of the material however are not suitable for longevity due to poor surface finish. The second main topic is multi-scale modeling which predicts the behavior of a system from multiple length scales. Homogenization theory is used to characterize material properties at a micro-scale. The micro scale properties are then sent to the macro scale for a higher level prediction of the ensemble structure. Each of the topics mentioned play large roles in engineering design and analysis.

\subsection{Additive Manufacturing}

The first mention of additive manufacturing (AM) can be traced back to Dr. Hideo Kodama in 1981 as he sought to file a patent for printing 3D models [21]. At this time the printing process would be referred to as "rapid prototyping" or RP [22]. Despite being a patent lawyer, Dr. Kodama did not fulfill the patent requirements on time and his request was denied [22]. However the first real breakthrough in AM wouldn't be until a few years later when Charles Hull developed and commercialized Stereolithography (SLA) [23]. The first models printed where made from a liquid polymer resin in combination with UV lasers. In 1989 fused deposition modeling (FDM) was invented by Scott Crump [24]. FDM is the most common manufacturing technique for AM today among enthusiasts and hobbyists thanks to companies like MakerBot ${ }^{\mathrm{TM}}[21,25]$. The late 1990's 
saw an opportunity to apply AM to medical scaffolds to grow organs however more research was needed [21]. The early 2000's saw big leaps in 3D printing technology; bringing it forward to the masses for both industrial and personal use. Today low-cost 3D printers have further popularized AMs potential and new technological advances continue to grow and improve the field [24].

As 3D printing technology evolved, so did the possibility of printing with multiple different materials. Early SLA was primarily for plastic based printing. Current technology can print objects using many different materials such as metal powder [26], sugar or food [27] and even sand [28, 29]. Metal casting operations have utilized 3D sand printing to develop molds with high accuracy [29]. The added freedom of 3D printed sand molds also allow for more complex geometries to be made with the advantage of tailoring the molds stiffness and permeability [29]. The advent of multi-material 3D printing has also helped to further open up many more opportunities for AM for engineering design. The first open source 3D printer available to the public "Fab@Lab" was multi-material by nature allowing hobbyists to experiment at their own free will and was the first commercial 3D food printer [30]. The very first edible items produced by the founders of "Fab@Home” were 3D printing cookies using dough [30]. Advancements on AM edibles have allowed many other types of food to be used to create impressive sculptures from chocolate, puree, jelly, cheese, mashed potatoes, sauces or powders. However the type of food is limited to the printing technique. Powdered based foods used selective laser sintering [31] to bond together food particles, extrusion methods [32] are 
used for moderately viscous foods and inkjet printing [33] for highly viscous foods. Many of the same techniques applied to powder foods are also extensions of those applied to metal powders.

Metal powder printing is a highly developed field in AM [26]. The AM printing process begins with a three-dimensional digital model whose geometric information is sent to the printer. Afterwards the object is built layer by layer with additional support structures for printing stability and accuracy. Three main manufacturing processes exist for metallic powder based prints and include selective laser sintering/melting (SLS/SLM), direct metal laser sintering (DMLS) and electron beam melting (EBM). Powder printing processes fall under the category of "Powdered Based Fusion" (PBF) where particles are fused together with high thermal energy [26]. PBF operates by melting a layer of metal powder on a platform by using a high powered laser. For each new layer; the platform moves downward so that a new even layer of powder is placed. The next layer is then melted by the laser and the platform descends once more. The thickness of the layer can be between 20-60 $\mu \mathrm{m}$. This process continues until the object is fully printed. Extracting the final component is performed by removing the excess powder from the printing area to reveal the finished object. The major differences between SLM, SLS and DMLS are related to the nature and texture of the metal powder and not by the manufacturing process.

In addition to powder based methods, Direct Energy Deposition methods (DED) will fuse metal powder or wire to a base material by inducing concentrated thermal gradients [26]. 
DED has multiple sub-branches leading to laser engineered net shaping (LENS), direct metal deposition (DMD) and electron free form fabrication (EBFFF) [26]. The main difference between DED and PBF is that DED uses a powder injection system with a controllable fiber laser. DED is typically used in repair applications such as cold gas spraying or high velocity oxygen fuel spraying [26]. EBFFF will focus a beam of electrons to create a molten pool on a metallic substrate [26]. Rapid plasma deposition (RPD) will melt metal wires so that the droplets fall onto the substrate in an argon atmosphere and is defined as a multi-layering printing process. When considering the printing resolution, DED is much worse than $\mathrm{PBF}$ and retooling is often required to improve surface finish [26].

Light weighing is another attractive reason for using AM. An optimization contest was held by GE in 2013 to design a new aircraft engine bracket. Finalists had reduced to be 70\%-80\% lighter than the original design [26]. Research conducted by Matthew Tomlin [34] studied weight minimization on an Airbus A320 nacelle hinge bracket and achieved a total mass saving of $64 \%$ by both topology and material selection. Another notable example of additive manufacturing in the aerospace industry is seen with fuel nozzle development for combustion chambers by GE [35]. The nozzle component was able to save assembly time by limiting the number of weld-able pieces from 20 to 2 [36]. The nozzle tip also had 15 percent less mass than the previous design [35]. Additive manufacturing is able to save on material waste by only using the required amount of material for each print. Traditional manufacturing techniques often remove material from 
a larger piece contributing to unneeded material waste. Less material waste will also save on energy demands during manufacturing.

It is known that additive manufacturing relaxes geometric manufacturing constraints due to its layering approach. Highly complex geometries can be manufactured such as lattice truss structures. Cellular materials have untapped potential in engineering design in heat management [37, 38], electrical properties [39], energy absorbance [40, 41] and light weighing abilities [42]. Research on lattice structures have been used to develop extremely light PIN installation equipment to be used in thin-walled aerospace work pieces [42]. The combinatory optimization technique of multilateral with lattice materials had produced a failsafe design that is $9.1 \%$ lighter than traditional topology optimization and sizing techniques. Lattice structures have also been researched in the aerospace industry for wing leading edge de-icing systems. Bici et al have proposed using lattice sandwich cores as heat exchangers for de-icing the leading edge [43]. The leading edge was optimized for weight, lattice dimension, thermal properties, natural frequency, stiffness and stress. Conforming cooling channels are a promising idea and more research is needed to fully develop this concept. AM technologies coupled with structural optimization opens new territories for engineers to explore.

There are notable disadvantages however when considering additive manufacturing. One disadvantage is related to post-process clean up times. The scaffolds or supports must be removed and sanded appropriately. In some cases, not all of the support material can be 
removed leading the prints with higher mass than expected. Sanding is also required for satisfactory surface finishes.

Another disadvantage is resolution size and the size of the part. Economically, higher resolutions require more printing time and will generally be more suitable for smaller geometric objects or prints. There is also a maximum size that can be printed as the object cannot be larger than the printing space. The final disadvantage is material selection may be limited when designing a component for AM and the material properties tend to be weaker [44].

\subsection{Multi-Scale Design and Optimization}

Multi-scale refers to problems dealing with multiple scale lengths such as a micro-scale and a macro-scale. The scale lengths could be geometric or time based. In multi-physics problems, the scales lengths (or meso-scale) may begin at the quantum level up to atomic arrangements and then end at larger scales of an automobile part [45]. The constituents of the problem at each scale is simultaneously designed, analyzed and modeled through simulations. Engineering, chemistry, mathematics, physics and computer sciences are all scientific fields that have benefited from the application of multi-scale design (or modeling) techniques to solve hierarchical design problems. Multi-scale design has helped with fluid modeling [46], heterogeneous materials [47], polymer design [45], protein synthesis [48], chemical reactions [49], universe or cosmic simulations [50, 51] and industrial manufacturing [52]. Modeling aspects refer to the physical interpretations at each length scale by their simulation methods. The bridging of information between 
scales is an important concept as each scale is analyzed based on the reactions of either the smallest or largest meso-scale [53]. Interactions between scales however are not always addressed due to simulations being locked into a specific scale. The lack of physical inter-scaling behavior is a well-known disadvantage of multi-scale models and is termed by Batterman as the "tyranny of scales" [51, 54]. Major advantages of multi-scale design include financial savings, reducing trial-and error iterations, lower development costs, the possibly to develop new materials, increasing product quality, improving design process and reducing the number of costly large scale experiments [53].

The origins of multi-scale design is said to have been conceived for nuclear testing in the early 1990's [53]. The US Department of Energy had begun performing simulation based design by using multi-scale modeling to reduce cost and improve safety and reliability for nuclear material testing, management and usage. Parallel computing was also a main contributor to the adoption of multi-scale modeling as computing power rapidly increased. Not only was computing power significantly better, but it also allowed for more degrees of freedom in simulations for more accurate predictions at various length scales [53]. As multi-scale modeling method improved throughout the 90's, it was clear that there was a potential for simulation-based design. The workflow methods developed by the DOE and other research labs at the time were eventually adapted to other industries such as aerospace, automotive and structural systems. Proliferation of multiscale modeling paradigms was also pushed by a rapid growth in scientific papers on the subject and from access to online journals [53]. 
There are many examples of multi-scale modeling and optimization applications within the literature. Kmiecik explains how modeling protein systems can benefit from multiscale modeling by using coarse grain systems [55]. The advantage is to avoid the complexities of simulating all-atomic systems which have millions of degrees of freedom by substituting atomic arrangements with larger scale protein models. Coarse grain models are mainly used to study protein folding mechanisms which are crucial to the function of organic living systems [55]. The smallest length scale may start at the quantum level of the structure of a single atom. The coarse-grained level is a subset of the meso-scale or aggregate of multiple protein systems which include the interactions of protein folding and global motions. Multi-scale modeling in structural biology has also allowed for the prediction of protein complexes [56, 55].

In the medical field, bone has been modeled extensively using multi-scale strategies. The effects of aging and fracture of cortical bones have been studied by Ho Ba Tho et al [57]. The results of their research had shown consistent behavior at the different levels. The macroscopic level and osteon lamellae elastic behavior was within the range found in literature data. Predictions at the microscopic levels of interstitial lamellae were slightly lower than reports results in the literature [57]. Other simulations of human bone have been performed by Katz et al to model calcified tissues at bone-tissue interface [58]. Scales lengths for Katz research go down to the Nano-scale of collagen molecules. The largest scale consists of the femoral cordial bone [58]. Results of Katz simulations and research were obtained by applying the finite element method. The discussed bone 
modeling techniques used homogenization theory to quantify the basic behavior of the micro-scale material and for bridging the length scales together [53].

Zeng et al have shown how multi-scale modeling and simulation of polymer Nanocomposites are achieved in the literature [45]. The advantages of these materials are from the enhanced reinforcements of nanoparticles. Zeng et al explain their assumptions and scaling strategies when developing multi-scale models for single fiber polymers; predictions and simulations are performed on the molecular level, the smallest length scale. The next meso-scale models equivalent truss structures and arrangements of the atoms as their bond strength. The highest levels are equivalent continuum models. Lebel and Therriault applied multi-scale modeling for polymer-based nano-composite structures [52]. These types of materials have applications in micro-electro-mechanical systems, organic electronics and in high performance structures for aerospace. Three major lengths scales had been studied, nano, micro and macro. The Nanoscale analyzed the molecular bonds of a polymer material known as a single-walled carbon nanotube (SWNT). The micro-scale contained the fabrication and control over the build orientations of the SWNTs. Macroscale levels analyzed a three-dimensional nano-composite with orientation information of the microscale structures and resin properties. SWNT Nano fibers had shown to have exceptionally higher stiffness than unloaded polyurethane micro fibers [52]. Nano fibers had on average up to $64 \%$ higher strength as much as 15 times higher stiffness. 
Optimization problems in composite material engineering also benefit from the multiscale paradigm. An example can be found in the work of Zuo et al who simultaneously optimized the micro and macrostructure for maximum natural frequencies [59]. The optimization problem is fine tuned to determine the best material layout in a unit cell for a specific application. Simultaneous optimization of both micro and macro scales requires a large number of design variables. Optimization methods for material patterns often implement gradient based topology optimization techniques. In the case of Zuo et al, a BESO optimization algorithm [60] was selected to determine the optimal material.

Lattice or cellular material has also been subjected to multi-scale design philosophies. Homogenization theory is used to characterize lattice or cellular material properties. Bendsoe et al used porous material for optimal material placement and developed the SIMP optimization method [61]. Micro-scale behavior was governed by relative densities of the lattice topology to obtain graded or FGM structures. The research of Xia applied multi-scale workflows for analysis between the global and local scales by superimposing macro strains onto the local RVE [62]. Homogenization theory has been crucial for the development of bridging information between scales in lattice optimization and FGMs [63].

From an optimization point of view, a multi-scale optimization is a global optimization problem. A key feature of searching for the global optima is the risk of settling into a local optima resulting in sub-optimal solutions. This poses many challenges for engineers because many engineering problems are non-convex and contain objective functions with 
considerable roughness [64]. This problem is made worse in multi-scale optimization due to dramatic differences between scale lengths or between the local and the global nature of the problem. Protein docking and folding are examples with high non-linearity [64]. Solid mechanics approaches for cellular material design and prediction also have similar problems however it is outside the scope of this thesis [64].

\subsection{Multi-Objective Optimization}

When the optimization problem contains multiple objectives, there is no single optimal answer to the problem. For multiple objectives, the answer is often a set of optimal solutions which describes a trade-off curve or Pareto frontier. Mathematically speaking, there are an infinite number of optimal solutions along the Pareto frontier [65].

Multiple objective optimizations follow the same principles of regular optimization. This means that the Karush-Kuhn-Tucker (KKT) conditions are met for each objective with respect to its constraints [14]. There exist many types of multi-objective optimization schemes such as genetic algorithms and gradient based methods. For the purpose of this thesis report, only gradient based strategies are considered as they are generally better suited for large scale problems with expensive cost functions.

The quality of a solution is determined by its dominance over the set of obtained solutions. Dominance between optimal points can be tested by comparing the solutions obtained between each other. A dominant solution is no worse than another solution if all objectives are better than the other solutions objectives. A dominated solutions objectives 
contain at least one objective strictly worse than a dominant solution [66]. This forms the basis of what is known as a Pareto Optimal solution.

There also exists a set of non-dominated solutions that are not dominated by any member of the solution set. The non-dominated solution set is part of the entire feasible decision space and is referred to as the Pareto-Optimal set. The boundary of the Pareto-Optimal set is known as the Pareto-Optimal front and is the main goal for multiple-objective optimizations. Therefore, locating as many solutions along the Pareto-Optimal front is the common goal for multiple-objective problems. In other words, the Pareto-optimal front consists of solutions to which one objective cannot be made better without making the other objectives worse. Many strategies and algorithms exist to help determine an optimal set of solutions such as classic gradient based methods or evolutionary algorithms.

Genetic algorithms or evolutionary algorithms differ from gradient based strategies as they operate on a set of candidate solution were as gradient based will operate on a single candidate solution at a time. There are two main types of genetic algorithms which function on varying definitions of elitism to select the candidate set. An example for evolutionary algorithms includes the widely used NSGA II [67].

A more straightforward approach to tackling multiple objectives is to merge the objective together into a single objective function. Each objective function is assigned a prescribed weight. By varying the weights, whose summation is equal to one, then solving the new optimization problem; it is possible to obtain the entire Pareto front. This method allows 
for gradient based methods to be applied when solving for design points along the Pareto front. A disadvantage to this method is its difficulty to derive concave Pareto fronts [14]. As stated in the overview section of this chapter, gradient based strategies are adopted for the manuscript to perform the multiple objective topology optimizations as a method of design space exploration.

\subsection{Asymptotic Homogenization}

Asymptotic homogenization is the process of determining the properties of a material from a micro-scale onto a macro-scale level. This method is commonly used to derive the effective mechanical properties of composite or porous materials. It can also be extended to determine homogenized properties for three-dimensional lattice materials as well. Homogenization is favorable for complex microstructures as it reduces the computation costs during FEM simulations.

Some notable uses for numerical homogenization in engineering had been done by Hollister in 2005 [68]. Homogenization of porous bones structures was performed and the same techniques were applied to foam metals. Recent studies using asymptotic homogenization have been able to produce complex geometries with the use of topology optimization for negative Poisson ratio materials [69]. Composite material design has also greatly benefited by the application of homogenization techniques as material testing is costly and time consuming [70]. Advancements in the design and analysis of composite materials through homogenization has led to the development of high performing materials such as graphite-epoxy and fiber-reinforces plastics [71]. Other homogenization 
methods have used Bloch's theorem and the Cauchy-Born hypothesis for two dimensional cases [72]. Other research lead by Hutchinson and Fleck homogenized the properties of periodic truss structures for kagome and similar lattices [72].

Analytical methods have been proposed in the literature to estimate the bulk properties of composite materials. One of the oldest methods is the "Rule of Mixtures". If the designer knows the volume fractions of each material in the composite, they can determine the bulk stiffness, shear and Poisson properties. The spatial distribution in orientation however is not taken into consideration unless additional calculations are made to the mechanical properties beforehand. Mechanics of material approaches have also been applied for simple cases such as a plate with a hole in the center. Beams and rods had been previously used to define the homogenized properties for lattice material. Simple closed form analytical solutions have been derived however they are limited to very strict assumptions [73].

Homogenization methods rely on a so called representative volume element or RVE, this is identical to a repeating unit cell. The definition of an RVE is a repeating section of a porous medium that can be used to define the entire structure. The RVE can be subjected to multiple independent cases of uniform boundary displacement or traction fields. The overall properties of the material are then estimated based on the RVE's response of the imposed fields. In a two dimensional case for example there could be either three independent traction, three displacement or tractions fields applied on the boundaries of RVE. Homogenization also assumes the average strain energy of the RVE and original 
material is equal when they are submitted to the same special boundary conditions [74]. This is used to relate the macro and microscopic scales. The scales and illustrations of homogenization are shown in Figure 2.
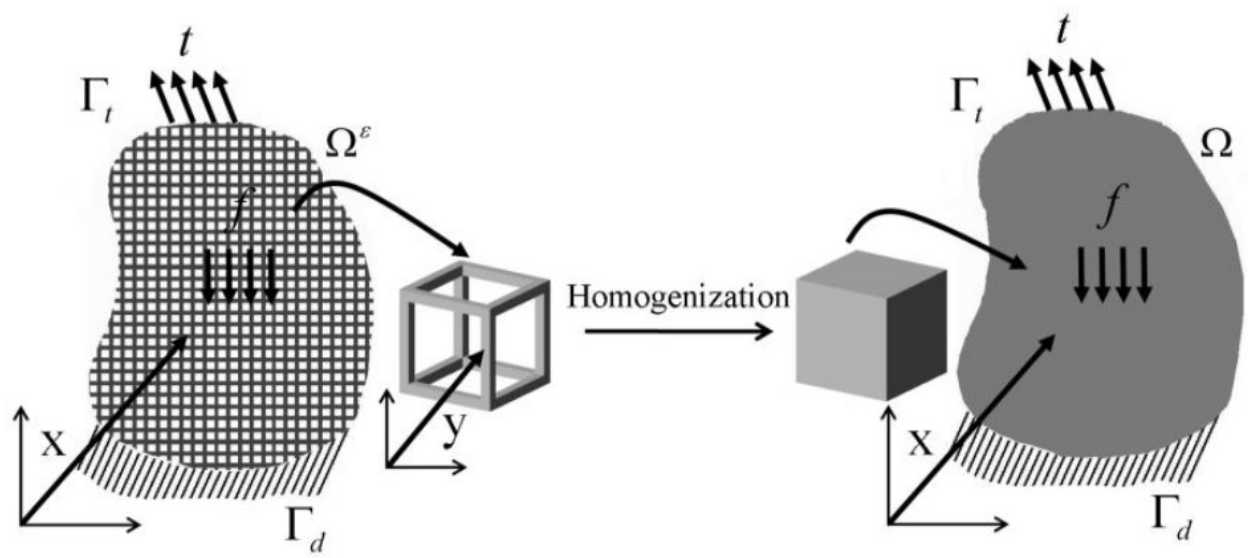

Figure 2 : Illustration of Homogenization - Microscale (Y) to Macroscale (X) [75]

Hooke's law can be applied to elastic mediums. The energy absorbed by elastic strain of the RVE can be measured with the equation [76]:

$$
C=\frac{1}{2} D_{i j k l} \varepsilon_{i j} \varepsilon_{k l}
$$

where $C$ is the strain energy, $D_{i j k l}$ is a fourth order tensor or constitutive tensor for elastic material and $\varepsilon$ is the strain. As a reminder to the reader, the elasticity tensor will map the second order strain tensors to the second order stress tensors. Hooke's law is also only valid when the periodic medium is subjective to elastic deformation.

\subsection{Topology Optimization}

Modern structural optimization has existed in much the same amount of time as finite element analysis [77]. The first structural optimization was done on beam elements in a finite element model. Sizing optimization was generally the main tool for structural 
design. Solution methods for these problems where driven by the philosophy of a fully stressed design (FSD). When a structure has all of its members under its maximum allowed stress, the structure is said to be using all of its material in the most efficient way possible. Nowadays this philosophy has been met with criticism as to whether or not the optimal topology of a structure can be obtained by FSD [77].

Topology optimization has evolved to include a wide variety of strategies to determine the optimal layout of material in a design space. The engineer is no longer limited to sizing of predetermined geometry but is able to redesign a part by only requiring the geometry limits and boundary conditions. The use of topology optimization has further gained popularity because of additive manufacturing where the manufacturing constraints are more relaxed and previously unseen structural topologies can now become a reality. The push for more sophisticated topology optimization methods has seen five strategies to determine the optimal material layout. New approaches to topology optimization have led to the creation of the SIMP [69], Level-Set [78], Super Shape [79], Bubble [80] and Phase field [81] methods.

The primary topology optimization strategy in this thesis will focus on the methods for material redistribution techniques. Material redistribution uses a finite element meshes elements as the design variables to determine the optimal placement of material for a given objective. The SIMP method is the optimization method within the optimization framework of this manuscript and is explained in the proceeding section of this chapter. 


\subsubsection{SIMP}

Bendsoe et al pioneered the SIMP method for topology optimization. The SIMP method relies of changing the relative densities of elements within an FEM model to minimize or maximize a given objective while satisfying the constraints. Hence, the design variables for each SIMP optimization are the relative densities of the design elements. SIMP is regarded as a material redistribution method because design variables can turned on and off within the design space. A drawback to this method is the development of intermediate design variables. Generally an optimal design should consist of black and white solution. Bendsoe suggested that the intermediate densities can be represented by a composite material [61].

A)

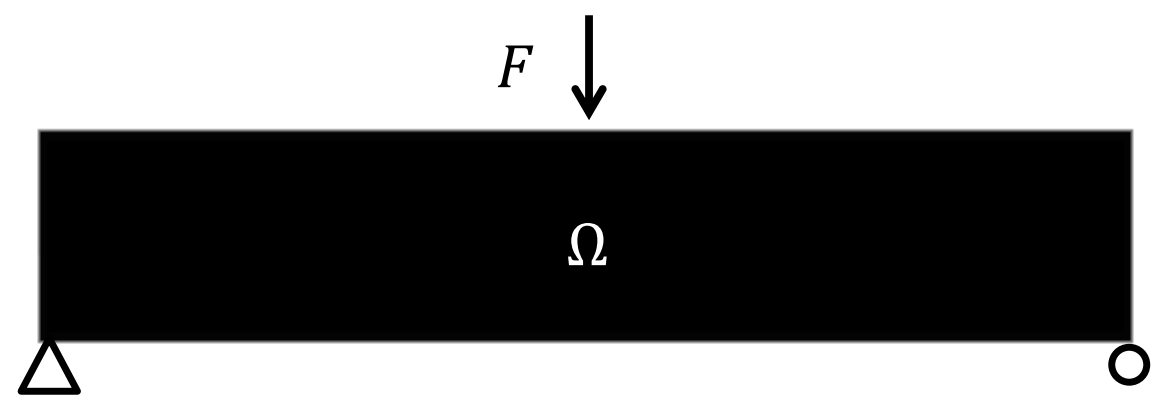

B)

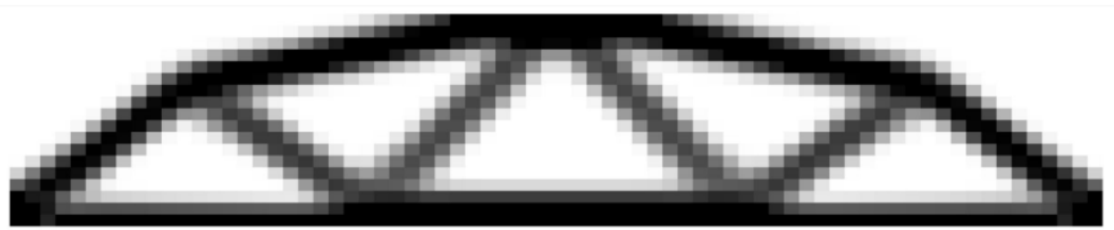

C)

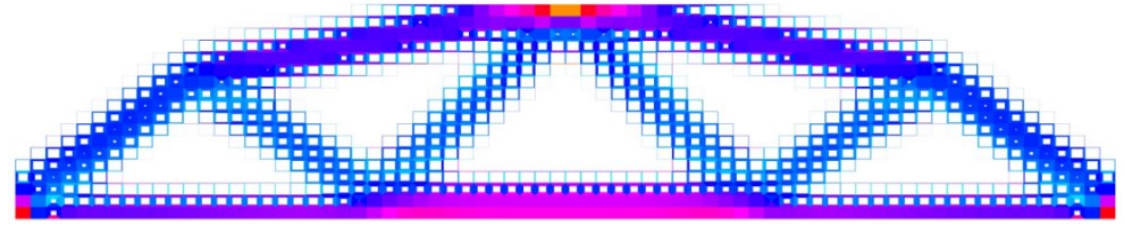

Figure 3 : SIMP Compliance Minimization Example with Mass Constraint (A) Before (B) After

SIMP (C) Equivalent SIMP with Cellular Material 
Today intermediate densities are seen to be cellular material with a lower relative density. The association of intermediate densities with cellular material has gained attention with the application of asymptotic homogenization of composites and lattice materials to generate equivalent material properties for a specified relative density. An example of the application of homogenized material can be seen in Figure 3. Homogenized properties are easily adapted to the SIMP formulation and form the basis of lattice optimizations [82].

\subsection{Lattice Optimization}

There are two underlying optimization strategies to consider so that lattice material can be integrated into a particular design or structure. The first seeks to determine the optimal cellular material by asymptotic homogenization. The second type will optimize material layout for a structure using cellular material of varying relative densities to achieve a graded structure. Both methods rely on SIMP or BESO topology optimization techniques. The lattice optimization in this thesis is mainly concerned with optimal material layouts as opposed to developing material with extreme properties.

Graded structures have been a subject of research for some time in structural optimization $[10,63]$. Functionally graded materials or FGM are a class of cellular materials which contain porous medium with gradual changes in density [10]. FGM can be either periodic or non-periodic. An example of non-periodic FGM is metallic foams whose porosity is controlled in specific areas to obtain the desired design. Two classifications of FGM exists to categorize their structure; discontinuous and continuous. Discontinuous FGM 
will have stepwise changes in microstructure similar to a composite material. Interfaces are also present in discontinuous FGMs. Continuous FGM on the other hand contain gradual changes in microstructure and are the cellular material of interest in this manuscript. An example of FGM with constant relative density, also known as ordinary lattice material, is shown in Figure 4.

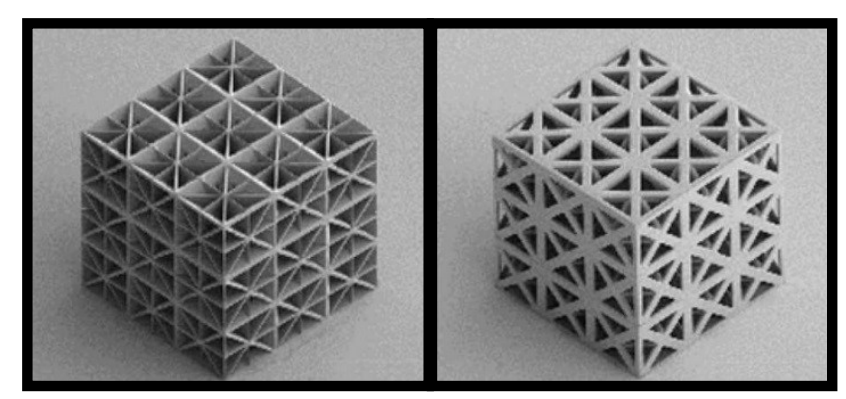

Figure 4 : Example of Lattice Material [83]

Some advantages of FGM are their ability to potentially lower the residual stress level in printed parts [10]. This was noticed by $\mathrm{K}$. Pietrzak et al when $\mathrm{Al}_{2} \mathrm{O}_{3}-\mathrm{Cr}$ FMG was inserted between a layer of $\mathrm{Al}_{2} \mathrm{O}_{3}$ and steel. The AM printed assembly's total residual stress reduced by almost one half $[10,37]$. The reduction is residual stresses is due to the tailored microstructure to facilitate better heat management [37]. Thermal gradient control via FGM has been observed by researchers in the aerospace industry in areas of combustion chambers. Some FGM can withstand very high temperatures of up to 2000k which current traditionally high performing composite materials are unable to survive. Naotake imagined using FGM to either replace the current composites in high temperature areas or by minimizing delamination due to heat damage [84]. However recent research into ceramic matrix composites surpasses Naotake's theorized thermal 
applications of FGM by reaching temperatures of almost 2500K [85]. Nevertheless FGM contain untapped potential in heat management.

Recent research into graded lattice structures has been of particular importance in the biomedical field. One notable area is the field of femoral prosthetic implants. When applied to orthopedic implants; the porous nature of lattice materials is beneficial to bone growth or reabsorption, reduced interface failure and light-weightiness [86]. Another advantage for orthopedic implant applications is reduced stress shielding effects allowing the bone to regrow [87, 88]. Essentially, Osseo-integration is encouraged with functionally graded materials by reducing the stiffness of the implant [89]. Many proposed design have been implemented from two dimensional inserts to three dimensional femoral implants [86]. Currently design for bone implants operate on a trade-off curve balancing interfacial stresses with reabsorbed bone mass. Gonzalez optimized femur implants with reabsorbed bone mass between $38 \%$ and $54 \%$ with decreased maximum the interfacial stress between $22 \%$ and $64 \%$ [90]. Future developments into orthopedic implants will require better quality printing techniques to minimize defects and standardized testing to ensure longevity [86].

Mechanical engineering design has also benefited from the use of functionally graded materials. Jing et al applied FGM to the weight minimization of gears coupled with goal oriented optimization techniques. The proposed design by Jing et al. had a reduced mass of 32 percent and improved stiffness of five percent by integrating FGM into the gear teeth [91]. The improved properties of the gears can help reduce noise and decrease fuel 
consumption. Other research by Alshabatat had used FGM to optimize the vibration characteristics of a plate. The results Alshabatat's research had shown the natural frequencies of the plate had increased and noise response decreased by $22.4 \mathrm{~dB}$ when compared to non-FGM plates [92]. Current research trends in mechanical design point towards improving noise reduction, light weight designs and modified stiffness. While industry examples in mechanical engineering are few, it proves that there is still more research that can be done in practical applications of FGM in mechanical design.

Optimization techniques have been applied to further maximize the mechanical responses of functionally graded structures. For thermo mechanical responses, analytical solutions have been derived and used as a basis to determine optimal properties [93]. Other more traditional methods use topology optimization techniques such as SIMP to determine optimal distribution of FGM densities. SIMP optimization methods have been applied to obtain graded structures that function favorably in such conditions as thermo mechanical cyclic loadings [94], static stress [95], buckling [96], femur implant objectives [86] and even Eigen-modes for piezoelectric behavior [97]. Homogenization of the FGM is thus a required step before employing material redistribution strategies.

Another popular method for optimization of FGM is by beam sizing truss structures. For lattice topologies, a collection of one dimensional Euler beams can be used to represent the lattice topology in a design space. An important assumption for Euler beams is that the resulting aspect ratio of the beams must remain slender for accurate mechanical 
behavior. Using one-dimensional beams will allow for microscopic optimization of the struts directly instead of a homogenized average.

One example in the literature used one-dimensional beams to optimize the stiffness of an aircraft hinge [98]. A mixture of solid elements and lattice beams constituted the component and commercial software was used to optimize the beam sizes with respect to a displacement constraint. It was found that when more lattice material was added to the hinge, the compliance increased [98]. An overall benefit from the lattice was measured to be at most $14 \%$ for large displacements [98]. Another example of beam sizing for optimal graded lattice structures is seen in the work of Smith et al with a novel layout optimization [99]. Smith's optimization routine would find the optimal placement of beams or struts and then perform sizing optimization on the new structure. It was seen that as the number of beams increased for stress constrained optimization, the overall mass converged towards a minimum value [99]. Chen et al have also used Euler beams for lattice optimization [100]. A parametric design to map out the Euler beams was used to model the structure followed by a beam sizing optimization. The optimized structure from Chen et al's research has an $11 \%$ improvement in the stiffness compared to the uniform gradient assembly [100]. Industry applications have also adopted the Euler beam approach for lattice material optimization. The commercial software Altair Optistruct has a lattice optimization module which is been commonly used by engineers for FGM [101]. The software will convert pre-meshed elements into equivalent lattice structures comprised of Euler beams. 
There are certain advantages for using one dimensional Euler beams; they are both computationally cheap and accurate within the assumed limits. Beam elements can also be used for buckling modeling in graded lattice structure optimization if higher order elements are used. The behavior of lattice structures have been studied from both an analytical perspective using Euler beam theory and from FEM approaches (simulations). Many engineering studies have shown modeling straight truss lattices can be used to predict lattice material behavior [102]. Ushijima et al found that the differences between their analytical predictions of Euler-based lattice materials when compared to FEM simulations showed no discernible difference in accuracy [102].

\subsection{Fatigue}

Fatigue plays an important role in engineering design. Often the main mode of failure is due to fracture and not static failure. Understanding the main mechanism for fatigue failure is thus very important. This section presents a literature overview of the mechanical fracture process for fatigue failure. The following section provides mathematical models for fatigue prediction inside the high cycle regime. The final section illustrates the prediction method for fatigue through finite element method for high cycle fatigue prediction.

Fatigue failure occurs through cumulative damage as a result of crack initiation and growth on the surface due to cyclic loads at magnitudes well below the materials yield strength $[13,12]$. Many different load cases exist, such as repeated, cyclic or random 
(stochastic) [13]. A defining characteristic of failure resulting from fatigue loadings is the occurrence of a sudden rupture as seen in Figure 5. During static load cases, the mechanical component or material will deflect first before failure similar to a ductile fracture. The rapid fracture of fatigue failure is mostly brittle in nature such that a clean cut can be seen at the point of failure. Stephens et al. estimate that failure due to fatigue is the main cause of $50 \%$ to $90 \%$ of all mechanical failures [103]. The prominence of fatigue failures prompts engineers to consider fatigue both at the design stage and when assessing the overall performance of a structure.

Fracture due to fatigue occurs in multiple steps or stages. First stage is crack nucleation or initiation followed by crack propagation in crystallographic directions along close packed slip planes. The second stage is the evolution of the cracks as micro-cracks become macro-sized. Plateaus begin to form in the second stage perpendicular to the direction of maximum cyclic tensile stress. Beach marks or clamshell marks are named after the plateau region generated in the second stage which can be discerned by their wavy nature. The final stage, stage three is when the material can no longer support the loads and results in a sudden brittle fracture. Most of the crack propagation to failure life is spent in stage two [1]. An outline of the steps and the effects on the surface is given in Figure 5, starting with initiation due to defects (stage 1), then crack growth and propagation (stage 2) followed by fracture (stage 3 ).

Crack initiation of fatigue cracks originates at stress concentrations. These include sharp corners and discontinuities that exist in the stressed regions. Hence mechanical design of 
such parts is important to avoid fatigue failure, particularly at regions under tensile stresses. Surface quality of the mechanical component or material is also crucial as scratches and highly rough surfaces can contribute to crack initiation sites and cause premature failure [104].

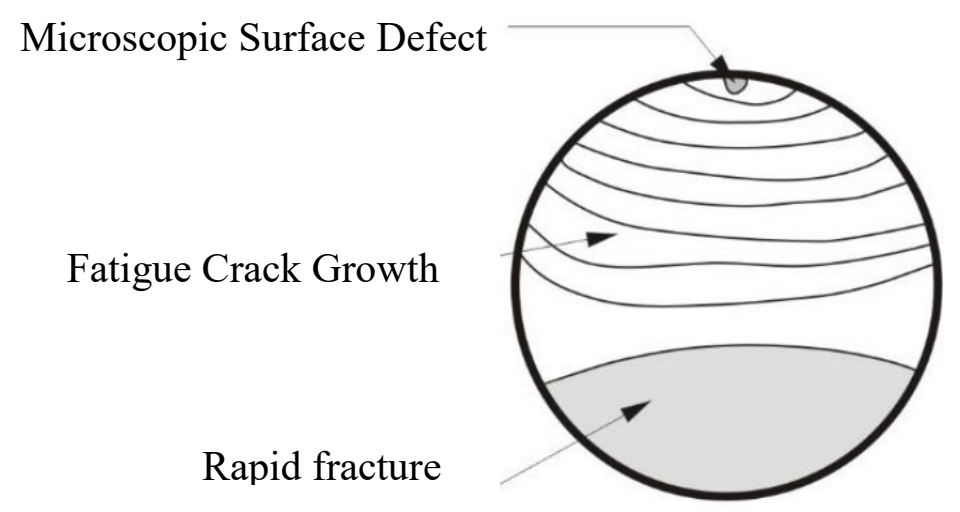

Figure 5 : Appearance of the fatigue fracture surface [105]

Because fatigue crack propagation occurs in the direction perpendicular to the maximum tensile stresses, adding layers of compressive residual stress are able to counter-act crack propagation. Such process often involves cold working the surface of the material and imposing residual strains. Cold working or work hardening is the process of strengthening a metal by plastic deformation in the absence of heat at room temperature [106]. The crystalline structure is subjected to high strains due to the increase in dislocations. The increase in dislocation density creates an environment where it is difficult for the dislocations to move, increasing the strength of the material to failure. Cold working will result in increased hardness with reduced surface roughness and ductility. 
For many non-ferrous metals such as titanium, there is no predefined endurance limit. By convention, the numbers of cycles to failure at $10^{7}$ or $10^{8}$ cycles is used to define an endurance limit [107]. Relationships between the fatigue, yield strength, ultimate tensile strength and hardness have been a popular choice for characterizing the fatigue strength [108]. Many empirical relationships have been developed to help estimate fatigue strength based on both Vickers hardness and ultimate tensile strength. This phenomenon has been heavily studied for steels such that contributions to the fatigue strength occur solely through the hardness of the material. The relationship between hardness and fatigue strength at $10^{7}$ cycles for non-ferrous materials has weaker correlation than for steels. However, approximations by hardness still give good results within $12 \%$ depending on the metal. It could be said that the hardness of microstructure may be viewed as an important factor for controlling the fatigue strength for both non-ferrous and ferrous metals [108]. Increasing the surface hardness of metallic components would then be beneficial for longer lasting engineering parts. The measured hardness is often much higher after cold working treatments as new surface dislocations will impede the deformation of the tool tip, creating shallower indentations. The higher dislocation density will also make the formation of persistent slip bands more difficult; delaying crack initiation [4].

Many surface treatments to cold work materials exist such as shot peening, ultrasonic impact and ball burnishing. Increasing the fatigue life of titanium alloys is advantageous as they are used in many high-risk engineering environments and industries where safety 
is prioritized. Aerospace structures [16], prosthetic implants [109] and automotive components [110] have integrated titanium material into their designs due to its high specific strength. Extending the life of such titanium components through cold working treatments can be advantageous for both economical and safety reasons.

\subsubsection{Predicting Fatigue}

The phenomenon of fatigue failure was first discovered by the German railway engineer August Wöhler while studying the effects of cyclical loads on train axels in 1842 [111]. It was found that a logarithmic relationship between the number of cycles to failure and the mean of the applied stress existed. This is known as a stress-life (S-N) or Wöhler curve and is widely used for engineering applications for when long fatigue lives (High-Cycle Fatigue) are to be expected. There exist three other fatigue life methods and include the strain-life method, linear-elastic fracture mechanics method and energy based methods [13]. This manuscript will primarily focus on the fatigue life predictions using the stresslife approach.

Critical plane method is another approach for predicting fatigue lives. This method searches for the plane that will generate the most damage. The predicted lives are more accurate however the procedure is computationally expensive and often creates nonconvex optimization problems [112]. This method is best used for complex multi-axial fatigue loadings and when non-proportional loads are applied. For more stable optimizations, fatigue lives will need to be considered using a uniaxial amplitude stress 
method. A final method is from calculating the fatigue in the frequency domain [113]. Fatigue life derived from frequency is outside of the scope of this thesis.

\subsubsection{Stress-Life Method}

Fatigue failure is a fairly complex failure mode. Many factors such as manufacturing defects and stress concentrations can initiate crack nucleation and can be difficult to control. Due to these difficulties and high variance, the development of high cycle S-N curves will often contain a high degree of scatter. The final curve will most likely shift the fitted curve between the mean stress and the cycles to failure two standard deviations below the mean. This helps ensure that the predicted life is within a 95 percent confidence interval.

The consequence of using S-N curves for predicting fatigue life is that there are only valid for the same applied loading. For example, is a fatigue test runs with a stress ratio of 0.5 , the results of the test cannot be directly compared to those with a stress ratio of 1 . This problem will require a correction factor when comparing different $\mathrm{S}-\mathrm{N}$ curves with different stress ratios together. The loading direction is also important and must be factored into the conversion process. There are many different types of loads and are categorized as proportional and non-proportional loadings.

\subsubsection{S-N Curve}

A stress-life curve or S-N curve is an experimentally derived equation from uniaxial load histories. The curve functions by relating the number of cycles to failure based on an 
alternating stress state. S-N curves have multiple sections to define low and high cycle regimes. Low cycles are typically categorized when failure occurs between 1 and $10^{4}$ cycles. Plastic deformation is expected in low cycle fatigue and the ultimate strength of the material is the failure criterion when the number of cycles to failure is equal to one. High cycle fatigue occurs from $10^{4}$ up to $10^{7}$ cycles and the material behavior is expected to be elastic. For some materials there is an assumed endurance limit after $10^{7}$ cycles where as long as the alternating stress is below this value, no damage will occur and the part will survive indefinitely. Metals such as iron or copper have an endurance limit however non-ferrous materials such as magnesium are said to have no well-defined endurance limit [111]. The notion of an endurance limit for metallic materials has come into question by Bathias and Miller $[114,115]$. This has led to research into the giga cycle or very high cycle regimes. Very high and giga cycle regimes however are more costly to experimentally validate and are not normally a concern as engineering components are often exchanged before reaching a giga-number of life cycles.

When the fatigue behavior is within the elastic region, the relationship between the stress and strain remains linear. In a plastic region, the shape of the object will begin to change and the material will experience high stress levels. When plastic deformation is expected, then an SN curve should not be used due to the influencing factors of shape change and potential strain-hardening [12]. Strain-Life approaches are best used in high stress environments. 
Equations to best fit S-N curves can be defined in many ways. One of these formulations is the Basquin curve which is a power law relationship between the logs of the stress to the log of the number of cycles to failure for each fatigue regime.

$$
\varpi^{b} \sigma_{S N 1}=\sigma_{\mathrm{e}}
$$

where $\varpi$ is the number of cycles above $10^{4}$ and $\sigma_{\mathrm{e}}$ is an alternating stress or endurance limit on the $\mathrm{SN}$ curve, $\sigma_{S N 1}$ is the stress of the $\mathrm{SN}$ curve at 1 cycle and $\mathrm{b}$ is the fatigue strength exponent (or Basquin exponent) and is calculated from:

$$
b=\frac{\log \left(\varpi_{1}\right)-\log \left(\varpi_{2}\right)}{\left(\log \left(\sigma_{\mathrm{e} 2}\right)-\log \left(\sigma_{\mathrm{e} 1}\right)\right)}
$$

The constant $\sigma_{S N 1}$ can be found analytically if the number of cycles to failure is known for a specific alternating stress. $\sigma_{S N 1}$ is calculated in equation 2.3 .

$$
\sigma_{S N 1}=\frac{\sigma_{\mathrm{e}}}{\varpi_{o}^{b}}
$$

\subsubsection{Consideration of Multi-Axial Loadings and Equivalent Stresses}

When multiple fatigue loads are applied to a structure, equivalent amplitude and mean stresses must be calculated. There are three types of fatigue problems; completely reversing simple loads, fluctuating simple loads and a combination of loading modes. Completely reversing and fluctuating simple loads are easily handled with an S-N diagram where the alternating stress is converted to life. This requires one type of loading and the midrange stress must be appropriate for the S-N curve. The second type has a fluctuating load history with a special criterion to relate mean and amplitude stresses. The final type is a combination containing bending, torsion and axial stress loads [13]. 
A failure criterion is needed to properly gauge which stress states are more important in a multi-axial fatigue problem. Equivalent stress theories exist such as the maximum principal stress theory, maximum shear stress theory (Tresca) and the octahedral shear stress theory von-Mises. All methods provide extensions of the static yield criteria for fatigue. Von-Mises is best used when the material exhibits ductile behavior. When brittle behavior is expect the maximum principal stress criterion or Tresca theory are the favorable yield criterions [13]. The failure modes for titanium are expected to be ductile; hence a von-Mises failure criterion for the fatigue lives will be used.

For improved accuracy, the equivalent stresses used in this thesis are the signed vonMises. The mean stresses are affected by the sign and can signify either tension or compression. For general cases, the equivalent von-Mises amplitude and mean stresses can be found using equations 2.17 and 2.18 [13];

$$
\begin{gathered}
\sigma_{v m_{a}}=\frac{1}{\sqrt{2}} \sqrt{\left(\sigma_{11_{a}}-\sigma_{22_{a}}\right)^{2}+\left(\sigma_{22_{a}}-\sigma_{33_{a}}\right)^{2}+\left(\sigma_{33_{a}}-\sigma_{11_{a}}\right)^{2}+6\left(\sigma_{44_{a}}^{2}+\sigma_{55_{a}}^{2}+\sigma_{66_{a}}^{2}\right)} \\
\sigma_{v m_{m}}=\frac{1}{\sqrt{2}} \sqrt{\left(\sigma_{11_{m}}-\sigma_{22_{m}}\right)^{2}+\left(\sigma_{22_{m}}-\sigma_{33_{m}}\right)^{2}+\left(\sigma_{33_{m}}-\sigma_{11_{m}}\right)^{2}+6\left(\sigma_{44_{m}}^{2}+\sigma_{55_{m}}^{2}+\sigma_{66_{m}}^{2}\right)}
\end{gathered}
$$

where $\sigma_{\mathrm{vm}}$ is the von Mises stress amplitude and $\sigma_{\mathrm{vm}_{\mathrm{m}}}$ is the von-Mises mean stress. To take into account the compressive stresses the signed von-Mises criterion is used for the mean stress term. This will alter the calculation of the mean stress and is defined in equation 2.6.

$$
\zeta=\operatorname{sign}\left(\frac{1}{3}\left(\sigma_{11_{m}}+\sigma_{22 m}+\sigma_{22 m}\right)+\sigma_{\text {res }}\right)
$$




$$
\sigma_{\text {sgnvm }_{m}}=\zeta \cdot \sigma_{v m_{m}}
$$

where $\sigma_{\text {sgnvm }}$ is the signed von-Mises mean stress.

The sign for the mean stress could also be found from the largest magnitude of the principle stresses. The absolute maximum principal stress is widely used in finite element software to determine the sign of the signed von-Mises terms [116]. The equivalent stress from the von-Mises method is found by applying an appropriate mean stress correction function such as the Modified Goodman criteria.

\subsubsection{Failure criteria and Mean Stress Correction from Fluctuating Loads}

There are many methods to determine if failure will occur due to a uniaxial cyclical load. Some of these methods use a safety factor to determine if the material is sufficiently resistant enough for the engineering loads. A safety factor can be calculated based on three methods of failure analysis; the Soderberg criteria, the Gerber criteria, modified Goodman criteria and the ASME-elliptic method. The formulas for each criterion are written below:

$$
\begin{gathered}
\text { Soderberg line }: \frac{\sigma_{a}}{\sigma_{\mathrm{e}}}+\frac{\sigma_{m}}{\sigma_{y}}=\frac{1}{S} \\
\text { Gerber }: \frac{S \cdot \sigma_{a}}{\sigma_{\mathrm{e}}}+\left(\frac{S \cdot \sigma_{m}}{\sigma_{u t}}\right)^{2}=1 \\
\text { ASME - Elliptic }:\left(\frac{\sigma_{a}}{\sigma_{\mathrm{e}}}\right)^{2}+\left(\frac{\sigma_{m}}{\sigma_{y}}\right)^{2}=\frac{1}{S^{2}} \\
\text { Modified Goodman }: \frac{\sigma_{a}}{\sigma_{\mathrm{e}}}+\frac{\sigma_{m}}{\sigma_{u t}}=\frac{1}{S}
\end{gathered}
$$


where $S$ is the safety factor, $\sigma_{m}$ is the mean stress, $\sigma_{a}$ is the stress amplitude or alternating stress, $\sigma_{y}$ is the yield stress of the material, $\sigma_{u t}$ is the ultimate tensile strength of the material and $\sigma_{\mathrm{e}}$ is the endurance limit of the material at $10^{7}$ cycles. The endurance limit can be exchanged with any other stress level to suit the engineer's needs. When a lower number of cycles are used as the failure criterion, the Goodman diagram line becomes steeper [117]. The four equations above are only valid if the mean stresses are below the yield of ultimate strength of the material. If a tensile stress larger than the failure limit of the material is expected, then the component will to fail during its first cycle. Likewise compressive mean stresses will only extend the overall life of the part by decreasing the equivalent alternating stress.

Equations can be rearranged to solve for an equivalent reversible stress to calculate the lives for each cycle. Rearranging the above equations give:

$$
\begin{gathered}
\text { Soderberg line }\left\{\tau_{S}(\vec{\sigma})\right\}: \sigma_{\text {rev }}=S \frac{\sigma_{a} \sigma_{y}}{\sigma_{y}-S \sigma_{m}} \\
\text { Gerber }\left\{\tau_{G}(\vec{\sigma})\right\}: \sigma_{\text {rev }}=\frac{S \sigma_{a} \sigma_{u t}^{2}}{\sigma_{u t}^{2}-\left(\sigma_{m} S\right)^{2}} \\
\text { ASME - Elliptic }\left\{\tau_{E}(\vec{\sigma})\right\}: \sigma_{\text {rev }}= \pm S \sigma_{a} \sigma_{y} \sqrt{\frac{1}{\left(\sigma_{m} S\right)^{2}+\sigma_{y}^{2}}} \\
\text { Modified Goodman }\left\{\tau_{M G}(\vec{\sigma})\right\}: \sigma_{r e v}=S \frac{\sigma_{a} \sigma_{u t}}{\sigma_{u t}-\sigma_{m} S}
\end{gathered}
$$

where $\sigma_{\text {rev }}$ is the equivalent reversible stress and $\tau(\vec{\sigma})$ is the mean stress correction the function. The advantage of using the above criteria is that the material yielding and safety factors can be taken into account. The above equations are only valid when the mean 
stress is below the yield or ultimate strengths. For the purpose of this thesis, only the modified Goodman line is considered for fatigue analysis and subsequent optimizations.

\subsubsection{Rainflow Counting Algorithms}

The Rainflow algorithm was created by T. Endo and M. Matsuishi in 1968 [118]. It is

named the Rainflow algorithm because of its resemblance to rain flowing down a Japanese pagoda roof. The premise of this algorithm is to create representative sections of a random load history into appropriate load reversals. The goal of a cycle counting algorithm to determine the number of cycles (a damaging event for fatigue) and stress ranges. The Rainflow counting algorithm looks at the peaks and valleys of a variable uniaxial load history (maximums and minimums). From those peaks and valleys, a new mean and amplitude scaling factor is derived for each of the individual load reversals. The number of load reversals is also found. In addition, scaling factors can also be found in relation to the maximum load or stress. The results of the Rainflow algorithm can then be used to calculate the fatigue damage by using Miner's rule. Similarly, each Rainflow cycle can be used to calculate crack increments in linear fracture mechanics. In addition to the pagoda roof method, there exist cycle counting methods such as the three [119] and four point method [120]. A pseudo code for the three point method which calculates the number of cycles is given in Appendix A.1 to illustrate its implementation [121]. 


\subsubsection{Palmgren Miner Cumulative Damage Hypotheses}

The Palmgren Miner cumulative damage hypothesis states that the summation of damage from variable single reversals can be done by the summation of the inverse of the individual cycles. The formulation for damage accumulation can be calculated from:

$$
H=\sum_{i=1}^{k} \frac{n_{i}}{\varpi_{i}}
$$

Where $k$ is the number of loads (or reversals), $n_{i}$ is the number of cycles at a given load level and $\varpi_{i}$ is the number of cycles before failure at that load level. If $H$ is above one, then failure ensures. Rainflow counting can be used for each of the $k$ number of cycles to calculate the total damage from a stress profile.

The number of cycles for the load history until failure can also be calculated from Miner's rule. The equation for the number of load history repetitions is:

$$
\varpi_{\text {total }}=\frac{H}{\sum_{i=1}^{k} \frac{n_{i}}{\varpi_{i}}}
$$

where $\varpi_{\text {total }}$ is the number of repetitions of the load history until failure and $H$ is the failure criterion determined by experiment (typically between 0.7 to 2.2 and averages around 1). The total number of cycles until failure can be calculated by multiplying the number of time block repetitions by the number of reversals in the load time $\operatorname{history}\left(\sum n_{i}\right)$.

\subsection{Ultrasonic Impact Treatment}

Studies have shown that Ultrasonic Impact Treatment (UIT) improves the surface finish and fatigue properties in the field of post-welding [11]. UIT is a process in which an 
indenter vibrating at ultrasonic speeds slides over a surface. This treatment deforms the surface while inducing and redistributing residual stress in the part resulting in enhanced fatigue life [122]. UIT devices operate by inducing plastic deformation from the indenter or impact needle by first exciting a transducer by a controlled voltage input. The power source directly controls the oscillations exhibited by the transducer which sends its frequencies to the sonotrode (ultrasonic horn) [122]. The cold working and softening of the surface results in high plastic deformation generating large compressive residual stresses while at the same time reducing its roughness.

Figure 6B displays the diagram of a UIT device. The arrow pointing from 1 is the piezoelectrical converter. The arrow at 2 indicates the sonotrode and 3 is the specific impactor (or needle). The sub diagram at 5 describes the movement cycle of the impactor inside the end piece shown in 4 .
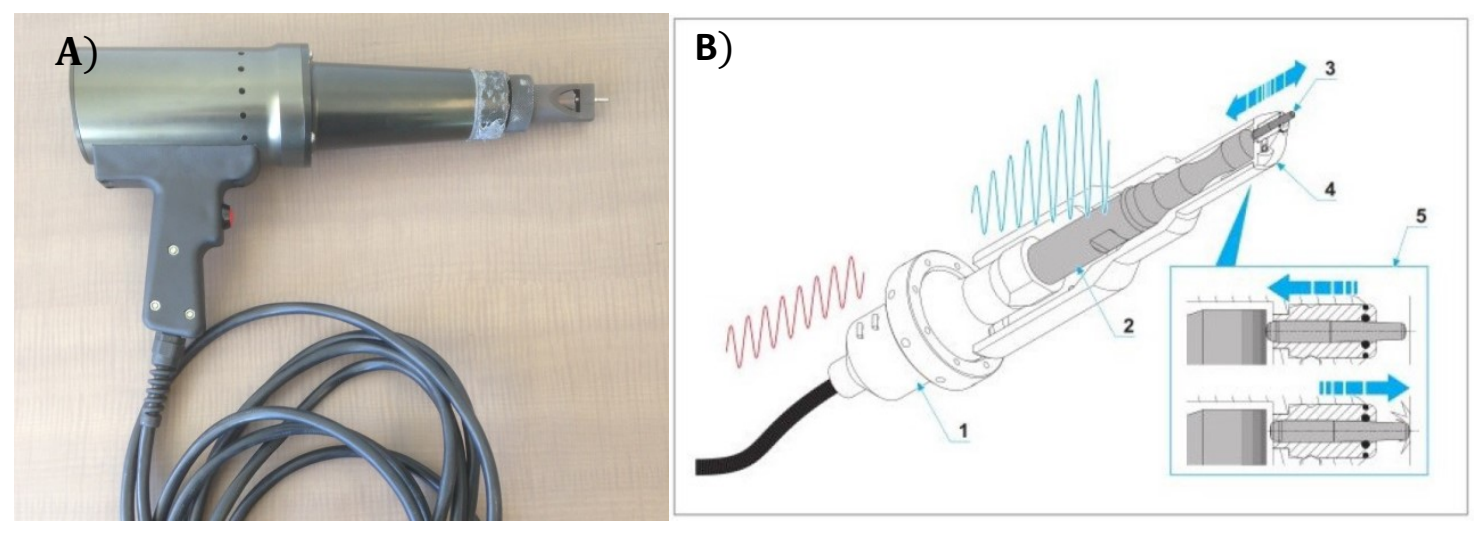

Figure 6 : (A) UIT Device (B) UIT Diagram [123]

The research of E. Statnikov et al. [11] compared a variety of methods that improve the fatigue life of welded joints. These methods included Ultrasonic impact treatment (UIT), hammer peening, shot peening and TIG dressing. An improvement of $65 \%$ was observed 
in the UIT treated joints. In B.N. Mordyul et al. [124] investigation of the enhancements that occur in the surface layer of ultrasonic impacted specimens, it was concluded that the compressive residual stresses and work hardening of the surface layer attributed to the improvement in fatigue properties of processed specimens. A.I. Dekhtyar et al. [125] concluded that at high stress levels, UIT-processed Ti-6Al-4V has a fatigue life that is twice of the pristine (untreated) samples, and a roughness reduction Ra of $75 \%$. While UIT is widely used in fatigue improvement of welded joints, the aim is to treat the surface of DMLS Ti-6Al-4V to enhance the fatigue life as a virtue of improved roughness, increased hardness and induced compressive residual stresses.

\subsubsection{UIT and Work Hardening}

Work hardening is defined by the strengthening or toughening of a material by cold working [124]. Plastic deformation at the surface of a material is able to increase the number of dislocations while disorientating and entangling local slip systems [12]. The addition of dislocations will often hinder the motion of other dislocations improving the strength of the material. The favorable increase in strength however is limited by the decreased ductility of the material.

The effects of work hardening of a material can be evaluated by analyzing the dislocation density at the surface of the material. X-ray diffraction (XRD) and transmission electron microscopy (TEM) are such methods used to measure and compare the dislocation density at the surface of a material [126]. Etching the surface of a material may also allow for dislocation measurement and motion by analyzing the formation of small pits or 
etch pits. Holes are formed during etching and attack regions surrounding the dislocations due to the higher strain fields. The results of the etching allow the observer to see were the dislocations are intercepting the material surface [127].

The surface microstructure of a work hardened material can also be used to evaluate cold working. The effect of plastic deformation due to cold work on a crystalline material will cause grain refinements. Fine grain materials will have a longer fatigue life especially at low plastic strain amplitudes [12]. Some cold working procedures such as rolling can cause grain refinement of to fall into the nanometer range [128]. The researches of Tanaka and Mura have developed a theoretical model to evaluate the effects of fatigue crack nucleation life based on grain size and dislocation slip [129]. Their findings have shown that as the grain size decreases the fatigue crack nucleation life will begin to increase. The Tanaka-Mura model has later been refined by $\mathrm{Wu}$ to omit the grain size and introduce dimensionless strain [130].

Due to the stochastic nature of some of the cold working process, including UIT; the crystallographic planes of the refined grains will be randomly oriented at the surface. XRD can be used to quantify the amount of surface softening resulting from UIT and shot peening by comparing peak intensities and peak widths [131]. Similarly, XRD can be used to measure the residual stresses on the surface of a crystalline material. Residual stresses are particularly important as large compressive stresses on the surface are beneficial to longer fatigue lives [124]. 
Observing the surface of a material can also indicate its effectiveness against fatigue failure. The concentration of crack initiation sites on the surface play a major role in controlling fatigue life. High surface porosity indicates an increased risk for crack initiation sites which is detrimental for fatigue resistance. Reduction in roughness and increase in hardness are also measures of cold working and effects on surface porosity [132].

Direct plastic deformation will increase the density of dislocations by a proportion equal to the square root of the shear stress [133]. Dislocations are often activated by three main mechanisms notably homogeneous nucleation, grain boundary initiation and from interface differences between the crystal lattice and the surface. During cold working, dislocations are added to the surface through multiplication and can be explained by the Frank-Read source [12].

Hardness measurements may also be used to characterize the cold working of the surface. Vickers and Rockwell measurement tests are the most used methods and involve creating an indentation on the metallic surface using a tool tip. The depth of the indentation will reflect the materials hardness. It is said that higher hardness reflects increased dislocations density preventing further plastic deformation [108] . 


\section{Chapter 3: DMLS Ti-6Al-4V Material Characterization}

The section reviews the experimental work used to characterize the material properties of DMLS Ti-6Al-4V. The material properties to be characterized are the young's modulus, yield and ultimate yield strength and the fatigue properties SN curve. Both tensile tests and fatigue tests were performed. The Poisson ratio was not calculated and was assumed to be approximately 0.33 , an average for titanium alloys. The first sub section goes into detail about the chemical properties of the Ti-6Al-4V power used to build the metallic specimens. The second sub section explains the procedure to calculate the young's modulus and tensile strengths. The final section gives an overview on deriving the fatigue properties for the titanium material. A novel UIT application procedure is also developed in the final section and an SN curve for a UIT treated titanium specimen is also derived.

\subsection{Experimental Design Flowchart}

The first goal in this thesis is to calculate the mechanical and fatigue properties of the printed material. In Figure 7, the properties of elastic modulus and ultimate yield strength are found from tensile testing. The Poisson ratio was not experimentally derived and company data was used as reference. An SN curve for the material with and without the novel UIT treatment will also be derived. The fatigue improvements of both curves are analyzed and compared. 


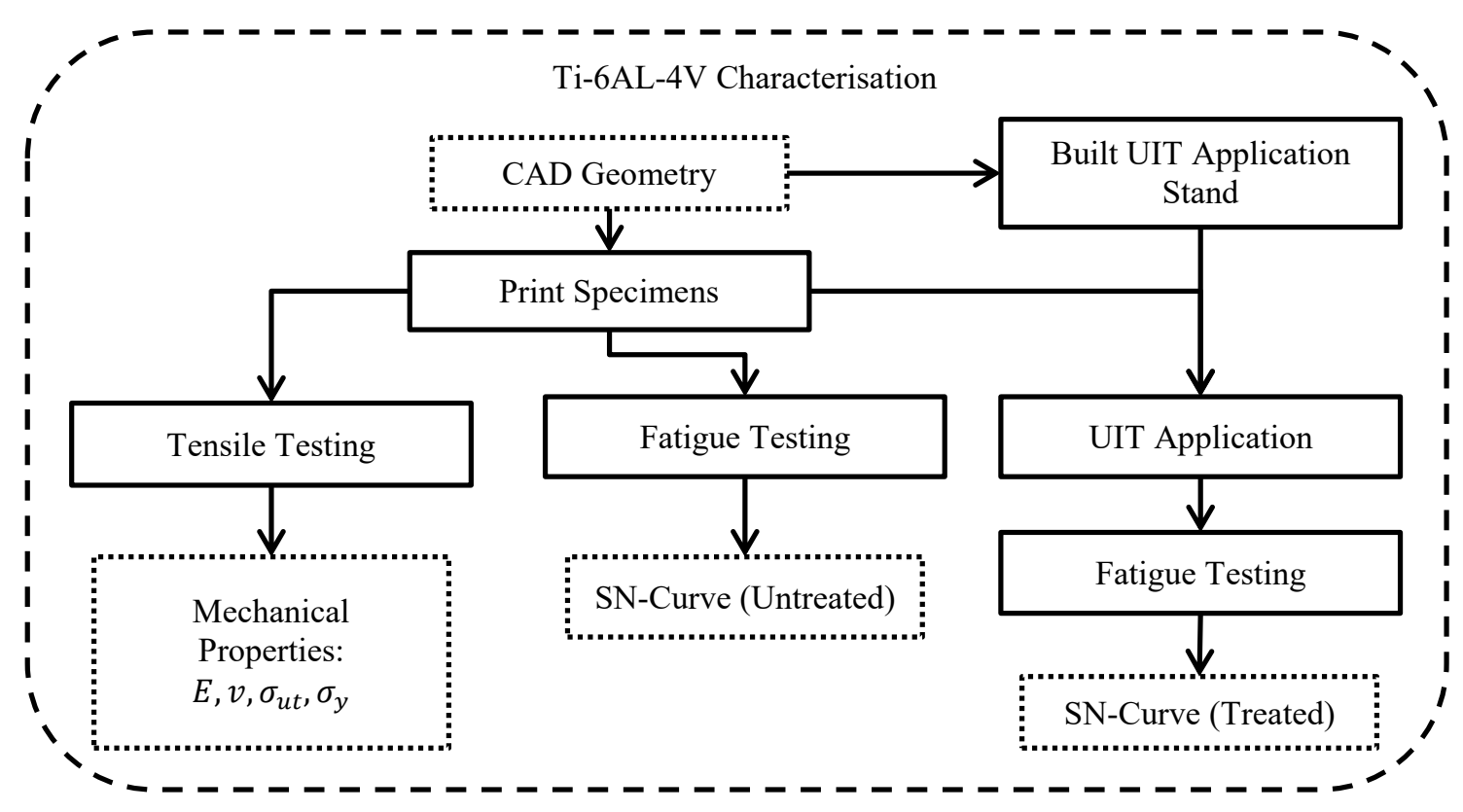

Figure 7 : Block Diagram for Material Characterization of DMLS Ti-6AL-4V

\subsection{Ti-6AL-4V DMLS Chemical Properties}

All titanium dog bone specimens were built by OmniSint-160, a metal sinter, using Ti6Al-4V grades 23 powder from AP\&C following the EOS M290/400W standard DMLS parameters. The particle size ranged from 15-23 microns and the layers were 60-microns thick. The specimens were heat treated in accordance to AMS 2801 to relieve the stress induced by the rapid melting and cooling that takes place during the printing process [134]. Specimens were treated at $800^{\circ} \mathrm{C}$ for 2 hours in an argon atmosphere. Figure $14 \mathrm{~b}$ shows the printing orientation, where the printing platform lies in the $x-y$ plane. A single 2D layer is formed on the platform when the laser beam sinters particles starting from the left end and moving towards the right end of the dog bone in Figure 13. When a layer is completed, the platform is leveled to a new elevation in the z-axis allowing a new layer to be added on top of the previous one. This build direction ensures that the grain 
boundaries are parallel to the application of force, maximizing the materials resistance to fatigue failure [135]. Wire EDM is a precise cutting technique that minimizes the need for excessive post processing machining. Wire cutting was used to breakaway supports and remove the specimens from the platform [136]. Lastly, the edges were polished using an emery cloth of grades 120 and 220.

The chemical distribution and powder size of the initial pre-sintered material are presented in Table 1 and Figure 8 below. The average sizes of the un-melted powder range in between 15 and $45 \mu \mathrm{m}$. The chemical composition and particle distribution is given by the manufacturer AP\&C [137].

Table 1 : Chemical Composition of Ti-6Al-4V Grade 23 Power, AP\&C [137]

\begin{tabular}{|c|l|l|l|l|}
\hline \multicolumn{1}{|c|}{ Element } & \multicolumn{1}{|c|}{ Content in wt\% } & \multicolumn{1}{|c|}{ Element } & \multicolumn{1}{c|}{ Content in wt\% } \\
\hline $\mathrm{Ti}$ & balance & $\mathrm{Fe}$ & $0.05-0.20$ \\
\hline $\mathrm{Al}$ & $5.50-6.50$ & $\mathrm{Cu}$ & $<0.10$ \\
\hline $\mathrm{V}$ & $3.50-4.50$ & $\mathrm{Sn}$ & $<0.10$ \\
\hline $\mathrm{O}$ & $0.10-0.12$ & $\mathrm{Y}$ & $<0.005$ \\
\hline $\mathrm{N}$ & 0.02 & Other max. each & 0.1 \\
\hline $\mathrm{C}$ & 0.02 & Other max. total & 0.4 \\
\hline & 0.01 & & \\
\hline
\end{tabular}

Figure 8 : Ti-6Al-4V Powder Size Distribution [137] 
The density of the material is given in Table 2, as well as the company ratings for the tensile and ultimate strength. Both horizontal and vertical build direction properties are given.

Table 2: Company Rating for Material Properties [137]

\begin{tabular}{|l|c|}
\hline \multicolumn{1}{|c|}{ Property (Heat Treated) } & Value \\
\hline \hline Density & $2.66 \mathrm{~g} / \mathrm{cm}^{3}$ \\
\hline Modulus of Elasticity (XY) & $109 \mathrm{GPa}$ \\
\hline Modulus of elasticity $(\mathrm{Z})$ & $113.5 \mathrm{GPa}$ \\
\hline Tensile Strength $(\mathrm{XY})$ & $1075 \mathrm{MPa}$ \\
\hline Tensile Strength $(\mathrm{Z})$ & $1080 \mathrm{MPa}$ \\
\hline Yield Strength (XY) & $1000 \mathrm{MPa}$ \\
\hline Yield Strength (Z) & $1005 \mathrm{Mpa}$ \\
\hline Elongation at Break (XY) & $13 \mp 3 \%$ \\
\hline Elongation at Break (Z) & $15 \mp 4 \%$ \\
\hline
\end{tabular}

Table 2 shows the mechanical properties. There is very little deviation in the stiffness. An isotropic material assumption can be made in this case. Isotropic assumption will simplify the fatigue behavior of the material as anisotropic behavior does not need to be accounted for.

\subsection{Material Tensile Properties}

Multi-physic optimizations require the material properties to be known. For metallic materials, the young's modulus, Poisson ratio and tensile strength are required. The starting point on the $\mathrm{SN}$ curve is also equal to the failure of at one cycle; the equivalent stress would be the ultimate tensile strength. For the mentioned criteria, tensile tests are needed to be performed to gather the necessary data for the multi-scale workflow for additively manufacture titanium components. The procedure, tensile specimen 
dimensions and calculations for the tensile properties are shown in the proceeding section.

\subsubsection{Specimen Dimensions}

The specimen dimensions closely followed the standards of ASTM E8/E8M-13 [138].

Flat rectangular dog bones where used for the tensile tests. The tensile specimens require specific dimensions for the standard; these are shown in the figure below:

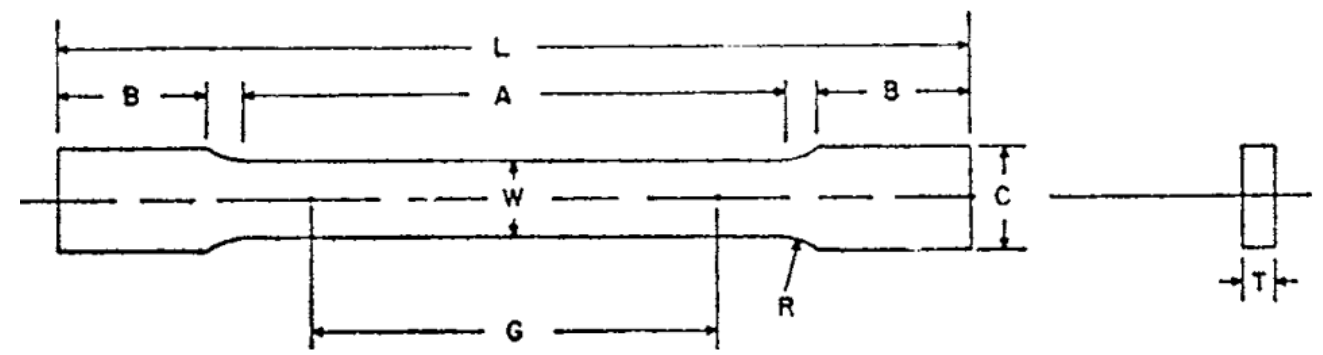

Figure 9: Tensile Specimen Dimension Standards [138]

Figure 9 displays the dimensions in relation to variables. The dimensions of the tensile specimen are displayed in Table 3.

Table 3 : Tensile Specimen Dimensions

\begin{tabular}{|c|c|}
\hline \multicolumn{1}{|c|}{ Dimension } & Value \\
\hline \hline A - Length of Reduced Section & $32 \mathrm{~mm}$ \\
\hline B - Length of Grip Section & $37.92 \mathrm{~mm}$ \\
\hline C - Width of grip section, approximate & $10 \mathrm{~mm}$ \\
\hline W - Width & $6 \mathrm{~mm}$ \\
\hline T - Thickness & $3.16 \mathrm{~mm}$ \\
\hline $\mathrm{R}-$ Radius of fillet, min & $6 \mathrm{~mm}$ \\
\hline L - Overall Length & $116.78 \mathrm{~mm}$ \\
\hline $\mathrm{G}-$ Gauge Length & $25 \mathrm{~mm}$ \\
\hline
\end{tabular}

\subsubsection{Tensile Testing Methodology}


Five tensile specimens were tested and each tensile specimen is named using a convention. Each specimen is named T-NT-XX. The first letter T stands for tensile, the second pair of letters stands for not treated (NT). The last two numbers (XX) are the identification number of the tensile specimen. The tensile tests were performed on a servo-hydraulic 810 Material Test System (max. load 100 kN), MTS [139]. The rate of pull or strain rate was set to be $0.5 \mathrm{~mm} / \mathrm{min}$ and is continuously monitored by the MTS for a consistent pull rate. The grip pressure and the ends of the tensile specimens were set to be 800 psi.

The cross section of each specimen was measured at the center of the gauge length. By measuring the cross-section; the engineering stresses and engineering tensile strength can be calculated from the tensile data. The specimens have a rectangular cross section and the area is calculated by the product of the thickness (T) and the width (W).

The yield strength of the ADM metal is to be found by using an offset method with 0.2 percent of the strain. The intersection of the offset line and the stress-strain curve is the value of the yield strength. The maximum strain before plastic deformation is found by the yield strength positions strain using the offset method. The ultimate tensile strength id found by dividing the maximum force applied to the specimen during tensile testing by the original cross-sectional area of the specimen. The final length of the specimen is calculated by measuring the changes in lengths of the gauge lengths of the specimens. The change in lengths is sued to calculate the strain at failure. 
The modulus of elasticity is found by linear regression of the elastic section's slope of the stress and strain curve. An average of the calculated slopes is taken to calculate the modulus of elasticity of the material. The maximum displacement in the elastic region for calculating the slope was assumed to be $0.75 \mathrm{~mm}$. The experimental modulus of elasticity is then compared to the company rating of the material properties.

\subsubsection{Stress Strain Curve Data}

The draw data for the tensile tests are shown below with the engineering stress plotted against the engineering strain.

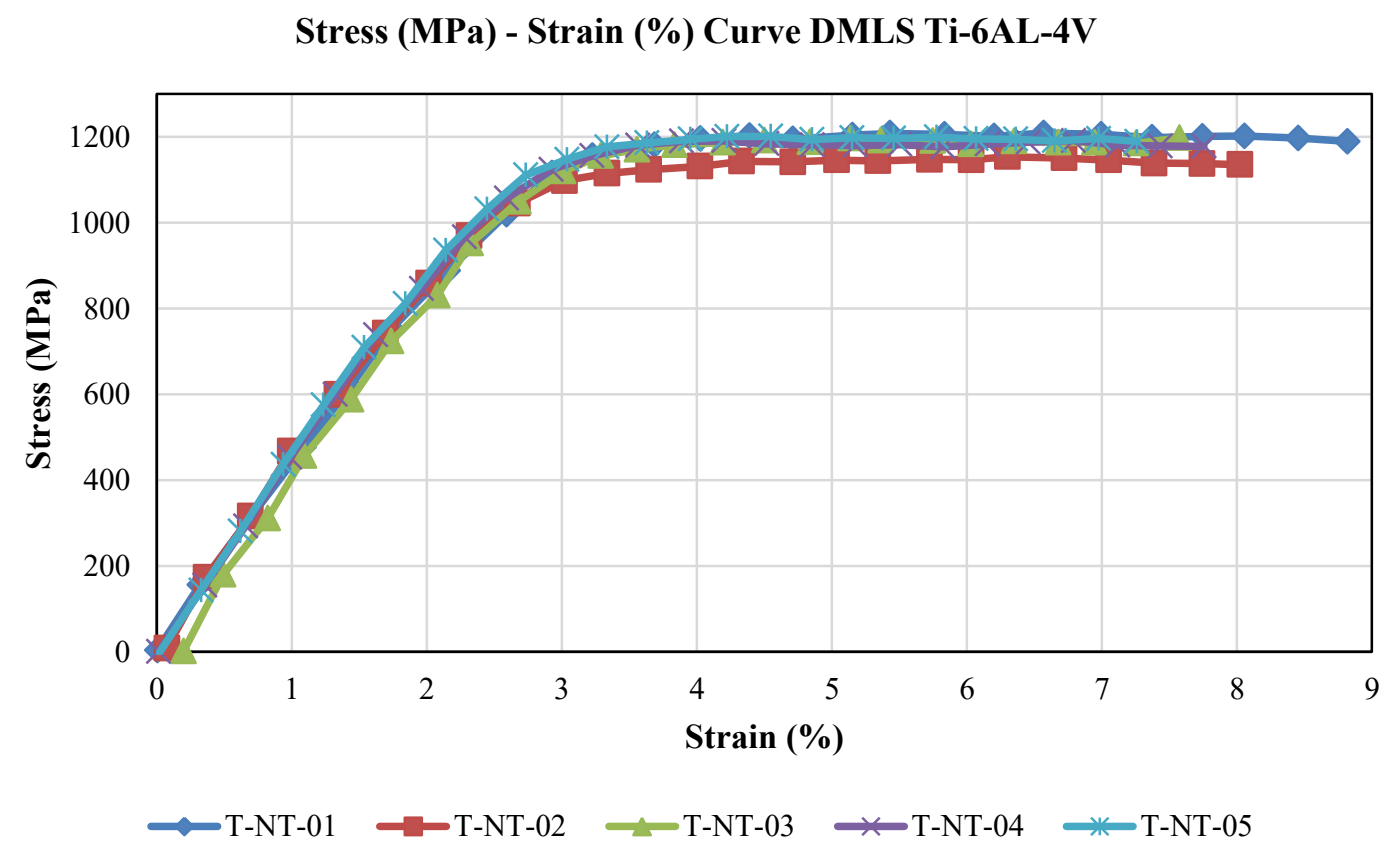

Figure 10 : Engineering Stress (MPa) - Strain (\%) Curve DMLS Ti-6AL-4V

A summary of the experimentally derived material properties are displayed in the next table: 
Table 4 : Material Properties Summary

\begin{tabular}{|l|c|c|}
\hline \multicolumn{1}{|c|}{ Property } & Value & Standard Error \\
\hline Elastic (Young's) Modulus (GPa) & 113.58 & 5.7 \\
\hline Yield Strength (0.2\%) (Mpa) & 1077.09 & 26.69 \\
\hline Ultimate Tensile Strength (Mpa) & 1209.92 & 23.38 \\
\hline Yield Point Strain & $2.71 \mathrm{e}-2$ & $1.26 \mathrm{e}-3$ \\
\hline
\end{tabular}

\subsubsection{Material Property Calculations}

The young's modulus of elasticity, yield strength and ultimate yield strength are derived in this section. First to be calculated is the ultimate tensile strength, followed by the modulus and then the yield strength properties. The ultimate yield strength for each specimen is calculated by taking the maximum force registered by the MTS and then dividing it by the original cross-sectional area. The equation below is applied:

$$
\sigma_{U T S}=\frac{F_{\text {max }}}{A_{\text {original }}}
$$

where $F_{\text {max }}$ is the maximum force and $A_{\text {original }}$ is the original cross-sectional area of the tensile specimen at the gauge area.

The data for the maximum force and cross-sectional area is given in the table below for each tensile specimen.

Table 5 : Max Force and Cross-Sections

\begin{tabular}{|l|c|c||c|c|c||}
\hline & T-NT-01 & T-NT-02 & T-NT-03 & T-NT-04 & T-NT-05 \\
\hline \hline Maximum Force (kN) & 23.19 & 22.74 & 23.29 & 22.92 & 22.88 \\
\hline Cross-Sectional Area (mm $\left.{ }^{2}\right)$ & 18.92 & 19.44 & 19.16 & 18.88 & 18.68 \\
\hline Ultimate Yield Strength (Mpa) & 1225.92 & 1169.3 & 1215.08 & 1213.96 & 1225.32 \\
\hline
\end{tabular}

The ultimate yield stress was then calculated to be on average 1209.92 Mpa. 
The modulus of elasticity was calculated from the slope of the engineering stress to engineering strain in the elastic region. This region was assumed to be within a displacement of $0 \mathrm{~mm}$ to $0.75 \mathrm{~mm}$. This is assumed due to the shape of the Stress-Strain graph. The displacement data was converted to strain using the equation below:

$$
\varepsilon=\frac{L_{\text {original }}-L_{\text {final }}}{L_{\text {original }}}=\frac{\Delta L}{L_{\text {original }}}
$$

where $L_{\text {original }}$ is the original length of the tensile specimens reduced section and $\Delta L$ is the difference between the new length and the original length.

The engineering stress is calculated in the elastic region with the proceeding equation:

$$
\sigma=\frac{F}{A_{\text {original }}}
$$

Where $\mathrm{F}$ is the measured force and $A_{\text {original }}$ is the original cross section.

The modulus is calculated from the following relation:

$$
E=\frac{\sigma}{\varepsilon}=\frac{\Delta \sigma}{\Delta \varepsilon}
$$

where $\sigma$ is stress and $\varepsilon$ is the strain.

The modulus values are then obtained from linear regression of the engineering stress to strain graph. The slopes of the elastic regions where then used to determine the yield strengths. An alternative method to calculate the modulus is to find the slope between the force and then displacements and then multiply the slope by the ratio of the original length to the original area. The elastic range was taken to be between displacements of $0 \mathrm{~mm}$ to $0.71 \mathrm{~mm}$. The modulus as a result of the raw strain data is displayed in Table 6 . 
Table 6 : Young's Modulus Results from Raw MTS Strain Data

\begin{tabular}{|l|c|c|c|c||c|}
\hline & T-NT-01 & T-NT-02 & T-NT-03 & T-NT-04 & T-NT-05 \\
\hline \hline Elastic Modulus (GPa) & 115.09 & 111.18 & 106.02 & 113.95 & 121.63 \\
\hline
\end{tabular}

The moduli have an average of $113.58 \mathrm{GPa}$. This value is close to the company rating for elastic stiffness and to other modulus measurements for titanium alloys in the literature [140]. The yield strength is found using the offset method. The yield strength is calculated by offsetting the modulus equation by $0.2 \%$ strain. The intercept with the stress-strain curve will determine the yield strength. A Savitzky-Golay filter was passed through the force data to remove noise $[141,142]$. The order of the Savitzky-Golay filter was set to 3 with a frame length of 5 . The results for the yield strengths and yield point elongations are given in the table below:

Table 7 : Yield Strength and Yield Elongation

\begin{tabular}{|l|c|c|c|c|c||}
\hline & T-NT-01 & T-NT-02 & T-NT-03 & T-NT-04 & T-NT-05 \\
\hline \hline Yield Strength (Mpa) & 1061.34 & 1044.48 & 1114.44 & 1076.14 & 1089.03 \\
\hline Yield Point Strain & $2.72 \mathrm{e}-2$ & $2.60 \mathrm{e}-2$ & $2.92 \mathrm{e}-2$ & $2.64 \mathrm{e}-2$ & $2.66 \mathrm{e}-2$ \\
\hline
\end{tabular}

The average yield strength was calculated to be $1077.09 \mathrm{MPa}$. The average strain to yield was 2.71e-2.

\subsubsection{Material Property Discussion}

The curve in Figure 10 shows that minimal necking occurs in the plastic region of the stress-strain curve before fracture. The plastic flow region was also seen to be very large which suggests the DMLS Ti-6AL-4V is a ductile material [13]. The engineering stress and strain graphs also showed that the material can withstand large amounts of energy as the area under the curve is quite large. 
The young's modulus; when the raw strain measurements were used gave results that were close to those of the literature. The error for the derived modulus value was less than one percent when compared to the company rating. The yield and ultimate tensile strength were also measured to be on average $10 \%$ higher. Errors for data collection may be caused by the strain rate chosen and the alignment of the tensile specimen with the clamps/grips. The first sample had a higher UTS compared to the others and had a different strain rate. This means that the rate of pull significantly affects the results. Nevertheless, the yield strength of the material is very high and also has good ductility which will be beneficial for engineering designs that aim to minimize mass.

\subsection{Untreated and UIT Treated Fatigue Behavior of DMLS Ti-6AL-4V}

It has been previously stated in this manuscript that the fatigue properties for additively manufactured metals have been known to have poor fatigue behavior. To enhance the fatigue behavior of DMLS Ti-6AL-4V; UIT will be applied to the surface of the material. The compressive strength will increase the fatigue life of the material. It is then crucial to characterize the fatigue behavior of the material before and after the surface treatment to properly gauge the effects of UIT. This section will explain the methods and calculations for two SN curves, one treated and an untreated curve. The two curves will then be used to compare the effects of UIT on multi-scale design optimizations.

\subsubsection{Experimental Methodology Overview}

In order to develop S-N curves for both the untreated Titanium and UIT treated titanium materials, a device would need to be built to properly apply the UIT treatment. It is also 
imperative that the surface treatment be as uniform possible as uneven treatments will cause large scatter.

The samples would also need to be prepared for fatigue testing. Many samples had scratches and notches which would cause erratic fatigue lives at equal cyclic stresses. Milling the sides of the specimens helped remove theses imperfections. The milling would also improve the metal performance due to hardening of the cut surface however there was no other alternative. Figure 11 shows the clean-up procedure on the fatigue specimens. Figure 11A shows the side of the sample were the support structure where grown and is significantly rougher than the top section in Figure 11B.

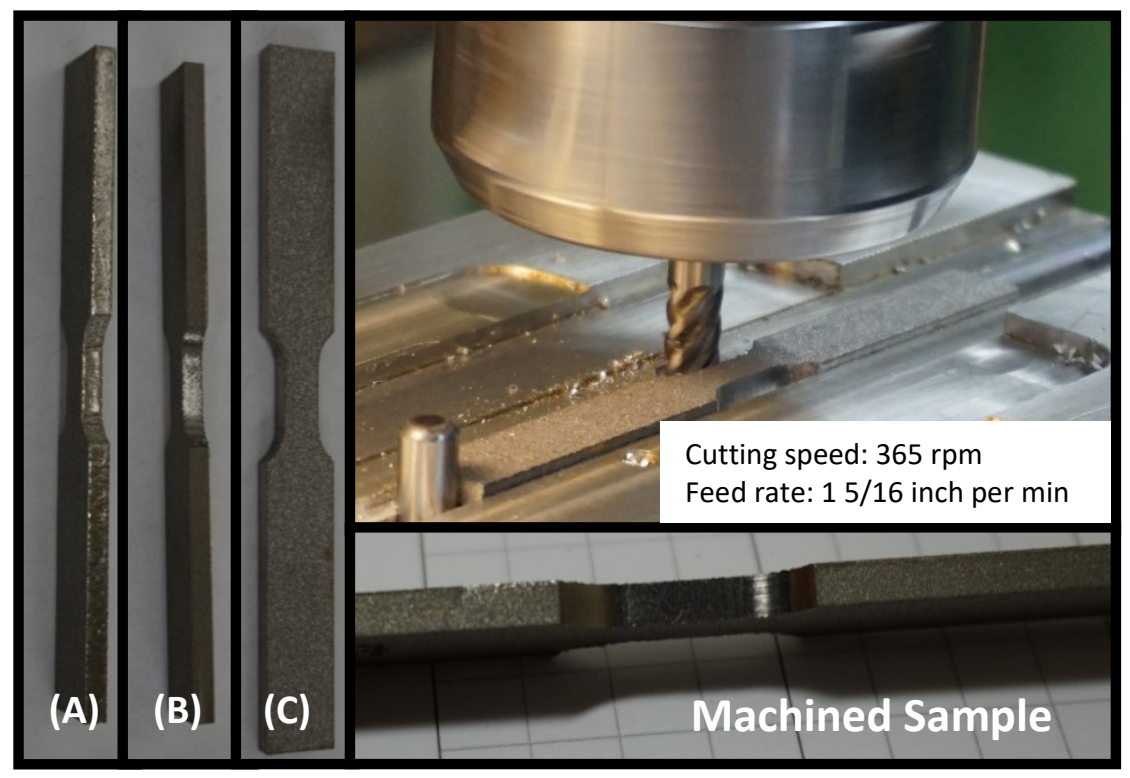

Figure 11 : Machining Fatigue Specimens (A) Pristine Sample Side Bottom (B) Pristine Sample Side Top (C) Machined Sample

It was decided that the application device to be a spring loaded system and that the UIT appliance would be attached to a $\mathrm{CNC}$ machine. The titanium samples were placed on the 
spring loaded device to which the CNC machine would control the UIT appliance along a scanning path and press down on the spring loaded system to apply the treatment evenly. Eighteen samples will be used to determine the SN curve for the untreated case and nine treated samples where used for the optimal fatigue curve. A batch of three to four samples were used for each tested stress level and the logarithmic average was taken for a mean fatigue life at each data point. The pearl string method was then used to obtain the S-N curves following the standards of the DIN 5100.

The SN curve will contain both the high cycle regime and the low cycle's regime. The high cycles will be found from fatigue tests to determine the slope of the Basquin curve. The low cycle regime is found from the previous tensile test data where the failure at one cycle is the ultimate tensile strength. The transition point between the low cycle regime and the high cycle regime will be at $10^{4}$ cycles.

\subsubsection{UIT Device and Application}

The UIT device displayed in Figure 13a is typically used as a hand-held tool for postwelding processing. It is equipped with slots for 4 impactors, but for the purposes of this experiment, only one impactor was used as illustrated in Figure $12 \mathrm{~b}$. To provide

automated control, a custom-built fixation is used to attach the device to the spindle of a $\mathrm{CNC}$ Mill. Figure 13c shows the treatment path programmed to minimize the surface roughness while obtaining a uniformly deformed surface. 
a)

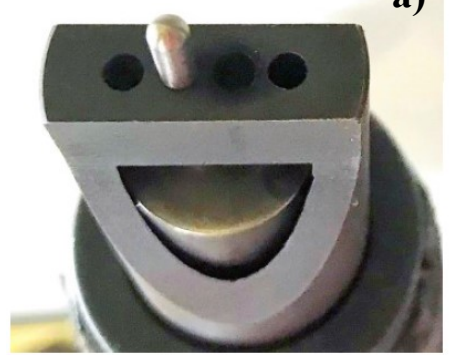

b)

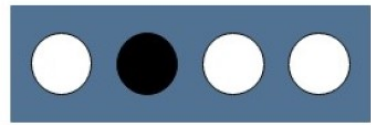

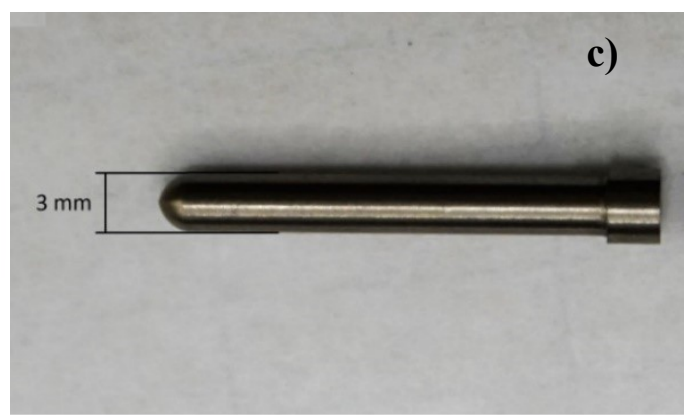

Figure 12 : Impact Needle Position in UIT Device

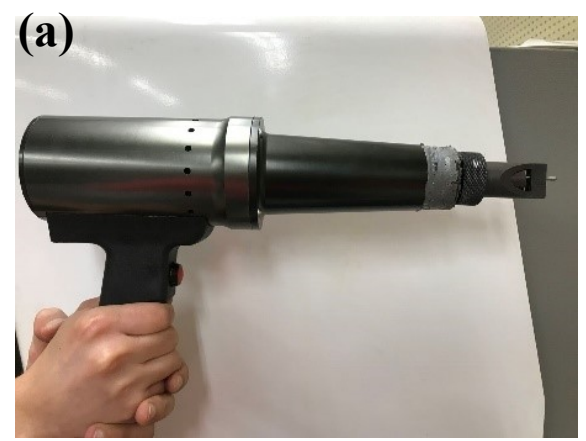

(b)

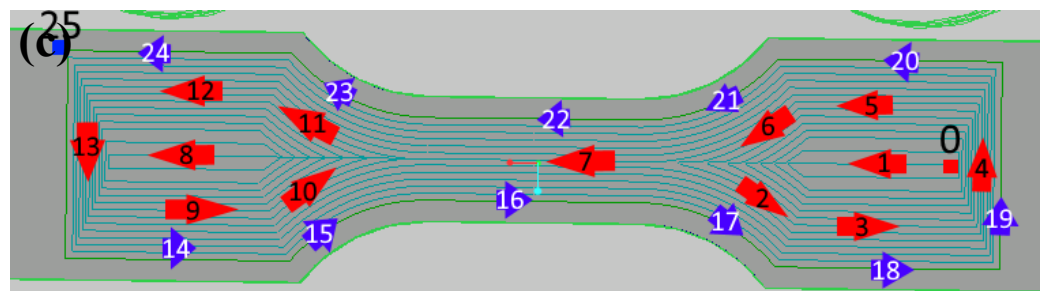

(d)
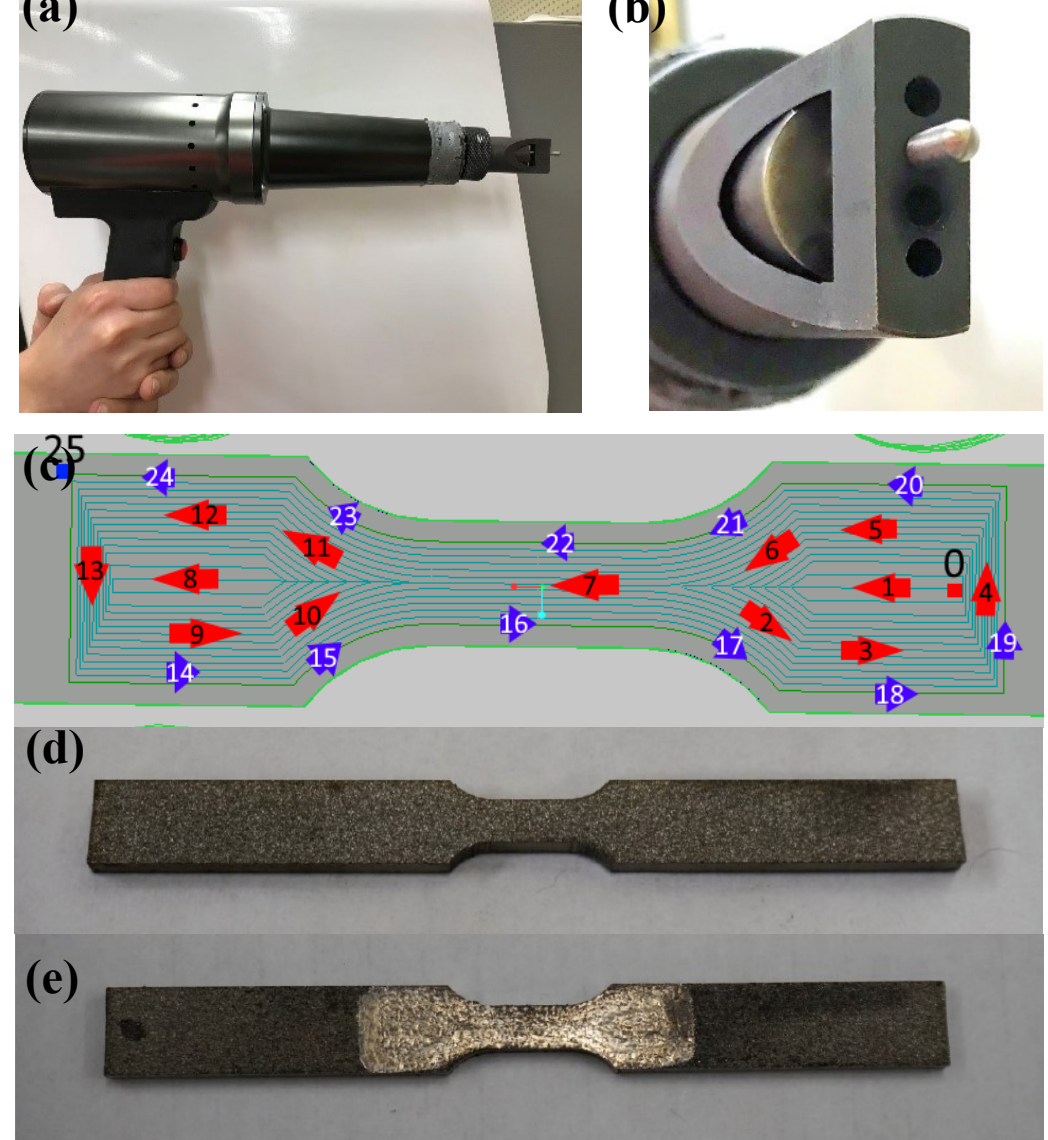

Figure 13: (a) Side View (b) Front View of UIT device (c) Schematic of treatment path astarting point, \ inner local spirals, outer global spiral, a end point (d) Pristine Machined Sample (e) UIT Machined Sample

The scanning speed of the CNC machine was set to $1000 \mathrm{~mm} / \mathrm{min}$. This speed was chosen as it was deemed to be to best speed for optimal coverage for the treatment from 
previous experiments and testing. The experimental procedure for the scan speed is given in the Appendix D . Each line represents a scan path. The spacing between the scans is known as an interval. Amplitude control of the device is controlled by a control box. The amplitude was set to 57 percent of 40 micrometers during testing [11]. The interval for treating titanium alloys typically ranges between 10 and $70 \mu \mathrm{m}[143,144,4]$. The path contours were chosen to increase outwards at $71.1 \mu \mathrm{m}$ intervals to match previous efforts and research into surface treatments on titanium [122]. In other words the spacing's in Figure 13C are constant.

The teal pattern in Figure 13c is the programmed treatment path followed by the device's indenter/needle. Impact begins at the red arrow " 1 " and ends at the blue square " 25 ". Each line represents a scan path and the spacing between the scans is referred to as the interval. The spiral shape of the scanning path was designed to allow the material to spread evenly outwards from the center of the surface and to ensure a relatively even and symmetrical treatment. However, no research has been conducted to determine if the scanning path shape has any effect on surface quality or distribution of stresses at the surface [11]. The influence of the scanning path will be outside of the scope of this thesis.

\subsubsection{Ti-6AL-4V DMLS Sample Dimensions}

Flat sheet fatigue specimens were manufactured in accordance to ASTM E606 for strain fatigue testing, taking in account the critical length that prevents the jaws of the material test system (MTS) from colliding. 


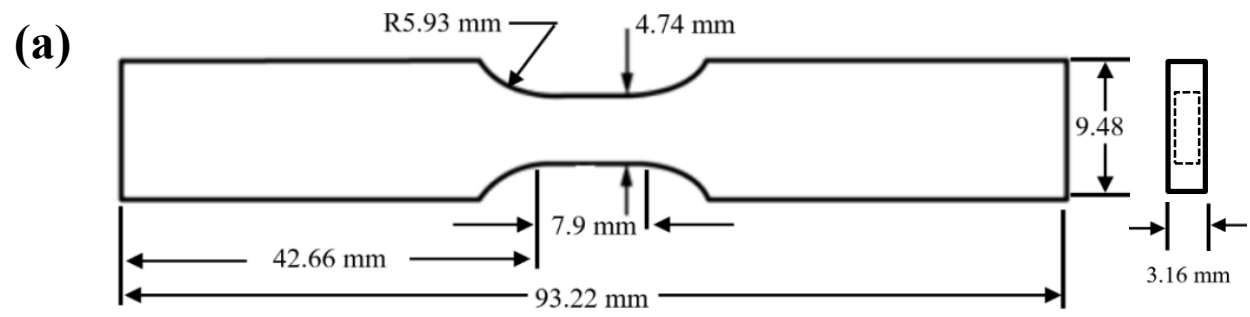

(b)

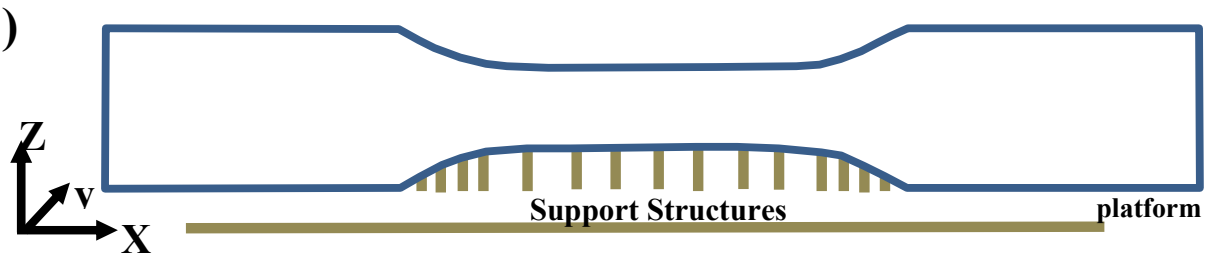

Figure 14 : (a) Schematic of flat sheet fatigue specimen with rectangular cross-section (b) Orientation of the specimens during DMLS manufacturing process

Figure 14a illustrates the dimensions of the dog bone in millimeters. The thickness for the fatigue specimens is the same as the tensile specimens. Figure $14 \mathrm{~b}$ displays the build orientation and the location of the support structures.

\subsubsection{Specimen Fixture}

To apply UIT, the Ti-6Al-4V specimens were clamped to an aluminum plate that is supported by two steel rods and two linear bearings as illustrated in Figure 15a. The plate can freely slide along the rods and its motion range is limited to two springs placed between the plate and the end of the supporting rods. Compressing the springs allows for a constant static force to be applied onto the samples during treatment. By pushing the UIT device into the plate and compressing the springs a certain distance, the amount of static force can be determined. Aluminum cutting fluid was used to lubricate the rods so that the plate could freely move by the springs. For treating the titanium specimens, a static force of $30 \mathrm{~N}$ was applied. This force was selected as it was seen to give the optimal 
performance increase and coverage of the treatment when combined with a scanning speed of $1000 \mathrm{~mm} / \mathrm{min}$. Selecting the best force setting was determined through experimentation and is shown in Appendix D .

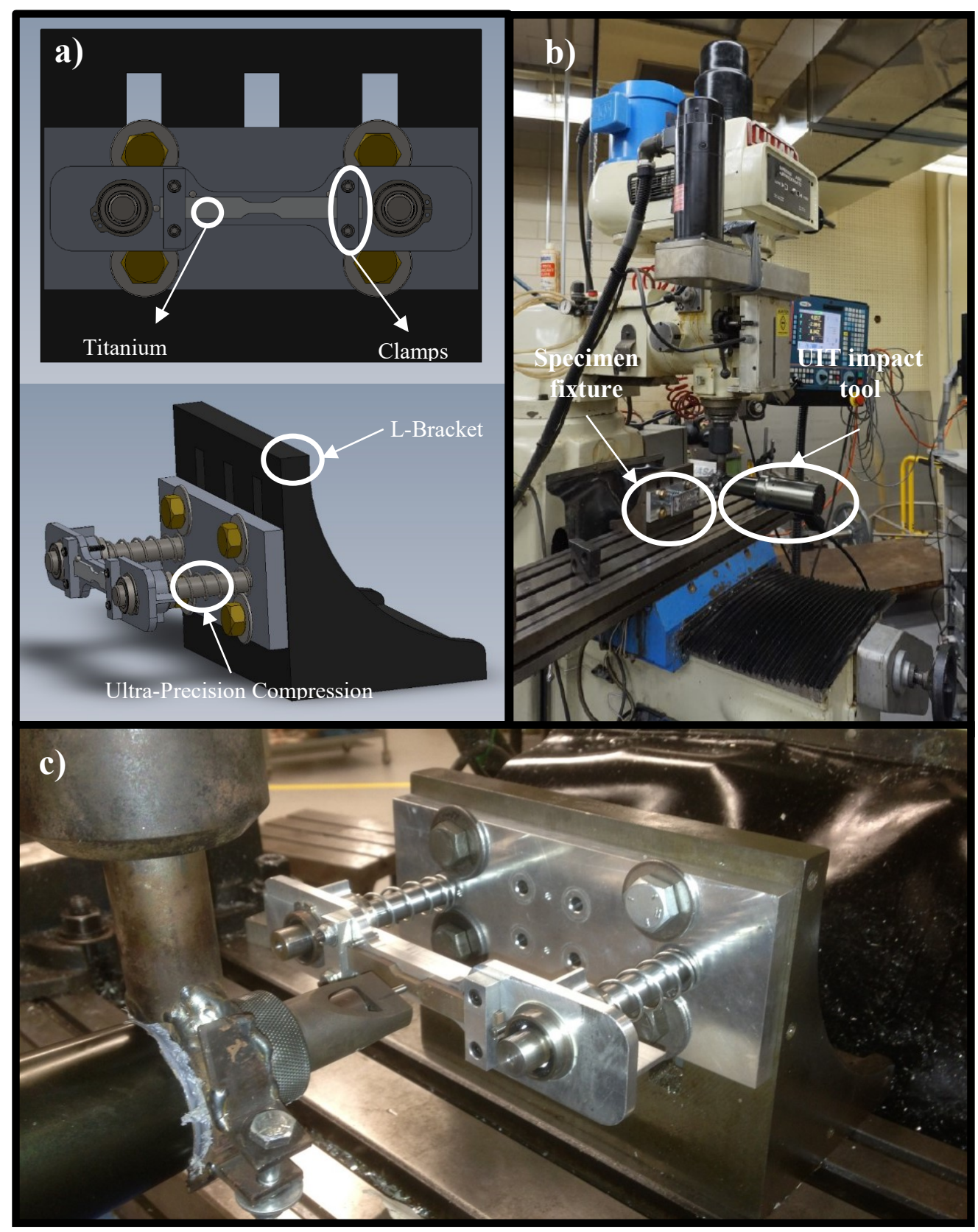

Figure 15: (a) CAD of UIT Fixture Set-Up (b) CNC machine and Fixture Assembly (c) Close up of assembly 


\subsubsection{Fatigue Testing}

Fatigue tests were undertaken with a servo-hydraulic 810 Material Test System (max. load $100 \mathrm{kN}$ ), MTS [139]. Fatigue testes followed the standards of the DIN 50100 [145] at The untreated S-N curve was built using 6 different stress amplitudes; 75Mpa, $100 \mathrm{MPa}, 125 \mathrm{MPa}, 150 \mathrm{MPa}, 175 \mathrm{MPa}$ and $200 \mathrm{MPa}$. The treated $\mathrm{S}-\mathrm{N}$ curve used $550 \mathrm{MPa}$, 400MPa and 250Mpa stress levels to assemble the curve. The chosen $\mathrm{R}$ value was 0 for each of the fatigue tests. A short tapered sinusoidal waveform with the amplitude rising to the maximum load was chosen for the first few cycles to smoothen the starting process of the MTS. According to DIN 50100, the load cycles belonging to the tapered sinus pattern up to a difference of $1.5 \%$ of the load amplitude and the last cycle right before the fracture were subtracted from the total number of cycles. All fatigue tests were conducted at a temperature range of 20.4 to $24.2{ }^{\circ} \mathrm{C}$ and a humidity range of 17 to $68 \%$. UIT specimens were also measured before and after UIT treatment to evaluate the extent of the surface deformations. The grip sections of the specimens were clamped in the clamping jaws with a pressure of $800 \mathrm{psi}(5.516 \mathrm{MPa})$ and tested with a constant frequency of $25 \mathrm{~Hz}$.

\subsubsection{SN Curve Calculations}

The stress-life curves for the additively manufactured titanium material were constructed based on the standards of the DIN 50100 [145]. The pearl string method was used to determine the S-N curve for the untreated specimens. The mean for the number of cycles to failure for a given stress value are the primary values extracted by the fatigue 
experiments. A total of 16 untreated fatigue specimens were used to acquire the untreated S-N curve. Nine treated specimens were used to derive the treated S-N curve.

Because of the lack of empirical values for the DLMS Ti-6Al-4V in terms of fatigue life estimation through experimentation; the standard deviation of the population $\left(S_{\log \varpi, G G}\right)$ and the quotient of the means (distance of the load levels in the direction of the number of cycles, $\frac{\varpi_{50 \%, 2}}{\varpi_{50 \%, 1}}$ were initially assumed to be 0.2 and 50 , respectively. The value $\varpi_{50 \%, 1}$ is equal to 20000 load cycles and $\varpi_{50 \%, 2}$ is equal to 1 million load cycles. The methodology of the treated curve follows the same steps as the untreated stress-life curve but modified for 9 samples $(h)$ instead of 16 .

Due to having 16 untreated specimens for characterizing the untreated S-N curve, the scatter band of the mean-value $\left(\mathrm{T}_{\mathrm{m}}\right)$ was estimated to be 1.745 . While the permissible error band for the number of specimens required for an estimation of the mean $\varpi_{50 \%, G G}$ with assumed population standard deviation of 0.20 and an $80 \%$ confidence interval is between $24.3 \%$ and $32.1 \%$. Assuming $\varpi_{50 \%, G G}$ is equal to 100000 cycles, then $80 \%$ of the sample means should be expected to be between 75700 and 132100 load cycles.

An assumed logarithmic normal distribution was used to develop the slopes of the S-N curves. The general formula for logarithmic regression is represented in equation 4.5:

$$
\log \varpi=\log \mathfrak{C}-\mathcal{K} \cdot \log \sigma_{a}
$$

where $\varpi$ is the number of cycles to failure, $\sigma_{a}$ is the stress amplitude, $\mathcal{K}$ is the slope of the stress-life curve and $\mathfrak{C}$ is a constant for the description of the position of the High Cycles Fatigue (HCF) S-N curve at $\varpi=1$ cycles. The methodology 
for deriving the equation constants is shown in the Appendix C . Calculating the standard deviation was done by shifting every single test result to the same common load level $F_{a, f i c t}$, or accordingly stress level $\sigma_{a, f i c t}$, with the number of load cycles $\varpi_{i, f i c t}$, based on the assumption that the standard deviation at each load level $i$ is identical. Shifting is carried out parallel to the beforehand obtained regression line.

$$
\varpi_{i, f i c t}=\varpi_{i} \cdot\left(\frac{F_{a, f i c t}}{F_{a, i}}\right)^{-\mathcal{K}}
$$

where $\varpi_{i, f i c t}$ is the number of load cycles at some fictitious load level, $\varpi_{i}$ is the number of cycles at some common level, $F_{a, f i c t}$ and $F_{a, i}$ are the fictitious and common load levels, respectively and $k$ is the slope of the stress-life curve.

The mean of the number of load cycles is calculated with equation (4.7).

$$
\varpi_{50 \%, f i c t}=\frac{1}{n} \sum \log \left(\varpi_{i, f i c t}\right)
$$

where $N_{50 \% \text {,fict }}$ is the mean number of cycles to failure at $F_{a, f i c t}$, a fictitious applied load. Thus, estimating the standard deviation corrected for small sample sizes will use equation (4.8).

$$
\tilde{s}_{\log N}=\frac{h-1.74}{h-2} \cdot \sqrt{\frac{1}{h-2} \cdot \sum\left(\log \left(\varpi_{i, f i c t}\right)-\log \left(\varpi_{50 \%, f i c t}\right)\right)^{2}}
$$

where $\tilde{s}_{\log N}$, is the standard deviation and $h$ is the number of samples being tested.

Finally, the scatter band, which is the ratio of the number of cycles at a failure probability of $90 \%$, to the failure probability of $10 \%\left(\varpi_{10 \%}\right)$ is then calculated with equation (3.9). 


$$
\mathrm{T}_{\mathrm{N}}=\frac{\varpi_{90 \%}}{\varpi_{10 \%}}=10^{2.564 \cdot \tilde{s}_{\log N}}
$$

where $T_{N}$ is the scatter band, $\varpi_{90 \%}$ is the number cycles at $90 \%$ probability of failure and $\varpi_{10 \%}$ the number of cycles at $10 \%$ probability of failure. The failure band results and calculation methods are presented in Appendix C .

It is important to have reliable SN-curves so that designs are safe and trustworthy. Fatigue failure is expected to occur as a function of a logarithmic distribution along the SN-curve. The probability of failure is equal to $50 \%$ along the regression line or at the center of the scatter band. For a given stress level, the probability of failure increases as the number of cycles to failure increases. To ensure the predicted lives are conservative and to remove uncertainty; an offset is applied to the regression line equal to at least two standard deviations from the mean. The standard deviations are those calculated from $\tilde{S}_{\log N}$ (equation (4.8)).

A survivability rating of $99.99 \%$ is chosen to calculate the final SN-Curve. Survivability is the inverse of the probability to failure and determines the distance of the downward (or upward) shift of the regression line. Both the un-shifted SN curve and modified SN curve are developed, compared and shown in Figure 16. This will allow the designer to choose an appropriate safety factor for their design without worrying about unintentionally over designing. Calculations to shift the regression line are shown in Appendix C .

The Basquin equation will also be derived from the logarithm regression. This is because the optimization workflow uses the Basquin equation to calculate the life. Both the low 
cycle regime and high cycle regime curves will be developed. The low cycle is created by connecting the ultimate tensile strength to the beginning of the high cycle regime. The Basquin equation for life prediction is represented by the formula below:

$$
\varpi=\left(\frac{\sigma_{a}}{\sigma_{S N 1}}\right)^{1 / b}
$$

where $\sigma_{S N 1}$ is the intercept on the $\mathrm{S}-\mathrm{N}$ curve stress axis. The Basquin equation can be found from the logarithmic equation be rearranging the terms:

$$
\begin{gathered}
\mathrm{b}=1 /-\mathcal{K} \\
\sigma_{S N 1}=\mathfrak{C}^{-b}
\end{gathered}
$$

Taking the inverse of both sides gives the Basquin equation as used in the multi-scale design workflow:

$$
\varpi=\left(\frac{\sigma_{a}}{\left(\mathfrak{c} \frac{1}{\mathcal{K}}\right.}\right)^{-\mathcal{K}}
$$

\subsubsection{Final SN Curve}

The combined curves of the approximated low cycle regime and the high cycle regime for the DMLS Ti-6AL-4V metal are given in Figure 16. The low cycle regime starts at 1 cycle with the alternating stress equal to the ultimate tensile strength and ends at $10^{4}$ cycles. The ultimate tensile strength is the same for both SN curves because it is not assumed that the mechanical properties (stiffness) are affected by surface treatments. The endurance limit increased by $25 \%$. On average, a $200 \%$ increase in fatigue life across all stress levels can be seen by comparing the untreated line to the treated S-N curve. The experimentally driven relationships for both stress-life curves are presented in Table 8; 
Table 8: S-N Curve Logarithmic Regression Equations (99.99\% Survivability)

\begin{tabular}{|l|c|c||}
\hline \multicolumn{1}{|c|}{ Curve } & Equation(s) & $\begin{array}{c}\text { Knee Point of Max } \\
\text { Stress (MPa) }\end{array}$ \\
\hline \hline Untreated & $\log \varpi=\log \left(1.01133 \cdot 10^{14}\right)-3.7836 \cdot \log \sigma_{a}$ & 200 \\
\hline Treated & $\log \varpi=\log \left(3.85033 \cdot 10^{15}\right)-4.22 \cdot \log \sigma_{a}$ & 250 \\
\hline
\end{tabular}

The scatter band TN from the test results of the untreated and treated curves are 2.458 and 2.613 respectively. Lower stress ratios will have longer fatigue lives for specimens due to lower peak tensile stresses. There was much larger scatter in the treated samples, possibly due to slightly different treatments and initial residual stresses in the fatigue specimens. Another cause of scatter is fluctuations in humidity, which is known for influencing the fatigue strength behavior [146]. Additional causes of scatter are also due to uneven geometries in the tensile specimens. It is also noted that very few samples were tested in the high cycle region for the endurance limit. This is because of an oversight for the number of needed samples to test. The equivalent Basquin curve equations for the high cycle regime are given in Table 9.

Table 9 : Equivalent Basquin Curve Equations (99.99\% Survivability)

\begin{tabular}{|c||c||c|}
\hline Curve & Equation(s) & $\begin{array}{c}\text { Knee Point of Max } \\
\text { Stress (MPa) }\end{array}$ \\
\hline \hline Untreated & $\varpi=\left(\frac{\sigma_{a}}{5028.94}\right)^{-3.7836}$ & 200 \\
\hline Treated & $\sigma_{a}=5028.94(\varpi)^{-0.2643}$ & \\
\hline & $\varpi=\left(\frac{\sigma_{a}}{4923.34}\right)^{-4.22}$ & 250 \\
\hline
\end{tabular}


S-N Curve by Logarithmic Regression (DIN5100) - DMLS Ti-

6Al-4V - $(R=0)$ - (Survivability \%)

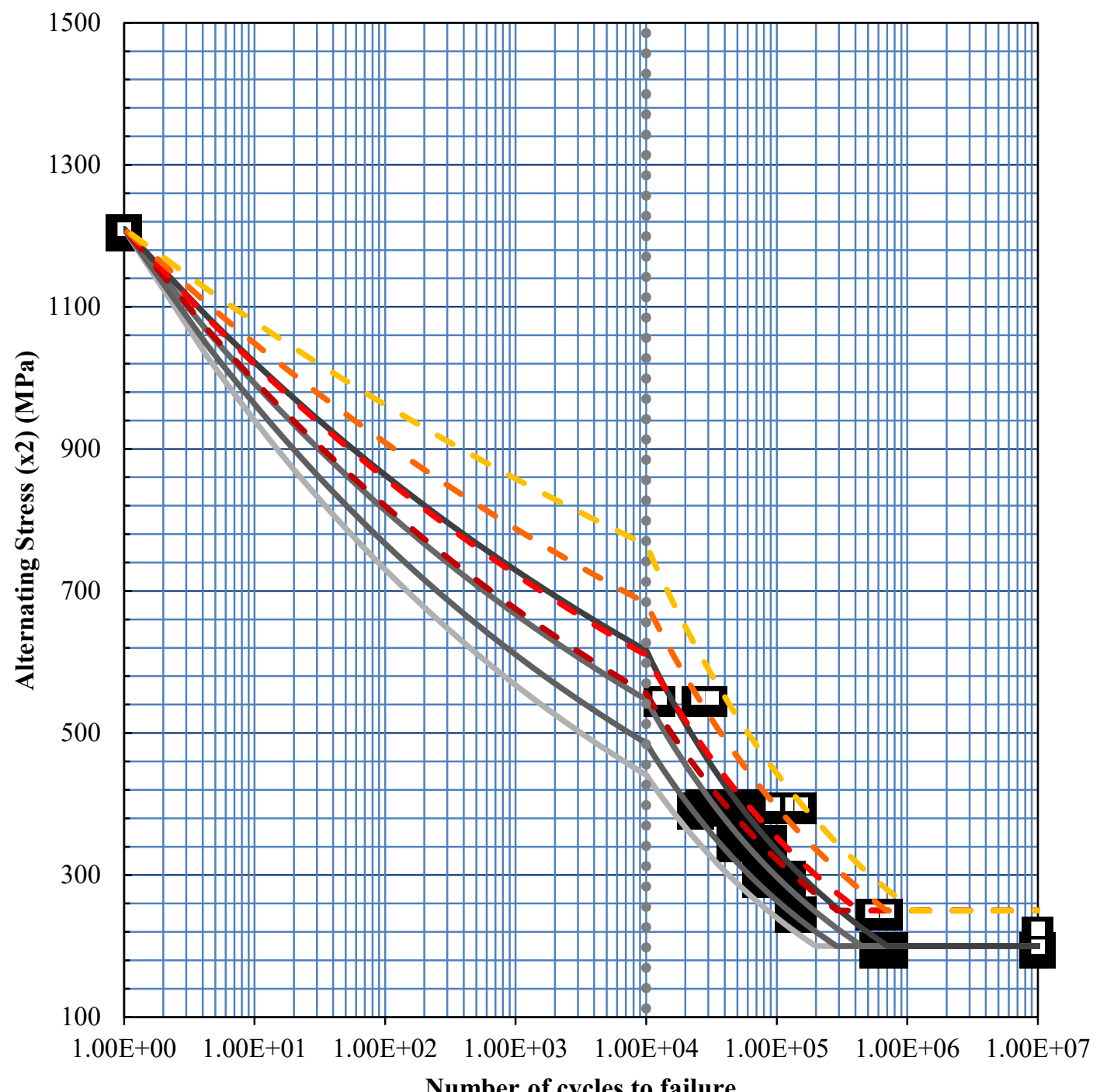

$\begin{array}{ll}\text { Untreated Data } & \square \quad \text { Treated Data } \\ \text { Low to High Cycle Transition } & \\ -90 \% \text { Untreated } & -50 \% \text { Untreated } \\ 90 \% \text { Unted } & -59.99 \% \text { Treated } \\ 10 \% \text { Untreated } & -\quad-90 \% \text { Treated } \\ \text { Transition Point } & -\quad-10 \% \text { Treated }\end{array}$

Figure 16: Logarithmic Regression SN Curve $(R=0)$ for Treated and Untreated DMLS Ti-6AL-4V A 


\section{S-N Curve by Logarithmic Regression (DIN5100) - DMLS Ti- 6Al-4V - (R=0) - (Survivability \%)}

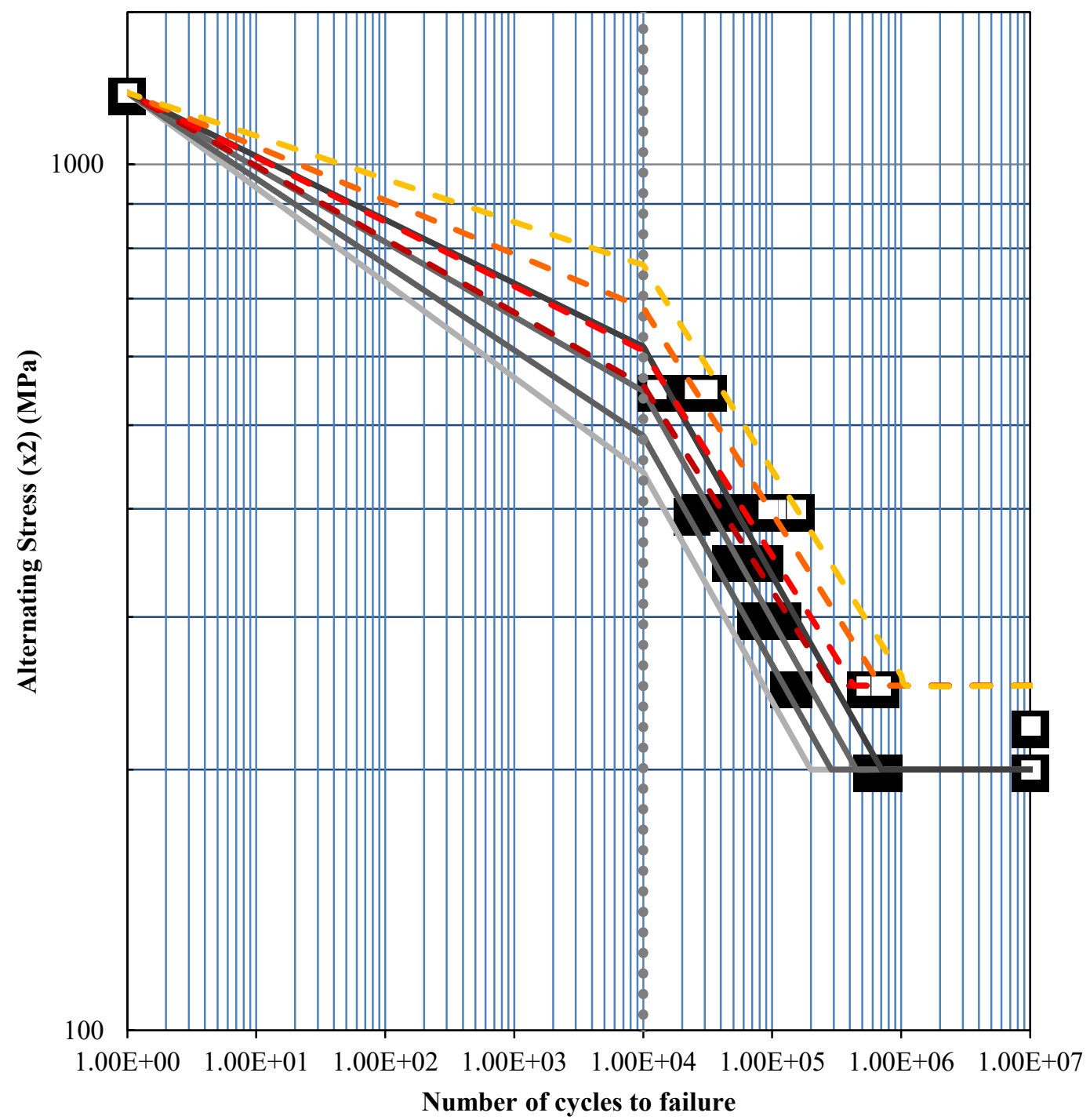

\begin{tabular}{ll}
- Untreated Data & $\square \quad$ Treated Data \\
Low to High Cycle Transition & $-99.99 \%$ Untreated \\
\hline $90 \%$ Untreated & $-50 \%$ Untreated \\
\hline 10\% Untreated & $-\quad 99.99 \%$ Treated \\
- Transition Point & $--90 \%$ Treated \\
$-50 \%$ Treated & $-\quad-10 \%$ Treated
\end{tabular}

Figure 17 : Logarithmic Regression SN Curve $(R=0)$ for Treated and Untreated DMLS Ti-6AL-4V B 


\subsection{Closing Statements}

This section presented experimental derivations of new material properties. The stiffness of the material was recorded to be $113 \mathrm{GPa}$. This was similar to the recorded modulus of the company. The ultimate tensile strength was measured to be $1209.92 \mathrm{MPa}$ and the yield strength was recorded at $1077 \mathrm{MPa}$. The results show promising material properties and for developing light-weight optimized topologies with high fatigue failure resistance. The fatigue properties were poor for the untreated specimens. UIT proved to increase the endurance limit by $25 \%$. The high survivability chosen for the SN curves limits the amount of time inside the high cycle fatigue; however the low cycle lifetime will be omitted during topology and sizing optimizations. The UIT surface treatment will be able to save mass in the optimization section as will be seen later.

To illustrate safe design principles, a survivability rating for fatigue is presented in the final SN-Curve graphs. Due to the probabilistic nature of fatigue failure, a scatter band is calculated based on the standard deviation of the mean regression line. Failure is least likely to occur as you move further left from the mean regression line. A $99.99 \%$ survivability SN-Curve is calculated for both untreated and treated Ti-6AL-4V DMLS materials. The high survivability rate will also minimize the amount of uncertainty when predicting fatigue lives.

The high cycle regime assumes elastic deformation from the repeated loadings [13]; an highest alternating stress of $10^{4} \mathrm{MPa}$ is well below the yield stress and therefore validates the initial fatigue behavior assumptions given in Chapter 3.4.1. 


\section{Chapter 4: Asymptotic Homogenization}

Cellular materials possess many unique characteristics and capturing these qualities requires sophisticated techniques. Periodic structures are geometrically complex and computationally expensive to analyze. Averaging techniques are therefore essential to reduce computation time for optimizations while maintaining accuracy of the physical response of the material. This chapter will present the reader with information about asymptotic homogenization and its applications to topology optimization. The first section is an overview and literature review of asymptotic homogenization. The next section will go over the mathematical principles of the process to obtain relative properties for lattice materials. The last section will describe the interpolation method employed in the multi-scale workflow to update the relative properties during topology optimizations.

\subsection{Numerical Asymptotic Homogenization}

Numerical asymptotic homogenization is used to determine relative mechanical properties for unit cells of three-dimensional lattice truss structures. The process is explained in Figure 18. The relative properties are a function of the relative density. Hence for any given lattice truss structure, the correct macro mechanical properties can be extracted. These properties are interpolated from a table during lattice topology optimizations. Micro-scale information is passed to the macro-scale in a form of homogenized properties. The modulus and Poisson ratios are modified during the multi- 
objective topology optimizations. These properties are then used to find the optimal graded structure for maximum fatigue strength to weight ratio.

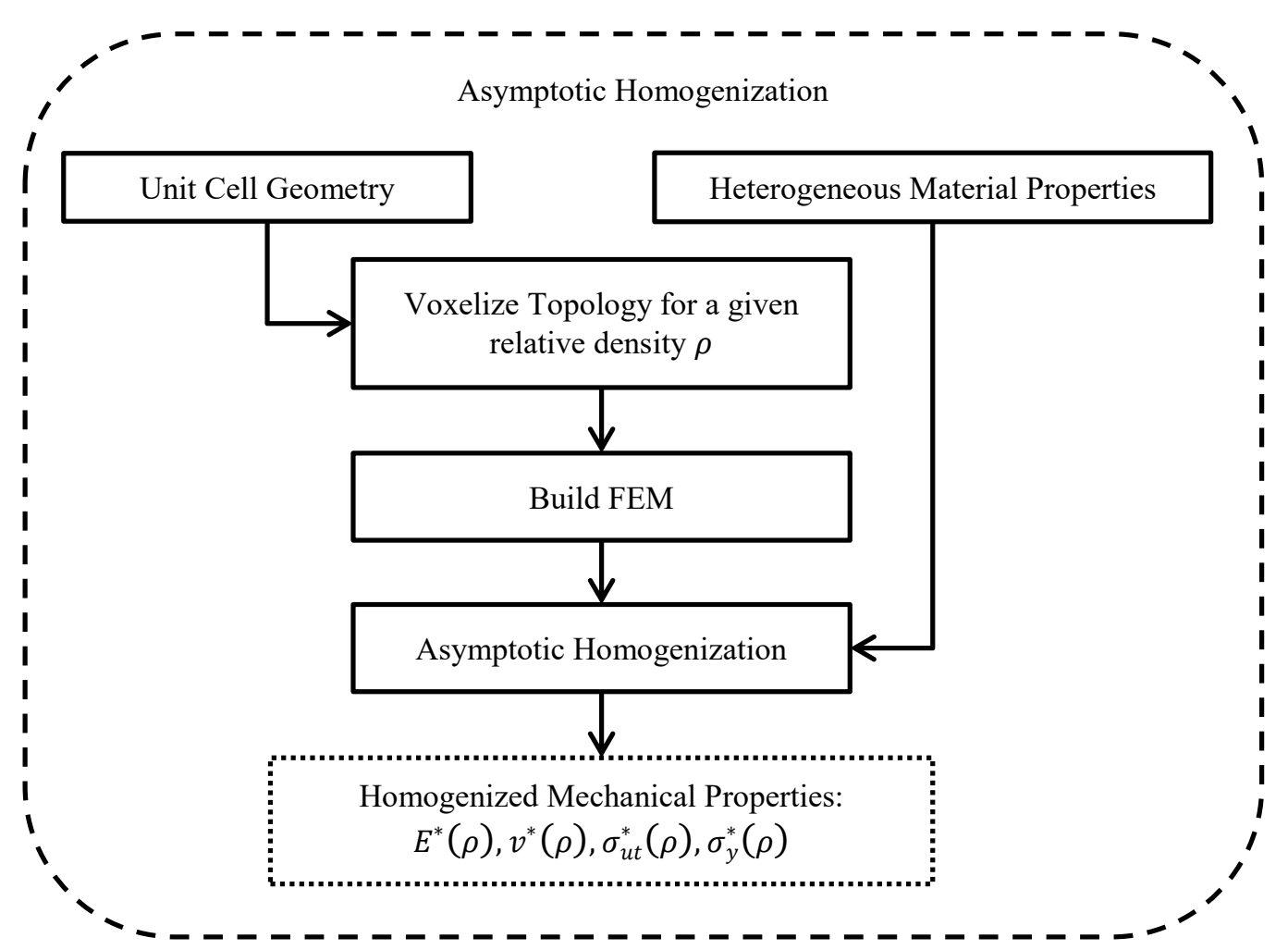

Figure 18 : Block Diagram for Asymptotic Homogenization Workflow

\subsection{Homogenization Method for Elastic Materials}

The theory of homogenization is related to the development of partial differential equations with non-constant coefficients [147]. Asymptotic homogenization assumes that each field quantity depends on two scale lengths, namely the microscopic (local) and macroscopic (global) levels [95]. For elastic materials, the displacements, strains, stiffness and stresses are assumed to vary smoothly at the global level while behave periodically at the local level [147]. The field quantity of a unit cell is assumed to be the 
superposition of macro scale quantity and a small periodic fluctuation [148]. The displacement field for example can be expanded into a power series of the form:

$$
\mathrm{U}_{\mathrm{i}}^{\mathcal{N}}\left(\chi_{\mathrm{i}}, \mathrm{y}_{\mathrm{i}}\right)=\mathrm{U}_{\mathrm{o}_{\mathrm{i}}}\left(\chi_{\mathrm{i}}, \mathrm{y}_{\mathrm{i}}\right)+\mathcal{N} \mathrm{U}_{1_{\mathrm{i}}}\left(\chi_{\mathrm{i}}, \mathrm{y}_{\mathrm{i}}\right)+\mathcal{N}^{2} \mathrm{U}_{2 \mathrm{i}}\left(\chi_{\mathrm{i}}, \mathrm{y}_{\mathrm{i}}\right)+\cdots, \quad \mathrm{y}_{\mathrm{i}}=\frac{\chi_{\mathrm{i}}}{\mathcal{N}}
$$

where $U_{i}^{\mathcal{N}}$ is the exact value of the field variable, $U_{o_{i}}$ is the macroscopic average value of the field variable, $\mathrm{U}_{1_{\mathrm{i}}}$ and $\mathrm{U}_{2_{\mathrm{i}}}$ are perturbations in the field variables due to the microstructure, $\chi$ is the global variable coordinate system, $y_{i}$ is the local coordinate system and $\eta$ is a scale factor or ratio for the size of the period and relates the global to the local levels.

Early developments in homogenization theory had found that the macroscopic displacements from the asymptotic expansion converge to an average displacement [149]. Other research indicated that as the local scale decreased relative to the global scale the mechanical behavior of the homogenized material more closely reflected the true behavior of the material [150]. Overall, the accuracy of the homogenization depends on $\mathcal{N}$ and the application of periodic boundary conditions.

Periodic properties are represented by a unit cell or RVE. The boundaries of the RVE are periodic $(\mathrm{PBC})$ and allow for the solutions of homogenized properties. The RVE allows for both micro-scale and macro-scale analysis by applying a solid mechanics approach to relate the coordinate systems together.

\subsection{Methodology}

The work of Dong G et al. [151] and Andreassen et al. [152] was adopted to define the homogenized properties of several lattice unit cells. The methodology of their work 
utilizes numerical methods of homogenization to solve for relative Lame's parameters. The relative modulus and Poisson ratio can then be extracted from the relative Lame values. Dong et al adapted their implementation from a two-dimensional homogenization formulation written by Andreassen and Andreasen to compute a homogenize elasticity tensor [152]. By obtaining the relative Lame's parameters for each relative density, it is possible to obtain the relative young's modulus, Poisson ratio and the shear modulus as well. Bendsoe defines the homogenized stiffness tensor by the integration over the area or volume of the RVE [69]. The general equation for this method is as follows:

$$
D_{i j k l}^{H}=\frac{1}{|V|} \int_{V_{s}} D_{i j p m} M_{p m k l} d V=\frac{1}{|V|} \int_{V} D_{p q r s}\left(\varepsilon_{p q}^{0(i j)}-\varepsilon_{p q}^{(i j)}\right)\left(\varepsilon_{r s}^{0(k l)}-\varepsilon_{r s}^{(k l)}\right) d V
$$

where $D_{\text {pqrs }}$ is the locally varying elasticity tensor, $M_{p m k l}$ is the local structure tensor, $|V|$ is the volume of the unit cell. $\varepsilon_{p q}^{0(i j)}$ are prescribed macroscopic (average) strain fields, while the locally varying strain fields $\varepsilon_{p q}^{(i j)}$ is defined as:

$$
\varepsilon_{p q}^{(i j)}=\varepsilon_{p q}\left(U^{i j}\right)=\frac{1}{2}\left(U_{p, q}^{i j}+U_{q, p}^{i j}\right)
$$

$U^{k l}$ is the displacement field which can be found by the assumption of equal strain energies. The displacement field is calculated by the following equation:

$$
\int_{V} D_{i j p q} \varepsilon_{i j}\left(\mathcal{V}_{\text {virtual }}\right) \varepsilon_{p q}\left(U^{k l}\right) d V=\int_{V} D_{i j p q} \varepsilon_{i j}\left(\mathcal{V}_{\text {virtual }}\right) \varepsilon_{p q}^{0(k l)} d V \quad \forall \mathcal{V}_{\text {virtual }} \in V
$$

where $\mathcal{V}_{\text {virtual }}$ is the virtual displacement field. 


\subsubsection{Relative Constitutive Matrix}

The elasticity tensor or constitutive matrix can be calculated by applying unit strain fields in each principle direction. Hence for a two-dimensional problem, three strain fields must be applied vertical, horizontal and shear stresses. A three-dimensional problem requires six strains to be applied and solved. The strains must be applied on the periodic boundary conditions. The macro displacements for each strain direction are found using the finite element method:

$$
K U^{k l}=F^{k l}
$$

where $U^{k l}$ is constrained to be periodic with respect to the RVE and $K$ is the global stiffness matrix. Periodicity can be enforced by a penalty approach, or from assigning equal node numbers to the opposing boundary nodes [69]. Multi-point constraints may also be used for periodicity.

The global stiffness matrix is the summation of the finite elements. In the implementation of Andreassen, the individual finite elements are constructed so that it is a linear combination of the lames parameters [152].

$$
K=\sum_{e=1}^{n} K_{e}=\sum_{e=1}^{n} \int_{V_{e}} B_{e}{ }^{T} D_{e} B_{e} d V_{e}
$$

where $K_{e}$ is the local stiffness matrix, $B_{e}$ is the strain displacement matrix for the element, $D_{e}$ is the constitutive matrix of the element. 
The force vector to solve for the macroscopic displacements or volumetric straining can be calculated from:

$$
F^{k l}=\sum_{e=1}^{n} \int_{V_{e}} B_{e}^{T} D_{e} \bar{\varepsilon}^{k l} d V_{e}
$$

For two dimensional problems, the strains $\overline{\varepsilon^{k l}}$ are selected to be:

$$
\varepsilon_{1}^{k l}=\varepsilon_{11}=\left[\begin{array}{lll}
1 & 0 & 0
\end{array}\right]^{T}, \quad \varepsilon_{2}^{k l}=\varepsilon_{22}=\left[\begin{array}{lll}
0 & 1 & 0
\end{array}\right]^{T}, \quad \varepsilon_{3}^{k l}=\varepsilon_{12}=\left[\begin{array}{lll}
0 & 0 & 1
\end{array}\right]^{T}
$$

The same is applied for a three dimensional problem;

$$
\begin{array}{lllll}
\varepsilon_{1}^{k l} & =\varepsilon_{11}=\left[\begin{array}{llllll}
1 & 0 & 0 & 0 & 0 & 0
\end{array}\right]^{T,} & \varepsilon_{2}^{k l}=\varepsilon_{22}=\left[\begin{array}{llllll}
0 & 1 & 0 & 0 & 0 & 0
\end{array}\right]^{T} \\
\varepsilon_{3}^{k l}=\varepsilon_{33}=\left[\begin{array}{llllll}
0 & 0 & 1 & 0 & 0 & 0
\end{array}\right]^{T,} & \varepsilon_{4}^{k l}=\varepsilon_{12}=\left[\begin{array}{llllll}
0 & 0 & 0 & 1 & 0 & 0
\end{array}\right]^{T} \\
\varepsilon_{5}^{k l}=\varepsilon_{23}=\left[\begin{array}{llllll}
0 & 0 & 0 & 0 & 1 & 0
\end{array}\right]^{T,} & \varepsilon_{6}^{k l}=\varepsilon_{31}=\left[\begin{array}{llllll}
0 & 0 & 0 & 0 & 0 & 1
\end{array}\right]^{T}
\end{array}
$$

The displacements are obtained by imposing periodic boundary conditions on the RVE boundary. The resulting displacements for each strain case are then used to calculate the homogenized constitutive matrix. There are two possible methods to construct the homogenized constitutive matrix. The first method is by calculating the local structure tensor for each element using the periodic displacement fields and then applying the equation below:

$$
D_{i j k l}^{H}=\frac{1}{|V|} \int_{V_{S}} D_{i j p m} \mathbb{M}_{p m k l} d V
$$

Another simpler method for calculating the homogenized constitutive matrix is to first collect the strains for each element and subtract them from the macro volumetric strain vectors. The homogenized constitutive matrix is then the sum of the products of the 
original elasticity matrices multiplied by their modified elemental strains. The summation of the contributions of each element to the homogenized constitutive matrix is written as:

$$
D_{i j k l}^{H}=\frac{1}{|V|} \sum_{e=1}^{n} \int_{V_{e}} D_{e_{i j k l}}\left(I-B_{e} U_{i j_{e}}{ }^{k l}\right)^{T} d V_{e}
$$

where $I$ is the identity matrix, $B_{e}$ is the strain-displacement matrix of the element, $n$ is the

number of elements, and $U_{i j_{e}}{ }^{k l}$ is the resulting displacements where each row contains the periodic displacements for each macroscopic strain field case.

The homogenized elasticity matrix is the summation of the contribution of all elements in the RVE mesh. The above equations can then be differentiated with respect to the design variables within the RVE for topology optimizations whose aim is to design a cellular material with particular characteristics in mind [70].

\subsubsection{Relative Yield Strength}

In addition to homogenized elasticity matrices, homogenization can calculate the relative yield stress of a periodic medium. This was performed for two dimensional lattice materials by Pasini et al [95]. The effective or relative stress tensor of an elastic medium is given as:

$$
\overline{\sigma_{l j}}=\mathrm{D}_{\mathrm{ijkl}}^{\mathrm{H}} \overline{\varepsilon_{\mathrm{kl}}}
$$

where $\overline{\sigma_{1 j}}$ is the effective stress tensor, $D_{i j k l}^{H}$ is the homogenized constitutive matrix and $\overline{\varepsilon_{\mathrm{kl}}}$ is the average strain tensor. 
It is assumed the equal strain energies between the RVE and the homogenized material are equivalent. The average stress $\left(\overline{\sigma_{\mathrm{lj}}}\right)$ or average strain $\left(\overline{\varepsilon_{\mathrm{kl}}}\right)$ in RVE are defined by the average of the stress or strain tensors over the volume of RVE:

$$
\overline{\sigma_{l j}}=\frac{1}{V} \int_{V} \sigma_{i j} d V, \quad \overline{\varepsilon_{k l}}=\frac{1}{V} \int_{V} \varepsilon_{k l} d V
$$

This is possible due to the assumption of equal internal strain energies. These quantities can be directly calculated from Finite element analysis and then using a volume average of the elements. A more computationally efficient method of correlating averages stress or strain is by calculating the total contributions on the boundaries. The average strain is defined by the applied displacements at the boundary while the average stresses are defined by the self-equilibrated applied tractions [153]. The local RVE strain and the average strains can be written with the transformation tensor below:

$$
\varepsilon_{\mathrm{ij}}=\mathbb{M}_{\mathrm{ijkl}} \overline{\varepsilon_{\mathrm{kl}}}, \quad \mathbb{M}_{\mathrm{ijkl}}=\frac{1}{2}\left(\delta_{\mathrm{ik}} \delta_{\mathrm{jl}}+\delta_{\mathrm{il}} \delta_{\mathrm{jk}}\right)-\varepsilon_{\mathrm{ij}}^{* \mathrm{kl}}
$$

where $\delta_{\mathrm{ij}}$ is the Kronecker delta and $\varepsilon_{\mathrm{ij}}$ is the microscopic (periodic) strains in the microstructure. The local and average strains are substituted into the macroscopic equilibrium equation [150].

The relation between the average stress and micro stresses can be calculated using the local structure tensor. The microscopic stress distribution can be derived from:

$$
\sigma_{i j}=D_{i j k l} \mathbb{M}_{k l m n} \overline{\varepsilon_{m n}}
$$

where $\sigma_{i j}$ is the microscopic stress tensor and $\overline{\varepsilon_{m n}}$ is the average strain tensor. The microscopic stress tensor can also be calculated from: 


$$
\sigma_{i j}=D_{i j k l} \mathbb{M}_{k l m n}\left(D_{r s m n}^{H}\right)^{-1} \overline{\sigma_{r s}}
$$

Where $\overline{\sigma_{r s}}$ is the macroscopic stress distribution in the RVE.

Relative failure of the medium can is determined using the formulation of Pasini et al [95].

$$
{\overline{\sigma_{l \jmath}}}^{y}=\frac{\sigma_{y s}}{\max \left\{\sigma_{v m}\left(\bar{\sigma}_{l \jmath}\right)\right.} \bar{\sigma}^{{ }_{l \jmath}}{ }_{v m}
$$

Where ${\overline{\sigma_{l j}}}^{y}$ is the yield strength factor for given macro stress, $\sigma_{y s}$ is the reference yield strength, $\sigma_{v m}\left(\overline{\sigma_{l j}}\right)$ is the von-Mises stress distribution within the unit cell domain corresponding the macroscopic stress $\overline{\sigma_{l \jmath}}$ and ${\overline{\sigma_{l}}}_{v m}$ is the von-Mises stress of the input stress vector.

The yield strength surface can be derived by testing multiple unit macro stress vectors and extracting the highest stress state from the micro structure. For a two dimensional problem; three stresses must be analyzed. A three dimensional problem has six stress states. For the purpose of this thesis, six unit stresses are used to determine the yield strengths and multi-axial properties are calculated by interpolation. The six stress cases are unit tests in the normal directions and shear directions. The vectors for the test cases are shown below:

$$
\begin{array}{llllll}
\overline{\sigma_{x x}} & =\left[\begin{array}{llllll}
1 & 0 & 0 & 0 & 0 & 0
\end{array}\right]^{T}, & \overline{\sigma_{y y}} & =\left[\begin{array}{llllll}
0 & 1 & 0 & 0 & 0 & 0
\end{array}\right]^{T} \\
\overline{\sigma_{z z}} & =\left[\begin{array}{llllll}
0 & 0 & 1 & 0 & 0 & 0
\end{array}\right]^{T}, & \overline{\sigma_{x y}} & =\left[\begin{array}{llllll}
0 & 0 & 0 & 1 & 0 & 0
\end{array}\right]^{T} \\
\overline{\sigma_{y z}} & =\left[\begin{array}{llllll}
0 & 0 & 0 & 0 & 1 & 0
\end{array}\right]^{T}, & \overline{\sigma_{x z}} & =\left[\begin{array}{llllll}
0 & 0 & 0 & 0 & 0 & 1
\end{array}\right]^{T}
\end{array}
$$


The yield properties are therefore estimated as a function of the input normalized stress vector and of the relative density of the lattice topology. Interpolation and estimation methods are explained in Appendix F .

It was noted by Arabnejad and Pasini that extracting the maximum micro-stresses from an imposed macro stress will often be much higher than expected due to stress discontinuities [73]. The errors in the finite element method and the imposed volumetric strains can be attributed to the discontinuities which cause the yield stress to decrease by almost forty percent when the relative density drops by one percent. To ensure conservative estimates while obtaining meaningful interpretations of internal stresses in the RVE; relative yield factors for relative densities above seventy percent are found by a high order polynomial regression. The regression will refit the data so that the drop in yield strength is smoother. Comparisons between the raw data and the fitted data can be seen in Appendix F .

\subsection{Homogenization Results}

The mechanical properties of eight lattice structures where calculated as a function of the relative density. The definition of the relative density is described as the ratio of volume inside the RVE to the total volume of the RVE. The chosen lattice topologies include CUBIC, BCC, FCC, diamond and the Octet truss. Using the methodology developed by Dong G et al [151], the homogenization was applied to a voxelated representation of the lattice structure. Additional calculations were made to acquire the relative yield strength by calculating local structure tensors and solving for six failure factors. The yield strength 
in the axial and shear directions was calculated for a number of different relative densities. The average of all six failure criterions are shown in the results as the average relative yielding. The average normal stresses at yielding and the average shear stresses for yielding are also shown in the graphs. A single unit cell (RVE) for a specific lattice topology was analyzed for the homogenization technique.

The relative Poisson ratio was seen to change based on the heterogeneous materials initial Poisson ratios. The results shown will present the relative properties (modulus, Poisson ratio, yield strength) for materials with a Poisson ratio of 0.3 . The relative young's modulus on the other hand was independent of the materials properties. The mesh discretization of the RVE was 50 hexahedron elements in the $\mathrm{X}, \mathrm{Y}$ and $\mathrm{Z}$ directions. The homogenized properties are assumed to be isotropic. Because of the isotropic assumption, many of the unit cells have very similar mechanical properties.

Additional numerical data for the homogenized properties are presented in Appendix $\mathrm{F}$.
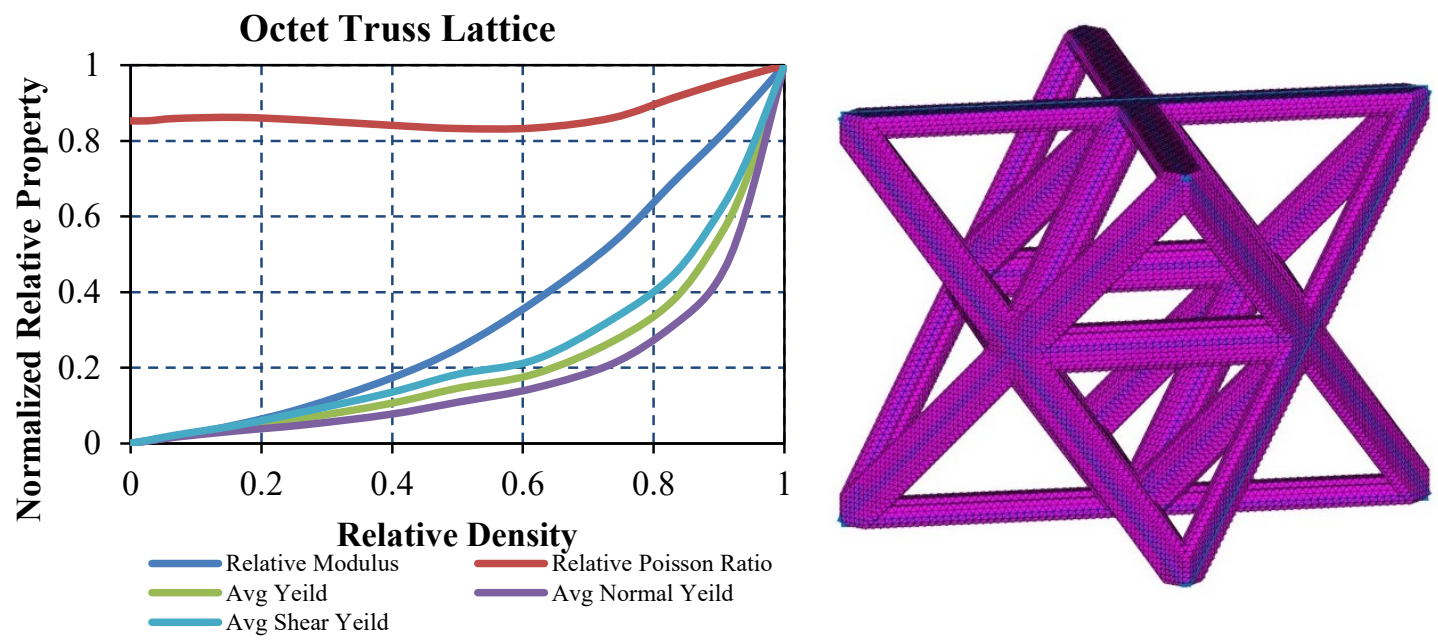

Figure 19: Octet Truss Lattice Relative Properties 

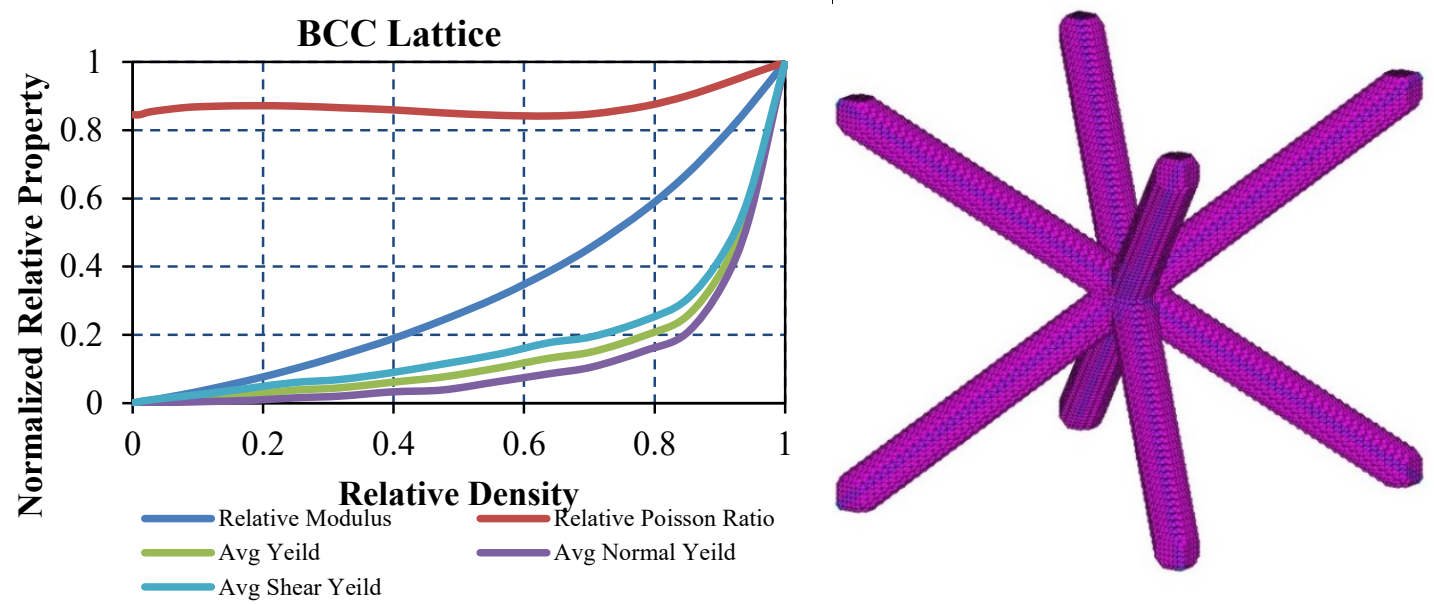

Figure 20: BCC Lattice Relative Properties
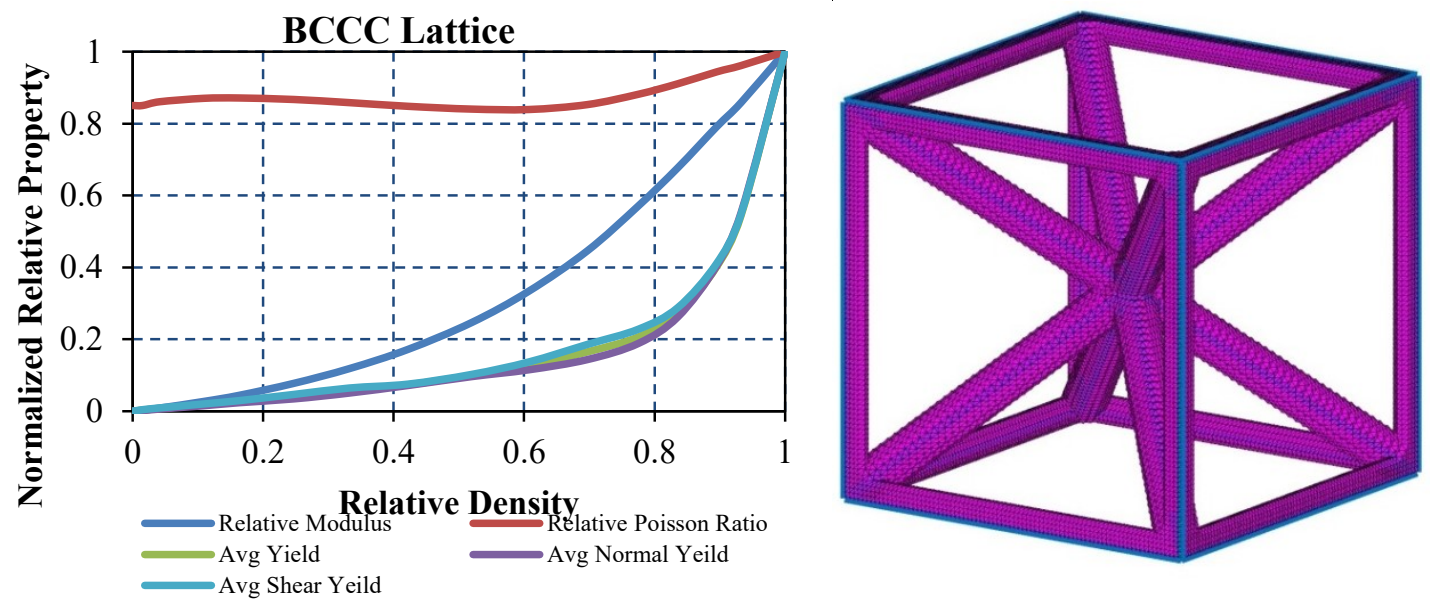

Figure 21 : BCCC Lattice Properties
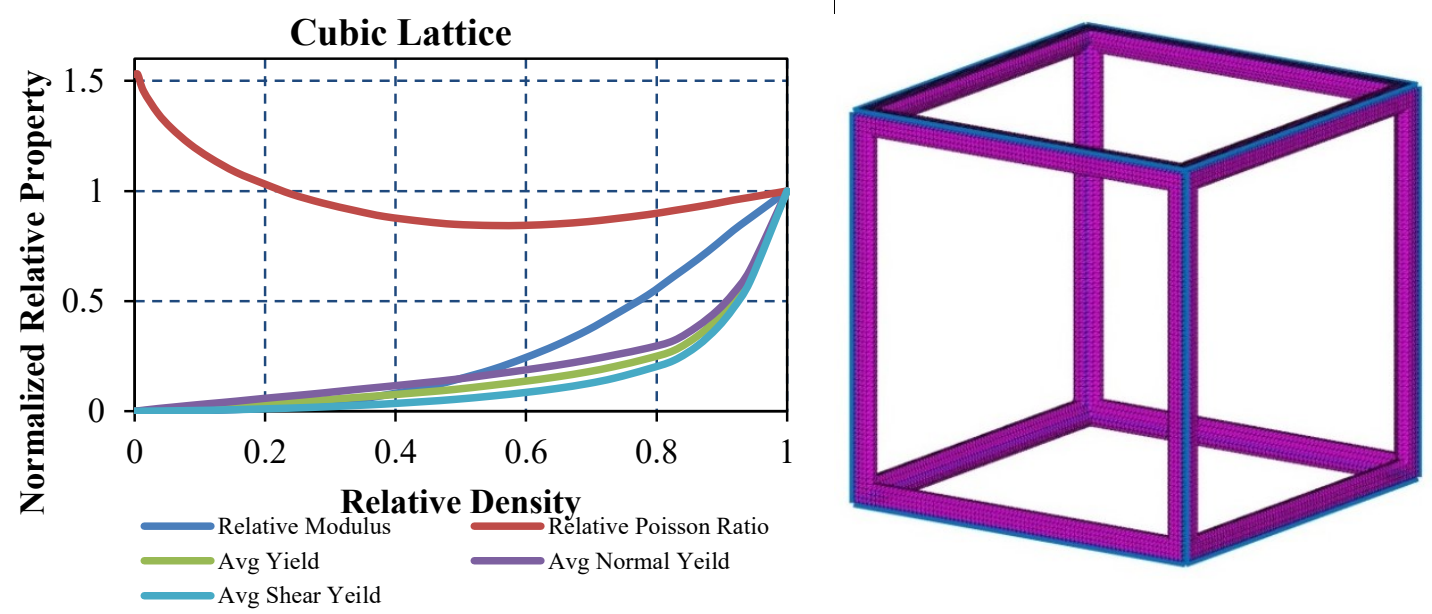

Figure 22 : Cubic Lattice Relative Properties 


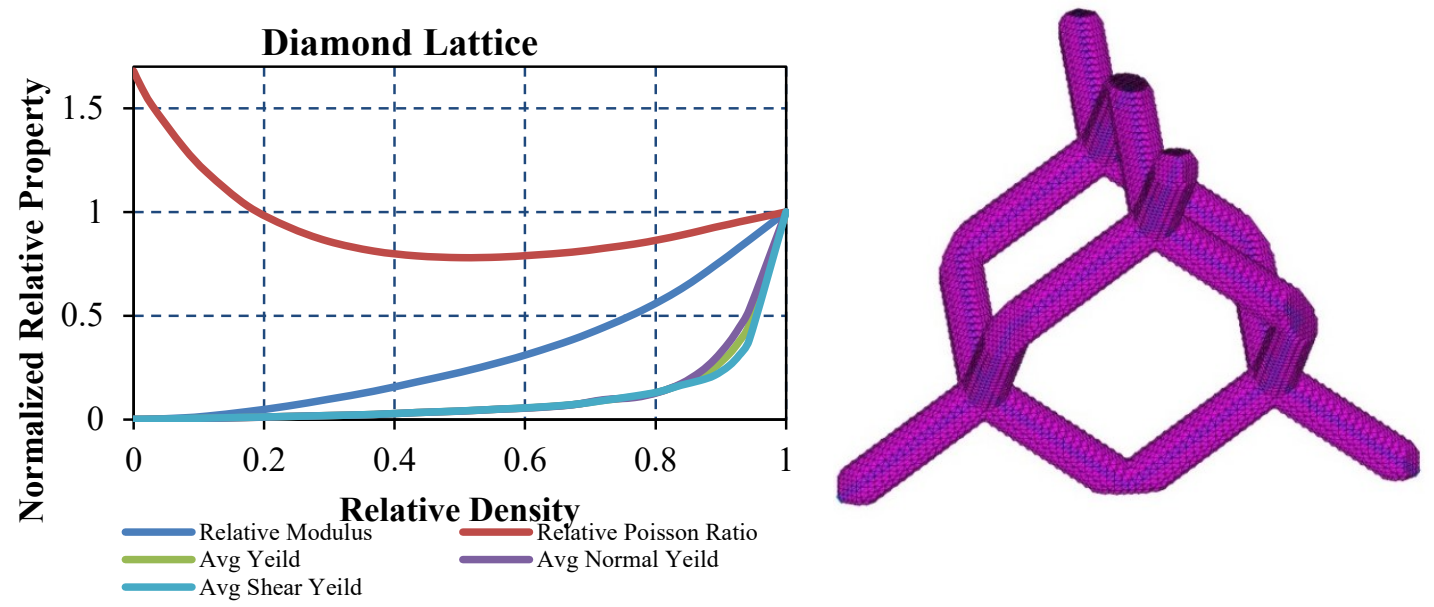

Figure 23 : Diamond Lattice Relative Properties
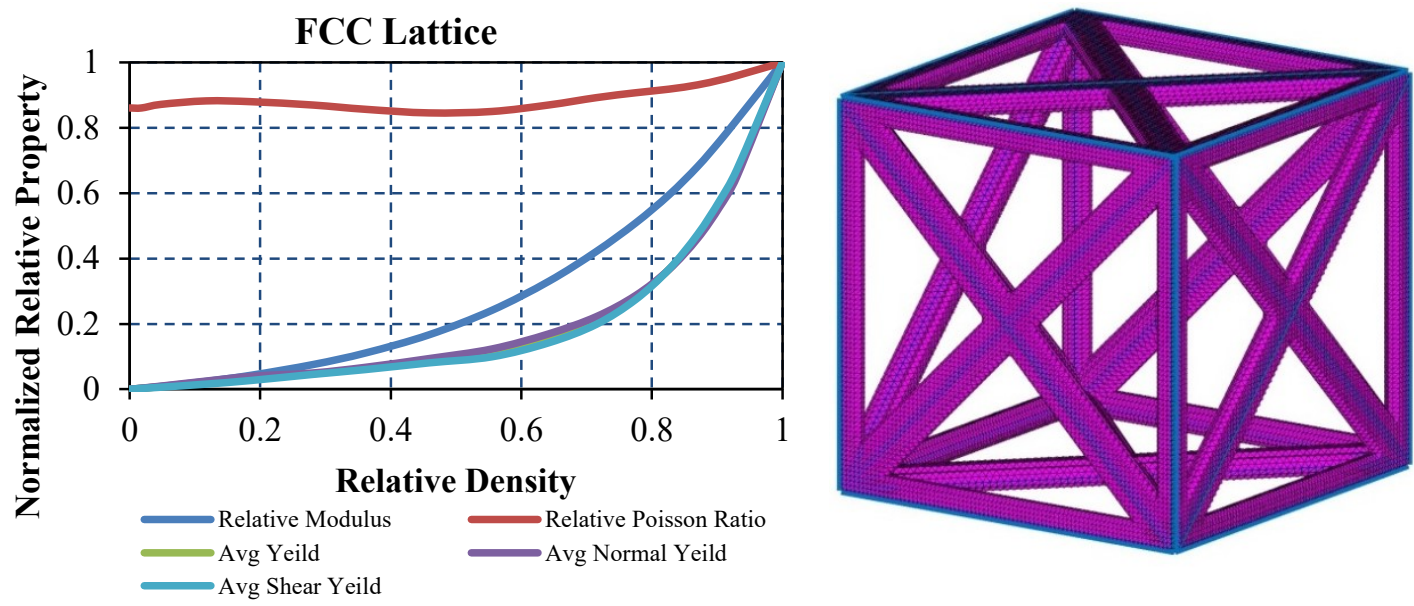

Figure 24 : FCC Relative Properties
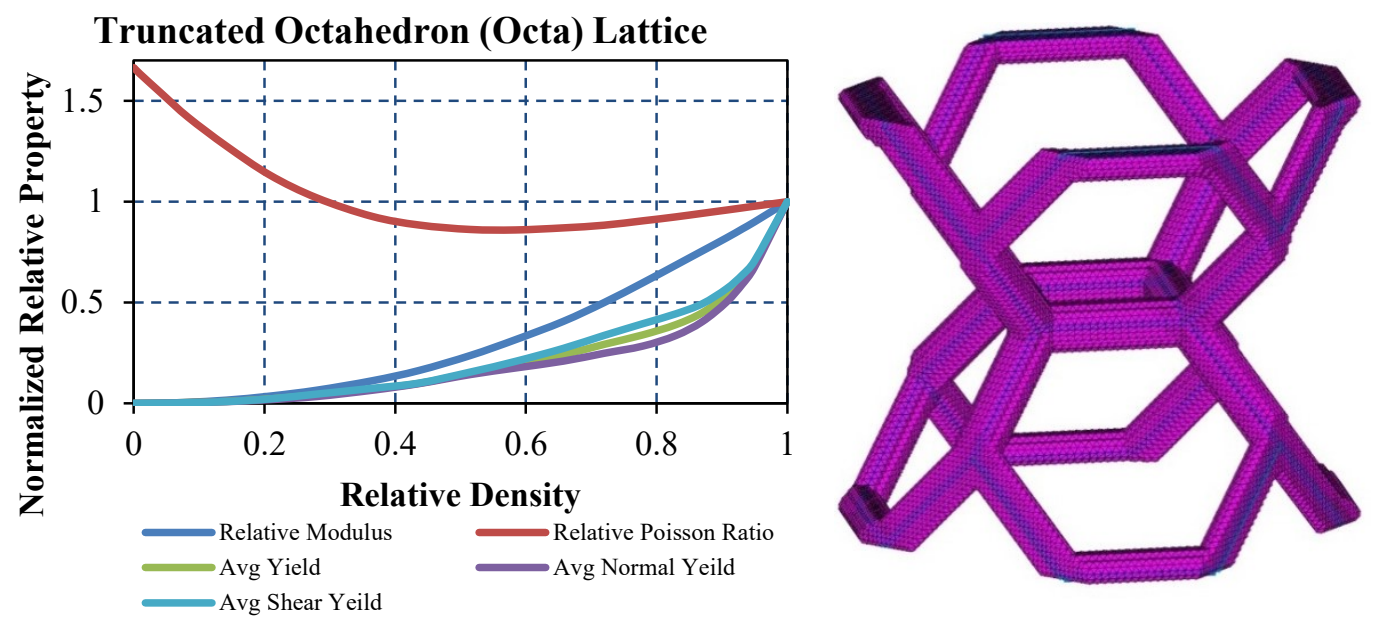

Figure 25 : Truncated Octahedron (Octa) Lattice Relative Properties 

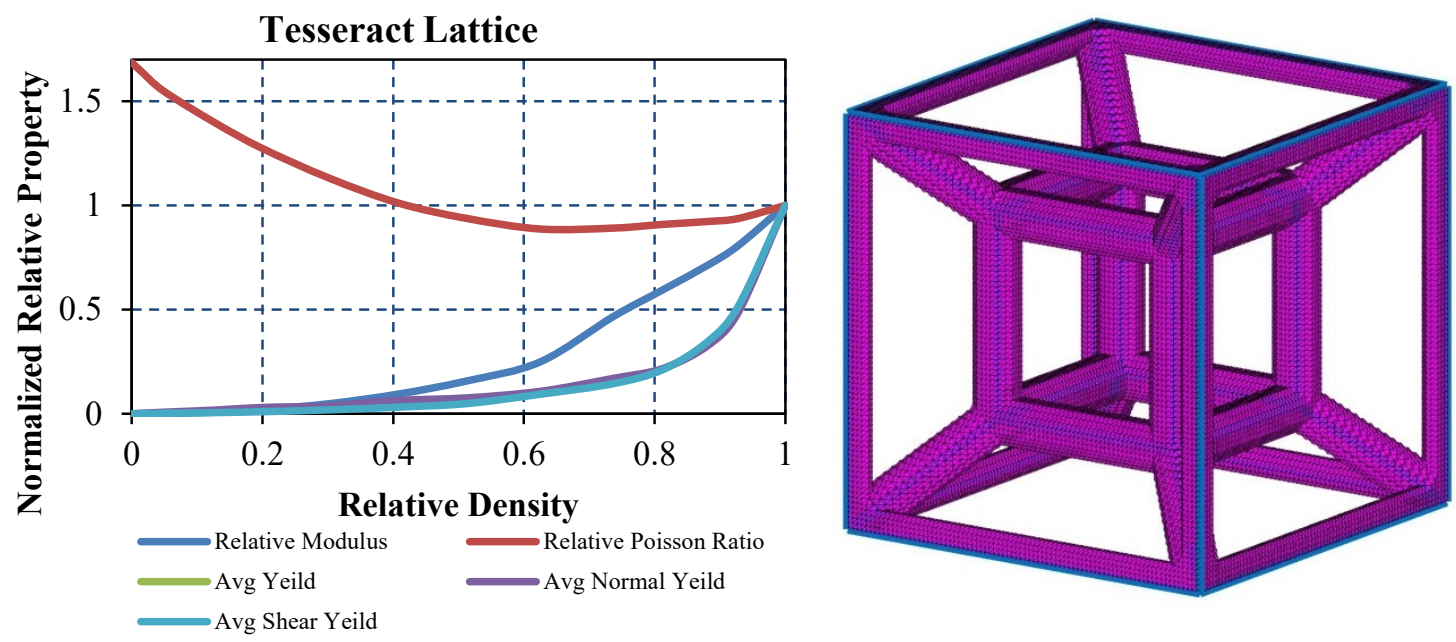

Figure 26 : Tesseract Lattice Relative Properties

\subsubsection{Comments on Homogenization Results}

For engineers it can be challenging to determine which lattice structure is best suited for their application. The research presented in this chapter calculated the relative modulus, Poisson ratio and yield strengths of some lattice topologies. However electrical, thermal conductivity and energy absorption are other properties that can be quantified. The multifunctionality of lattice structures adds complexity to design decisions [154]. One method to determine the applicability of lattice structures is by considering the lattice as a homogenous material and to then apply material selection techniques. Ashby's method is a popular strategy for selecting the correct material which satisfies the designer's needs for cost, weight and performance [155].

Material selection is a powerful tool to determine the optimal material for a given application [155]. Ashby's method will plot all possible materials onto a graph to compare mechanical properties such as stiffness, density, thermal conductivity, cost and 
etc [155]. By plotting various lattice materials at equivalent densities to compare their properties, a suitable lattice configuration can be found. The material chart could for example plot the compressive stiffness versus the density and the material with the best ratio would be selected as the optimal choice.

Another technique to determine the best lattice topology for a given task is by its structural categorization. For elastic responses, structural lattices can be categorized as either stretching or bending dominated [156]. Cubic and truncated octahedron lattices are known to be a bending dominated lattice because when they are subjected to point loads the bending moments are transferred to the struts. Lattices subjected to high bending forces will fail earlier than those under tension [157] and are seen to have increasing Poisson ratios as the relative density decreases. Stretching dominated lattices are those such as the octet truss and tend to be more weight efficient structures [156]. By comparing the relative Poisson ratios for each lattice topology as an isotropic material, one can determine if it is best suited for structural application as stretching dominated structures are ideal. However in contrast to bending dominated lattices, stretching dominated lattices perform worst in buckling and are less attractive when energy absorption is considered [156]. 


\section{Chapter 5: Structural Optimization}

\subsection{Topology Optimization}

Topology optimization is a field whose goal is to determine to optimal geometry for a given load case. This chapter presents an overview of solution methods for the various strategies in topology optimizations. Particular emphasis will be made for gradient based methodologies as they are more flexible when considering multiple constraints and objectives. The first section presents traditional problem formulations for gradient based optimization methods followed by filtering techniques and solutions methods.

\subsubsection{General Problem Formulation}

There are many methods for tackling topology optimization problems. Depending on the engineer's preference, they may implement the level set method or rely on material redistribution [62]. This thesis is mainly concerned with optimization by material redistribution which modifies the element material properties in a finite element mesh to minimize a certain objective. The mathematical statement for topology optimizations by density redistribution (SIMP) can be written as;

$$
\begin{gathered}
\text { minimize: } \psi(\rho, U(\rho)) \\
\text { subject to: }\left\{\begin{array}{c}
K(\rho) U=F \\
\mathcal{G}_{i}(\rho, U)<0 \\
h_{i}(\rho, U)=0 \\
0<\rho_{\min } \leq \rho \leq 1
\end{array}\right.
\end{gathered}
$$

where $\psi(\rho, U(\rho))$ is the objective or response function, $\rho$ is the vector of design variables (relative density in this problem), $U$ is the displacement vector and is a state 
variable, $K(\rho)$ is the global stiffness matrix as a function of the design variables, $F$ is the force vector, $\rho_{\min }$ is a lower value limit to avoid singularities, $\mathcal{G}_{i}$ is the set of inequality constraints and $h_{i}$ is a set of equality constraints.

The first material distribution technique for topology optimization is the SIMP method; also known as Solid Isotropic Microstructures with Penalization. SIMP is an optimization method which modifies the global stiffness matrix by varying each of the design elements young's modulus (or stiffness). The SIMP formulation is the most popular implementation for topology optimization due to the pioneering success of Bendsoe and Sigmund [61]. For implementing the SIMP methodology, the design variables are known to be the relative density and penalizes the stiffness terms using the formulation in equation (7.2);

$$
E_{e}\left(\rho_{e}\right)=E_{e}^{o} \rho_{e}^{p}
$$

where $p$ is the penalization factor and is greater than $1, E_{e}\left(\rho_{e}\right)$ is the penalized stiffness and $E_{e}^{O}$ is the original stiffness of an element $e$.

Bendsoe and Sigmund remark that the SIMP model can satisfy the Hashin-Shtrikman bound for two phase materials model if the penalization factor $(p)$ satisfies the following criteria [69]:

$$
\begin{gathered}
p \geq \max \left\{\frac{2}{1-v^{0}}, \frac{4}{1+v^{0}}\right\} \text { (2D-case) } \\
p \geq \max \left\{15 \frac{1-v^{0}}{7-5 v^{0}}, \frac{3}{2} \frac{1-v^{0}}{1-2 v^{0}}\right\} \text { (3D-case) }
\end{gathered}
$$

where $v^{0}$ is the Poisson ratio. The interpolation method for the SIMP method provides areas of grey which questions the physical permissibility of such a material. If the above 
Hashin-Shtrikman constraints for the penalization power can be met, then the intermediate design variables can be interpreted in much the same way as a layered composite material or as another solid material. The above equation implies that for a two-dimensional case, the smallest penalization factor is about three. For a threedimensional case, the smallest penalization factor is two however Bendsoe and Sigmund suggest a minimum value of three be chosen for topology optimizations.

There are many more interpolation methods for material redistribution methods. For this thesis, only the SIMP method and a modified interpolation scheme for homogenized lattice materials will be used.

\subsubsection{Filtering}

Filtering is used to enforce a length-scale in the problem and to ensure meshindependency. Another key feature of filtering is to avoid checker boarding which predominantly arises when first order FE meshes are used. Checker boarding can be seen in Figure 27A. Many types of filtering algorithms exist; the most notable ones are the sensitivity filter and the density filter. Figure 27B illustrates the differences compared to Figure 27A when a filter is applied.

The sensitivity filter was first introduced by Sigmund and will directly modify the sensitivity of the objectives and constraint functions $[158,159]$. The original design variables are unchanged. The design sensitivities are altered using the sensitivity by means of the following equation: 


$$
\frac{\widehat{\partial \psi}}{\partial \rho_{e}}=\frac{1}{\max \left(\tau, \rho_{e}\right) \sum_{i \in N_{e}}^{N_{e}} w_{e i}} \cdot\left(\sum_{i \in N_{e}}^{n_{e}} w_{e i} \rho_{i} \frac{\partial \psi}{\partial p_{i}}\right)
$$

where $\tau$ is some minimal density value to avoid singularities, $p_{e}$ is the design variables, $w_{e i}$ is a convolution weighted distance factor, $\psi$ is the response function whose sensitivities are to be modified and $\mathrm{N}_{\mathrm{e}}$ is the set of all elements whose centers lie within a distance of $r_{\min }$ from the center of the element $e$ under consideration. The linear weights $\mathrm{w}_{\mathrm{ei}}$ are calculated using the following definition;

$$
w_{e i}=\max \left(0, \frac{\mathcal{R}_{\text {min }}-\mathcal{R}_{e i}}{\mathcal{R}_{\min }}\right)
$$

Where $\mathcal{R}_{\min }$ and $\mathcal{R}_{e j}$ are user defined variables to enforce minimum member size and the distance between centroids of elements $e$ and $i$ respectively.

A)

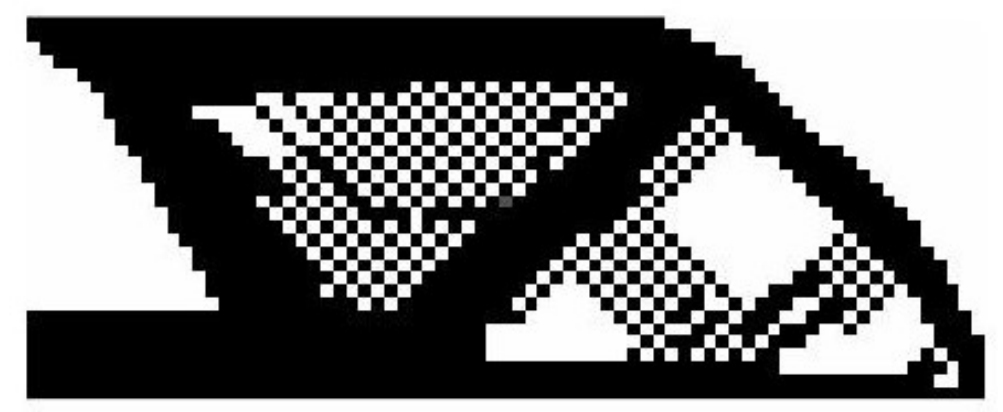

B)

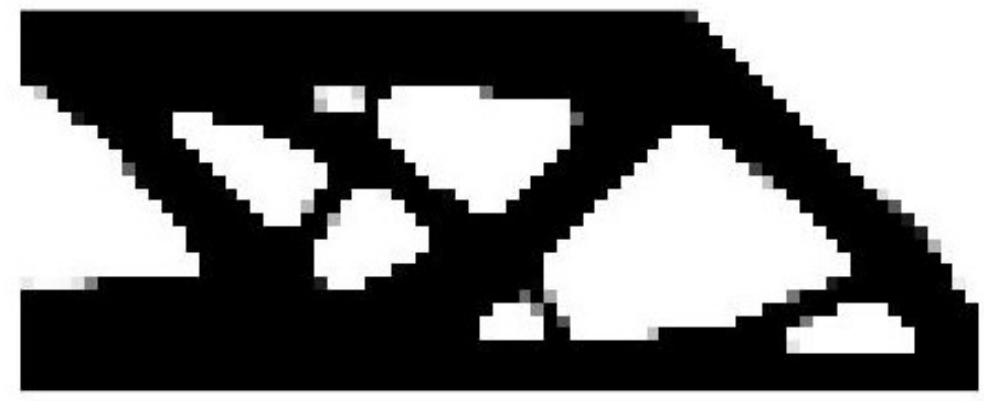

Figure 27 : (A) No Filter (B) With Filter 
In addition to the sensitivity filter there also exists the density filter which modifies the design variables by a weighted average of the nearby elemental densities. The projected element density of an element $\rho_{e}$ is written as;

$$
\widetilde{\rho_{e}}=\frac{\sum_{i \epsilon N_{e}} w_{e i} \rho_{i}}{\sum_{i \epsilon N_{e}} w_{e i}}
$$

Because the design variables are updated due to the filter, the sensitivities of the objective functions must be modified as well. To compute the sensitivity of the objective function $\psi$ with respect to the independent design variables $\mathrm{p}_{\mathrm{j}}$, we use the chain rule:

$$
\frac{\partial \psi}{\partial \rho_{j}}=\sum_{e \in N_{j}} \frac{\partial \psi}{\partial \widetilde{\rho_{e}}} \frac{\partial \widetilde{\rho_{e}}}{\partial \rho_{j}}=\sum_{e \in N_{j}} \frac{1}{\sum_{i \in N_{j}} w_{e i}} w_{j e} \frac{\partial \psi}{\partial \widetilde{\rho_{e}}}
$$

When implementing the density filter, a symmetrical distance matrix can be calculated firsthand. This is done by calculating the convolution weight factors of each element with respect to the other elements or design variables. The filtered design variables then become a matrix multiplication between the vector of the original design variables and the convolution weight matrix. The modified sensitivities can be obtained the same method by multiplying the current gradient by the convolution weight matrix.

In this thesis, only the density filter will be considered for optimization purposes. This is because of its simplicity and speed of computation. Having to consider only one filter will also limit the number of design cases to consider.

\subsubsection{Solution Methods}

In topology optimizations, there exists a wide variety of optimization methods. These methods take first order information from the optimization responses and 
correspondingly update the design variables. For general optimizations cases there is the Method of Moving Asymptotes (MMA) as well as other sequential approximation algorithms such as SQP for optimizations. Many other strategies have been used to search for the optimal structural layout in a topology optimization problem. For instance, the optimality criterion has been used for material constrained problems by exploiting the KKT conditions of optimality [14]. Another strategy is BESO with operates similarly to the optimality criteria method [62].

For the purposes of this paper, the MMA [69] gradient based method is used as objective information and constraints must be interchangeable to be used inside the multi-objective framework.

\subsubsection{Method of Moving Asymptotes}

The method of moving asymptotes or MMA is a popular optimization algorithm in the field of topology optimization. Its popularity stems from its ability to be both versatile and an efficient large scale optimizer [69]. MMA was first introduced by Svanberg to tackle large scale structural optimization problems with an emphasis on stress constraints [160]. This optimization algorithm is favorable due its ability to solve problems with a large number of constraints with a moderate amount of constraints. Svanberg has also stated that one of the advantages of MMA is that it is easy to implement and use [160].

The algorithm is an iterative procedure which creates separable convex sub problems based on the objective function and its constraints. The main criterion is that the functions themselves be smooth and twice continuously differentiable. The convex sub 
problems are generated from first order information from the input functions and information from previous steps (iterations).

The steps for implementing MMA follow the same general methodology for any sequential approximation algorithm. The only difference is the implementation of the sub problem itself. The general steps for the sequential approximation are explained again for consideration of the MMA algorithm [160].

1. Choose a starting point $\mathrm{x}^{0}$ for current iteration $I=0$

2. At the current point calculate $f_{i}\left(x^{I}\right)$ and $\nabla f_{i}\left(x^{I}\right)$ where $i=0,1, \ldots$ ㅁ

3. Calculate the moving asymptote bounds $\mathcal{U}_{i}^{I}$ and $l_{i}^{I}$

4. Generate the MMA sub problem $\mathbb{Q}^{I}$

5. Solve $\mathbb{Q}^{I}$ using the primal-dual algorithm and go to step 1 with the optimal solution $x_{*}^{I}$ obtained from $\mathbb{Q}^{I}$, set $\mathrm{I}=\mathrm{I}+1$

If the original optimization problem is formulated as:

$$
\begin{gathered}
\min f_{0}(x) \\
\text { subject to: } f_{i}(x) \leq 0, \quad i=1, \ldots, \text {, ता } \\
x \in \mathbb{S}
\end{gathered}
$$

Then the sub problem for the method of moving asymptotes will approximate the original problem as follows:

$$
\min f_{0}^{k}(x)+\varrho_{0} z+\sum_{i=1}^{\mathrm{m}}\left(c_{i} y_{i}+\frac{1}{2} \mathcal{D}_{i} y_{i}^{2}\right)
$$




$$
\begin{aligned}
& f_{i}^{I}(x)-\varrho_{i} z-y_{i} \leq 0 \quad i=1, \ldots \ldots, \text {, ㄴ } \\
& \sigma_{j}^{I} \leq x_{j} \leq \nVdash_{j}^{I} \quad j=1, \ldots \ldots, \text { m } \\
& \text { subject to: } \quad y_{i} \geq 0 \quad i=1, \ldots \ldots \text {, wn } \\
& z \geq 0 \\
& x_{j}^{\min } \leq x_{j} \leq x_{j}^{\max } \quad j=1, \ldots \ldots, \mathbb{R}
\end{aligned}
$$

Where $f_{0}$ is the approximated objective function, $f_{i}$ are the approximated functions, $a_{0}$, $\varrho a_{i}, c_{i}, \mathcal{D}_{i}$ are real numbers which satisfy $\varrho_{0}>0, \varrho_{i} \geq 0, c_{i} \geq 0, \mathcal{D}_{i} \geq 0, c_{i}+\mathcal{D}_{i}>0$ for all $i$, and also $\varrho_{i} c_{i}>\varrho_{0}$ for all I with $a_{i}>0 . \sigma_{j}^{I}$ is the lower bound (asymptote) and $\Psi_{j}^{I}$ is the upper bound which are determine through iterations where $I>0 . x^{\min }$ and $x^{\max }$ and the original box constraints for the optimizations problem. $\mathrm{m}$ is the number of design variables and un ist he number of differential functions. Also, $z \in \mathbb{R}, x \in \mathbb{R}^{\mathbb{m}}$ and $y \in \mathbb{R}^{\text {叫 }}$. The approximating functions $f_{i}^{I}$ are evaluated as:

$$
f_{i}^{I}(x)=њ_{i}^{I}+\sum_{j=1}^{\mathrm{m}}\left(\frac{Д_{i j}^{I}}{u_{j}^{I}-x_{j}}+\frac{\mathrm{Ч}_{i j}^{I}}{x_{j}^{I}-l_{j}^{I}}\right), \quad i=0,1, \ldots \text { шा }
$$

where $Д_{i j}^{k}, q_{i j}^{k}$ and $r_{i}^{k}$ are obtained from:

$$
\begin{gathered}
Д_{i j}^{I}=\left(\mathcal{U}_{j}^{I}-x_{j}^{I}\right)^{2}\left(1.001\left(\frac{\partial f_{i}}{\partial x_{j}}\left(x^{I}\right)\right)^{+}+0.001\left(\frac{\partial f_{i}}{\partial x_{j}}\left(x^{I}\right)\right)^{-}+\frac{10^{-5}}{x_{j}^{\text {max }}-x_{j}^{\text {min }}}\right) \\
\mathrm{\Psi}_{i j}^{I}=\left(x_{j}^{I}-l_{j}^{I}\right)^{2}\left(1.001\left(\frac{\partial f_{i}}{\partial x_{j}}\left(x^{I}\right)\right)^{+}+0.001\left(\frac{\partial f_{i}}{\partial x_{j}}\left(x^{I}\right)\right)^{-}+\frac{10^{-5}}{x_{j}^{\text {max }}-x_{j}^{\text {min }}}\right) \\
\leftrightarrow_{i}^{I}=f_{i}\left(x^{I}\right)-\sum_{j=1}^{\mathrm{m}}\left(\frac{Д_{i j}^{I}}{\mathcal{U}_{j}^{I}-x_{j}}+\frac{\mathrm{\Psi}_{i j}^{I}}{x_{j}^{I}-l_{j}^{I}}\right)
\end{gathered}
$$


where $\left(\frac{\partial f_{i}}{\partial x_{j}}\left(x^{I}\right)\right)^{+}$is equal to $\max \left\{\frac{\partial f_{i}}{\partial x_{j}}\left(x^{I}\right), 0\right\}$ and $\left(\frac{\partial f_{i}}{\partial x_{j}}\left(x^{I}\right)\right)^{-}$is equal to $\max \left\{-\frac{\partial f_{i}}{\partial x_{j}}\left(x^{I}\right), 0\right\}$ and the bounds $\sigma_{j}^{I}$ and $\varkappa_{j}^{I}$ are calculated from:

$$
\begin{array}{ccc}
\sigma_{j}^{I}=\min \left\{x_{j}^{\min },\right. & +0.1\left(x_{j}^{I}-l_{j}^{I}\right), & \left.x_{j}^{I}-0.5\left(x_{j}^{\max }-x_{j}^{\min }\right)\right\} \\
\varkappa_{j}^{I}=\min \left\{x_{j}^{\max },\right. & -0.1\left(u_{j}^{I}-x_{j}^{I}\right), & \left.x_{j}^{I}+0.5\left(x_{j}^{\max }-x_{j}^{\min }\right)\right\}
\end{array}
$$

The asymptotes $U_{j}^{k}$ and $L_{j}^{k}$ have their own updating scheme. When I is equal to 1 and 2;

$$
\begin{gathered}
l_{j}^{I}=x_{j}^{I}-0.5\left(x_{j}^{\text {max }}-x_{j}^{\text {min }}\right) \\
\mathcal{u}_{j}^{I}=x_{j}^{I}+0.5\left(x_{j}^{\text {max }}-x_{j}^{\text {min }}\right)
\end{gathered}
$$

When $\mathrm{k}$ is greater than 2 ;

$$
\begin{gathered}
l_{j}^{I}=x_{j}^{I}-\mathcal{Y}_{j}\left(x_{j}^{(I-1)}-l_{j}^{(I-1)}\right) \\
u_{j}^{I}=x_{j}^{I}+\mathcal{Y}_{j}\left(u_{j}^{(I-1)}-x_{j}^{(I-1)}\right)
\end{gathered}
$$

And finally $\mathcal{Y}_{j}$ is equal to;

$$
\mathcal{Y}_{j}=\left\{\begin{array}{l}
0.7 \text { if }\left(x_{j}^{I}-x_{j}^{(I-1)}\right)\left(x_{j}^{(I-1)}-x_{j}^{(I-2)}\right)<0 \\
1.2 \text { if }\left(x_{j}^{I}-x_{j}^{(I-1)}\right)\left(x_{j}^{(I-1)}-x_{j}^{(I-2)}\right)>0 \\
1 \text { if }\left(x_{j}^{I}-x_{j}^{(I-1)}\right)\left(x_{j}^{(I-1)}-x_{j}^{(I-2)}\right)=0
\end{array}\right.
$$

The sub problem is solved by means of a primal-dual algorithm. Due to the large number of steps and checks involved in the algorithm; a detailed explanation can be found in the paper written by Svanberg [160]. 


\subsection{Lattice Optimization}

Lattice optimization procedures are identical to SIMP topology optimization method. The major difference comes from the modifications to the constitutive matrices of the finite elements to reflect material properties of homogenized lattice materials. An additional step in the multi-scale design procedure is to convert low density elements into a collection of tapered Euler beams. This transforms the original optimization problem into a new one. The new problem allows the design to assess the microscopic behavior more accurately and fine tune the properties in those sections. The conversion into microtapered beams however is a trade-off between accuracy and modeling efficiency. This is because beam elements are most accurate when they have high aspect ratios. This subchapter will present the reader with mathematical principles to obtain graded structures with embedded cellular materials.

\subsubsection{Sensitivity Analysis for Lattice SIMP Optimizations}

In a SIMP topology optimization the optimum is found by modifying the designable elements young's modulus based on their relative density. For a Lattice SIMP optimization, both the young's modulus and Poisson ratio of the design elements are continuously modified. The sensitivity analysis for structural responses in a Lattice SIMP and SIMP optimization are the same with the exception of the differentiation of the local stiffness matrix with respect to the relative density (design variable). The new total derivative of the local stiffness matrices are presented in this section. 
In Lattice SIMP optimization the relative density directly changes the properties of the constitutive matrices using relative property functions. The relative property functions are based on the interpolating methodology outlined in Appendix D. Because the change in the stiffness does not depend only on the young's modulus as it does in the regular SIMP formulation, the change in the Poisson ratio must be taken into account. This requires the sensitivities of the relative property functions to be included into the differentiation of the responses. For the sensitivities of the relative property functions; they are found by calculating the gradient of fitted high-order polynomials over a subsection of the homogenized lattice data. These sensitivities are then inserted into the constitutive matrix to determine the total gradients for the changes in the local stiffness matrix with respect to the design variables.

The sensitivity for the local stiffness matrix depends on two relative property functions; $\Gamma(\rho)$ which is the change of relative modulus and $\Lambda(\rho)$ which is the change in relative Poisson ratio. Both $\Gamma(\rho)$ and $\Lambda(\rho)$ are functions in terms of the relative density $\rho$. This will modify the local stiffness matrix of the elements. Therefore, the local stiffness matrices $K_{e}$ can be reformulated as:

$$
K_{e}\left(p_{e}\right)=K_{e}\left(\Gamma\left(p_{e}\right), \Lambda\left(p_{e}\right)\right)
$$

Since $\Gamma(\rho)$ and $\Lambda(\rho)$ both explicitly rely on the relative density, then the sensitivity of $\mathrm{K}_{\mathrm{e}}$ with respect to the design variable can be determined with the following expression [82]:

$$
\frac{\partial K_{e}}{\partial p_{e}}=\int_{V} B_{e} \cdot \frac{d D_{e}}{d p_{e}} \cdot B_{e} \cdot d V
$$


Where $\mathrm{B}_{\mathrm{e}}$ is the strain-displacement matrix element type, $V$ is the volume (or area in 2D) and $D_{e}$ is the elements constitutive material stiffness matrix. $B_{e}$ is independent of the design variable as is constant. The material stiffness matrix in three dimensions is given as:

$$
D=\frac{E}{(1+v)(1-2 v)} \cdot\left[\begin{array}{cccccc}
1-v & v & v & 0 & 0 & 0 \\
v & 1-v & v & 0 & 0 & 0 \\
v & v & 1-v & 0 & 0 & 0 \\
0 & 0 & 0 & 1-2 v & 0 & 0 \\
0 & 0 & 0 & 0 & 1-2 v & 0 \\
0 & 0 & 0 & 0 & 0 & 1-2 v
\end{array}\right]
$$

The modified material matrix $\mathrm{D}$ when $\Gamma(\rho)$ and $\Lambda(\rho)$ are inserted becomes:

$$
\begin{aligned}
& D\left(p_{e}\right) \\
& =\frac{E \cdot \Gamma\left(p_{e}\right)}{\left(1+v \cdot \Lambda\left(p_{e}\right)\right)\left(1-2 v \cdot \Lambda\left(p_{e}\right)\right)} \\
& {\left[\begin{array}{cccccc}
1-v \cdot \Lambda\left(p_{e}\right) & v \cdot \Lambda\left(p_{e}\right) & v \cdot \Lambda\left(p_{e}\right) & 0 & 0 & 0 \\
v \cdot \Lambda\left(p_{e}\right) & 1-v \cdot \Lambda\left(p_{e}\right) & v \cdot \Lambda\left(p_{e}\right) & 0 & 0 & 0 \\
v \cdot \Lambda\left(p_{e}\right) & v \cdot \Lambda\left(p_{e}\right) & 1-v \cdot \Lambda\left(p_{e}\right) & 0 & 0 & 0 \\
0 & 0 & 0 & 1-2 v \cdot \Lambda\left(p_{e}\right) & 0 & 0 \\
0 & 0 & 0 & 0 & 1-2 v \cdot \Lambda\left(p_{e}\right) & 0 \\
0 & 0 & 0 & 0 & 0 & 1-2 v \cdot \Lambda\left(p_{e}\right)
\end{array}\right]}
\end{aligned}
$$

The derivative of the material stiffness matrix $\mathrm{D}$ can then be found with the chain rule:

$$
\frac{d D_{e}}{d p_{e}}=\frac{\partial D_{e}}{\partial \Gamma} \frac{\partial \Gamma}{\partial p_{e}}+\frac{\partial D_{e}}{\partial \Lambda} \frac{\partial \Lambda}{\partial p_{e}}
$$

\subsubsection{Stress Sensitivity Modifications}

When dealing with stress responses, the change in the elastic properties does not affect the contributions to the stress components. Many numerical examples of this phenomenon are explained in Appendix E.2.2. Proposed solutions to the challenges with stress responses are also explained in Appendix E.2.2 and include the " $q_{p}$ " method or the 
epsilon relaxation method [161]. The solutions to stress inconveniences aim to relax the feasible region so that the global optimum can be achieved.

The nature of using homogenized properties as an interpolation method for a topology optimization allows for alternative strategies when dealing with stress based structural responses. For a given relative density of a unit cell topology; there exists a relative stress value that can be used to scale the elemental stresses to an appropriate value. The relative stress properties can therefore be implemented into the stress response for the lattice optimizations. This strategy had been implemented with great success by Pasini et al who homogenized properties of two dimensional lattice structures to derive properties of relative modulus, Poisson ratio, shear modulus and yield stress [95]. Pasisni et al. included the microscopic stress measurements in the evaluation of the stresses to scale the problem accordingly [95]. This highlights an advantage of this method in that it is conceptually easier to visualize and interpret than the previous stress response modification methods. The stress scaling by homogenized stress scalars will be adopted for this thesis. The scalar function is the inverse of the average relative yield function developed in the homogenization chapter.

The modification to the stress response by including a relative stress function is written as such:

$$
\sigma_{e}\left(\rho_{e}\right)=\sigma_{e}\left(\rho_{e}, \vartheta\left(\rho_{e}, v_{0}\right)\right)
$$

where $\sigma_{e}$ is the elemental stress as a function for the relative density, $\rho_{e}$ is the relative density of the element, $v_{0}$ is the original Poisson ratio of the heterogeneous material and 
$\vartheta$ is a function for the relative increase in the stress measured by the relative density and Poisson ratio. As stated before the relative increases in the stress is governed by the inverse of the average relative yielding.

The elemental stress can also be written explicitly as a function of the relative density and is shown below:

$$
\sigma_{e}\left(\rho_{e}\right)=D\left(\rho_{e}\right) B U \vartheta\left(\rho_{e}\right)
$$

The sensitivities of the stress function with respect to the relative density can be found using the product rule:

$$
\frac{d \sigma_{e}}{d \rho_{e}}=\frac{\partial \sigma_{e}}{\partial \rho_{e}} \vartheta\left(\rho_{e}\right)+\sigma_{e} \frac{\partial \vartheta}{\partial \rho_{e}}
$$

The explicit form of the sensitivities using the adjoint method is then written as:

$$
\frac{d \sigma_{e}}{d \rho_{e}}=\left[\frac{\partial D_{e}}{\partial \rho_{e}} B_{e} U_{e}-\lambda_{e}^{T}\left(\frac{d K_{e}}{d \rho_{e}} U_{e}\right)\right] \vartheta\left(\rho_{e}\right)+\left[D_{e} B_{e} U_{e}\right] \vartheta\left(\rho_{e}\right)^{\prime}
$$

The adjoint vector is found by solving the system:

$$
\lambda=K^{-1} \frac{d \sigma}{d U}
$$

\subsubsection{Fatigue Sensitivity Modifications}

Following the same principles of homogenized stress properties for unit cells described in chapter 5.2.1.1; the macro damage can be converted into micro damage using the relative yield function. The modification the sensitivity of the cumulative damage is performed by introducing the relative stress factor as a function of relative density into the 
sensitivities. The modified fatigue equation by the introduction of stress scalars are calculated in the equations below. The damage is recalculated as:

$$
H=\sum_{i=1}^{k} \frac{n_{i}}{\varpi_{i}}
$$

The life per cycle in the above equation is found with the equations below:

$$
\begin{gathered}
\varpi=\ell\left(\sigma_{\mathrm{e}}\right)=\frac{\sigma_{S N 1}}{\sigma_{\mathrm{e}}^{b}} \\
\sigma_{\mathrm{e}}=\tau(\sigma, \vartheta)=\tau\left(\sigma_{a}\left(\rho_{e}\right) \vartheta\left(\rho_{e}\right), \sigma_{m}\left(\rho_{e}\right) \vartheta\left(\rho_{e}\right)\right)
\end{gathered}
$$

The resulting sensitivities using the adjoint method for the life and damage are as follows using both the product rule and the chain rule:

$$
\frac{d H_{e}}{d \rho_{e}}=\left(\sum_{i=1}^{k}\left[\frac{\partial H_{e}}{\partial \ell} \frac{\partial \ell}{\partial \tau} \frac{\partial \tau}{\partial \sigma_{e_{i}}}\right]\right) \frac{\partial \sigma_{e_{i}}}{\partial \sigma_{e}}\left(\frac{\partial \sigma_{e}}{\partial \rho_{e}} \vartheta\left(\rho_{e}\right)+\sigma_{e}\left(\rho_{e}\right) \frac{\partial \vartheta}{\partial \rho_{e}}\right)-\lambda_{e}^{T}\left[\frac{d K_{e}}{d \rho_{e}} U_{e}\right]
$$

The adjoint vector is found by solving the following system:

$$
\lambda=K^{-1} \sum_{i=1}^{k}\left[\frac{\partial H_{e}}{\partial \ell} \frac{\partial \ell}{\partial \tau} \frac{\partial \tau}{\partial \sigma_{e_{i}}}\right]\left(\frac{\partial \sigma_{e_{i}}}{\partial \sigma_{e}} \frac{\partial \sigma_{e}}{\partial U} \vartheta\left(\rho_{e}\right)\right)
$$

The contributions of the relative stress scalars are included in the adjoint load.

\subsubsection{Lattice Beam Optimizations}

Sizing optimizations on a collection of beam elements is performed to fine tune the microscopic scale of the lattice structure. This method is applied to an area that has been converted from a volume of graded relative densities to a structure of beam segments. To obtain a graded lattice structure from the micro-struts; the beams are assumed to follow classical Euler-Bernoulli beam theory [162]. Mechanical properties for micro-lattice 
structures have been studied extensively with FEM simulations closely modeling classical beam theory $[162,163,164]$.

\subsubsection{Problem Formulation for Lattice Beam Optimization}

Tapered beam elements with constant slope are used as replacements for the lattice struts [101]. Sensitivity analysis of the global stiffness matrix is controlled by the crosssectional areas at the joining nodes of each tapered beam. This reduces the number of design variables to consider by focusing on commonly shared joints (nodes) as opposed to the ends of each individual beam. Lattice beam optimization can be described best as a sizing optimization problem where the joint thicknesses are optimized for a given objective function. The general problem statement for mass reduction for a lattice structure is given below;

$$
\begin{aligned}
& \text { minimize: } f(\vec{A}) \\
& \text { subject to: }\left\{\begin{array}{c}
K(A) U=F \\
\mathcal{G}_{i}\left(A_{i}\right) \leq 0 \\
h_{i}\left(A_{i}\right)=0 \\
A_{\min } \leq A_{i} \leq A_{\max } \\
\rho_{i} \cdot L_{i}>0
\end{array}\right.
\end{aligned}
$$

where $f(A)$ is the objective function, $\rho_{i}$ is the density of each beam, $A_{i}$ is the vector of cross-sectional areas of the tapered beam elements, $L_{i}$ is the length of each tapered beam, $U$ is the displacement vector, $K(A)$ is the global stiffness matrix as a function of crosssectional areas, $F$ is the force vector, $A_{\min }$ is the minimum cross-sectional area to avoid singularities, $A_{\max }$ is a pre-determined maximum area for the tapered beams and $\mathcal{G}_{i}$ is a set of inequality constraints and $h_{i}$ is a set of equality constraints. 
For mass minimization of micro-lattice structures; stress constraints combined with a buckling criterion are normally used together [101]. This is because buckling is the main initialization of failure for some lattice topologies, particularly those that are bending dominant [165]. However, buckling is not considered in this manuscript during lattice beam sizing optimizations because it is outside the scope and will reduce computational costs.

\subsubsection{Lattice Beam Sensitivity Analysis}

The optimization responses will depend on the cross-sectional areas of tapered beams. During optimizations, the design variables are the radii of the lattice struts. Hence the sensitivities are then converted from area-based design variables to radii-based design variables.

Due to the cross-sectional areas are coupled; a modification of the sensitivities must be applied. For the purposes of this thesis, the beam optimizations are performed in Altair Optistruct. This is because the micro-scale optimizations are single objective optimizations and industry software is much more accurate than MATLAB run algorithms. Nevertheless, the objective functions and their sensitivities are presented in the proceeding sections.

\subsubsection{Mass}

The mass is calculated using the volume of the beam multiplied by the density. 


$$
M(r)=\frac{1}{2} \pi \sum_{e=1}^{\mathcal{E}}\left(r_{e, 1}^{2}+r_{e, 2}^{2}\right) L_{e} d_{e}
$$

where $\mathcal{E}$ is the number of elements, $M$ is the total mass as a function of the radii, $d$ is the density, $L$ is the length of the element and $r$ is the radii or design variables at the ends of the tapered beams.

The mass function can be represented with a matrix И. Тhe dimensions of the matrix are $\mathrm{n}$ by $\mathrm{m}$ where $\mathrm{n}$ is the number of elements and $\mathrm{m}$ is the number of design variables. The entries in the matrix are formed by the equation below:

$$
Y_{i, j}=\left\{\begin{array}{lr}
d_{e} L_{e}, & \text { if } e_{i} \ni r_{j} \\
0, & \text { otherwise }
\end{array}\right.
$$

The sensitivity with respect to the cross sectional areas are:

$$
\frac{\partial M}{\partial \rho_{e}}=\pi \mathrm{H} \overrightarrow{\mathrm{r}}
$$

\subsubsection{Fatigue}

The fatigue life and cumulative damage of a tapered beam element is calculated using the stresses coupled with a mean stress correction factor. The equation for damage calculation in a tapered beam is shown below:

$$
H=\sum_{i=1}^{k} \frac{n_{i}}{\ell\left(\sigma_{e q_{i}}\right)}
$$

where the local equivalent stress $\sigma_{e q}$ is calculated using the formulation and the local stress $\sigma_{i}$ can be found in shown in Appendix $\mathrm{C}, \ell\left(\sigma_{e q}\right)$ is functional to calculate the number of cycles to failure for a particular load and $\mathrm{k}$ is the number of cycles in the 
fatigue history. The damage sensitivity of a tapered beam using the adjoint method by changing its radii in general form is shown below:

$$
\frac{d H}{d r_{j}}=\left.\frac{\partial H}{\partial r_{j}}\right|_{U=\text { const }}+\lambda^{T}\left(\frac{d F}{d r_{j}}-\frac{d K}{d r_{j}} U\right)
$$

Because linear static stresses are assumed the derivative of the force vector $\frac{d F}{d r_{j}}$ is equal to zero. The adjoint vector $\lambda$ is found from:

$$
\lambda=\frac{\partial H_{e}}{\partial U} K^{-1}=\sum_{i=1}^{k}\left[\frac{\partial H}{\partial \ell} \frac{\partial \ell}{\partial \tau} \frac{\partial \tau}{\partial \sigma_{i}} \frac{\partial \sigma_{i}}{\partial \sigma_{e}} \frac{\partial \sigma_{e}}{\partial U}\right] K^{-1}=\sum_{i=1}^{k}\left[\frac{\partial H}{\partial \ell} \frac{\partial \ell}{\partial \tau} \frac{\partial \tau}{\partial \sigma_{i}} \frac{\partial \sigma_{i}}{\partial \sigma_{e}} E_{e} B_{e} \mathbb{T}_{e}\right] K^{-1}
$$

where $\tau$ is the equivalent stress functional, $\sigma_{i}$ is the mean and amplitude stresses at load cycle $\mathrm{i}$ and $\sigma_{e}$ is the reference stress in the beam. The implicit sensitivities are calculated by the summation of all tapered beams implicit sensitivities which are connected to the analyzed design variable. The implicit sensitivities are shown below:

$$
\left.\frac{\partial H_{e}}{\partial r_{j}}\right|_{\text {implict }}=\sum_{i=1}^{e} \lambda_{i}^{T}\left(-\frac{d K_{i}}{d r_{j}} U_{i}\right)
$$

where $e$ is the set of elements attached to design variable $r_{j}$.

The explicit sensitivity for the damage is calculated using the equation below:

$$
\left.\frac{d H_{e}}{d r_{j}}\right|_{\text {explicit }}=\frac{\partial H_{e}}{\partial r_{j}}=\sum_{i=1}^{k}\left[\frac{\partial H}{\partial \ell} \frac{\partial \ell}{\partial \tau} \frac{\partial \tau}{\partial \sigma_{i}} \frac{\partial \sigma_{i}}{\partial \sigma_{e}} E_{e} \frac{\partial B_{e}}{\partial r_{j}} \mathbb{T}_{e} U_{e}\right]
$$

The total sensitivity for damage in a tapered beam is therefore:

$$
\frac{d H_{e}}{d r_{j}}=\sum_{i=1}^{k}\left[\frac{\partial H}{\partial \ell} \frac{\partial \ell}{\partial \tau} \frac{\partial \tau}{\partial \sigma_{i}} \frac{\partial \sigma_{i}}{\partial \sigma_{e}} E_{e} \frac{\partial B_{e}}{\partial r_{j}} \mathbb{T}_{e} U_{e}\right]+\sum_{i=1}^{e} \lambda_{i}^{T}\left(-\frac{d K_{i}}{d r_{j}} U_{i}\right)
$$


The damage sensitivities can also be reformulated into an aggregate function such as the P-norm. Similarly to the stress sensitivities for the tapered beams, the number of design variables to evaluate reduces dramatically. The adjoint vector for a P-norm aggregate of damage in tapered beams is derived in the following equation:

$$
\lambda=\frac{1}{P}\left(\sum_{i=1}^{e} H_{i}^{P}\right)^{\frac{1}{P}-1}\left\{\sum_{i=1}^{e}\left[H_{i}^{P-1} \frac{\partial H_{i}}{\partial U}\right] P\right\} K^{-1}
$$

The implicit terms are calculated from:

$$
\left.\frac{d H_{P U}}{d r_{j}}\right|_{\text {implicit }}=\sum_{i=1}^{e} \lambda_{i}^{T}\left(-\frac{d K_{i}}{d r_{j}} U_{i}\right)
$$

The explicit terms are calculated with:

$$
\left.\frac{d H_{P U}}{d r_{j}}\right|_{\text {explicit }}=\frac{1}{P}\left(\sum_{i=1}^{e} H_{i}^{P}\right)^{\frac{1}{P}-1} \sum_{i=1}^{e}\left[H_{i}^{P-1} \frac{\partial H_{e}}{\partial r_{j}}\right] P
$$

The combined sensitivity is:

$$
\frac{d H_{P U}}{d r_{j}}=\frac{1}{P}\left(\sum_{i=1}^{e} H_{i}^{P}\right)^{\frac{1}{P}-1} \sum_{i=1}^{e}\left[H_{i}^{P-1} \frac{\partial H_{i}}{\partial r_{j}}\right] P+\sum_{i=1}^{e} \lambda_{i}^{T}\left(-\frac{d K_{i}}{d r_{j}} U_{i}\right)
$$

where $e$ is the set of elements associated with the design variable $r_{j}$. 


\section{Chapter 6: Automated CAD from Topology Optimizations}

The multi-scale design workflow presented in this thesis relies on automated model building. Topology optimization results are post-processed so that low density areas are converted into one-dimensional beam elements. The conversion is performed to achieve two objectives. The first objective is to create an FEM so that sizing optimizations can be performed. The second objective is to create an STL of the final structure. A journal paper was written describing the lattice meshing algorithm method and can be read in Appendix $\mathrm{H}$. The lattice meshing article is titled "Conformal wireframe nets for trimmed symmetric unit cells in functionally graded lattice materials" and is currently under review for the International Journal for Computational Methods in Engineering Science \& Mechanics. This chapter presents the methodology for creating the FEM models with integrated lattice structures and for producing the equivalent STL representation.

\subsubsection{ISO-Surface Extraction}

ISO-surfaces are computer graphics techniques for data visualization [166]. An ISOsurface is a surface representation of an ISO-line (or surface) of constant values. For topology optimization, the constant line values would be the optimal relative densities of the elements. The output surfaces can therefore be directly converted into engineering designs for post-processing. To extract the surface there exist several algorithms which can yield accurate representations of the final design. The marching cubes algorithm is commonly used to generate three dimensional meshes from volume data [167]. Other 
algorithms also expand upon the marching cubes algorithm to include the marching tetrahedral [168] and the asymptotic decider [169].

The marching cubes (marching squares in two-dimensions) algorithm is described in four steps [170]. Firstly, the shape must be represented as a collection of points inside the object and include discrete bounds for the object. When the two conditions are met, the marching cubes algorithm can begin. The first step is to divide the shape into an arbitrary amount of cubes by voxelization. Each cube is classified as either interior, exterior or on the boundary of the object. If a cube lies on the boundary, where some of the corners are inside of the shape and other corners lie outside, then a line (or plane in 3D) must pass through it. Surfaces must pass through the boundary cubes so that the intersecting edges of the cube are in between corners of opposite classification [170]. The surfaces must pass through the cube and create intersections. Each intersection from the cubes must then be connected together. The output of the algorithm is a three-dimensional triangulated surface that can be easily converted into an STL object. However, the mesh quality of the surface however is very poor. If post-processing finite element analysis is to be done on the ISO-surface; cumbersome re-meshing and user-intervention is required. Many other robust algorithms exist such as OPTISTRUCT's OSSmooth program [171]. The capability of OSSmooth allows for the generation of ISO-surfaces for both FEA reanalysis and STL geometries. It provides an accurate ISO-surface in an efficient timeframe. The ISO-surface will carve-out profiles using the finite elements form an input mesh so that tetra-meshing can be applied. This software is particularly usefully 
when transitioning from the results of a lattice optimization to a structure with tapered beams in areas of low density.

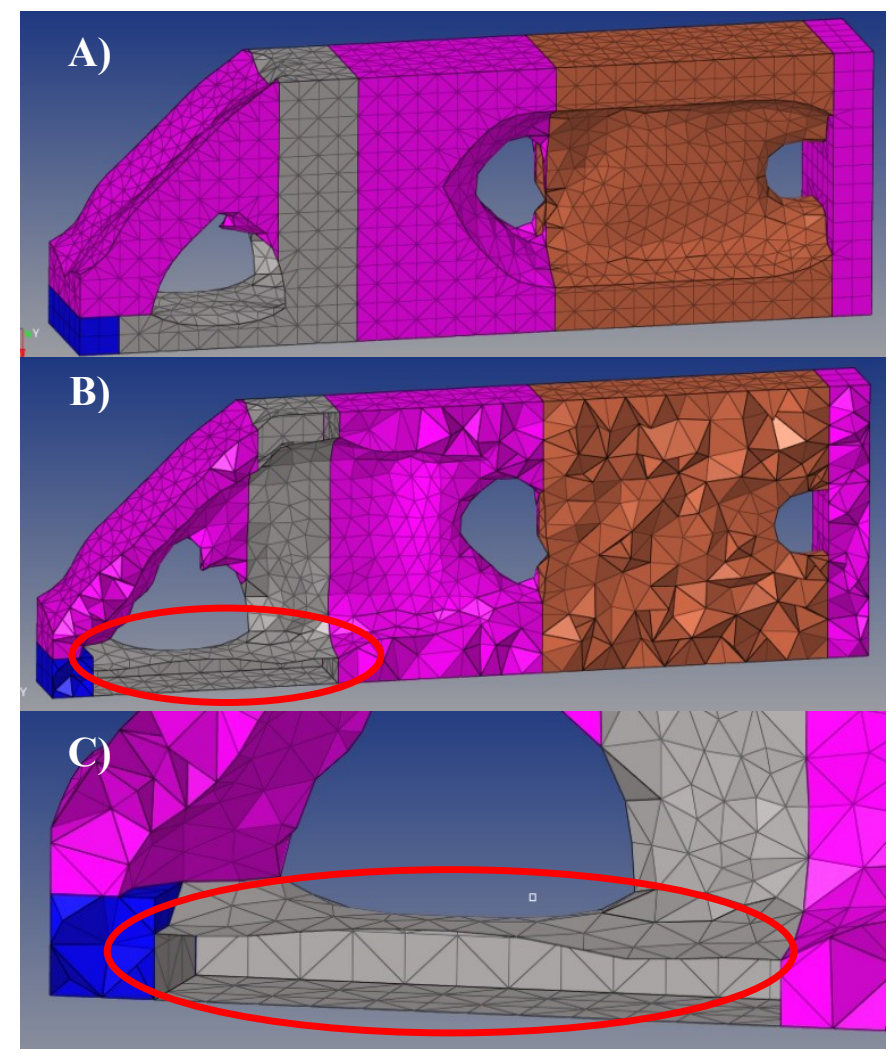

Figure 28 : Example of ISO-Surface Extraction Problems with OSSMooth (A) Iso-Surface (B) CrossSection of Meshed Areas (circled area shows meshing error) (C) Zoomed Section displaying failed mesh is empty with no tetrahedrons

The main issue when using OSSmooth for FEA-Reanalysis is the risk of un-meshable ISO-surfaces (or non-manifold). A manifold surface is defined as a closed mesh with no intersections or duplicate triangles. The ISO-surface extractor may fail to generate a manifold ISO-surface during post-processing. HYPERMESH ${ }^{\mathrm{TM}}$ has many features to automate mesh refinements however; no satisfying solution or workflow could be found for the goals in this manuscript. An example of failed re-meshing due to a non-manifold 
ISO-surface is shown in Figure 28a and Figure 28b. Figure 28a shows an ISO-Surface extraction from OSSmooth after a topology optimization with a relative density threshold of $30 \%$. In Figure $28 \mathrm{~b}$ the circled area is seen to be non-manifold and thus could not be meshed with tetrahedrons. Figure $28 \mathrm{c}$ shows the empty volume that could not be remeshed for FEA and would require user post processing to alleviate meshing errors. The multi-scale design workflow requires full automation hence user interaction must be removed entirely.

It is important that the re-meshing process produces error-free meshes because highly complex ISO surfaces are expected to be post processed. Any chance of encountering meshing errors is unacceptable especially in an optimization environment. To ensure that ISO-surfaces can be generated reliably for STL generation and FEA re-analysis; a new workflow is proposed inside MATLAB. The new workflow allows the creation both “.bdf" (NASTRANTM) and ".fem" (HYPERMESH ${ }^{\mathrm{TM}}$ ) files for FEA analysis and for lattice beam sizing optimizations. In addition, STL models will also be automatically generated for 3D printing the final results. It is important to have a robust and reliable method to generating the ISO-surfaces as the results from the multi-objective topology optimizations need to be post-processed automatically.

\subsubsection{MATLAB Methodology for Lattice Embedded ISO-Surfaces}

One of the main phases in the multi-scale design workflow in this thesis is concerned with the application and optimization of lattice truss structures. Lattice beams can be embedded into the ISO-surface FEM by using additional Boolean operations and from 
incorporating the trimmed lattice structure. The procedure is similar to that for the development of an ISO-surface for a regular topology optimization with the addition of a "lattice design space". The element density threshold does not convert elements below that value into voids but into lattice beams.

The goal of this step is to create a finite element model for beam sizing optimizations. In addition, the geometric accuracy of the solid regions in the FEM is relaxed for more reliable reconstruction of the solid domain. The overall steps for creating the lattice embedded FEM models are given below:

1. Lattice Topology Optimization Post-Processing

2. Voxelization and Surface Reconstruction

3. ISO-Surface Generation

4. Lattice Embedded FEM Creation

5. Contact Surface Creation

6. STL Representation

The steps defined above are explained further in the subsections of this chapter.

\subsubsection{Lattice Topology Optimization Post-Processing}

The optimization results are processed such that elements below the relative density threshold are converted in lattice material (beam elements). The elements affected belong to the design space. Similar to the method in the regular ISO surface reconstruction, the FEM is split into the design space and non-design space. 
Within the design space there will be a "design space solid domain" and a "design space lattice domain". The solid domain is the regions where elements have relative densities above the threshold and the lattice domain is for elements which relative densities below the threshold. That surface will then be sent to the surface reconstruction stage to be used for Boolean operations to obtain the final solid domain and lattice domain surfaces. The "entire design space faces" and "non-design space faces" are also extracted for Boolean operations.

\subsubsection{Voxelization and Surface Reconstruction}

Surface reconstruction is performed on the design space solid faces. This generates the "new design space solid faces" which is sent for Boolean operations. The design space solid faces are voxelized and then sent to a marching cube algorithm for surface reconstruction. Afterwards the surface is smoothed to remove jagged edges and is then checked for manifoldness. If the new surface is manifold then it is used to divide the design space faces into the "updated design space solid faces" and the "lattice design space faces".

\subsubsection{ISO-Surface Generation}

ISO-surface generation for lattice embedded topologies follows the same principles as in the ordinary ISO-surface generation. However, there is an additional step which extracts the lattice design space faces. 


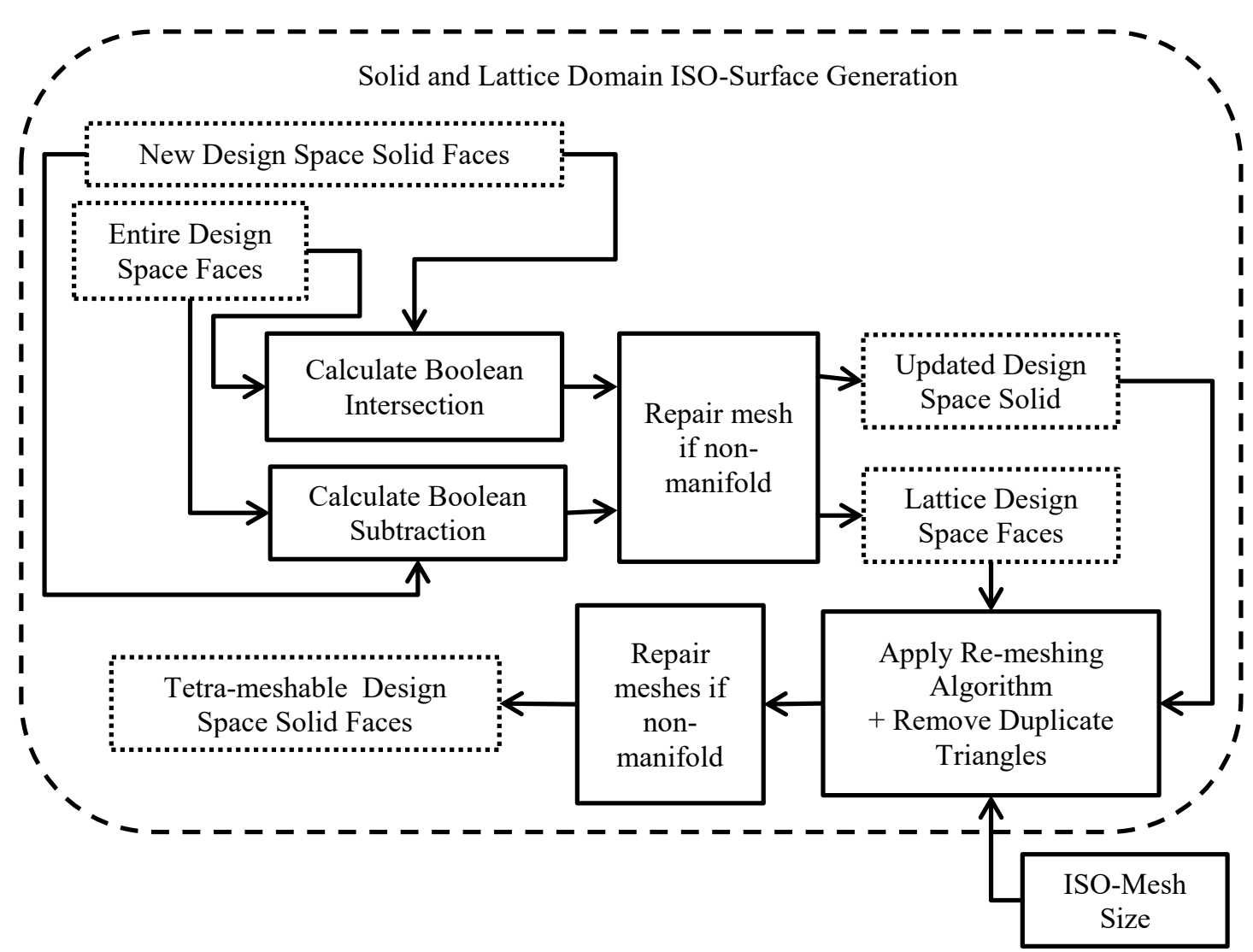

Figure 29 : Block Diagram Solid and Lattice Domain ISO-Surface Generation

The "updated design space solid faces" is obtained by the intersection between the "new design space solid faces" and the "entire design space faces". The "Lattice design space solid faces" is calculated by the subtraction of the "new design space solid faces" from the "entire design space faces" surface. The subtraction operation is to ensure the boundaries between the two domains are equivalent without the risk of introducing nonmanifold elements.

For each of the computed surfaces from the Boolean operations, they must be checked for manifoldness. If errors in the mesh are found they are corrected using automated mesh 
cleaning algorithms in the ISO2MESH toolbox [172]. Producing the tetra-meshable triangulated surfaces also require manifoldness to avoid singular elements.

The "Patch Remesher" algorithm is then applied to the "updated design space solid faces" so that the solid domain can be tetra-meshed [173]. The block diagram for the meshing and splitting process is shown in Figure 29.

\subsubsection{Lattice Embedded FEM Creation}

The step in the ISO-surface generation phase produces the lattice beams to be appended to the FEM. The lattice beams are created using the trimmed lattice algorithm. The lattice is to appear in the "lattice design space faces". The information for the size and type of unit cell is required before creating the trimmed lattice. If a lattice net is selected to connect the cut struts together, then it is applied along the "lattice design space faces" boundary.

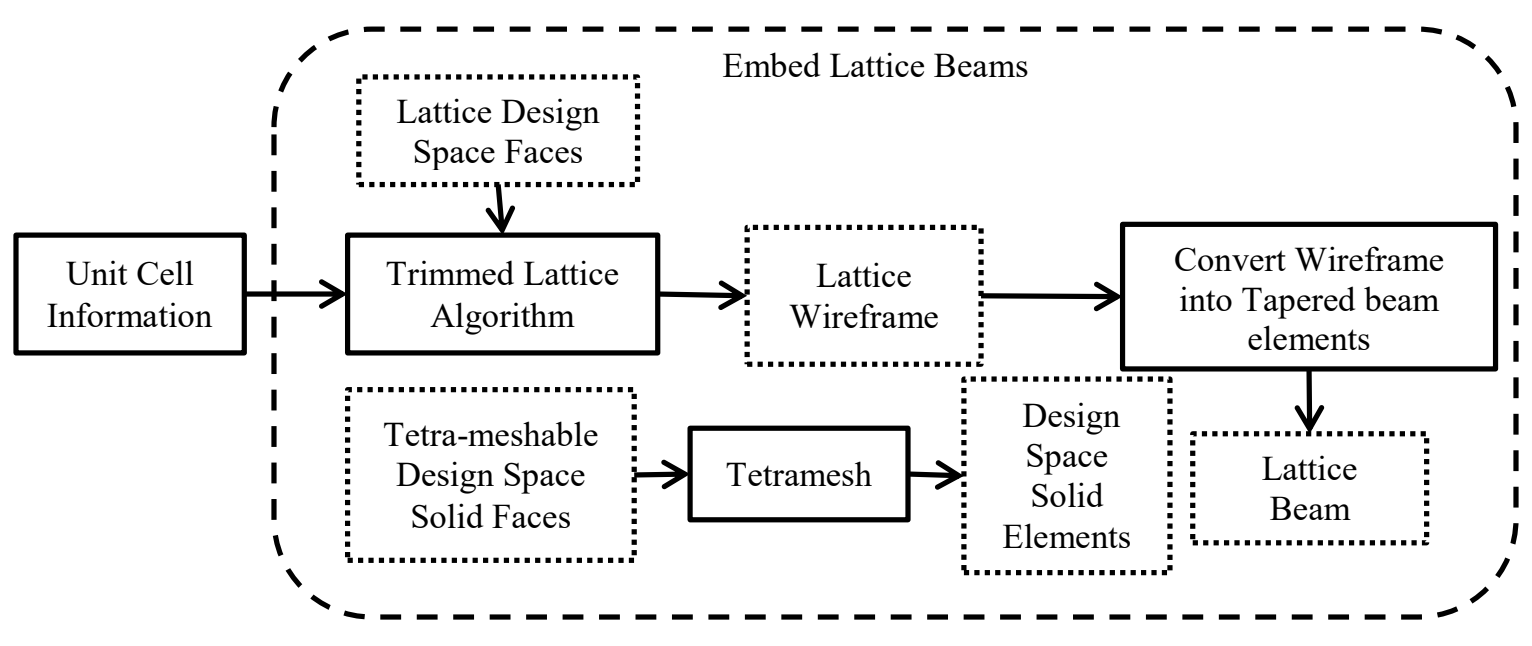

Figure 30 : Block Diagram for Embed Lattice Beams 
After the trimmed lattice algorithm has output a trimmed lattice, the lattice wireframe is converted into a collection of beam elements. The new beam elements are then connected to the design and non-design elements with a contact surface in the contact surface creation step. The steps are outlined in Figure 30.

The final step in this section is to tetra-mesh the "tetra-meshable solid design faces". If the entire design space is to be converted into lattice, then tetra-meshing is omitted. Much like the beam elements, the solid tetrahedron elements are to be connected by a contact surface to the beams, solid shell elements and non-design space elements.

\subsubsection{Contact Surface Creation}

Contact surfaces are used to combine the design space tetra-mesh to shell elements and the lattice beam elements. This is done so that it minimizes meshing modeling complexities. The design space solid elements are connected to the non-design space elements using a contact surface. The solid design elements are the master set while the nodes in the non-design elements are part of the slave set. The lattice beams are connected by a contact surface to the solid elements where the nodes in the lattice beams are the slave set and the solid elements are the master set. If a lattice net is used to connect the cut struts together, then any beams whose nodes are doubly connected in a slave set are removed from the FEM. The master elements are found by slightly enlarging the "lattice design space faces" and locating solid element nodes inside the enlarged volume. Elements who have any of their attached nodes found in the enlarged volume form the new master set to connect to the lattice beam nodes. 


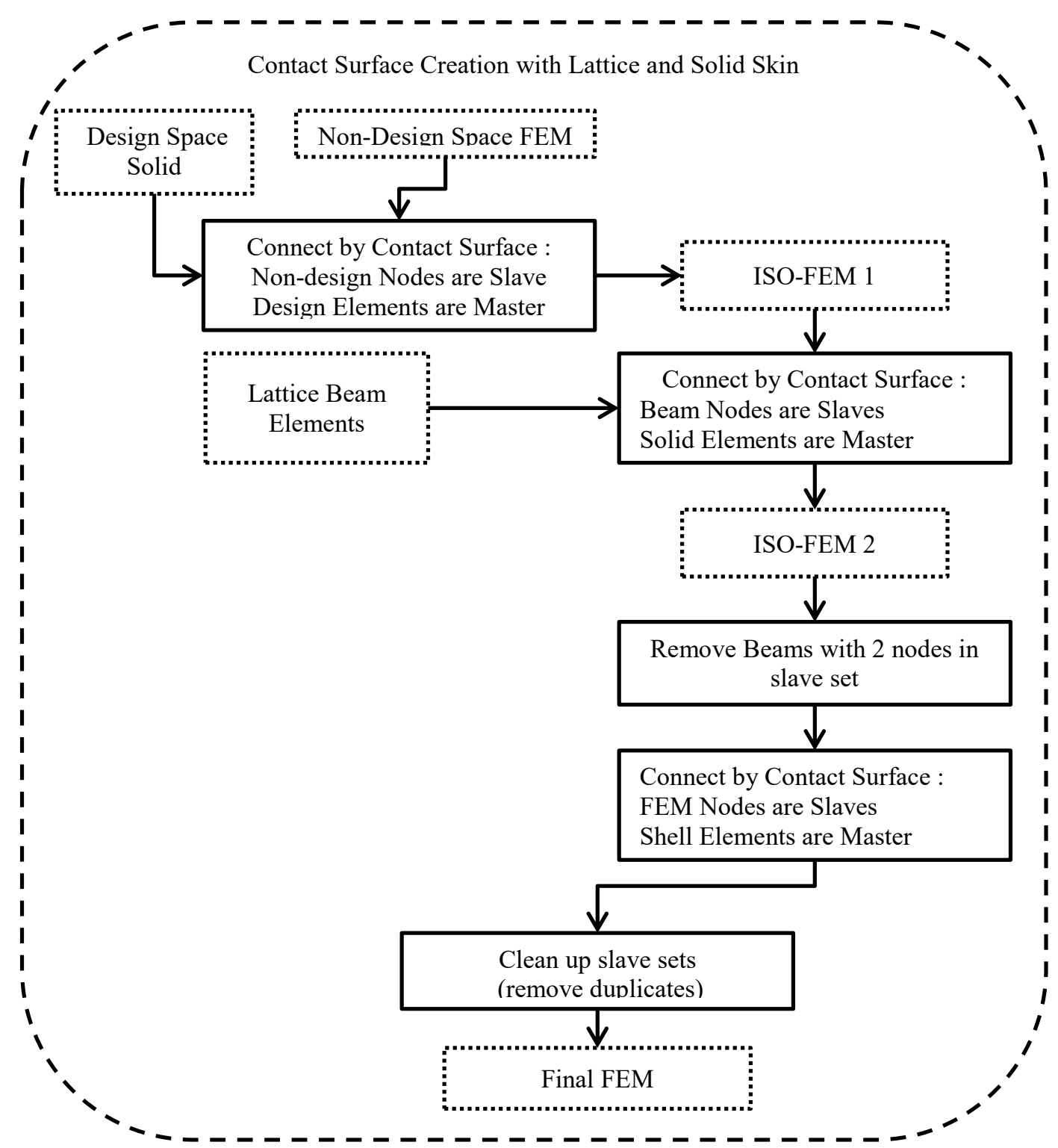

Figure 31 : Block Diagram for Contact Surface $\bar{C} \overline{\text { reation }} \overline{\text { with }} \overline{\text { Lattice }} \overline{-} \overline{\text { nnd }} \overline{\text { Solid Skin }}$

Once the master and slave sets have been created, common nodes in slave sets are removed. This is to avoid finite element errors. The slave sets are analyzed in order of creation, for example; if a node placed in the first slave set " $\mathrm{A}$ " is present in another slave set " $\mathrm{B}$ ", then the duplicate is removed from slave set "B". A block diagram illustrates the 
contact surface creation in Figure 31. The final step after locating slave nodes and master elements is writing the NASTRANTM and/or OPTISRTUCT ${ }^{\mathrm{TM}}$ contact surface cards. Defining the contact cards is required so that the FEM can be sent to the FE solver.

\subsubsection{STL Representation}

The STL creation for lattice embedded topologies is typically done after a beam sizing optimization. An STL file can be created by using the "updated solid design space faces" and the "non-design space faces".

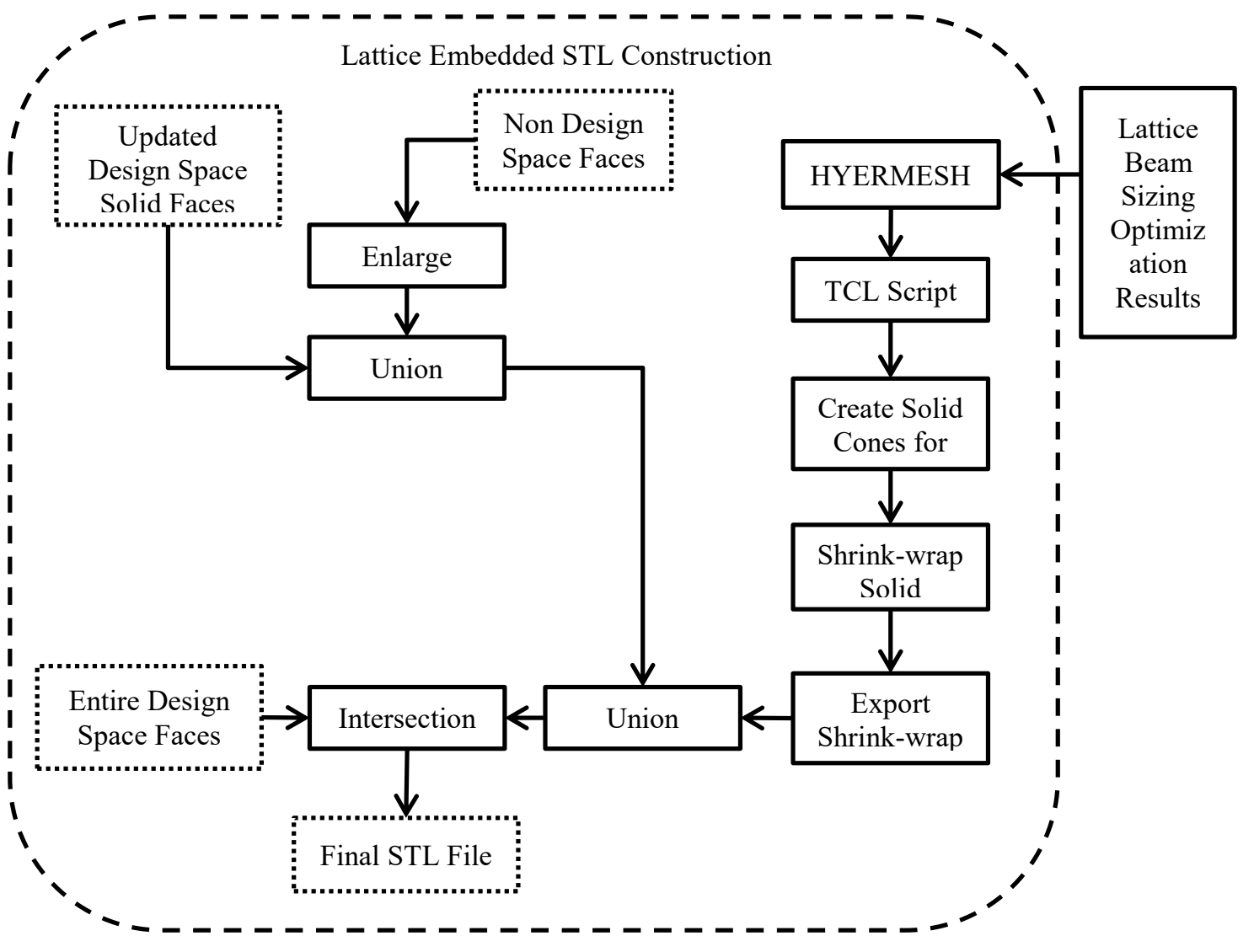

Figure 32: Lattice STL File Creation 
Similar to a regular ISO surface STL creation, the non-design space faces are slightly enlarged and added to the "updated solid-design space faces". The updated solid-design space faces are those after the voxelization stage and surface reconstruction. After which the intersection between the two face groups is taken and the non-lattice region is created. A block diagram shown in Figure 32 describes the STL creation process for lattice embedded topologies.

The lattice beams must be converted into an STL representation as well and is done using HYPERMESH ${ }^{\mathrm{TM}}$. A TCL script is used to automate the conversion between the onedimensional tapered beam elements into solid cones. Once solid beam representations have been generated, a shrink-wrap is applied onto the solid surface. Shrink-wrapping is a process where an object is voxelized and a surface re-construction algorithm is applied to create a closed surface. The shrink-wrapped lattice beams are then converted into an STL and exported into MATLAB. Additional mesh repairing is performed on the mesh to ensure it is manifold. A final Boolean operation is applied onto the lattice STL by a union between it and the optimized topology STL followed by an intersection between the union and the entire design space faces.

\subsubsection{Numeric Examples}

Four examples are shown in Figure 33 which illustrates the automated modeling output after a topology optimization. The lattice topology chosen for these examples is a diamond lattice. Figure $33 \mathrm{a}$ and $\mathrm{b}$ shows the results of a lattice optimization with $\mathrm{a}$ conforming lattice net as a skin to connect the trimmed lattice structure. 

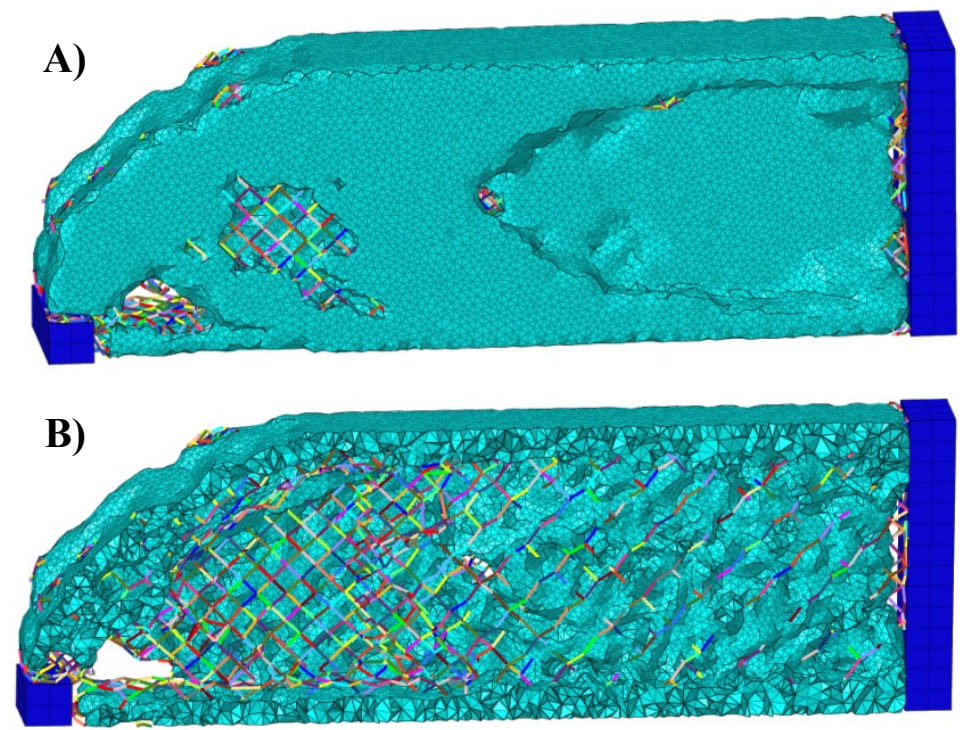

Figure 33 : Lattice Embedded ISO-Surface Results (a) Lattice-Net (b) Lattice-Net Cross-Section The solid blue elements in Figure 33 display the non-design space while the cyan elements are the solid elements within the design space. Tapered beam elements are line elements and are seen in the inside of the structure. Each of the linear elements is associated with a unique property and is thus color-coded at random. Individual properties are given to the linear elements so that they can be sized appropriately in structural optimizations. 


\section{Chapter 7: Case Study and Industrial Multi-Scale Design}

Two case studies will be presented in this section. This first case study will validate the proposed optimization gradients by performing a multi-objective optimization between the weight and the cumulative damage on a so called "L-bracket". Methods of lattice optimization will be compared to traditional methods for the first case study. The beneficial effects of UIT will also be compared by using the maximum damage results between the Pareto frontiers.

A second case study will apply the proposed methods to optimize an aircraft hinge for minimum weight with maximum fatigue life. Pareto frontier for lattice material subjected to mass minimization and life maximization is generated. The addition of lattice material will also be incorporated into the design. The material properties of the lattice material will not have the optimized material characteristics as it is not possible to apply UIT on cellular material. A comparison is also made to quantify the accuracy of the final model when linear beam elements are used to model the lattice structure when compared to when three dimensional elements are used to model the lattice.

\subsection{Multiple-Objective Gradient Based Optimization}

For gradient based multiple objective optimization, there exists three basic formulations which combine the objective functions into together. These methods include the weighted sum method, weighted metric method and the constrained epsilon method. The optimal solution for all objectives is called the utopia point and is a fictional state where all objectives are at their minimum value. The multi-objective workflow presented in this 
thesis only refers to the constrained epsilon method and hence is explained in the main text.

\subsubsection{Constrained Epsilon Method}

This method will convert all but one objective into an inequality constraint. For each new constraint added to the optimization problem, a set of constraint values (epsilon) will produce a new sub problem to solve. Each sub problem will constrain the transformed objectives to a value specified by epsilon. The process continues until all weighted factors for the transformed objectives have been processed. The results can then be plotted to show the Pareto frontier. One advantage of this method is that it can be applied to both convex and non-convex optimization problems. A disadvantage is that a maximization and minimization of each objective must be done so that the epsilon values are within the appropriate range of the optimization problem.

A multiple-objective optimization problem can then be transformed from the original form of:

$$
\begin{gathered}
\min f_{1}(x), f_{2}(x) \ldots f_{n}(x) \\
\text { subject to; } \begin{array}{c}
\mathcal{G}_{i}(x) \leq 0, \quad i=1,2, \ldots, \mathbb{r}_{e q} \\
h_{i}(x)=0, \quad i=1,2, \ldots, \mathbb{m}_{e q}
\end{array}
\end{gathered}
$$

To a transformed form with a single objective and new inequality constraints:

$$
\begin{gathered}
\min f_{1}(x) \\
f_{i+1}(x)-\vec{\varepsilon} \leq 0, \quad i=1,2, \ldots, n-1 \\
\text { subject to; } \quad \mathcal{G}_{i}(x) \leq 0, \quad i=1,2, \ldots, \mathbb{r}_{e q} \\
h_{i}(x)=0, \quad i=1,2, \ldots, \mathrm{m}_{e q}
\end{gathered}
$$


Where $\vec{\varepsilon}$ is a list of weights to constrain the transformed objective functions.

\subsection{Multi-Objective Optimization Problem Definition and Sensitivities}

The problem statement for the multiple objective optimizations can be represented in the following system of equations below:

$$
\begin{gathered}
\min \|H(\vec{\rho})\| \\
\frac{M(\vec{\rho})}{M_{\text {total }}}-\vec{\varepsilon} \leq 0, \\
0<\rho_{\text {min }} \leq \rho \leq 1 \\
K(\vec{\rho}) U=F
\end{gathered}
$$

where $\|H(\vec{\rho})\|$ is the p-norm of 2 for the elemental damage, $M(\vec{\rho})$ is the mass of the Lbracket as a function of relative densities, $\vec{\varepsilon}$ is the vector of mass constraints for the multi-objective optimization and $\vec{\rho}$ is the design variables of relative densities. The norm of the damage is measured because it is able to represent he damage as a single objective. The mass is calculated with the formulation.

$$
M(\vec{p})=\sum_{e=1}^{N} v_{e} d_{e} \rho_{e}
$$

where $v_{e}$ is the volume of the design element, $\mathrm{d}_{\mathrm{e}}$ is the original density of the design element and $p_{e}$ is the relative density of element e.

The gradient for a mass response can therefore be written as:

$$
\frac{\partial M}{\partial \rho_{e}}=v_{e} d_{e}
$$

The method for determining the mass fraction and its sensitivities is found by dividing the total mass of the design space. 
The cumulative damage when calculated with an aggregate function is expressed as:

$$
\|H(\vec{\rho})\|=\left.\frac{\partial \psi_{H_{P U}}}{\partial \rho_{e}}\right|_{U=\text { const }}-\lambda^{T} \frac{d K}{d \rho} U
$$

where,

$$
\begin{gathered}
\left.\frac{\partial \psi_{H_{P U}}}{\partial \rho_{e}}\right|_{U=\text { const }}=\frac{1}{P}\left(\sum_{j=1}^{N} H(\vec{\rho})^{P}\right)^{\frac{1}{P}-1} \sum_{j=1}^{N}\left[H(\vec{\rho})^{P-1} \frac{H_{j}(\vec{\rho})}{\partial \rho_{e}}\right] P \\
\lambda=K^{-1}\left[\frac{1}{P}\left(\sum_{j=1}^{N} H_{j}^{P}\right)^{\frac{1}{P}-1} \sum_{j=1}^{N}\left[H_{j}^{P-1} \frac{\partial H_{j}}{\partial U}\right] P\right]
\end{gathered}
$$

where $P$ is equal to 2 and the relative stress influence is included in the calculate of the damage for lattice material only. For solid material optimization, the " $q_{p}$ " method is applied [174]. The SIMP method is also applied as a stiffness penalization metric when lattice material is not required for the optimization procedure. Lattice is applied in the aircraft hinge case study while both solid material and lattice material are compared in the L-bracket case study.

\subsection{L-Bracket}

The L-bracket is a common case study for stress constrained topology optimization [175]. The ninety degree kink, shown in Figure 34, creates a stress concentration making this particular case useful for stress based topology optimizations. Normally engineers would round off sharp corners to avoid stress singularities but with stress and fatigue 
minimization techniques that is not needed as the optimization will generate the optimal geometry around the kink.

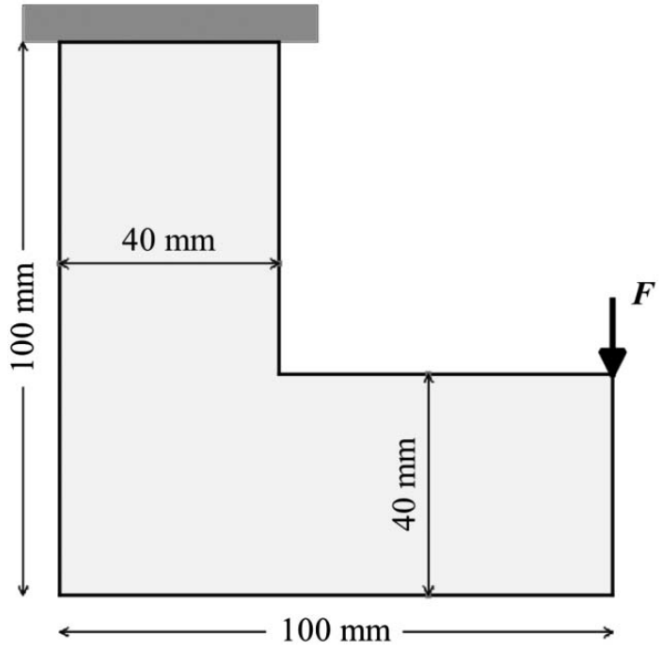

Figure 34: Block Diagram of the L-Bracket [175]

Four Pareto frontier plots for mass fraction and maximum damage are generated. Two plots will include lattice material while another two plots will contain solid material only. The multi-objective analysis will be performed using the constrained epsilon method where the damage will be minimized while the amount of material is constrained. The total number of points on the fronts is limited to twelve cases excluding the extreme cases of full and zero mass (or volume). The damage will be calculated as the norm of all elemental damages and is equivalent to a p-norm of two.

\subsubsection{Problem Definition}

The applied force on the L-bracket is $100 \mathrm{~N}$ and the model is mesh with 640 bilateral square elements. The fatigue history for the L-bracket is given in Figure 35. The 
reference stresses calculated are the signed-Von-Mises and the Modified Goodman line is used for multi-axial tress corrections.

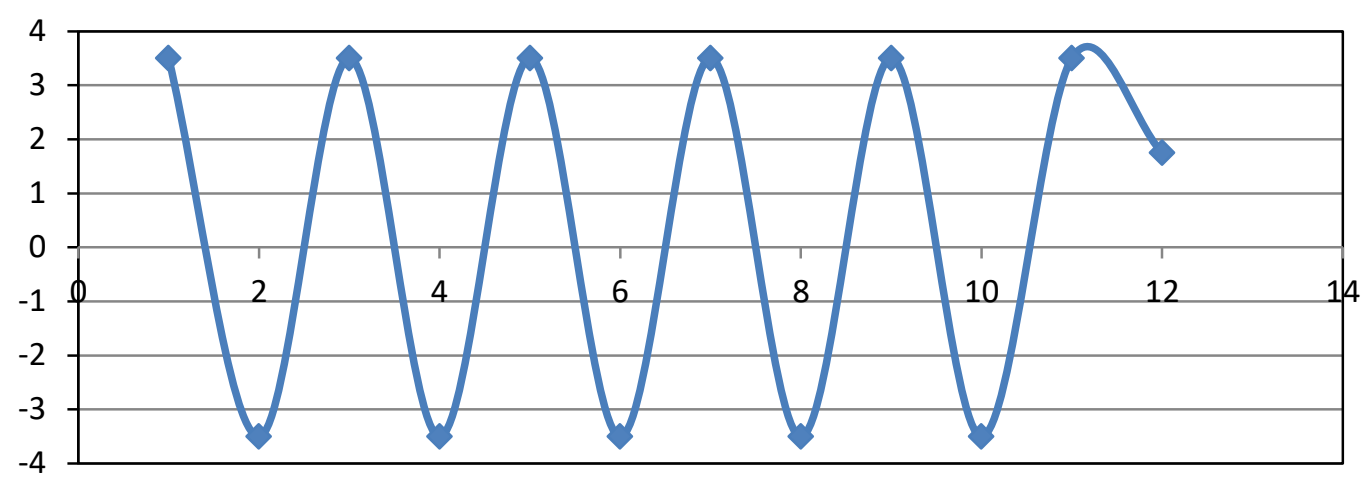

Figure 35 : Fatigue History for L-bracket (Force Multiplier)

The selected yield strengths and ultimate tensile strengths for the material are $1080 \mathrm{MPa}$ and $1200 \mathrm{MPa}$. The modulus was set to be 114GPa. The lattice topology chosen for this case study is the cubic lattice. Finally, the method of moving asymptotes (MMA) was selected to determine the optimal design points.

\subsubsection{Results}

The Pareto frontier for the non-treated cases shows higher damage when compared to the treated versions when the mass fraction is above fifty percent. This is expected because the fatigue properties are enhanced with the UIT treatment. However the untreated curve produced lower damage topologies when the mass constraint fell below fifty percent, shown in Figure 36. These differences are due to the different slopes of the SN-curves. The untreated slope is steeper but has a higher intercept whereas the treated $\mathrm{SN}$-curve has a lower intercept but flatter slope. The differences in the SN-curve equation cause the untreated models to have lower damage in the low cycle regime. However because SN- 
curves are only valid in the high cycle regime (1000 cycles or more); the low cycle section of the Pareto frontier can be omitted for fatigue improvement comparisons. The low and high cycles regimes are indicated in Figure 36, all points to the left of the transition line is in the high cycle regime while points to the right are in the low cycle regime. The fatigue limit was omitted for the multi-objective optimization and is an additional factor which may have contributed to the untreated material outperforming the treated material.

\section{$\log ($ Cumulative Damage) vs. Mass Fraction}

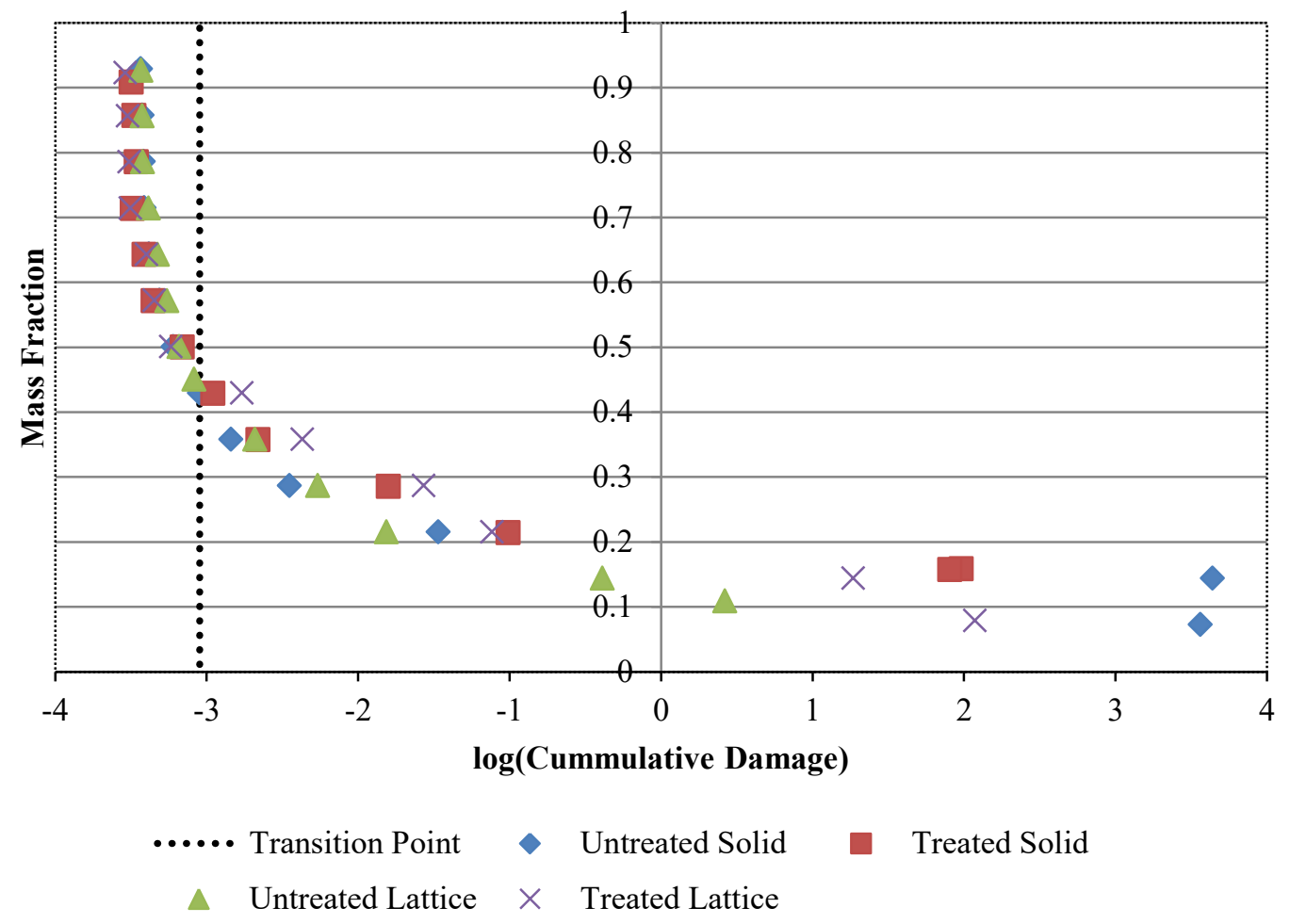

Figure $36: \log ($ Damage $)$ vs. Mass Fraction

The different the topologies for the L-Bracket case study are seen in Figure 37. For the non-lattice material; the fatigue objective successfully avoided the kink in the L-bracket. 
A ' $\mathrm{V}$ ' shape connecting the force location to the vertical members was formed in Figure $37 \mathrm{a}$ and $\mathrm{b}$. The results for the cubic lattice material did not avoid the kink in the L-bracket as seen in Figure 37D, and resulted in higher cumulative damage than the treated solid material shown in Figure 37b.
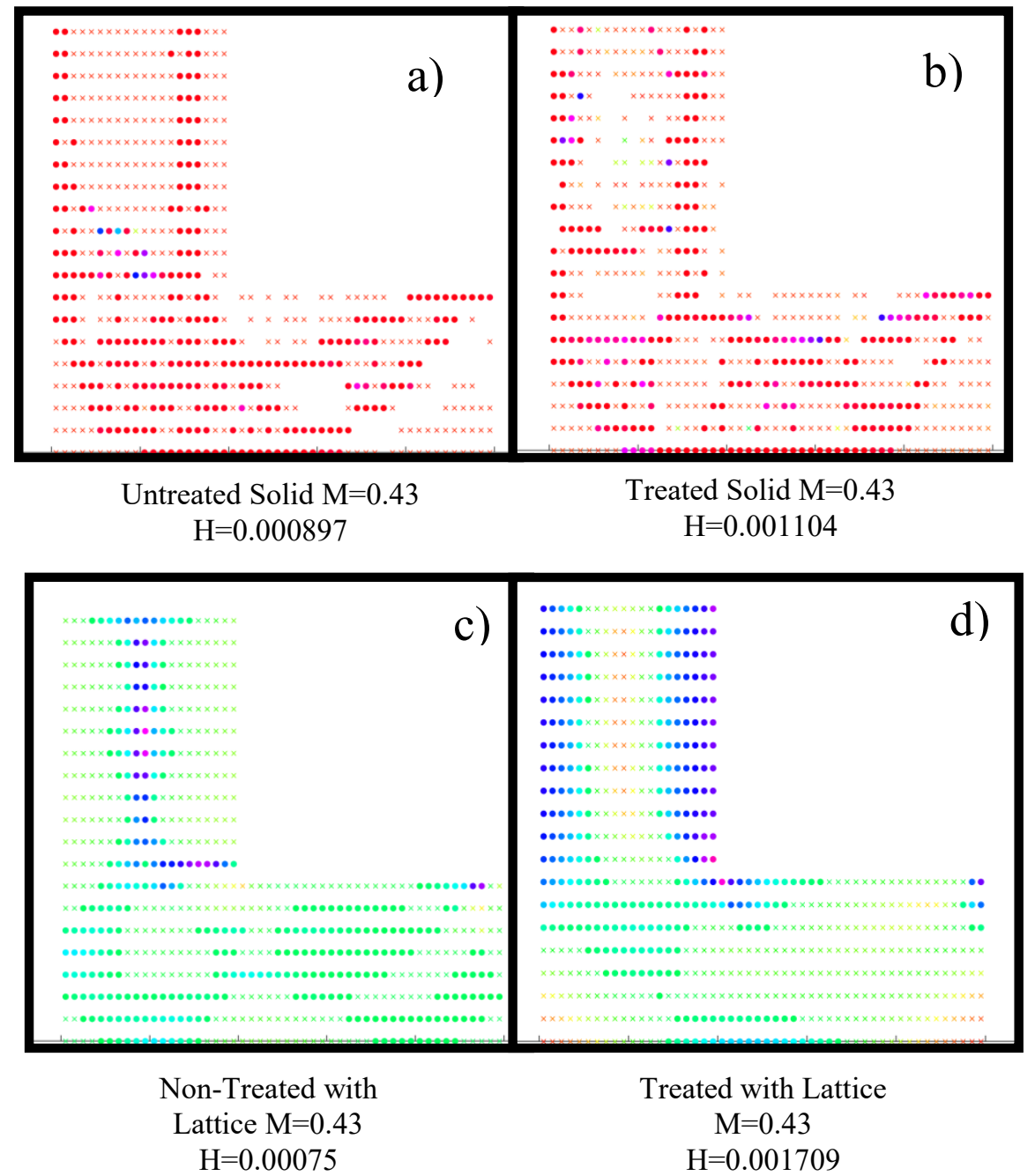

Figure 37 : L-Bracket Optimized Topologies at 43\% Mass Fraction $($ Dot = Solid, ' $X$ ' = Lattice)

The untreated lattice case on the other hand, Figure 37c, does not have a high density of material around the kink, suggesting that the stress concentration should be avoided. All 
the displayed topologies in Figure 37 have different material layouts due to the nature of gradient descent algorithms and their sensitivities to the initial starting point.

The topologies for the solid cases are more discrete when compared to the lattice optimized topologies. The high degree of discreteness is expected because the stiffness interpolation function is steeper and the stresses are more heavily penalized. The lattice optimized topologies showed high porosity and more damage was seen in the treated lattice material when compared to the solid treated material in the low cycle regime. The porosity is also expected as a functionally graded material is used for the stiffness penalization. The FGM also affects the Poisson ratio and alters the macros stresses in the L-bracket into higher micro stresses and minimizes the effects of stress discontinuities. The relaxed stresses in FGM can be seen in Figure 37c and d. Figure 37 shows the spectrum of relative densities. When the material is close to full density, the color is red and as the densities drops to zero (void material) it becomes dark pink. Green colors indicate relative densities near forty percent. As seen in Figure 37, there are many possible solutions due to the non-linear nature of topology optimization. The final topology is sensitive to the starting point and trust-region size. The existence of many local optima requires multi-objective programming to sort out the possible load paths for a given problem as seen in this case study. To determine if a solution is truly unique, then the same solution should arise if a finer mesh size is used, similar to a mesh convergence study $[63,159]$. 


\subsection{Aircraft Hinge Case Study}

The following case study will demonstrate the applicability of the proposed method for high lifecycle AM components. The two objectives are the mass fraction of the design space and the minimum fatigue life. A cubic lattice will be chosen as the lattice structure of choice because it is computationally cheaper than the other types. The mechanical properties of the cubic lattice are generally weaker, seen in Figure 22, than say an FCC lattice however, determining the optimal lattice for fatigue optimizations is outside the scope of this research. A Pareto frontier of the part will be generated and the design closest to the utopia point will be chosen for a beam sizing optimization. The model will be rebuilt following the methodology outlined in Chapter 6 and linear beam elements will be used to represent the lattice structure. After beam sizing optimizations, the accuracy of the final model is then compared to a fully meshed model consisting of solely three dimensional elements. Advantages and disadvantages are discussed at the end of this case study.

\subsubsection{Design Workflow}

General multi-objective topology optimizations combine objectives together and perform a minimization on the joined functions. Filtering is used to prevent checker boarding; an FEM numerical instability in topology optimizations. When the relative change during iterations is below a certain threshold, then convergence is said to be achieved. Multiple optimizations are performed for different weight assigned to the objective functions. The results of the optimizations are then displayed in a Pareto frontier. 


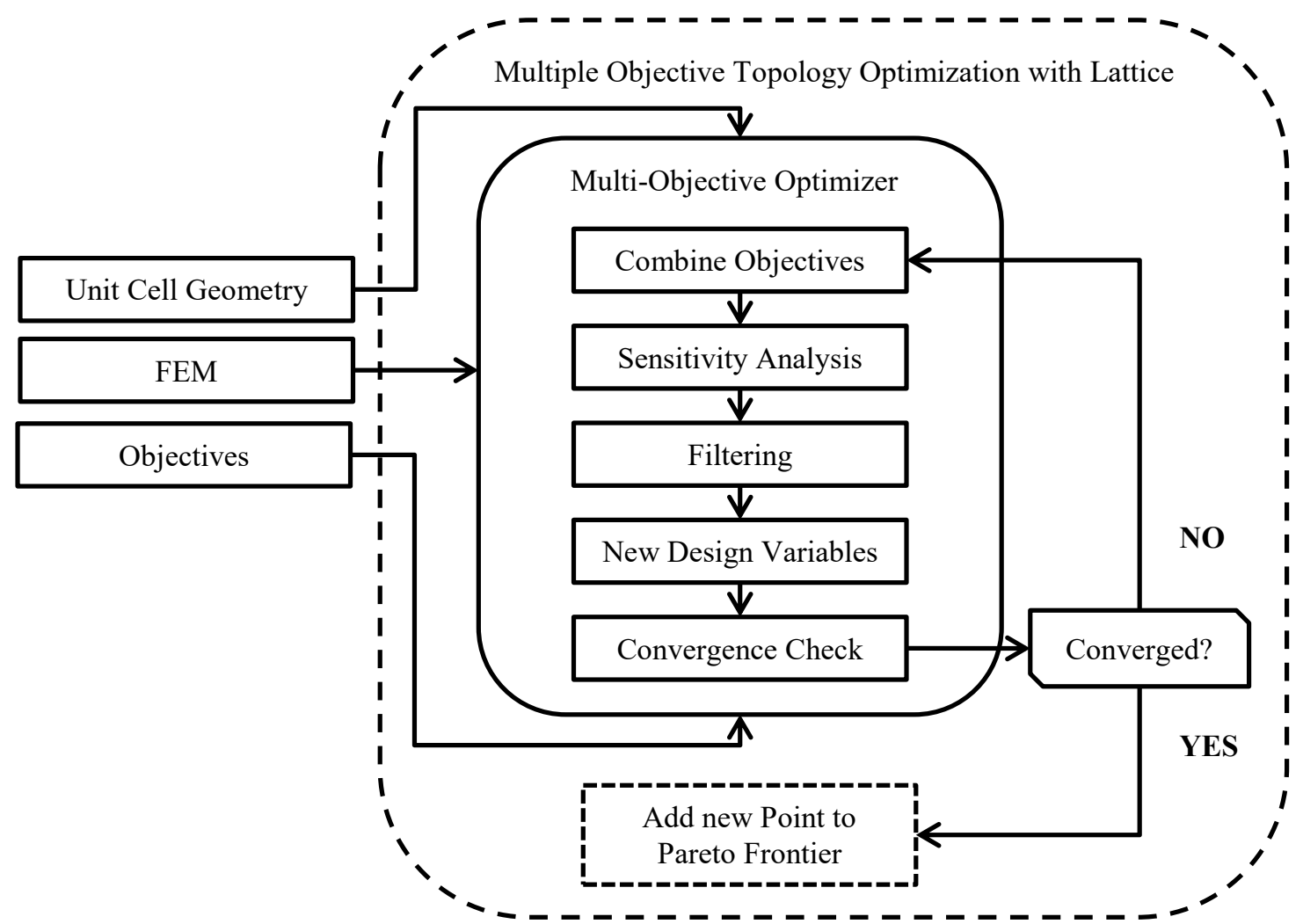

Figure 38 : Block Diagram for Multiple Objective Topology Optimizations with Lattice Material

The post-processing step is performed on the solution closest to the utopia point found in from the third goal. The homogenized lattice areas are converted into lattice truss structures. Global optimization techniques are used to determine what percentage of the design should remain lattice and which should remain solid material. The percentage control of lattice to solid is used as a defining variable for the global optimization step. Regions of the optimized structure within a certain relative density threshold are converted into lattice struts based on the global variables. After linear tapered beams are inserted into the design and are optimized for minimum mass with a constraint on the fatigue life equal to the Pareto equivalent solution. To obtain a graded structure from the 
sizing optimizations, the design variables are the shared cross-sections of joined tapered beams. Results of the sizing optimizations are saved to a database used to construct a surrogate model (or response surface) which is then used to locate the global optimum. The process continues to build and optimize models until a convergence criterion is met by the global response surface. The global optimum result is then converted into an STL file for 3D printing. A block diagram to summarize the multi-scale workflow is given in Figure 39.

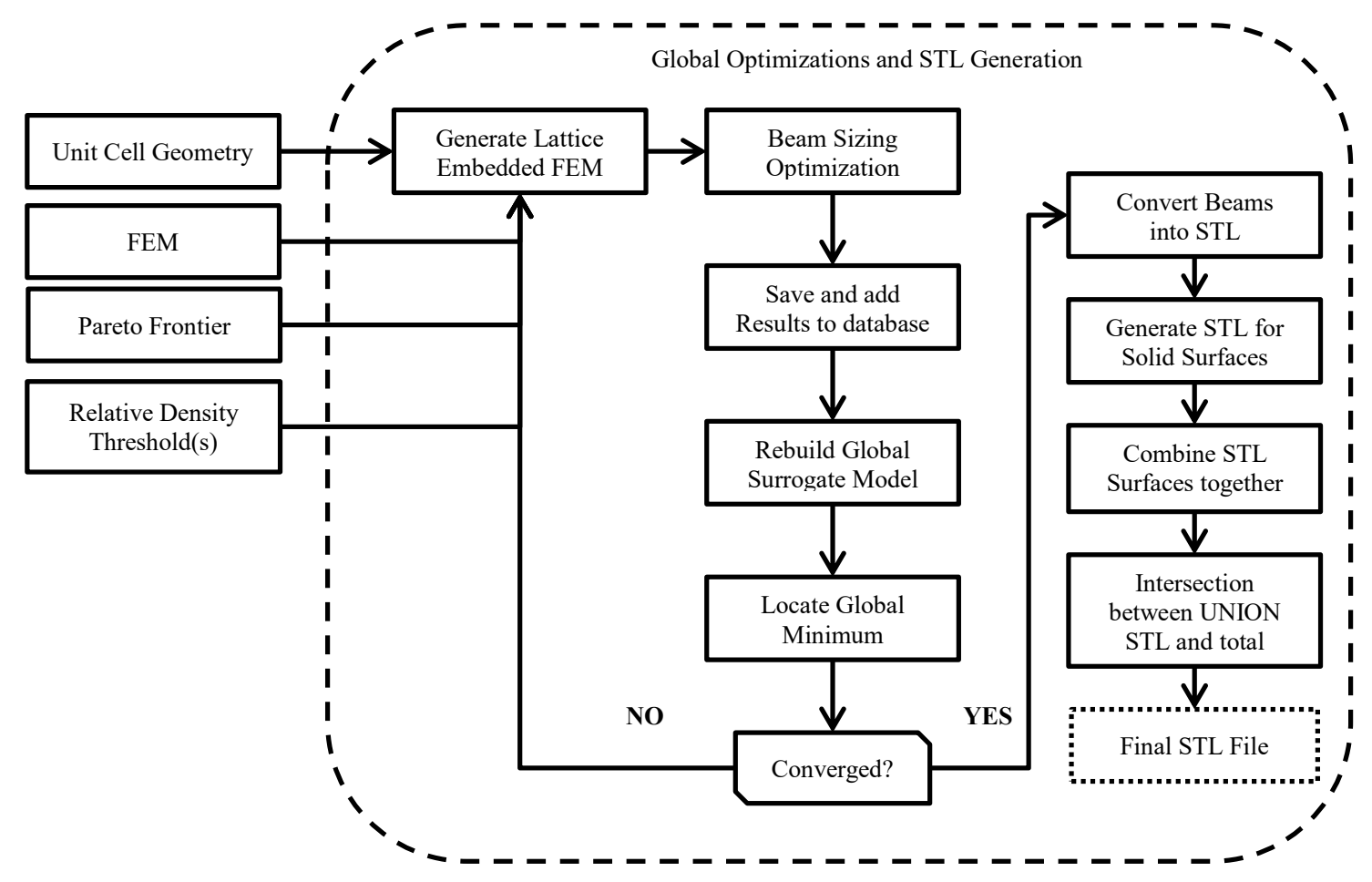

Figure 39 : Block Diagram for Global Optimization and Post-Processing Results

The block diagram in Figure 39 is applied to produces many testable models. The sizing optimizations also help with modeling the lattice beams for 3D printing because the optimized beam elements can be converted into STL representation. The size of the unit cell in the lattice regions is also independent of the mesh and hence an additional 
optimization is needed so that the struts sizes are acceptable. The fatigue properties of the lattice beams are those associated with the untreated titanium samples. This is because UIT cannot be applied on lattice material without severely damaging it. However if the lattice has sufficient thickness then UIT may be successful on the outer struts. Lattice material with UIT applications is outside the scope of this manuscript. After convergence of the global optimization a validation procedure which converts the linear tapered beams into three dimensional elements is performed to evaluate the accuracy of linear beams as a method to model the lattice structures.

\subsubsection{Surrogate Modeling and Global Optimization}

Global optimization seeks to find the global optimum among many local optimums. Typical gradient based optimization algorithms are highly susceptible to find the closest local optimum regardless if it is global or local. To ensure the solution of a global minimum, genetic algorithms are often employed as the search method of choice. Some of these algorithms include NSGA II [67] or simulated annealing [176]. The disadvantage of genetic algorithms is a high number of function evaluations making them unsuitable for computationally expensive black-box functions. However, meta-models or surrogate models are relatively cheap to run and can be used to approximate an expensive blackbox function using as few function evaluations as possible.

Surrogate models take on many forms such as radial basis functions [177], kriging, curve fitting, neural networks, support vector machines, random forests [178] and e.t.c. The popularity of surrogate models has increased in engineering applications especially for 
simulation based optimizations. The surrogate model selected for this thesis is a radial basis function $[177,179]$. RBF are useful because they are continuously differentiable and smooth. These models are quick to train and use making them ideal to be coupled with genetic algorithms. An advantage of radial basis functions is that they operate on least squares fitting approach. Radial basis functions do not require unique response output for each unique independent variable combination unlike kriging and neural networks.

The global optimization in this thesis relies on two definitions, global response, global design variable and local design variables. Global response and design variables are the dependent and independent variables of the surrogate model while local design variables are those from the simulations or sub optimizations. Figure 40 illustrates the distinction between the global and local variable and their effects on the global optimization procedure .The local design variables are defined after a topology optimization when low density regions are converted into linear tapered beam elements. The joined crosssectional areas of the tapered beams become the local design variables. Global optimization is then used to fine tune the micro-structure.

A global optimization step is performed after the topology optimization stage. The goal for the global optimization is to determine to optimal volume of lattice material which will reduce the greatest amount of mass. The optimal volume is controlled by the global variable ISO-element threshold. An additional lattice rotation variable will also be considered. 


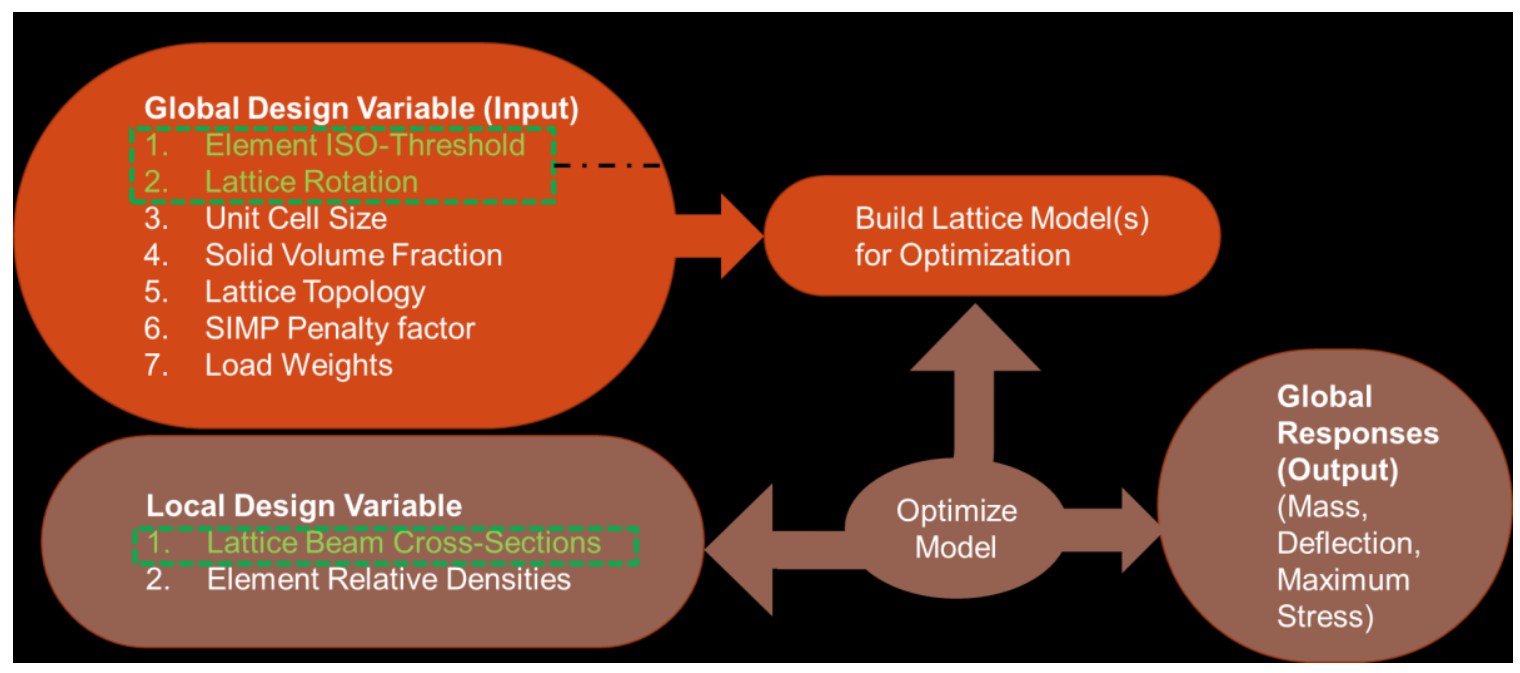

Figure 40 : Global vs. Local Design Variable Distinction

Cross validation is required to determine the best surrogate modeling settings. For example, if training data is being fit with a radial basis function whose kernel is a polynomial, then the degree of the polynomial would be the model setting to be optimized. Determining the correct polynomial degree will avoid over-fitting the training data so that extrapolation or interpolation along the data is more accurate. In other words, cross-validation generalizes the surrogate model so that it is able to predict solutions more closely.

Global optimization on surrogate models without cross-validation could also be stuck in a local minimum even if more points are added to the training data. This occurs because new solutions are added to the database so that the surrogate model can more accurately describe the solution space. Without generalization, all of the new solutions could be 
clumped together in a single dimension space and extrapolation will be unable to predict better solutions.

The surrogate model is trained using the element ISO-threshold and lattice rotation global variables while the output is controlled by the final mass of the beam sizing optimization. The parameters for constructing the surrogate model are optimized by a leave-one-out cross validation strategy [180]. The volume fraction of lattice material will limit the amount of solid material in the new design space and automatic meshing tools will create lattice beams of a certain topology in the lattice space. The newly meshed model is then optimized in the next section and its results are saved and added to the current surrogate model. An iterative procedure follows as multiple models are tested and built based on the global minimum of the surrogate model. Other global variables can be included such as the unit cell height or lattice topology however they are not included to save computation time.

The design paradigm for global optimization with surrogate models requires a certain number of iterations to build a database for response surface fitting. Afterwards the new model can be searched with a genetic algorithm to local the global optimum. The new optimum is then used to rebuild a new model and then optimize the tapered beams for minimum mass. This procedure continues until a convergence criterion is reached as shown in Figure 39. The final outcome of the global optimization is then validated with three-dimensional elements and stress and damage fields are compared. 


\subsubsection{Problem Definition}

In Figure 41, the design space is colored gray, while the non-design space is colored in orange) The chosen material is titanium Ti6Al4V alloy and its material properties can be found in Chapter 2.
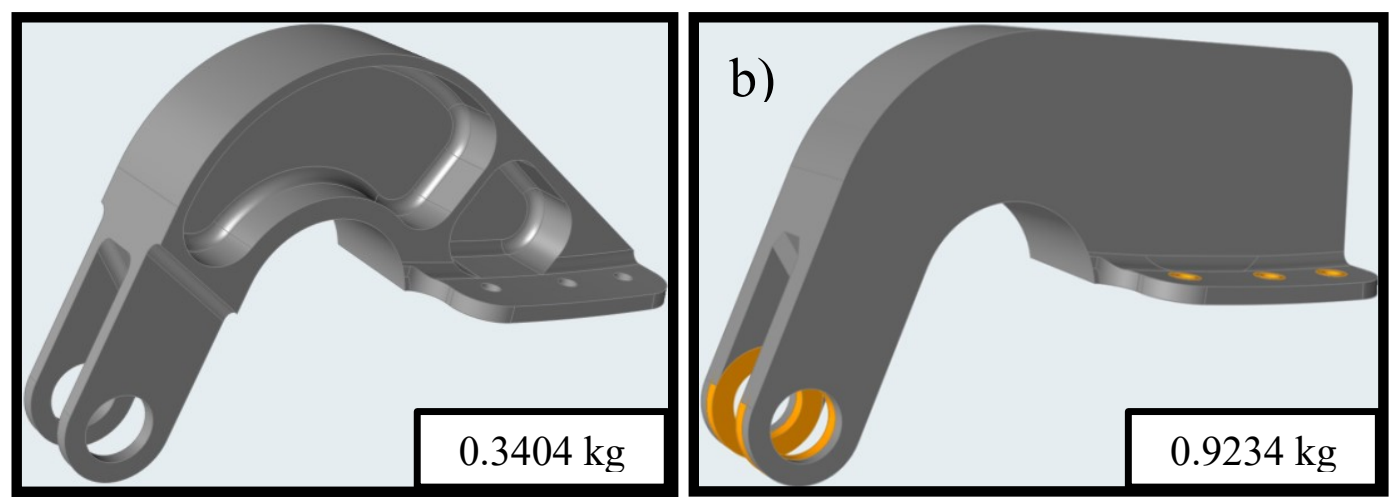

Figure 41 : Aircraft Hinge (a) Original Design, (b) Expanded Design Space $($ Grey $=$ Design, Orange = Non-design)

The original design of the aircraft hinge is shown in Figure 41A. To locate a new local optimum in the design space, the lightening holes were filled and the back height increased. Three load cases are applied on to the aircraft hinge and are illustrated in Figure 42 and magnitudes are displayed in Table 10.

Table 10: Loading Conditions for Hinge

\begin{tabular}{|c|c|}
\hline Loading & Force (lbs) \\
\hline Tensile & 3000 \\
\hline Compression & 3300 \\
\hline Skewed Compression $\left(11.6^{\text {o }}\right.$ offset $)$ & 2400 \\
\hline
\end{tabular}




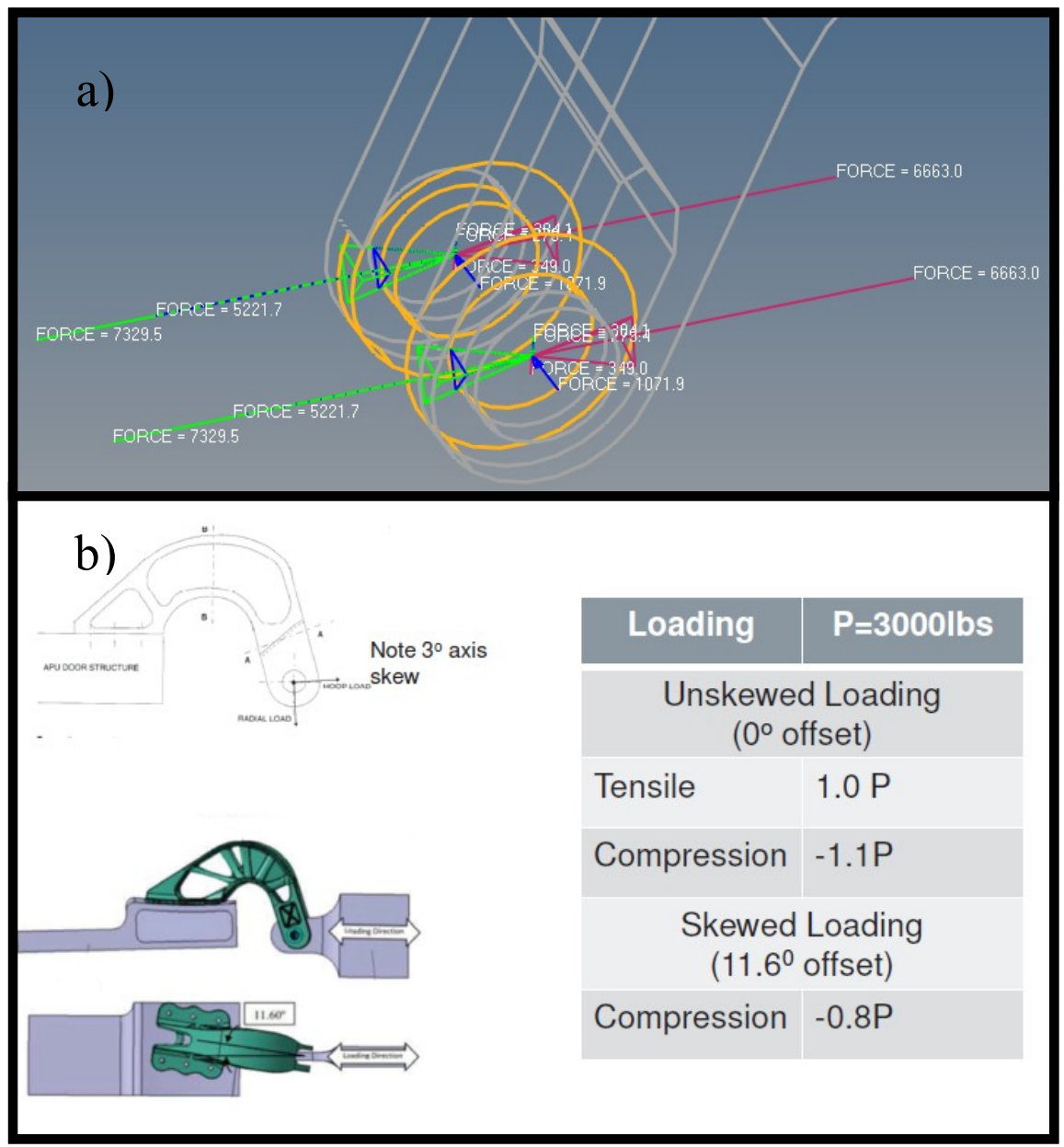

Figure 42 : Loading Conditions of (a) Newtons shown in Hypermesh (b) Schematic The fatigue history is given in Figure 43 below:

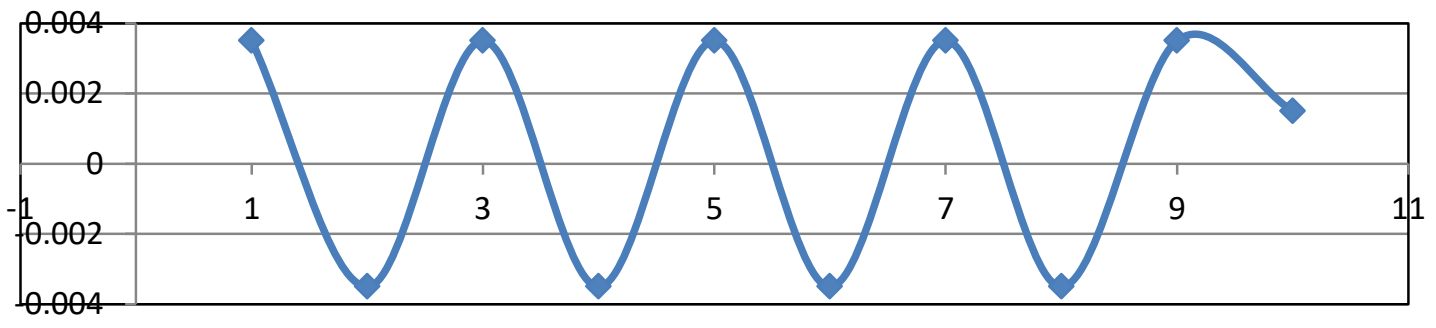

Figure 43 : Aircraft hinge Fatigue History (Force Multiplier) 
Due to multiple static load cases, the fatigue life objective will only consider the compression load case. The fatigue is measured by the norm of the damage among all elements in the FEM mesh. The multi-objective problem is the same as the previous case study of the L-bracket and the constrained epsilon method is used to derive the specific damage metric along the Pareto front. Due to the heavy computational expense, 5 designs are generated at mass fractions $0.6,0.5,0.4,0.3$ and 0.2. Two Pareto curves are generated; one with a density filter and another without the filter. The best design from both curves is selected for the second stage global lattice optimization. The filter radius was set to $3.75 \mathrm{~mm}$. A cubic lattice is chosen as the lattice topology for the multiobjective optimizations.

\subsubsection{Pareto Frontier}

The optimization results are shown in Figure 44 and Figure 45. The design closest to the utopia point when the filter is applied is design 2 with a volume fraction of 0.5 . The best point when no filter is used is given to be the first design with a volume fraction of 0.6 . The utopia points for both fronts are when the mass is equal to zero and when the damage is at is its minimum possible value.

Figure 44 shows the evolution of the design; the base collects more material at the bottom than at the front. The heaviest design tends to have a hollow lung while lighter designs have a more homogenous gradient. The large radius caused the transition between voids and solid material to be much more spread out. Due to this effect, convergence was more difficult to achieve hence each design was limited to 250 iterations each. 


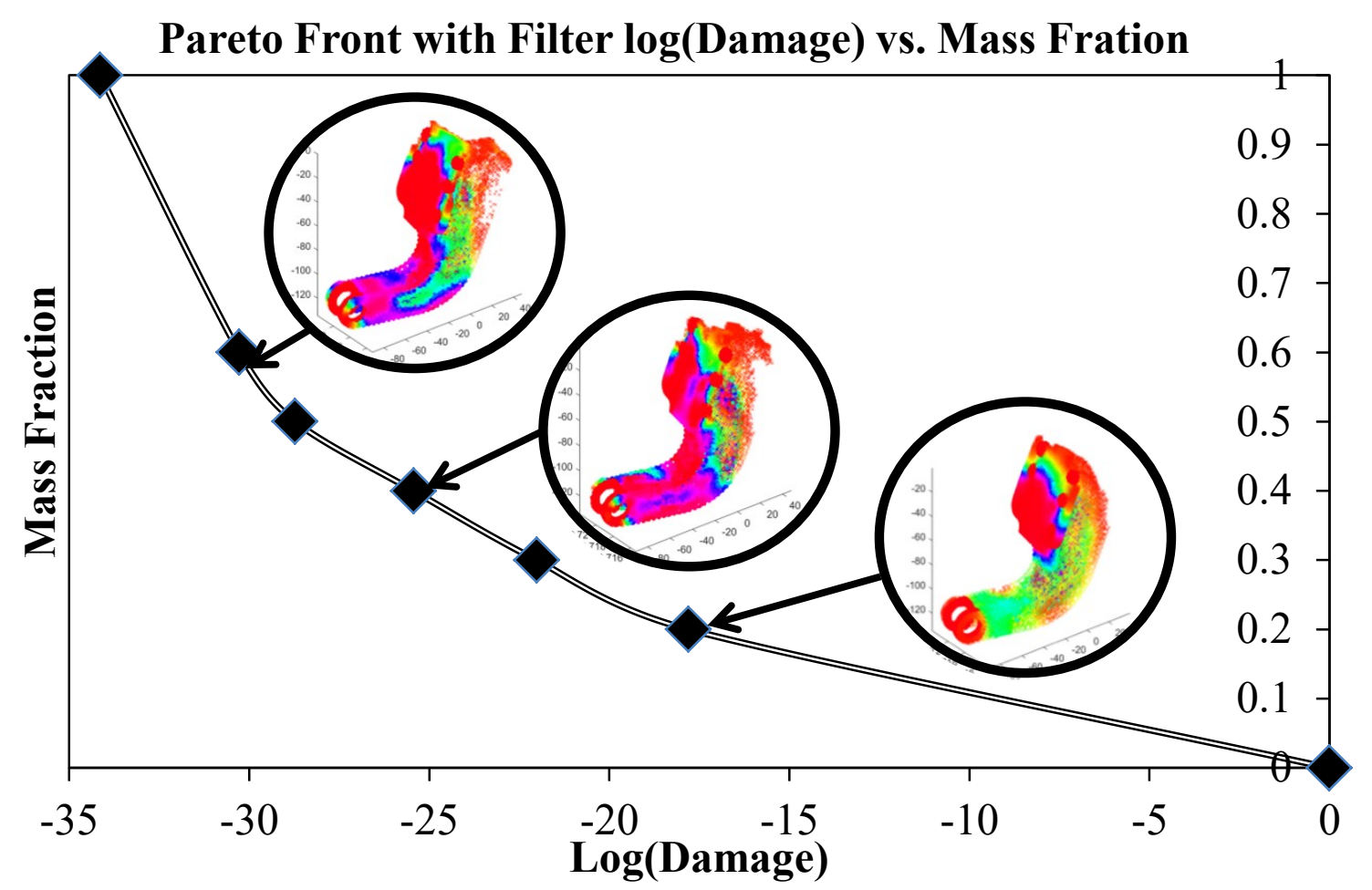

Figure 44 : Pareto with Frontier for Aircraft Hinge

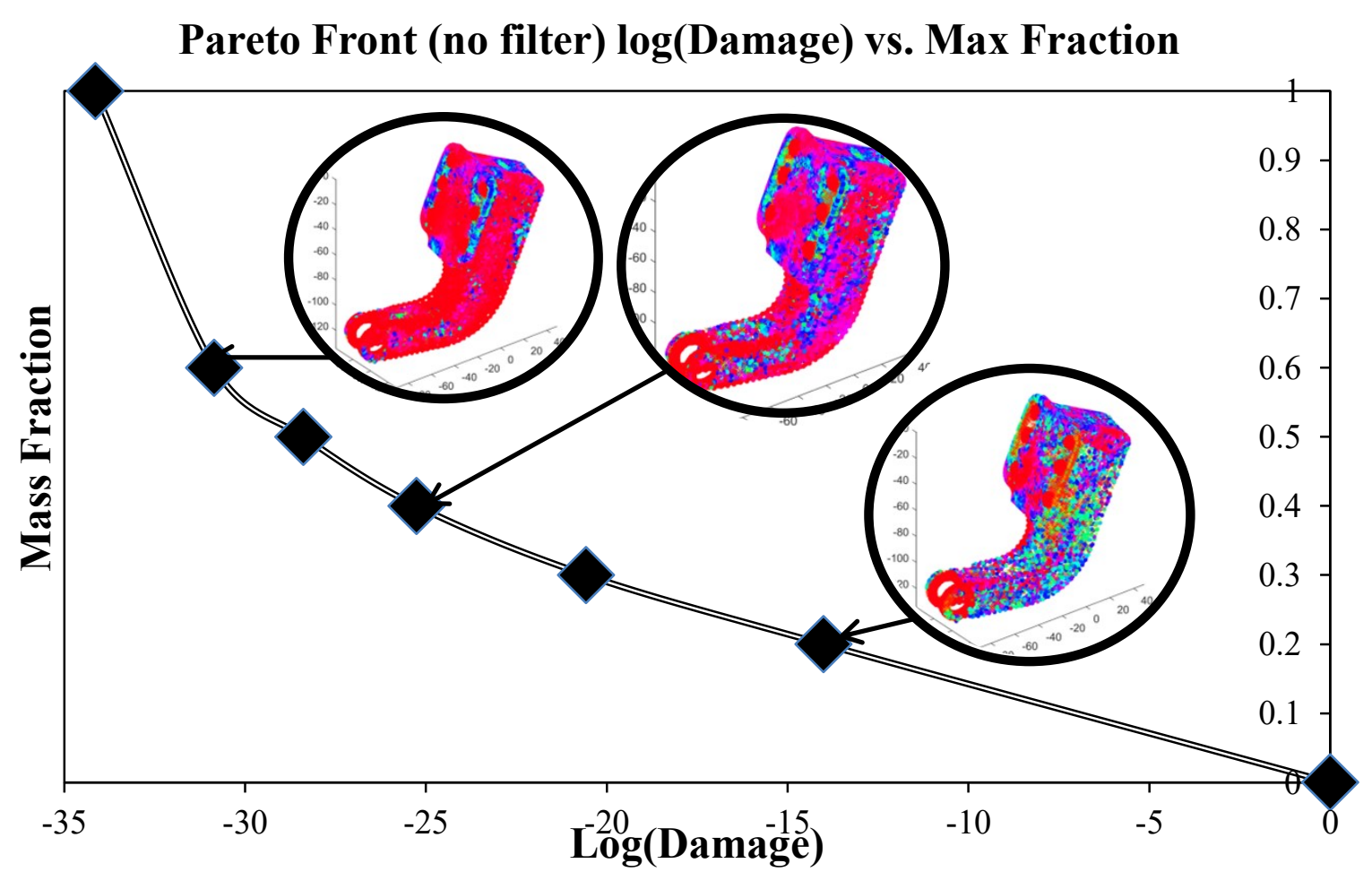

Figure 45 : Pareto Frontier with no Filter for Aircraft hinge 
Figure 45 displays the solutions without the density filter. Checker boarding can be seen at lower mass fractions while it is less prominent fort higher mass fractions. The results for a mass fraction of 0.6 show a hollow hinge as the optimal topology. A messy load path is observed for a volume fraction of 0.2 . The designs between both extremes contain some to minimal checker-boarding. The damage for the design without the filter tended to have lower cumulative damage than those with the filter. This makes sense as the filter adds an additional constraint to the problem which would increase the global minima.

The design for a mass fraction of 0.6 with no filter is chosen for global lattice optimization of the microstructure. This design was seen to have more favorable load paths. This structure will be re-meshed low density elements with lattice beams and then sent for beam sizing optimizations.

\subsubsection{Lattice Beam Sizing}

The lattice model will have all relative densities smaller than the ISO-threshold converted into tapered Euler beams. The original aim of this step was to minimize the mass subjected to a fatigue constraint; however the numerical solver Optistruct is unable to calculate the fatigue lives of linear tapered beam elements. Therefore an equivalent signed von-Mises stress constraint is used instead. The minimum cumulative damage for the current design is measured to be 7.13E-14. The equivalent alternating von-Mises stress would be equal to $0.943 \mathrm{MPa}$ using the UIT $\mathrm{SN}$ - curve, assuming failure occurs when damage reaches a value of 1 . For design purposes, the stress constraint will be equal to $1 \mathrm{MPa}$ so that the minimum life of the aircraft bracket is equal to approximately 
$5 \times 10^{14}$ cycles. The compressive load step is multiplied by the maximum force amplitude multiplier of 0.0035 to convert static load case into a pseudo fatigue load case.

The global optimization sub problems are defined in the system of equations below:

$$
\begin{gathered}
\text { minimize: } M_{\text {total }}(r) \\
\text { subject to: }\left\{\begin{array}{c}
K(A) U=F \\
\sigma_{V M}(\vec{r}) \leq 1 \\
r_{i} \leq r_{\max } \\
r_{\min } \leq r_{i} \\
d_{i} \cdot L_{i}>0
\end{array}\right.
\end{gathered}
$$

The minimum radius for the lattice struts is $0.1 \mathrm{~mm}$ while the maximum size is set to $1.6 \mathrm{~mm}$. The unit cell size of the lattice is set to $7 \mathrm{~mm}$. The global problem will consist of modifying the element ISO-threshold which builds the sub-problem that is sent to Optistruct for sizing optimization. The kernel chosen for the radial basis function is a poly-harmonic spline whose order (highest exponent) is optimized by a leave-one out validation scheme. Global convergence is assumed when the global step size is smaller than a specified value.

A global design variable of interest is the rotation of the lattice topology around the $\mathrm{x}$ axis. The alignment was chosen so that the model is oriented in the best possible position for $3 \mathrm{D}$ printing. However the orientation is subject to change after lattice optimization. The axis in relation to the aircraft hinge is presented in the figure below: 


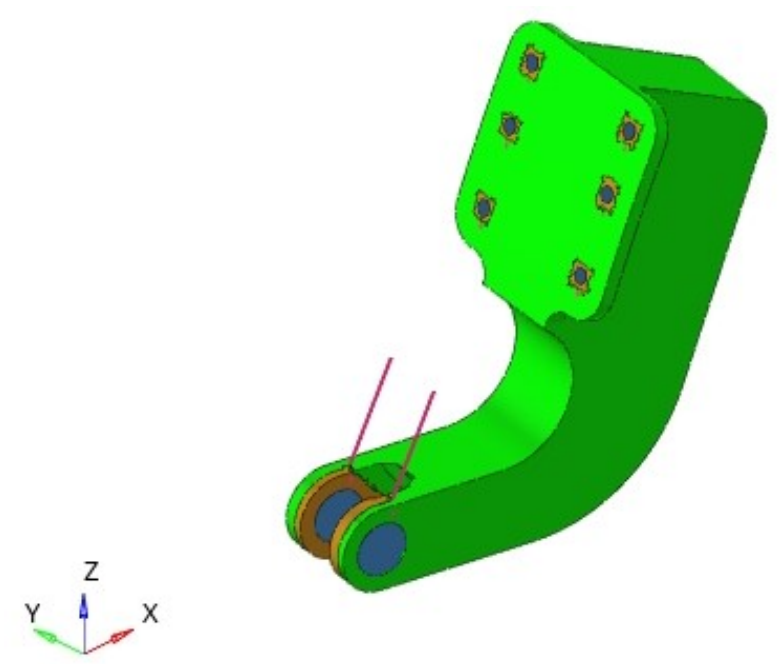

Figure 46 : Axis Alignment with Relation to Model for Lattice Rotation along X-Axis

The results of the globally optimized beam sizing operation reduced the mass from 0.9234 to $0.4218 \mathrm{~kg}$, the optimal ISO-surface value was 0.95 and the optimal lattice rotation angle along the $\mathrm{x}$-axis was approximately 32 degrees.
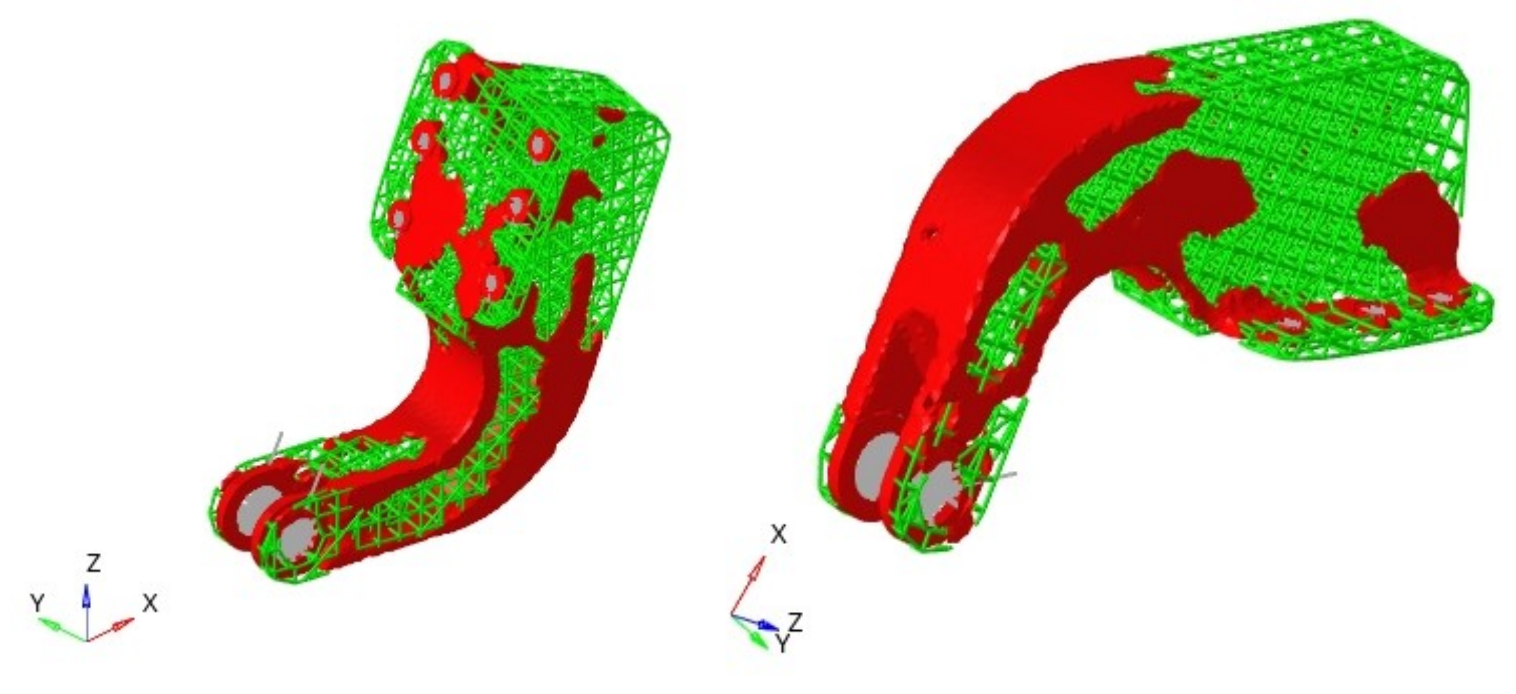

Figure $47:$ Final design $($ Lattice $=$ Green $),($ Red $=$ Solid $)$, ISO $=0.95$, Rotation $=32^{\circ}$

The surrogate model plot is given in Figure 48. It can be seen that the radial basis function model follows the global output well. The RBF assumes that the constraints are 
satisfied however if the constraint is broken then a large mass is obtained, to which the genetic algorithm will search elsewhere in the global design space. Combining the constraint violation with the mass function could yield a weighted response to simultaneously determine the smallest error and mass.

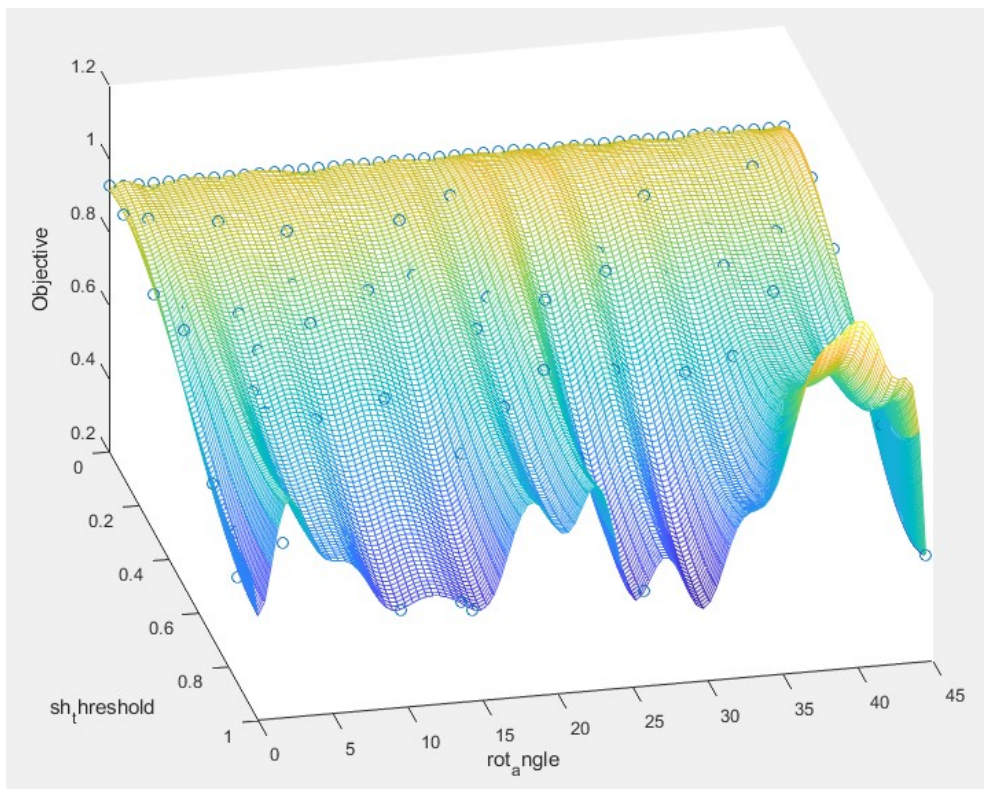

Figure 48 : Surrogate Model Surface (Mass) $($ Z-Axis = Mass)

As seen in Figure 48, more training points are needed to fully map out the global design space for the aircraft hinge. However a global minimum was found without requiring all the necessary information.

\subsubsection{FEM Validation Stage}

To properly asses the accuracy of the model with beams representing the lattice, a three dimensional model is generated. The three dimensional version of the lattice model is generated using the following steps: 
1. Upload the optimized model into Hypermesh

2. Convert the linear beams into a solid cone (surface)

3. Apply a shrink-wrap (voxelization + marching cubes algorithm) onto the cones and the three dimensional elements simultaneously.

4. Convert the surface obtained in (3) into a triangulated surface and export it as an STL.

5. Upload the STL from (4) into MATLAB and apply a Boolean intersection between (4) and the original design space.

6. Re-mesh (5) using a MATLAB re-meshing algorithm.

7. Tetra-mesh (6) and export the elements as a separate file.

8. Attach the new tetra elements from (7) to the non-design space elements using a contact surface using Hypermeshs auto contact tool.

The STL representation of the new hinge is given in Figure 49. The shrink wrap successfully merged the solid and lattice beams together into a single closed and manifold mesh. The design space is re-meshed and connected to the non-design space elements using a contact surface which is then sent to be analyzed for stress, fatigue and deflection. 


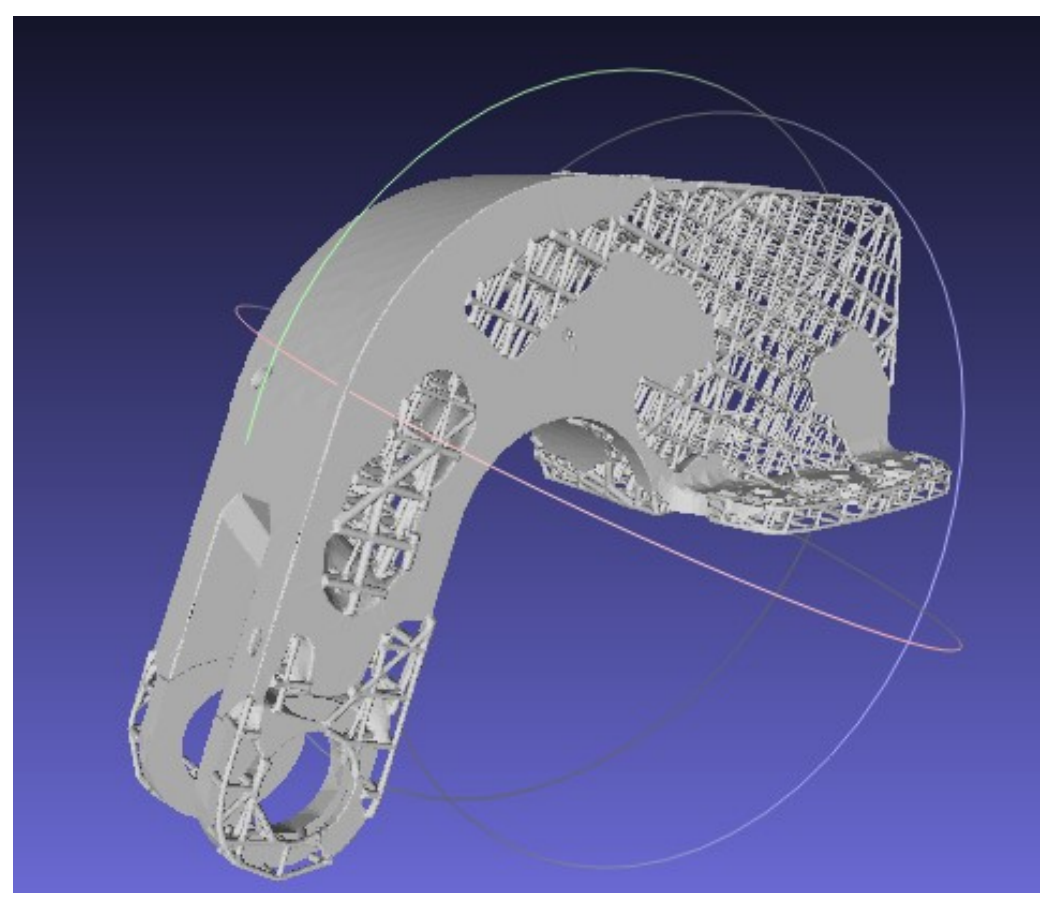

Figure 49 : STL Representation of the Aircraft Hinge

Constructing the 3D model using the above mentioned steps tends to generate heavier models. This is because fillets are added between the lattice struts and between the strut and the solid domain however, using a very fine mesh size during the shrink-wrap can alleviate this problem.

Static analysis for the three dimensional model reveals lower stresses in the lattice struts than previously measured compared to their one-dimensional beam representation. However in some cases the stresses are under-estimated. The stress where about $60 \%$ less intensive than the 2D model as the peak stresses where measured to be $7 \mathrm{MPa}$ while they were $3 \mathrm{MPa}$ as $3 \mathrm{D}$ elements. The beams related to the conforming net are seen to be the most stressed as seen in Figure 51 and Figure 52. The conforming net contains the dominant load paths throughout the lattice structure. 
The deflection of the model with beam elements is also much smaller than the three dimensional model. This may be due to stiff beam element representation and large element sizes to reduce the computation time. For more accurate deflections in the 3D model it would have been better to use second order tetrahedrons.

The fatigue life was seen to be well above the minimum damage predicated by the topology optimizations. For both the 2D model and the 3D model, no fatigue failure was recorded. Therefore the optimization was able to successfully reduce the mass of the initial design while maintaining as long service life. Better accuracy can be achieved if a different linear element was used which more accurately predicted the mechanical behavior of the lattice struts.

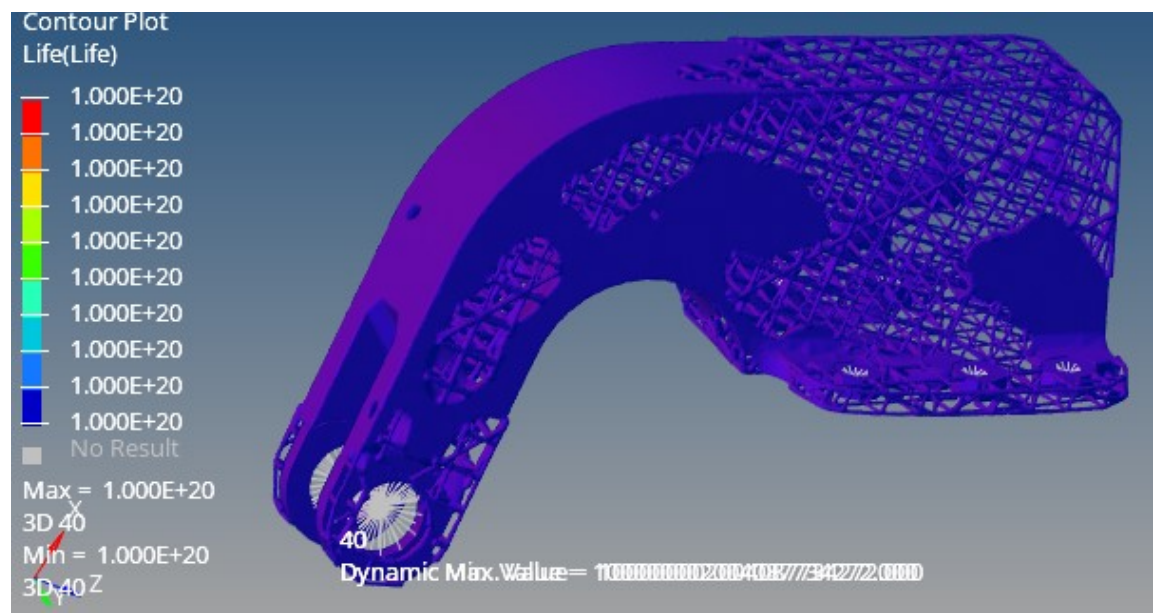

Figure 50 : Fatigue life Contour of 3D Model 


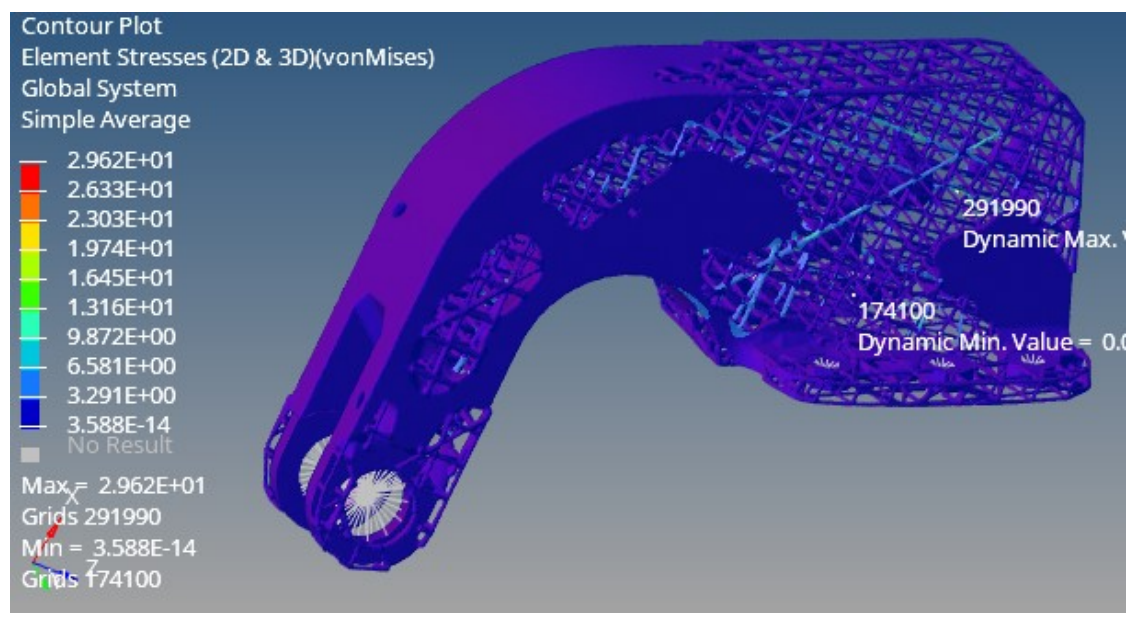

Figure 51 : Alternating Stress Von-Mises Contour

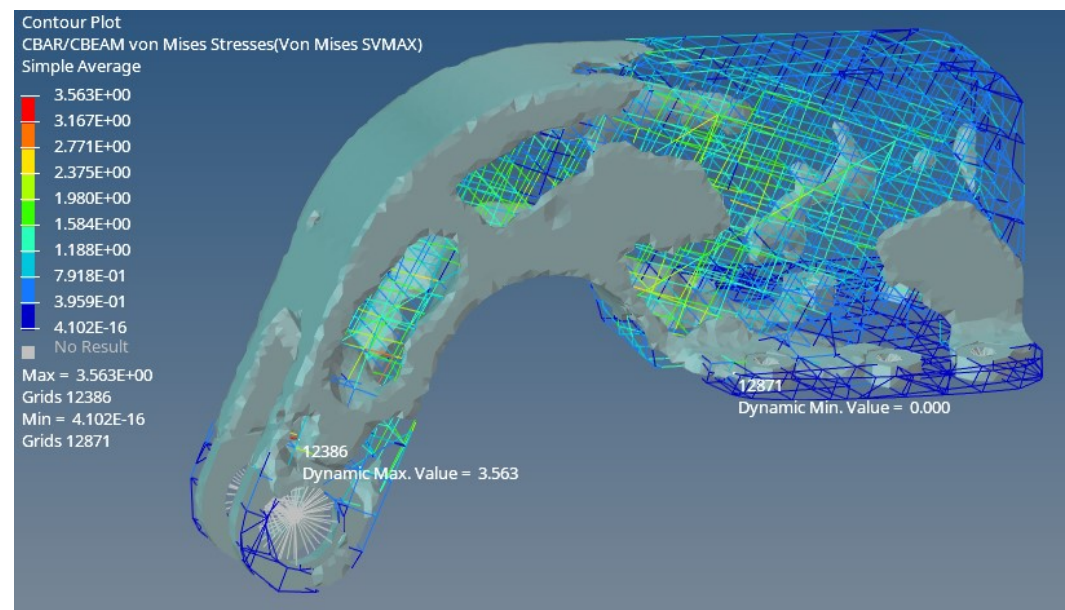

Figure 52 : Alternating Stress for Beam Model (Tapered Beams)

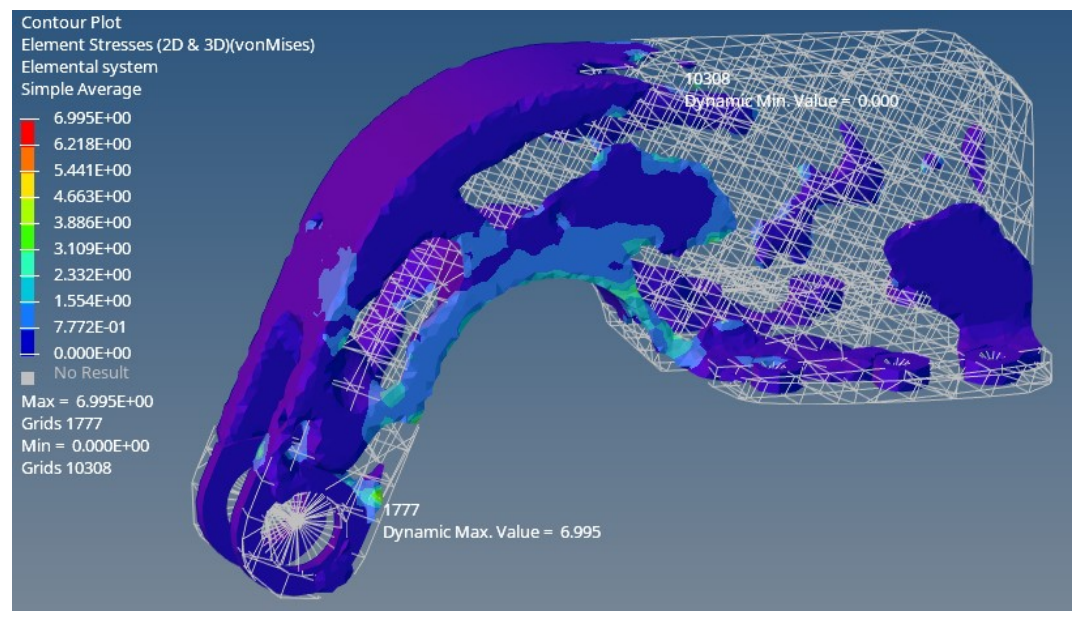

Figure 53 : Alternating Stress for Beam Model (3D Elements) 


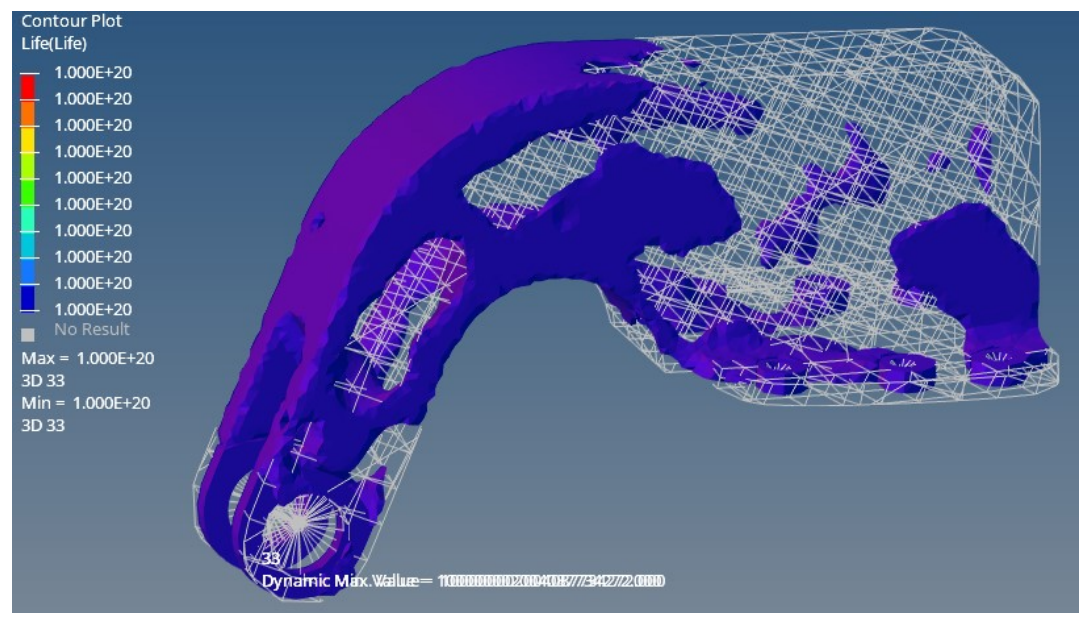

Figure 54 : Fatigue Live Contour for Beam Model

Table 11 below illustrates the differences between the model with the linear tapered beams and the three dimensional elements. Overall, there is more mass saving potential for the hinge as the fatigue lives are still very high. The specific life of the new hinge is considerably higher than the original model due to having lower mass but equivalent fatigue life (infinite). The final mass was also much lighter in the 3D version. This is due to the lack of overlapping beam elements in the $2 \mathrm{D}$ model which are removed during the remodeling.

Table 11: Results Comparisons

\begin{tabular}{|c|c|c|}
\hline & Beams Model & 3D Element Model \\
\hline \hline Deflection & -1.79775 & -2.00631 \\
\hline $\begin{array}{c}\text { Max Alternating } \\
\text { Stress }\end{array}$ & $\begin{array}{c}6.995(3 \mathrm{D} \text { Elements) } \\
3.563 \text { (Beam Elements) }\end{array}$ & $2.962 \mathrm{MPa}$ \\
\hline Fatigue Life & $1 \mathrm{E}+20$ (infinite) & $1 \mathrm{E}+20$ (infinite) \\
\hline Mass & $0.4218 \mathrm{~kg}$ & $0.4222 \mathrm{~kg}$ \\
\hline
\end{tabular}


In an industrial setting, the next validation step, which is not part of the scope of this thesis, is to simulate the $3 \mathrm{D}$ printing process and evaluate any spring-back due to thermal contraction and expansion.

\subsubsection{Linear vs. Solid Beam Comparison}

The maximum stresses from the solid beams are compared to the von-Mises stress in the tapered beam element. The comparison is shown in Figure 55. It is seen that accuracy at high stresses is poor. The inaccuracies could be a result of not using a fine enough mesh to model the very fine lattice struts.

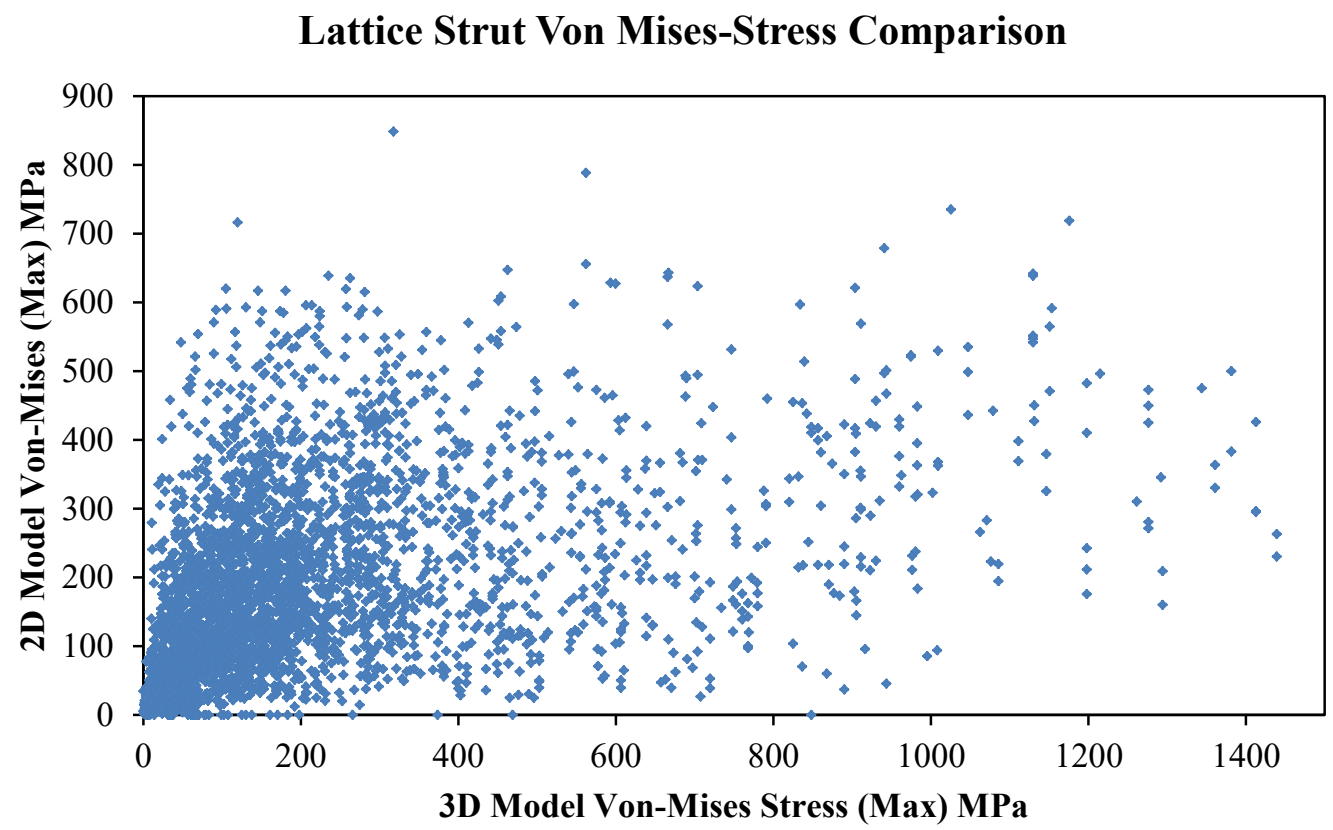

Figure 55 : Maximum Von-Mises Comparison

An additional correlation study is performed to measure the accuracy of the strut stresses. Table 12 shows that the maximum stress, with a correlation of 0.428 , is the best metric to 
assess the differences between the two fem models. The minimum stress and mean stresses where not well correlated with the linear beam stresses.

Table 12 : Correlation Table

\begin{tabular}{|c|c|}
\hline Stress (3D) & Correlation with 2D Von-Mises Stresses \\
\hline \hline Max 3D Element Stress & 0.428564 \\
\hline Mean 3D Element Stress & 0.215826 \\
\hline Min 3d Element Stress & -0.36177 \\
\hline
\end{tabular}

Overall, the best correlation is still very poor and better modeling of the lattice struts as linear elements is needed. If accuracy improves than the lattice beam sizing optimization will become more useful for design and optimizing AM components.

\subsubsection{Comments}

It is seen that the final model is highly dependent on the initial topology optimization. The quality of the relative density distribution is important. The final model had a significant reduction in mass while maintaining high fatigue tolerances. This was possible using a two stage optimization procedure. The first applied multiple objective optimizations to search the design space for a suitable load-path configuration. A utopia point was located and compared to the other possible topologies. The selected topology was refined with tapered beam sizing optimization or by re-meshing lattice areas with linear beam elements. The accuracy of the results was very poor however. Sizing optimizations in the second stage is recommended with strict constraints on stress or fatigue if possible. 
Some degree of asymmetry can be seen in Figure 47 as material was more likely to accumulate on the right side of the hinge. This can be due to an incomplete optimization if convergence was not reached. However topology optimization with fatigue is very nonlinear therefore the geometry obtained could be a result of locating a local minimum. A trade-off is usually taken between the quality of the optimization and the amount of computation time required to reach a solution. As the number of design variables increases so does the number of iterations and resulting optimization time will increase exponentially. If multiple optimizations are needed, then it may not always be feasible to reach a strict convergence on the optimization routine due to feasible time constraints. A final comment about the asymmetry in the topology optimized part could be a result of the already asymmetric design space.

Additional work is required such that the component can be placed in the aircraft. These include experimental testing and design of support structures. Thermo-mechanical printing simulations may also be performed to analyze spring-back. Beneficial uses of AM component arise due to their ability to perform multiple functions with minimum weight. In the case of the aircraft hinge case study, the weight was reduced while infinite life was met. Feasibility of the design in a real world setting however requires additional analysis such as experimental testing. Effects of buckling, vibration and thermal effects on the part where not considered in the design process. These analyses must be performed before consideration on an aircraft can be made. 


\section{Chapter 8: Conclusion}

A method of improving the fatigue life of additively manufactured components is presented. A surface treatment called ultrasonic impact treatment (UIT) is applied to titanium alloy to improve the materials fatigue characteristics. A $25 \%$ increase in the fatigue limit was found when UIT is applied. The slope of the treated materials' Basquin curve in the high cycle regime was calculated to be less than the untreated curve. Sufficient work hardening is the main mechanism which slows down crack growth on the surface extending the work life of the treated material. The beneficial fatigue properties of UIT can be directly translated to AM parts with FGM. Topology optimizations show that materials with better fatigue properties result in longer life cycles for equivalent mass structures. When topology optimization is combined with lattice optimization; greater mass savings can be achieved while still maintaining a longer work life.

Asymptotic homogenization is used to calculate equivalent isotropic material properties such as the modulus, Poisson ratio and relative failure of lattice structures in threedimensions. Topology optimization is combined with the isotropic lattice properties used to determine the optimal lattice placement in a design space. Case study results on the Lbracket show that improvements in fatigue properties are not always acquired using lattice material. These results are due to high stress concentrations within the cubic lattice structure. The relative yielding as a function of the relative density for lattice topologies was derived using asymptotic homogenization. There were no large differences between 
the change in the macro stress direction and the relative increase in micro stress. Only six stress directions were tested however the entire yield surface was not calculated. Methods of developing automated lattice meshing workflows were also presented. Trimmed lattices offer more control over the internal lattice structure with little user intervention. The new lattice meshing tool allows for more accurate lattice representation in closed volumes which will improve the mechanical accuracy of topology optimizations with FGM.

An aircraft hinge was developed with a mass fraction of sixty percent to with minimum damage. A two scale optimization reduces the mass by $51 \%$. The first stage was a global optimization on the macro scale level where the unit cell properties are inserted into the design space. The macro scale results in a functionally graded material for additively manufactured parts. A new meshing algorithm is employed to convert the lattice domains into linear beam elements within the design space using a trimmed lattice. The second step procedure will perform micro-scale optimization on the lattice struts themselves. A global lattice optimization strategy was selected and a response surface was used to locate the optimal lattice building parameters and the optimal beam cross-sectional areas. The final part was then converted into a printable file and then analyzed for accuracy using the finite element method with 3D elements. The full cycle shows that large mass savings are possible while maintaining long fatigue lives for AM parts. The optimized design was shown to have infinite life. 


\subsection{Future Work}

Experimental SN-curves for DMLS Ti-6AL-4V were derived. More accurate representation for the $\mathrm{SN}-$ Curves can be derived if more experimental data is acquired. The fatigue tests had too few treated samples to properly reflect the SN properties. The large scatter bandwidth resulted in overlap between the two SN-curves.

Homogenization of relative yield properties of lattice materials are calculated for six macro stress directions. In the literature, the micro stresses are calculated by a yielding surface which is a function of the macro stress and the relative density of the lattice topology. If a yielding surface for all potential macro stress combinations had been calculated then the lattice optimizations have benefited the additional accuracy. The three dimensional lattice structure yield surface would be future work and is easily implemented. A surrogate model can be employed to calculate the conversion between the macro and micro multi-axial stress values where the independent variables are the relative density and stress tensor.

Another area for improvement is the lattice beam sizing optimization without reliance on Optistruct. In-house methods for beam sizing would significantly improve the workflow because more memory efficient numerical optimization can be performed. Internal calculations of the tapered beams will also allow for fatigue to be calculated from the linear elements as Optistruct is unable to calculate fatigue from linear elements. Workflow updating with Nastran is another possibility for fatigue calculations on linear elements. 
The global optimization methodology for the optimal element ISO threshold is a very time consuming strategy. An advantage of surrogate modeling is it allows for expensive fitness functions to be approximated and optimized efficiently. The majority of the optimization time is spent locally rather than globally. The response surface for the global response illustrates that only a few iteration $\mathrm{s}$ is needed to intelligently map the entire design space. Future implementations will consider additional global design variables to achieve better global fitness measures.

Converting the linear beam elements into $3 \mathrm{D}$ elements has a few issues namely; the minimum resolution and element size. As the printing resolution decreases, so does the required element size. This causes the number of degrees of freedom in the model to increase exponentially and lengthen computation time. Typically the minimum beam radius for a lattice should be larger than the four times minimum resolution of the printer. To avoid large number of elements, the mesh is approximated through either voxelization or patch-meshing (edge collapse) algorithms. The fine details of the original geometry are omitted for more robust mesh algorithm but less accurate geometries. To avoid long analysis times, mesh-less finite element methods can be explored.

The accuracy of the lattice beams as linear elements was also analyzed. The modeling comparison shows discrepancies; the stresses are underestimated when linear elements are used. Knowing this in advance, the constraints can be relaxed for the beams increasing the feasible design space. The deflections were also seen to be much higher 
with linear elements than with solid elements. Lattice structure analysis is more easily performed with mesh-less finite element method and is proposed as future work. Software such as SimSolid (Altair) can perform linear and bucking analysis using the mesh free method and is significantly faster than the meshed method. The downside is that automation with mesh free software is not yet available to engineers.

Design process for developing an aerospace component using 3D printing technology is presented. Manufacturing and post-printing processes where not included in the design process. To ensure the part can be ready for installation within an aircraft numerous steps are required and include analysis for buckling and vibrations to ensure a safe design. Printing supports and thermal-mechanical management in the design stage is needed as well. Supports structures can be used to influence the heat released from the part during printing and avoid high internal residual stresses and warping. Orientation of the part for best print is also needed. In addition, post-printing processing such as machining holes for the bolts and support removal is required before installation. Heat treatment to remove internal residual stresses is an important step to both increase the parts longevity and improve ductility. Experimental test to assess the number of cycles to failure for the part is also required to compare the finite element prediction to real life.

The total design cycle for an AM part was not presented in this thesis. An additional step to simulate the printing process and evaluate the thermal-mechanical response and spring-back can be investigated. Spring-back occurs when the printed part cools down in such a way that warping occurs. This mainly occur when $3 \mathrm{D}$ printing metal components. 
Evaluation of the supports structure must also be done and was left-out of the design cycle as it was not part of the scope for this thesis. Each step requires validation through simulation and if predictions display unwanted behavior of the part; modifications and further design checks are needed.

Other areas of future improvement include refinements for the lattice meshing algorithm to include non-symmetric unit cells. Numerical artifacts still persist within the lattice meshing tool and additional work is needed to remove those errors. One final comment for future development is to analyze the fatigue minimization of the L-bracket considering other lattice topologies to see if improvements in the fatigue performance can be achieved. 


\section{Bibliography}

[1] L. K. Narayan, Computer Aided Design and Manufacturing, New Delhi: Prentice Hall of India, 2008.

[2] M. Bendsoe and C. A. Mota Soares, Topology Design of Structures, Dortdretch: Kluver Academic , 1993.

[3] Altair, "What is Altair Inspire?," Altair, 2020. [Online]. Available:

https://solidthinking.com/product/inspire/. [Accessed 20 July 2020].

[4] H. Zhang, R. Chiang, H. Qin, Z. Ren, X. Hou, D. Lin, G. L. Doll, V. K. Vasudevan, Y. Dong and C. Ye, "The effects of ultrasonic nanocrystal surface modification on the fatigue performance of 3D-printed Ti64," International Journal of Fatigue, vol. 103, pp. 136-146, 2017.

[5] J. Lindermann, C. Buque and F. Appel, "Effect of shot peening on fatigue performance of a lamellar titanium aluminide alloy," Acta Materialia, vol. 54, no. 4, pp. 1155-1164, 2006.

[6] G. D. Revenkar, R. Shetty, S. S. Rao and V. Neelakanth, "Wear resistance enhancement of titanium alloy (Ti-6Al-4V) by ball burnishing process," Journal of Materials Research and Technology, vol. 6, no. 1, pp. 13-32, 2017.

[7] R. K. Nalla, I. Altenberger, U. Noster, G. Y. Liu, B. Scholtes and R. O. Ritchie, "on the Influence of Mechanical Surface Treatments - Deep Rolling and Laser Shock Peening On the Fatigue behavior of Ti-6Al-4V at Ambient and Elevated Temperatures," Materials Science and Engineering, vol. 355, no. 1-2, pp. 216-230, 2003. 
[8] O. Scott-Emuakpor, T. George, M.-H. H. Shen, C. J. Cross and J. Calcaterra, "Development of an Improved High Cycle Fatigue Criterion," Journal of Engineering for Gas Turbines and Power, vol. 129, pp. 162-169, 2007.

[9] I. I. Kornilov, "Heat resistance of titanium alloys," Metal Science and Heat Treatment, vol. 5, pp. 73-77, 1963.

[10] C. Zhang, F. Chen, Z. Huang, M. Jia, G. Chen, Y. Ye, Y. Lin, W. Liu, B. Chen, Q. Shen, L. Zhang and E. J. Lavernia, "Additive manufacturing of functionally graded materials: A review," Materials Science \& Engineering A, vol. 764, p. 138209, 2019.

[11] E. S. Statnikov, V. O. Muktepavel and A. Blomqvist, "'Comparison of Ultrasonic Impact Treatment (UIT) and Other Fatigue Life Improvement Methods," Welding in the World, vol. 46, no. 3-4, pp. 20-32, 2002.

[12] X. Wu, Deformation and Evolution of Life in Crystalline Materials: An Integrated CreepFatigue Theory, Ottawa: CRC Press, 2018.

[13] J. K. Nisbett and R. G. Budynas, Shigley's Mechanical Engineering Design, 10th Edition, McGraw-Hill Education, 2015.

[14] S. Boyd and L. Vandenberghe, Convex Optimization, New York: Cambridge Univeristy Press, 2009.

[15] C. Liu, D. Chen, M. R. Hill, M. N. Tran and J. Zou, "Effects of ultrasonic impact treatment on weld microstructure, hardness, and residual stress," Materials Science and Technology, vol. 33, no. 14, pp. 1601-1609, 2017.

[16] I. Inagaki, T. Takechi, Y. Shirai and N. Ariyasu, "Application and Features of Titanium 
for the Aerospace Industry," Nippon steel \& Sumitomo Metal Technical Report, vol. 106, 2014.

[17] N. S. Mahmoodi, M. Rastgaar and M. Ahmadian, "Active Vibration Control of Aerospace Structures Using a Modified Positive Position Feedback Method," Proceedings of the American Control Conference, 2009.

[18] N. Hrabe, T. Gnäupel-Heroldb and T. Quinn, "Fatigue Properties of a Titanium Alloy (Ti-6Al-4V) Fabricated Via Electron Beam Melting 9EBM): Effects of Internal Defects and Residual Stress," International Journal of Fatigue, vol. 94, no. 2, pp. 202-210, 2017.

[19] J. Jianchao, X. Xun and J. Stringer, "Suppoer Structures for Additive Manufacturing: A Review," Journal of Manufacturing and Materials Processing, vol. 2, no. 64, 2018.

[20] STRATASYS, "GETTING THE MOST OUT OF METAL 3D PRINTING: UNDERSTANDING DESIGN \& PROCESS CONTROLS FOR DMLS," STRATASYS, [Online]. Available: https://www.stratasysdirect.com/content/white_papers/DMLS_White_Paper_201509_v3. pdf. [Accessed 8 November 2017].

[21] G. Schotte, "A Brief History of Additive Manufacturing," TRIMECH, 4 October 2019. [Online]. Available: https://blog.trimech.com/a-brief-history-of-additive-manufacturing. [Accessed 27 April 2020].

[22] J. Flynt, "A Detailed History of 3D Printing," 3DInsider, [Online]. Available: https://3dinsider.com/3d-printing-history/. [Accessed 27 April 2020].

[23] H. Schleifenbaum, A. Diatlov, C. Hinke, J. Bultmann and H. Voswinckel, "Direct 
photonic production: Towards high speed additive manufacturing of individualized goods," Production Engineering, vol. 5, no. 4, pp. 359-371, 2001.

[24] T. Wohlers and T. Gornet, "History of Additive Manufacturing," Wohlers Report 2014, 2014. [Online]. Available: http://www.wohlersassociates.com/history2014.pdf.

[Accessed 27 April 2020].

[25] "MAKERBOT," MakerBot Industries, [Online]. Available: https://www.makerbot.com/. [Accessed 27 April 2020].

[26] T. Duda and L. V. Raghavan, "3D Metal Printing Technology," IFAC-PapersOnLine, vol. 49, no. 29, pp. 103-110, 2016.

[27] J. Sun, Z. Peng, L. Yan, J. Y. Hsi Fuh and G. S. Hong, "3D food printing an innovative way of mass customization in food fabrication," International Journal of Bioprinting, vol. 1, no. 1, pp. 27-38, 2015.

[28] K. Plewa, "Is a sand 3D printer the future of Additive Manufacturing?," Sculpteo, 17 July 2019. [Online]. Available: https://www.sculpteo.com/blog/2019/07/17/is-a-sand-3dprinter-the-future-of-additive-manufacturing/. [Accessed 27 April 2020].

[29] J. Walker, E. Harris, C. Lynagh, A. Beck, R. Lonardo, B. Vuksanovich, J. Theil, K. Rogers, B. Conner and E. MacDonald, "3D Printed Smart Molds for Sand Casting," International Journal of Metalcasting, vol. 12, pp. 785-796, 2018.

[30] E. Malone and H. Lipson, "Fab@Home: The Personal Desktop Fabricator Kit," in Proceedings of the 17th Solid Freeform Fabrication Symposium, Austin, Texas, 2006.

[31] D. Southerland, P. Walters and D. Huson, "Edible 3D printing," Society for Imaging 
Science and Technology, vol. 2011, no. 2, pp. 819-822, 2011.

[32] Z. Liu, M. Zhang, B. Bhandari and C. Yang, "Impact of rheological properties of mashed potatoes on 3D printing," Journal of Food Engineering, vol. 220, pp. 76-82, 2018.

[33] F. Pallotino, L. Hakola, C. Costa, F. Antonucci, S. Figorilli, A. Seisto and P. Menesatti, "Printing on food or food printing: a review," Food and Bioprocess Technology, vol. 9, no. 5 , pp. $725-733,2016$.

[34] M. Tomlin and J. Meyer, "Topology Optimization of an Additive Layer Manufactured (ALM) Aerospace Part," in The 7th Altair CAE Technology Conference 2011, 2011.

[35] "New manufacturing milestone: 300,000 additive fuel nozzles," GE Additive, 04 October 2018. [Online]. Available: https://www.ge.com/additive/stories/new-manufacturingmilestone-30000-additive-fuel-nozzles. [Accessed 28 April 2020].

[36] P. Zelinski, "Video: The Additive Nozzle's Origin Story," Additive Manufacturing, 27 November 2015. [Online]. Available: https://www.additivemanufacturing.media/blog/post/video-the-additive-nozzles-originstory. [Accessed 28 April 2020].

[37] K. Pietrzak, D. Kaliński and M. Chmielewski, "Interlayer of A12O3-Cr functionally graded material for reduction of thermal stresses in alumina-heat resisting steel joints," Journal of the European Ceramic Society, vol. 27, no. 2-3, pp. 1281-1286, 2007.

[38] J. Zhou, P. Shrotriya and W. Soboyejo, "On the deformation of aluminum lattice block structures: from struts to structures," Mechanics of Materials, vol. 36, no. 8, pp. 723-737, 2004. 
[39] T. Maconachie, M. Leary, B. Lozanovski, X. Zhang, M. Qian, O. Faruque and M. Brandt, "SLM lattice structures: Properties, performance, applications and challenges," Materials \& Design, vol. 183, p. 108137, 2019.

[40] Y. Du, D. Gu, L. Xi, D. Dai, T. Gao, J. Zhu and C. Ma, "Laser additive manufacturing of bio-inspired lattice structure: Forming quality, microstructure and energy absorption behavior," Materials Science and Engineering: A, vol. 773, p. 138857, 2020.

[41] K. Davami, M. Mohsenizadeh, M. Munther, T. Palma, A. Beheshti and K. Momeni, "Dynamic Energy Absorption Characteristics of Additively-Manufactured ShapeRecovering Lattice Structures," Materials Research Express, vol. 6, p. 045302, 2019.

[42] M. Calabrese, T. Primo and A. Del Prete, "Lattice structures integration with conventional topology optimization," in AIP Conference Proceedings, 2017.

[43] M. Bici, S. Brischetto, F. Campana, C. G. Ferro, C. Secli, S. Varetti, P. Maggiore and A. Mazza, "Development of a multifunctional panel for aerospace use through SLM additive manufacturing," 11th CIRP Conference on Intelligent Computation in Manufacturing Engineering, CIRP ICME '17, vol. 67, pp. 215-220, 2018.

[44] S. Fried, "Additive Manufacturing: Advantages and Disadvantages for PCB Prototyping," Nano Dimension, 18 April 2019. [Online]. Available: https://www.nanodi.com/blog/2019-additive-manufacturing-advantages-and-disadvantages-for-pcbprototyping. [Accessed 28 April 2020].

[45] Q. Zeng, A. Yu and G. Lu, "Multiscale modeling and simulation of polymer nanocomposites," Progress in Polymer Science, vol. 33, no. 2, pp. 191-269, 2008. 
[46] M. O. Steinhauser, Computational Multiscale Modeling of Fluids and Solids: Theory and Applications, New York: Springer Science \& Business Media, 2007.

[47] J. T. Oden, K. Vemaganti and N. Moes, "Hierarchical modeling of heterogeneous solids," Computer Methods in Applied Mechanics and Enigneering, vol. 172, no. 1-4, pp. 3-25, 1999.

[48] L. Micheal, "Birth and Future of Multiscale Modeling for Macromolecular Systems (Nobel Lecture)," Angewandte Chemie International Edition, vol. 53, no. 38, pp. 10006$10018,2014$.

[49] S. Adamson, V. Astapenko, I. Chernysheva, V. Chorkov, M. Demisky, G. Demchenko, A. Demura, A. Demyanov, N. Dyatko, A. Eletzkii, A. Knizhnik, I. Kochetov, A. Napartovich, E. Rykova, L. Sukhanov, S. Umanskii, A. Vetchinkin, A. Zaitsevskii and B. Potapkin, "Multiscale multiphysics nonempirical approach to calculation of light emission properties of chemically active nonequilibrium plasma: application to Ar-GaI3 system," Journal of Physics D: Applied Physics, vol. 40, no. 13, pp. 3857-3881, 2007.

[50] L. T. Lliev, "Multi-scale simulations of Cosmic Reionization," Barcelona Supercomputing Center Annual Report 2015, 2015.

[51] M. Massimi, "Three problems about multi-scale modelling in cosmology," Studies in History and Philosophy of Modern Physics, vol. 64, pp. 26-38, 2018.

[52] L. L. Lebel and D. Therriault, "Multiscale Manufacturing of Three-Dimensional Polymer-Based Nanocomposite Structures," Advances in Diverse Industrial Applications of Nanocomposites, pp. 409-428, 2011. 
[53] M. F. Horstemeyer, "Multiscale Modeling: A Review," in Practical Aspects of Computational Chemistry, New York, Springer, 2009, pp. 87-135.

[54] R. Batterman, "The Tyranny of Scales," in The Oxford Handbook of Philosophy of Physics, OUP, 2013.

[55] S. Kmiecik, D. Gront, M. Kolinski, L. Wieteska, A. E. Dawid and A. Kolinski, "CoarseGrained Protein Models and Their Applications," Chemical Reviews, vol. 116, no. 14, pp. 7898-7936, 2016.

[56] E. Spiga, M. T. Degiacomi and M. D. Peraro, "Chapter Three - New strategies for integrative dynamic modeling of macromolecular assembly," Advances in Protein Chemistry and Structural Biology, vol. 96, pp. 77-111, 2014.

[57] M.-C. Ho Ba Tho, C. Stolz, M. Vanleene, S. Bensamoun, J.-M. Treutenaere and C. Rey, "Multi-scale Characterization and Modelling of Human Cortical Bone," MRS Proceedings, vol. 898, pp. 0898-L05-15, 2005.

[58] J. L. Katz, A. Misra, S. Paulette, Y. Wang, S. Bumrerraj, T. Nomura, S. J. Eppel and M. Tabib-Azar, "Multiscale mechanics of hierarchical structure/property relationships in calcified tissues and tissue/material interfaces," Materials Sicence and Engineering, vol. 27, no. 3, pp. 450-468, 2007.

[59] Z. H. Zuo, X. Huang, J. H. Rong and M. Y. Xie, "Multi-scale design of composite materials and structures for maximum natural frequencies," Materials \& design, vol. 51, pp. 1023-1034, 2013.

[60] X. Yang, Y. Xie, G. Steven and O. Querin, "Bidirectional evolutionary method for 
stiffness optimization method," Structural Multidisciplinary Optimization, vol. 37, pp. 430-8, 1999.

[61] M. P. Bendsøe and O. Sigmund, "Optimal shape design as a material distribution problem," Structural Optimization, vol. 1, pp. 193-202, 1989.

[62] L. Xia, Multiscale Structural Topology Optimization, London: ISTE Press - Elsevier; 1 edition, 2016.

[63] G. Allaire, The Homogenization Method for Topology and Shape Optimization, Vienna: Springer, 1997, pp. 101-133.

[64] A. Lucia and P. A. DiMaggio, "Multi-Scale Optimization," European Symposium on Computer-Aided Process Engineering - 14, pp. 1093-1098, 2004.

[65] S. Sudhoff, "Lecture 9: Multi-Objective Optimization," [Online]. Available: https://engineering.purdue.edu/ sudhoff/ee630/Lecture09.pdf. [Accessed 25 April 2020].

[66] A. Messac, Optimization in Practice with Matlab for Engineering students and Professionals, New York: Cambridge Press, 2013.

[67] K. Deb, A. Pratap, S. Agarwal and T. Meyarivan, "A Fast and Elitist Multiobjective Genetic Algorithm: NSGA-II," IEEE Transactions of Evolutionary Computation, vol. 6, no. 2, pp. 182-197, 2002.

[68] S. Hollister, "Porous scaffold design for tissue engineering," Nature Materials, vol. 4, pp. $518-524,2005$.

[69] M. P. Bendsoe and O. Sigmund, Topology Optimization : Theory, Methods and Applications, New York: Springer, 1999. 
[70] A. Radman, Bi-directional Evolutionary Structural Optimization (BESO) for Topology Optimization of Material's Microstructure, Melbourne: RMIT Univeristy, 2013.

[71] S. Ryu, S. Lee, J. Jung, J. Lee and Y. Kim, "Micromechanics-Based Homogenization of the Effective Physical Properties of Composites With an Anisotropic Matrix and Interfacial Imperfections," Frontiers in Materials: Rising Stars, 01 March 2019.

[72] R. Hutchinson and N. Fleck, "The Structural Performance of the Periodic Truss," Journal of the Mechanics and Physics of Solids, vol. 54, no. 4, pp. 756-782, 2006.

[73] S. Arabnejad and D. Pasini, "Mechanical properties of lattice materials via asymptotic homogenization and comparison with alternative homogenization methods," International Journal of Mechanical Sciences, vol. 77, pp. 249-262, 2013.

[74] F. Moravec and S. Roman, "Numerical Computing of Elastic Homogenized coefficients for periodic fibrous tissue," Applied and Computational Mechanics, vol. 3, pp. 141-152, 2009.

[75] D. Pasini and S. Arabnejad, "Mechanical properties of lattice materials via asymptotic homogenization and comparison with alternative homogenization methods," International Journal of Mechanical Sciences, vol. 77, pp. 249-262, 2013.

[76] M. P. Bendsoe, Optimization of Structural Topology Shape, and Material, New York: Springer, 1995.

[77] G. I. Rozvany, Structural Design Via Optimality Criteria: The Prager Approach to Structural Optimization, London: Kluwer Academic Publishers, 1989.

[78] V. J. Challis, "A Discrete Level-Set Topology Optimization Code Written in Matlab," 
Structural Multidisciplinary Optimization, vol. 41, pp. 453-464, 2010.

[79] H. Smith and J. A. Norato, "A MATLAB code for topology optimization using the geometry projection method," Structural and Multidisciplinary Optimization, 2020.

[80] H. Eschenauer, V. Kobelev and A. Schumacher, "Bubble Method for Topology and Shape Optimization of Structures," Structural Optimization, vol. 8, pp. 42-51, 1994.

[81] B. Bourdin and A. Chambolle, "The phase-field method in optimal design," IUTAM Symposium on Topological Design Optimization of Structures, Machines and Materials, pp. 207-215, 2006.

[82] Y. Wang, H. Xu and D. Pasini, "Multiscale isogeometric topology optimization for lattice materials," Computer Methods in Applied Mechanics and Engineering, vol. 316, pp. 568-585, 2017.

[83] T. Boissonneault, "New plate-lattice architectures could enable stiffest 3D printed structures," 3D Printing Media Network, 19 December 2018. [Online]. Available: https://www.3dprintingmedia.network/plate-lattice-architectures/. [Accessed 20 August 2020].

[84] N. Noda, "Thermal Dtresses in Functionally Graded Materials," Journal of Thermal stresses, vol. 22, no. 4-5, pp. 477-512, 1999.

[85] D. Levy, "Ceramic matrix composites take flight in LEAP jet engine," Science X Network, 4 January 2017. [Online]. Available: https://phys.org/news/2017-01-ceramicmatrix-composites-flight-jet.html. [Accessed 19 April 2020].

[86] D. Mahmoud and M. A. Elbestawi, "Lattice Structures and Functionally Graded 
Materials Applications in Additive Manufacturing of Orthopedic Implants: A Review," Journal of Manufacturing and Materials Processing, vol. 1, no. 2, pp. 1-19, 2017.

[87] K. Hazlehurst, C. Wang and M. Stanford, "The potential application of a Cobalt Chrome Molybdenum femoral stem with functionally graded orthotropic structures manufactured using Laser Melting technologies.," Medical Hypotheses, vol. 81, pp. 1096-1099, 2013.

[88] H. Frost, "Skeletal structural adaptations to mechanical usage (SATMU): 3. The hyaline cartilage modeling problem," The Anatomical Record, vol. 226, no. 4, pp. 423-432, 1990.

[89] J. Van der Stok, O. P. Van der Jagt, S. A. Yavari, M. F. Haas, J. H. Waarsing, H. Jahr, M. V. Van Lieshout, P. Patka, J. A. Verhaar, A. A. Zadppor and H. Weinans, "Selective laser melting-produced porous titanium scaffolds regenerate bone in critical size cortical bone defects.," Journal of Orthopaedic Research, vol. 31, no. 5, pp. 792-799, 2012.

[90] F. J. QUEVEDO GONZÁLEZ, "Computational Design of Functionally Graded Hip Implants by Means of Additively Manufactured Porous Materials," École de Technologie Supérieure, 2016.

[91] S. Jing, H. Zhang, J. Zhou and G. Song, "Optimum weight design of functionally graded material gears," Chinese Journal of Mechanical Engineering, vol. 28, no. 6, pp. 11861193, 2015.

[92] N. Alshabatat, K. Myers and K. Naghshineh, "Design of in-plane functionally graded material plates for optimal vibration performance," Noise Control Engineering Journal, vol. 64 , no. 2, pp. 268-278, 2016.

[93] Z. Wang, Q. Zhang, L. Xia, J. Wu and P. Liu, "Stress Analysis and Parameter 
Optimization of an FGM Pressure Vessel Subjected to Thermo-Mechanical Loadings," Procedia Engineering, vol. 130, pp. 374-389, 2015.

[94] G. Maciejewski and Z. Mroz, "Optimization of functionally gradient materials under cyclic thermal and mechanical loading," in ECCOMAS Special Interest Conference: Numerical Heat Transfer 2012, Gliwice-Wroclaw, 2012.

[95] D. Pasini, A. Moussa and A. Rahimizadeh, "Stress-Constrained Topology Optimization for Lattice Materials," Encyclopedia of Continuum Mechanics, 2018.

[96] B. Yi, Y. Zhou, G. H. Yoon and K. Saitou, "Topology Optimization of FunctionallyGraded Lattice Structures with Buckling Constraints," Computer Methods in Applied Mechanics and Engineering, vol. 354, pp. 593-619, 2019.

[97] W. M. Rubio and E. C. Nelli Silva, "Toward design of Functionally Graded Piezoelectric Ultrasonic Motors using topology optimization," 2009 IEEE International Ultrasonics Symposium, Rome, pp. 2592-2595, 2009.

[98] V. Dakshnamoorthy and R. M. Taylor, "Automated Lattice Optimization of Hinge Fitting with Displacement Constraint," Solid Freeform Fabrication 2016: Proceedings of the 27th Annual International, pp. 2123-2138, 2016.

[99] C. Smith, M. Gilbert, I. Todd and F. Derguti, "Application of Layout Optimization to the Design of Additively Manufactured Metallic Components," Structural and Multidisciplinary Optimization, vol. 54, no. 2, p. 1297-1313, 2016.

[100] W. Chen, X. Zheng and S. Liu, "Finite-Element-Mesh Based Method for Modeling and Optimization of Lattice Structures for Additive Manufacturing," Materials, vol. 11, no. 
11, p. 2073, 2018.

[101] Altair, "Lattice Structure Optimization," in HyperWorks Solvers, 2017.

[102] K. Ushijima, W. J. Cantwell and D. H. Chen, "Prediction of the mechanical properties of micro-lattice structures subjected to multi-axial loading," International Journal of Mechanical Sciences, vol. 68, pp. 47-55, 2013.

[103] R. I. Stephens, A. Fatemi, R. R. Stephens and H. O. Fuchs, Metal FAtigue in Engineering, 2nd Edition, Palo Alto: John Wiley \& Sons, 2000.

[104] T. Yuri, Y. Ono and T. Ogata, "Effects of surface roughness and notch on fatigue properties for Ti-5Al-2.5Sn ELI alloy at cryogenic temperatures," Science and Technology of Advanced Materials, vol. 4, no. 4, pp. 291-299, 2003.

[105] J. M. Djokovic, R. R. Nikolic, J. Bujnak and B. Hadzima, "Estimate of the steel bridges fatigue life by application of the fracture mechanics," IOP Conference Series Materials Science and Engineering, vol. 419, no. 1, 2018.

[106] T. Bell, "How Cold Working Strengthens Metal," the balance, 25 January 2019. [Online]. Available: https://www.thebalance.com/what-is-cold-working-2340011. [Accessed 24 February 2019].

[107] Y. Murakami, "1.1.2 Nonferrous Metals," in Metal Fatigue: Effects if Small Defects and Nonmetallic Inclusions, Elsevier, 2002, p. 4.

[108] Y. Murakami, Metal Fatigue: Effects of Small Defects and Nonmetallic Inclusions, Elsevier, 2002.

[109] J. Tillander, K. Hagberg, L. Hagberg and R. Branemark, "Osseointegrated Titanium 
Implants for Limb Prostheses Attachments: Infectious Complications," Clinical Orthopaedics and Related Research, vol. 468, no. 10, pp. 2781-2788, 2010.

[110] D. Peacock, "Titanium for Automotive Applications," Azo Materials, 20 June 2001. [Online]. Available: https://www.azom.com/article.aspx?ArticleID=553. [Accessed 24 February 2019].

[111] C. A. "What is a SN-Curve?," Siemens, 29 August 2019. [Online]. Available: https://community.sw.siemens.com/s/article/what-is-a-sn-curve. [Accessed 1 March 2020].

[112] D. Taylor, "CHAPTER 11 - Multiaxial Loading: Fracture and Fatigue Under Complex Stress States," in The Theory of Critical Distances, Elsevier, 2070, pp. 213-233.

[113] J. W. Lee, G. H. Yoon and H. S. Jeong, "Topology optimization considering fatigue life in the frequency domain," Computers \& Mathematics with Applications, vol. 70, no. 8, pp. 1852-1877, 2015.

[114] C. Batias, "There is no infinite fatigue life in matallic materials," FAtigue \& Fracture of Engineering Materials \& Structures, vol. 22, no. 7, pp. 559-565, 1999.

[115] K. Miller and O'donnell, "The Fatigue Limit and its Elimination," Fracture of Engineering Materials and Structures, vol. 22, no. 7, pp. 545-557, 1999.

[116] Altair University and M. Goelke, Learn Fatigue Analysis with Altair Optistruct 2nd Edition, Altair, 2020.

[117] e. "Glossary," Altair, [Online]. Available: https://www.efatigue.com/glossary/. [Accessed 25 February 2020]. 
[118] T. Endo, M. Matsuishui, M. Koichi, T. Kiyohum and K. Kakuichi, "Damage evaluation of metals for random or varying loading — three aspects of rain flow method," Mechanical Behavior of Materials, vol. 1, pp. 371-380, 1974.

[119] M. Barkey, "Rainflow Cycle Counting part 3/3--Fortran Program," Youtube, 8 January 2018. [Online]. Available: https://www.youtube.com/watch?v=TIMKbyw3u4I. [Accessed 25 February 2020].

[120] C. Amzallag, J. P. Gerey, J. L. Robert and J. Bahuaud, "Standardization of the rainflow counting method for fatigue analysis," International Journal of Fatigue, vol. 16, no. 4, pp. 287-293, 1994.

[121] Y. L. Lee and D. Taylor, "Cycle Counting Techniques," in Fatigue Testing and Analysis, Elsevier, 2005, pp. 77-102.

[122] E. S. Statnikov, O. V. Korolkov and V. N. Vityazev, "Physics and Mechanism of Ultrasonic Impact," Ultrasonics, vol. 44, pp. e533-e508, 2006.

[123] P. Lefevre, "Fatigue life improvement of welded structure by ultrasonic needle peening," 2016. [Online]. Available: http://www.sonats-et.com/telechargements_44download.html. [Accessed 16 June 2018].

[124] B. N. Mordyuk and G. I. Prokopenko, "Ultrasonic Impact Peening for the Surface Properties’ Management," Journnal of Sound and Vibration, vol. 308, no. 3-5, pp. 855$866,2007$.

[125] A. I. Dektyar, B. N. Mordyuk, D. G. Savvakin, V. I. Bondarchuk, I. V. Moiseeva and N. I. Khripta, "Enhanced fatigue behavior of powder metallurgy $\mathrm{Ti}-6 \mathrm{Al}-4 \mathrm{~V}$ alloy by 
applying ultrasonic impact treatment," Materials Science and Engineering: A, vol. 641, pp. 348-359, 2015.

[126] T. Metzger, R. Höpler, E. Born, O. Ambacher, M. Stutzmann, R. Stömmer, M. Schuster, H. Göbel, S. Christiansen, M. Albrecht and H. P. Strunk, "Defect structure of epitaxial GaN films determined by transmission electron microscopy and triple-axis X-ray diffractometry," Philosophical Magazine A, vol. 77, no. 4, pp. 1013-1025, 1998.

[127] D. J. Stirland, G. J. Rees and A. Ritson, "The relationship between etch pit density and dislocation density for (001)GaAs," Journal of Crystal Growth, vol. 79, no. 1-3, pp. 493$502,1986$.

[128] B. Hu, G. Trotter, I. Baker, M. K. Miller, L. Yao, S. Chen and Z. Cai, "The Effects of Cold Work on the Microstructure and Mechanical Properties of Intermetallic Strengthened Alumina-Forming Austenitic Stainless Steels," Metallurgical and Materials Transactions A, vol. 46, no. 8, pp. 3773-3785, 2015.

[129] K. Tanaka and T. Mura, "A Dislocation Model for Fatigue Crack Initiation," Journal of Applied Mechanics, vol. 48, no. 1, pp. 97-103, 1980.

[130] X. Wu, "On Tanaka-Mura's fatigue crack nucleation model and validation," Fatigue Fracture Engineering Material \& Structures, vol. 41, no. 4, pp. 894-899, 2018.

[131] A. I. Dekhtyar, B. N. Mordyuk, D. G. Savvakin, V. I. Bondarchuk, I. V. Moiseeva and N. I. Khripta, "Enhanced fatigue behavior of powder metallurgy Ti-6Al-4V alloy by applying ultrasonic impact treatment," Materials Science and Engineering: A, vol. 641, pp. 348-359, 2015. 
[132] P. S. Maiya and D. E. Busch, "Effect of surface roughness on low-cycle fatigue behavior of type 304 stainless steel," Metallurgical Transactions A, vol. 6, 1975.

[133] "Dislocation," Wikipeidia, 6 November 2018. [Online]. Available: https://en.wikipedia.org/wiki/Dislocation\#cite_note-8. [Accessed 2 March 2019].

[134] C. Qiu, N. J. Adkins and M. M. Attallah, "Microstructure and tensile properties of selectively laser-melted and of HIPed laser-melted Ti-6Al-4V," Materials Science and Engineering: A, vol. 578, pp. 230-239, 2013.

[135] A. Baca, R. Konecana, G. Nicoletto and L. Kunz, "Influence of Build Direction on the Fatigue Behaviour of Ti6A14V Alloy Produced by Direct Metal Laser Sintering," Material Today: Proceedings, vol. 3, no. 4, pp. 921-924, 216.

[136] D. E. Dimla, N. Hopkinson and H. Rothe, "Investigation of complex rapid EDM electrodes for rapid tooling applications," International Journal of Advanced Manufacturing Technology, vol. 23, no. 3-4, pp. 249-255, 2004.

[137] "AP\&C a GE Additive Company," [Online]. Available: https://www.advancedpowders.com/. [Accessed 20 August 2020].

[138] A. International, ASTM E8/E8M-13, Standard Test Methods for Tension Testing of Metallic Materials, West Conshohocken: ASTM, 2013.

[139] "MTS $810 \& 858$ Test Systems," MTS, 2006. [Online]. Available: https://www.upc.edu/sct/documents_equipment/d_77_id-412.pdf. [Accessed 21 July 2019].

[140] "Titanium Properties," SGS Solid Carbide Tools, [Online]. Available: https://kyocera- 
sgstool.co.uk/titanium-resources/titanium-information-everything-you-need-toknow/titanium-properties/. [Accessed 21 August 2020].

[141] S. J. Orfanidis, Introduction to Signal Processing, Englewood Cliffs, NJ: Prentice Hall, 1996.

[142] R. Schafer, "“What Is a Savitzky-Golay Filter? [Lecture Notes]."," IEEE Signal Processing Magazine 28, pp. 111-117, July 2011.

[143] C. Ma, M. T. Andani, H. Qin, N. S. Moghaddam, H. Ibrahim, A. Jahdakbar, A. Amerinatanzi, Z. Ren, H. Zhang, G. L. Doll, Y. Dong, M. Elahinia and C. Ye, "Improving surface finish and wear resistance of additive manufactured nickel-titanium by ultrasonic nano-crystal surface modification," Journal of Materials Processing Technology, vol. 249, pp. 433-440, 2017.

[144] C. Ma, Y. Dong and C. Ye, "Improving Surface Finish of 3D-Printed Mateals by Ultrasonic Nanocrystal Surface Modification," Proceeding CIRP, vol. 45, pp. 319-322, 2016.

[145] DIN 50100 : Load Controlled Fatigue testing - Execution and Evaluation of Cyclic Tests at Constant Load Amplitudes on Metallic Speciemns and Components, Berlin: D.I.F.N.E.V.G.N. Standard, 2016.

[146] R. Zheng, E. Han and W. Ke, "Effect of Temperature and Relative humidity on Fatigue Crack Propagation Behavior of Az61 Megnisium Alloy," Materials Science Forums, Vols. 546-549, pp. 409-412, 2007.

[147] B. Hassani and E. Hinton, "Homogenization Theory for Media with Periodic Structures," 
in A Review of Homogenization and Topology Optimization I, 1998.

[148] D. Li, N. Dai, Y. Tang, G. Dong and Y. F. Zhao, "Design and Optimization of Graded Cellular Structures with Triply Periodic Level Surface-based Topological Shapes," Journal of Mechanical Design, vol. 141, no. 7, 2019.

[149] N. Bakhvalov and G. Panasenko, Homogenization: averaging processes in periodic media, Dordrecht: Kluwer Academic Publishers, 1989.

[150] S. Hollister and N. Kikuchi, "A Comparison of Homogenization and Standard Mechanics Analyses for Periodic Porous Composites," Computational Mechanics, vol. 10, pp. 7395, 1992.

[151] G. Dong, Y. Tang and Y. F. Zhao, "A 149 Line Homogenization Code for ThreeDimensional Cellular Materials Written in matlab," Journal of Engineering Materials and Technology, vol. 141, no. 1, p. 011005, 2018.

[152] E. Andreassen and C. S. Andreasen, "How to determine composite material properties using numerical homogenization," Computational Materials Science, vol. 83, pp. 488495, 2014.

[153] S. Nemat-Nasser and M. Hori, Micromechanics: Overall Properties of Heterogeneous Materials, North Hooland, 1993.

[154] P. Liu and G. Chen, "Pourous Materials," Processing and Applications, pp. 189-220, 2014.

[155] M. F. Ashby, Materials Selection in Mechanical Design 5th Edition, Oxford England: Elsevier, 2016. 
[156] V. Deshprande, M. Ashby and N. Fleck, "Foam topology: bending versus stretching dominated architectures," Acta Materialia, vol. 49, no. 6, pp. 1035-1040, 2001.

[157] M. F. Ashby and L. J. Gibson, Cellular Solids, Structure and Properties, Oxford England: Pergamon Press, 1988.

[158] O. Sigmund, "A 99 line topology optimization code written in Matlab," Struct Multidisc Optim, vol. 21, pp. 120-127, 2001.

[159] O. Sigmund, "On the Design of Compliant Mechanisms using topology Optimization," Mechanics of Structures and Machines, vol. 25, no. 4, pp. 493-524, 1997.

[160] K. Svanberg, "The method of moving asymptotes - a new method for structural optimization," International Journal for Numerical Methods in Engineering, vol. 24, no. 2, pp. 359-373, 1987.

[161] M. Bruggi, "On an alternative approach to stress constraints relaxation in topology optimization," Structural Multidisciplianry Optimization, vol. 36, no. 2, pp. 125-141, 2008.

[162] K. Ushijima, W. Cantwell and D. Chen, "Prediction of the mechanical properties of micro-lattice structures subjected to multi-axial loads," Internaltional Journal of Mechanical Sciences, vol. 68, pp. 47-55, 2013.

[163] K. Ushijima, W. J. Cantwell, M. S. Tsopanos and M. Smith, "An Investigation inot the compressive properties of stainles steel micro-lattice structures," Journal of Sandwich Structuress \& Materials, vol. 13, no. 3, pp. 303-329, 2010.

[164] K. Ushijima, W. Cantwell and D. Chen, "Estimation of the compressive and shear 
responses of three-dimensional micro-lattice structures," Procedia Engineering, vol. 10, pp. 2441-2446, 2011.

[165] M. F. Ashby, "The properties of foams and lattices," Philosophical Transactions of the Royal Society, pp. 15-30, 2005.

[166] C. D. Hansen and C. R. Johnson, Visualization handbook, New York: Academic Press, 2004.

[167] W. E. Lorensen and E. H. Cline, "Marching Cubes: A High Resolution 3D Surface Construction Algorithm," Computer Graphics, vol. 21, no. 4, 1987.

[168] D. Akio and K. Akio, "An Efficient Method of Triangulating Equi-Valued Surfaces by Using Tetrahedral Cells," IEICE Transactions of Information and Systems, Vols. E74-D, no. $1,1991$.

[169] N. M. Gregory and B. Hamann, "The Asymptotic Decider: Resolving the Ambiguity in Marching Cubes," Proceedings of the 2nd conference on Visualization '91 (VIS '91), pp. 83-91, 1991.

[170] B. Anderson, "An Implementation of the Marching Cubes[1] Algorithm," Carleton College, 2004. [Online]. Available:

http://www.cs.carleton.edu/cs_comps/0405/shape/marching_cubes.html\#2. [Accessed 20 April 2020].

[171] "Altair," Altair Engineering, 2020. [Online]. Available: https://www.altair.com/. [Accessed 20 April 2020].

[172] Q. Fang, "Iso2Mesh," MATLAB File Echange, 19 July 2018. [Online]. Available: 
https://www.mathworks.com/matlabcentral/fileexchange/68258-iso2mesh/. [Accessed 21 April 2020].

[173] M. "Patch Remesher," MATLAB Central File Exhange, 14 December 2015. [Online]. Available: https://www.mathworks.com/matlabcentral/fileexchange/49691-patchremesher. [Accessed 21 April 2020].

[174] P. Duysinx and M. P. Bendsøe, "Topology optimization of continuum structures with local stress constraints," International Journal for Numerical Methods in Engineering, vol. 43, no. 8, p. 1453-1478, 1998.

[175] K. Nabaki, J. Shen and X. Huang, "Stress Minimization of Structures Based on Bidriectional Evolutionary Procedure," Journal of Structural Engineering, vol. 145, no. 2, pp. 04018256-14, 2019.

[176] M. Pincus, "Letter to the Editor-A Monte Carlo Method for the Approximate Solution of Certain Types of Constrained Optimization Problems," Operations Research, vol. 18, no. 6, pp. 1225-1228, 1970.

[177] V. Skala, "A practical use of radial Basis functions interpolation and approximation," Investigation Operational, vol. 37, no. 2, 2016.

[178] A. S. Krishna Dasari and P. A. Chaddad, "Random Forest Surrogate Models to Support Design Space Exploration in Aerospace Use-Case," Artificial Intelligence Applications and Innovations (AIAI 2019), pp. 532-544, 2019.

[179] M. D. Buhmann, Radial Basis Functions: Theory and Implementations, Cambridge: The Press Syndicate of the University of Cambridge, 2003. 
[180] J. Yuan, Y.-M. Li, C.-L. Liu and X. F. Zha, "Leave-One-Out Cross-Validation Based Model Selection for Manifold Regularization," Advances in Neural Networks - ISNN 2010. ISNN 2010. Lecture Notes in Computer Science, vol. 6063.

[181] C. Bliss, "The method of probits," Science, vol. 79, pp. 38-39, 1934.

[182] N. H. Kim, "Sensitivity Analysis," in Encyclopedia of Aerospace Engineering, Ed R. Blockey and W. Shyy (Eds), Chichester, John Wiley and Sons Ltd, 2010, pp. 5251-5264.

[183] A. Verbart, M. Langelaar and F. v. Keulen, "A unified aggregation and relaxation approach for stress-constrained topology optimization," Structural and Multidisciplinary Optimization, vol. 55, no. 2, pp. 663-679, 2017.

[184] E. Holmberg, B. Torstenfelt and A. Klarbring, "Stress constrained topology optimization," Structural and Multidisciplinary Optimization, vol. 48, no. 1, p. 33-47, 2013.

[185] J. Oest and E. Lund, "Topology optimization with finite-life fatigue constraints," Structural and Multidisciplinary Optimization, vol. 56, no. 1, pp. 1045-1059, 2017.

[186] A. B. Lambe, G. J. Kennedy and J. R. Martinis, "An evaluation of constraint aggregation strategies for wing box mass minimization," Structural and Multidisciplinary Optimization, vol. 55, no. 1, pp. 257-277, 2017.

[187] G. Kreisselmeier and R. Steinhauser, "Systematic Control Design by Optimizing a Vector Performance," in Symposium on Computer-Aided Design of Control Systems, Zurich, 1979.

[188] X. Tang, "Shape Functions of Tapered Beam-Column Elements," Computers \& 
Structures, vol. 46, no. 5, pp. 943-953, 1993.

[189] J. R. Banerjee, "EXACT BERNOULLI-EULER STATIC STIFFNESS MATRIX FOR A RANGE OF TAPERED BEAM_COLUMNS," International Journal for Numerical Methods in Engineering, vol. 23, pp. 1615-1628, 1986.

[190] X. Tang, "Second-order tapered beam-column elements," Computers \& Structures, vol. 46, no. 5, pp. 931-941, 1993.

[191] B. V. Hooreweder and J.-P. Kruth, "Advanced fatigue analysis of metal lattice structures produced by Selective Laser Melting," CIRP Annals - Manufacturing Technology, 2017.

[192] M. Kazuhisa, "Structures and Mechanical Properties of Natural and Synthetic Diamonds," Diamond Films and Technology, vol. 8, no. 3, pp. 153-172, 1999.

[193] W. j. D. Callister and D. G. Rethwiseh, Materials Science and Engineering: An Introduction 8th Edition, New York: john Wiley \& Sons, Inc., 2010.

[194] A. O. Aremu, J. J. Brennan-Craddock, A. Panesar, I. A. Ashcroft, R. M. Hague, R. D. Wildman and C. Tuck, "A voxel-based method of constructing and skinning conformal and functionally graded lattice structures suitable for additive manufacturing," Additive Manufacturing, vol. 13, pp. 1-13, 2017.

[195] J. Feng, J. Fu, Z. Lin, C. Shang and B. Li, "A review of the design methods of complex topology structures for 3D printing," Visual Computing for Industry, Biomedicine, and Art, 2018.

[196] Y. Tang, A. Kurtz and Y. F. Zhao, "Bidirectional Evolutionary Structural Optimization (BESO) based design method for lattice structure to be fabricated by additive 
manufacturing," Computer-Aided Design, vol. 69, no. 1, pp. 91-101, 2015.

[197] M. Burns, Automated Fabrication, Prentice Hall, 1993.

[198] J. Tuszynski, "in_polyhedron," MATLAB Central File Exchange, 20 August 2018. [Online]. Available: https://www.mathworks.com/matlabcentral/fileexchange/48041in_polyhedron. [Accessed 15 December 2019].

[199] Sven, "inpolyhedron - are points inside a triangulated volume?," MATLAB CENTRAL File Exchange, 12 November 2015. [Online]. Available: Sven (2020). inpolyhedron - are points inside a triangulated volume? (https://www.mathworks.com/matlabcentral/fileexchange/37856-inpolyhedron-arepoints-inside-a-triangulated-volume), MATLAB Central File Exchange. Retrieved January 12, 2020. . [Accessed 12 January 2020].

[200] D. Frisch, "point2trimesh( ) - Distance Between Point and Triangulated Surface," MATLAB Central File Exchange, 25 September 2016. [Online]. Available: https://www.mathworks.com/matlabcentral/fileexchange/52882-point2trimesh-distancebetween-point-and-triangulated-surface. [Accessed 25 Januaray 2020].

[201] M. W. Jones, "3D Distance from a Point to a Triangle," Swansea, 1995.

[202] J. Tuszynski, "Triangle/Ray Intersection," MATLAB Central File Exchange, 18 May 2018. [Online]. Available: https://www.mathworks.com/matlabcentral/fileexchange/33073-triangle-ray-intersection. [Accessed 15 December 2019].

[203] T. Moller and B. Trumbore, "Fast, Minimum Storage Ray-Triangle Intersection," Journal 
of Graphics Tools, vol. 2, no. 1, pp. 21-28, 1997.

[204] J. v. den Berg and u. , "Calculate Rotation Matrix to align Vector A to Vector B in 3d?, URL (version: 2016-09-01): https://math.stackexchange.com/q/476311," StackExchange, 26 August 2013. [Online]. Available:

https://math.stackexchange.com/questions/180418/calculate-rotation-matrix-to-alignvector-a-to-vector-b-in-3d/897677\#897677. [Accessed 18 May 2020].

[205] J. B. Kuipers, Quaternions and Rotation Sequences: A Primer with Applications to Orbits, Aerospace and Virtual Reality, Princeton University Press, 2002.

[206] J. Tuszynski, "Surface Intersection," MATLAB Central File Exchange, 1 December 2014. [Online]. Available:

https://www.mathworks.com/matlabcentral/fileexchange/48613-surface-intersection. [Accessed 15 December 2019].

[207] "featureEdges," MATLAB, [Online]. Available: https://www.mathworks.com/help/matlab/ref/triangulation.featureedges.html. [Accessed 12 January 2020]. 


\section{Appendices}

\section{Appendix A : Fatigue Analysis}

\section{A.1 Cycle Counting : Three Point Method}

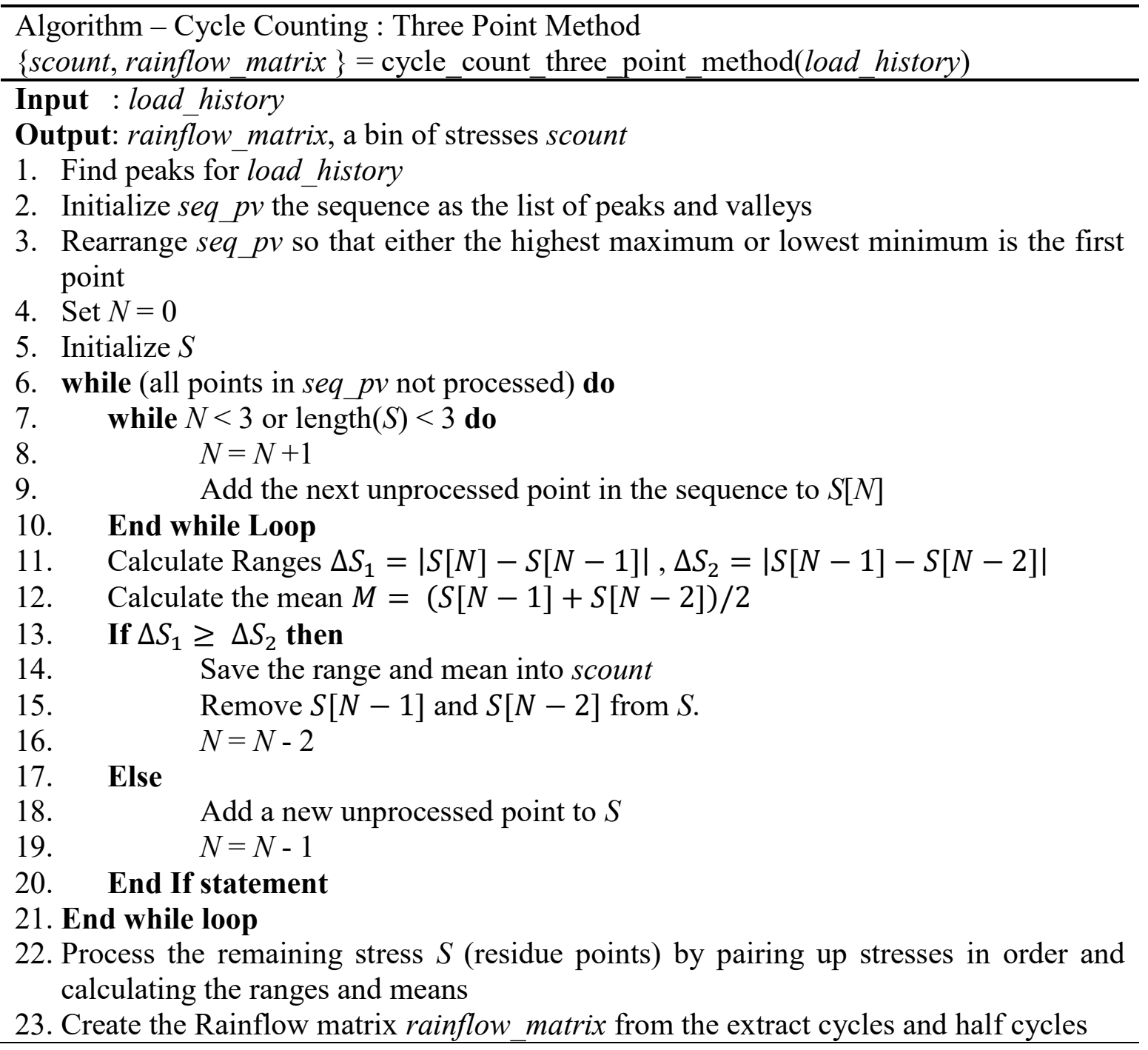

\section{A.2 Fatigue Simulation Using Finite Element Method}

Finite element simulations can be used to predict fatigue lives and calculate damage. Problems are discretized into elements where each element is a sub problem of the main problem. Sub 
problems are a collection of linear equations that when solved, predict the behavior of the problem in a localized sub space. The process of fatigue analysis and prediction is therefore applied on each element.

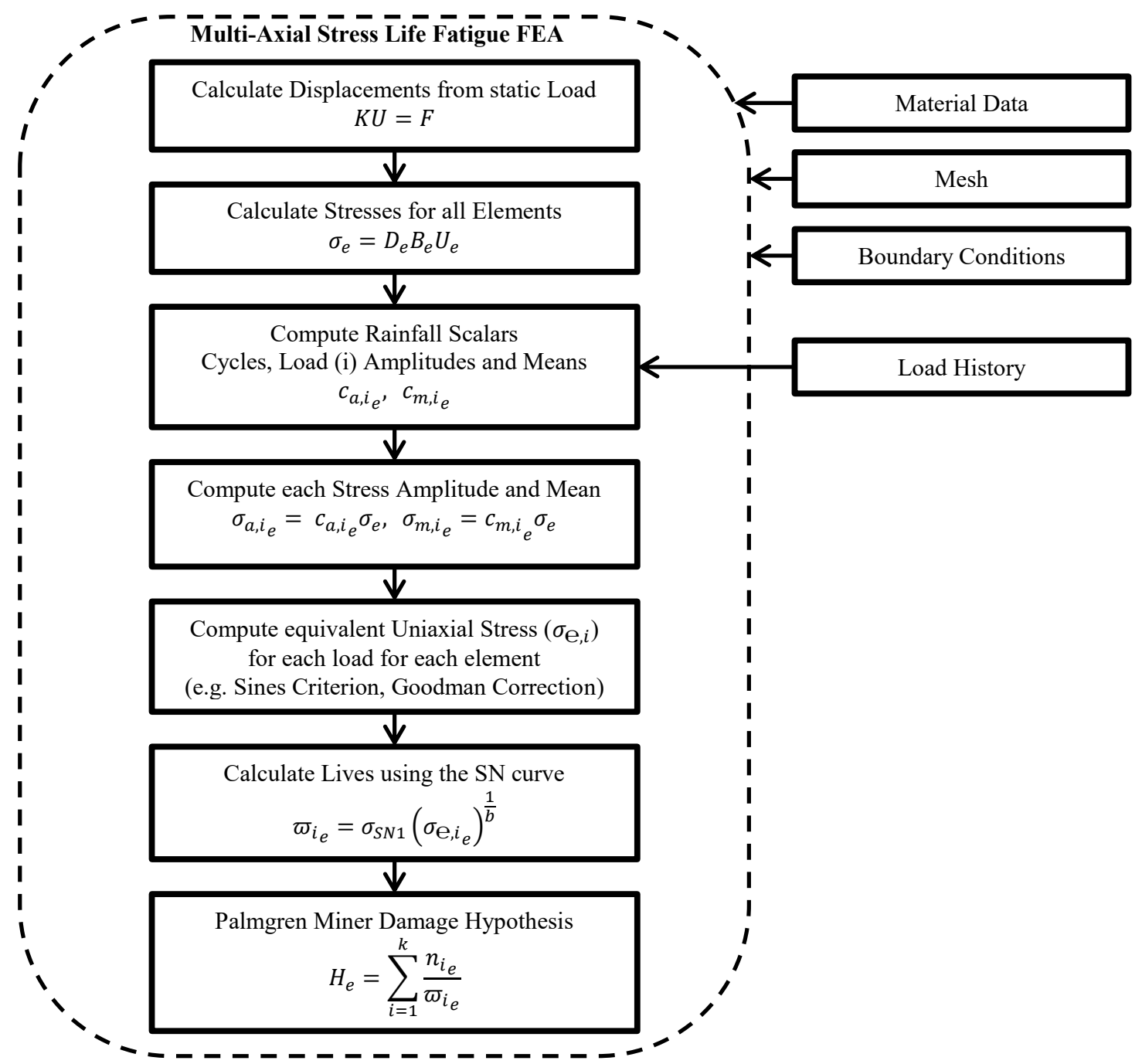

Figure 56 : Finite Element Simulation for Multi-Axial Fatigue Prediction of Proportional Loads

In finite element analysis, the reference load is used to acquire the reference displacements for the nodes. The reference displacements are then used to determine the reference stresses for ach load case. Because the equilibrium equation assumes linear elasticity between the stresses and the displacements, scalar multiplication can be used to calculate the fatigue stresses. This means 
that the mean and amplitude stress components for each element and each load reversal can be found by calculating a reference stress.

The Finite element method will be used to predict the fatigue life of a proportionally loaded structure using the stress life approach. If non proportional loadings are applied, then an alternate method for predicting the fatigue life needs to be considered. This problem is beyond the scope of this thesis. The procedure for proportional multi-axial fatigue is presented in Figure 56. 
Appendix B : Test Data

\section{B.1 Tensile Test Results}

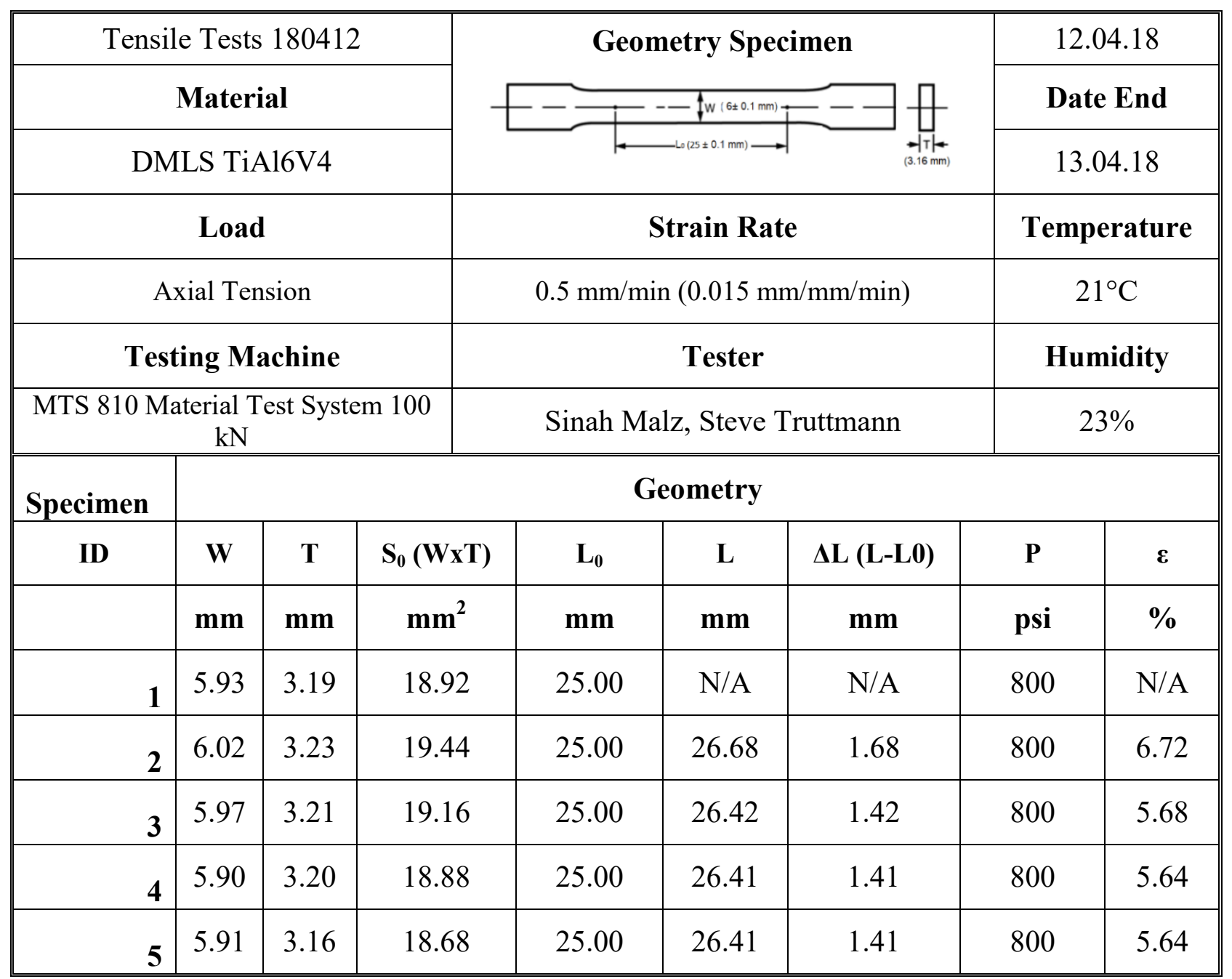




\section{B.2 Fatigue Test Results}

\begin{tabular}{|c|c||c|c|}
\hline \multicolumn{2}{|c||}{ Treated Specimens } & \multicolumn{2}{c|}{ Untreated Specimens } \\
\hline \hline Stress Level & Cycles to Failure & Stress Level & Cycles to Failure \\
\hline 550 & $3.17 \mathrm{E}+04$ & 396 & 23944 \\
\hline 550 & $1.26 \mathrm{E}+04$ & 400 & 27658 \\
\hline 400 & $1.31 \mathrm{E}+05$ & 400 & 40195 \\
\hline 400 & $1.52 \mathrm{E}+05$ & 400 & 58523 \\
\hline 400 & $9.26 \mathrm{E}+04$ & 350 & 47903 \\
\hline 250 & $6.71 \mathrm{E}+05$ & 350 & 62468 \\
\hline 250 & $5.32 \mathrm{E}+05$ & 350 & 87405 \\
\hline 550 & 24828.86 & 300 & 120702 \\
\hline 250 & 695225.1 & 300 & 75065 \\
\hline 225 & $1.00 \mathrm{E}+07$ & 300 & 105016 \\
\hline 200 & $1.00 \mathrm{E}+07$ & 300 & 103511 \\
\hline & & 250 & 143968 \\
\hline & & 250 & 133783 \\
\hline & & 250 & 147518 \\
\hline & & 200 & 319230.4 \\
\hline & & 200 & 593327 \\
\cline { 3 - 4 } & & 150 & 10000000 \\
\hline & & & 3255149 \\
\hline
\end{tabular}




\section{Appendix C : SN-Curve Equation Methodology}

The methodology presented for calculating the general terms for the $\mathrm{SN}$-curve is given in this section. The equation is given as:

$$
\log \varpi=\log \widetilde{K}-\mathcal{K} \cdot \log \sigma_{a}
$$

where $\varpi$ is the number of cycles to failure, $\sigma_{a}$ is the stress amplitude, $\mathcal{K}$ is the slope of the stress-life curve and $\mathfrak{C}$ is a constant for the description of the position of the High Cycles Fatigue (HCF) S-N curve at $\varpi=1$ cycles.

The constants in the above equation are found using the formula below:

$$
\begin{gathered}
\mathcal{K}=-\frac{h \sum(\log (\varpi) \log (\sigma))-\left(\sum \log (\varpi)\right)\left(\sum \log (\sigma)\right)}{h \sum\left(\log (\sigma)^{2}\right)-\left(\sum \log (\sigma)\right)^{2}} \\
\mathfrak{C}=10^{\frac{1}{h}\left(\sum \log (\varpi)-\mathcal{K} \sum \log (\sigma)\right)}
\end{gathered}
$$

The formula for predicting the number of cycles for a given stress can be re-arranged into the following equation:

$$
\varpi=\mathfrak{c} \sigma_{a}^{-\mathcal{K}}
$$

The above equation is built for $50 \%$ survivability. Two bands are calculated for a $90 \%$ and a $10 \%$ survivability rating. The percentages reflect the standard deviations away from the mean of 50\%. A total of three SN-Curves are calculated and are scaled base on a fictitious load at $400 \mathrm{MPa}$ which is the mean of the largest and smallest stress level [145]. The quantile level for failure probability is determined by the quantile function and is associated with the standard normal distribution (probit function) and is shown in Table 13 [181]. 
Table 13 : Quantile Distribution

\begin{tabular}{|c|c|}
\hline Failure Probability (\%) & Quantile \\
\hline 0.001 & -3.09023 \\
\hline 5 & -1.64485 \\
\hline 10 & -1.282 \\
\hline 15.9 & -1 \\
\hline 50 & 0 \\
\hline 84.1 & 1 \\
\hline 90 & 1.282 \\
\hline 95.99 & 1.64485 \\
\hline
\end{tabular}

\section{C.1 Untreated Curve Derivation}

The untreated SN-Curve is calculated below; Table 14 displays the tabulated data to compute the constants for the logarithmic regression model and for the Basquin equation.

Table 14 : Tabular Data for Untreated SN-Curve Derivation A

\begin{tabular}{|c|c|c|c|c|c||}
\hline$\sigma_{a}$ & $\varpi$ & $\log \left(\sigma_{a}\right)$ & $\log (\varpi)$ & $\log \left(\sigma_{a}\right) * \log (\varpi)$ & $\log \left(\sigma_{a}\right)^{2}$ \\
\hline 400 & 27658 & 2.60 & 4.44 & 11.56 & 6.77 \\
\hline 400 & 40195 & 2.60 & 4.60 & 11.98 & 6.77 \\
\hline 400 & 20996 & 2.60 & 4.32 & 11.25 & 6.77 \\
\hline 400 & 58523 & 2.60 & 4.77 & 12.40 & 6.77 \\
\hline 396 & 23944 & 2.60 & 4.38 & 11.38 & 6.75 \\
\hline 350 & 47903 & 2.54 & 4.68 & 11.91 & 6.47 \\
\hline 350 & 62468 & 2.54 & 4.80 & 12.20 & 6.47 \\
\hline 350 & 87405 & 2.54 & 4.94 & 12.57 & 6.47 \\
\hline 300 & 120702 & 2.48 & 5.08 & 12.59 & 6.14 \\
\hline 300 & 75065 & 2.48 & 4.88 & 12.08 & 6.14 \\
\hline 300 & 105016 & 2.48 & 5.02 & 12.44 & 6.14 \\
\hline 300 & 103511 & 2.48 & 5.01 & 12.42 & 6.14 \\
\hline 250 & 143968 & 2.40 & 5.16 & 12.37 & 5.75 \\
\hline 250 & 133783 & 2.40 & 5.13 & 12.29 & 5.75 \\
\hline 250 & 147518 & 2.40 & 5.17 & 12.39 & 5.75 \\
\hline 200 & 741511 & 2.30 & 5.87 & 13.51 & 5.29 \\
\hline & & 40.04147 & 78.249233 & 195.3346 & 100.3373 \\
\hline
\end{tabular}

Using the tabular data above, the $\mathrm{SN}$-curve constants for $50 \%$ survivability are calculated as: 


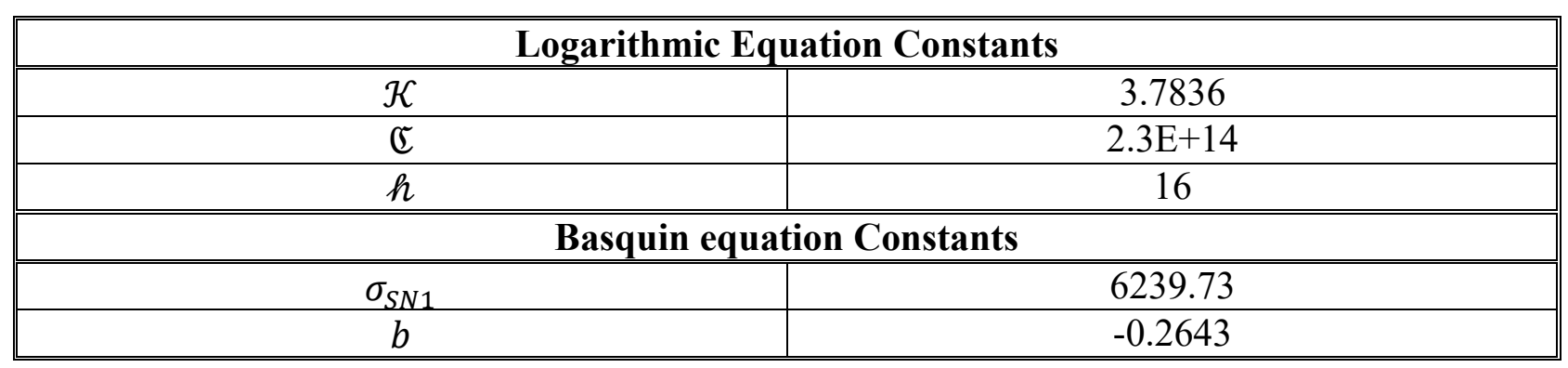

The survivability at $90 \%$ is determined by calculating $\tilde{s}_{\log N}$ for a particular scatter band deviation. The scatter band of the mean-value $\left(T_{m}\right)$ was estimated to be 1.745 for $90 \%$. The required tabular data for calculating $\tilde{s}_{\log N}$ is given in the table below:

Table 15 : Tabular Data for Untreated SN-Curve Derivation B

\begin{tabular}{|c|c|c|c|}
\hline $\boldsymbol{\sigma}_{\text {fict }}$ & $\boldsymbol{\varpi}_{\boldsymbol{i} \text { fict }}$ & $\log \left(\boldsymbol{\varpi}_{\boldsymbol{i} \text {,fict }}\right)$ & $\left(\log \left(\boldsymbol{\varpi}_{\boldsymbol{i} \text {,fict }}\right)-\log \left(\boldsymbol{\sigma}_{\text {fict }}\right)\right)^{\mathbf{2}}$ \\
\hline 400 & 27658 & 4.441821 & 0.005243 \\
\hline 400 & 40195 & 4.604172 & 0.008089 \\
\hline 400 & 20996 & 4.322137 & 0.0369 \\
\hline 400 & 58523 & 4.767327 & 0.064057 \\
\hline 400 & 23050.59 & 4.362682 & 0.022967 \\
\hline 400 & 28903.11 & 4.460945 & 0.002839 \\
\hline 400 & 37691.16 & 4.576239 & 0.003845 \\
\hline 400 & 52737.33 & 4.722118 & 0.043217 \\
\hline 400 & 40644.02 & 4.608997 & 0.008981 \\
\hline 400 & 25276.66 & 4.40272 & 0.012435 \\
\hline 400 & 35362.07 & 4.548538 & 0.001177 \\
\hline 400 & 34855.29 & 4.542269 & 0.000786 \\
\hline 400 & 24319.7 & 4.385958 & 0.016454 \\
\hline 400 & 22599.21 & 4.354093 & 0.025644 \\
\hline 400 & 24919.38 & 4.396537 & 0.013852 \\
\hline 400 & 53844.51 & 4.731141 & 0.04705 \\
\hline \hline
\end{tabular}

The values for the band equations are presented below for the untreated SN-Curve:

\begin{tabular}{|c|c|}
\hline$\tilde{S}_{\log N}$ & 0.15238 \\
\hline$T_{N}$ & 2.45864 \\
\hline
\end{tabular}


The constants for the SN-Curve bands for both 10\% and 90\% survivability (probability of no failure) are calculated form the equation below [145]:

$$
\mathfrak{C}_{\%}=10^{\left(\varpi_{50 \%, f i c t} \mp T_{N, \%} \tilde{s}_{\log N}\right)}
$$

The results are given in the table below; percentages are the survivability rating:

Table 16 : Model Parameter Comparisons for Untreated SN Curve

\begin{tabular}{|c|c||}
\hline \multicolumn{3}{|c|}{$\mathbf{9 0 \%}$ Survivability, $\boldsymbol{T}_{\boldsymbol{N}, \%}=\mathbf{1 . 2 8 2}$} \\
\hline $\mathfrak{C}_{90 \%}$ & $1.45914 \mathrm{E}+14$ \\
\hline$\sigma_{S N 1}, 90 \%$ & 5540.33 \\
\hline \hline \multicolumn{3}{|c|}{$\mathbf{1 0 \%}$ Survivability, $\boldsymbol{T}_{\boldsymbol{N}, \%}=\mathbf{1 . 2 8 2}$} \\
\hline $\mathfrak{C}_{10 \%}$ & $3.358638+14$ \\
\hline$\sigma_{S N 1}, 10 \%$ & 7027.42 \\
\hline \hline \multicolumn{2}{|c|}{$\mathbf{9 9 . 9 9 \%}$ Survivability, $\boldsymbol{T}_{\boldsymbol{N}, \%}=\mathbf{5}$} \\
\hline $\mathfrak{C}_{99.99 \%}$ & $1.01133 \mathrm{E}+14$ \\
\hline$\sigma_{S N 1}, 99.99 \%$ & 5028.94 \\
\hline
\end{tabular}

The fatigue strength exponents $(\mathcal{K}, b)$ are equivalent to the $50 \%$ survivability model (nonshifted).

\section{C.2 UIT SN-Curve Derivation}

The section shows the calculations for deriving the treated $\mathrm{SN}$-curve.

Table 17 : Tabular Data for UIT SN-Curve Derivation A

\begin{tabular}{||c||c|c|c||c||c||}
\hline$\sigma_{a}$ & $\varpi$ & $\log \left(\sigma_{a}\right)$ & $\log (\varpi)$ & $\log \left(\sigma_{a}\right) * \log (\varpi)$ & $\log \left(\sigma_{a}\right)^{2}$ \\
\hline \hline 550 & $3.17 \mathrm{E}+04$ & 2.74 & 4.50 & 12.34 & 7.51 \\
\hline 550 & $1.26 \mathrm{E}+04$ & 2.74 & 4.10 & 11.24 & 7.51 \\
\hline 400 & $1.31 \mathrm{E}+05$ & 2.60 & 5.12 & 13.31 & 6.77 \\
\hline 400 & $1.52 \mathrm{E}+05$ & 2.60 & 5.18 & 13.49 & 6.77 \\
\hline 400 & $9.26 \mathrm{E}+04$ & 2.60 & 4.97 & 12.92 & 6.77 \\
\hline 250 & $6.71 \mathrm{E}+05$ & 2.40 & 5.83 & 13.97 & 5.75 \\
\hline 250 & $5.32 \mathrm{E}+05$ & 2.40 & 5.73 & 13.73 & 5.75 \\
\hline 550 & 24828.86 & 2.74 & 4.39 & 12.04 & 7.51 \\
\hline 250 & 695225.1 & 2.40 & 5.84 & 14.01 & 5.75 \\
\hline \hline \multicolumn{2}{|c|}{ SUM } & 23.22 & 45.65733 & 117.0499 & 60.09126 \\
\hline
\end{tabular}


Using the tabular data above, the SN-curve constants for $50 \%$ survivability are calculated as:

\begin{tabular}{|c|c||}
\hline \multicolumn{3}{|c|}{ Logarithmic Equation Constants } \\
\hline $\mathcal{K}$ & 4.2212 \\
\hline $\mathfrak{c}$ & $9.20566 \mathrm{E}+15$ \\
\hline $\boldsymbol{h}$ & 9 \\
\hline \hline \multicolumn{3}{|c|}{ Basquin equation Constants } \\
\hline$b$ & 6052.591 \\
\hline$\sigma_{S N 1}$ & -0.2369 \\
\hline
\end{tabular}

The survivability at $90 \%$ is determined by calculating $\tilde{S}_{\log N}$ for a particular scatter band deviation. The fictitious load is the mean between the lowest and the highest stress. The scatter band of the mean-value $\left(\mathrm{T}_{\mathrm{m}}\right)$ was estimated to be 1.745 for $90 \%$. The required tabular data for calculating $\tilde{S}_{\log N}$ is given in the table below:

Table 18 : Tabular Data for UIT SN-Curve Derivation B

\begin{tabular}{||c|c|c|c||}
\hline $\boldsymbol{\sigma}_{\text {fict }}$ & $\boldsymbol{\varpi}_{\boldsymbol{i} \text { fict }}$ & $\log \left(\boldsymbol{\varpi}_{\text {i,fict }}\right)$ & $\left(\log \left(\boldsymbol{\varpi}_{\text {i,fict }}\right)-\log \left(\boldsymbol{\sigma}_{\text {fict }}\right)\right)^{\mathbf{2}}$ \\
\hline \hline 400 & 121757.5655 & 5.085496 & 0.011039 \\
\hline 400 & 48270.41041 & 4.683681 & 0.08806 \\
\hline 400 & 95224.78117 & 4.97875 & $2.82 \mathrm{E}-06$ \\
\hline 400 & 130768 & 5.116501 & 0.018516 \\
\hline 400 & 152224 & 5.182483 & 0.040826 \\
\hline 400 & 92614 & 4.966677 & 0.000189 \\
\hline 400 & 92332.36394 & 4.965354 & 0.000227 \\
\hline 400 & 73183.29648 & 4.864412 & 0.01346 \\
\hline 400 & 95611.26275 & 4.980509 & $6.37 \mathrm{E}-09$ \\
\hline \hline \multicolumn{2}{|c|}{} & 44.82386 & 0.172319 \\
\hline
\end{tabular}

The values for the band equations are presented below for the untreated SN-Curve:

\begin{tabular}{|c|c|}
\hline$\tilde{S}_{\log N}$ & 0.156898 \\
\hline$T_{N}$ & 2.61353 \\
\hline
\end{tabular}


The constants for the SN-Curve bands for both 5\% and 95\% survivability are calculated from the equations presented in Chapter 3.4. The results are given in the table below; percentages are the survivability rating (probability of survival):

Table 19 : Model Parameter Comparisons for UIT SN Curve

\begin{tabular}{|c|c||}
\hline \multicolumn{3}{|c|}{$\mathbf{9 0 \%}$ Survivability, $\boldsymbol{T}_{\boldsymbol{N}, \%}=\mathbf{1 . 2 8 2}$} \\
\hline $\mathfrak{C}_{90 \%}$ & $5.69526 \mathrm{E}+15$ \\
\hline$\sigma_{S N 1}, 90 \%$ & 5401.57 \\
\hline \hline \multicolumn{3}{|c|}{$\mathbf{1 0 \%}$ Survivability, $\boldsymbol{T}_{\boldsymbol{N}, \%}=\mathbf{1 . 2 8 2}$} \\
\hline $\mathfrak{C}_{10 \%}$ & $1.48798 \mathrm{E}+16$ \\
\hline$\sigma_{S N 1}, 10 \%$ & 6782.08 \\
\hline \hline \multicolumn{2}{|c|}{$\mathbf{9 9 . 9 9 \%}$ Survivability, $\boldsymbol{T}_{\boldsymbol{N}, \%}=\mathbf{5}$} \\
\hline $\mathfrak{C}_{99.99 \%}$ & $3.85033 \mathrm{E}+15$ \\
\hline$\sigma_{S N 1}, 99.99 \%$ & 4923.34104 \\
\hline
\end{tabular}

The fatigue strength exponents $(\mathcal{K}, b)$ are equivalent to the $50 \%$ survivability model (nonshifted). 


\section{Appendix D : UIT Optimal Settings Study}

This appendix sections will summarize the design of experiment to determine the optimal settings for optimal UIT coverage and increase in fatigue life. The data presented in this section is part of the journal paper titled "Experimental Optimization for Fatigue Life Maximization of Additively Manufactured Ti-6Al-4V Alloy Employing Ultrasonic Impact Treatment". This paper is still awaiting review as of the completion of this thesis. A matrix testing two major UIT settings is developed to include the static force and the scan speed. The combination which results in the best fatigue performance is used to treat the titanium samples.

A unidirectional stress-controlled fatigue test was conducted according to DIN 50100 [145]. Data scatter is assumed to fall within a logarithmic normal distribution. Table 20 displays the fatigue life of Ti-6Al-4V specimens treated at different parameters. The average fatigue life of each parameter is plotted in Figure 57. Evidently, samples treated at $30 \mathrm{~N}$ and $2000 \mathrm{~mm} / \mathrm{min}$ had the highest fatigue life. The next best treatment is for a static force of $30 \mathrm{~N}$ and a scanning speed of $1000 \mathrm{~mm} / \mathrm{min}$, showing a trend that if the scan speed is increased, so does the treatments efficacy of improving fatigue resistance. However, for $40 \mathrm{~N}$ and $2000 \mathrm{~mm} / \mathrm{min}$, the fatigue life improvement was seen to be much worse. This is probably due to overtreatment and parameters at $30 \mathrm{~N}$ and $2000 \mathrm{~mm} / \mathrm{min}$ are right before the effects of unwanted surface damage. The worst treatment however was the $20 \mathrm{~N}$ and $500 \mathrm{~mm} / \mathrm{min}$ scan speed. This makes sense as the treatment is less effective when the applied force is small and shear forces caused by the scan speed are at their lowest. 
Table 20 : Average fatigue life at different UIT parameters

\begin{tabular}{|c|c|c|c|}
\hline $\begin{array}{c}\text { Scanning Speed } \\
{[\mathbf{m m} / \mathbf{m i n}]}\end{array}$ & $\mathbf{2 0 0 0}$ & $\mathbf{1 0 0 0}$ & $\mathbf{5 0 0}$ \\
\hline Force [N] & \multicolumn{3}{|c|}{} \\
\hline \hline $\mathbf{4 0}$ & $5.22 \times 10^{4}$ & $7.78 \times 10^{4}$ & $5.82 \times 10^{4}$ \\
\hline $\mathbf{3 0}$ & $1.22 \times 10^{5}$ & $8.96 \times 10^{4}$ & $6.55 \times 10^{4}$ \\
\hline $\mathbf{2 0}$ & $1.11 \times 10^{5}$ & $6.91 \times 10^{4}$ & $4.59 \times 10^{4}$ \\
\hline
\end{tabular}

As shown in Figure 57, there is an increase in fatigue life between $20 \mathrm{~N}$ and $30 \mathrm{~N}$ followed by a dip at $40 \mathrm{~N}$. The reduction in improvement in fatigue life is due to over treatment and possible surface damage. The surface begins to develop cracks and the density of fatigue crack initiation sites increases. The increased probability of micro cracks which develop into macro-cracks during fatigue loading resulted in worsened performance. Furthermore, the extent of reduction in fatigue life between specimens treated under a static load of $30 \mathrm{~N}$ and $40 \mathrm{~N}$ is significant at higher scanning speeds and subtle at lower scanning speeds.

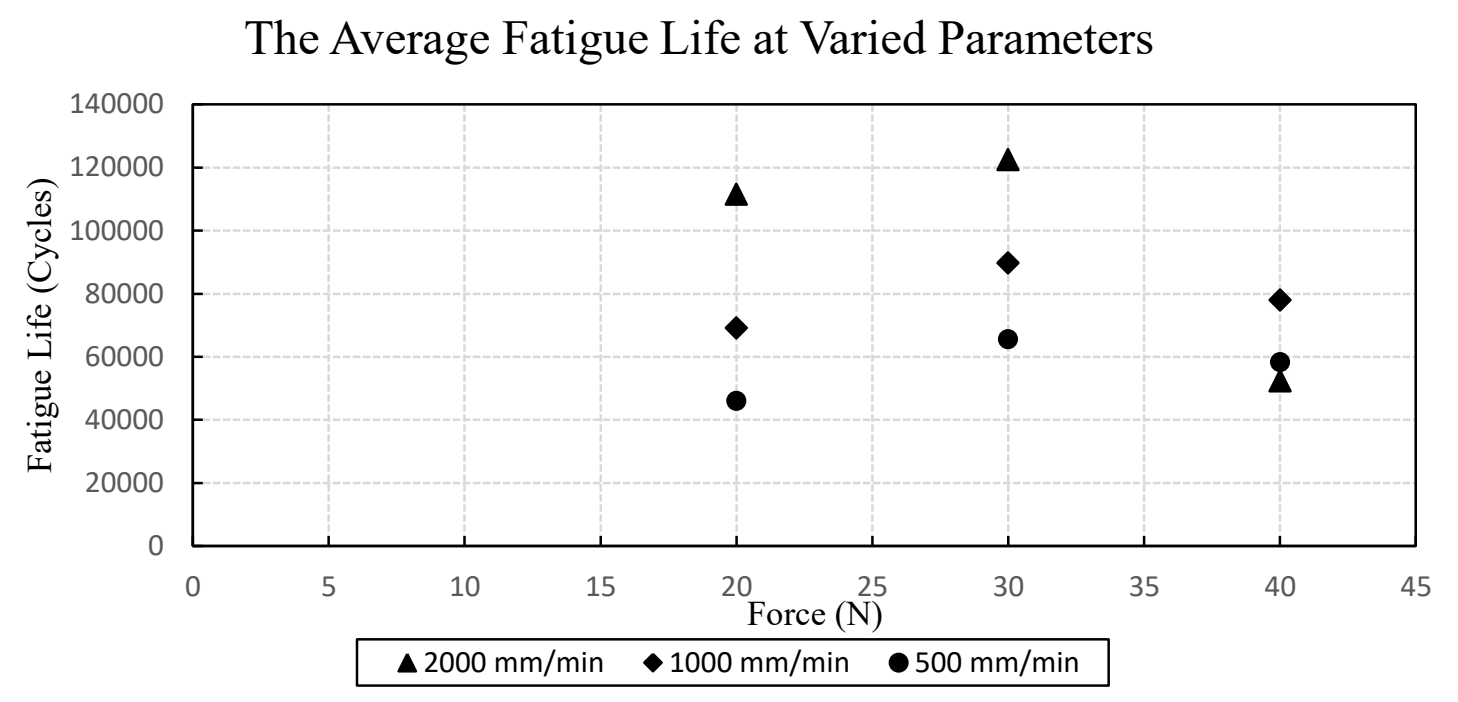

Figure 57 : Fatigue life at 400 MPa for different UIT Parameters 
To maximize the fatigue life of the titanium samples, a simple regression model of the observed improvements was created to locate a new possible maximum. The new maximum would then be a new testing point to validate the regression model. If the validation is proved to be successful and the new fatigue lives of the new parameters are shown to be significantly higher, then the new UIT parameters will be used to develop the optimized S-N curve.

The improvements in fatigue were characterized as the ratio of the observed logarithmic average of the treated to the untreated fatigue lives at each UIT parameter. The experimental data showed the fatigue life enhancement was strongly correlated with the average power input. The average power input is characterized by the product between the static force $(\mathrm{N})$ and scanning speed $(\mathrm{mm} / \mathrm{min})$. Therefore, the improvement ratio is to be estimated using the average power input. To determine the best regression model for generalizing the dataset, a set of high order polynomial functions were built, tested and compared to one another. The model with the least mean squared error is used to predict the maximum UIT parameters. High order regression models of up to 4 were considered to describe the data.

Comparison using cross-validation of the multiple polynomial fits was performed using a leaveone-out analysis scheme. Hence, for each model, an error metric is calculated based on building the model with $\mathrm{n}-1$ points, where $\mathrm{n}$ is the number of data points, and assessing the mean squared error between the model's prediction and the removed data point. The process is repeated for each data point. The average error for each model is used for comparison and model selection. An additional data point was added to the dataset to represent zero improvement when the static force is zero. The models were also generated so that the intercept would be zero. A total of 10 data points was used to develop the regression models. 
Table 21: Dataset and UIT Treatment Summary

\begin{tabular}{|l|c|c|c||c|c|c|c||}
\hline \multicolumn{7}{|c|}{ Dataset for Regression Model } \\
\hline \hline Treatment & $\begin{array}{c}\text { Force } \\
(\mathbf{N})\end{array}$ & $\begin{array}{c}\text { Speed } \\
(\mathbf{m m} / \mathbf{m i n})\end{array}$ & Improvement & Treatment & $\begin{array}{c}\text { Force } \\
(\mathbf{N})\end{array}$ & $\begin{array}{c}\text { Speed } \\
(\mathbf{m m} / \mathbf{m i n})\end{array}$ & Improvement \\
\hline \hline $20 / 500$ & 20 & 500 & 1.144 & $30 / 2000$ & 30 & 2000 & 3.049 \\
\hline $20 / 1000$ & 20 & 1000 & 1.852 & $40 / 500$ & 40 & 500 & 1.450 \\
\hline $20 / 2000$ & 20 & 2000 & 2.775 & $40 / 1000$ & 40 & 1000 & 1.952 \\
\hline $30 / 500$ & 30 & 500 & 1.632 & $40 / 2000$ & 40 & 2000 & 1.301 \\
\hline $30 / 1000$ & 30 & 1000 & 2.233 & Untreated & 0 & - & 0.000 \\
\hline
\end{tabular}

The selected model with the least error and the most evenly distributed residuals was the third order polynomial model. Training of the final model was performed using a leave-two-out cross validation method. Two points were randomly removed from the dataset without replacement. The final third order polynomial with the least error was then selected as the final model and is plotted as a dotted line in Figure 58.

Improvement Ratio (\%) vs. Static Force (N) x Scanning Speed (mm/min)

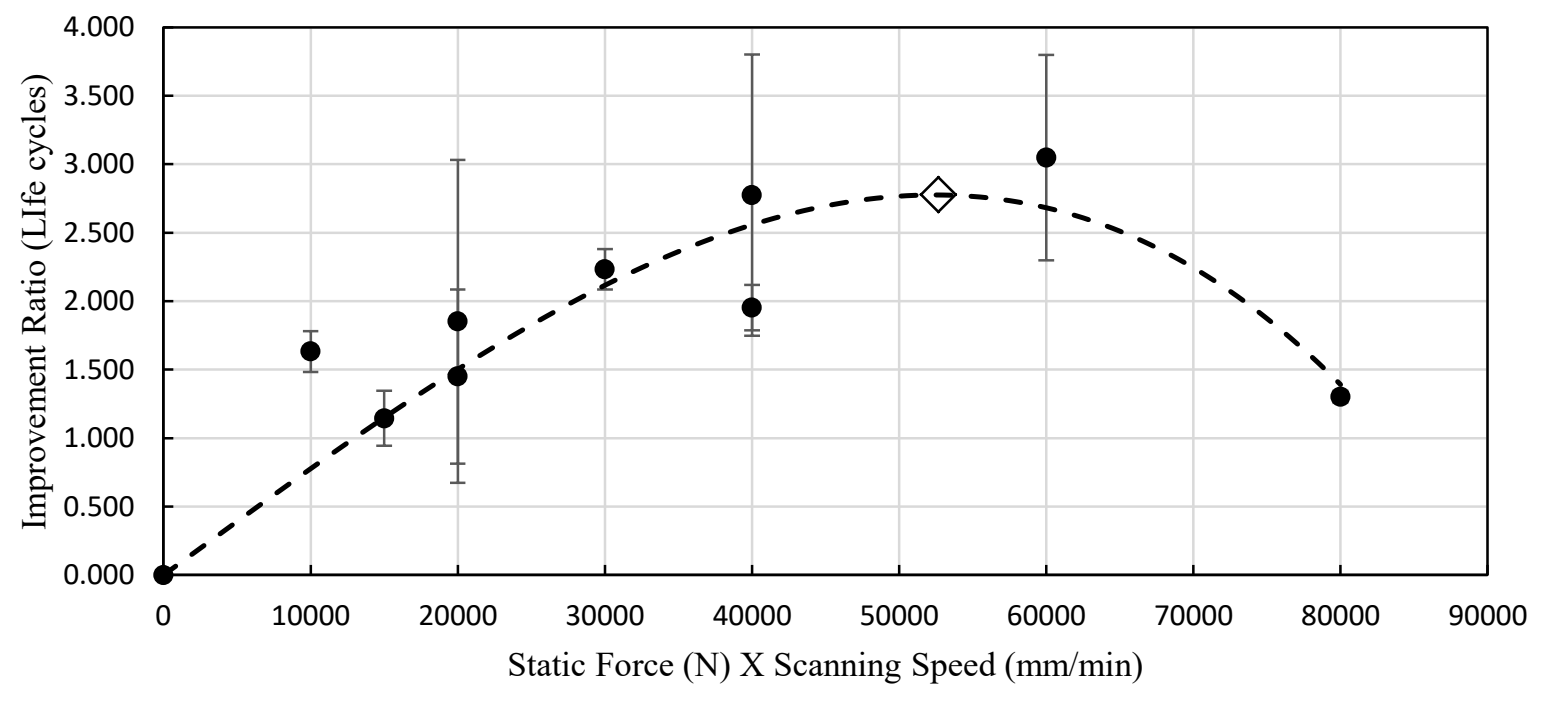

Figure 58: Improvement Ratio (\%) vs. Static force (N) times Scanning Speed (mm/min) 
By interpolating the third order polynomial, the maximum improvement in fatigue life can be found. The increase in fatigue life was seen to be minimal compared to the current solution. The static force would be $26.3 \mathrm{~N}$ using a scanning speed of $2000 \mathrm{~mm} / \mathrm{min}$. The expected improvement in fatigue would only improve by $3.27 \%$ when compared to the improvement ratio at $30 \mathrm{~N}$ and $2000 \mathrm{~mm} / \mathrm{min}$. Due to the small predicted increase, it was not deemed practical to validate the prediction because of the nature of scatter in fatigue results and uncertainties in the model. Hence, the UIT parameters for the optimize S-N curve was chosen to be the originally observed $30 \mathrm{~N}$ and $2000 \mathrm{~mm} / \mathrm{min}$ settings. 


\section{D.1 Experimental Data}

\begin{tabular}{|c|c|c|c|c|c|c|c|c|c|c||}
\hline $\begin{array}{c}\text { Force } \\
(\mathbf{N})\end{array}$ & $\begin{array}{c}\text { Speed } \\
(\mathbf{m m} / \\
\mathbf{m i n}\end{array}$ & $\boldsymbol{\varpi}$ & $\mathbf{W}_{\mathbf{0}}$ & $\begin{array}{c}\mathbf{T}_{\mathbf{o}} \\
(\mathbf{m m})\end{array}$ & $\begin{array}{c}\mathbf{W}_{\mathbf{2}} \\
(\mathbf{m m})\end{array}$ & $\begin{array}{c}\mathbf{T}_{\mathbf{2}} \\
(\mathbf{m m})\end{array}$ & $\begin{array}{c}\text { Area } \\
\left(\mathbf{m m}^{2}\right)\end{array}$ & $\begin{array}{c}\text { Mean } \\
\mathbf{L o a d}(\mathbf{N})\end{array}$ & $\begin{array}{c}\text { Amplitude } \\
(\mathbf{M P a})\end{array}$ & $\begin{array}{c}\text { Mean } \\
\text { Stress } \\
(\mathbf{M P a})\end{array}$ \\
\hline \hline 20 & 1000 & 48213 & 4.77 & 3.25 & 4.90 & 3.10 & 15.2 & 3038 & 400 & 200 \\
\hline 20 & 1000 & 46822 & 4.77 & 3.27 & 4.86 & 3.14 & 15.3 & 3052 & 400 & 200 \\
\hline 20 & 1000 & 147219 & 4.69 & 3.26 & 4.72 & 3.11 & 14.7 & 2940 & 400 & 200 \\
\hline 20 & 1000 & 92736 & 4.69 & 3.26 & 4.78 & 3.10 & 14.8 & 2958 & 400 & 200 \\
\hline 20 & 2000 & 73896 & 4.78 & 3.25 & 4.84 & 3.11 & 15.1 & 3010 & 400 & 200 \\
\hline 20 & 2000 & 156231 & 4.76 & 3.28 & 4.88 & 3.11 & 15.2 & 3036 & 400 & 200 \\
\hline 20 & 2000 & 120402 & 4.73 & 3.27 & 4.82 & 3.15 & 15.2 & 3036 & 400 & 200 \\
\hline 20 & 500 & 46033 & 4.81 & 3.28 & 4.90 & 3.11 & 15.2 & 3048 & 400 & 200 \\
\hline 30 & 1000 & 84825 & 4.74 & 3.27 & 4.86 & 3.13 & 15.2 & 3042 & 400 & 200 \\
\hline 30 & 1000 & 88517 & 4.69 & 3.26 & 4.74 & 3.11 & 14.7 & 2948 & 400 & 200 \\
\hline 30 & 1000 & 96431 & 4.73 & 3.27 & 4.88 & 3.12 & 15.2 & 3045 & 400 & 200 \\
\hline 30 & 2000 & 130768 & 4.76 & 3.27 & 4.82 & 3.11 & 15.0 & 2998 & 400 & 200 \\
\hline 30 & 2000 & 92614 & 4.73 & 3.28 & 4.80 & 3.15 & 15.1 & 3024 & 400 & 200 \\
\hline 30 & 2000 & 152224 & 4.76 & 3.24 & 4.92 & 3.10 & 15.3 & 3044 & 400 & 200 \\
\hline 30 & 500 & 61505 & 4.83 & 3.24 & 4.92 & 3.11 & 15.3 & 3060 & 400 & 200 \\
\hline 30 & 500 & 70042 & 4.75 & 3.22 & 4.87 & 3.11 & 15.1 & 3030 & 400 & 200 \\
\hline 40 & 1000 & 83389 & 4.74 & 3.25 & 4.87 & 3.11 & 15.1 & 3030 & 400 & 200 \\
\hline 40 & 1000 & 73920 & 4.74 & 3.29 & 4.81 & 3.16 & 15.2 & 3040 & 400 & 200 \\
\hline 40 & 2000 & 53644 & 4.73 & 3.26 & 4.86 & 3.11 & 15.1 & 3022 & 400 & 200 \\
\hline 40 & 2000 & 51068 & 4.78 & 3.25 & 4.86 & 3.12 & 15.2 & 3032 & 400 & 200 \\
\hline 40 & 500 & 35210 & 4.78 & 3.29 & 4.85 & 3.15 & 15.3 & 3056 & 400 & 200 \\
\hline 40 & 500 & 65397 & 4.76 & 3.27 & 4.85 & 3.12 & 15.1 & 3026 & 400 & 200 \\
\hline 40 & 500 & 86082 & 4.72 & 3.28 & 4.85 & 3.13 & 15.2 & 3036 & 400 & 200 \\
\hline
\end{tabular}




\section{Appendix E : Topology Optimization Sensitivity Analysis}

In structural optimization, there are two methods for calculating the gradients of the objective functions with respect to the design variables. One of these methods is called the direct method. The direct method will calculate the derivatives of the entire structural response as well as the intermediate quantities. The derivative calculates are obtained by the performance measures from the chain rule. The second method is the adjoint method which is dependent on an associated adjoint problem and a performance measure. The sensitivities obtained for the performance measure include the structural and adjoint responses.

Another important topic for sensitivity analysis is both the explicit and implicit solutions to some problems. Explicit solutions are derivatives that are expressed solely on the variables used to describe the function. An implicit solution is the partial differential solutions to the function expressed by variables not used to describe the function. Such examples of implicit solutions are the displacements of a structure with a design variable being the relative density of an element or the width of a bar. These implicit solutions lend themselves to the results of the equilibrium equation when the finite element method is used.

For some of the objective functions, the sensitivities can be calculated based on their explicit and implicit terms. If the structural response is called $\psi(U, \vec{\rho})$ and relies of the state variable $\mathrm{U}$ and the design variables $\rho$ then the sensitivity of the response can be found with [182]:

$$
\frac{d \psi}{d \rho}=\left.\frac{\partial \psi}{\partial \rho}\right|_{U=\text { const }}+\left(\left.\frac{\partial \psi}{\partial U}\right|_{\rho=\text { const }}\right)^{T} \frac{d U}{d \rho}
$$

Where the only unknown term is $\frac{d U}{d \rho}$ and can be found using the direct and adjoint methods. The majority of the time they can be found by differentiating the equilibrium equation for static 
analyses. For other types of analyses, the gradients will require a different approach (such as vibrations) to calculating the implicit sensitivities of the displacements.

\section{E.1 Adjoint Method for Sensitivity Analysis}

The adjoint method uses the performance measure coupled with the structural response to derive the gradients. The advantage of this method is the number of function evaluations is equal to the number of responses and not by the number of design variables. This makes certain responses such as stress more easily computed when compared to the direct method. The adjoint method will also calculate the sensitivities without explicitly calculating the sensitivity of the state variables. The adjoint method avoids the implicit calculations by forming a state equation or adjoint equation. The adjoint equation for a static analysis can be written as:

$$
K(\vec{\rho}) \lambda=\frac{\partial \psi}{\partial U}
$$

This comes from the direct sensitivity global equation where the solution to the inverse of the stiffness matrix and the derivative of the performance measure with respect to the displacements is equal to some vector.

$$
\lambda=\left(\left.\frac{\partial \psi}{\partial U}\right|_{\rho_{e}=\text { const }}\right)^{T} K(\vec{\rho})^{-1}
$$

where $\mathrm{K}$ is the global stiffness matrix, the adjoint load is defined as $\frac{\partial \psi}{\partial U}$ (the derivative of the cost function with respect to the displacements) and the adjoint solution is $\lambda$. This is relation is possible because of the symmetry in the global stiffness matrix [2]. It is important to note that the adjoint solution is not dependent on the design variables but by the performance measure. The adjoint load signifies another advantage for using the adjoint method for calculating sensitivities because the adjoint load only needs to be calculated once. For each design variable, the global 
stiffness matrix requires only one inversion in which it can then be used to calculate all the required adjoint solutions. When the adjoint solution is calculated then the sensitivity for the performance measure can be found with the following equation:

$$
\frac{d \psi}{d \rho}=\left.\frac{\partial \psi}{\partial \rho}\right|_{U=\text { const }}+\lambda^{T}\left(\frac{d F}{d \rho}-\frac{d K}{d \rho} U\right)
$$

The equation above is called the adjoint sensitivity global equation. This formulation is more closely related to the performance measure. It is useful to note that the derivatives of the displacements with respect to the design variables do not need to be computed for this method because of the substitution of $\lambda$ into the direct sensitivity global equation.

\section{E.2 Stress Based Structural Responses}

The stress can be computed using Hooke's law where the elasticity matrix is multiplied by the strain.

$$
\vec{\sigma}=D \varepsilon
$$

Where $\vec{\sigma}$ is the vector of principle stresses, D is the constitutive material stiffness matrix and $\varepsilon$ is the strain vector.

In finite element software, for solid elements, the strain is calculated using a strain -displacement matrix which converts the displacements into a strain. The stresses are then expressed as:

$$
\overrightarrow{\sigma_{e}}=D_{e}\left(\rho_{e}\right) B_{e} U_{e}
$$

Where $\vec{\sigma}_{e}$ is the vector of principle stresses for element e, $\mathrm{D}_{\mathrm{e}}$ is the constitutive material stiffness matrix or element e and $\varepsilon_{e}$ is the strain vector for element e. The constitutive matrix is typically a function of the relative density in SIMP topology optimization problems. Another fact to note is that the state equation for the stress is the equilibrium equation. 


\section{E.2.1 Stress Sensitivities from the Adjoint Method}

The adjoint method requires the adjoint sensitivity global equation shown below:

$$
\frac{\overrightarrow{d \sigma_{e}}}{d \rho_{e}}=\left.\frac{\partial \sigma_{e}}{\partial \rho_{e}}\right|_{U=\text { const }}+\lambda^{T}\left(\frac{d F}{d \rho}-\frac{d K}{d \rho} U\right)
$$

From the direct sensitivity analysis we already know that $\frac{d F}{d \rho}$ is equal to zero and that:

$$
\left.\frac{\partial \sigma}{\partial \rho_{e}}\right|_{U=c o n s t}=\frac{\partial D_{e}}{\partial p_{e}} B_{e} U_{e}
$$

The adjoint vector can be found from:

$$
\lambda=\frac{\partial \sigma}{\partial U} K^{-1}
$$

Where $\frac{\partial \sigma}{\partial U}$ can be calculated from:

$$
\frac{\partial \sigma}{\partial U}=D_{e} B_{e}
$$

The final sensitivities for the adjoint method becomes:

$$
\frac{\overrightarrow{d \sigma_{e}}}{d \rho_{e}}=\frac{\partial D_{e}}{\partial \rho_{e}} B_{e} U_{e}-\lambda^{T}\left(\frac{d K}{d \rho_{e}} U\right)
$$

\section{E.2.2 Considerations and Numerical Problems for Stress Responses}

Stress constrained optimization problems are known to have vanishing constraints. This means that an optimal solution to the problem may require one design variable to be gone completely. In other words, the optimal solutions may lie in a lower dimension space. This phenomenon can be seen in a 3 bar truss problem where the optimal solution is found when the center bar is removed [183]. Similar problems also appear in topology optimization as when the relative 
density is zero, so is the stress measurement. Another factor to consider for SIMP like topology optimizations is that stress is not related to the material properties and alternative methods need to be used when developing meaningful stress measurements for structural responses.

Many solutions have been proposed for the numerical instabilities of stress constraints. One solution is to treat the elements at intermediate densities as a rank-2 layered composite. This allows for the microscopic stresses to be meaningful throughout the optimization procedure [183]. This can be achieved by making the microscopic stresses inversely proportional to the relative density and having the constitutive matrix a function of both the relative density and by an exponent $q$. The new formulation will modify the measurement of the stress components to be:

$$
\overrightarrow{\sigma_{e}}=\frac{\sigma_{e}\left(\rho_{e}\right)}{\rho_{e}^{q}}
$$

where the exponent $q$ is chosen so that the stress conditions imposed by a rank-2 can be met. For a rank-2 composite condition to be satisfied, the stress must converge to a finite non-zero stress as the relative density goes to zero. In a SIMP optimization scheme, this condition however, can only be fulfilled if the exponent $q$ is equal to the penalty factor. The stress at the microscopic level in a SIMP Optimization is calculated as:

$$
\overrightarrow{\sigma_{e}}=\left.D_{e}\right|_{\rho_{e}=1} \varepsilon_{e}
$$

where $\left.D_{e}\right|_{\rho_{e}=1}$ is the constitutive matrix at full density (relative density is equal to one) and $\varepsilon_{e}$ is the strain of the element under the influence of the design variables.

The proposed methodology for the stress function however does have a drawback. The stress will converge to a finite value when the relative density goes to zero; this may violate any stress constraint if the stress function is applied as a constraint. In addition, this effect causes 
discontinuities in the stress function. To fix this, the stress will need to converge to zero as the relative density reaches zero. To do this the exponent q must be smaller than the SIMP penalty factor. This is equivalent to the " $q_{p}$ " method proposed by Duynix and Bendose [174]. The qp method expects a relation between $\mathrm{q}$ and the SIMP penalty factor $\mathrm{p}$ such that $q_{p}=p-q$. The modified formulation is given below as:

$$
\overrightarrow{\sigma_{e}}=\rho_{e}^{q_{p}} D_{e} \varepsilon_{e}
$$

where $p$ is the stiffness penalization factor for SIMP topology optimizations and $q$ is the stress penalization variable. This formulation for the stresses ensures that the stress at zero density is zero and that the stress is inversely proportional to the relative density. This formulation was shown to work well by Bruggi as it avoids stress singularities [161]. For most applications, $q_{p}$ is equal to 0.5 [184] and generally acts as a stress penalization term. This method ensures that the penalizing of stress is not equal to the penalization of stiffness which would lead to an all void design [185].

The sensitivity analysis for stress penalization is formulated using the adjoint method. The partial derivative of the penalized stress term with respect to the design variable (relative density) is found to be:

$$
\left.\frac{\partial \overrightarrow{\sigma_{e}}}{\partial \rho_{e}}\right|^{q_{p}}=\left(q_{p}-1\right) \rho_{e}{ }^{q_{p}-1} D_{e} B_{e} U_{e}
$$

The partial derivative for the penalized stress with respect to the displacements is calculated as:

$$
\left.\frac{\partial \overrightarrow{\sigma_{e}}}{\partial U}\right|^{q_{p}}=\rho_{e}^{q_{p}} D_{e} B_{e}
$$

The adjoint vector is found from solving the system of equations: 


$$
\lambda=\left.\frac{\partial \overrightarrow{\sigma_{e}}}{\partial U}\right|^{q_{p}} K^{-1}
$$

The final sensitivity as derived by the adjoint method is:

$$
\left.\frac{d \overrightarrow{\sigma_{e}}}{d \rho_{e}}\right)^{q_{p}}=\left(q_{p}-1\right) \rho_{e} q_{p}^{-1} D_{e} B_{e} U_{e}+\lambda_{e}^{T}\left(\frac{\partial K}{\partial \rho_{e}} U\right)
$$

The stress penalization by the $q_{p}$ method applied in this manuscript will multiply the stress term by a correction function. This correction function aims to recalculate the stresses so that its value fits that of the penalization term. The equation below explains the modifications for stress penalization:

$$
\left.\overrightarrow{\sigma_{e}}\right\rangle^{q_{p}}=\rho_{e}^{q_{p}} D_{e} B_{e} U_{e}=f_{c}\left(\rho_{e}\right) D_{e} B_{e} U_{e}
$$

The correction function depends on the type of interpolation used for the optimization, these are given below as:

$$
\left.f_{c}\left(\rho_{e}\right)\right\rangle_{S I M P}=\rho_{e}^{q-p}
$$

The new sensitivities for the stress by the adjoint method will then take the form of:

$$
\left.\frac{\overrightarrow{d \sigma_{e}}}{d \rho_{e}}\right)^{q_{p}}=\left(f_{c} \frac{\partial D_{e}}{\partial \rho_{e}}+\frac{\partial f_{c}}{\partial \rho_{e}} D_{e}\right) B_{e} U_{e}-\lambda_{e}^{T}\left(\frac{d K_{e}}{d \rho_{e}} U_{e}\right)
$$

The adjoint vector is solved using the system below:

$$
\lambda=\frac{\partial \sigma\rangle^{q_{p}}}{\partial U} K^{-1}
$$

Where the derivative of the penalized stress with respect to the displacements becomes:

$$
\frac{\partial \sigma\rangle^{q_{p}}}{\partial U}=f_{c}\left(\rho_{e}\right) D_{e} B_{e}
$$

The partial derivatives for the correction function with respect to the design variable (relative density) are given below for each interpolation method: 


$$
\left.\frac{\partial f_{c}}{\partial \rho_{e}}\right\rangle_{S I M P}=(q-p) \rho_{e}^{q-p-1}
$$

\section{E.2.3 Von Mises Stress}

For a general three dimensional case, the von-Mises stress can be calculated with the following equation:

$$
\sigma_{V M}=\sqrt{\frac{\left[\left(\sigma_{11}-\sigma_{22}\right)^{2}+\left(\sigma_{22}-\sigma_{33}\right)^{2}+\left(\sigma_{33}-\sigma_{11}\right)^{2}+6\left(\sigma_{12}^{2}+\sigma_{23}^{2}+\sigma_{31}^{2}\right)\right]}{2}}
$$

Where $\sigma_{i j}$ are the principle and shear stresses.

To find the corresponding sensitivities for a von-Mises criterion of an element e, the chain rule must be applied.

$$
\frac{d \sigma_{V M_{e}}}{d \rho_{e}}=\frac{\partial \sigma_{V M_{e}}}{\partial \sigma_{e}} \frac{\partial \sigma_{e}}{\partial \rho_{e}}
$$

The sensitivities for each of the von-Mises sum stresses are shown below:

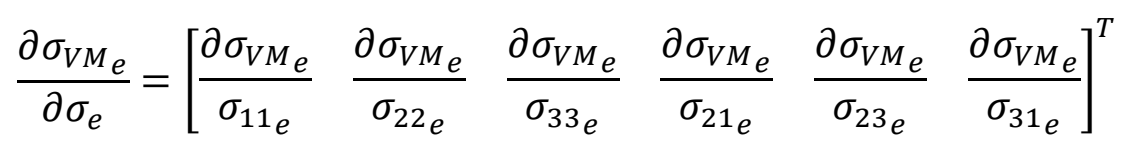

The individual components are derived to be:

$$
\begin{aligned}
& \frac{\partial \sigma_{V M_{e}}}{\sigma_{11_{e}}}=\frac{1}{2 \sigma_{V M_{e}}} \frac{4 \sigma_{11_{e}}-2 \sigma_{22_{e}}-2 \sigma_{33_{e}}}{2} \\
& \frac{\partial \sigma_{V M_{e}}}{\sigma_{22}}=\frac{1}{2 \sigma_{V M_{e}}} \frac{4 \sigma_{22_{e}}-2 \sigma_{11_{e}}-2 \sigma_{33_{e}}}{2} \\
& \frac{\partial \sigma_{V M_{e}}}{\sigma_{33_{e}}}= \frac{1}{2 \sigma_{V M_{e}}} \frac{4 \sigma_{33_{e}}-2 \sigma_{11_{e}}-2 \sigma_{22_{e}}}{2} \\
& \frac{\partial \sigma_{V M_{e}}}{\sigma_{21_{e}}}=\frac{1}{2 \sigma_{V M_{e}}} 6 \sigma_{21_{e}}
\end{aligned}
$$




$$
\begin{aligned}
& \frac{\partial \sigma_{V M_{e}}}{\sigma_{23_{e}}}=\frac{1}{2 \sigma_{V M_{e}}} 6 \sigma_{23} \\
& \frac{\partial \sigma_{V M_{e}}}{\sigma_{31_{e}}}=\frac{1}{2 \sigma_{V M_{e}}} 6 \sigma_{31_{e}}
\end{aligned}
$$

The derivative of the stress with respect to the design variable can be found in the stress gradient

derivations in appendix E.2. The derivative with respect to the displacements is found by using the chain rule on the von-Mises definition. The last term are the stress components with respect to displacement and are derived in appendix E.2.

\section{E.3 Fatigue Based Structural Responses}

Fatigue can be represented by either the total cumulative damage or cycles to failure. For StressLife prediction methods, these are both calculated by Palmgren Miner's cumulative damage hypotheses. The number of life cycles can be calculated from the inverse of the damage formulation.

$$
\varpi=1 / H=1 /\left[\sum_{i=1}^{k} \frac{n_{i}}{\ell\left(\sigma_{e q_{i}}\right)}\right]=\left(\sum_{i=1}^{k} \frac{n_{i}}{\ell\left(\sigma_{e q_{i}}\right)}\right)^{-1}
$$

where $H$ is the total damage and is shown in the equation below:

$$
H=\sum_{i=1}^{k} \frac{n_{i}}{\varpi_{i}}
$$

The total damage is re-written as a function of the equivalent stresses. The number of cycles to failure for a particular load $\left(\varpi_{i}\right)$ is represented by a function $\ell\left(\sigma_{e q}\right)$ where $\sigma_{e q}$ is the equivalent stress. 


$$
H=\sum_{i=1}^{k} \frac{n_{i}}{\ell\left(\sigma_{e q_{i}}\right)}
$$

The equivalent stresses themselves are a function of the mean and amplitudes stresses which are a function of the initial element (reference) stresses. The high cycle fatigue regime assumes elastic deformations; the mean and amplitude stresses are calculated using the load scalars from the loading history. This is seen in the next equations below:

$$
\begin{aligned}
& \overrightarrow{\sigma_{m}}=c_{m} \vec{\sigma} \\
& \overrightarrow{\sigma_{a}}=c_{a} \vec{\sigma}
\end{aligned}
$$

Where $\vec{\sigma}$ is the reference stress from a static solution and $c_{m}$ and $c_{a}$ are calculated from the mean and load amplitudes from the time history by a rainflow cycle counting algorithm.

The reference stresses are calculated by either computing the von-Mises, signed von-Mises stress or by the Sines method. The equivalent stress for the Sines method is calculated with:

$$
\sigma_{\text {eq }}=c_{a} \sigma_{v m}+c_{m} \frac{1}{\sqrt{2}} \beta\left(\sigma_{11}+\sigma_{22}+\sigma_{33}\right)
$$

The equivalent stresses can also be calculated from a mean stress correction formula such as the modified Goodman line or Gerber curve. However, the mean stresses must be less than the ultimate or yield strengths. In the event of mean stresses which are greater than the failure criteria, a step response function is substituted for the mean stresses to avoid negative equivalent stresses. The step response formulation is given below:

$$
S \sigma_{m}=\left(\sigma_{y}-\mathfrak{s}\right)\left(1-e^{\left(-\frac{\mathcal{B} S \sigma_{m}}{\sigma_{y}}\right)}\right), \quad \text { if } S \sigma_{m}>r \sigma_{y}
$$

where, $\sigma_{y}$ is the yield strength of the material, $\mathfrak{s}>0$ is a small value $\approx 1 e^{-16}$ to avoid numerical instabilities and the variable $\mathcal{B}$ is equal to the equation below: 


$$
\mathcal{B}=-\log (1-r) / r, \quad 0<r<1
$$

where $r$ is the transition ratio between the linear section of the mean stresses and the beginning of the step function. To avoid negative values when large safety factors are used, the yield is multiplied by the safety factor. The equations for the mean stress corrections then take on a new form:

$$
\begin{aligned}
& \text { New M. Goodman }\left\{\tau_{M G}^{*}(\vec{\sigma})\right\}: \sigma_{\text {rev }}= \begin{cases}\frac{S \sigma_{a} \sigma_{u t}}{\sigma_{u t}-S \sigma_{m}}, & \text { if } S \sigma_{m} \leq r \sigma_{u t} \\
\frac{S \sigma_{a}}{\sigma_{u t}-\left(\sigma_{u t}-\mathfrak{s}\right)\left(1-e^{\left(-\frac{\mathcal{B S} \sigma_{m}}{\sigma_{u t}}\right)}\right)}, & \text { if } S \sigma_{m}>r \sigma_{u t}\end{cases} \\
& \text { New Soderberg }\left\{\tau_{S}^{*}(\vec{\sigma})\right\}: \sigma_{\text {rev }}= \begin{cases}\frac{S \sigma_{a} \sigma_{y}}{\sigma_{y}-S \sigma_{m}} & \text { if } S \sigma_{m} \leq r \sigma_{y} \\
\frac{S \sigma_{a}}{\sigma_{y}-\left(\sigma_{y}-\mathfrak{s}\right)\left(1-e^{\left(-\frac{B S \sigma_{m}}{\sigma_{y}}\right)}\right)}, \text { if } S \sigma_{m}>r \sigma_{y}\end{cases}
\end{aligned}
$$

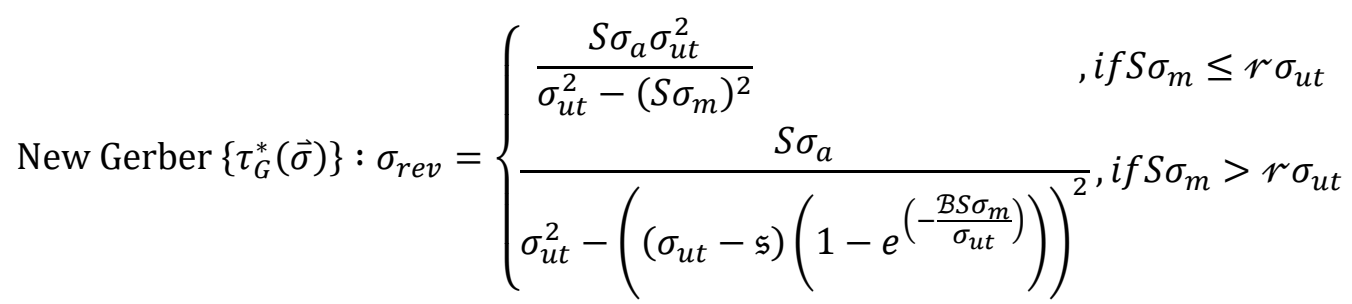

$$
\begin{aligned}
& \text { New ASME Elliptic }\left\{\tau_{E}^{*}(\vec{\sigma})\right\}: \sigma_{\text {rev }}= \begin{cases}S \sigma_{a} \sigma_{y} \sqrt{\frac{1}{\left(\sigma_{m} S\right)^{2}+\sigma_{y}^{2}}} & , \text { if } S \sigma_{m} \leq r \sigma_{y} \\
S \sigma_{a} \sqrt{\frac{1}{\left(\left(\sigma_{y}-\mathfrak{s}\right)\left(1-e^{\left(-\frac{\mathcal{B S} \sigma_{m}}{\sigma_{y}}\right)}\right)\right)^{2}+\sigma_{y}^{2}}}, \text { ifS } \sigma_{m}>r \sigma_{y}\end{cases}
\end{aligned}
$$

\section{E.3.1 Partial Derivatives}

The individual partial derivatives for each of the components are calculated as follows, first is the partial derivative of the damage equation with respect to the $\mathrm{SN}$ interpolation function. 


$$
\frac{\partial H}{\partial \ell}=-\frac{n_{i}}{\ell^{2}}
$$

The partial derivative of the SN interpolation function will depend on the interpolation method used. When the Basquin equation is adopted, the partial derivative of the interpolation function with respect to the mean stress correction function is:

$$
\frac{\partial \ell}{\partial \tau}=\left(\frac{1}{b \sigma_{S N 1}}\right)\left(\frac{\tau\left(\overrightarrow{\sigma_{l}}\right)}{\sigma_{S N 1}}\right)^{\frac{1}{b}-1}
$$

The third and fourth partial derivative component for the equivalent stresses is also case dependent. For the case of the Sines method; the partial derivative of the Sines method with respect to the mean and amplitude stress components is:

$$
\begin{aligned}
\tau_{\text {sines }}\left(\overrightarrow{\sigma_{l}}\right) & =\sigma_{a_{i}}+\sigma_{m_{i}} \\
\frac{\partial \tau_{\text {sines }}}{\partial \sigma_{i}} & =\left[\begin{array}{ll}
1 & 1
\end{array}\right]
\end{aligned}
$$

For the other mean stress correction functions, the sensitivities with respect to the equivalent means and amplitudes are:

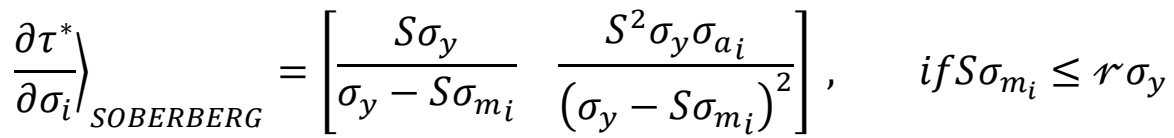

$$
\begin{aligned}
& \left.\frac{\partial \tau^{*}}{\partial \sigma_{i}}\right\rangle_{G E R B E R}=\left[\frac{S \sigma_{u t}^{2}}{\sigma_{u t}^{2}+\sigma_{m_{i}}^{2} S^{2}} \quad 2 \frac{S^{3} \sigma_{u t}^{2} \sigma_{a_{i}} \sigma_{m_{i}}}{\left(\sigma_{u t}^{2}+\sigma_{m_{i}}^{2} S^{2}\right)^{2}}\right], \quad \text { if } S \sigma_{m_{i}} \leq r \sigma_{u t} \\
& \left.\frac{\partial \tau^{*}}{\partial \sigma_{i}}\right\rangle_{\text {ELLIPTICAL }}=\left[\frac{S \sigma_{y}}{\sqrt{\left(\sigma_{y}^{2}+\sigma_{m_{i}}^{2} S^{2}\right)}} \frac{S^{3} \sigma_{y} \sigma_{a_{i}} \sigma_{m_{i}}}{\sqrt{\left(\sigma_{y}^{2}+\sigma_{m_{i}}^{2} S^{2}\right)^{3}}}\right], \quad \text { if } S \sigma_{m_{i}} \leq r \sigma_{y} \\
& \left.\frac{\partial \tau^{*}}{\partial \sigma_{i}}\right\rangle_{M G O O D M A N}=\left[\frac{S \sigma_{u t}}{\sigma_{u t}-S \sigma_{m_{i}}} \quad \frac{S^{2} \sigma_{u t} \sigma_{a_{i}}}{\left(\sigma_{u t}-S \sigma_{m_{i}}\right)^{2}}\right], \quad \text { if } S \sigma_{m_{i}} \leq r \sigma_{u t}
\end{aligned}
$$


When the mean stresses are larger than the ultimate or yield strengths of the material, the mean stress correction lines partial derivatives take the form of:

$$
\begin{aligned}
& \left.\frac{\partial \tau^{*}}{\partial \sigma_{i}}\right)_{\text {MGOODMAN }}=\left[\frac{S \sigma_{u t}}{\mathfrak{s}+e^{\left(-\frac{\mathcal{B S} \sigma_{m_{i}}}{\sigma_{u t}}\right)}\left(\sigma_{u t}-\mathfrak{s}\right)} \frac{S^{2} \sigma_{a_{i}} \mathcal{B} e^{\left(-\frac{\mathcal{B} S \sigma_{m_{i}}}{\sigma_{u t}}\right)}\left(\sigma_{u t}-\mathfrak{s}\right)}{\left(\sigma_{u t}+\left(e^{\left(-\frac{\mathcal{B} S \sigma_{m_{i}}}{\sigma_{u t}}\right)}-1\right)\left(\sigma_{u t}-\mathfrak{s}\right)\right)^{2}}\right], \text { if } S \sigma_{m_{i}}>r \sigma_{u t} \\
& \left.\frac{\partial \tau^{*}}{\partial \sigma_{i}}\right|_{\text {SOBERBERG }}=\left[\frac{S \sigma_{y}}{\mathfrak{s}+e^{\left(-\frac{\mathcal{B S} \sigma_{m_{i}}}{\sigma_{y}}\right)}\left(\sigma_{y}-\mathfrak{s}\right)} \frac{S^{2} \sigma_{a_{i}} \mathcal{B} e^{\left(-\frac{\mathcal{B} S \sigma_{m_{i}}}{\sigma_{y}}\right)}\left(\sigma_{y}-\mathfrak{s}\right)}{\left(\sigma_{y}+\left(e^{\left(-\frac{\mathcal{B} S \sigma_{m_{i}}}{\sigma_{y}}\right)}-1\right)\left(\sigma_{y}-\mathfrak{s}\right)\right)^{2}}\right], \text { if } S \sigma_{m_{i}}>r \sigma_{y} \\
& \left.\frac{\partial \tau^{*}}{\partial \sigma_{i}}\right)_{G E R B E R}=\left[\frac{-S \sigma_{u t}^{2}}{\left(e^{\left(-\frac{B S \sigma_{m_{t}}}{\sigma_{u t}}\right)}-1\right)^{2}\left(\sigma_{u t}-\mathfrak{s}\right)^{2}-\sigma_{u t}^{2}} \frac{2 \sigma_{u t} S^{2} \mathcal{B} \sigma_{a_{i}} \sigma_{m_{i}} e^{\left(-\frac{B \sigma_{m_{i t}}}{\sigma_{u t}}\right)}\left(e^{\left(-\frac{B S \sigma_{m_{m}}}{\sigma_{u t}}\right)}-1\right)\left(\sigma_{u t}-\mathfrak{s}\right)^{2}}{-\left(\left(e^{\left(-\frac{B S \sigma_{m_{i}}}{\sigma_{u t}}\right)}-1\right)^{2}\left(\sigma_{u t}-\mathfrak{s}\right)^{2}-\sigma_{u t}^{2}\right)^{2}}\right], i f S \sigma_{m_{i}}>r \sigma_{u t} \\
& \left.\frac{\partial \tau^{*}}{\partial \sigma_{i}}\right\rangle_{\text {ELIPTICAL }}=\left[\sqrt{\sqrt{\left(e^{\left(-\frac{B S \sigma_{m_{i}}}{\sigma_{y}}\right)}-1\right)^{2}\left(\sigma_{y}-\mathfrak{s}\right)^{2}+\sigma_{y}^{2}}} \frac{S^{2} \sigma_{a i} B e^{\left(-\frac{B S \sigma_{m_{i}}}{\sigma_{y}}\right)}\left(e^{\left(-\frac{B S \sigma_{m_{i}}}{\sigma_{y}}\right)}-1\right)\left(\sigma_{y}-\mathfrak{s}\right)^{2}}{\left.\left(e^{\left(-\frac{B S \sigma_{m_{i}}}{\sigma_{y}}\right)}-1\right)^{2}\left(\sigma_{y}-\mathfrak{s}\right)^{2}+\sigma_{y}^{2}\right)^{3 / 2}}\right], i f S \sigma_{m_{i}}>r \sigma_{y}
\end{aligned}
$$

The partial derivatives of the von-Mises stresses with respect to the principal stress components are calculated in appendix E.2.3. The derivative of the equivalent stress components for the Sines method with respect to the stress components is:

$$
\begin{gathered}
\frac{\partial \sigma_{i}}{\partial \sigma}=\left[\begin{array}{c}
\frac{\partial \sigma_{a_{i}}}{\partial \sigma} \\
\frac{\partial \sigma_{m_{i}}}{\partial \sigma}
\end{array}\right] \\
\frac{\partial \sigma_{i}}{\partial \sigma}=\left[\begin{array}{lllllll}
c_{a_{i}} \frac{\partial \sigma_{V M}}{\sigma_{11}} & c_{a_{i}} \frac{\partial \sigma_{V M}}{\sigma_{22}} & c_{a_{i}} \frac{\partial \sigma_{V M}}{\sigma_{33}} & c_{a_{i}} \frac{\partial \sigma_{V M}}{\sigma_{12}} & c_{a_{i}} \frac{\partial \sigma_{V M}}{\sigma_{23}} & c_{a_{i}} \frac{\partial \sigma_{V M}}{\sigma_{31}} \\
c_{m_{i}} \frac{1}{\sqrt{2}} \beta & c_{m_{i}} \frac{1}{\sqrt{2}} \beta & c_{m_{i}} \frac{1}{\sqrt{2}} \beta & 0 & 0 & 0
\end{array}\right]
\end{gathered}
$$


If the sum of the signed von-Mises stresses is used, then the partial derivatives of the equivalent stress components with respect to the signed von-Mises equation is:

$$
\frac{\partial \sigma_{i}}{\partial \sigma}=\left[\begin{array}{cccccc}
c_{a_{i}} \frac{\partial \sigma_{V M}}{\sigma_{11}} & c_{a_{i}} \frac{\partial \sigma_{V M}}{\sigma_{22}} & c_{a_{i}} \frac{\partial \sigma_{V M}}{\sigma_{33}} & c_{a_{i}} \frac{\partial \sigma_{V M}}{\sigma_{12}} & c_{a_{i}} \frac{\partial \sigma_{V M}}{\sigma_{23}} & c_{a_{i}} \frac{\partial \sigma_{V M}}{\sigma_{31}} \\
\zeta_{i} c_{m_{i}} \frac{\partial \sigma_{V M}}{\sigma_{11}} & \zeta_{i} c_{m_{i}} \frac{\partial \sigma_{V M}}{\sigma_{22}} & \zeta_{i} c_{m_{i}} \frac{\partial \sigma_{V M}}{\sigma_{33}} & \zeta_{i} c_{m_{i}} \frac{\partial \sigma_{V M}}{\sigma_{12}} & \zeta_{i} c_{m_{i}} \frac{\partial \sigma_{V M}}{\sigma_{23}} & \zeta_{i} c_{m_{i}} \frac{\partial \sigma_{V M}}{\sigma_{31}}
\end{array}\right]
$$

where $\zeta_{i}$ is the sign of the hydrostatic stress for load $\mathrm{i}$.

The remaining derivatives of the stress components with respect to the design variables and the displacements are calculated in appendix E.2.

\section{E.3.2 Damage Sensitivity from the Adjoint Method}

The sensitivity for cumulative damage by the adjoint method uses the adjoint sensitivity global equation shown below:

$$
\frac{\partial H}{d \rho}=\left.\frac{\partial H}{\partial \rho}\right|_{U=\text { const }}+\lambda^{T}\left(\frac{d F}{d \rho}-\frac{d K}{d \rho} U\right)
$$

The direct sensitivity analysis assumed that $\frac{d F}{d \rho}$ is equal to zero. The adjoint vector $\lambda$ is found from:

$$
\begin{gathered}
\lambda=\frac{\partial H}{\partial U} K^{-1} \\
\lambda=\sum_{i=1}^{k}\left[\frac{\partial H}{\partial \ell} \frac{\partial \ell}{\partial \tau} \frac{\partial \tau}{\partial \sigma_{i}} \frac{\partial \sigma_{i}}{\partial \sigma} \frac{\partial \sigma}{\partial U}\right] K^{-1}
\end{gathered}
$$

The adjoint sensitivities are thus;

$$
\frac{d H}{d \rho}=\sum_{i=1}^{k}\left[\frac{\partial H}{\partial \ell} \frac{\partial \ell}{\partial \tau} \frac{\partial \tau}{\partial \sigma_{i}} \frac{\partial \sigma_{i}}{\partial \sigma} \frac{\partial \sigma}{\partial \rho}\right]-\lambda^{T}\left(\frac{d K}{d \rho} U\right)
$$

where the displacement vector $U$ is obtained from the reference load. 
From an element basis, the individual derivatives can be written as:

$$
\frac{d H_{e}}{d \rho_{e}}=\sum_{i=1}^{k}\left[\frac{\partial H_{e}}{\partial \ell} \frac{\partial \ell}{\partial \tau} \frac{\partial \tau}{\partial \sigma_{e_{i}}} \frac{\partial \sigma_{e_{i}}}{\partial \sigma_{e}} \frac{\partial \sigma_{e}}{\partial \rho_{e}}\right]-\lambda_{e}{ }^{T}\left[\frac{d K_{e}}{d \rho_{e}} U_{e}\right]
$$

\section{E.3.3 Modifications for Life Sensitivity}

The sensitivity for the number of life cycles can be determined using the same derivations used for the cumulative damage. The equation for life is:

$$
\varpi=\left(\sum_{i=1}^{k} \frac{n_{i}}{\ell\left(\sigma_{e q_{i}}\right)}\right)^{-1}
$$

The direct and adjoint sensitivity analysis require partial derivatives of the structural response with respect to the design variable and the displacements. These partial derivatives are shown in equation $\mathrm{X}$ and $\mathrm{X}$ :

$$
\begin{aligned}
& \frac{\partial \varpi}{\partial \rho}=-\frac{1}{H^{2}} \sum_{i=1}^{k}\left[\frac{\partial H}{\partial \ell} \frac{\partial \ell}{\partial \tau} \frac{\partial \tau}{\partial \sigma_{i}} \frac{\partial \sigma_{i}}{\partial \sigma} \frac{\partial \sigma}{\partial \rho}\right] \\
& \frac{\partial \varpi}{\partial U}=-\frac{1}{H^{2}} \sum_{i=1}^{k}\left[\frac{\partial H}{\partial \ell} \frac{\partial \ell}{\partial \tau} \frac{\partial \tau}{\partial \sigma_{i}} \frac{\partial \sigma_{i}}{\partial \sigma} \frac{\partial \sigma}{\partial U}\right]
\end{aligned}
$$

On an element basis, the total derivatives for the direct method is:

$$
\frac{d \varpi_{e}}{d \rho_{e}}=-\frac{1}{H_{e}{ }^{2}}\left[\sum_{i=1}^{k}\left[\frac{\partial H}{\partial \ell} \frac{\partial \ell}{\partial \tau} \frac{\partial \tau}{\partial \sigma_{i}} \frac{\partial \sigma_{i}}{\partial \sigma} \frac{\partial \sigma}{\partial \rho}\right]+\left(\sum_{i=1}^{k}\left[\frac{\partial H}{\partial \ell} \frac{\partial \ell}{\partial \tau} \frac{\partial \tau}{\partial \sigma_{i}} \frac{\partial \sigma_{i}}{\partial \sigma} \frac{\partial \sigma}{\partial U}\right]\right)^{T}\left[\frac{\partial U}{\partial \rho_{e}}\right]\right]
$$

The adjoint sensitivities on an element basis for the number of cycles to failure is calculated from the following equations: 


$$
\frac{d \varpi_{e}}{d \rho_{e}}=-\frac{1}{H_{e}^{2}}\left[\sum_{i=1}^{k}\left[\frac{\partial H}{\partial \ell} \frac{\partial \ell}{\partial \tau} \frac{\partial \tau}{\partial \sigma_{i}} \frac{\partial \sigma_{i}}{\partial \sigma} \frac{\partial \sigma}{\partial \rho}\right]-\lambda_{e}{ }^{T}\left[\frac{d K_{e}}{d \rho_{e}} U_{e}\right]\right]
$$

The adjoint vector is equivalent to that of the damage and is calculated from:

$$
\lambda=\sum_{i=1}^{k}\left[\frac{\partial H}{\partial \ell} \frac{\partial \ell}{\partial \tau} \frac{\partial \tau}{\partial \sigma_{i}} \frac{\partial \sigma_{i}}{\partial \sigma} \frac{\partial \sigma}{\partial U}\right] K^{-1}
$$

\section{E.4 Aggregate Functions}

An aggregate function is a method used to lump multiple functions together. This is useful for stress constraint and fatigue constraints as there could be as many constraints as design variables. The most notable methods are the Kreisselmeier-Steinhauser function and the P-norm method by Duysinx and Sigmund [183]. The major benefit of using an aggregate function is to help decrease the computational costs during sensitivity analyses. Aggregate functions can also help smooth out the structural response so that it is possible to find the global optimum using gradient based optimization techniques [186]. The smoothing properties are especially useful for largescale applications with the presence of noisy gradients such as stress or fatigue structural responses.

\section{E.4.1 Overview}

One aggregation technique employs the P-norm or the P-mean. This assumes that the functions are non-negative to start with. The regular P-norm method is written as:

$$
\psi_{P U}=\left(\sum_{i=1}^{N} f_{i}(\vec{\rho})^{P}\right)^{1 / P}
$$


where $f_{i}(\vec{\rho})$ is the collection of functions defined by the design variables and $\mathrm{P}$ is the norm factor. The P-mean is nearly identical to the P-norm and is formulated as:

$$
\psi_{P L}=\left(\frac{1}{N} \sum_{i=1}^{N} f_{i}(\vec{\rho})^{P}\right)^{1 / P}
$$

The P-mean will act as a lower bound to the problem while the P-norm will act as an upper bound for the local maximum function values [183].

A second aggregate function also used in structural optimization is the Kreisselmeier-Steinhauser [187] (or KS for short) and is defined as:

$$
\psi_{K U}=\frac{1}{P} \ln \left(\sum_{i=1}^{N} e^{P f_{i}(\vec{\rho})}\right)
$$

The KS function works as an upper bound for the local maximum function values. When $\mathrm{P}$ is larger than zero, the maximum values will be overestimated. The overestimation can be remedied by first calculating the maximum value for all functions. The difference can be calculated as [183]:

$$
\frac{1}{P} \ln \left(N e^{P f_{\max }}\right)-f_{\max }=\frac{1}{P} \ln (N)
$$

By subtracting this value from the original KS function, the lower bound formulation can be derived and is:

$$
\psi_{K L}=\frac{1}{P} \ln \left(\frac{1}{N} \sum_{i=1}^{N} e^{P f_{i}(\vec{\rho})}\right)
$$

For optimal designs in an efficient number of iterations for gradient based optimizations, the $\mathrm{P}$ value should be within 20 to 40 [183]. As the P value increases, so does the non-linearity of the aggregate function. 
The sensitivities of the aggregate functions can be found by applying the chain rule. For the PNorm aggregate functions, the gradients are with respect to the individual functions is obtained from:

$$
\begin{gathered}
\frac{d \psi_{P U}}{d f}=\frac{1}{P}\left(\sum_{i=1}^{N} f_{i}(\vec{\rho})^{P}\right)^{\frac{1}{P}-1}\left(\sum_{i=1}^{N}\left[f_{i}(\vec{\rho})^{P-1} \frac{\partial f_{i}}{\partial f}\right]\right) P \\
\frac{d \psi_{P L}}{d f}=\frac{1}{P}\left(\frac{1}{N} \sum_{i=1}^{N} f_{i}(\vec{\rho})^{P}\right)^{\frac{1}{P}-1} \frac{1}{N}\left(\sum_{i=1}^{N}\left[f_{i}(\vec{\rho})^{P-1} \frac{\partial f_{i}}{\partial f}\right]\right) P
\end{gathered}
$$

The KS sensitivities can be found in a similar manner:

$$
\begin{gathered}
\frac{d \psi_{K U}}{d f}=\frac{1}{P} \frac{1}{\sum_{i=1}^{N} e^{P f_{i}(\vec{\rho})}}\left(\sum_{i=1}^{N}\left[e^{P f_{i}(\vec{\rho})} \frac{\partial f_{i}}{\partial f}\right]\right) P \\
\frac{d \psi_{K L}}{d f}=\frac{1}{P} \frac{1}{\frac{1}{N} \sum_{i=1}^{N} e^{P f_{i}(\vec{\rho})}}\left(\frac{1}{N} \sum_{i=1}^{N}\left[e^{P f_{i}(\vec{\rho})} \frac{\partial f_{i}}{\partial f}\right]\right) P
\end{gathered}
$$

Another form of aggregate function is a summation of all the individual responses. This is not morally used unless a compliance structural response is requested. Nevertheless it is able to reduce the number of structural responses down to one single function with many benefits for reducing computational costs during optimizations. Hence the summation of a structural response is written as:

$$
\psi_{S}=\sum_{i=1}^{N} f_{i}(\vec{\rho})
$$

The corresponding sensitivities are calculated by the summation of all the individual derivatives of the responses: 


$$
\frac{d \psi_{S}}{d f}=\sum_{i=1}^{N} \frac{\partial f_{i}}{\partial f}
$$

The same principles for a summation can be also be applied to an average function over the individual structural responses. This is done by dividing the performance measure and gradients by the number of individual responses.

\section{E.4.2 Clustering Strategies}

One common method coupled with aggregation functions is by clustering or dividing the design variables into groups and then applying the aggregation onto the clusters. Aggregate clustering results in new structural responses equal to the number of desired clusters. This technique has been implemented for stress based constraints in topology optimization and successfully used by Holmberg et al [184] and Pasini et al [95].

Each group or cluster is sorted based on its response values, either by ascension or descending order. The advantage of clustering is that it creates multiple responses that are able to improve the accuracy for large scale problems with many constraints. This is because global approximations may under or overestimate the true value of the lumped functions. The under and over estimation arises from a collection of functions where only a few responses are significantly higher than the rest. The smaller functions values will dampen out the larger responses to under estimate the true maximum of the lumped response. In other words, both global and local approximations are possible by clustering.

When the number of clusters is equal to one, the response is equal to the global approximation first explained in section 7.5.2. When the number of clusters is equal to the number of design variables, then the problem evaluates everything locally and is computationally expensive. 


\section{E.4.3 Aggregation Application to Von-Mises Stresses}

When considering a global stress evaluation as a structural response, an aggregation of multiple stress functions is required. The Von-Mises stress can be lumped together as a single function which helps reduce the computational costs during optimizations. The adjoint method will be used to acquire the sensitivities of the aggregate function. This is because it is much more efficient to compute than the direct method.

By applying the chain rule, the full sensitivities can be determined for the aggregate function. The adjoint sensitivity global equation is:

$$
\frac{d \psi_{V M}}{d \rho}=\left.\frac{\partial \psi_{V M}}{\partial \rho}\right|_{U=\mathrm{const}}+\lambda^{T}\left(\frac{d F}{d \rho}-\frac{d K}{d \rho} U\right)
$$

For the aggregate von-Mises stress, the force vector is a constant so it is equal to zero. The gradient with respect to the design variables is found with:

$$
\begin{aligned}
& \left.\frac{\partial \psi_{V M_{P U}}}{\partial \rho_{e}}\right|_{U=\text { const }}=\frac{\partial \psi_{V M_{P U}}}{\partial \rho_{e}}=\frac{1}{P}\left(\sum_{i=1}^{N} \sigma_{V M_{i}}(\vec{\rho})^{P}\right)^{\frac{1}{P}-1} \sum_{i=1}^{N}\left[\sigma_{V M_{i}}(\vec{\rho})^{P-1} \frac{\sigma_{V M_{i}}(\vec{\rho})}{\partial \rho_{e}}\right] P \\
& \left.\frac{\partial \psi_{V M_{P L}}}{\partial \rho_{e}}\right|_{U=\text { const }}=\frac{\partial \psi_{V M_{P L}}}{\partial \rho_{e}}=\frac{1}{P}\left(\frac{1}{N} \sum_{i=1}^{N} \sigma_{V M_{i}}(\vec{\rho})^{P}\right)^{\frac{1}{P}-1} \frac{1}{N} \sum_{i=1}^{N}\left[\sigma_{V M_{i}}(\vec{\rho})^{P-1} \frac{\sigma_{V M_{i}}(\vec{\rho})}{\partial \rho_{e}}\right] P \\
& \left.\frac{\partial \psi_{V M_{K U}}}{\partial \rho_{e}}\right|_{U=\text { const }}=\frac{\partial \psi_{V M_{K U}}}{\partial \rho_{e}}=\frac{1}{P} \frac{1}{\sum_{i=1}^{N} e^{P \sigma_{V M_{i}}(\vec{\rho})}} \sum_{i=1}^{N}\left[e^{P \sigma_{V M_{i}}(\vec{\rho})} \frac{\sigma_{V M_{i}}(\vec{\rho})}{\partial \rho_{e}}\right] P \\
& \left.\frac{\partial \psi_{V M_{K L}}}{\partial \rho_{e}}\right|_{U=\text { const }}=\frac{\partial \psi_{V M_{K L}}}{\partial \rho_{e}}=\frac{1}{P} \frac{1}{\frac{1}{N} \sum_{i=1}^{N} e^{P \sigma_{V M_{i}}(\vec{\rho})}} \frac{1}{N} \sum_{i=1}^{N}\left[e^{P \sigma_{V M_{i}}(\vec{\rho})} \frac{\sigma_{V M_{i}}(\vec{\rho})}{\partial \rho_{e}}\right] P
\end{aligned}
$$

The derivatives of the von-Mises stress with respect to the design variables can be found in chapter C.2. The adjoint load $\frac{\partial \psi}{\partial U}$ can be calculated using the equations below for each of the possible aggregate functions explained in the previous section: 


$$
\begin{gathered}
\frac{\partial \psi_{V M_{P U}}}{\partial U}=\frac{1}{P}\left(\sum_{i=1}^{N} \sigma_{V M_{i}}^{P}\right)^{\frac{1}{P}-1} \sum_{i=1}^{N}\left[\sigma_{V M_{i}}^{P-1} \frac{\partial \sigma_{V M_{i}}}{\partial U}\right] P \\
\frac{\partial \psi_{V M_{P L}}}{\partial U}=\frac{1}{P}\left(\frac{1}{N} \sum_{i=1}^{N} \sigma_{V M_{i}}^{P}\right)^{\frac{1}{P}-1} \frac{1}{N} \sum_{i=1}^{N}\left[\sigma_{V M_{i}}^{P-1} \frac{\partial \sigma_{V M_{i}}}{\partial U}\right] P \\
\frac{\partial \psi_{V M_{K U}}}{\partial U}=\frac{1}{P} \frac{1}{\sum_{i=1}^{N} e^{P \sigma_{V M_{i}}}} \sum_{i=1}^{N}\left[e^{\left.P \sigma_{V M_{i}} \frac{\partial \sigma_{V M_{i}}}{\partial U}\right] P}\right. \\
\frac{\partial \psi_{V M_{K L}}}{\partial U}=\frac{1}{P} \frac{1}{\frac{1}{N} \sum_{i=1}^{N} e^{P \sigma_{V M_{i}}}} \frac{1}{N} \sum_{i=1}^{N}\left[e^{\left.P \sigma_{V M_{i}} \frac{\partial \sigma_{V M_{i}}}{\partial U}\right] P}\right.
\end{gathered}
$$

In a general case, the sensitivities due to the displacements for the von-Mises stresses can be calculated as:

$$
\frac{\partial \sigma_{V M_{i}}}{\partial U}=\frac{\partial \sigma_{V M_{i}}}{\partial \sigma_{i}} \frac{\partial \sigma_{i}}{\partial U}=\frac{\partial \sigma_{V M_{i}}}{\partial \sigma_{i}} D_{i} B_{i}
$$

Where $\frac{\partial \sigma_{V M_{i}}}{\partial \sigma}$ is a row vector of sensitivities of the von-Mises stress with respect to the principle and shear stresses and are calculated in chapter E.2.3.

\section{E.4.4 Application to Cumulative Damage and Life}

A global structural response is advantageous when it comes to reducing the number of responses. In much the same philosophy for an aggregate function for the stresses, the cumulative damage and life equations can be lumped together into a single function. By combining all the fatigue responses into one function, the computational cost sensitivity analysis will decrease 
dramatically. Using one response will allow the use of the adjoint method to calculate the sensitivities. The adjoint sensitivity global equation is:

$$
\frac{d \psi}{d \rho}=\left.\frac{\partial \psi}{\partial \rho}\right|_{U=\text { const }}+\lambda^{T}\left(\frac{d F}{d \rho}-\frac{d K}{d \rho} U\right)
$$

The first partial derivative for the performance measure with respect to the design variables is as follows:

$$
\begin{gathered}
\left.\frac{\partial \psi_{H_{P U}}}{\partial \rho_{e}}\right|_{U=\text { const }}=\frac{\partial \psi_{H_{P U}}}{\partial \rho_{e}}=\frac{1}{P}\left(\sum_{j=1}^{N} H(\vec{\rho})^{P}\right)^{\frac{1}{P}-1} \sum_{j=1}^{N}\left[H(\vec{\rho})^{P-1} \frac{H_{j}(\vec{\rho})}{\partial \rho_{e}}\right] P \\
\left.\frac{\partial \psi_{H_{P L}}}{\partial \rho_{e}}\right|_{U=\text { const }}=\frac{d \psi_{H_{P L}}}{\partial \rho_{e}}=\frac{1}{P}\left(\frac{1}{N} \sum_{j=1}^{N} H(\vec{\rho})^{P}\right)^{\frac{1}{P}-1} \frac{1}{N} \sum_{j=1}^{N}\left[\sigma_{V M_{i}}(\vec{\rho})^{P-1} \frac{H_{j}(\vec{\rho})}{\partial \rho_{e}}\right] P \\
\left.\frac{\partial \psi_{H_{K U}}}{\partial \rho_{e}}\right|_{U=\text { const }}=\frac{\partial \psi_{H_{K U}}}{\partial \rho_{e}}=\frac{1}{P} \frac{1}{\sum_{i=1}^{N} e^{P H_{j}(\vec{\rho})}} \sum_{j=1}^{N}\left[e^{P H_{j}(\vec{\rho})} \frac{H_{j}(\vec{\rho})}{\partial \rho_{e}}\right] P \\
\left.\frac{\partial \psi_{H_{K L}}}{\partial \rho_{e}}\right|_{U=\text { const }}=\frac{\partial \psi_{H_{K L}}}{\partial \rho_{e}}=\frac{1}{P} \frac{1}{\frac{1}{N} \sum_{j=1}^{N} e^{P H_{j}(\vec{\rho})}} \frac{1}{N} \sum_{j=1}^{N}\left[e^{P H_{j}(\vec{\rho})} \frac{H_{j}(\vec{\rho})}{\partial \rho_{e}}\right] P
\end{gathered}
$$

The derivative of the cumulative damage function with respect to the design variables can be found in chapter E.3.

The adjoint vector is calculated by the adjoint load $\frac{\partial \psi}{\partial U}$. The load is the derivative of the performance measure with respect to the displacements. The equations below demonstrate the derivations:

$$
\frac{\partial \psi_{H_{P U}}}{\partial U}=\frac{1}{P}\left(\sum_{j=1}^{N} H_{j}^{P}\right)^{\frac{1}{P}-1} \sum_{j=1}^{N}\left[H_{j}^{P-1} \frac{\partial H_{j}}{\partial U}\right] P
$$




$$
\begin{gathered}
\frac{\partial \psi_{H_{P L}}}{\partial U}=\frac{1}{P}\left(\frac{1}{N} \sum_{j=1}^{N} H_{j}^{P}\right)^{\frac{1}{P}-1} \frac{1}{N} \sum_{j=1}^{N}\left[H_{j}^{P-1} \frac{\partial H_{j}}{\partial U}\right] P \\
\frac{\partial \psi_{H_{K U}}}{\partial U}=\frac{1}{P} \frac{1}{\sum_{j=1}^{N} e^{P H_{j}}} \sum_{j=1}^{N}\left[e^{P H_{j}} \frac{\partial H_{j}}{\partial U}\right] P \\
\frac{\partial \psi_{H_{K L}}}{\partial U}=\frac{1}{P} \frac{1}{\frac{1}{N} \sum_{j=1}^{N} e^{P H_{j}}} \frac{1}{N} \sum_{j=1}^{N}\left[e^{P H_{j}} \frac{\partial H_{j}}{\partial U}\right] P
\end{gathered}
$$

The derivative of the damage function with respect to the displacements can be found in chapter E.3.2. The same process can be applied for a lumped life response. Life is obtained by multiplying the damage sensitivities by a factor of $-\frac{1}{H_{e}^{2}}$, where $\mathrm{H}_{\mathrm{e}}$ is the damage for the element e. 


\section{E.5 Local Stiffness and Constitutive Matrix}

The sensitivities for the local stiffness matrix depend largely on the type of interpolation function used. Most of the time the design variable will be the relative density which will modify the young's modulus. Because the young's modulus is a multiplicative factor, the local stiffness matrix is penalized purely based on the value of the young's modulus. Therefore for any stiffness matrix that is a function of the young's modulus; it can be written as:

$$
K_{e}\left(\rho_{e}\right)=\frac{E_{e}\left(\rho_{e}\right)}{E_{e}^{O}}\left[\int_{V} B_{e} \cdot D_{e}^{O} \cdot B_{e} \cdot d V\right]
$$

where $K_{e}\left(\rho_{e}\right)$ is the local stiffness matrix as a function of the relative density, $E_{e}\left(\rho_{e}\right)$ is the modulus as a function of the relative density, $E_{e}^{O}$ is the original young's modulus of the element assuming that the material properties are isotropic, $B_{e}$ is the strain displacement matrix, $D_{e}^{O}$ is the constitutive matrix of the material (which includes the material modulus) and $\mathrm{V}$ is the volume of the element.

The sensitivity of the stiffness matrix with respect to the design variables is then:

$$
\frac{\partial K_{e}}{\partial \rho_{e}}=\frac{\partial E_{e}}{\partial \rho_{e}} \frac{1}{E_{e}^{O}}\left[\int_{V} B_{e} \cdot D_{e}^{O} \cdot B_{e} \cdot d V\right]
$$

where $\frac{\partial E_{e}}{\partial \rho_{e}}$ is largely dependent on the interpolation method. When a SIMP modification is used for a topology optimization, the sensitivity of the young's modulus with respect to the relative density is equal to:

$$
\left.\frac{\partial E_{e}}{\partial \rho_{e}}\right\rangle_{S I M P}=p \rho_{e}^{p-1} E_{e}^{O}
$$

where $\mathrm{p}$ is the penalty factor and $E_{e}^{O}$ is the unmodified element young's modulus of element e. 


\section{E.6 Finite Difference vs. Analytical Sensitivity Comparisons}

The following section will demonstrate the accuracy of the derived sensitivities by using the finite difference method to numerically solve for the sensitivities for each optimization type.

The model used is a $2 \mathrm{D}$ cantilever beam with a triangle mesh shown in the picture below:

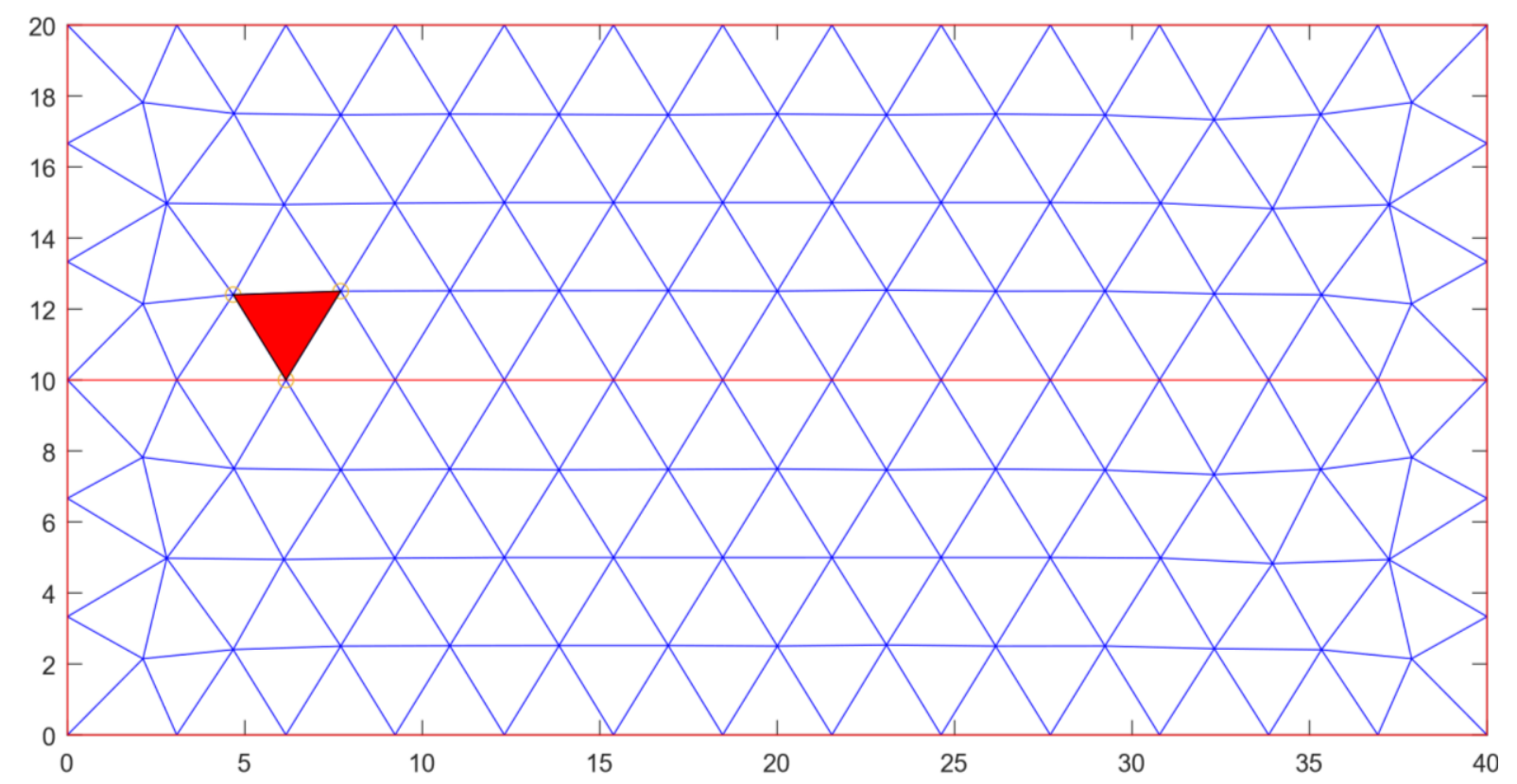

Figure 59 : test Mesh for Sensitivity Analysis

The left side is fully constrained in the $\mathrm{x}$ and $\mathrm{y}$ direction with a point load pointing upwards located at the node $(40,10)$. Each element has two degrees of freedom; hence plate elements are used in the FEM example. A single element highlighted in red is updated in the model by changing its relative density from 0.005 to 0.98 . The analytical and numerically calculated gradient is calculated for each response. A BCC lattice is used for the LATTICE_SIMP and the LATTICE_TRUE sensitivities. The material is titanium with a Poisson ratio of 0.31 and modulus of $114000 \mathrm{MPa}$. The plate thickness is one. 


\section{E.6.1 SIMP Damage with PNORM}

The penalization term for the SIMP sensitivities is set to 5. The exponent for the PNORM is equal to 2 in the following sensitivities.

\begin{tabular}{|c|c|c|c|c|}
\hline \multicolumn{5}{|c|}{ SIMP DAMAGE (Unmodified) } \\
\hline \hline Relative Density & Forward Difference & Backwards Difference & Central Difference & Analytical \\
\hline 0.005 & $-6.08 \mathrm{E}-06$ & $-6.66 \mathrm{E}-07$ & $-3.37 \mathrm{E}-06$ & $-3.48 \mathrm{E}-08$ \\
\hline 0.12687 & $4.00 \mathrm{E}-05$ & $7.08 \mathrm{E}-05$ & $5.54 \mathrm{E}-05$ & $5.53 \mathrm{E}-05$ \\
\hline 0.24875 & 0.035825 & 0.035789 & 0.035807 & 0.035795 \\
\hline 0.37062 & -0.02199 & -0.00336 & -0.01267 & -0.01683 \\
\hline 0.4925 & 0.22431 & 0.22434 & 0.22433 & 0.22433 \\
\hline 0.61438 & -0.00018 & -0.00019 & -0.00019 & -0.00019 \\
\hline 0.73625 & 0.029629 & 0.02962 & 0.029624 & 0.029622 \\
\hline 0.85813 & -0.00064 & -0.00064 & -0.00064 & -0.00064 \\
\hline 0.98 & 0.000204 & 0.000195 & 0.000199 & 0.000212 \\
\hline
\end{tabular}

\begin{tabular}{|c|c|c|c|c|}
\hline \multicolumn{6}{|c|}{ SIMP DAMAGE (with Stress Penalization (0.5)) } \\
\hline Relative Density & Forward Difference & Backwards Difference & Central Difference & Analytical \\
\hline 0.005 & $1.91 \mathrm{E}+14$ & $8.61 \mathrm{E}+14$ & $5.26 \mathrm{E}+14$ & $7.29 \mathrm{E}+08$ \\
\hline 0.12687 & $1.08 \mathrm{E}+08$ & $1.08 \mathrm{E}+08$ & $1.08 \mathrm{E}+08$ & $1.08 \mathrm{E}+08$ \\
\hline 0.24875 & $-3.49 \mathrm{E}+07$ & $-3.49 \mathrm{E}+07$ & $-3.49 \mathrm{E}+07$ & $-3.49 \mathrm{E}+07$ \\
\hline 0.37062 & $-2.06 \mathrm{E}+16$ & $4.56 \mathrm{E}+16$ & $1.25 \mathrm{E}+16$ & $8.69 \mathrm{E}+15$ \\
\hline 0.4925 & -8119.8 & -8119.8 & -8119.8 & -8119.7 \\
\hline 0.61438 & $-1.79 \mathrm{E}+17$ & $-1.79 \mathrm{E}+17$ & $-1.79 \mathrm{E}+17$ & $-4.54 \mathrm{E}+19$ \\
\hline 0.73625 & $-5.00 \mathrm{E}+19$ & $-5.00 \mathrm{E}+19$ & $-5.00 \mathrm{E}+19$ & $-5.04 \mathrm{E}+19$ \\
\hline 0.85813 & $-2.94 \mathrm{E}+18$ & $-2.94 \mathrm{E}+18$ & $-2.94 \mathrm{E}+18$ & $-4.11 \mathrm{E}+18$ \\
\hline 0.98 & $4.22 \mathrm{E}+18$ & $4.23 \mathrm{E}+18$ & $4.22 \mathrm{E}+18$ & $3.69 \mathrm{E}+18$ \\
\hline
\end{tabular}

\begin{tabular}{||c|c|c|c|c||}
\hline \multicolumn{5}{|c|}{ SIMP DAMAGE (with Yield Strength Penalization (1.5)) } \\
\hline \hline Relative Density & Forward Difference & Backwards Difference & Central Difference & Analytical \\
\hline \hline 0.005 & $2.41 \mathrm{E}-06$ & $-2.19 \mathrm{E}-06$ & $1.11 \mathrm{E}-07$ & $5.06 \mathrm{E}-11$ \\
\hline 0.12687 & 0.32093 & 0.32093 & 0.32093 & 0.32094 \\
\hline 0.24875 & 0.062518 & 0.062471 & 0.062494 & 0.062493 \\
\hline 0.37062 & 0.13701 & 0.13699 & 0.137 & 0.137 \\
\hline 0.4925 & 0.016025 & 0.016025 & 0.016025 & 0.016025 \\
\hline 0.61438 & -0.02797 & -0.02791 & -0.02794 & -0.02789 \\
\hline 0.73625 & 0.002872 & 0.002893 & 0.002882 & 0.002877 \\
\hline
\end{tabular}




\begin{tabular}{|c|l|l|l|l||}
\hline 0.85813 & 0.025009 & 0.025009 & 0.025009 & 0.025009 \\
\hline 0.98 & 0.051821 & 0.051866 & 0.051843 & 0.051858 \\
\hline
\end{tabular}

\begin{tabular}{||c|c|c|c|c||}
\hline \multicolumn{5}{|c|}{ SIMP DAMAGE (with SN-Curve Intercept Penalization) } \\
\hline \hline Relative Density & Forward Difference & Backwards Difference & Central Difference & Analytical \\
\hline \hline 0.005 & $-1.46 \mathrm{E}-06$ & $2.87 \mathrm{E}-05$ & $1.36 \mathrm{E}-05$ & $1.48 \mathrm{E}-07$ \\
\hline 0.12687 & -0.01425 & -0.01413 & -0.01419 & -0.01408 \\
\hline 0.24875 & -9.3431 & -9.3436 & -9.3434 & -9.3429 \\
\hline 0.37062 & 0.037887 & 0.037892 & 0.03789 & 0.037873 \\
\hline 0.4925 & -0.02218 & -0.02575 & -0.02396 & -0.02278 \\
\hline 0.61438 & -2.1137 & -2.114 & -2.1139 & 232.73 \\
\hline 0.73625 & 0.53043 & 0.53109 & 0.53076 & 0.52982 \\
\hline 0.85813 & -0.00051 & 0.000351 & $-7.97 \mathrm{E}-05$ & $-4.24 \mathrm{E}-06$ \\
\hline 0.98 & -0.00682 & -0.00688 & -0.00685 & -0.00686 \\
\hline
\end{tabular}

\section{E.6.2 Lattice Sensitivities with PNORM}

A p-norm value of 2 was selected for the following sensitivities. The penalization function is equal to the relative yield function.

\begin{tabular}{|c|c|c||c|c||}
\hline \multicolumn{5}{|c|}{ Lattice DAMAGE (Unmodified) } \\
\hline \hline Relative Density & Forward Difference & Backwards Difference & Central Difference & Analytical \\
\hline 0.005 & 0.005618 & 0.005618 & 0.005618 & 0.005618 \\
\hline 0.12687 & -0.00082 & -0.00082 & -0.00082 & -0.00082 \\
\hline 0.24875 & 0.000172 & 0.000172 & 0.000172 & 0.000172 \\
\hline 0.37062 & $-2.00 \mathrm{E}-05$ & $-2.02 \mathrm{E}-05$ & $-2.01 \mathrm{E}-05$ & $-2.01 \mathrm{E}-05$ \\
\hline 0.4925 & $-6.94 \mathrm{E}-05$ & $-6.91 \mathrm{E}-05$ & $-6.93 \mathrm{E}-05$ & $-6.93 \mathrm{E}-05$ \\
\hline 0.61438 & 0.000103 & 0.000103 & 0.000103 & 0.000103 \\
\hline 0.73625 & 0.00019 & 0.00019 & 0.00019 & 0.00019 \\
\hline 0.85813 & 0.000712 & 0.000712 & 0.000712 & 0.000712 \\
\hline 0.98 & 0.000494 & 0.000494 & 0.000494 & 0.000494 \\
\hline
\end{tabular}

\begin{tabular}{||c|c|c||c|c||}
\hline \multicolumn{5}{|c||}{ Lattice DAMAGE (with Stress Penalization) } \\
\hline \hline Relative Density & Forward Difference & Backwards Difference & Central Difference & Analytical \\
\hline \hline 0.005 & -8530.7 & -8531.6 & -8531.1 & -8531.1 \\
\hline 0.12687 & $-3.72 \mathrm{E}+05$ & $-3.72 \mathrm{E}+05$ & $-3.72 \mathrm{E}+05$ & $-3.72 \mathrm{E}+05$ \\
\hline
\end{tabular}




\begin{tabular}{|c|c|c|c|c||}
\hline 0.24875 & $3.44 \mathrm{E}+12$ & $3.43 \mathrm{E}+12$ & $3.44 \mathrm{E}+12$ & $3.44 \mathrm{E}+12$ \\
\hline 0.37062 & 0 & 0 & 0 & $-7.78 \mathrm{E}+05$ \\
\hline 0.4925 & $7.65 \mathrm{E}+06$ & $7.65 \mathrm{E}+06$ & $7.65 \mathrm{E}+06$ & $7.65 \mathrm{E}+06$ \\
\hline 0.61438 & 3.3913 & 3.3903 & 3.3908 & 3.3907 \\
\hline 0.73625 & $3.90 \mathrm{E}+05$ & $3.90 \mathrm{E}+05$ & $3.90 \mathrm{E}+05$ & $3.90 \mathrm{E}+05$ \\
\hline 0.85813 & $-3.52 \mathrm{E}+07$ & $-3.52 \mathrm{E}+07$ & $-3.52 \mathrm{E}+07$ & $-3.52 \mathrm{E}+07$ \\
\hline 0.98 & $-3.62 \mathrm{E}+14$ & $-3.62 \mathrm{E}+14$ & $-3.62 \mathrm{E}+14$ & $-3.62 \mathrm{E}+14$ \\
\hline
\end{tabular}

\begin{tabular}{||c|c|c|c|c||}
\hline \multicolumn{5}{|c||}{ Lattice DAMAGE (with Yield Penalization) } \\
\hline \hline Relative Density & Forward Difference & Backwards Difference & Central Difference & Analytical \\
\hline \hline 0.005 & 0.004683 & 0.004683 & 0.004683 & 0.004683 \\
\hline 0.12687 & 0 & 0 & 0 & $-5.90 \mathrm{E}+11$ \\
\hline 0.24875 & $-1.80 \mathrm{E}+09$ & $-1.80 \mathrm{E}+09$ & $-1.80 \mathrm{E}+09$ & $-1.80 \mathrm{E}+09$ \\
\hline 0.37062 & $-8.17 \mathrm{E}-05$ & $-8.20 \mathrm{E}-05$ & $-8.18 \mathrm{E}-05$ & $-8.19 \mathrm{E}-05$ \\
\hline 0.4925 & 0.001043 & 0.001043 & 0.001043 & 0.001043 \\
\hline 0.61438 & 0.001286 & 0.001286 & 0.001286 & 0.001286 \\
\hline 0.73625 & -0.0129 & -0.0129 & -0.0129 & -0.0129 \\
\hline 0.85813 & -0.00015 & -0.00015 & -0.00015 & -0.00015 \\
\hline 0.98 & 0.008334 & 0.008334 & 0.008334 & 0.008334 \\
\hline
\end{tabular}

\begin{tabular}{||c|c|c|c|c||}
\hline \multicolumn{5}{|c|}{ Lattice DAMAGE (with SN-Curve Intercept Penalization) } \\
\hline \hline Relative Density & Forward Difference & Backwards Difference & Central Difference & Analytical \\
\hline \hline 0.005 & -638.33 & -638.4 & -638.36 & -638.36 \\
\hline 0.12687 & -18.675 & -18.682 & -18.678 & -18.679 \\
\hline 0.24875 & 18.76 & 18.668 & 18.714 & 18.718 \\
\hline 0.37062 & -725.44 & -725.35 & -725.39 & -725.25 \\
\hline 0.4925 & -163.99 & -164.09 & -164.04 & -164.11 \\
\hline 0.61438 & -91125 & -91163 & -91144 & -91154 \\
\hline 0.73625 & $-8.30 \mathrm{E}+05$ & $-8.30 \mathrm{E}+05$ & $-8.30 \mathrm{E}+05$ & $-8.30 \mathrm{E}+05$ \\
\hline 0.85813 & -96520 & -96524 & -96522 & -96517 \\
\hline 0.98 & -75.513 & -75.584 & -75.549 & -75.535 \\
\hline
\end{tabular}




\section{Appendix F : Numerical Homogenization Data and Interpolation Methods}

During multi-scale optimizations, the change in the material properties is directly related to the derived homogenized properties. This occurs during SIMP-like optimizations where the change in the stiffness matrix is directly related to the relative young's modulus and Poisson ratios. The homogenized properties are extracted for a number of different initial Poisson ratios and a single young's modulus. This is because the young's modulus is a multiplicative scalar and the relative modulus is independent of the Poisson ratio.

The yield strength properties are also a factor of the relative density and of the input stresses and can be determined by two possible interpolation strategies. Both interpolation methods are discussed. The first method requires two interpolation steps while the first only requires information about the average relative yielding. For both methods the relative yield strengths for uniaxial stress states are interpolated given the values of the modulus and Poisson ratio. Additional steps are generally required to determine equivalent stresses when multi-axial stress states are imposed.

\section{F.1 Interpolation Methods}

The homogenized properties are written to a look-up table. During optimizations, the relative properties are obtained by interpolating the table from a given relative density, unit cell topology and Poisson ratio. The interpolation scheme has two steps; the first step locates the required table rows which have information regarding the unit cell and its mechanical properties. An additional filter is used on the collected rows for find the rows containing the materials Poisson ratio. Then a temporary database is made from an interpolation function. The second step is used to fit the temporary database with a high order polynomial function. The regression fitting is mainly used 
to smooth out the interpolation and remove numerical noise. The newly fit polynomial function is also useful because it can have its gradient calculated for the relative properties as a function of the relative density. High order information is needed for the lattice topology optimizations. . A polynomial of order six was used for the regression fit in step two. The temporary database is set to 100 points to be fit by the polynomial function.

\section{F.2 Multi-axial Yield Strength Interpolation}

There are two possible methods described in this section for interpolating relative yield strengths for multi-axial stresses. The first is a two-step interpolation approach to determine the relative yield properties for lattice material. The first step will interpolate the uniaxial strength factors using the methodology presented in chapter F.1. The yield factor for multi-axial stress input is calculated by interpolating between the uniaxial stress factors. The input stress state is normalized and therefore stress states of zero cannot be used. The second interpolation step is then performed by computing a weighted average of the yield strength terms with the normalized macro stress. This interpolation method assures differentiability which is important for the optimization routines. The second stage interpolation for the first described method can be represented by the equation below:

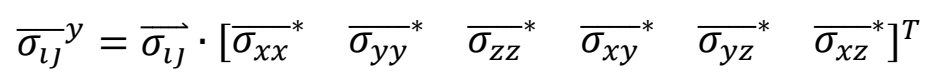

where ${\overline{\sigma_{l \jmath}}}^{y}$ is the relative yield strength, ${\overline{\sigma_{l \jmath}}}$ is the input macro stress on the RVE and ${\overline{\sigma_{l \jmath}}}^{*}$ are the relative yield strength coefficients for a given relative density and Poisson ratio. The evaluated constants assume the failure criterion is the von-Mises stress. 
The second interpolation method is a simpler approach and uses an average yielding criterion. The average relative yield is determined in the same manner as the young's modulus and the Poisson ratio described in chapter F.1. The average yielding is the mean of all six yielding factors for a given relative density and Poisson ratio. The trade-off to using this method is reduced accuracy for better computational simplicity and efficiency. This interpolation strategy for the stresses is better suited for topologies whose yielding criterions are all very similar such as the diamond lattice in Figure 23. The average relative yield strength will be used as the method of choice in the topology optimizations due to reduced computational cost. 


\section{F.3 Effective Mechanical Properties}

\begin{tabular}{|c|c|c|c|c|c|c||}
\hline \hline \multicolumn{7}{|c||}{ Octet Truss Relative Properties (Poisson ratio = 0.3) } \\
\hline \hline Density & Radius & D/L & $\mathbf{1}^{\text {st }}$ Lame & $\mathbf{2}^{\text {nd }}$ Lame & Poisson Ratio & Modulus \\
\hline \hline 0 & 0 & 0 & 0 & 0 & 0.85307 & 0 \\
\hline 0.026752 & 0.02 & 0.04 & 0.004666 & 0.006676 & 0.85307 & 0.006449 \\
\hline 0.068096 & 0.04 & 0.08 & 0.013196 & 0.018601 & 0.85923 & 0.017997 \\
\hline 0.1655 & 0.06 & 0.12 & 0.037466 & 0.052446 & 0.86212 & 0.050777 \\
\hline 0.26445 & 0.08 & 0.16 & 0.068322 & 0.097278 & 0.85505 & 0.094024 \\
\hline 0.39642 & 0.1 & 0.2 & 0.12072 & 0.1776 & 0.84143 & 0.1711 \\
\hline 0.50074 & 0.12 & 0.24 & 0.17391 & 0.26131 & 0.83264 & 0.25121 \\
\hline 0.61888 & 0.14 & 0.28 & 0.26171 & 0.39165 & 0.8343 & 0.37667 \\
\hline 0.73779 & 0.16 & 0.32 & 0.39032 & 0.54767 & 0.86114 & 0.53012 \\
\hline 0.82906 & 0.18 & 0.36 & 0.56788 & 0.70291 & 0.91315 & 0.68882 \\
\hline 0.89498 & 0.2 & 0.4 & 0.71351 & 0.80968 & 0.94884 & 0.80012 \\
\hline 0.9399 & 0.22 & 0.44 & 0.82776 & 0.88837 & 0.97154 & 0.88254 \\
\hline 1 & 0.26 & 0.52 & 1 & 1 & 1 & 1 \\
\hline \hline
\end{tabular}

\begin{tabular}{|c|c|c|c|c|c|c||}
\hline \multicolumn{7}{|c|}{ Body Centered Cubic (BCC) Lattice Relative Properties (Poisson ratio $=\mathbf{0 . 3})$} \\
\hline \hline Density & Radius & D/L & $\mathbf{1}^{\text {st }}$ Lame & $\mathbf{2}^{\text {nd }}$ Lame & Poisson Ratio & Modulus \\
\hline 0 & 0 & 0 & 0 & 0 & 0.84568 & 0 \\
\hline 0.010816 & 0.02 & 0.04 & 0.002057 & 0.002996 & 0.84568 & 0.002889 \\
\hline 0.02848 & 0.04 & 0.08 & 0.006192 & 0.008826 & 0.85459 & 0.00853 \\
\hline 0.078016 & 0.06 & 0.12 & 0.019008 & 0.026351 & 0.86615 & 0.025537 \\
\hline 0.1168 & 0.08 & 0.16 & 0.030521 & 0.041928 & 0.86994 & 0.040669 \\
\hline 0.18208 & 0.1 & 0.2 & 0.051767 & 0.070746 & 0.87211 & 0.068658 \\
\hline 0.24928 & 0.12 & 0.24 & 0.076267 & 0.10455 & 0.87082 & 0.10143 \\
\hline 0.31802 & 0.14 & 0.28 & 0.10388 & 0.14403 & 0.8661 & 0.13958 \\
\hline 0.3975 & 0.16 & 0.32 & 0.13807 & 0.19423 & 0.86007 & 0.18796 \\
\hline 0.47584 & 0.18 & 0.36 & 0.17561 & 0.25249 & 0.85098 & 0.2438 \\
\hline 0.55955 & 0.2 & 0.4 & 0.22061 & 0.32257 & 0.84398 & 0.31096 \\
\hline 0.63789 & 0.22 & 0.44 & 0.27228 & 0.4001 & 0.84191 & 0.3855 \\
\hline 0.7047 & 0.24 & 0.48 & 0.32908 & 0.47634 & 0.84819 & 0.45965 \\
\hline 0.78496 & 0.26 & 0.52 & 0.42585 & 0.58416 & 0.87055 & 0.56671 \\
\hline 0.84832 & 0.28 & 0.56 & 0.53662 & 0.68533 & 0.90021 & 0.66955 \\
\hline 0.90438 & 0.3 & 0.6 & 0.67437 & 0.79103 & 0.93528 & 0.77922 \\
\hline 0.94547 & 0.32 & 0.64 & 0.80111 & 0.87699 & 0.9635 & 0.8696 \\
\hline 1 & 0.36 & 0.72 & 1 & 1 & 1 & 1 \\
\hline
\end{tabular}




\begin{tabular}{||c|c|c||c|c|c|c||}
\hline \multicolumn{6}{|c|}{ Body Centered Cubic with Cubic (BCCC) Lattice Relative Properties (Poisson ratio $=\mathbf{0 . 3})$} \\
\hline \hline Density & Radius & D/L & $\mathbf{1}^{\text {st }}$ Lame & $\mathbf{2}^{\text {nd }}$ Lame & Poisson Ratio & Modulus \\
\hline \hline 0 & 0 & 0 & 0 & 0 & 0.8501 & 0 \\
\hline 0.015232 & 0.02 & 0.04 & 0.002119 & 0.003054 & 0.8501 & 0.002948 \\
\hline 0.041152 & 0.04 & 0.08 & 0.006476 & 0.009101 & 0.86051 & 0.008808 \\
\hline 0.10854 & 0.06 & 0.12 & 0.020421 & 0.028046 & 0.87005 & 0.027205 \\
\hline 0.16346 & 0.08 & 0.16 & 0.033386 & 0.045747 & 0.87101 & 0.044385 \\
\hline 0.24698 & 0.1 & 0.2 & 0.057882 & 0.080096 & 0.86692 & 0.077636 \\
\hline 0.33222 & 0.12 & 0.24 & 0.086777 & 0.12265 & 0.85812 & 0.11863 \\
\hline 0.42208 & 0.14 & 0.28 & 0.12314 & 0.17838 & 0.84788 & 0.17212 \\
\hline 0.51942 & 0.16 & 0.32 & 0.17349 & 0.25599 & 0.84018 & 0.24655 \\
\hline 0.60736 & 0.18 & 0.36 & 0.23443 & 0.34686 & 0.83905 & 0.33397 \\
\hline 0.69683 & 0.2 & 0.4 & 0.32234 & 0.4616 & 0.85265 & 0.44591 \\
\hline 0.77536 & 0.22 & 0.44 & 0.43965 & 0.58742 & 0.88148 & 0.57136 \\
\hline 0.83565 & 0.24 & 0.48 & 0.55522 & 0.6915 & 0.9106 & 0.67724 \\
\hline 0.89498 & 0.26 & 0.52 & 0.69694 & 0.80113 & 0.94358 & 0.7907 \\
\hline 0.93242 & 0.28 & 0.56 & 0.7864 & 0.8659 & 0.96114 & 0.85813 \\
\hline 1 & 0.32 & 0.64 & 1 & 1 & 1 & 1 \\
\hline
\end{tabular}

\begin{tabular}{||c|c|c|c|c|c|c||}
\hline \multicolumn{7}{|c||}{ Cubic Lattice Relative Properties (Poisson ratio = 0.3) } \\
\hline \hline Density & Radius & D/L & $\mathbf{1}^{\text {st }}$ Lame & $\mathbf{2}^{\text {nd }}$ Lame & Poisson Ratio & Modulus \\
\hline \hline & 0 & 0 & 0 & 0 & 1.5297 & 0 \\
\hline 0.004672 & 0.02 & 0.04 & $3.06 \mathrm{E}-05$ & $4.11 \mathrm{E}-06$ & 1.5297 & $4.61 \mathrm{E}-06$ \\
\hline 0.013696 & 0.04 & 0.08 & 0.000166 & $3.65 \mathrm{E}-05$ & 1.4532 & $4.03 \mathrm{E}-05$ \\
\hline 0.035456 & 0.06 & 0.12 & 0.000745 & 0.000258 & 1.3538 & 0.000279 \\
\hline 0.056256 & 0.08 & 0.16 & 0.001621 & 0.00072 & 1.2859 & 0.000768 \\
\hline 0.084352 & 0.1 & 0.2 & 0.003187 & 0.001785 & 1.2136 & 0.001873 \\
\hline 0.11507 & 0.12 & 0.24 & 0.005471 & 0.003673 & 1.1514 & 0.003801 \\
\hline 0.15526 & 0.14 & 0.28 & 0.009469 & 0.007611 & 1.0852 & 0.00776 \\
\hline 0.20083 & 0.16 & 0.32 & 0.015059 & 0.013962 & 1.03 & 0.014059 \\
\hline 0.24038 & 0.18 & 0.36 & 0.02144 & 0.022216 & 0.98573 & 0.022142 \\
\hline 0.28787 & 0.2 & 0.4 & 0.030515 & 0.03491 & 0.94554 & 0.034471 \\
\hline 0.33843 & 0.22 & 0.44 & 0.04284 & 0.053355 & 0.9106 & 0.052254 \\
\hline 0.38298 & 0.24 & 0.48 & 0.056536 & 0.075072 & 0.88406 & 0.073063 \\
\hline 0.4439 & 0.26 & 0.52 & 0.078829 & 0.11056 & 0.86131 & 0.10702 \\
\hline 0.49005 & 0.28 & 0.56 & 0.10122 & 0.14627 & 0.84888 & 0.14117 \\
\hline 0.54771 & 0.3 & 0.6 & 0.13415 & 0.19644 & 0.84338 & 0.18934 \\
\hline 0.59802 & 0.32 & 0.64 & 0.1719 & 0.2513 & 0.84404 & 0.24226 \\
\hline 0.64666 & 0.34 & 0.68 & 0.21651 & 0.31175 & 0.85038 & 0.30099 \\
\hline 0.69453 & 0.36 & 0.72 & 0.2714 & 0.38099 & 0.86094 & 0.36877 \\
\hline
\end{tabular}




\begin{tabular}{|c|c|c|c|c|c|c||}
\hline 0.73542 & 0.38 & 0.76 & 0.33136 & 0.45068 & 0.8741 & 0.43759 \\
\hline 0.78656 & 0.4 & 0.8 & 0.41604 & 0.54087 & 0.89284 & 0.52749 \\
\hline 0.82656 & 0.42 & 0.84 & 0.50309 & 0.62518 & 0.91152 & 0.61241 \\
\hline 0.86253 & 0.44 & 0.88 & 0.588 & 0.7009 & 0.92868 & 0.68936 \\
\hline 0.89306 & 0.46 & 0.92 & 0.67202 & 0.77063 & 0.94456 & 0.76077 \\
\hline 0.9175 & 0.48 & 0.96 & 0.74807 & 0.82888 & 0.95858 & 0.82096 \\
\hline 0.9433 & 0.5 & 1 & 0.82257 & 0.88344 & 0.97125 & 0.87758 \\
\hline 1 & 0.54 & 1.08 & 1 & 1 & 1 & 1 \\
\hline
\end{tabular}

\begin{tabular}{|c|c|c|c|c|c|c||}
\hline \multicolumn{7}{|c|}{ Diamond Lattice Relative Properties (Poisson ratio = 0.3) } \\
\hline \hline Density & Radius & D/L & $\mathbf{1}^{\text {st }}$ Lame & $\mathbf{2}^{\text {nd }}$ Lame & Poisson Ratio & Modulus \\
\hline \hline 0 & 0 & 0 & 0 & 0 & 1.6786 & 0 \\
\hline 0.010688 & 0.02 & 0.04 & 0.002036 & 0.00011 & 1.6086 & 0.000126 \\
\hline 0.028 & 0.04 & 0.08 & 0.005918 & 0.000893 & 1.5143 & 0.000999 \\
\hline 0.076096 & 0.06 & 0.12 & 0.016669 & 0.006744 & 1.3126 & 0.007231 \\
\hline 0.11309 & 0.08 & 0.16 & 0.025352 & 0.01532 & 1.1881 & 0.015985 \\
\hline 0.17446 & 0.1 & 0.2 & 0.04031 & 0.037437 & 1.0293 & 0.037691 \\
\hline 0.23638 & 0.12 & 0.24 & 0.054934 & 0.065463 & 0.92879 & 0.064388 \\
\hline 0.29795 & 0.14 & 0.28 & 0.070708 & 0.099805 & 0.85866 & 0.09655 \\
\hline 0.36784 & 0.16 & 0.32 & 0.089596 & 0.14172 & 0.81122 & 0.13555 \\
\hline 0.43373 & 0.18 & 0.36 & 0.11334 & 0.18961 & 0.78791 & 0.18033 \\
\hline 0.50134 & 0.2 & 0.4 & 0.14032 & 0.2396 & 0.77943 & 0.2274 \\
\hline 0.56125 & 0.22 & 0.44 & 0.17094 & 0.28986 & 0.7823 & 0.2753 \\
\hline 0.61139 & 0.24 & 0.48 & 0.20329 & 0.33715 & 0.79151 & 0.32093 \\
\hline 0.67197 & 0.26 & 0.52 & 0.2507 & 0.40164 & 0.80592 & 0.38365 \\
\hline 0.72058 & 0.28 & 0.56 & 0.30201 & 0.46224 & 0.82493 & 0.44357 \\
\hline 0.76784 & 0.3 & 0.6 & 0.36167 & 0.52731 & 0.84517 & 0.50847 \\
\hline 0.8048 & 0.32 & 0.64 & 0.42169 & 0.58501 & 0.86586 & 0.5669 \\
\hline 0.83939 & 0.34 & 0.68 & 0.49135 & 0.64623 & 0.88803 & 0.62953 \\
\hline 0.86854 & 0.36 & 0.72 & 0.56392 & 0.70518 & 0.90892 & 0.69036 \\
\hline 0.89002 & 0.38 & 0.76 & 0.62599 & 0.75223 & 0.92536 & 0.73927 \\
\hline 0.91229 & 0.4 & 0.8 & 0.6911 & 0.80101 & 0.94019 & 0.78996 \\
\hline 0.92848 & 0.42 & 0.84 & 0.74467 & 0.83848 & 0.95203 & 0.82919 \\
\hline 0.9433 & 0.44 & 0.88 & 0.79293 & 0.87178 & 0.96174 & 0.86408 \\
\hline 1 & 0.48 & 0.96 & 1 & 1 & 1 & 1 \\
\hline & & & & & & \\
\hline
\end{tabular}




\begin{tabular}{|c|c|c|c|c|c|c|c||}
\hline \multicolumn{7}{|c|}{ Face Centered Cubic (FCC) Lattice Relative Properties (Poisson ratio = 0.3) } \\
\hline \hline Density & Radius & $\mathbf{D} / \mathbf{L}$ & $\mathbf{1}^{\text {st }}$ Lame & $\mathbf{2}^{\text {nd }}$ Lame & Poisson Ratio & Modulus \\
\hline \hline 0 & 0 & 0 & 0 & 0 & 0.86076 & 0 \\
\hline 0.018112 & 0.02 & 0.04 & 0.002305 & 0.003237 & 0.86076 & 0.003133 \\
\hline 0.047744 & 0.04 & 0.08 & 0.006527 & 0.008929 & 0.8717 & 0.008664 \\
\hline 0.11731 & 0.06 & 0.12 & 0.018496 & 0.024657 & 0.88243 & 0.023988 \\
\hline 0.18707 & 0.08 & 0.16 & 0.033134 & 0.044433 & 0.87997 & 0.043202 \\
\hline 0.28122 & 0.1 & 0.2 & 0.056877 & 0.07818 & 0.8697 & 0.075829 \\
\hline 0.36154 & 0.12 & 0.24 & 0.08107 & 0.11499 & 0.85662 & 0.11119 \\
\hline 0.45856 & 0.14 & 0.28 & 0.11867 & 0.1728 & 0.84568 & 0.16665 \\
\hline 0.55501 & 0.16 & 0.32 & 0.17377 & 0.25039 & 0.85008 & 0.24173 \\
\hline 0.64262 & 0.18 & 0.36 & 0.24852 & 0.34124 & 0.87014 & 0.33101 \\
\hline 0.71488 & 0.2 & 0.4 & 0.33222 & 0.43239 & 0.89237 & 0.42165 \\
\hline 0.77504 & 0.22 & 0.44 & 0.41422 & 0.51994 & 0.90736 & 0.50883 \\
\hline 0.82618 & 0.24 & 0.48 & 0.49595 & 0.60609 & 0.91842 & 0.59468 \\
\hline 0.87002 & 0.26 & 0.52 & 0.58476 & 0.69242 & 0.93141 & 0.68146 \\
\hline 0.90387 & 0.28 & 0.56 & 0.67302 & 0.76882 & 0.94613 & 0.75926 \\
\hline 0.93024 & 0.3 & 0.6 & 0.75327 & 0.83226 & 0.95974 & 0.82453 \\
\hline 1 & 0.34 & 0.68 & 1 & 1 & 1 & 1 \\
\hline
\end{tabular}

\begin{tabular}{||c|c|c|c|c|c|c|c||}
\hline \multicolumn{7}{|c|}{ Truncated Octahedron (Octa) Lattice Relative Properties (Poisson ratio = 0.3) } \\
\hline \hline Density & Radius & D/L & $\mathbf{1}^{\text {st }}$ Lame & $\mathbf{2}^{\text {nd }}$ Lame & Poisson Ratio & Modulus \\
\hline \hline 0 & 0 & 0 & 0 & 0 & 1.6645 & 0 \\
\hline 0.009216 & 0.02 & 0.04 & 0.001932 & $5.70 \mathrm{E}-05$ & 1.6345 & $6.54 \mathrm{E}-05$ \\
\hline 0.043392 & 0.04 & 0.08 & 0.009765 & 0.00127 & 1.5337 & 0.001426 \\
\hline 0.082944 & 0.06 & 0.12 & 0.019238 & 0.005034 & 1.4191 & 0.005521 \\
\hline 0.1417 & 0.08 & 0.16 & 0.033064 & 0.015189 & 1.2759 & 0.016156 \\
\hline 0.20813 & 0.1 & 0.2 & 0.049357 & 0.034947 & 1.1322 & 0.036013 \\
\hline 0.27994 & 0.12 & 0.24 & 0.068431 & 0.065214 & 1.0192 & 0.065503 \\
\hline 0.3648 & 0.14 & 0.28 & 0.095437 & 0.11418 & 0.92715 & 0.11227 \\
\hline 0.43622 & 0.16 & 0.32 & 0.12635 & 0.16757 & 0.88458 & 0.16311 \\
\hline 0.51533 & 0.18 & 0.36 & 0.17565 & 0.24573 & 0.86238 & 0.23792 \\
\hline 0.58368 & 0.2 & 0.4 & 0.23178 & 0.32601 & 0.86012 & 0.31549 \\
\hline 0.65741 & 0.22 & 0.44 & 0.30561 & 0.41944 & 0.87034 & 0.40689 \\
\hline 0.71962 & 0.24 & 0.48 & 0.38652 & 0.51374 & 0.88366 & 0.49995 \\
\hline 0.77875 & 0.26 & 0.52 & 0.48445 & 0.61121 & 0.90525 & 0.59785 \\
\hline 0.82944 & 0.28 & 0.56 & 0.57986 & 0.69763 & 0.92487 & 0.68553 \\
\hline 0.86976 & 0.3 & 0.6 & 0.66468 & 0.76591 & 0.94258 & 0.75576 \\
\hline
\end{tabular}




\begin{tabular}{||c|c|c|c|c|c|c||}
\hline 0.90694 & 0.32 & 0.64 & 0.74779 & 0.82845 & 0.95864 & 0.82054 \\
\hline 0.93139 & 0.34 & 0.68 & 0.80826 & 0.87164 & 0.96959 & 0.86553 \\
\hline 0.94957 & 0.36 & 0.72 & 0.85558 & 0.90411 & 0.97781 & 0.89948 \\
\hline 1 & 0.4 & 0.8 & 1 & 1 & 1 & 1 \\
\hline
\end{tabular}

\begin{tabular}{|c|c|c|c|c|c|c||}
\hline \multicolumn{7}{|c|}{ Tesseract Lattice Relative Properties (Poisson ratio $=\mathbf{0 . 3}$ ) } \\
\hline \hline Density & Radius & $\mathbf{D} / \mathbf{L}$ & $\mathbf{1}^{\text {st }}$ Lame & $\mathbf{2}^{\text {nd }}$ Lame & Poisson Ratio & Modulus \\
\hline 0 & 0 & 0 & 0 & 0 & 1.685 & 0 \\
\hline 0.014464 & 0.02 & 0.04 & 0.001878 & $4.08 \mathrm{E}-05$ & 1.6429 & $4.69 \mathrm{E}-05$ \\
\hline 0.048848 & 0.04 & 0.08 & 0.007905 & 0.000908 & 1.5482 & 0.001023 \\
\hline 0.12749 & 0.06 & 0.12 & 0.023981 & 0.006973 & 1.396 & 0.007611 \\
\hline 0.19848 & 0.08 & 0.16 & 0.039099 & 0.017996 & 1.2753 & 0.019139 \\
\hline 0.30057 & 0.1 & 0.2 & 0.065579 & 0.046356 & 1.1328 & 0.047777 \\
\hline 0.40717 & 0.12 & 0.24 & 0.096782 & 0.09392 & 1.012 & 0.09418 \\
\hline 0.51238 & 0.14 & 0.28 & 0.13689 & 0.15971 & 0.9375 & 0.15741 \\
\hline 0.62534 & 0.16 & 0.32 & 0.19419 & 0.25619 & 0.88675 & 0.24949 \\
\hline 0.73677 & 0.18 & 0.36 & 0.36636 & 0.47841 & 0.891 & 0.46638 \\
\hline 0.81378 & 0.2 & 0.4 & 0.48766 & 0.6095 & 0.90915 & 0.59672 \\
\hline 0.88251 & 0.22 & 0.44 & 0.60236 & 0.72807 & 0.92295 & 0.71512 \\
\hline 0.92832 & 0.24 & 0.48 & 0.70223 & 0.8197 & 0.93729 & 0.80783 \\
\hline 1 & 0.28 & 0.56 & 1 & 1 & 1 & 1 \\
\hline
\end{tabular}




\section{F.4 Effective Stresses (Raw Data)}

\begin{tabular}{|c|c|c|c|c|c||c|c||}
\hline \multicolumn{9}{||c|}{ Octet Truss Lattice Raw Effective Stresses at Yielding (Poisson ratio= 0.3) } \\
\hline \hline \multirow{2}{*}{ Density } & $\boldsymbol{\sigma}_{\boldsymbol{x} \boldsymbol{y}}^{\boldsymbol{y}}$ & $\boldsymbol{\sigma}_{\boldsymbol{y} \boldsymbol{y}}$ & $\boldsymbol{\sigma}_{\boldsymbol{z z}}^{\boldsymbol{y}}$ & $\boldsymbol{\sigma}_{\boldsymbol{x y}}^{\boldsymbol{y}}$ & $\boldsymbol{\sigma}_{\boldsymbol{y} \boldsymbol{y}}^{\boldsymbol{y}}$ & $\boldsymbol{\sigma}_{\boldsymbol{x} \boldsymbol{z}}^{\boldsymbol{y}}$ & $\boldsymbol{\sigma}_{\boldsymbol{a v g}}^{\boldsymbol{y}}$ \\
\hline \hline 0 & 0 & 0 & 0 & 0 & 0 & 0 & 0 \\
\hline 0.026752 & 0.006634 & 0.006653 & 0.00665 & 0.008178 & 0.008171 & 0.008175 & 0.00741 \\
\hline 0.068096 & 0.016412 & 0.016456 & 0.016862 & 0.021663 & 0.021683 & 0.021686 & 0.019127 \\
\hline 0.1655 & 0.033445 & 0.033418 & 0.033174 & 0.048539 & 0.048542 & 0.048541 & 0.040943 \\
\hline 0.26445 & 0.048836 & 0.049203 & 0.049143 & 0.083504 & 0.083509 & 0.083501 & 0.066283 \\
\hline 0.39642 & 0.076538 & 0.076458 & 0.076563 & 0.13386 & 0.13386 & 0.13386 & 0.10519 \\
\hline 0.50074 & 0.10894 & 0.1089 & 0.10888 & 0.18292 & 0.18292 & 0.18292 & 0.14591 \\
\hline 0.61888 & 0.14653 & 0.14651 & 0.14648 & 0.22152 & 0.22152 & 0.22152 & 0.18401 \\
\hline 0.73779 & 0.21108 & 0.21108 & 0.21111 & 0.32909 & 0.32909 & 0.32909 & 0.27009 \\
\hline 0.82906 & 0.31011 & 0.31011 & 0.31013 & 0.44365 & 0.44365 & 0.44365 & 0.37688 \\
\hline 0.89498 & 0.42169 & 0.4217 & 0.42174 & 0.47371 & 0.47371 & 0.47371 & 0.44771 \\
\hline 0.9399 & 0.46484 & 0.46485 & 0.46487 & 0.50718 & 0.50718 & 0.50718 & 0.48602 \\
\hline 0.96026 & 0.50249 & 0.50249 & 0.5025 & 0.52788 & 0.52788 & 0.52788 & 0.51519 \\
\hline 1 & 1 & 1 & 1 & 1 & 1 & 1 & 1 \\
\hline
\end{tabular}

\begin{tabular}{||c|c|c|c|c|c|c|c||}
\hline \multicolumn{8}{||c|}{ Body Centered Cubic (BCC) Lattice Raw Effective Stresses at Yielding (Poisson ratio = 0.3) } \\
\hline \hline Density & $\boldsymbol{\sigma}_{\boldsymbol{x} \boldsymbol{y}}^{\boldsymbol{y}}$ & $\boldsymbol{\sigma}_{\boldsymbol{y} \boldsymbol{y}}$ & $\boldsymbol{\sigma}_{\boldsymbol{z z}}^{\boldsymbol{y}}$ & $\boldsymbol{\sigma}_{\boldsymbol{x} \boldsymbol{y}}^{\boldsymbol{y}}$ & $\boldsymbol{\sigma}_{\boldsymbol{y z}}^{\boldsymbol{y}}$ & $\boldsymbol{\sigma}_{\boldsymbol{x} \boldsymbol{z}}^{\boldsymbol{y}}$ & $\boldsymbol{\sigma}_{\boldsymbol{a v g}}^{\boldsymbol{y}}$ \\
\hline 0 & 0 & 0 & 0 & 0 & 0 & 0 & 0 \\
\hline 0.010816 & 0.000385 & 0.000383 & 0.00036 & 0.006635 & 0.006635 & 0.006637 & 0.003506 \\
\hline 0.02848 & 0.001463 & 0.001486 & 0.001193 & 0.015161 & 0.015164 & 0.015206 & 0.008279 \\
\hline 0.078016 & 0.005727 & 0.005574 & 0.005924 & 0.037362 & 0.037368 & 0.037362 & 0.021553 \\
\hline 0.1168 & 0.010932 & 0.010931 & 0.011059 & 0.051131 & 0.051142 & 0.051131 & 0.031054 \\
\hline 0.18208 & 0.020069 & 0.020204 & 0.020088 & 0.07038 & 0.07039 & 0.080781 & 0.046985 \\
\hline 0.24928 & 0.037191 & 0.037299 & 0.037193 & 0.10669 & 0.10669 & 0.10669 & 0.07196 \\
\hline 0.31802 & 0.050891 & 0.05128 & 0.051134 & 0.14468 & 0.14468 & 0.14468 & 0.097891 \\
\hline 0.3975 & 0.083283 & 0.083457 & 0.083313 & 0.17732 & 0.17732 & 0.17732 & 0.13034 \\
\hline 0.47584 & 0.098452 & 0.098245 & 0.098107 & 0.21331 & 0.21331 & 0.21331 & 0.15579 \\
\hline 0.55955 & 0.1609 & 0.16038 & 0.16081 & 0.27561 & 0.27561 & 0.27561 & 0.21816 \\
\hline 0.63789 & 0.22801 & 0.22961 & 0.22857 & 0.32666 & 0.32666 & 0.32666 & 0.2777 \\
\hline 0.7047 & 0.28312 & 0.27563 & 0.28309 & 0.38234 & 0.38234 & 0.38234 & 0.33148 \\
\hline 0.78496 & 0.40234 & 0.39198 & 0.40239 & 0.46368 & 0.46368 & 0.46368 & 0.43129 \\
\hline 0.84832 & 0.46004 & 0.46153 & 0.5082 & 0.65997 & 0.65997 & 0.65997 & 0.56828 \\
\hline 0.90438 & 0.60856 & 0.61309 & 0.61309 & 0.77082 & 0.77082 & 0.77082 & 0.6912 \\
\hline 0.94547 & 0.70872 & 0.6801 & 0.70217 & 0.85833 & 0.85833 & 0.85833 & 0.77766 \\
\hline 0.97619 & 0.81649 & 0.81649 & 0.81649 & 0.96509 & 0.96509 & 0.96509 & 0.89079 \\
\hline
\end{tabular}




\begin{tabular}{|l|l|l|l|l|l|l|l|}
\hline 1 & 1 & 1 & 1 & 1 & 1 & 1 & 1 \\
\hline
\end{tabular}

\begin{tabular}{|c|c|c|c|c|c|c|c|}
\hline \multicolumn{8}{|c|}{$\begin{array}{c}\text { Body Centered Cubic + Cubic (BCCC) Lattice Raw Effective Stresses at Yielding (Poisson ratio = } \\
0.3)\end{array}$} \\
\hline Density & $\sigma_{x x}^{y}$ & $\sigma_{y y}^{y}$ & $\sigma_{z z}^{y}$ & $\sigma_{x y}^{y}$ & $\sigma_{y z}^{y}$ & $\sigma_{x z}^{y}$ & $\sigma_{a v g}^{y}$ \\
\hline 0 & 0 & 0 & 0 & 0 & 0 & 0 & 0 \\
\hline 0.015232 & 0.002061 & 0.002061 & 0.002081 & 0.006594 & 0.006592 & 0.006596 & 0.004331 \\
\hline 0.041152 & 0.006218 & 0.006169 & 0.006324 & 0.014809 & 0.014827 & 0.014841 & 0.010531 \\
\hline 0.10854 & 0.021925 & 0.021968 & 0.021883 & 0.037828 & 0.037793 & 0.037782 & 0.029863 \\
\hline 0.16346 & 0.036448 & 0.036627 & 0.036575 & 0.059397 & 0.059376 & 0.059382 & 0.047967 \\
\hline 0.24698 & 0.056288 & 0.05887 & 0.056254 & 0.084881 & 0.084885 & 0.084882 & 0.07101 \\
\hline 0.33222 & 0.086244 & 0.086204 & 0.08614 & 0.1262 & 0.1262 & 0.12619 & 0.1062 \\
\hline 0.42208 & 0.18056 & 0.17937 & 0.18161 & 0.15978 & 0.1598 & 0.15978 & 0.17015 \\
\hline 0.51942 & 0.24917 & 0.24958 & 0.24971 & 0.20553 & 0.20556 & 0.20552 & 0.22751 \\
\hline 0.60736 & 0.2561 & 0.25611 & 0.25686 & 0.27611 & 0.27614 & 0.2761 & 0.26624 \\
\hline 0.69683 & 0.39206 & 0.3923 & 0.39163 & 0.35402 & 0.35403 & 0.35401 & 0.37301 \\
\hline 0.77536 & 0.4837 & 0.48385 & 0.48376 & 0.45082 & 0.45083 & 0.45082 & 0.4673 \\
\hline 0.83565 & 0.55587 & 0.57944 & 0.5798 & 0.5402 & 0.5402 & 0.5402 & 0.55595 \\
\hline 0.89498 & 0.67236 & 0.67228 & 0.69807 & 0.65199 & 0.65199 & 0.65199 & 0.66645 \\
\hline 0.93242 & 0.71727 & 0.76164 & 0.71736 & 0.75047 & 0.75047 & 0.75047 & 0.74128 \\
\hline 0.95834 & 0.82447 & 0.78224 & 0.75311 & 0.96069 & 0.96069 & 0.96069 & 0.87365 \\
\hline 0.97677 & 0.79442 & 0.7946 & 0.79707 & 0.99336 & 0.99336 & 0.99336 & 0.89436 \\
\hline 1 & 1 & 1 & 1 & 1 & 1 & 1 & 1 \\
\hline
\end{tabular}

\begin{tabular}{||c|c|c|c|c||c||c|c||}
\hline \multicolumn{8}{||c|}{ Cubic Lattice Raw Effective Stresses at Yielding (Poisson ratio = 0.3) } \\
\hline \hline \multirow{2}{*}{ Density } & $\boldsymbol{\sigma}_{\boldsymbol{x} \boldsymbol{y}}^{\boldsymbol{y}}$ & $\boldsymbol{\sigma}_{\boldsymbol{y} \boldsymbol{y}}^{\boldsymbol{y}}$ & $\boldsymbol{\sigma}_{\boldsymbol{z} \boldsymbol{z}}^{\boldsymbol{y}}$ & $\boldsymbol{\sigma}_{\boldsymbol{x} \boldsymbol{y}}^{\boldsymbol{y}}$ & $\boldsymbol{\sigma}_{\boldsymbol{y} \boldsymbol{y}}^{\boldsymbol{y}}$ & $\boldsymbol{\sigma}_{\boldsymbol{x} \boldsymbol{z}}^{\boldsymbol{y}}$ & $\boldsymbol{\sigma}_{\boldsymbol{a v g}}^{\boldsymbol{y}}$ \\
\hline \hline 0 & 0 & 0 & 0 & 0 & 0 & 0 & 0 \\
\hline 0.004672 & 0.001598 & 0.001601 & 0.001602 & 0.00012 & 0.000123 & 0.000122 & 0.000861 \\
\hline 0.013696 & 0.004831 & 0.004825 & 0.004828 & 0.000628 & 0.000628 & 0.000631 & 0.002728 \\
\hline 0.035456 & 0.016098 & 0.016103 & 0.016044 & 0.002252 & 0.002209 & 0.002205 & 0.009152 \\
\hline 0.056256 & 0.025491 & 0.025575 & 0.025538 & 0.00357 & 0.003568 & 0.003582 & 0.014554 \\
\hline 0.084352 & 0.038302 & 0.03844 & 0.038315 & 0.006188 & 0.006177 & 0.006172 & 0.022265 \\
\hline 0.11507 & 0.052684 & 0.052862 & 0.052662 & 0.009361 & 0.009351 & 0.009332 & 0.031042 \\
\hline 0.15526 & 0.073969 & 0.074259 & 0.073957 & 0.016411 & 0.016413 & 0.016367 & 0.045229 \\
\hline 0.20083 & 0.098163 & 0.098448 & 0.09812 & 0.025798 & 0.025812 & 0.025759 & 0.062017 \\
\hline 0.24038 & 0.11925 & 0.11976 & 0.11941 & 0.034499 & 0.034508 & 0.034458 & 0.076979 \\
\hline 0.28787 & 0.14741 & 0.14786 & 0.14766 & 0.047901 & 0.047914 & 0.047862 & 0.097767 \\
\hline
\end{tabular}




\begin{tabular}{||c|c|c|c|c|c|c|c||}
\hline 0.33843 & 0.17902 & 0.17928 & 0.17904 & 0.064841 & 0.064924 & 0.064807 & 0.12199 \\
\hline 0.38298 & 0.20654 & 0.20657 & 0.20644 & 0.081261 & 0.08129 & 0.081243 & 0.14389 \\
\hline 0.4439 & 0.24698 & 0.24713 & 0.24144 & 0.11123 & 0.11123 & 0.11121 & 0.1782 \\
\hline 0.49005 & 0.2827 & 0.28272 & 0.28264 & 0.13761 & 0.1376 & 0.1376 & 0.21015 \\
\hline 0.54771 & 0.3263 & 0.32117 & 0.32634 & 0.1816 & 0.1816 & 0.18159 & 0.2531 \\
\hline 0.59802 & 0.36614 & 0.36619 & 0.36628 & 0.22706 & 0.22705 & 0.22705 & 0.29663 \\
\hline 0.64666 & 0.39863 & 0.40699 & 0.40702 & 0.27349 & 0.27349 & 0.27349 & 0.33885 \\
\hline 0.69453 & 0.44997 & 0.44999 & 0.45 & 0.32039 & 0.32039 & 0.32039 & 0.38519 \\
\hline 0.73542 & 0.48864 & 0.47733 & 0.48864 & 0.37799 & 0.37798 & 0.37799 & 0.43143 \\
\hline 0.78656 & 0.5627 & 0.53927 & 0.53928 & 0.44539 & 0.44539 & 0.44539 & 0.49624 \\
\hline 0.82656 & 0.56082 & 0.61646 & 0.61648 & 0.50901 & 0.50901 & 0.50901 & 0.55347 \\
\hline 0.86253 & 0.61012 & 0.63293 & 0.63295 & 0.57585 & 0.57585 & 0.57585 & 0.60059 \\
\hline 0.89306 & 0.64617 & 0.69745 & 0.67338 & 0.62221 & 0.62221 & 0.62221 & 0.64727 \\
\hline 0.9175 & 0.70138 & 0.68946 & 0.6532 & 0.6783 & 0.6783 & 0.6783 & 0.67982 \\
\hline 0.9433 & 0.71547 & 0.71476 & 0.7085 & 0.72622 & 0.72622 & 0.72622 & 0.71956 \\
\hline 0.95789 & 0.73335 & 0.73335 & 0.76306 & 0.76297 & 0.76297 & 0.76297 & 0.75311 \\
\hline 0.97158 & 0.76607 & 0.76607 & 0.76607 & 0.85224 & 0.85224 & 0.85224 & 0.80915 \\
\hline 1 & 1 & 1 & 1 & 1 & 1 & 1 & 1 \\
\hline \hline
\end{tabular}

\begin{tabular}{||c|c|c|c|c|c|c|c||}
\hline \multicolumn{8}{|c|}{ Diamond Lattice Raw Effective Stresses at Yielding (Poisson ratio = 0.3) } \\
\hline \hline \multirow{2}{*}{ Density } & $\boldsymbol{\sigma}_{\boldsymbol{x} \boldsymbol{y}}$ & $\boldsymbol{\sigma}_{\boldsymbol{y} \boldsymbol{y}}^{\boldsymbol{y}}$ & $\boldsymbol{\sigma}_{\boldsymbol{z z}}^{\boldsymbol{y}}$ & $\boldsymbol{\sigma}_{\boldsymbol{x} \boldsymbol{y}}^{\boldsymbol{y}}$ & $\boldsymbol{\sigma}_{\boldsymbol{y z}}^{\boldsymbol{y}}$ & $\boldsymbol{\sigma}_{\boldsymbol{x} \boldsymbol{z}}^{\boldsymbol{y}}$ & $\boldsymbol{\sigma}_{\boldsymbol{a} \boldsymbol{g}}^{\boldsymbol{y}}$ \\
\hline \hline 0 & 0 & 0 & 0 & 0 & 0 & 0 & 0 \\
\hline 0.010688 & 0.00054 & 0.000534 & 0.000531 & 0.000661 & 0.000624 & 0.000741 & 0.000605 \\
\hline 0.028 & 0.002016 & 0.002021 & 0.001959 & 0.001741 & 0.001786 & 0.001857 & 0.001897 \\
\hline 0.076096 & 0.005171 & 0.005061 & 0.005173 & 0.005624 & 0.00566 & 0.005708 & 0.0054 \\
\hline 0.11309 & 0.007858 & 0.008093 & 0.007477 & 0.010253 & 0.010272 & 0.010311 & 0.009044 \\
\hline 0.17446 & 0.017971 & 0.018032 & 0.017482 & 0.020297 & 0.020304 & 0.020351 & 0.019073 \\
\hline 0.23638 & 0.026956 & 0.027064 & 0.025964 & 0.027844 & 0.027818 & 0.027812 & 0.027243 \\
\hline 0.29795 & 0.034894 & 0.034961 & 0.035404 & 0.037607 & 0.037568 & 0.037551 & 0.036331 \\
\hline 0.36784 & 0.045893 & 0.04584 & 0.045774 & 0.050661 & 0.050648 & 0.050621 & 0.04824 \\
\hline 0.43373 & 0.057894 & 0.058725 & 0.058755 & 0.072891 & 0.072937 & 0.072881 & 0.06568 \\
\hline 0.50134 & 0.0725 & 0.072413 & 0.072423 & 0.088838 & 0.088852 & 0.088825 & 0.080642 \\
\hline 0.56125 & 0.089519 & 0.089462 & 0.089476 & 0.10665 & 0.10667 & 0.10665 & 0.098071 \\
\hline 0.61139 & 0.10554 & 0.10547 & 0.1055 & 0.12687 & 0.12689 & 0.12688 & 0.11619 \\
\hline 0.67197 & 0.13398 & 0.13389 & 0.13388 & 0.15557 & 0.15557 & 0.15557 & 0.14474 \\
\hline 0.72058 & 0.17346 & 0.17346 & 0.17336 & 0.19285 & 0.19285 & 0.19286 & 0.18314 \\
\hline 0.76784 & 0.25904 & 0.25905 & 0.25895 & 0.26601 & 0.26601 & 0.26601 & 0.26251 \\
\hline 0.8048 & 0.30339 & 0.3034 & 0.30335 & 0.34804 & 0.34804 & 0.34805 & 0.32571 \\
\hline
\end{tabular}




\begin{tabular}{||c|c|c|c|c|c|c|c||}
\hline 0.83939 & 0.40847 & 0.40872 & 0.4082 & 0.32706 & 0.34084 & 0.32707 & 0.37006 \\
\hline 0.86854 & 0.45455 & 0.45452 & 0.45345 & 0.38382 & 0.38382 & 0.38382 & 0.419 \\
\hline 0.89002 & 0.4871 & 0.48826 & 0.48825 & 0.42692 & 0.42693 & 0.42693 & 0.4574 \\
\hline 0.91229 & 0.51036 & 0.51035 & 0.51035 & 0.54531 & 0.54531 & 0.54532 & 0.52783 \\
\hline 0.92848 & 0.552 & 0.54852 & 0.55199 & 0.65879 & 0.65879 & 0.6588 & 0.60482 \\
\hline 0.9433 & 0.60809 & 0.59459 & 0.59399 & 0.75002 & 0.75002 & 0.75003 & 0.67446 \\
\hline 0.9559 & 0.73298 & 0.66299 & 0.73298 & 0.81771 & 0.81771 & 0.81772 & 0.76368 \\
\hline 0.96381 & 0.75679 & 0.75208 & 0.72614 & 0.84598 & 0.84598 & 0.84598 & 0.79549 \\
\hline 0.9744 & 0.85218 & 0.82693 & 0.85556 & 0.88929 & 0.88929 & 0.88929 & 0.86709 \\
\hline 1 & 1 & 1 & 1 & 1 & 1 & 1 & 1 \\
\hline
\end{tabular}

\begin{tabular}{||c|c|c|c|c|c||c|c||}
\hline \multicolumn{6}{||c|}{ Face Centered Cubic (FCC) Lattice Raw Effective Stresses at Yielding (Poisson ratio= 0.3) } \\
\hline \hline \multirow{2}{*}{ Density } & $\boldsymbol{\sigma}_{\boldsymbol{x} \boldsymbol{y}}$ & $\boldsymbol{\sigma}_{\boldsymbol{y} \boldsymbol{y}}$ & $\boldsymbol{\sigma}_{\boldsymbol{z z}}^{\boldsymbol{y}}$ & $\boldsymbol{\sigma}_{\boldsymbol{x} \boldsymbol{y}}^{\boldsymbol{y}}$ & $\boldsymbol{\sigma}_{\boldsymbol{y z}}^{\boldsymbol{y}}$ & $\boldsymbol{\sigma}_{\boldsymbol{x} \boldsymbol{y}}^{\boldsymbol{y}}$ & $\boldsymbol{\sigma}_{\boldsymbol{a v g}}^{\boldsymbol{y}}$ \\
\hline \hline 0 & 0 & 0 & 0 & 0 & 0 & 0 & 0 \\
\hline 0.018112 & 0.00341 & 0.003359 & 0.003439 & 0.002599 & 0.002599 & 0.002601 & 0.003001 \\
\hline 0.047744 & 0.009685 & 0.009571 & 0.009752 & 0.006336 & 0.006338 & 0.006335 & 0.008003 \\
\hline 0.11731 & 0.025155 & 0.025244 & 0.025394 & 0.015857 & 0.015835 & 0.015848 & 0.020556 \\
\hline 0.18707 & 0.03974 & 0.039702 & 0.040094 & 0.027354 & 0.027145 & 0.027157 & 0.033532 \\
\hline 0.28122 & 0.049879 & 0.05 & 0.049905 & 0.045645 & 0.045624 & 0.045635 & 0.047781 \\
\hline 0.36154 & 0.067295 & 0.067413 & 0.067402 & 0.060438 & 0.06039 & 0.06039 & 0.063888 \\
\hline 0.45856 & 0.093869 & 0.093867 & 0.09386 & 0.080786 & 0.080786 & 0.080777 & 0.087324 \\
\hline 0.55501 & 0.12332 & 0.12339 & 0.12341 & 0.099467 & 0.099471 & 0.099459 & 0.11142 \\
\hline 0.64262 & 0.16998 & 0.17002 & 0.17005 & 0.14263 & 0.14263 & 0.14263 & 0.15632 \\
\hline 0.71488 & 0.26697 & 0.26708 & 0.26702 & 0.21047 & 0.21047 & 0.21047 & 0.23875 \\
\hline 0.77504 & 0.31762 & 0.31762 & 0.31765 & 0.24185 & 0.24185 & 0.24185 & 0.27974 \\
\hline 0.82618 & 0.3448 & 0.34482 & 0.34483 & 0.27435 & 0.27435 & 0.27435 & 0.30958 \\
\hline 0.87002 & 0.37952 & 0.37957 & 0.37955 & 0.29511 & 0.29511 & 0.29511 & 0.33733 \\
\hline 0.90387 & 0.42697 & 0.42701 & 0.427 & 0.33225 & 0.33225 & 0.33225 & 0.37962 \\
\hline 0.93024 & 0.46065 & 0.46068 & 0.46068 & 0.37899 & 0.37899 & 0.37899 & 0.41983 \\
\hline 0.95008 & 0.48044 & 0.48045 & 0.48045 & 0.41902 & 0.41902 & 0.41902 & 0.44973 \\
\hline 0.9655 & 0.50019 & 0.50019 & 0.50019 & 0.46002 & 0.46002 & 0.46002 & 0.48011 \\
\hline 0.97728 & 0.5305 & 0.5305 & 0.5305 & 0.50505 & 0.50505 & 0.50505 & 0.51777 \\
\hline 1 & 1 & 1 & 1 & 1 & 1 & 1 & 1 \\
\hline \hline
\end{tabular}

\begin{tabular}{|c||c|c|c|c|c||c|c||}
\hline \multicolumn{6}{||c|}{ Truncated Octahedron (Octa) Lattice Raw Effective Stresses at Yielding (Poisson ratio =0.3) } \\
\hline \hline \multirow{2}{*}{ Density } & $\sigma_{x \boldsymbol{x}}^{\boldsymbol{y}}$ & $\sigma_{y y}^{\boldsymbol{y}}$ & $\sigma_{z z}^{\boldsymbol{y}}$ & $\sigma_{x y}^{\boldsymbol{y}}$ & $\sigma_{y z}^{\boldsymbol{y}}$ & $\sigma_{x z}^{y}$ & $\sigma_{a v g}^{\boldsymbol{y}}$ \\
\hline \hline 0 & 0 & 0 & 0 & 0 & 0 & 0 & 0 \\
\hline
\end{tabular}




\begin{tabular}{||c|c|c|c|c|c|c|c||}
\hline 0.009216 & 0.000261 & 0.000371 & 0.000329 & 0.000681 & 0.000427 & 0.000309 & 0.000396 \\
\hline 0.043392 & 0.001384 & 0.002367 & 0.002901 & 0.001695 & 0.001527 & 0.001391 & 0.001877 \\
\hline 0.082944 & 0.003877 & 0.005482 & 0.007401 & 0.003635 & 0.003478 & 0.003365 & 0.00454 \\
\hline 0.1417 & 0.008726 & 0.010593 & 0.01208 & 0.009196 & 0.009113 & 0.009053 & 0.009794 \\
\hline 0.20813 & 0.018543 & 0.022614 & 0.02482 & 0.022243 & 0.022205 & 0.022143 & 0.022095 \\
\hline 0.27994 & 0.034177 & 0.041198 & 0.041398 & 0.046309 & 0.046274 & 0.046232 & 0.042598 \\
\hline 0.3648 & 0.065381 & 0.068886 & 0.068753 & 0.074754 & 0.074755 & 0.074736 & 0.071211 \\
\hline 0.43622 & 0.098045 & 0.098444 & 0.098422 & 0.10067 & 0.10067 & 0.10066 & 0.099486 \\
\hline 0.51533 & 0.14326 & 0.14348 & 0.1436 & 0.16675 & 0.16675 & 0.16674 & 0.1551 \\
\hline 0.58368 & 0.17549 & 0.17559 & 0.17562 & 0.20817 & 0.20817 & 0.20817 & 0.19187 \\
\hline 0.65741 & 0.20976 & 0.2098 & 0.20985 & 0.27297 & 0.27297 & 0.27297 & 0.24139 \\
\hline 0.71962 & 0.24857 & 0.24855 & 0.24857 & 0.33697 & 0.33697 & 0.33697 & 0.29277 \\
\hline 0.77875 & 0.29456 & 0.29456 & 0.29459 & 0.40217 & 0.40217 & 0.40217 & 0.34837 \\
\hline 0.82944 & 0.32905 & 0.32904 & 0.32906 & 0.44426 & 0.44426 & 0.44426 & 0.38665 \\
\hline 0.86976 & 0.41756 & 0.41756 & 0.41757 & 0.49375 & 0.49375 & 0.49375 & 0.45566 \\
\hline 0.90694 & 0.44855 & 0.44855 & 0.44855 & 0.52828 & 0.52828 & 0.52828 & 0.48841 \\
\hline 0.93139 & 0.47541 & 0.47541 & 0.47542 & 0.52053 & 0.52053 & 0.52053 & 0.49797 \\
\hline 0.94957 & 0.50095 & 0.50095 & 0.50095 & 0.53379 & 0.53379 & 0.53379 & 0.51737 \\
\hline 0.96659 & 0.52681 & 0.52681 & 0.52681 & 0.53141 & 0.53141 & 0.53141 & 0.52911 \\
\hline 0.97786 & 0.56447 & 0.56447 & 0.56447 & 0.55765 & 0.55765 & 0.55765 & 0.56106 \\
\hline 1 & 1 & 1 & 1 & 1 & 1 & 1 & 1 \\
\hline
\end{tabular}

\begin{tabular}{||c|c|c|c|c|c||c|c||}
\hline \multicolumn{9}{||c|}{ Tesseract Lattice Raw Effective Stresses at Yielding (Poisson ratio = 0.3) } \\
\hline \hline \multirow{2}{*}{ Density } & $\boldsymbol{\sigma}_{\boldsymbol{x} \boldsymbol{y}}^{\boldsymbol{y}}$ & $\boldsymbol{\sigma}_{\boldsymbol{y} \boldsymbol{y}}$ & $\boldsymbol{\sigma}_{\boldsymbol{z z}}^{\boldsymbol{y}}$ & $\boldsymbol{\sigma}_{\boldsymbol{x} \boldsymbol{y}}$ & $\boldsymbol{\sigma}_{\boldsymbol{y z}}^{\boldsymbol{y}}$ & $\boldsymbol{\sigma}_{\boldsymbol{x} \boldsymbol{z}}^{\boldsymbol{y}}$ & $\boldsymbol{\sigma}_{\boldsymbol{a v g}}^{\boldsymbol{y}}$ \\
\hline \hline 0 & 0 & 0 & 0 & 0 & 0 & 0 & 0 \\
\hline 0.014464 & 0.002075 & 0.002073 & 0.002074 & 0.000267 & 0.000267 & 0.000277 & 0.001172 \\
\hline 0.048848 & 0.00749 & 0.007541 & 0.007111 & 0.00258 & 0.002427 & 0.002396 & 0.004925 \\
\hline 0.12749 & 0.017835 & 0.018045 & 0.01789 & 0.005411 & 0.005178 & 0.005736 & 0.011682 \\
\hline 0.19848 & 0.030167 & 0.029546 & 0.02918 & 0.010083 & 0.009988 & 0.009869 & 0.019806 \\
\hline 0.30057 & 0.038028 & 0.037364 & 0.037936 & 0.018961 & 0.018803 & 0.018729 & 0.028304 \\
\hline 0.40717 & 0.06379 & 0.064004 & 0.064666 & 0.03184 & 0.031855 & 0.031799 & 0.047992 \\
\hline 0.51238 & 0.076247 & 0.076162 & 0.076572 & 0.048882 & 0.048941 & 0.048854 & 0.062609 \\
\hline 0.62534 & 0.10778 & 0.10784 & 0.10765 & 0.10353 & 0.1036 & 0.1035 & 0.10565 \\
\hline 0.73677 & 0.16988 & 0.16979 & 0.16964 & 0.14586 & 0.14587 & 0.14586 & 0.15782 \\
\hline 0.81378 & 0.24697 & 0.24697 & 0.24682 & 0.20055 & 0.20055 & 0.20053 & 0.22373 \\
\hline 0.88251 & 0.30796 & 0.30795 & 0.30778 & 0.29349 & 0.29349 & 0.29346 & 0.30069 \\
\hline 0.92832 & 0.28838 & 0.28831 & 0.2883 & 0.37342 & 0.37342 & 0.37342 & 0.33087 \\
\hline 0.9687 & 0.44501 & 0.44504 & 0.44507 & 0.45613 & 0.45613 & 0.45613 & 0.45059 \\
\hline
\end{tabular}




\begin{tabular}{|l|l|l|l|l|l|l|l|}
\hline 1 & 1 & 1 & 1 & 1 & 1 & 1 & 1 \\
\hline
\end{tabular}




\section{F.5 Effective Stresses (Curve Fitted)}

\begin{tabular}{|c|c|c|c|c|c||c|c||}
\hline \multicolumn{8}{|c|}{ Octet Truss Lattice Curve Fitted Effective Stresses at Yielding (Poisson ratio = 0.3) } \\
\hline \multirow{2}{*}{ Density } & $\boldsymbol{\sigma}_{\boldsymbol{x} \boldsymbol{y}}^{\boldsymbol{y}}$ & $\boldsymbol{\sigma}_{\boldsymbol{y} \boldsymbol{y}}$ & $\boldsymbol{\sigma}_{\mathbf{z z}}^{\boldsymbol{y}}$ & $\boldsymbol{\sigma}_{\boldsymbol{x} \boldsymbol{y}}^{\boldsymbol{y}}$ & $\boldsymbol{\sigma}_{\boldsymbol{y z}}^{\boldsymbol{y}}$ & $\boldsymbol{\sigma}_{\boldsymbol{x} \boldsymbol{z}}^{\boldsymbol{y}}$ & $\boldsymbol{\sigma}_{\boldsymbol{a v g}}^{\boldsymbol{y}}$ \\
\hline \hline 0 & 0 & 0 & 0 & 0 & 0 & 0 & 0 \\
\hline 0.026752 & 0.006634 & 0.006653 & 0.00665 & 0.008178 & 0.008171 & 0.008175 & 0.00741 \\
\hline 0.068096 & 0.016412 & 0.016456 & 0.016862 & 0.021663 & 0.021683 & 0.021686 & 0.019127 \\
\hline 0.1655 & 0.033445 & 0.033418 & 0.033174 & 0.048539 & 0.048542 & 0.048541 & 0.040943 \\
\hline 0.26445 & 0.048836 & 0.049203 & 0.049143 & 0.083504 & 0.083509 & 0.083501 & 0.066283 \\
\hline 0.39642 & 0.076538 & 0.076458 & 0.076563 & 0.13386 & 0.13386 & 0.13386 & 0.10519 \\
\hline 0.50074 & 0.10894 & 0.1089 & 0.10888 & 0.18292 & 0.18292 & 0.18292 & 0.145913 \\
\hline 0.61888 & 0.14653 & 0.14651 & 0.14648 & 0.22152 & 0.22152 & 0.22152 & 0.184013 \\
\hline 0.73779 & 0.21108 & 0.21108 & 0.21111 & 0.32909 & 0.32909 & 0.32909 & 0.27009 \\
\hline 0.82906 & 0.31011 & 0.31011 & 0.31013 & 0.44365 & 0.44365 & 0.44365 & 0.376883 \\
\hline 0.89498 & 0.42169 & 0.4217 & 0.42174 & 0.59677 & 0.59677 & 0.59677 & 0.53031 \\
\hline 0.9399 & 0.604428 & 0.604428 & 0.604428 & 0.739513 & 0.739513 & 0.739513 & 0.687743 \\
\hline 1 & 1 & 1 & 1 & 1 & 1 & 1 & 1 \\
\hline \hline
\end{tabular}

\begin{tabular}{||c|c|c|c|c|c|c|c||}
\hline \multicolumn{6}{||c|}{ Body Centered Cubic (BCC) Lattice Curve Fitted Effective Stresses at Yielding (Poisson ratio = } \\
\hline \hline Density & $\sigma_{\boldsymbol{x} \boldsymbol{y}}^{\boldsymbol{y}}$ & $\boldsymbol{\sigma}_{\boldsymbol{y y}}^{\boldsymbol{y}}$ & $\boldsymbol{\sigma}_{\boldsymbol{z z}}^{\boldsymbol{y}}$ & $\boldsymbol{\sigma}_{\boldsymbol{x y}}^{\boldsymbol{y}}$ & $\boldsymbol{\sigma}_{\boldsymbol{y z}}^{\boldsymbol{y}}$ & $\boldsymbol{\sigma}_{\boldsymbol{x} \boldsymbol{y}}^{\boldsymbol{y}}$ & $\boldsymbol{\sigma}_{\boldsymbol{a v g}}^{\boldsymbol{y}}$ \\
\hline \hline 0 & 0 & 0 & 0 & 0 & 0 & 0 & 0 \\
\hline 0.010816 & 0.000191 & 0.000193 & 0.00018 & 0.004484 & 0.004484 & 0.004484 & 0.002336 \\
\hline 0.02848 & 0.000719 & 0.000742 & 0.000579 & 0.009657 & 0.009657 & 0.009657 & 0.005168 \\
\hline 0.078016 & 0.002884 & 0.002779 & 0.00304 & 0.020018 & 0.020018 & 0.020018 & 0.01146 \\
\hline 0.1168 & 0.004756 & 0.00473 & 0.004907 & 0.028318 & 0.028318 & 0.028318 & 0.016558 \\
\hline 0.18208 & 0.007944 & 0.008059 & 0.007895 & 0.04474 & 0.04474 & 0.04474 & 0.026353 \\
\hline 0.24928 & 0.015507 & 0.015639 & 0.01555 & 0.060712 & 0.060712 & 0.060712 & 0.038139 \\
\hline 0.31802 & 0.020459 & 0.020571 & 0.020512 & 0.069104 & 0.069104 & 0.069104 & 0.044809 \\
\hline 0.3975 & 0.032843 & 0.032859 & 0.032818 & 0.089428 & 0.089428 & 0.089428 & 0.061134 \\
\hline 0.47584 & 0.038635 & 0.038644 & 0.038606 & 0.11468 & 0.11468 & 0.11468 & 0.076654 \\
\hline 0.55955 & 0.062923 & 0.062938 & 0.062939 & 0.14301 & 0.14301 & 0.14301 & 0.102972 \\
\hline 0.63789 & 0.08593 & 0.085937 & 0.085979 & 0.17703 & 0.17703 & 0.17703 & 0.131489 \\
\hline 0.7047 & 0.10608 & 0.10607 & 0.10606 & 0.195046 & 0.195046 & 0.195046 & 0.150558 \\
\hline 0.78496 & 0.15369 & 0.1537 & 0.1537 & 0.24285 & 0.24285 & 0.24285 & 0.198273 \\
\hline 0.84832 & 0.203794 & 0.203794 & 0.203794 & 0.302524 & 0.302524 & 0.302524 & 0.253159 \\
\hline 0.90438 & 0.347715 & 0.347715 & 0.347715 & 0.436916 & 0.436916 & 0.436916 & 0.392315 \\
\hline 0.94547 & 0.544559 & 0.544559 & 0.544559 & 0.61126 & 0.61126 & 0.61126 & 0.57791 \\
\hline
\end{tabular}




\begin{tabular}{|l|l|l|l|l|l|l|l|}
\hline 1 & 1 & 1 & 1 & 1 & 1 & 1 & 1 \\
\hline
\end{tabular}

\begin{tabular}{|c|c|c|c|c|c|c|c|}
\hline \multicolumn{8}{|c|}{$\begin{array}{c}\text { Body Centered Cubic + Cubic (BCCC) Lattice Curve Fit. Effective Stresses at Yielding (Poisson } \\
\text { ratio }=0.3)\end{array}$} \\
\hline Density & $\sigma_{x x}^{y}$ & $\sigma_{y y}^{y}$ & $\sigma_{z z}^{y}$ & $\sigma_{x y}^{y}$ & $\sigma_{y z}^{y}$ & $\sigma_{x z}^{y}$ & $\sigma_{a v g}^{y}$ \\
\hline 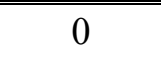 & 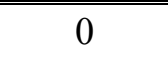 & 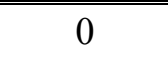 & 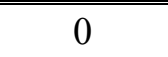 & 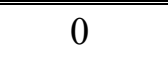 & 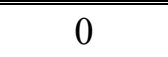 & 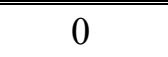 & 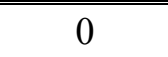 \\
\hline 0.015232 & 0.002048 & 0.002052 & 0.002076 & 0.004432 & 0.004431 & 0.004434 & 0.003245 \\
\hline 0.041152 & 0.006205 & 0.006127 & 0.006313 & 0.009343 & 0.009354 & 0.009363 & 0.007784 \\
\hline 0.10854 & 0.015384 & 0.015602 & 0.015316 & 0.02032 & 0.020301 & 0.020295 & 0.01787 \\
\hline 0.16346 & 0.02385 & 0.024106 & 0.023893 & 0.029668 & 0.029658 & 0.029661 & 0.026806 \\
\hline 0.24698 & 0.034455 & 0.034584 & 0.03454 & 0.046791 & 0.046793 & 0.046791 & 0.040659 \\
\hline 0.33222 & 0.050646 & 0.050537 & 0.050706 & 0.064669 & 0.064672 & 0.064667 & 0.05765 \\
\hline 0.42208 & 0.071696 & 0.071666 & 0.071828 & 0.075521 & 0.075528 & 0.075519 & 0.073626 \\
\hline 0.51942 & 0.096303 & 0.096314 & 0.096376 & 0.10241 & 0.10243 & 0.10241 & 0.099374 \\
\hline 0.60736 & 0.115395 & 0.118214 & 0.116805 & 0.13714 & 0.13715 & 0.13713 & 0.126972 \\
\hline 0.69683 & 0.143689 & 0.144973 & 0.144331 & 0.185755 & 0.185755 & 0.185755 & 0.165043 \\
\hline 0.77536 & 0.188822 & 0.182286 & 0.185554 & 0.228326 & 0.228326 & 0.228326 & 0.20694 \\
\hline 0.83565 & 0.262307 & 0.246665 & 0.254486 & 0.288323 & 0.288323 & 0.288323 & 0.271405 \\
\hline 0.89498 & 0.404064 & 0.381298 & 0.392681 & 0.41075 & 0.41075 & 0.41075 & 0.401716 \\
\hline 0.93242 & 0.55175 & 0.529157 & 0.540454 & 0.547935 & 0.547935 & 0.547935 & 0.544194 \\
\hline 1 & 1 & 1 & 1 & 1 & 1 & 1 & 1 \\
\hline
\end{tabular}

\begin{tabular}{||c|c|c|c||c||c||c|c||}
\hline \multicolumn{7}{||c|}{ Cubic Lattice Curve Fitted Effective Stresses at Yielding (Poisson ratio = 0.3) } \\
\hline \hline Density & $\boldsymbol{\sigma}_{\boldsymbol{x} \boldsymbol{y}}^{\boldsymbol{y}}$ & $\boldsymbol{\sigma}_{\boldsymbol{y} \boldsymbol{y}}$ & $\boldsymbol{\sigma}_{\mathbf{z z}}^{\boldsymbol{y}}$ & $\boldsymbol{\sigma}_{\boldsymbol{x} \boldsymbol{y}}^{\boldsymbol{y}}$ & $\boldsymbol{\sigma}_{\boldsymbol{y} \boldsymbol{z}}$ & $\boldsymbol{\sigma}_{\boldsymbol{x} \boldsymbol{z}}^{\boldsymbol{y}}$ & $\boldsymbol{\sigma}_{\boldsymbol{a v g}}^{\boldsymbol{y}}$ \\
\hline \hline 0 & 0 & 0 & 0 & 0 & 0 & 0 & 0 \\
\hline 0.004672 & $1.60 \mathrm{E}-03$ & $1.60 \mathrm{E}-03$ & $1.60 \mathrm{E}-03$ & $1.10 \mathrm{E}-04$ & $1.12 \mathrm{E}-04$ & $1.11 \mathrm{E}-04$ & $8.54 \mathrm{E}-04$ \\
\hline 0.013696 & $4.77 \mathrm{E}-03$ & $4.77 \mathrm{E}-03$ & $4.77 \mathrm{E}-03$ & $3.19 \mathrm{E}-04$ & $3.19 \mathrm{E}-04$ & $3.20 \mathrm{E}-04$ & $2.55 \mathrm{E}-03$ \\
\hline 0.035456 & 0.012173 & 0.0122 & 0.012151 & 0.001227 & 0.001195 & 0.001202 & 0.006691 \\
\hline 0.056256 & 0.01817 & 0.018203 & 0.018135 & 0.001873 & 0.001872 & 0.001879 & 0.010022 \\
\hline 0.084352 & 0.026389 & 0.026443 & 0.026391 & 0.003154 & 0.003148 & 0.003145 & 0.014778 \\
\hline 0.11507 & 0.034817 & 0.034889 & 0.034794 & 0.004688 & 0.004684 & 0.004674 & 0.019758 \\
\hline 0.15526 & 0.045613 & 0.045742 & 0.045543 & 0.007547 & 0.007547 & 0.007526 & 0.026586 \\
\hline 0.20083 & 0.059139 & 0.059361 & 0.059098 & 0.011159 & 0.011165 & 0.011142 & 0.035177 \\
\hline 0.24038 & 0.069697 & 0.069951 & 0.069668 & 0.014607 & 0.014611 & 0.01459 & 0.042187 \\
\hline 0.28787 & 0.083015 & 0.083239 & 0.082923 & 0.019582 & 0.019587 & 0.019566 & 0.051319 \\
\hline 0.33843 & 0.098631 & 0.0988 & 0.098596 & 0.026374 & 0.026387 & 0.02636 & 0.062525 \\
\hline 0.38298 & 0.11141 & 0.1116 & 0.11141 & 0.032842 & 0.032854 & 0.032835 & 0.072159 \\
\hline 0.4439 & 0.12976 & 0.12987 & 0.12978 & 0.044045 & 0.044047 & 0.04404 & 0.086924 \\
\hline
\end{tabular}




\begin{tabular}{||c|c|c|c|c|c|c|c||}
\hline 0.49005 & 0.14442 & 0.1445 & 0.14444 & 0.053757 & 0.053757 & 0.053754 & 0.099105 \\
\hline 0.54771 & 0.16709 & 0.16715 & 0.16712 & 0.069266 & 0.069265 & 0.069264 & 0.118193 \\
\hline 0.59802 & 0.18743 & 0.18745 & 0.18745 & 0.085234 & 0.085234 & 0.085233 & 0.136339 \\
\hline 0.64666 & 0.20857 & 0.20859 & 0.20858 & 0.10328 & 0.10328 & 0.10328 & 0.15593 \\
\hline 0.69453 & 0.23253 & 0.23253 & 0.2325 & 0.12571 & 0.12571 & 0.12571 & 0.179115 \\
\hline 0.73542 & 0.25579 & 0.25581 & 0.25577 & 0.15062 & 0.15062 & 0.15062 & 0.203205 \\
\hline 0.78656 & 0.28714 & 0.28715 & 0.28713 & 0.19178 & 0.19178 & 0.19178 & 0.23946 \\
\hline 0.82656 & 0.32126 & 0.32127 & 0.32127 & 0.231001 & 0.231001 & 0.231001 & 0.276134 \\
\hline 0.86253 & 0.385495 & 0.385495 & 0.385495 & 0.29718 & 0.29718 & 0.29718 & 0.341338 \\
\hline 0.89306 & 0.457518 & 0.457518 & 0.457518 & 0.379133 & 0.379133 & 0.379133 & 0.418325 \\
\hline 0.9175 & 0.535245 & 0.535245 & 0.535245 & 0.467812 & 0.467812 & 0.467812 & 0.501528 \\
\hline 0.9433 & 0.642168 & 0.642168 & 0.642168 & 0.589981 & 0.589981 & 0.589981 & 0.616074 \\
\hline 1 & 1 & 1 & 1 & 1 & 1 & 1 & 1 \\
\hline
\end{tabular}

\begin{tabular}{|c|c|c|c|c|c|c|c|}
\hline \multicolumn{8}{|c|}{ Diamond Lattice Curve Fitted Effective Stresses at Yielding (Poisson ratio $=0.3$ ) } \\
\hline Density & $\sigma_{x x}^{y}$ & $\sigma_{y y}^{y}$ & $\sigma_{z z}^{y}$ & $\sigma_{x y}^{y}$ & $\sigma_{y z}^{y}$ & $\sigma_{x z}^{y}$ & $\sigma_{\text {avg }}^{y}$ \\
\hline 0 & 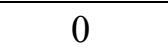 & 0 & 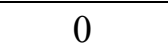 & 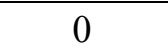 & 0 & 0 & 0 \\
\hline 0.010688 & 0.000309 & 0.000291 & 0.000306 & 0.000332 & 0.000332 & 0.000371 & 0.000324 \\
\hline 0.028 & 0.00088 & 0.000863 & 0.000863 & 0.001079 & 0.001108 & 0.001152 & 0.000991 \\
\hline 0.076096 & 0.002678 & 0.002614 & 0.002614 & 0.003687 & 0.00371 & 0.003741 & 0.003174 \\
\hline 0.11309 & 0.004572 & 0.004452 & 0.004452 & 0.006208 & 0.006218 & 0.006241 & 0.005357 \\
\hline 0.17446 & 0.008714 & 0.008499 & 0.008499 & 0.010115 & 0.010118 & 0.01014 & 0.009348 \\
\hline 0.23638 & 0.014252 & 0.014285 & 0.014285 & 0.013972 & 0.013959 & 0.013954 & 0.014118 \\
\hline 0.29795 & 0.018388 & 0.018461 & 0.018461 & 0.018273 & 0.018254 & 0.018245 & 0.018347 \\
\hline 0.36784 & 0.02356 & 0.02355 & 0.02355 & 0.023892 & 0.023885 & 0.023873 & 0.023718 \\
\hline 0.43373 & 0.032785 & 0.032796 & 0.032796 & 0.032917 & 0.032938 & 0.032912 & 0.032857 \\
\hline 0.50134 & 0.039935 & 0.039965 & 0.039965 & 0.040627 & 0.040634 & 0.040621 & 0.040291 \\
\hline 0.56125 & 0.049193 & 0.049201 & 0.049201 & 0.049541 & 0.049552 & 0.049542 & 0.049372 \\
\hline 0.61139 & 0.055829 & 0.055828 & 0.055828 & 0.057411 & 0.057419 & 0.057415 & 0.056622 \\
\hline 0.67197 & 0.070028 & 0.069961 & 0.069961 & 0.070929 & 0.070932 & 0.070932 & 0.070457 \\
\hline 0.72058 & 0.094756 & 0.088166 & 0.094677 & 0.091283 & 0.091285 & 0.091286 & 0.091909 \\
\hline 0.76784 & 0.105246 & 0.105246 & 0.105246 & 0.11253 & 0.11253 & 0.11254 & 0.10889 \\
\hline 0.8048 & 0.130722 & 0.130722 & 0.130722 & 0.13301 & 0.13301 & 0.13301 & 0.131866 \\
\hline 0.83939 & 0.172678 & 0.172678 & 0.172678 & 0.16483 & 0.16483 & 0.16484 & 0.168756 \\
\hline 0.86854 & 0.229282 & 0.229282 & 0.229282 & 0.18774 & 0.18774 & 0.18775 & 0.208513 \\
\hline 0.89002 & 0.288322 & 0.288322 & 0.288322 & 0.211402 & 0.211402 & 0.211402 & 0.249862 \\
\hline 0.91229 & 0.369661 & 0.369661 & 0.369661 & 0.257229 & 0.257229 & 0.257229 & 0.313445 \\
\hline 0.92848 & 0.444493 & 0.444493 & 0.444493 & 0.310381 & 0.310381 & 0.310381 & 0.377437 \\
\hline 0.9433 & 0.526692 & 0.526692 & 0.526692 & 0.380913 & 0.380913 & 0.380913 & 0.453802 \\
\hline
\end{tabular}




\begin{tabular}{|l|l|l|l|l|l|l|l|}
\hline 1 & 1 & 1 & 1 & 1 & 1 & 1 & 1 \\
\hline
\end{tabular}

\begin{tabular}{|c|c|c|c|c|c||c|c||}
\hline \multicolumn{6}{|c|}{ Face Centered Cubic (FCC) Lattice Curve Fitted Effective Stresses at Yielding (Poisson ratio = 0.3) } \\
\hline \hline Density & $\boldsymbol{\sigma}_{\boldsymbol{x} \boldsymbol{y}}$ & $\boldsymbol{\sigma}_{\boldsymbol{y} \boldsymbol{y}}$ & $\boldsymbol{\sigma}_{\boldsymbol{z z}}^{\boldsymbol{y}}$ & $\boldsymbol{\sigma}_{\boldsymbol{x} \boldsymbol{y}}$ & $\boldsymbol{\sigma}_{\boldsymbol{y} \boldsymbol{z}}^{\boldsymbol{y}}$ & $\boldsymbol{\sigma}_{\boldsymbol{x} \boldsymbol{y}}^{\boldsymbol{y}}$ & $\boldsymbol{\sigma}_{\boldsymbol{a} \boldsymbol{g}}^{\boldsymbol{y}}$ \\
\hline \hline 0 & 0 & 0 & 0 & 0 & 0 & 0 & 0 \\
\hline 0.018112 & 0.00341 & 0.003359 & 0.003439 & 0.002599 & 0.002599 & 0.002601 & 0.003001 \\
\hline 0.047744 & 0.009685 & 0.009571 & 0.009752 & 0.006336 & 0.006338 & 0.006335 & 0.008003 \\
\hline 0.11731 & 0.025155 & 0.025244 & 0.025394 & 0.015857 & 0.015835 & 0.015848 & 0.020556 \\
\hline 0.18707 & 0.03974 & 0.039702 & 0.040094 & 0.027354 & 0.027145 & 0.027157 & 0.033532 \\
\hline 0.28122 & 0.049879 & 0.05 & 0.049905 & 0.045645 & 0.045624 & 0.045635 & 0.047781 \\
\hline 0.36154 & 0.067295 & 0.067413 & 0.067402 & 0.060438 & 0.06039 & 0.06039 & 0.063888 \\
\hline 0.45856 & 0.093869 & 0.093867 & 0.09386 & 0.080786 & 0.080786 & 0.080777 & 0.087324 \\
\hline 0.55501 & 0.12332 & 0.12339 & 0.12341 & 0.099467 & 0.099471 & 0.099459 & 0.11142 \\
\hline 0.64262 & 0.16998 & 0.17002 & 0.17005 & 0.14263 & 0.14263 & 0.14263 & 0.156323 \\
\hline 0.71488 & 0.222283 & 0.222283 & 0.222283 & 0.198686 & 0.198686 & 0.198686 & 0.210485 \\
\hline 0.77504 & 0.286943 & 0.286943 & 0.286943 & 0.274421 & 0.274421 & 0.274421 & 0.280682 \\
\hline 0.82618 & 0.366336 & 0.366336 & 0.366336 & 0.36729 & 0.36729 & 0.36729 & 0.366813 \\
\hline 0.87002 & 0.461241 & 0.461241 & 0.461241 & 0.474062 & 0.474062 & 0.474062 & 0.467652 \\
\hline 0.90387 & 0.55785 & 0.55785 & 0.55785 & 0.577499 & 0.577499 & 0.577499 & 0.567675 \\
\hline 0.93024 & 0.651106 & 0.651106 & 0.651106 & 0.672857 & 0.672857 & 0.672857 & 0.661982 \\
\hline 1 & 1 & 1 & 1 & 1 & 1 & 1 & 1 \\
\hline
\end{tabular}

\begin{tabular}{|c|c|c|c|c|c|c|c|}
\hline \multicolumn{8}{|c|}{$\begin{array}{c}\text { Truncated Octahedron (Octa) Lattice Curve Fitted Effective Stresses at Yielding (Poisson ratio }= \\
0.3)\end{array}$} \\
\hline Density & $\sigma_{x x}^{y}$ & $\sigma_{y y}^{y}$ & $\sigma_{z z}^{y}$ & $\sigma_{x y}^{y}$ & $\sigma_{y z}^{y}$ & $\sigma_{x z}^{y}$ & $\sigma_{a v g}^{y}$ \\
\hline 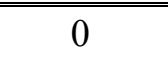 & 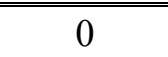 & 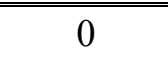 & 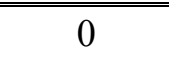 & 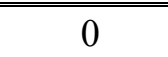 & 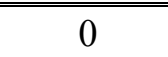 & 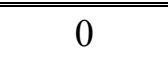 & 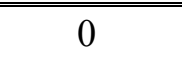 \\
\hline 0.009216 & $2.61 \mathrm{E}-04$ & $3.71 \mathrm{E}-04$ & $3.29 \mathrm{E}-04$ & $4.27 \mathrm{E}-04$ & $4.27 \mathrm{E}-04$ & $4.27 \mathrm{E}-04$ & $3.74 \mathrm{E}-04$ \\
\hline 0.043392 & 0.001384 & 0.002367 & 0.002901 & 0.001527 & 0.001527 & 0.001527 & 0.001872 \\
\hline 0.082944 & 0.003877 & 0.005482 & 0.007401 & 0.003478 & 0.003478 & 0.003478 & 0.004532 \\
\hline 0.1417 & 0.008726 & 0.010593 & 0.01208 & 0.009113 & 0.009113 & 0.009113 & 0.00979 \\
\hline 0.20813 & 0.018543 & 0.022614 & 0.02482 & 0.022205 & 0.022205 & 0.022205 & 0.022099 \\
\hline 0.27994 & 0.034177 & 0.041198 & 0.041398 & 0.046274 & 0.046274 & 0.046274 & 0.042599 \\
\hline 0.3648 & 0.065381 & 0.068886 & 0.068753 & 0.074755 & 0.074755 & 0.074755 & 0.071214 \\
\hline 0.43622 & 0.098045 & 0.098444 & 0.098422 & 0.10067 & 0.10067 & 0.10067 & 0.099487 \\
\hline 0.51533 & 0.14326 & 0.14348 & 0.1436 & 0.15577 & 0.15577 & 0.15577 & 0.149609 \\
\hline
\end{tabular}




\begin{tabular}{||c|c|c|c|c|c|c|c||}
\hline 0.58368 & 0.17549 & 0.17559 & 0.17562 & 0.20817 & 0.20817 & 0.20817 & 0.191868 \\
\hline 0.65741 & 0.20976 & 0.2098 & 0.20985 & 0.27297 & 0.27297 & 0.27297 & 0.241387 \\
\hline 0.71962 & 0.24857 & 0.24855 & 0.24857 & 0.33697 & 0.33697 & 0.33697 & 0.292767 \\
\hline 0.77875 & 0.284164 & 0.284164 & 0.284164 & 0.395 & 0.395 & 0.395 & 0.339582 \\
\hline 0.82944 & 0.336473 & 0.336473 & 0.336473 & 0.44426 & 0.44426 & 0.44426 & 0.390366 \\
\hline 0.86976 & 0.404807 & 0.404807 & 0.404807 & 0.49375 & 0.49375 & 0.49375 & 0.449278 \\
\hline 0.90694 & 0.502053 & 0.502053 & 0.502053 & 0.568339 & 0.568339 & 0.568339 & 0.535196 \\
\hline 0.93139 & 0.591156 & 0.591156 & 0.591156 & 0.637435 & 0.637435 & 0.637435 & 0.614296 \\
\hline 0.94957 & 0.673899 & 0.673899 & 0.673899 & 0.705029 & 0.705029 & 0.705029 & 0.689464 \\
\hline 1 & 1 & 1 & 1 & 1 & 1 & 1 & 1 \\
\hline
\end{tabular}

\begin{tabular}{|c|c|c|c|c|c|c|c|}
\hline \multicolumn{8}{|c|}{ Tesseract Lattice Curve Fitted Effective Stresses at Yielding (Poisson ratio $=0.3$ ) } \\
\hline Density & $\sigma_{x x}^{y}$ & $\sigma_{y y}^{y}$ & $\sigma_{z z}^{y}$ & $\sigma_{x y}^{y}$ & $\sigma_{y z}^{y}$ & $\sigma_{x z}^{y}$ & $\sigma_{a v g}^{y}$ \\
\hline 0 & 0 & 0 & 0 & 0 & 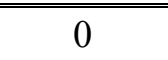 & 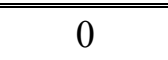 & 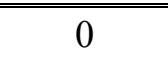 \\
\hline 0.014464 & $2.07 \mathrm{E}-03$ & $2.07 \mathrm{E}-03$ & $2.07 \mathrm{E}-03$ & $2.67 \mathrm{E}-04$ & $2.77 \mathrm{E}-04$ & $2.77 \mathrm{E}-04$ & $1.17 \mathrm{E}-03$ \\
\hline 0.048848 & 0.00749 & 0.007541 & 0.007111 & 0.00258 & 0.002396 & 0.002396 & 0.004919 \\
\hline 0.12749 & 0.017835 & 0.018045 & 0.01789 & 0.005411 & 0.005736 & 0.005736 & 0.011775 \\
\hline 0.19848 & 0.030167 & 0.029546 & 0.02918 & 0.010083 & 0.009869 & 0.009869 & 0.019786 \\
\hline 0.30057 & 0.038028 & 0.037364 & 0.037936 & 0.018961 & 0.018729 & 0.018729 & 0.028291 \\
\hline 0.40717 & 0.06379 & 0.064004 & 0.064666 & 0.03184 & 0.031799 & 0.031799 & 0.047983 \\
\hline 0.51238 & 0.076247 & 0.076162 & 0.076572 & 0.048882 & 0.048854 & 0.048854 & 0.062595 \\
\hline 0.62534 & 0.10778 & 0.10784 & 0.10765 & 0.093943 & 0.093943 & 0.093943 & 0.10085 \\
\hline 0.73677 & 0.16988 & 0.16979 & 0.16964 & 0.14455 & 0.14455 & 0.14455 & 0.15716 \\
\hline 0.81378 & 0.218221 & 0.218221 & 0.218221 & 0.213474 & 0.213474 & 0.213474 & 0.215847 \\
\hline 0.88251 & 0.329556 & 0.329556 & 0.329556 & 0.34938 & 0.34938 & 0.34938 & 0.339468 \\
\hline 0.92832 & 0.487677 & 0.487677 & 0.487677 & 0.51719 & 0.51719 & 0.51719 & 0.502433 \\
\hline 1 & 1 & 1 & 1 & 1 & 1 & 1 & 1 \\
\hline
\end{tabular}




\section{Appendix G : Tapered Beam Formulation}

The case for general tapered beams with circular cross-sections is developed. The shape function formulations for bending stiffness of tapered beams are those derived by Tang [188] and axial and torsional effects follow the methodology outlined by Banerjee [189]. The area along the length of the tapered beam is expressed with the following equation:

$$
A(x)=\pi\left(\left(r_{j}-r_{i}\right) \frac{x}{L}+r_{i}\right)^{2}
$$

\section{G.1 Axial Deformation}

The axial deformation or displacement equation can be found using the governing equation for axial deformation:

$$
\frac{d}{d x}\left(E A(x) \frac{d U}{d x}\right)=0
$$

The second order linear ordinary differential equation has boundary conditions are $U=U_{i}$ at $x=0$ and $U=U_{j}$ and $x=L$. This makes it possible to solve for the deformation $U$ at displacements $u_{i}$ and $u_{j}$. After taking the first integral of the partial differential equation we obtain the following equation [189]:

$$
E A(x) \frac{d U}{d x}=C_{1}
$$

Rearranging the above equation gives:

$$
E d U=\frac{C_{1}}{A(x)} d x=\frac{C_{1}}{\pi\left(\left(r_{j}-r_{i}\right) \frac{x}{L}+r_{i}\right)^{2}} d x
$$

Taking the integral a second time results in: 


$$
E U=-\frac{C_{1} L^{2}}{\pi\left(\left(x\left(r_{i}-r_{j}\right)-L r_{i}\right)\left(r_{i}-r_{j}\right)\right)}+C_{2}
$$

After applying the two boundary conditions, the terms $C_{1}$ and $C_{2}$ are solved and the terms $U_{i}$ and $U_{j}$ are collected and shown below:

$$
U_{i}=\frac{r_{i}(L-x)}{L r_{i}-\left(r_{i}+r_{j}\right) x}, \quad U_{j}=\frac{r_{j} x}{L r_{i}-\left(r_{i}+r_{j}\right) x}
$$

The resulting shape function for the axial displacement can then be formulated as:

$$
N_{\text {axial }}=\frac{1}{L r_{i}-\left(r_{i}+r_{j}\right) x}\left[r_{i}(L-x) \quad r_{j} x\right]
$$

The strain displacement matrix for the axial components can be determined from the derivative of the shape function with respect to the length of the beam. The resulting strain-displacement matrix is given in the equation below:

$$
B_{\text {axial }}=\frac{L r_{i} r_{j}}{\left(L r_{i}-\left(r_{i}+r_{j}\right) x\right)^{2}}\left[\begin{array}{ll}
-1 & 1
\end{array}\right]
$$

Using the principle of virtual work, the stiffness matrix can be derived from the integral in the proceeding equation:

$$
K_{\text {Tapered }}^{\text {Axial }}=\int_{0}^{V} B_{\text {axial }}^{T} E B_{\text {axial }} d V=\int_{0}^{L} \int_{0}^{A(x)} B_{\text {axial }}^{T} E B_{\text {axial }} d A d x
$$

The local stiffness matrix for the axial terms for the tapered beam element is finally given as:

$$
K_{\text {Tapered }}^{\text {Axial }}=\left[\begin{array}{ll}
K_{11}^{A} & K_{12}^{A} \\
K_{21}^{A} & K_{22}^{A}
\end{array}\right]=\frac{\pi E r_{i} r_{j}}{L}\left[\begin{array}{cc}
1 & -1 \\
-1 & 1
\end{array}\right]
$$

\section{G.2 Torsional Stiffness}

The governing first order differential equation for torsion is given below [189]: 


$$
\frac{d}{d x}\left(G J(x) \frac{d \theta}{d x}\right)=0
$$

where $\theta$ is the rotation of the tapered beam , $J(x)$ is the torsional constant or polar moment of inertia and $\mathrm{G}$ is shear modulus of the material.

The shape function for torsion can be found by imposing two boundary conditions at the end of the beam. The first boundary conditions is when $x=0$ and $\theta=\theta_{i}$, the second boundary conditions is $x=L$ and $\theta=\theta_{j}$. The polar moment of inertia for circular section can be expressed as a function to the two radii at the ends of the beam. The polar moment of inertia is given in the equation below:

$$
J(x)=\frac{\pi}{2}\left(\frac{\left(r_{j}-r_{i}\right) x}{L}+r_{i}\right)^{4}
$$

Taking the integral once gives:

$$
G J(x) \frac{d \theta}{d x}=C_{1}
$$

Taking the integral a second time after rearranging the terms results in:

$$
G d \theta=\frac{C_{1}}{J(x)} d x=\frac{C_{1}}{\pi\left(\left(r_{j}-r_{i}\right) \frac{x}{L}+r_{i}\right)^{4}} d x
$$

Taking the integral a second time results in:

$$
G \theta=-\frac{2 C_{1} L^{4}}{3 \pi\left(r_{i}-r_{j}\right)\left(L r_{i}+x\left(r_{j}-r_{i}\right)\right)^{3}}+C_{2}
$$

After applying the two boundary conditions, the terms $C_{1}$ and $C_{2}$ are solved and the terms $\theta_{i}$ and $\theta_{j}$ are collected and shown below: 


$$
N_{\text {torsion }}(x)=\left[\begin{array}{c}
\frac{L^{3}\left(r_{i}^{5}+r_{i}^{4} r_{j}+r_{i}^{3} r_{j}^{2}\right)-3 L\left(L r_{i}^{5} x+r_{i}^{5} x^{2}-r_{i}^{4} r_{j} x^{2}\right)-x^{3}\left(r_{i}^{5}+2 r_{i}^{4} r_{j}-r_{j}^{3} r_{j}^{2}\right)}{\left(L r_{i}+x\left(r_{j}-r_{i}\right)\right)^{3}\left(r_{i}^{2}+r_{i} r_{j}+r_{j}^{2}\right)} \\
\frac{r_{j}^{2} x\left(3 L r_{i}\left(L r_{i}+x\left(r_{j}-r_{i}\right)\right)+r_{i}^{2} x^{2}-2 r_{i} r_{j} x^{2}+r_{j}^{2} x^{2}\right)}{\left(L r_{i}+x\left(r_{j}-r_{i}\right)\right)^{3}\left(r_{i}^{2}+r_{i} r_{j}+r_{j}^{2}\right)}
\end{array}\right]^{T}
$$

The strain displacement matrix for the torsion within the tapered beam is the derivative of the shape function and is:

$$
B_{\text {torsion }}=\frac{3 L^{3} r_{i}^{3} r_{j}^{3}}{\left(L r_{i}+x\left(r_{j}-r_{i}\right)\right)^{4}\left(r_{i}^{2}+r_{i} r_{j}+r_{j}^{2}\right)}\left[\begin{array}{ll}
-1 & 1
\end{array}\right]
$$

Using the principle of virtual work, the element stiffness matrix for the torsion entries can be obtained.

$$
K_{\text {Tapered }}^{\text {Torsion }}=\int_{0}^{V} B_{\text {torsion }}^{T} G B_{\text {torsion }} d V=\int_{0}^{L} \int_{0}^{J(x)} B_{\text {torsion }}^{T} G B_{\text {torsion }} d A d x
$$

The local stiffness matrix for the axial terms for the tapered beam element is finally given as:

$$
K_{\text {Tapered }}^{\text {torsion }}=\left[\begin{array}{ll}
K_{11}^{T} & K_{12}^{T} \\
K_{21}^{T} & K_{22}^{T}
\end{array}\right]=\frac{3 \pi G r_{i}^{3} r_{j}^{3}}{2 L\left(r_{i}^{2}+r_{i} r_{j}+r_{j}^{2}\right)^{2}}\left[\begin{array}{cc}
1 & -1 \\
-1 & 1
\end{array}\right]
$$

\section{G.3 Bending Stiffness}

The bending properties of the beam can be determined using the governing equation for EulerBernoulli beam elements. A second order differential equation for bending is given for the bending effects to obtain relations for the deflection and rotations.

$$
\frac{d^{2}}{d x^{2}}\left(E I(x) \frac{d^{2} y}{d x^{2}}\right)=F_{d}
$$

where $\mathrm{E}$ is the modulus, $\mathrm{I}$ is the second moment of inertia, $\mathrm{y}$ is the deflection and $F_{d}$ is a distributed force per unit length along the beam. The second moment of area for a circular tapered beam is given as: 


$$
I(x)=\frac{\pi}{4}\left(\left(r_{j}-r_{i}\right) \frac{x}{L}+r_{i}\right)^{4}
$$

Following the methodology of Yang [188] for tapered beam elements, the deflection is found from the definition of the curvature of the beam:

$$
\frac{d^{2} y}{d x^{2}}=\frac{M(x)}{E I(x)}
$$

The moment equation of the beam is expressed as [188]:

$$
M(x)=\left(M_{i}+M_{j}\right) \frac{x}{L}-M_{i}
$$

By taking the integral of the curvature twice, the deflection equation with unknowns $\mathrm{C} 1, \mathrm{C} 2, M_{i}$ and $M_{j}$ is obtained. The full equation can be derived by applying four boundary conditions at each of the nodes. Hence for the deflections at each end, four boundary conditions are needed. By integration the curvature once, the rotations can be calculated with:

$$
\frac{d y}{d x}=C_{1}-\frac{2 L^{3}\left(\left(M_{i}+M_{j}\right) x-L M_{i}\right)^{2}\left(\frac{2 M_{i}\left(r_{i}-r_{j}\right)-3 r_{i}\left(M_{i}+M_{j}\right)}{L}+\frac{x\left(r_{i}-r_{j}\right)\left(M_{i}+M_{j}\right)}{L^{2}}\right)}{3 E \pi\left(M_{i} r_{j}+M_{j} r_{i}\right)^{2}\left(L r_{i}-r_{i} x+r_{j} x\right)^{3}}
$$

A second integration gives the deflection equation and is:

$$
y=C_{2}+C_{1} x+\frac{6 L^{3} x\left(M_{i}+M_{j}\right)-\frac{2 L^{4}\left(3 M r_{i}-M_{i} r_{j}+2 M_{j} r_{i}\right)}{r_{i}-r_{j}}}{3 E \pi\left(r_{i}-r_{j}\right)^{2}\left(L r_{i}-r_{i} x+r_{j} x\right)^{2}}+\frac{2 L x\left(M_{i}+M_{j}\right)^{3}}{3 E \pi\left(M_{i} r_{j}+M_{j} r_{i}\right)^{2}\left(r_{i}-r_{j}\right)^{2}}
$$

The deflection at node $i$ at its first degree of freedom is found with boundary conditions $x=0$, $=0, \frac{d y}{d x}=1$ and when $x=L, y=0, \frac{d y}{d x}=0$. The resulting equation for the rotations by solving for the unknown moments and integration constants is:

$$
y_{1 i}(x)=\frac{r_{i}(L-x)^{2}\left(L r_{i}+2 r_{j} x\right)}{L\left(L r_{i}-r_{i} x+r_{j} x\right)^{2}}
$$


The deflection at node $\mathrm{i}$ at its second degree of freedom is found with boundary conditions $x=$ $0, y=1, \frac{d y}{d x}=0$ and when $x=L, y=0, \frac{d y}{d x}=0$. The equation is:

$$
y_{2 i}(x)=\frac{r_{i}^{2} x(L-x)^{2}}{\left(L r_{i}-r_{i} x+r_{j} x\right)^{2}}
$$

The deflection at node $\mathrm{j}$ at its first degree of freedom is found with boundary conditions $x=$ $0, y=0, \frac{d y}{d x}=0$ and when $x=L, y=0, \frac{d y}{d x}=1$. The equation is:

$$
y_{1 j}(x)=\frac{r_{j} x^{2}\left(2 L r_{i}+L r_{j}-2 r_{i} x\right)}{L\left(L r_{i}-r_{i} x+r_{j} x\right)^{2}}
$$

The deflection at node $\mathrm{j}$ at its second degree of freedom is found with boundary conditions $x=$ $0, y=1, \frac{d y}{d x}=0$ and when $x=L, y=0, \frac{d y}{d x}=0$. The equation is:

$$
y_{2 j}(x)=-\frac{r_{j}^{2} x^{2}(L-x)}{\left(L r_{i}-r_{i} x+r_{j} x\right)^{2}}
$$

The shape function for the deflection is therefore:

$$
N(x)=\frac{1}{\left(L r_{i}-r_{i} x+r_{j} x\right)^{2}}\left[\begin{array}{c}
\frac{r_{i}(L-x)^{2}\left(L r_{i}+2 r_{j} x\right)}{L} \\
\frac{r_{i}^{2} x(L-x)^{2}}{L} x^{2}\left(2 L r_{i}+L r_{j}-2 r_{i} x\right) \\
-r_{j}^{2} x^{2}(L-x)
\end{array}\right]^{T}
$$

The shape function for the slope is:

$$
\frac{d N}{d x}=\frac{1}{\left(L r_{i}-r_{i} x+r_{j} x\right)^{3}}\left[\begin{array}{c}
\frac{-2 r_{i} r_{j} x(L-x)\left(2 L r_{i}+L r_{j}-r_{i} x+r_{j} x\right)}{L} \\
-r_{i}^{2}(L-x)\left(r_{j} x^{2}-r_{i} x^{2}-L^{2} r_{i}+2 L r_{i} x+L r_{j} x\right) \\
\frac{2 r_{i} r_{j} x(L-x)\left(2 L r_{i}+L r_{j}-r_{i} x+r_{j} x\right)}{L} \\
-r_{j}^{2} x\left(2 L^{2} r_{i}+r_{i} x^{2}-r_{j} x^{2}-3 L r_{i} x\right)
\end{array}\right]^{T}
$$


The strain displacement matrix is the second derivative of the deflection shape function and is shown below:

$$
B_{\text {bending }}=r_{d}(x) \frac{1}{\left(L r_{i}-r_{i} x+r_{j} x\right)^{4}}\left[\begin{array}{c}
2 L r_{i} r_{j}\left(2 r_{i}^{2} x-2 L r_{i}^{2}+2 r_{j}^{2} x-L r_{i} r_{j}+2 r_{i} r_{j} x\right) \\
2 L^{2} r_{i}^{2} r_{j}\left(2 r_{i} x-2 L r_{i}+r_{j} x\right) \\
-2 L r_{i} r_{j}\left(2 r_{i}^{2} x-2 L r_{i}^{2}+2 r_{j}^{2} x-L r_{i} r_{j}+2 r_{i} r_{j} x\right) \\
2 L^{2} r_{i} r_{j}^{2}\left(r_{i} x-L r_{i}+2 r_{j} x\right)
\end{array}\right]^{T}
$$

where $r_{d}(x)$ is the distance from the neutral axis to the top of the beam at a location $\mathrm{x}$. The stiffness matrix for the bending effects can be calculated using the principle of virtual work.

$$
K_{\text {Tapered }}^{\text {Bending }}=\int_{0}^{V} B_{\text {Bending }}^{\mathrm{T}} \mathrm{E}_{\text {Bending }} \mathrm{dV}=\int_{0}^{\mathrm{L}} \mathrm{B}_{\text {Bending }}^{\mathrm{T}} \mathrm{EI}(\mathrm{x}) \mathrm{B}_{\text {bending }} \mathrm{dx}
$$

The final stiffness matrix for the tapered beam element is:

$$
\begin{gathered}
K_{\text {Tapered }}^{\text {Bending }}=\left[\begin{array}{llll}
K_{11}^{B} & K_{12}^{B} & K_{13}^{B} & K_{14}^{B} \\
K_{21}^{B} & K_{22}^{B} & K_{23}^{B} & K_{24}^{B} \\
K_{31}^{B} & K_{32}^{B} & K_{33}^{B} & K_{34}^{B} \\
K_{41}^{B} & K_{42}^{B} & K_{43}^{B} & K_{44}^{B}
\end{array}\right] \\
K_{11}^{B}=-K_{13}^{B}=-K_{31}^{B}=K_{33}^{B}=\frac{E \pi r_{i} r_{j}\left(r_{i}^{2}+r_{i} r_{j}+r_{j}^{2}\right)}{L^{3}} \\
K_{12}^{B}=K_{21}^{B}=-K_{23}^{B}=-K_{32}^{B}=\frac{E \pi r_{i}^{2} r_{j}^{2}\left(2 r_{i}+r_{j}\right)}{2 L^{2}} \\
K_{14}^{B}=K_{41}^{B}=-K_{34}^{B}=-K_{43}^{B}=\frac{E \pi r_{i} r_{j}^{2}\left(r_{i}+2 r_{j}\right)}{2 L^{2}} \\
K_{24}^{B}=K_{42}^{B}=\frac{E \pi r_{i}^{2} r_{j}^{2}}{2 L} \\
K_{22}^{B}=\frac{E \pi r_{i}^{3} r_{j}}{L} \\
K_{44}^{B}=\frac{E \pi r_{i} r_{j}^{3}}{L}
\end{gathered}
$$




\section{G.4 3D Tapered Frame Beam Element}

The two-dimensional element can be expanded into a three dimensional frame element by modifying the bending terms. As explained by Tang [190], the bending properties in the Z-plane are mirrored for bending in the y plane. The local stiffness matrix is given as:

$$
K_{\text {Tapered }}^{3 D \text { Frame }}=\left[\begin{array}{cccccccccccc}
K_{11}^{A} & 0 & 0 & 0 & 0 & 0 & K_{12}^{A} & 0 & 0 & 0 & 0 & 0 \\
0 & K_{11}^{B} & 0 & 0 & 0 & K_{12}^{B} & 0 & K_{13}^{B} & 0 & 0 & 0 & K_{14}^{B} \\
0 & 0 & K_{11}^{B} & 0 & K_{23}^{B} & 0 & 0 & 0 & K_{13}^{B} & 0 & K_{23}^{B} & 0 \\
0 & 0 & 0 & K_{11}^{T} & 0 & 0 & 0 & 0 & 0 & K_{12}^{T} & 0 & 0 \\
0 & 0 & K_{23}^{B} & 0 & K_{22}^{B} & 0 & 0 & 0 & K_{12}^{B} & 0 & K_{24}^{B} & 0 \\
0 & K_{12}^{B} & 0 & 0 & 0 & K_{22}^{B} & 0 & K_{23}^{B} & 0 & 0 & 0 & K_{24}^{B} \\
K_{21}^{A} & 0 & 0 & 0 & 0 & 0 & K_{22}^{A} & 0 & 0 & 0 & 0 & 0 \\
0 & K_{31}^{B} & 0 & 0 & 0 & K_{32}^{B} & 0 & K_{33}^{B} & 0 & 0 & 0 & K_{32}^{B} \\
0 & 0 & K_{31}^{B} & 0 & K_{41}^{B} & 0 & 0 & 0 & K_{32}^{B} & 0 & K_{41}^{B} & 0 \\
0 & 0 & 0 & K_{21}^{T} & 0 & 0 & 0 & 0 & 0 & K_{22}^{T} & 0 & 0 \\
0 & 0 & K_{43}^{B} & 0 & K_{42}^{B} & 0 & 0 & 0 & K_{41}^{B} & 0 & K_{44}^{B} & 0 \\
0 & K_{41}^{B} & 0 & 0 & 0 & K_{42}^{B} & 0 & K_{43}^{B} & 0 & 0 & 0 & K_{44}^{B}
\end{array}\right]
$$

The associated displacement vector with the local stiffness matrix is given below:

$$
U=\left[\begin{array}{llllllllllll}
u_{x, i} & u_{y, i} & w_{z, i} & \theta_{x, i} & \theta_{y, i} & \theta_{z, i} & u_{x, j} & u_{y, j} & w_{z, j} & \theta_{x, j} & \theta_{y, j} & \theta_{z, j}
\end{array}\right]^{T}
$$




\section{G.5 Local Stiffness Matrix Sensitivities}

The sensitivities for tapered beam stiffness matrix and strain-displacement matrices are presented. The derivatives are taken with respect to the design variables and are the radii at the ends of the beam. For brevity, only the axial and torsional terms for the tapered beam element are presented. The derivation for the three dimensional tapered beam with circular cross section is given in Appendix G .

The derivative of the strain-displacement matrix for axial deformation is given as:

$$
B_{\text {axial }}=\frac{L r_{i} r_{j}}{\left(L r_{i}-\left(r_{i}+r_{j}\right) x\right)^{2}}\left[\begin{array}{ll}
-1 & 1
\end{array}\right]
$$

Where $\mathrm{L}$ is the length of the tapered beam, $r_{i}$ is the radius at node $i$ and $r_{j}$ is the radius at node $j$. The sensitivities of the strain-displacement matrix with respect to $r_{i}$ and $r_{j}$ are:

$\frac{d B_{\text {axial }}}{d r_{i}}=\frac{r_{j} x\left(r_{i}+r_{j}\right)-r_{i} r_{j} L^{2}}{\left(L r_{i}-\left(r_{i}+r_{j}\right) x\right)^{3}}\left[\begin{array}{ll}-1 & 1\end{array}\right], \quad \frac{d B_{\text {axial }}}{d r_{j}}=\frac{L^{2} r_{i}^{2}-L r_{i} x\left(r_{i}+r_{j}\right)}{\left(L r_{i}-\left(r_{i}+r_{j}\right) x\right)^{3}}\left[\begin{array}{ll}-1 & 1\end{array}\right]$

The local stiffness matrix for axial behavior in a tapered beam is:

$$
K_{\text {Tapered }}^{\text {Axial }}=\frac{\pi E r_{i} r_{j}}{L}\left[\begin{array}{cc}
1 & -1 \\
-1 & 1
\end{array}\right]
$$

It can be seen from the local stiffness matrix that when both radii are equal, the stiffness matrix becomes that of a bar element with constant area. The sensitivities for the local stiffness matrix for each radius are given as:

$$
\frac{d K_{\text {Tapered }}^{\text {Axial }}}{d r_{i}}=\frac{\pi E r_{j}}{L}\left[\begin{array}{cc}
1 & -1 \\
-1 & 1
\end{array}\right], \quad \frac{d K_{\text {Tapered }}^{\text {Axial }}}{d r_{j}}=\frac{\pi E r_{i}}{L}\left[\begin{array}{cc}
1 & -1 \\
-1 & 1
\end{array}\right]
$$

Torsion stiffness and strain-displacement matrix sensitivities can be calculated using the same method as the axial stiffness terms. The strain displacement matrix for torsion is given below: 


$$
B_{\text {torsion }}=\frac{3 L^{3} r_{i}^{3} r_{j}^{3}}{\left(L r_{i}+x\left(r_{j}-r_{i}\right)\right)^{4}\left(r_{i}^{2}+r_{i} r_{j}+r_{j}^{2}\right)}\left[\begin{array}{ll}
-1 & 1
\end{array}\right]
$$

The sensitivities of the strain-displacement matric with respect to each design variable are:

$$
\begin{aligned}
\frac{d B_{\text {torsion }}}{d r_{i}} & =\frac{3 L^{3} r_{i}^{2} r_{j}^{3}\left(L\left(3 r_{i}^{3}+2 r_{i}^{2} r_{j}+r_{i} r_{j}^{2}\right)-3 x\left(r_{i}^{3}+r_{i}^{2} r_{j}+r_{i} r_{j}^{2}+r_{j}^{3}\right)\right)}{\left(L r_{i}+x\left(r_{j}-r_{i}\right)\right)^{5}\left(r_{i}^{2}+r_{i} r_{j}+r_{j}^{2}\right)^{2}}\left[\begin{array}{ll}
-1 & 1
\end{array}\right] \\
\frac{d B_{\text {torsion }}}{d r_{j}} & =\frac{3 L^{3} r_{i}^{3} r_{j}^{2}\left(L\left(3 r_{i}^{3}+2 r_{i}^{2} r_{j}+r_{i} r_{j}^{2}\right)-3 x\left(r_{i}^{3}+r_{i}^{2} r_{j}+r_{i} r_{j}^{2}+r_{j}^{3}\right)\right)}{\left(L r_{i}+x\left(r_{j}-r_{i}\right)\right)^{5}\left(r_{i}^{2}+r_{i} r_{j}+r_{j}^{2}\right)^{2}}\left[\begin{array}{ll}
-1 & 1
\end{array}\right]
\end{aligned}
$$

The local stiffness matrix for the axial terms for the tapered beam element is:

$$
K_{\text {Tapered }}^{\text {torsion }}=\frac{3 \pi G r_{i}^{3} r_{j}^{3}}{2 L\left(r_{i}^{2}+r_{i} r_{j}+r_{j}^{2}\right)^{2}}\left[\begin{array}{cc}
1 & -1 \\
-1 & 1
\end{array}\right]
$$

The derivatives of the stiffness matric with respect to the radii are:

$$
\begin{aligned}
& \frac{d K_{\text {Tapered }}^{\text {torsion }}}{d r_{i}}=\frac{3 \pi G r_{i}^{2} r_{j}^{3}\left(r_{i}^{2}+2 r_{i} r_{j}+3 r_{j}^{2}\right)}{2 L\left(r_{i}^{2}+r_{i} r_{j}+r_{j}^{2}\right)^{2}}\left[\begin{array}{cc}
1 & -1 \\
-1 & 1
\end{array}\right] \\
& \frac{d K_{\text {Topered }}^{\text {torsion }}}{d r_{j}}=\frac{3 \pi G r_{i}^{3} r_{j}^{2}\left(3 r_{i}^{2}+2 r_{i} r_{j}+r_{j}^{2}\right)}{2 L\left(r_{i}^{2}+r_{i} r_{j}+r_{j}^{2}\right)^{2}}\left[\begin{array}{cc}
1 & -1 \\
-1 & 1
\end{array}\right]
\end{aligned}
$$

The sensitivities for the bending or flexural stiffness can be obtained following the same methodology outlined for the torsion and the axial effects. 


\section{Appendix H : Trimmed Lattice and Conformal Wireframe Nets}

Due to their superior specific properties, functionally graded lattice materials have been integrated into several applications in Aerospace, Biomedical, defense and automotive engineering. However, tessellating a periodic unit cell, the representative volume element, of a lattice material to fill up the design space in complex geometries has many challenges arising from their CAD modeling intricacy as well as interior lattice distortions around highly curved surfaces. One solution to the aforementioned inconveniences is by using trimmed lattice structures with a conformal lattice net as the periodicity is conserved without affecting the unit cell dimensions. This section presents a novel method for constructing a conformal lattice net as a wireframe of one-dimensional line segments. The construction of the net is applicable to symmetric unit cells while its generation is independent of the interior trimmed lattice topology. A novel projection filter is also discussed which projects an approximated pattern of the unit cell topology onto any closed triangulated surface. Advantages and disadvantages of the new meshing algorithm is discussed with numerical examples to display the proposed methods flexibility and robustness. The wireframe structure is easily transferred into a collection of onedimensional beam elements for micro-scale optimizations to obtain a functionally graded structure. A simple case study is presented to compare the effects of micro-scale optimization on trimmed lattices with and without lattice nets.

\section{H.1 Overview of Trimmed Lattice Structures}

A periodic cellular solid, also known as lattice material, is a micro-truss structure generated by tessellating a unit cell in $2 \mathrm{D}$ or $3 \mathrm{D}$ infinite periodicity. Lattice materials expand materials selection design space through providing meta-materials for advanced engineering applications 
spanning sandwich panels, energy absorption and insulation [157]. The high specific properties of cellular solids make them an attractive material to save weight and costs [155]. However, due to their complex geometries, manufacturing such micro structures was near impossible a few decades ago. Nevertheless, lattice solids have been gaining popularity due to the advancements in additive manufacturing.

Due to the complexity of the micro-structure of lattice materials, where it involves a huge number of elements, the possibility of solving all underlying equations in full details is very expensive and might be impossible. Accordingly, the laws of greatest importance are the principles of symmetry where the lattice is modelled through a Representative Volume Element (RVE), namely, the unit cell. Such concept in continuum mechanics finds its roots in solid state physics where a lattice is mainly concerned with replicating the strengths from atomic bonds commonly seen in metals and metallic alloys. Examples of atomic bonds and their strengths can be seen in diamonds where carbon atoms share all their valence electrons with other neighboring carbon atoms, they form what is called a diamond lattice. The structure of the diamond lattice is known to have the highest tensile strength among all atomic topologies and could be a good candidate for creating strong micro lattice structures [191, 192]. The amount of possibilities for lattice topologies is endless however, only symmetrical lattice structures with clear crystallographic planes are considered in this paper. The most common symmetric lattice topologies which are also analyzed in this paper include unit cells with Cubic and Body Centered Cubic (BCC), Face Centered Cubic (FCC) and diamond geometries [193].

There exist many methods for modeling lattice structures in CAD. These range from the utilization of voxels to implicit surface definitions to generate the interior and exterior lattice topology $[194,195,196]$. When it comes to implementing lattice structures into a design, there 
exist three main strategies [194]. Shown in Figure 60, for an empty designable domain there are many interior meshing strategies available. The first is a swept interior lattice which distorts the lattice to meet the curvature of the domain. The second is by directly meshing the design domain with elements and then converting those elements into the desired unit cell afterwards. The third is a trimmed interior where the lattice pattern is conserved (periodic) and is then truncated when it is outside of the design domain.

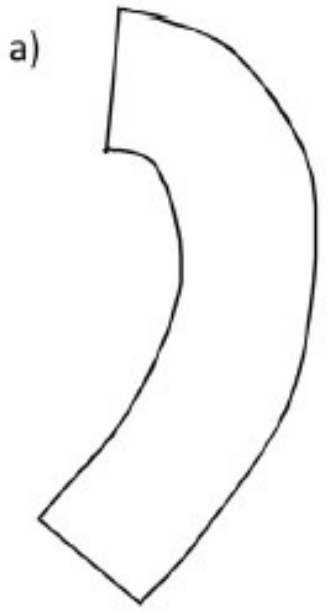

b)

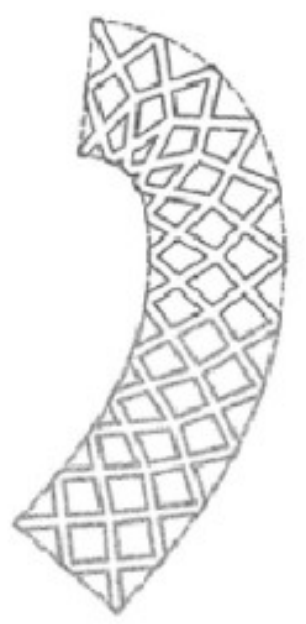

c)

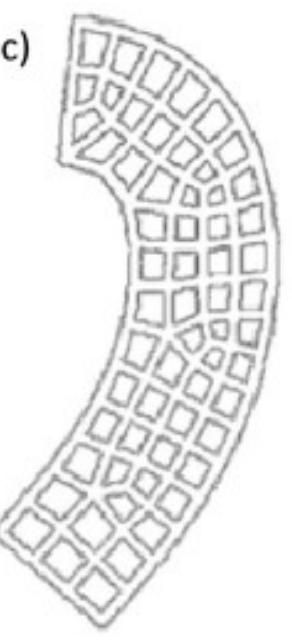

d)

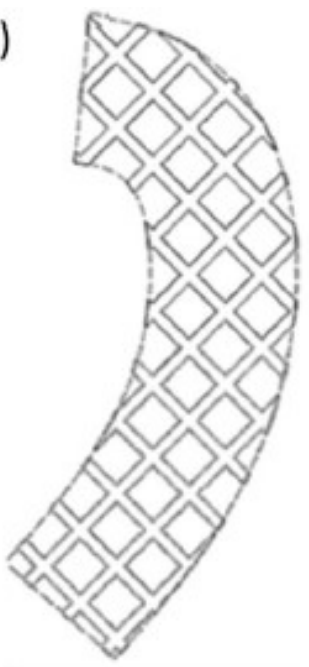

Figure 60 : Lattice Generation Methods a) Domain b) Swept C) Meshed d) Trimmed [194]

Sweeping methods require significant user intervention and panning and are very time consuming to produce. The problem is more apparent for complex geometries where transitions between sections does not guarantee a consistent unit cell size.

Meshed domains also require a user defined mesh, which can be time consuming. However, if the unit cell size is changed, then the entire mesh will be required to be redone. The unit cell size could also be inconsistent and is subject to warping in the vicinity of curved geometries. 
There are many advantages for swept and meshed lattice regions such as ease of application and limited complexity in modeling. The exterior of a domain can also be easily closed off using the correct lattice topology. However, connecting the exterior struts of the trimmed lattice is not as easily defined, especially in three-dimensional lattice configurations. An exterior mesh of structures connecting interior struts together is known as a conforming skin, lattice net or a conformal lattice net [194]. An illustration of such a lattice net is presented in Figure 61c.

a)

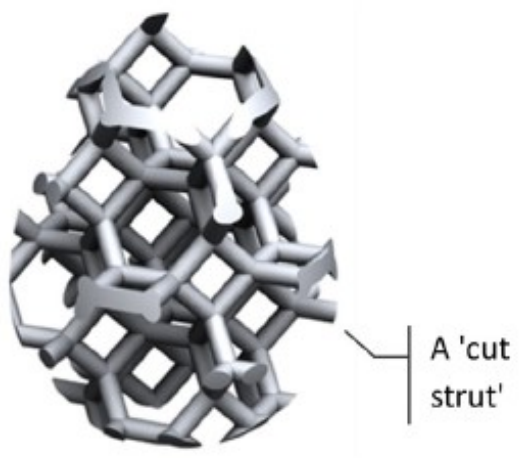

b)

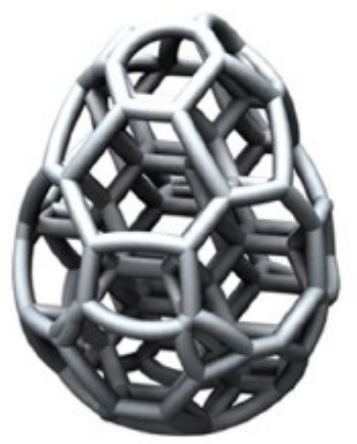

c)

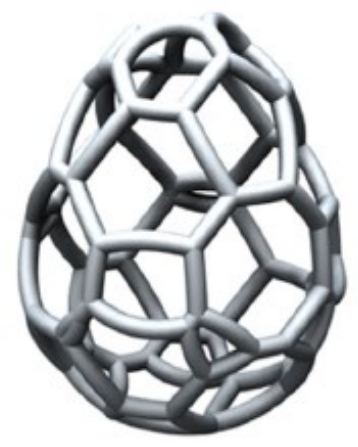

Figure 61: a) Trimmed Lattice Structure b) Lattice Structure with net-skin c) Net-Skin [194]

The works of Aremu et al. [194] have successfully developed an algorithm to generate a lattice net for any lattice unit cell. This method uses voxels to generate both the interior and exterior structures. Very few other algorithms can be found in literature that focuses on trimmed lattice structures and conformal lattice nets [10]. Therefore a methodology for building a conformal lattice net as a wireframe is explored in this paper.

There are several advantages for selecting a trimmed lattice over the other methods as explained in [194]. These advantages include the lack of user intervention when generating the mesh as it can be automated. Unit cells are not distorted due to curved geometries and can be oriented in any direction. In addition, the unit cell size is also independent of the geometry because geometry complexity is not a factor. 
Consistently sized unit cells are important to correctly reflect the properties of the lattice topologies. This is even more of a concern for topology optimizations of lattice structures that rely on asymptotic homogenization [95]. The relative properties from the homogenization rely on perfectly repeated unit cell properties. When distortions in the lattice representation occur, the simulated properties are no longer an accurate representation of the unit cell.

For multi-scale design, automation is very important when transitioning between the optimized lattice topology results and the beam representation for sizing optimization (micro-scale optimization). Due to the trimmed lattices ability to require very little user involvement, then it is an attractive method for modeling the lattice structures at the micro-scale. Hence, the timed lattices will be used for creating the lattice-beam structures as described in the multi-scale design workflow in chapter one. The one problem with the trimmed lattice structures is that there is no algorithm to connect the one-dimensional struts together for beam sizing optimization. Therefore, an algorithm for a wireframe lattice net will be developed for symmetrical unit cells. This text is organizes into seven sections. After this introduction section 2 will define mathematically a symmetric unit cell. In section 3 the methodology for developing an algorithm to create both an interior trimmed lattices structure and conforming lattice net is discussed. Section 4 explains the procedure to build an interior trimmed lattice structure without a lattice net. Section 5 presents the methodology for generating the conforming lattice net for the interior trimmed lattice structure. Numerical examples on common geometries for the lattice net are shown in section 6. A case study for optimizing the lattice wireframe for functionally graded structure is shown in section 7. Finally, section 8 discusses the advantages and disadvantages of the proposed workflow for a wireframe trimmed lattice structure with a conforming net. 


\section{H.2 Symmetrical Unit Cells}

There are four types of lattice symmetries including rotation, reflection, glide-reflection and translation [193]. Here, we use Bravais lattice symmetries to define the unit cell topology where a Bravais lattice can be defined as an infinite array of discrete points with arrangement that appears exactly the same from all symmetrical points of view. Position of the Bravais lattice points can be defined by a position vector $\vec{\Re}$ of the form:

$$
\vec{\Re}=\sum_{i=1}^{\mathfrak{m}} n_{i} \overrightarrow{\mathrm{a}_{l}}
$$

where $n_{i}$ are a set of integers or Miller indices and $\mathrm{a}_{i}$ are the periodicity of the unit cell while $m=3$ for 3D lattices [193].

a)

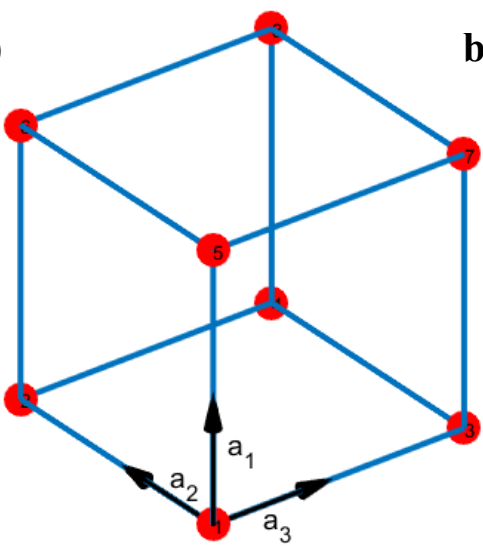

b)

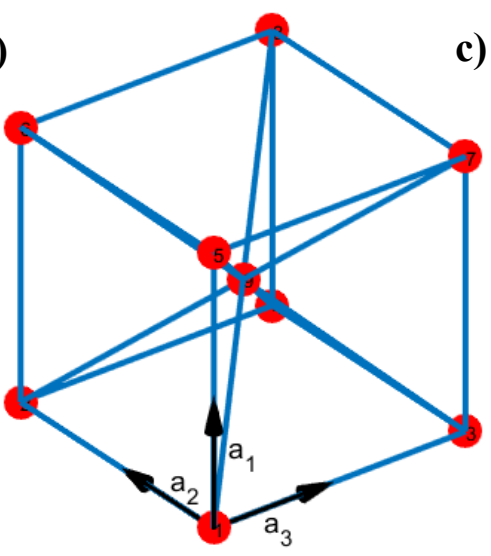

c)

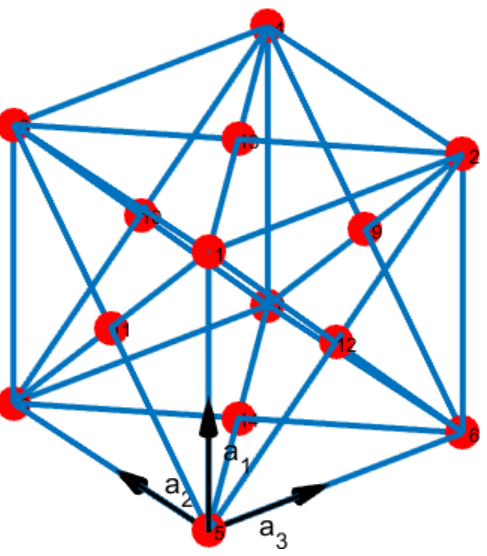

Figure 62 : Bravais Cubic Lattices (a) Cubic/Primitive (b) Body-Centered Cubic (BCC) (c) Face Centered Cubic (FCC)

On the other hand, a crystallographic plane in a symmetrical lattice can be defined using the Miller indices in crystal Bravais lattices. Miller indices are defined by the axis intercepts along the unit cell axes. As shown in Figure 63, for example, the cubic cell has three crystallographic planes which can be numbered using the Bravais system as (100), (010) and (001) [193]. 
In this manuscript, lattice unit cells are constructed by means of nodes and struts where a strut is a member that connects two nodes. Using the Bravais lattice terminology; a node in this paper is equivalent to a lattice point and is illustrated as a red point in Figure 62a. Nodes occur at the intersections of one or more struts and a strut is a line or member that is connected by two nodes. Crystallographic planes are defined when they can be drawn through a unit cell using only the nodes within that unit cell and that the resulting plane is either coplanar with the struts or intersects the nodes exclusively. A crystallographic plane may also be defined by symmetry elements within the unit cell as long as those planes pass through nodes.

a)

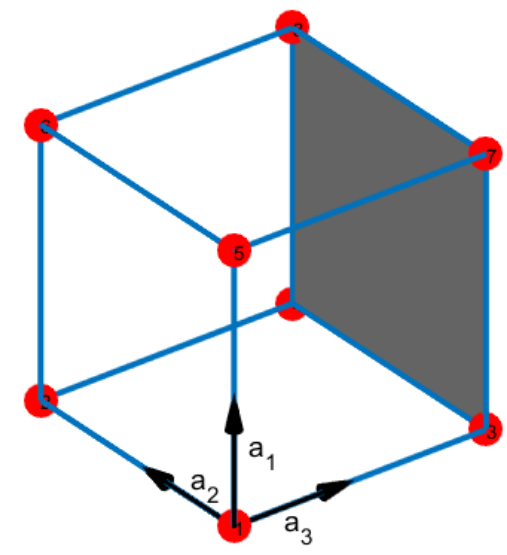

b)

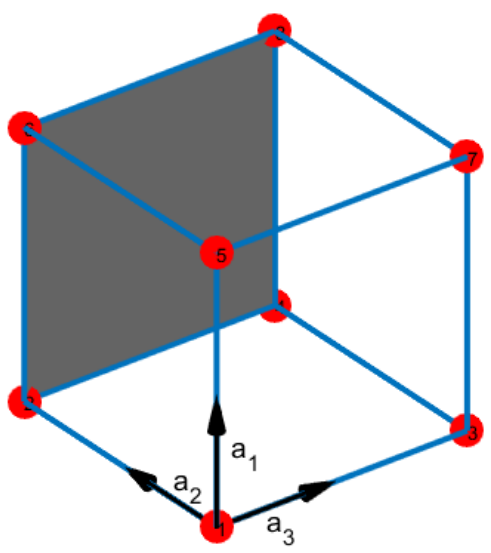

c)

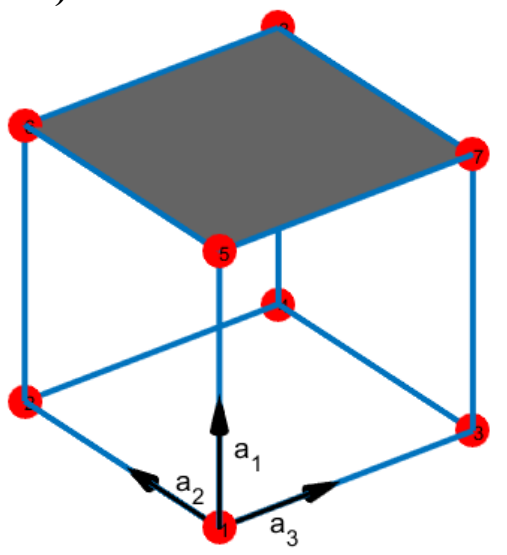

Figure 63 : Miller Indices $\left(n_{1}, n_{2}, n_{3}\right)$ Planes for Cubic System (a) (100) (b) (010) (c) (001)

The presented algorithm in this paper exploits the crystallographic planes by using them to connect cut struts where a cut strut is a section of the lattice inside a domain as illustrated in Figure 65.

While can be easily expanded to all types of symmetries, the proposed meshing algorithm is only concerned with unit cells with translational or reflection symmetries. This limits the algorithm to work exclusively with cubic lattices from the Bravais lattice family [193]. However, cubic symmetries can be easily adapted for many other types of lattice symmetries. As shown in Figure 
61, Cubic Bravais lattices have three topologies; including the simple cubic or the primitive structure, the Body-Centered Cube (BCC) and the Face Centered Cube (FCC). Symmetrical lattices that are also applicable for the presented lattice net algorithm include diamond and octettruss topologies as they have Bravais cubic symmetry as well.

\section{H.3 Algorithm for an Interior Trimmed Lattice}

This section presents an algorithm for generating an interior wireframe structure of a trimmed lattice. The methodology implements the work of Tang et al. [196] but replaces a functional volume (FV) with a closed surface that is composed of triangles such as the CAD geometry presented in StereoLithography (StL) files [197], as shown in Figure 64, the algorithm includes four stages.

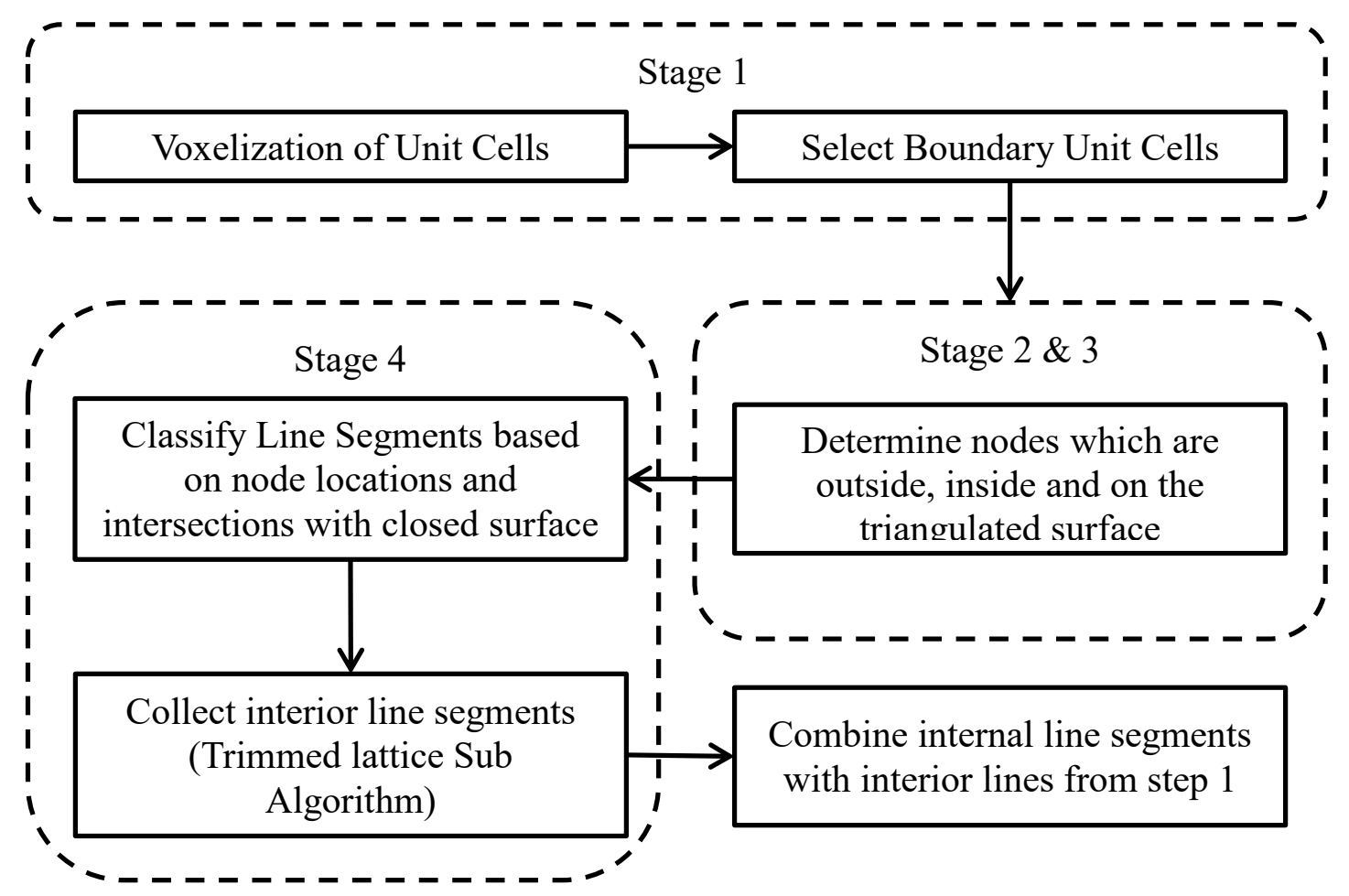

Figure 64 : Interior Trimmed Lattice Workflow 
The first stage for creating the interior trimmed lattice replicates a lattice unit cell to fill a volume. This can be achieved through voxelization [194] in a three-dimensional space. The advantage of using voxels to fill a closed volume is that the boundary voxels can be extracted to be used in the trimming section of the algorithm. By limiting the number of struts to be processed by ignoring those which are fully inside the volume increases the computational efficiency of the trimming algorithm. After trimming, the fully interior struts are combined with the cut struts to create the final trimmed lattice structure.

The second stage determines which nodes in the voxelated structure are outside or inside the closed surface. This is to group line segments based on the placement of the nodes in relation to the triangulated surface. The methods to sort the nodes are those formulated by Tuszynski [198] and Sven [199]. Ray-triangle intersection algorithms [200, 201, 202] will determine if the point is interior or exterior of a closed surface. If an even number of ray intersection is found, then the point is outside of the surface, the point is outside the closed surface if an odd number is calculated.

Ray-triangle intersections can be determined by evaluating the intersection point between the ray and the plane given by the triangles normal. If the intersection point is expressed in terms of barycentric coordinates for that triangle, then it is possible to classify whether or not the ray intersects the triangle. In a mathematical sense, a ray with direction $\mathfrak{D}$ and origin $o$ intersects a triangle with edge vertices $\mathbb{v}_{0}, \mathbb{v}_{1}$ and $\mathbb{v}_{2}$ if the following criteria shown in equations 11.2 and 11.3 are satisfied.

$$
\left\{t, w_{1}, w_{2},\left(1-w_{1}-w_{2}\right)\right\} \geq 0
$$




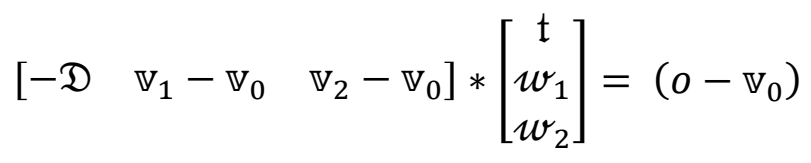

where $t$ is the parametric distance along the ray and $w_{1}$ and $w_{2}$ are components of bary-centric coordinate system for a triangle. In practice, Cramer's rule is used to solve for $t, w_{1}$ and $w_{2}$ [201]. Details of ray-search algorithms for interior/exterior point classification are shown in references $[199,198]$.

The third stage determines which nodes lay on the surface of the triangulated mesh. This stage is used for collecting information for classifying line segments in the fourth stage. Each node is projected onto the plane of each triangle and barycentric coordinates are used to check if the projected nodes lay within the triangles $[201,200]$. The criteria to determine if a node is within a triangle in three dimensions are:

$$
\begin{aligned}
& q=\left(\mathbb{v}_{0}-\mathbb{P}_{B}\right) \cdot \vec{n}
\end{aligned}
$$

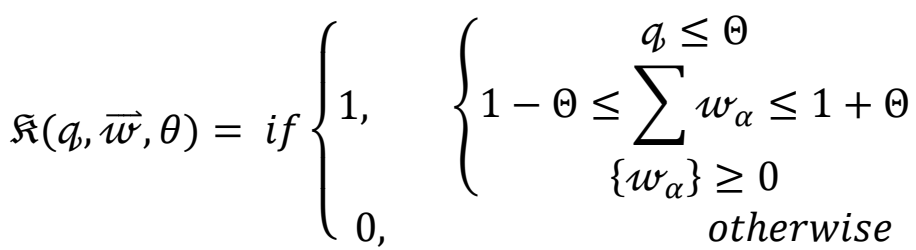

Where $\mathfrak{K}\left(q, \mathcal{W}_{\alpha}, \Theta\right)$ is a function to determine if the node lies on a surface, $\mathbb{v}_{0}$ is the first vertex of a triangle in $\mathbb{R}^{3}, \mathbb{P}_{B}$ is the node being evaluated, $\vec{n}$ is the unit normal of the triangle, $\Theta$ is the numerical tolerance, $w_{\alpha}$ is the barycentric coordinates of a point $\mathbb{P}_{A}$ for the triangle where $\alpha$ is equal to:

$$
\mathbb{P}_{A}=(q) \vec{n}-\mathbb{P}_{B}
$$

The fourth stage is the trimming algorithm which begins by evaluating each line segment from the voxelized structure and checks if an intersection occurs within it and the triangulated surface. Möller-Trumbore's ray-triangle intersection algorithm is adopted for this operation [203, 202]. 
During the trimming process, the nodes at the surface of the trimmed structure are also extracted to be used for constructing the lattice net.

After calculating all possible intersections between the line and the triangulated surface, the algorithm classifies the intersections depending on the locations of the nodes from the second and third stage. The type of class determines if the line should be cut, kept or rejected and which line segment pieces are to be retained. There are two main cases and eleven subcases for classifying line segments and is illustrated in Figure 65.

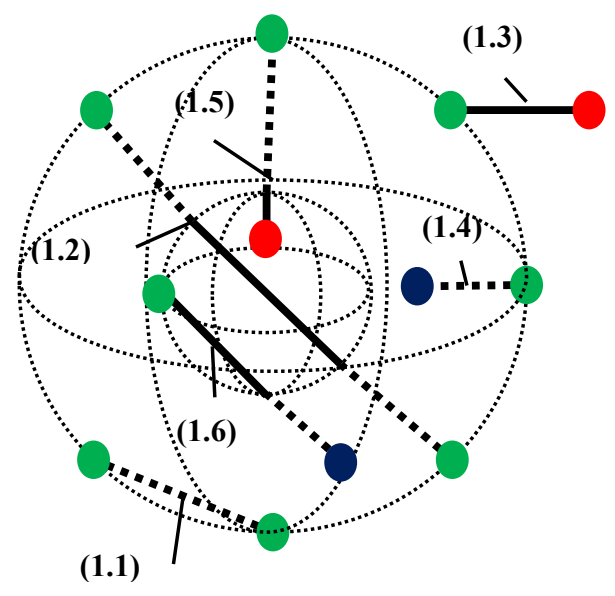

Case 1 : Node On Surface

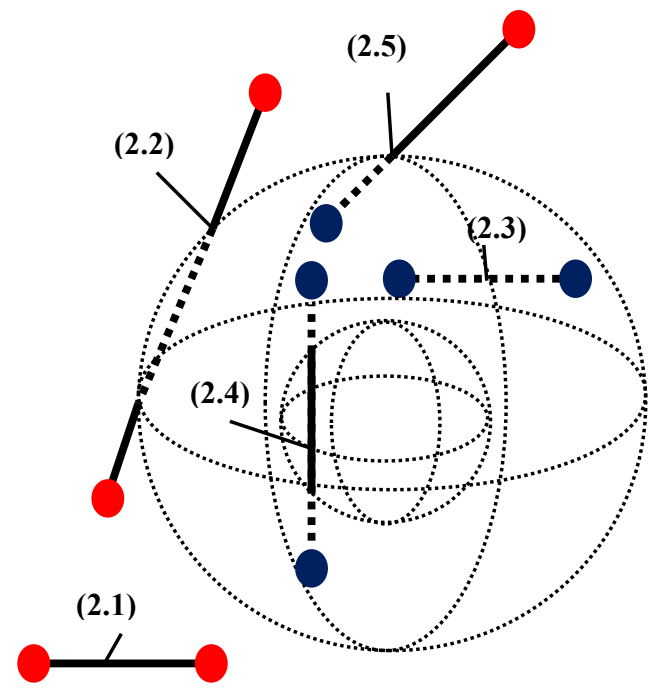

Case 2 : Nodes not On Surface

Figure 65 : Line Segment Classification within a Hollow Sphere (Red) Exterior Node (Blue) Interior Node

(Green) Surface Node (Dotted Line) Interior Segment (Solid Line) Exterior Segment

A hollow sphere is used to illustrate the different possibilities for a line segment intersection with a closed surface. Case 1 in Figure 65 shows six subcases where a node is on the surface of the triangulated surface while Case 2 contains five general subcases. The distinction between Case 1 and 2 is required because a node on the surface cannot be classified as either outside or inside the design space and the general method will fail. Dotted lines are line segments inside the hollow sphere and solid lines are located outside of the closed surface. The goal of the trimming and 
classification process is to retain the interior (dotted) line segments. The line subcase is used to determine a starting point (or reference node) for a pairing algorithm to determine the correct line segment pieces to be extracted. Intersection points and nodes at the surface are saved as surface nodes to build the lattice net.

Appendix I.1 contains algorithm 1 that exploits line segments classification given positions of the nodes and its intersections with the triangulated surface. Algorithm 1 uses Boolean logical arrays to sort the line segments efficiently. After sorting, the interior line segment pieces can be identified by grouping nodes without replacement based on their distance from a reference node. In addition, Appendix I.2 presents the process of selecting the proper reference node for collecting the interior line segment pieces where a reference node is either an intersection point or an interior point depending on the line segment case. After the final step, the interior line segments are retained as the final trimmed lattices structure. The entire algorithm for generating the interior struts and collecting surface nodes is shown in Appendix I.3.

\section{H.4 Algorithm for a Conformal Lattice Net for a Trimmed Symmetric Lattice}

For a given closed surface and an interior trimmed lattice, it is possible to connect the cut struts together using the common crystallographic planes of the unit cell. Closing open connections is important as unconnected struts bear no loads and only unnecessarily increase the weight and manufacturing time of the final design. Connecting the exterior lattice nodes also helps with ensuring that the entire volume is utilized.

Before initializing the lattice net generation algorithm, the center of rotation (for the triangulated surface), surface node coordinates (intersection between the truncated struts and the triangulated surface) and a seeding point is required. The seeding point is the minimum coordinates for a unit 
cell or voxel centroid. The seeding point is used to orient and correctly space the contours along the triangulated surface. The center of rotation is used for plane-surface contour collection which rotates the triangulated surface so its z-axis is equivalent to the plane normal direction of the unit cells crystallographic planes. In addition, information about the unit cell crystallographic planes is required and includes the inter-planar spacing between common planes and their associated unit normals. Figure 66 displays and categorizes the necessary information required to be passed to the lattice net algorithm.

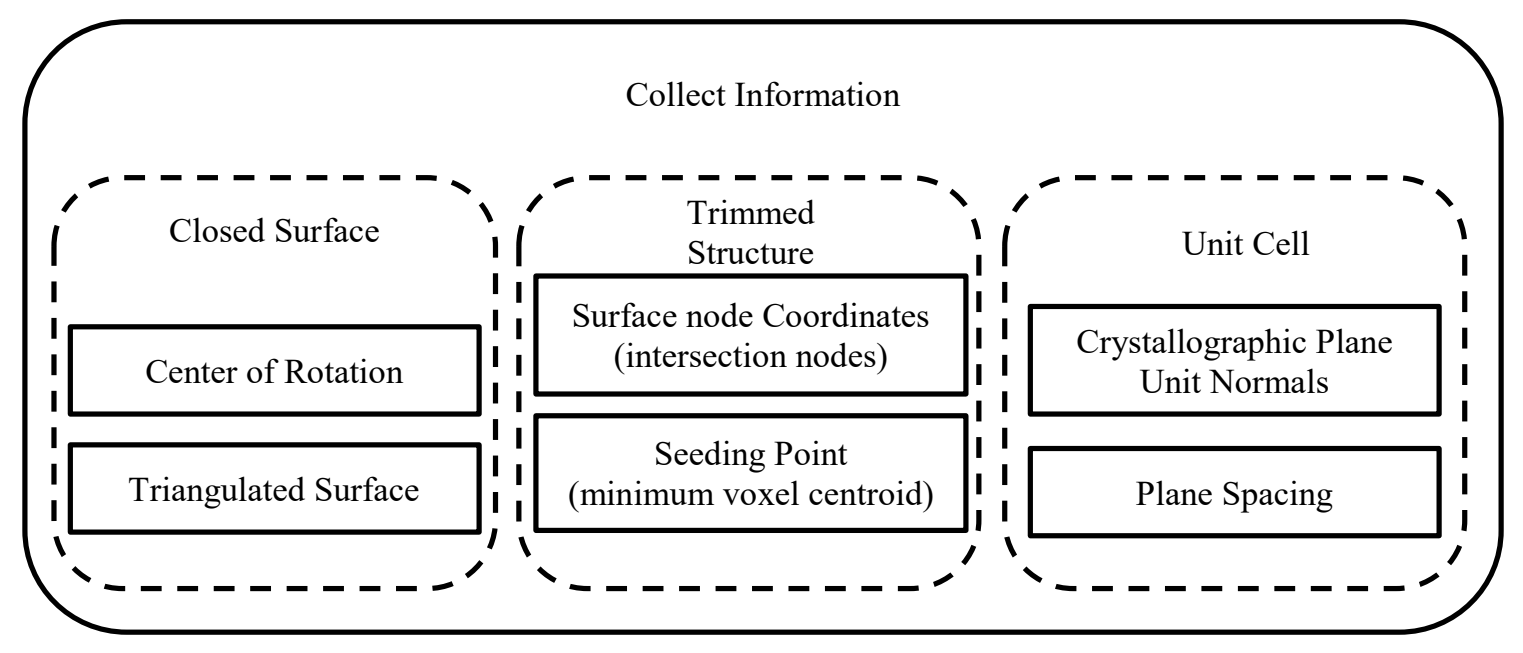

Figure 66 : Input Information for Lattice Net Generation

The lattice net generation algorithm contains five stages, shown in Figure 67; a contour collection stage, additional nodes from contour intersection and "important features" collections stage, then the lattice net generations stage which includes a projection filter, then a feature preservation stage followed by a final clean-up stage. A feature is a sharp angle on the triangulated surface to be retained in the lattice net during the contour collection stage. After generating the lattice net, a post processing step is applied to remove any duplicate line segments. The final cleanup step which collapses beams with less than three connections is done 
to simplify the wireframe structure. A pseudo code is developed for the whole process and can be found in Appendix I.7.

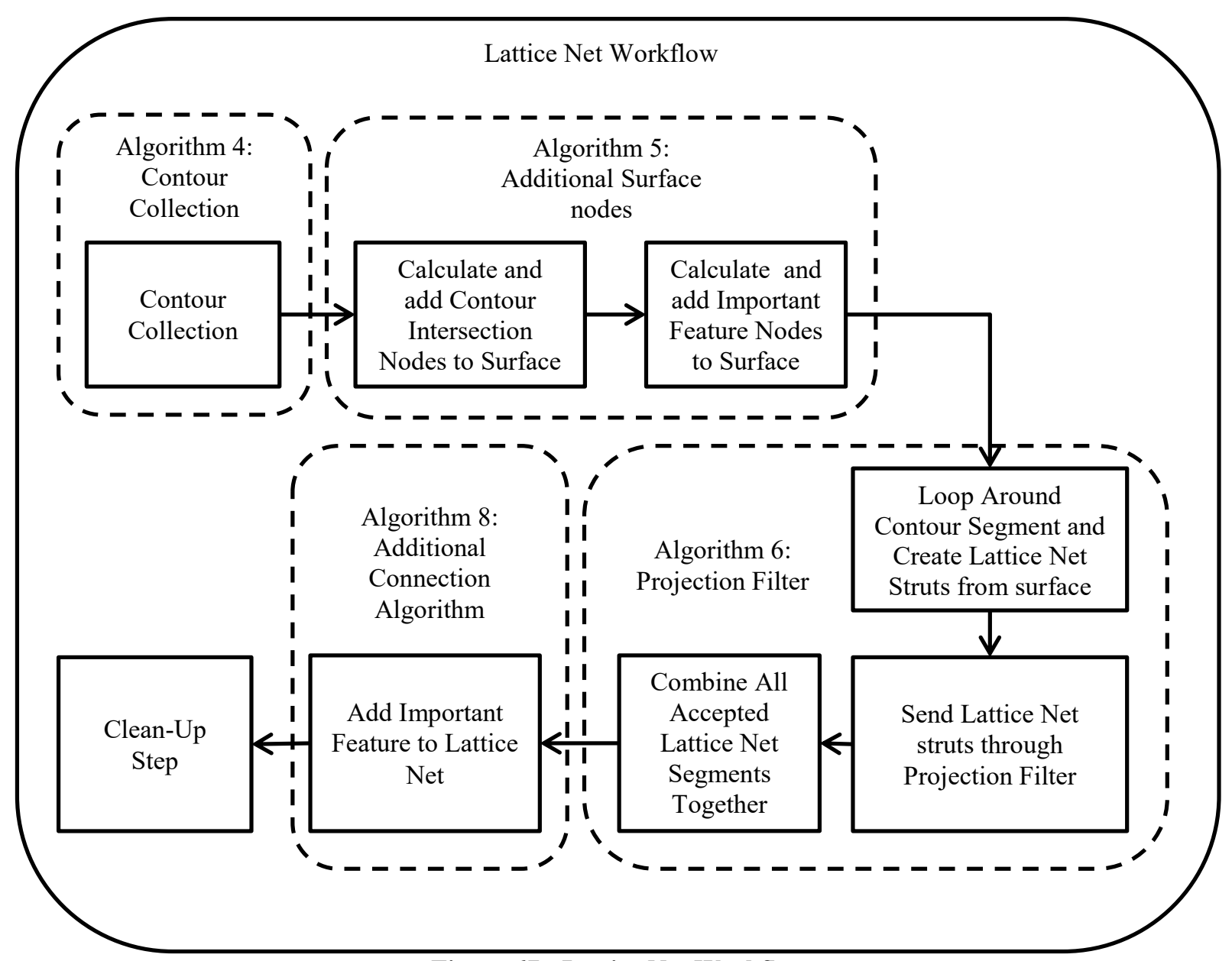

Figure 67 : Lattice Net Workflow

\section{H.4.1 Initial Contour Collection}

The first step collects the contours for all possible planes along the triangulated surface. The rotation center is used to rotate the triangulated surface so that the $\mathrm{z}$-axis matches the normal of a particular crystallographic plane as illustrated in Figure 68. A rotation matrix is calculated for each crystallographic plane normal and can be defined as [204, 205]: 


$$
Я_{i}=I+[\tau]_{\times}+[\tau]_{\times}^{2} \frac{1-\overrightarrow{\mathbb{V}_{l}} \cdot \overrightarrow{\mathbb{U}_{z}}}{\left\|\overrightarrow{\mathbb{V}_{l}} \times \overrightarrow{\mathbb{U}_{z}}\right\|^{2}}, \quad \mathrm{i}=1,2,3, \ldots \mathbb{k}
$$

where $Я_{i}$ is the rotation matrix to align $\overrightarrow{\mathbb{V}}_{i}$ with $\overrightarrow{\mathbb{U}}_{z}, I$ is the identity matrix, $[\tau]_{\times}$is a skewsymmetric cross product matrix between $\overrightarrow{\mathbb{V}}_{i}$ and $\overrightarrow{\mathbb{U}}_{z}$ and $i$ is a subscript for the $\mathbb{k}$ crystallographic planes. If both vectors $\overrightarrow{\mathbb{V}}$ and $\overrightarrow{\mathbb{U}}$ are equivalent, then the rotation matrix $\AA_{i}$ cannot be calculated and is instead replaced with the identity matrix. Another condition which results in a singular rotation matrix is when $\overrightarrow{\mathbb{V}}_{i}=-\overrightarrow{\mathbb{U}}_{z} ; Я_{i}$ must be replaced with $-I$. The vector $\overrightarrow{\mathbb{V}}_{i}$ is the crystallographic plane normal while $\overrightarrow{\mathbb{U}_{z}}$ is the direction of the $z$-axis and is $(0,0,1)$.

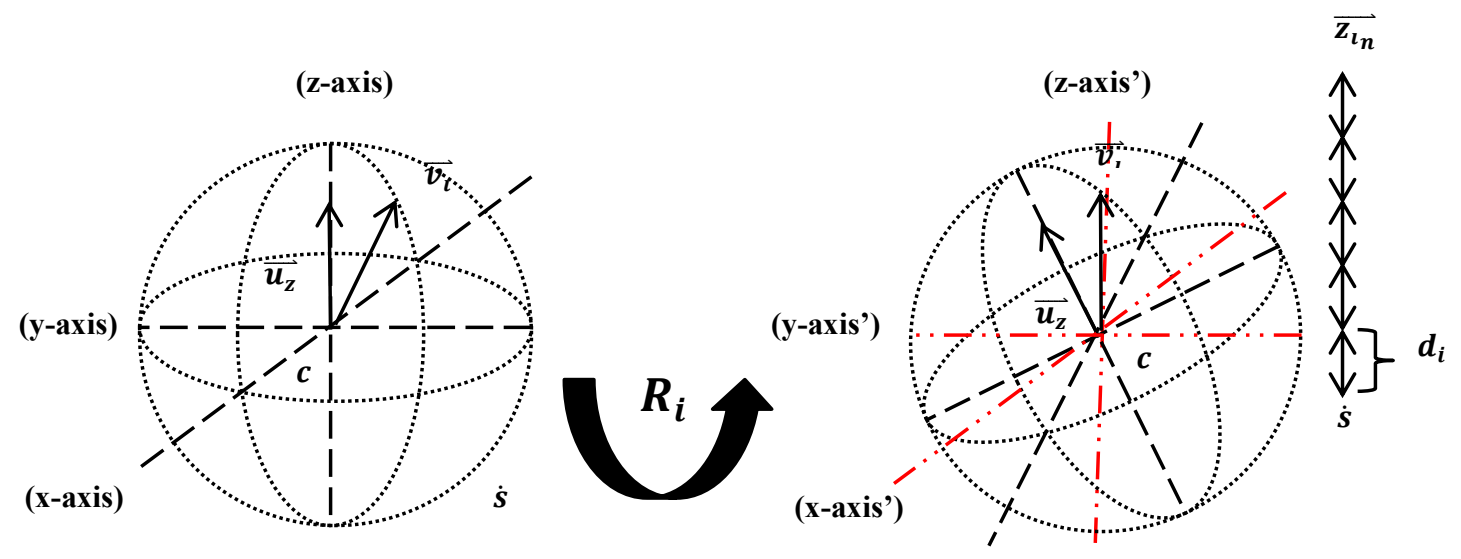

Figure 68: Axis Re-Alignment and Planar Elevations Calculations

Surface contours are collected at different elevations determined by crystallographic plane distances. The plane distances are the inter-planar distance between the same planes in a repeated unit cell structure. The planar elevations are calculated as;

$$
\begin{gathered}
s_{i}=Я_{i}(s-\mathfrak{c})+\mathfrak{c} \\
\overline{Z_{\iota_{n}}}=s_{i_{z}}+d_{i} n, \quad n=1,2,3 \ldots m
\end{gathered}
$$

where $\overline{Z_{l_{n}}}$ is a sequence of elevations, $d_{i}$ is the inter-planar distance and $s_{i}$ is the re-oriented seeding point for crystallographic plane $i . m$ is an integer number such that the maximum 
elevation is higher than the rotated triangulated surfaces highest surface node. $s$ is the original coordinate of the seeding point and $\mathrm{c}$ is the center of rotation.

a)

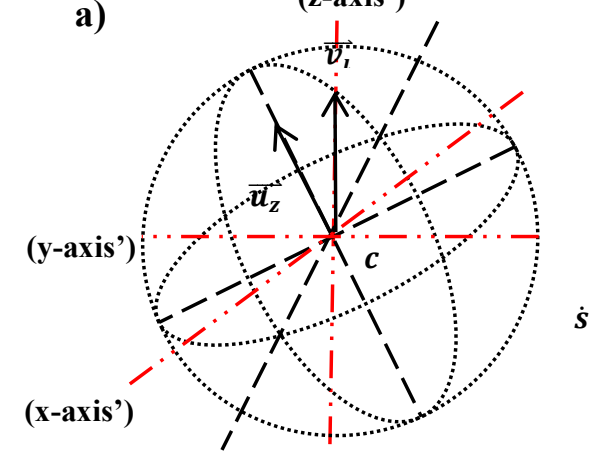

b)

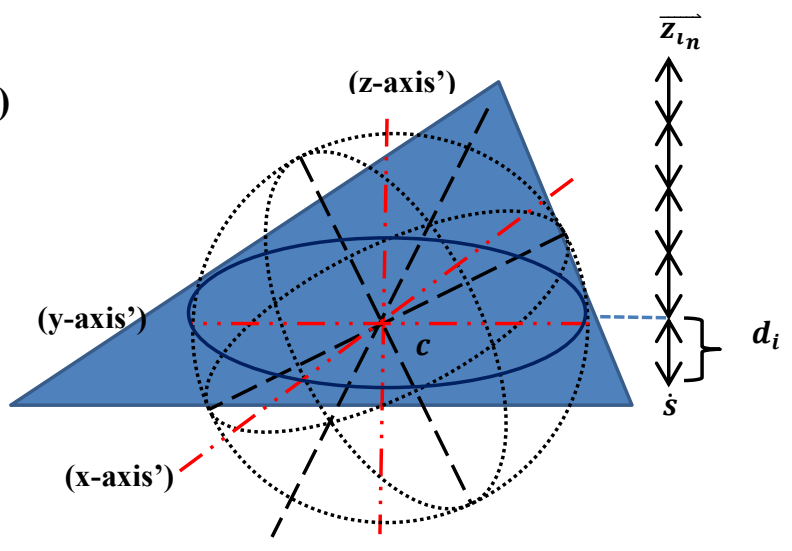

Figure 69: Surface Intersection (a) Orientation (b) Sphere Sliced by Triangle creates Contour at $Z_{i_{n}}=d_{i}$

Contour edges are computed using the Möller-Trumbore's algorithm for surface intersections $[203,206]$. Seen in Figure 69, one large equilateral triangle is used for slicing the triangulated surface; the contour edges are collected into organized lists to form a loop and then stored into an individual "bin" for each elevation and plane. For each contour, the nodes defining the path of the contour must be reoriented from the current $\mathrm{z}$-axis to their original axis using ${\mathrm{A}_{i}^{-1}}^{-1}$ about c. A pseudo code for this process can be seen in Appendix I.4.

\section{H.4.2 Additional Surface nodes Calculations}

Additional nodes are added to the input surface nodes calculated from the trimming algorithm. These nodes are calculated from the intersections between the plane slicing contours. This occurs for lattice topologies such as $\mathrm{BCC}$ where an ' $\mathrm{X}$ ' shape of the interior struts must be projected onto the surface of the volume and can be seen in Figure 71a. In addition to the intersections, important sharp features can also be preserved. Important features can also be retained by supplying an edge list of sharp features. For example, the edges of a cube, shown in Figure $71 \mathrm{~b}$ 
are sharp features that can be preserved and retained in the lattice net. In MATLAB, this can be done using the function "featureEdges" [207].

The additional nodes for both the contour intersection and important features are found by first rotating the contour lines and the important feature edges around the center of rotation $c$ so that the crystallographic plane normal points into the z-direction. This rotation strategy is the same as for the contour collection algorithm shown in equation 7 . The second step is to calculate the intersection nodes from the specified planar heights for the given rotation. The heights or elevations are calculated using equations 8 and 9. Edges in plane with the current plane or elevations are omitted. To find the intersection points, the feature and contour edges are converted into parameterized line formulated as:

$$
f(\mathrm{t})=\mathbb{P}_{1}+\mathrm{t} *\left(\mathbb{P}_{2}-\mathbb{P}_{1}\right)
$$

where $t$ is the independent variable, $\mathbb{P}_{1}$ and $\mathbb{P}_{2}$ are points along the line segment or edge. To solve for t; equation 10 can be rearranged as;

$$
\overrightarrow{\mathrm{t}_{l_{Z}}}=\left(\overrightarrow{Z_{l_{n}}}-\mathbb{P}_{1_{Z}}\right) /\left(\mathbb{P}_{2_{Z}}-\mathbb{P}_{1_{Z}}\right)
$$

where $\overline{Z_{l_{n}}}$ is a sequence of target elevations from the planar distances for a particular crystallographic plane $i$. A valid intersection occurs when $1 \geq \mathrm{t} \geq 0$. Valid intersection points are rotated about $\mathfrak{c}$ with $Я_{i}^{-1}$ and added to the "bin" (or list) of surface nodes bin with duplicate nodes removed. This bin of nodes is then used to generate the lattice net in the third stage. A pseudo code is written to further explain the method and is referred to as "Algorithm 5 : Additional Surface Nodes" in Appendix I.5. 


\section{H.4.3 Connecting the Lattice Net}

The proposed algorithm loops through all the contours and connect the cut struts together based on a collection of surface nodes acquired from the second stage. It is noted in this research paper that the surface nodes obtained by the intersection points calculated from the trimming algorithm are the same as those calculated from the contour-contour intersection nodes. For this reason it is possible to construct the net independently from the interior trimmed lattice.

a)
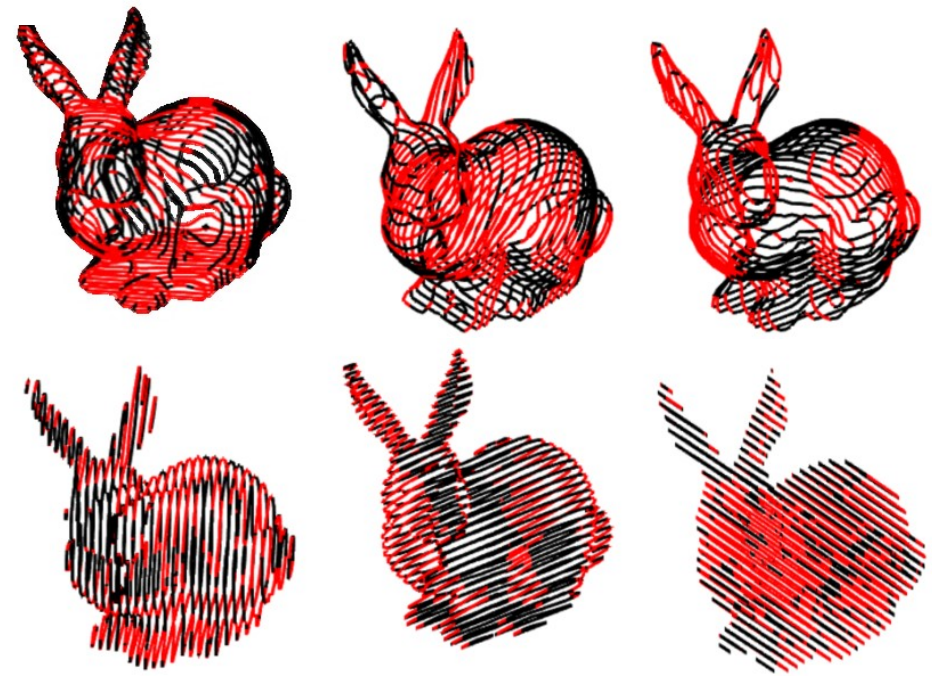

b)

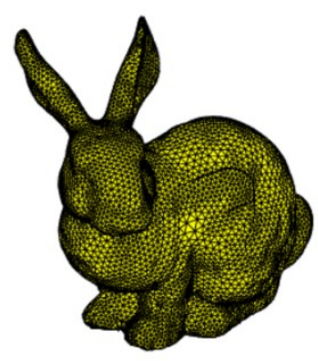

c)

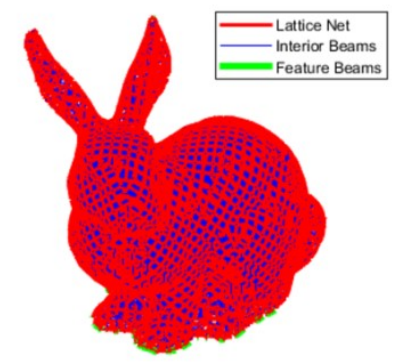

Figure 70 : (a) Filtering of Lattice Net Segments for each Crystallographic Plane of a BCC unit cell (b) Input Mesh and (c) Final Construction 
For a collection of contours for a specific crystallographic plane (i), the lattice net generation algorithm collects any surface nodes that lie within the specified contour loop into a list. That list is then converted into new line segments that represent wireframe sections of the lattice net.

When all the contours have been evaluated for the current plane, a projection filter is used to remove line segments that do not satisfy the unit cells strut orientation for that crystallographic plane. The filter also aims to remove zero-length line segments when projected into specific planes. If a line segment is accepted by the filter at certain angles, then it is retained in the lattice net. An illustration of what happens can be seen in Figure 70a for a BCC lattice. The red segments are the accepted sections from the filter while the black sections are the rejected pieces. The summation of all the accepted contour sections will create the final lattice net. An example of the final lattice net and it construction is seen in Figure 70c.

There are two possible filtering methods developed in this work. The first filter works by comparing the ratio of the projected normalized crystallographic plane normal into the $\mathrm{x}, \mathrm{y}$ and $\mathrm{z}$ planes with the projected length of the line segments normalized directions into the same $\mathrm{x} y$ and z planes. However, any projection plane can be used depending on the type of lattice topology or crystallographic family. Projection of a line onto a plane can be calculated as;

$$
\overrightarrow{\mathrm{g}}=\overrightarrow{\mathfrak{D}} \| \overrightarrow{\mathrm{b}}=\left(\overrightarrow{\mathrm{b}} \times\left(\frac{\overrightarrow{\mathfrak{D}} \times \overrightarrow{\mathfrak{b}}}{\|\overrightarrow{\mathfrak{b}}\|}\right)\right) \frac{1}{\|\overrightarrow{\mathfrak{b}}\|}
$$

where $\overrightarrow{\mathfrak{D}}$ is the normalized direction of the line or ray, $\overrightarrow{\mathrm{b}}$ is the plane unit normal and $\overrightarrow{\mathrm{g}}$ is the resulting line projection. For both discussed filters in the paper, the projected normal of the crystallographic plane is projected along with the list of line segments. 
If the line segment can satisfy the projection onto any of the three planes, then it is not rejected. A tolerance is given for the ratios to relax the acceptance criteria; a tighter tolerance would mean that the remaining line segments will need to strictly satisfy the projection lines. A tolerance of zero would work best if the curvature of the surface is zero such as a cube. For curved surface a higher tolerance is needed. Equation 13 is used to determine if a line segment will be accepted by the first filter.

$$
\text { Accept }=\left\{\begin{array}{lr}
\text { true, }, & 1-\Theta<\frac{\left\|\overrightarrow{\mathbb{g}_{z}}\right\|}{\left\|\overrightarrow{\mathrm{g}}_{i}\right\|}<1+\Theta \\
\text { false, } & \text { otherwise }
\end{array}\right.
$$

where $\vec{g}_{i}$ is the projected crystallographic plane normal onto the projection plane, $g_{z}$ is a projected line segment onto the projection plane and $\Theta$ is a tolerance level.

The second projection filter method compares the components of the projected segments $\overrightarrow{g_{z}}$ to $\vec{g}_{i}$ and $-\vec{g}_{i}$ as the filtering criterion. The criteria to determine if the line segment is accepted by the second filter type can be expressed as.

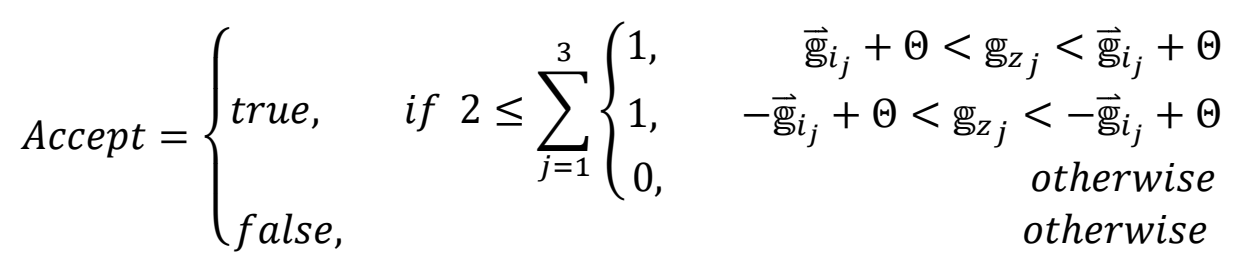

where $j$ indexes the dimensional entries of the vectors.

The second filter type cannot be applied to cubic unit cell crystallographic planes (100), (010) and (001) because all line segments will be rejected. However this method has shown to work very well with BCC unit cell topologies for planes (110), (101) and (011). The pseudo code for the projection filters are given in Appendix I.6. 


\section{H.5 Additional Connections for the Conformal Lattice Net}

After the initial construction of the lattice net, unit cells could be projected onto sharp corners or features. If the unit cell size does not precisely match the dimensions of the sharp geometries; a discontinuity between the struts will occur at the sharp feature. This occurs when the corner of a unit cell that is not placed directly at a vertex. Figure 71a shows a cube without any additional connections at the edges while Figure $71 \mathrm{~b}$ shows the addition of the important features into the lattice net. The added features allow structural loads to be transferred uniformly throughout the net eliminating discontinuities and improving the mechanical behavior of the lattice structure.

a)

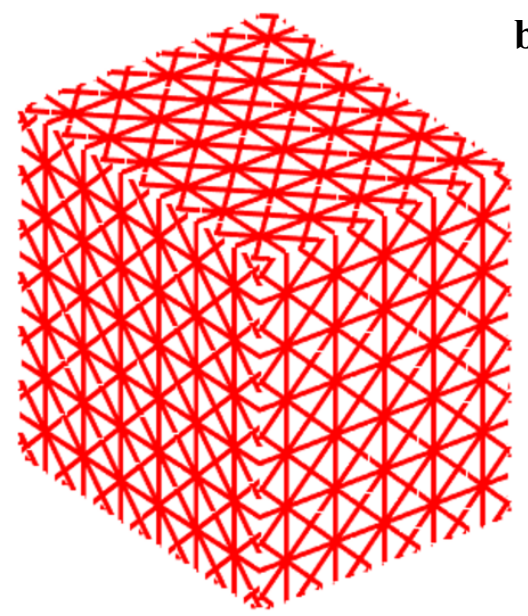

b)

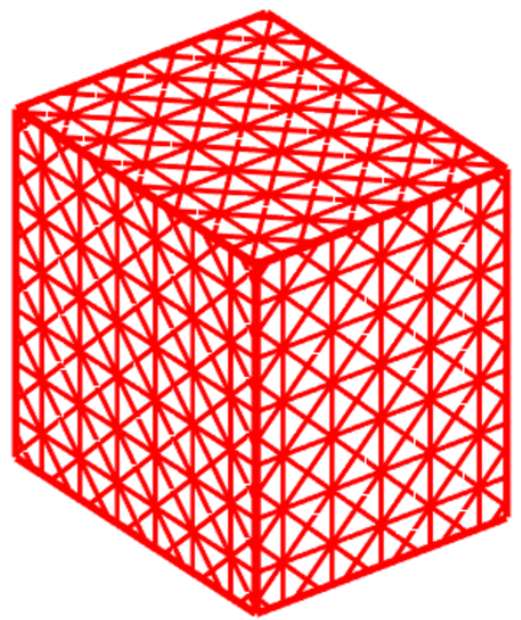

Figure 71: (a) Lattice net without preserved features and (b) Lattice net with preserved features

To promote better continuity in the lattice structure, sharp features of the geometry are extracted and added to the lattice net. This is done by collecting sharp edges of the input triangulated surface and then organizing those edges into open and closed loops. Loops are defined as edges connected to other edges based on a common node. A closed loop is a sequence of nodes where the first and final nodes are identical while an open loop has different nodes at each end. The 
nodes at intersections or junctions between the open and closed loops are also identified and are added to the list of lattice nodes. Junction points are found if a node is referenced more than twice from the extracted edges.

The new struts are created by following the open and closed loops and collecting any of the lattice net nodes or junction points that fall within the edges into an ordered list or sequence. If each node in the ordered lists is given unique identification, then the new struts can be created by connecting the nodes together from their positions in a list. A pseudo code is for the process is found in Appendix I.8 to explain the entire process.

\section{H.6 Clean Up Step}

The last step for the wireframe development includes a clean-up stage. Line segment connections are simplified by ensuring that all nodes are connected to at least three line segments. This does not include nodes that are used to conserve important features or sharp edges. After the net clean-up step, the interior trimmed lattice and lattice net are combined and any duplicate line segments are removed from the structure. In a structural sense, the clean-up step helps improve connectivity by removing non-loadbearing connections and creates more efficient load paths.

\section{H.7 Numerical Examples and Demonstrations}

The importance of filtering the struts is shown in Figure 72a and $b$. The initial surface contours create additional vertical cuts in Figure 72a on the front and top faces. Figure $72 b$ is the result of projection filtering to remove the unneeded contour lines. The effects of scaling by different unit cell sizes are shown in Figure 72e and f. The cylinder in Figure 72e contains small patterns where the unit cell is cut and produces non-uniform geometry radially. Figure $72 \mathrm{f}$ shows for smaller unit cell sizes the effects non-uniformity is diminished. Rotation of different sized unit 
cells is demonstrated in Figure 72c and d where the front faces pattern is rotated 30 degrees and has a net pattern slightly smaller than the top and side faces.

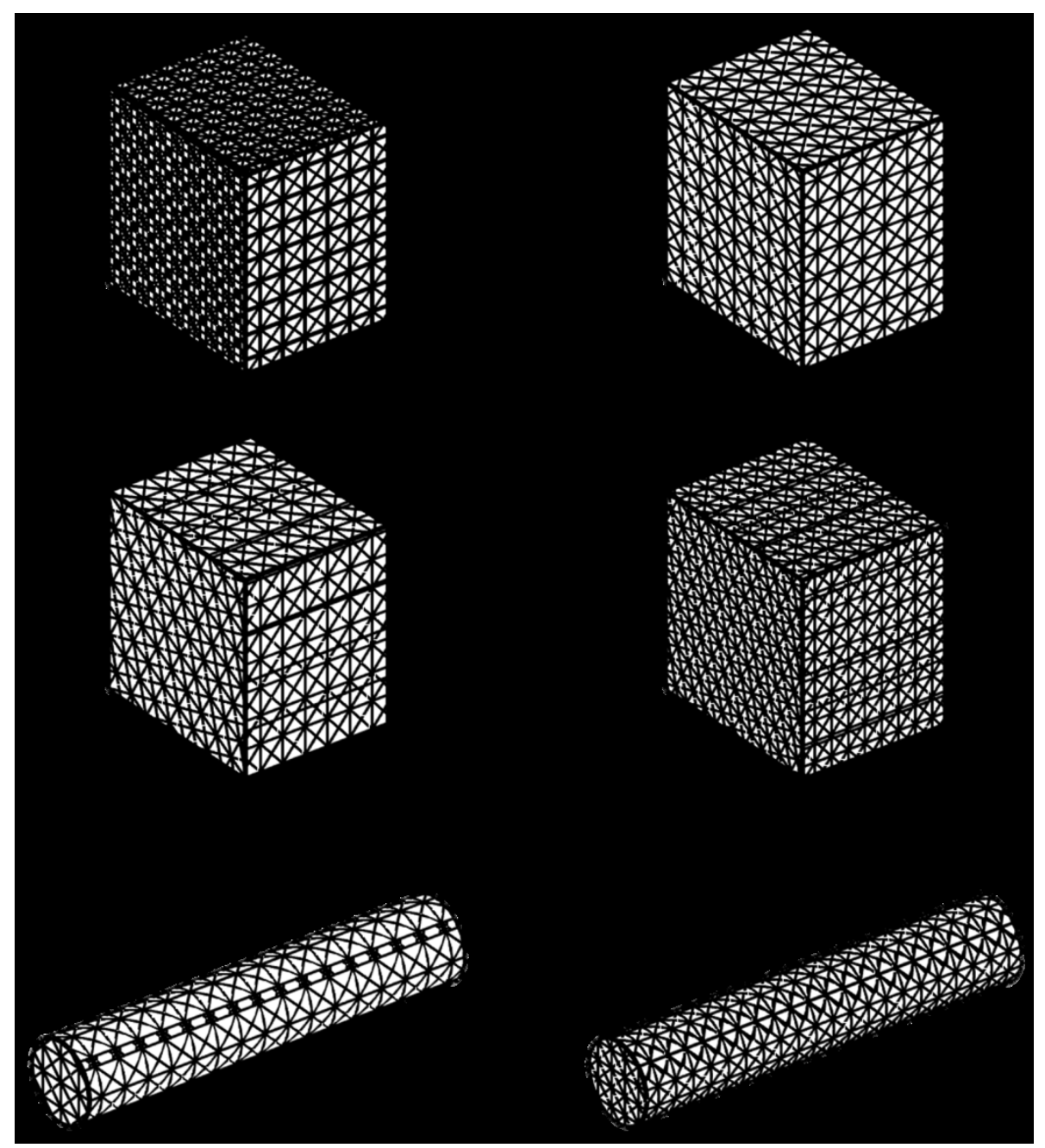

Figure 72 : FCC Net (a) No Filter (b) With filter applied (c,d) Cube with Lattice rotated $30^{\circ}$ with unit cell sizes 12 and $9(e, f)$ Cylinder with unit cell sizes 12 and 9

Six crystallographic planes are observed in cubic Bravais Lattices. FCC topology contains six plane types and is a superposition of the Cubic planes and the BCC planes. For some lattice configurations such as diamond (Figure 73a) and the octet truss (Figure 73d), the side profiles of these topologies are equivalent to the $\mathrm{BCC}$ and $\mathrm{FCC}$ unit cell respectively. Figure $73 \mathrm{~b}$ shows the 
front view of the diamond lattice is equivalent to that of the BCC lattice and its skin is meshed using crystallographic planes (110), (101) and (011) in Figure 73c.
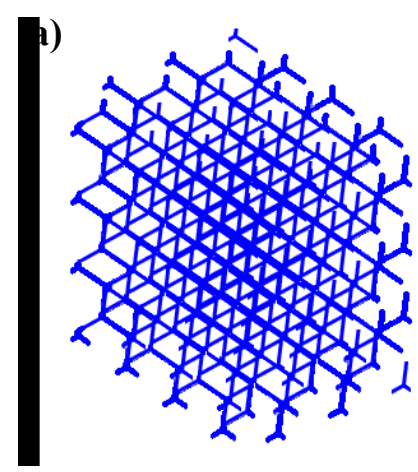

d)

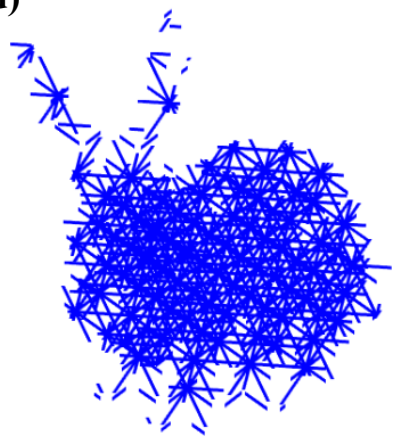

e)

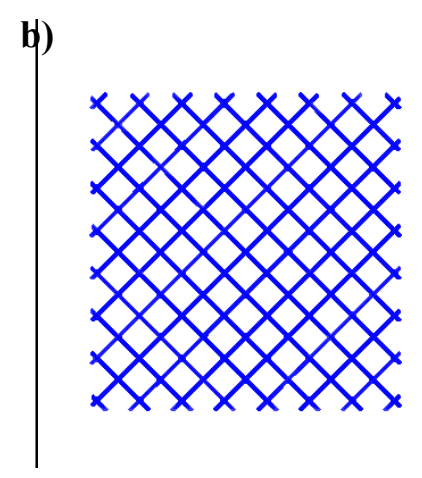

c)
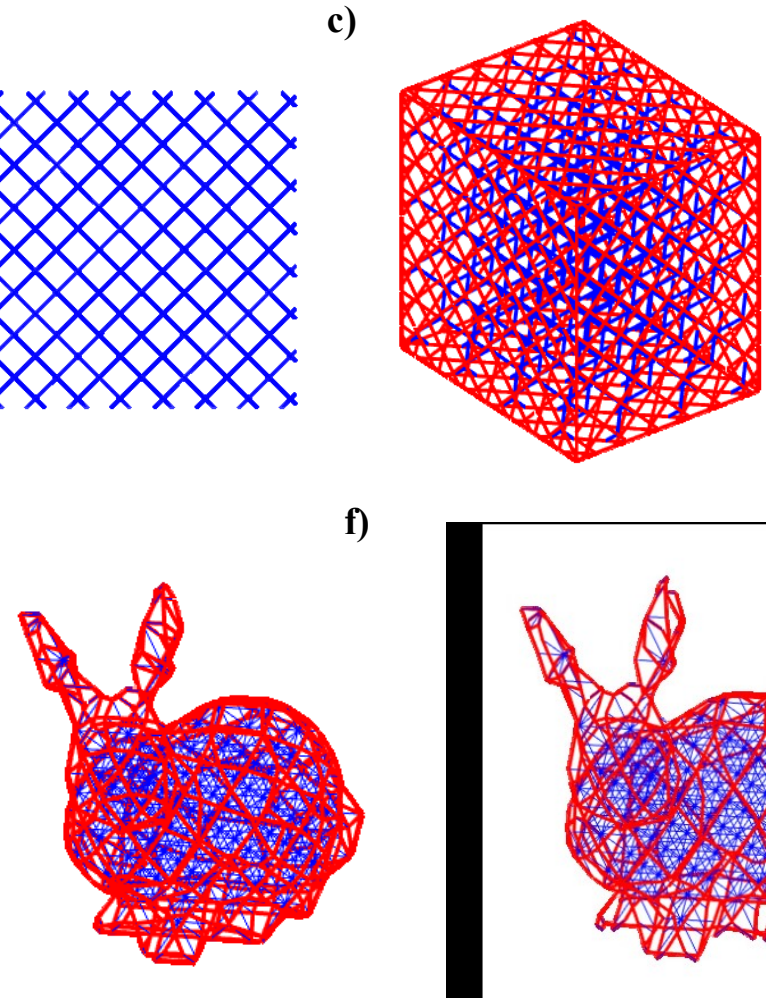

f)

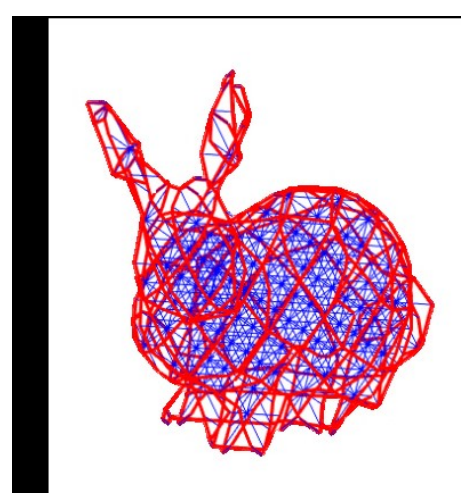

Figure 73 : (a) Interior Diamond Lattice (b) Front view of Diamond Lattice (c) Diamond Lattice net using $\{110\}$ (d) Bunny interior of Octet Truss (e) Octet Truss Lattice net with $\{111\}$ (f) Octet Truss Lattice Net with no filter and planes $(\overline{1} 11),(\overline{11}),(\overline{11} 1)$

Figure $73 \mathrm{f}$ displays a lattice net around the Stanford bunny model using only three crystallographic planes and no filter to connect an interior octet-truss lattice shown in Figure 73d. The resulting net is very similar to Figure $73 \mathrm{e}$ which had used all family planes from $\{111\}$ and the projection filter. This shows the possibility to omit the filter and select a few planes of symmetry to connect all the cut struts together of a trimmed lattice. 


\section{H.8 Advantages and Disadvantages of the Current Lattice Net Algorithm}

The main advantage of the lattice net algorithm is its ability to connect the struts of a symmetric unit cell of any size while maintaining the overall geometry of the triangulated surface. The ability to preserve important features is another advantage which helps promote continuity and shape. The lattice net construction is also very fast, even for a high number of surface nodes and plane intersections. Another crucial advantage is that connectivity is guaranteed among all cut struts, even if the lattice net does not follow the unit cell pattern very well. The final wireframe is also memory efficient due to being comprised of a list of nodal coordinates and can be easily converted into a finite element mesh for optimization applications. Another key factor about the lattice net algorithm is that its creation is independent of the interior trimmed lattice. This is because all the contour intersections produce the same intersection points from the lattice trimming section. The final advantage is that a BCC combined with a CUBIC lattice net can be used for many lattice topologies that share cubic symmetries such as the diamond lattice.

Disadvantages include the lack of support for non-symmetric lattices. Truncated octahedron lattice structures cannot be used for the lattice net to accurately capture the projected pattern of the lattice on a surface. However, a BCC and cubic lattice net can connect all the cut struts of this topology because it contains Bravais crystallographic planes. A final disadvantage is that it is not robust and depends on the tolerance level of the projection filter. For complex surfaces, the projection filter may fail to filter out or over filter connected struts. At sharp curvatures, the connected struts for the net may be rejected more easily by the filter. There is also an issue of impossible strut connections at highly curved cross sections. The issue of projection onto curved surfaces for lattice nets has been noted by Aremu et al. [194] in their own implementation of conforming lattice skins. Irregular strut connections arise from complex geometries such as 
saddle points which give the algorithm the most problems. However, for flat surfaces, such as a cube or rectangle, the algorithm works very well as seen Figure 71.

In conclusion, the lattice net algorithm while not perfect is quite flexible as only two or three crystallographic planes are needed to connect all the cut struts together for most symmetric topologies as seen in Figure $73 \mathrm{f}$.

\section{H.9 Lattice Net Case Study Example for Engineering Applications}

The purpose the lattice trimming algorithm is to generate a wireframe structure that can be embedded into any complex geometry. This wire frame geometry is then directly converted into a collection of beam elements in a finite element mesh for sizing optimizations. This section will demonstrate the performance of an optimized lattice structure meshed with different lattice topologies when a lattice net is or is not applied to the outside geometry. A simple Messerschmitt-Bolkow-Blohm (MBB) Beam [69] is used to compare the different lattice topologies and their performances when subjected to sizing optimizations.

\section{H.10 Problem Formulation}

The optimization problem is formulated such that design variables are the cross-section of the beam elements. Tapered beams are used in the finite element mesh with the common joints as a unified design variable for all connected tapered beams. The advantage of using tapered beam elements is that the unified joints will reduce the number of design variables during the optimization. The optimization problem will be referred to as a lattice beam optimization and is described by equation 11.15 . 


$$
\begin{gathered}
\text { minimize: } M_{\text {lattice }}(r)=\sum_{i=1}^{\varepsilon} \frac{1}{2} \rho_{i} \pi\left(r_{i, 1}^{2}+r_{i, 2}^{2}\right) L_{i} \\
\text { subject to: }\left\{\begin{array}{c}
K(r) U=F \\
\sigma_{i}\left(r_{i}, U_{i}\right) \leq \sigma_{\max } \\
r_{\min } \leq r_{i} \leq r_{\max } \\
\rho_{i}, L_{i}>0
\end{array}\right.
\end{gathered}
$$

Where $r_{i}$ is the cross sectional radii of the ends of the tapered beams, $K(r)$ is the global stiffness matrix as a function of the design variables, $\mathrm{F}$ is a constant force vector for static analysis, $\sigma_{\max }$ is the maximum allowable stress $(880 \mathrm{MPa}), \rho_{i}$ is the density of the material and $L_{i}$ is the length of the $i^{\text {th }}$ beam and $\mathcal{E}$ is the number of tapered beam elements. The objective $M_{\text {lattice }}(r)$ is measures the mass. The minimum radii for the optimization is $0.001 \mathrm{~mm}$ and the maximum radius is $0.5 \mathrm{~mm}$. Titanium was chosen as the material of choice and the young`s modulus is rated at $11400 \mathrm{MPa}$, Poisson ratio is 0.31 , density is $4.506 \mathrm{~g} / \mathrm{cm}^{3}$ and has a yield strength of 880 MPa.

The MBB Beam is meshed with different lattice topologies and includes Cubic, BCC, FCC and the octet truss. The MBB beam is a double supported beam with a vertical force applied at the center. Figure 74 shows the MBB mesh where the yellow elements represent the lattice domain to be replaced with a trimmed lattice structure and the brown elements represent the solid domain to attach forces and boundary conditions. The beams are then attached to the solid elements by tie contacts (contact surface) were the lattice beam nodes are the slave set and the solid elements as the master set. For test cases with a lattice net, any beam elements whose nodes 
are twice connected to the master set are removed. The optimizations for the case study will be done in Altair Optistruct using the "BIGOPT" optimization algorithm [101].

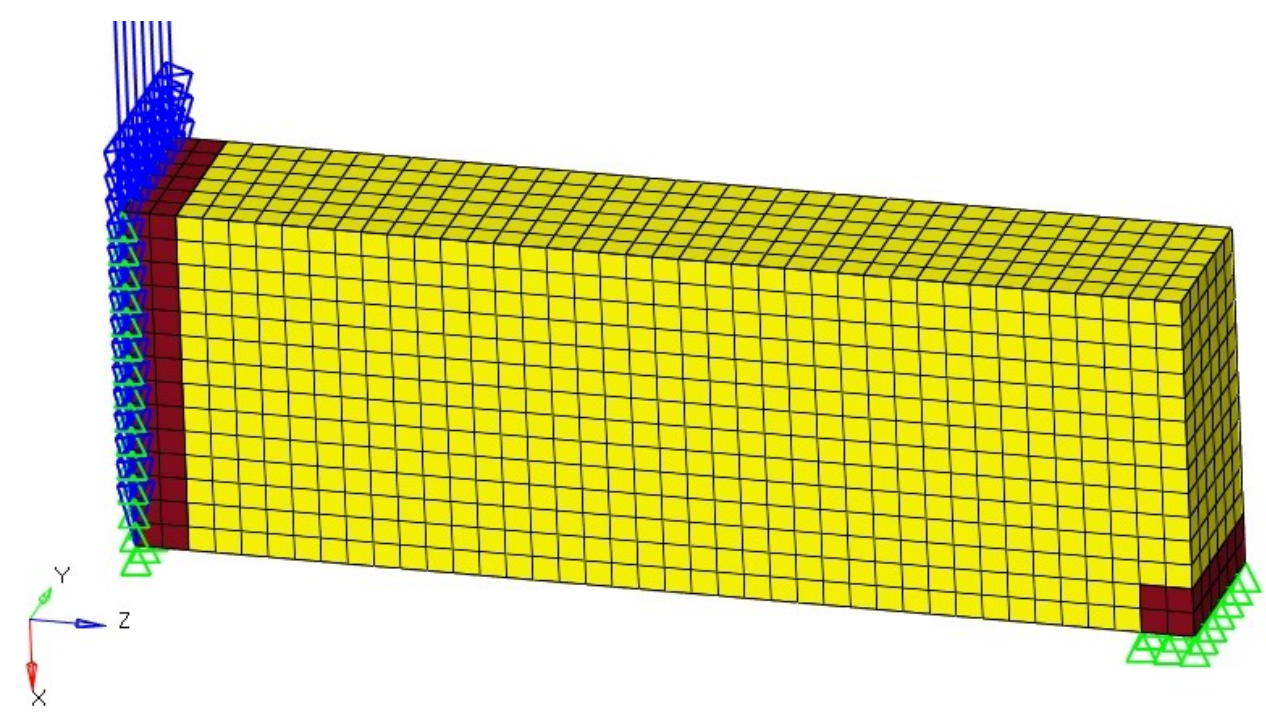

Figure 74 : MBB Initial Problem, Yellow = Design Space, Brown = Non-design Space

The dimensions of the MBB beam are $20 \mathrm{~mm}$ in the $\mathrm{x}$ direction, $10 \mathrm{~mm}$ in the $\mathrm{y}$ direction and $120 \mathrm{~mm}$ in the $\mathrm{z}$ direction. The boundary conditions are applied such that the side of the beam where the applied force is placed is free to move in the x-direction. The constraints applied on the side of the beam without applied loads are free to move in the z-direction. The applied force is $500 \mathrm{~N}$ in the $\mathrm{x}$-direction and will cause a maximum deflection of $0.0215 \mathrm{~mm}$ in the $\mathrm{x}$-direction with a maximum von-Mises stress of approximately $21 \mathrm{MPa}$. The models for the MBB beam meshed with lattices can be seen in Figure 75. The crystallographic planes, unit cell sizes and plane distances for the lattice net construction in this case study are presented in Table 22 . The projected planes for the filter were set to the $\mathrm{x} y$ and $\mathrm{z}$ planes. 
Table 22 : Lattice Net settings for Selected Topologies

\begin{tabular}{|c|c|c|c|c|c|}
\hline Topology & Unit Cell Size & \multicolumn{3}{|c|}{ Crystallographic Plane(s) } & $\begin{array}{c}\text { Plane } \\
\text { Distance }\end{array}$ \\
\hline \multirow{3}{*}{ CUBIC } & \multirow{3}{*}{1.9} & 1 & 0 & 0 & 1.9 \\
\hline & & 0 & 1 & 0 & 1.9 \\
\hline & & 0 & 0 & 1 & 1.9 \\
\hline \multirow{6}{*}{$\mathrm{BCC}$} & \multirow{6}{*}{3} & 0 & $1 / \sqrt{2}$ & $-1 / \sqrt{2}$ & 2.1213 \\
\hline & & 0 & $1 / \sqrt{2}$ & $1 / \sqrt{2}$ & 2.1213 \\
\hline & & $1 / \sqrt{2}$ & $1 / \sqrt{2}$ & 0 & 2.1213 \\
\hline & & $-1 / \sqrt{2}$ & $1 / \sqrt{2}$ & 0 & 2.1213 \\
\hline & & $1 / \sqrt{2}$ & 0 & $-1 / \sqrt{2}$ & 2.1213 \\
\hline & & $1 / \sqrt{2}$ & 0 & $1 / \sqrt{2}$ & 2.1213 \\
\hline \multirow{9}{*}{ FCC } & \multirow{9}{*}{3.8} & 0 & $1 / \sqrt{2}$ & $-1 / \sqrt{2}$ & 2.6870 \\
\hline & & 0 & $1 / \sqrt{2}$ & $1 / \sqrt{2}$ & 2.6870 \\
\hline & & $1 / \sqrt{2}$ & $1 / \sqrt{2}$ & 0 & 2.6870 \\
\hline & & $-1 / \sqrt{2}$ & $1 / \sqrt{2}$ & 0 & 2.6870 \\
\hline & & $1 / \sqrt{2}$ & 0 & $-1 / \sqrt{2}$ & 2.6870 \\
\hline & & $1 / \sqrt{2}$ & 0 & $1 / \sqrt{2}$ & 2.6870 \\
\hline & & 1 & 0 & 0 & 3.8 \\
\hline & & 0 & 1 & 0 & 3.8 \\
\hline & & 0 & 0 & 1 & 3.8 \\
\hline \multirow{6}{*}{$\begin{array}{l}\text { Octet } \\
\text { Truss }\end{array}$} & \multirow{6}{*}{3.8} & $-1 / \sqrt{3}$ & $1 / \sqrt{3}$ & $1 / \sqrt{3}$ & 2.1939 \\
\hline & & $1 / \sqrt{3}$ & $-1 / \sqrt{3}$ & $1 / \sqrt{3}$ & 2.1939 \\
\hline & & $-1 / \sqrt{3}$ & $-1 / \sqrt{3}$ & $1 / \sqrt{3}$ & 2.1939 \\
\hline & & $-1 / \sqrt{3}$ & $1 / \sqrt{3}$ & $-1 / \sqrt{3}$ & 2.1939 \\
\hline & & $-1 / \sqrt{3}$ & $-1 / \sqrt{3}$ & $-1 / \sqrt{3}$ & 2.1939 \\
\hline & & $1 / \sqrt{3}$ & $1 / \sqrt{3}$ & $-1 / \sqrt{3}$ & 2.1939 \\
\hline
\end{tabular}




\section{H.11 Case Study Results}

The results in Table 23 show that when a lattice net is added to the outside of a trimmed lattice structured; the optimizations with the net had a significantly lower final mass. This indicates that an added exterior lattice net is beneficial when creating lattice embedded geometries for trimmed lattices. A lower mass will therefore reduce printing time and save on material costs.

Table 23 : Lattice Net Case Study Results

\begin{tabular}{|l|c|c|c|c|c|c|}
\hline Topology & $\begin{array}{c}\text { Final Mass } \\
(\mathbf{k g})\end{array}$ & $\begin{array}{c}\text { Max } \\
\text { Deflection }\end{array}$ & $\begin{array}{c}\text { Max Stress } \\
(\mathbf{M P a})\end{array}$ & Net? & $\begin{array}{c}\text { Design } \\
\text { Variables }\end{array}$ & $\begin{array}{c}\text { Unit Cell } \\
\text { Size }\end{array}$ \\
\hline CUBIC & $1.0821 \mathrm{e}-05$ & 0.98556 & 880.0763 & No & 2443 & 1.9 \\
\hline CUBIC & $1.0061 \mathrm{e}-05$ & 1.11896 & 878.2104 & Yes & 2607 & 1.9 \\
\hline BCC & $1.7263 \mathrm{e}-05$ & 1.31736 & 880.2637 & No & 2397 & 3 \\
\hline BCC & $1.3831 \mathrm{e}-05$ & 0.701368 & 878.8573 & Yes & 3543 & 3 \\
\hline FCC & $1.0247 \mathrm{e}-05$ & 0.875091 & 878.3241 & No & 1931 & 3.8 \\
\hline FCC & $5.4147 \mathrm{e}-06$ & 0.805261 & 823.5212 & Yes & 3474 & 3.8 \\
\hline Octet Truss & $8.7844 \mathrm{e}-06$ & 1.10066 & 870.5327 & No & 2703 & 3.8 \\
\hline Octet Truss & $7.3769 \mathrm{e}-06$ & 0.724165 & 872.1389 & Yes & 3011 & 3.8 \\
\hline
\end{tabular}

The topology with the lowest mass was the FCC topology. This could be a result of it having more favorable strut directions and connections and a larger unit cell size than the other topologies for this specific load case. The maximum stress in the FCC final design was also much lower than the constraint maximum which means that the final mass could potentially be much lower. Figure 75 displays the final optimized designs; the beam size distribution was fairly homogenous among the test cases and the majority of the beam's radii fell between 0.24 and 0.26 $\mathrm{mm}$. The specific stiffness for the net-based optimization cases where much higher than those without the net. The octet truss however had decreased its specific stiffness as the results of adding the lattice net. However, specific strength had improved significantly for all cases from the addition of the lattice net. 
a)

b)

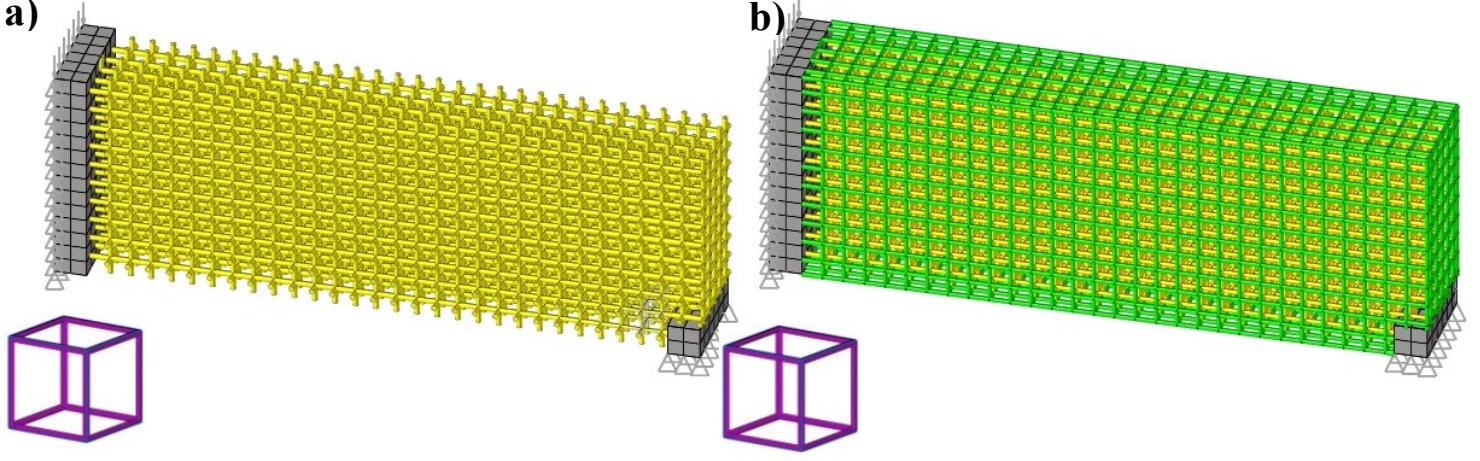

c)

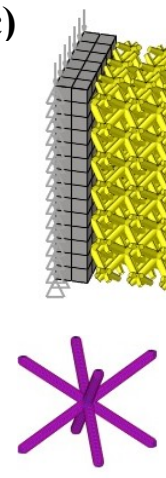

e)

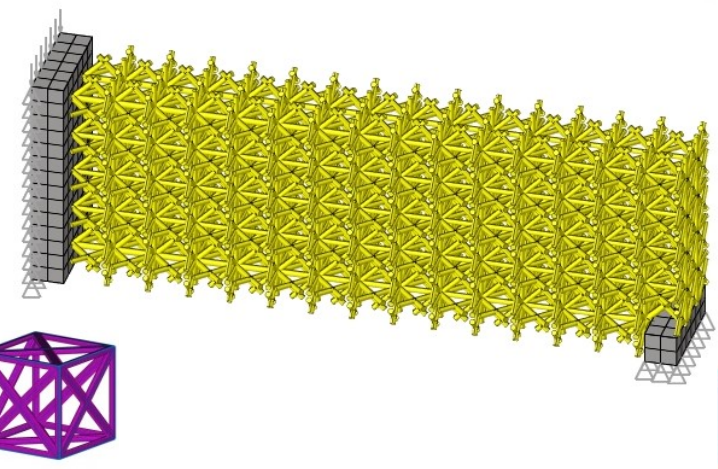

g)

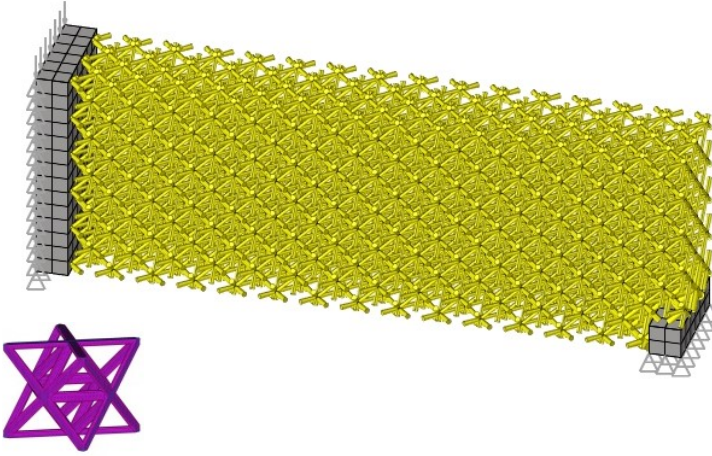

d)

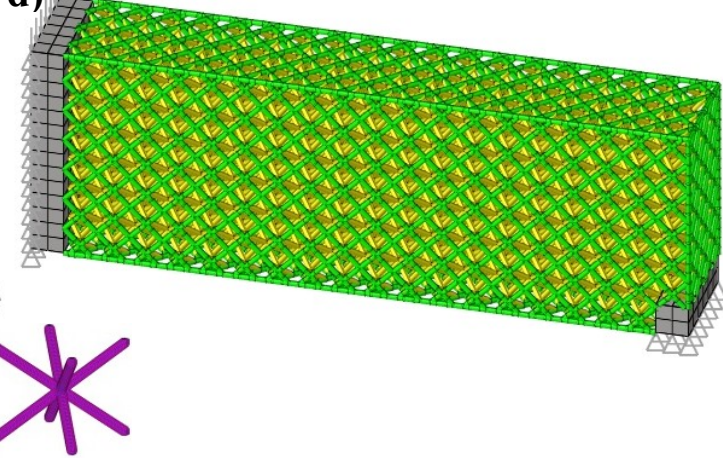

f)

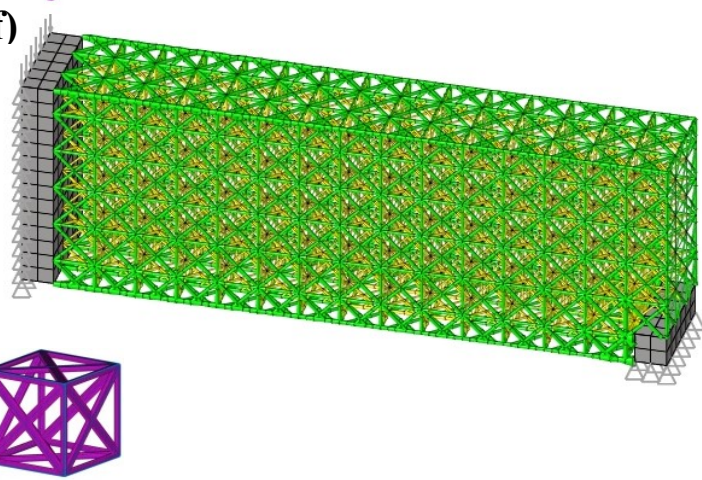

h)

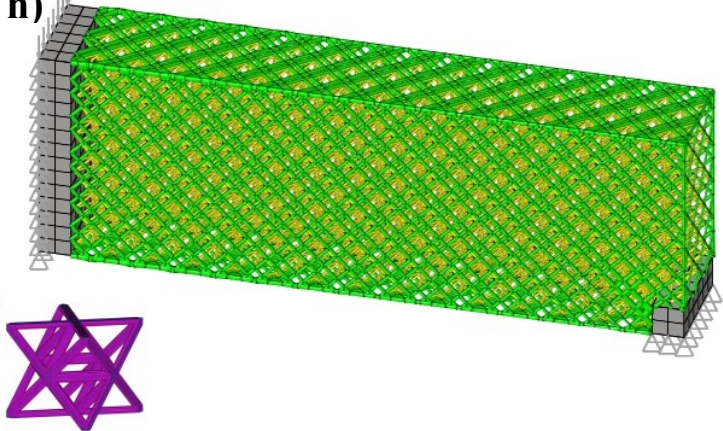

Figure 75: Optimized Results $($ Yellow $=$ Trimmed Interior Lattice, Green $=$ Lattice Net, Purple $=$ Single Unit Cell) (a) Cubic without net (b) Cubic with net (c) BCC without net (d) BCC with net (e) FCC without net (f) FCC with net (g) Octet truss without net (h) Octet truss with net 


\section{H.12 Closing Statements}

A method for creating a trimmed wrieframe lattice was developed. This method provides a list of surface nodes from the trimming operation to be used for constructing the lattice net. In addition, a novel method for constructing a conformal lattice net as a wireframe is presented. The generated lattice net is created by using the crystallographic planes from symmetric unit cell topologies. The lattice net algortihm functions by using contours from plane slicing at calculated intervals to connect surface nodes together which are then paired up and converted into struts. A projection filter is added to remove uneeded connections and produce an approximate projection of the unit cell onto the surface of a closed triangulated surface. The proposed algortihm is able to produce a net for any complex geometry and construction of the net is independent of the interior trimmed structure.

An engineering comparison for microscale optimization of functionaly graded materials with and without lattice nets was also done. For topologies such as cubic, BCC and FCC; the addtion of a lattice net was beneficial during the micro-scale optimizations. The FCC topology performed best with the net. The performance improvement of the added net shows that the overal mass of the lattice structure was lower yeilded higher specific strength and stiffness. 


\section{Appendix I : Trimmed Lattice Pseudo Codes}

\section{I.1 Algorithm 1: Trimmed Lattice Case Sorting}

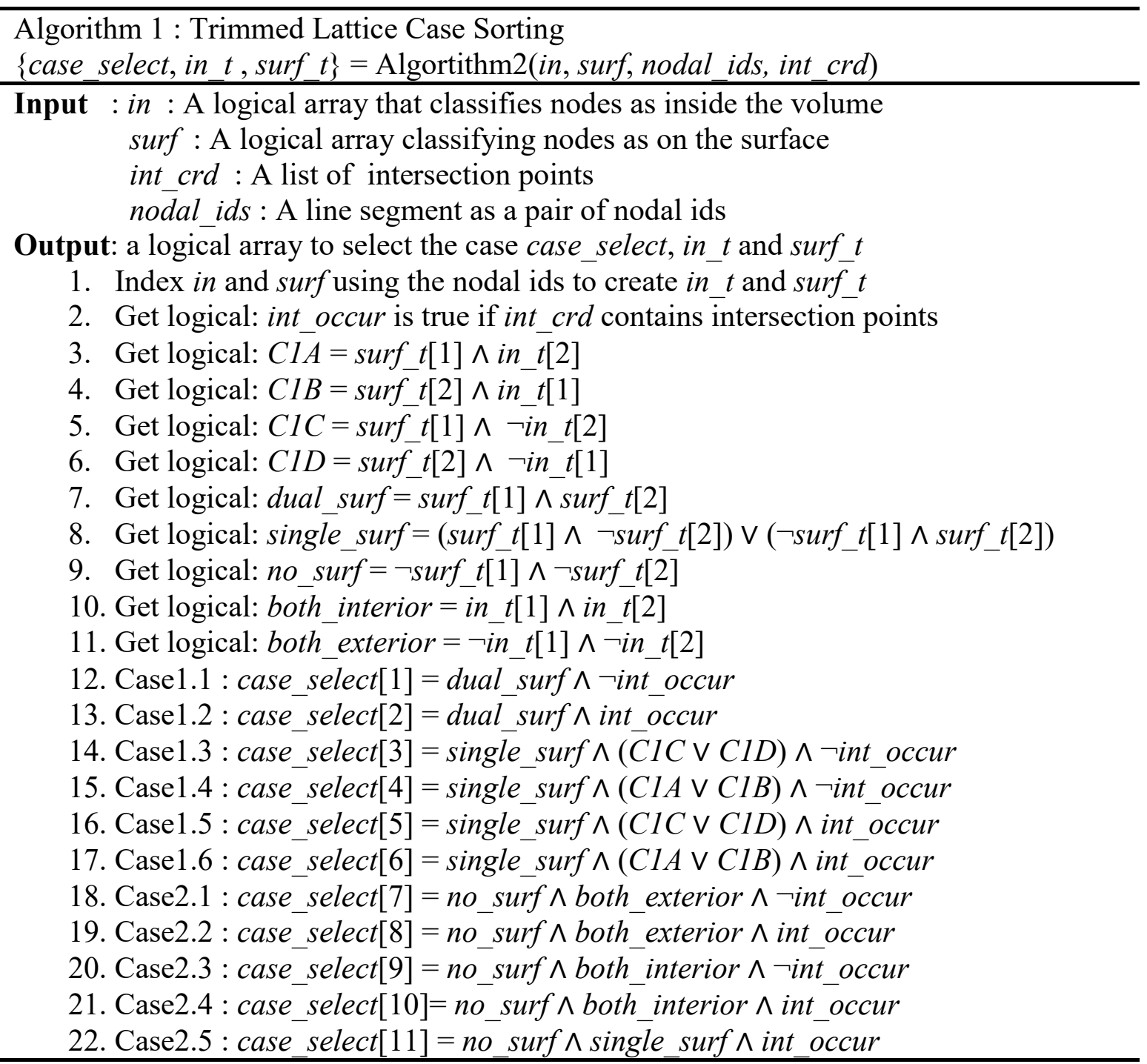




\section{I.2 Algorithm 2: Trimmed Lattice Sub Algorithm}

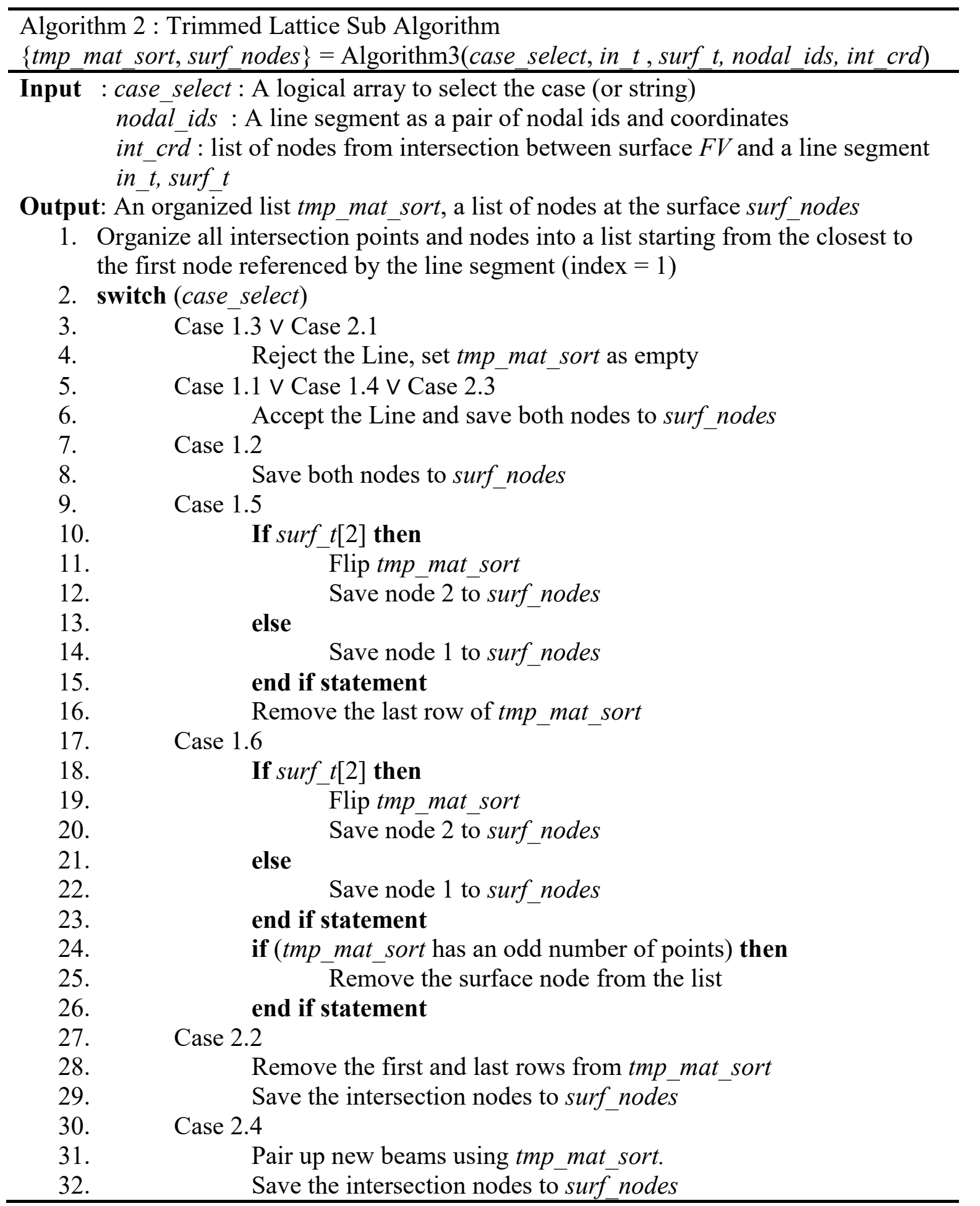




\begin{tabular}{|c|c|}
\hline 33. & Case 2.5 \\
\hline 34. & If $i n \_t[2]$ then \\
\hline 35 . & Flip tmp_mat_sort \\
\hline 36. & end if statement \\
\hline 37. & Save the intersection nodes to surf_nodes \\
\hline 38. & Remove the last row from $t m p \_m a t \_s o r t$ \\
\hline 39. & End case statement \\
\hline 40. & new beams using tmp mat sort \\
\hline
\end{tabular}

\section{I.3 Algorithm 3 : Trimmed Lattice Algorithm}

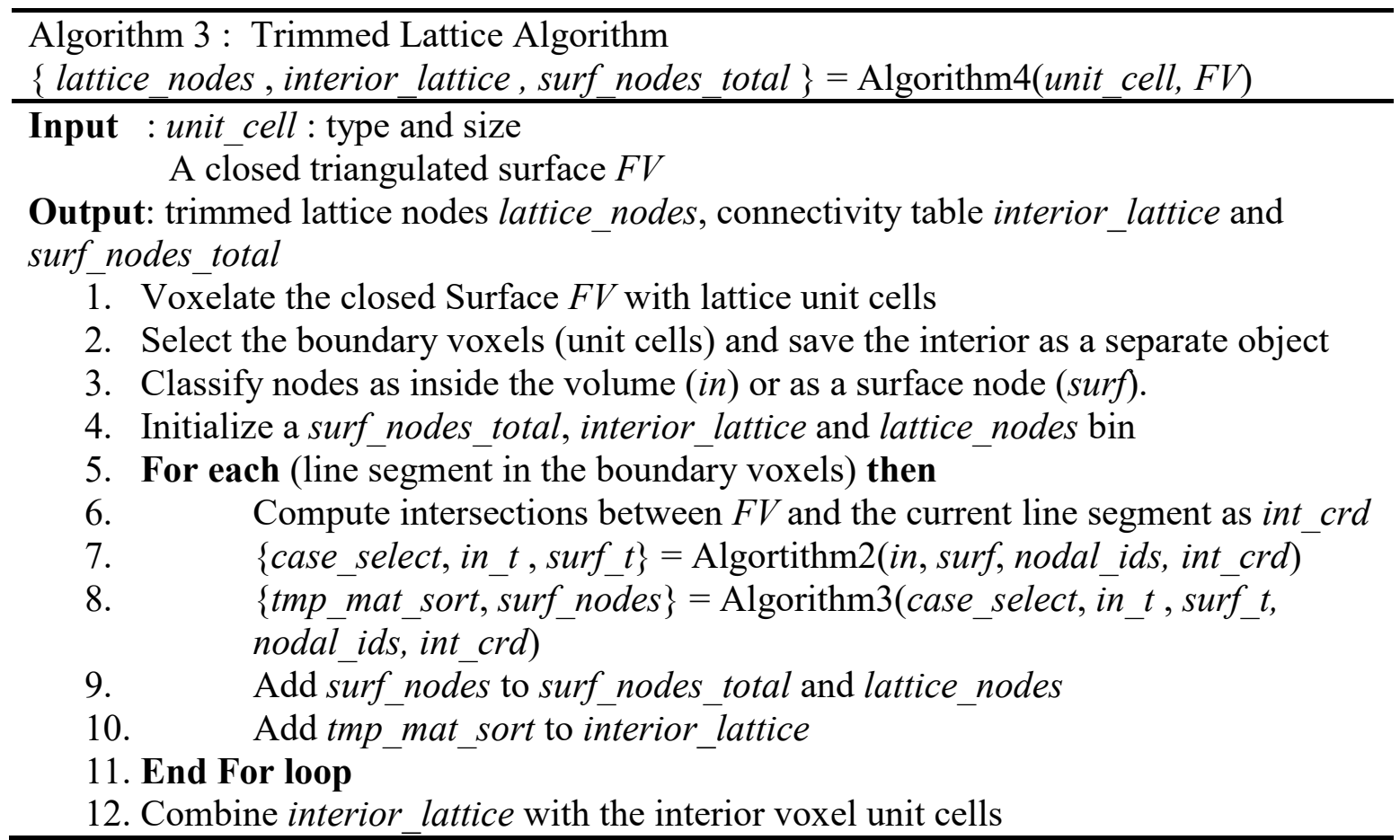

\section{I.4 Algorithm 4 : Contour Collection}

\begin{tabular}{l} 
Algorithm $4:$ Contour Collection \\
$\{$ contour_bin,edge_nodes $\}=$ Algorithm5(seeding_point,FV,unit_cell) \\
\hline Input : unit_cell : crystallographic planes and planar distances \\
A closed triangulated surface $F V$ and center of rotation rot \\
The minimum coordinates from voxel centroids seeding_point \\
Output: contours bin called contour_bin and a set of nodes called edge_nodes \\
1. Initialize contour_bin to store results for each crystallographic plane
\end{tabular}




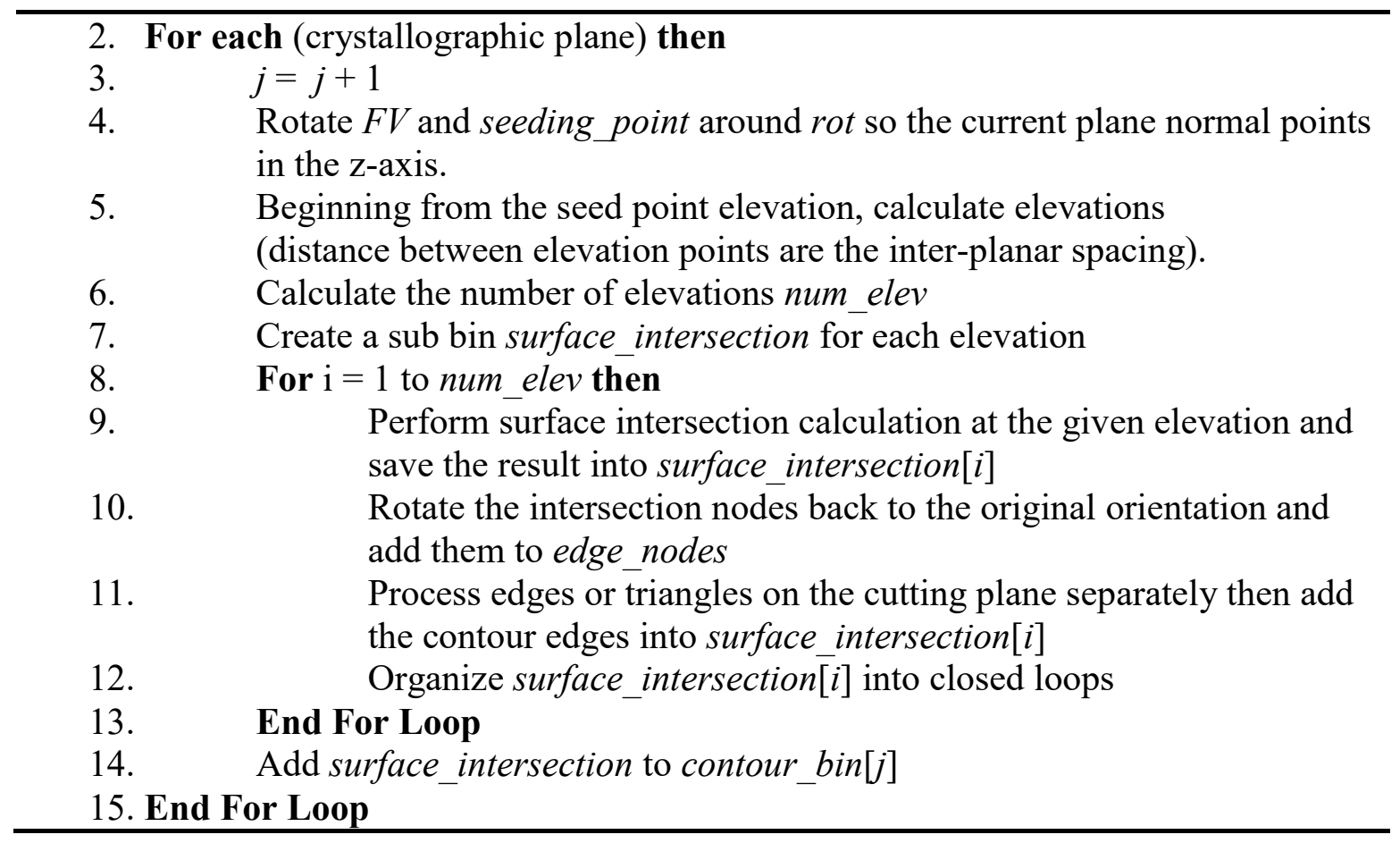

\section{I.5 Algorithm 5 : Additional Surface Nodes}

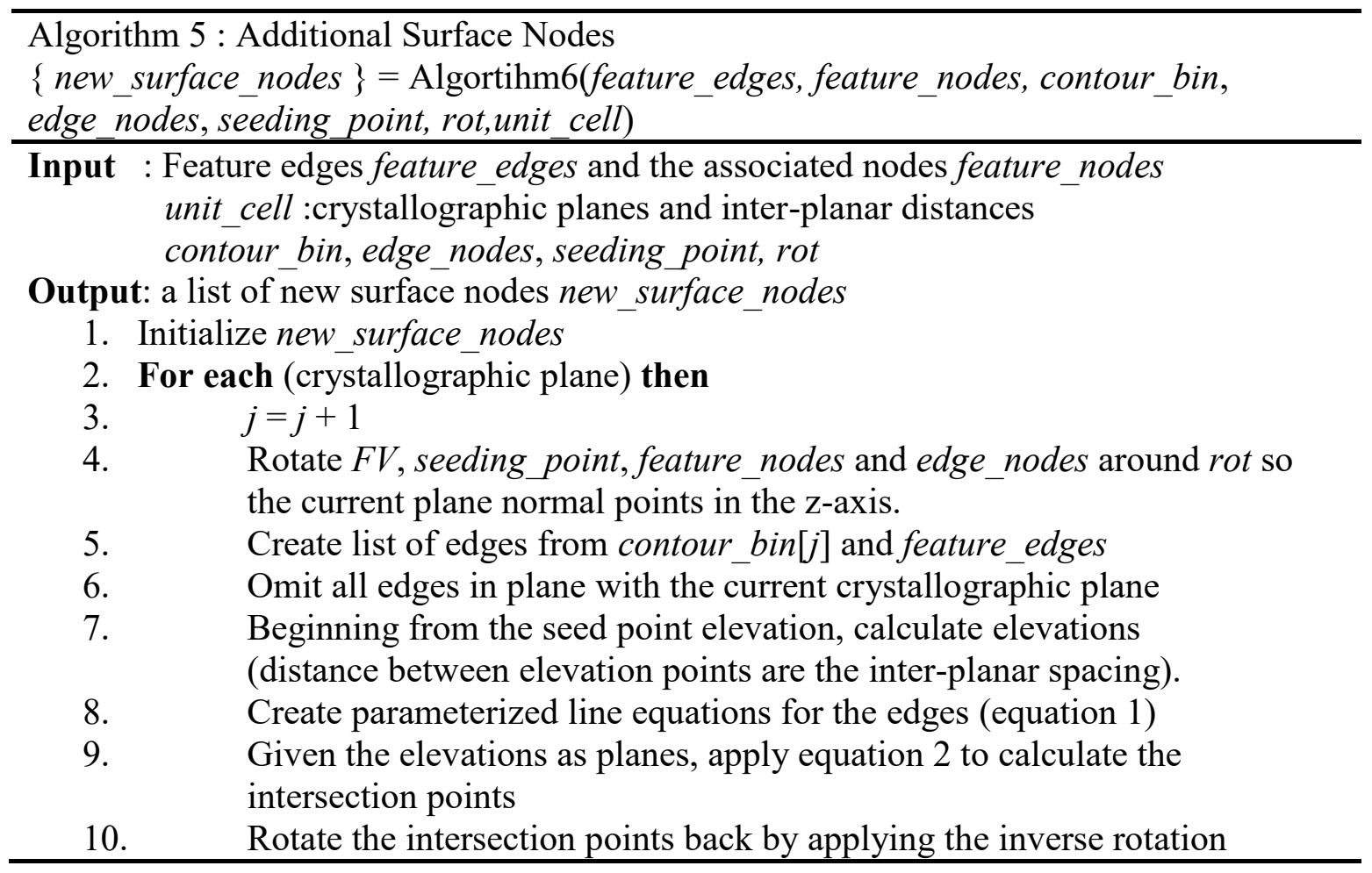


11. Add the new points to new_surface_nodes

12. End For Loop

\section{I.6 Algorithm 6 : Projection Filter}

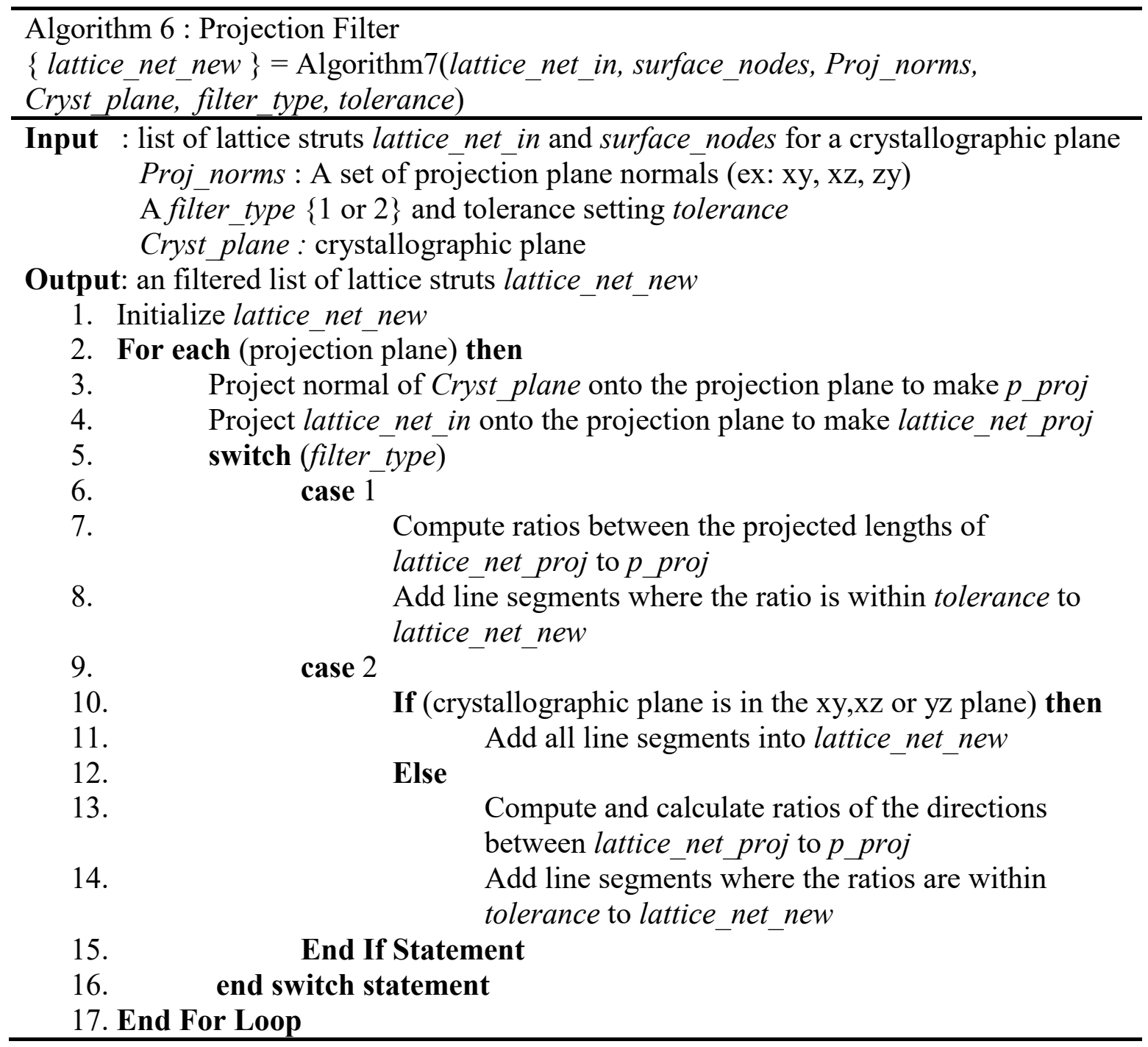




\section{I.7 Algorithm 7 : Lattice Net}

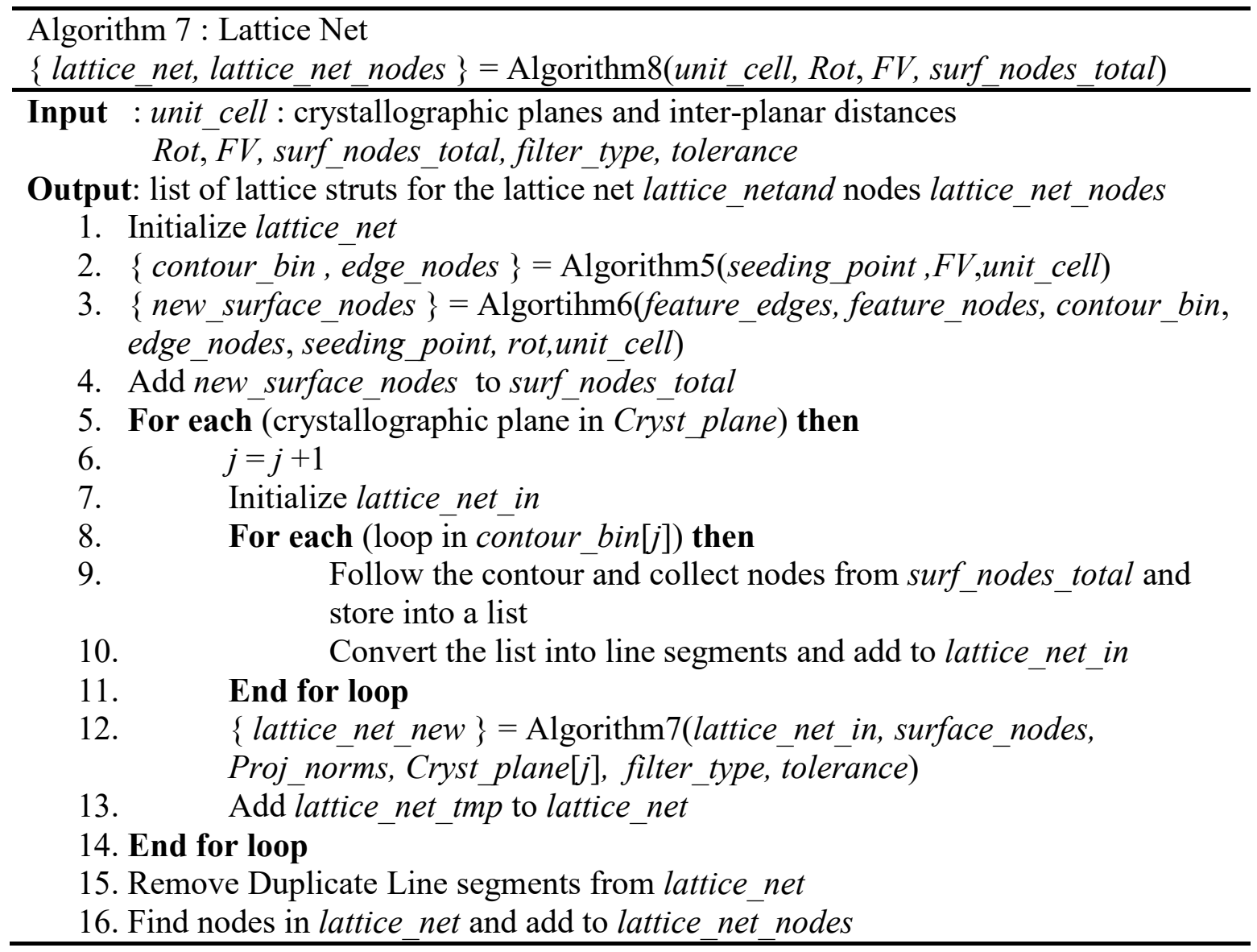

\section{I.8 Algorithm 8 : Additional Connections Algorithm}

\begin{tabular}{l} 
Algorithm 8: Additional Connections Algorithm \\
\{lattice_net, lattice_net_nodes $\}=$ Algortihm9(Feature_edges, lattice_net, \\
feature_nodes, lattice_net_nodes) \\
\hline Input : Feature_edges, lattice_net, feature_nodes, lattice_net_nodes \\
Output: Updated lattice_net and lattice_net_nodes with integrated feature edges \\
1. Sort the edge list from Feature_edges into closed or open loops called loop \\
2. Organize the loops in loop to be in order (edges connected) \\
3. Add nodes from feature_nodes referenced more than twice into lattice_net_nodes \\
4. Initialize new_line_segment as an empty bin \\
5. for each $($ loop) do \\
6. Collect nodes from lattice_net_nodes along the current loop to form a list \\
7. $\quad$ Turn the list into line segments \\
8. Add the new line segments into new_line_segment
\end{tabular}


9. End For Loop

10. Add new line segment into lattice net

\section{I.9 Algorithm 9: in_polyhedron (Ray-Search Algorithm for interior and Exterior Points}

Algorithm 9 : in_polyhedron (Ray-Search Algorithm for interior and Exterior Points) $\{$ in $\}=\operatorname{Algortihm1}($ point,$F V)$

Input : point : A list of points called $F V$ : A closed triangulated surface

Output: a logical array were true is a point located inside the closed surface called in

1. Determine the number of points for classification, save as num points

2. Initialize logical array of false operators equal to the number of points called in

3. For $i=1$ to num points do

4. Shoot a randomly directed $(d)$ infinite ray out of the query point which does not hit an edge of a triangular face

5. Evaluate each triangular face with edge vertices $v 0, v 1, v 2$ and assess the intersection criteria 1 and 2

6. Save number of intersections for the query point as count

7. If $($ count $\bmod 2)=0$ then

8 . Set $i n[i]$ to true

9. $\quad$ End if statement

10. End For loop 


\section{Appendix J : Case Study Data}

\section{J.1 L - Bracket}

\begin{tabular}{||c|c||c|c||c|c||c|c||}
\hline \multicolumn{9}{|c|}{ Pareto Frontier Data - L-Bracket } \\
\hline \hline \multicolumn{2}{|c|}{ Untreated Solid } & \multicolumn{2}{|c|}{ Treated Solid } & Untreated Lattice & \multicolumn{2}{c||}{ Treated Lattice } \\
\hline \hline Damage & $\begin{array}{c}\text { Mass } \\
\text { Fraction }\end{array}$ & Damage & $\begin{array}{c}\text { Mass } \\
\text { Fraction }\end{array}$ & Damage & $\begin{array}{c}\text { Mass } \\
\text { Fraction }\end{array}$ & Damage & $\begin{array}{c}\text { Mass } \\
\text { Fraction }\end{array}$ \\
\hline \hline 0.000368 & 0.92864 & 0.000319 & 0.908 & 0.000335 & 0.92617 & 0.00029 & 0.92301 \\
\hline 0.000377 & 0.85729 & 0.000332 & 0.85702 & 0.000342 & 0.85619 & 0.000301 & 0.85606 \\
\hline 0.000384 & 0.78593 & 0.000344 & 0.78559 & 0.000345 & 0.78565 & 0.000309 & 0.78544 \\
\hline 0.000386 & 0.71457 & 0.000324 & 0.71344 & 0.000375 & 0.71441 & 0.000315 & 0.71435 \\
\hline 0.000415 & 0.64321 & 0.000388 & 0.64283 & 0.000431 & 0.64279 & 0.000403 & 0.64306 \\
\hline 0.000489 & 0.57186 & 0.000443 & 0.57137 & 0.000499 & 0.57177 & 0.000452 & 0.57186 \\
\hline 0.000604 & 0.5005 & 0.000691 & 0.5 & 0.0006 & 0.5005 & 0.000582 & 0.5005 \\
\hline 0.000897 & 0.42914 & 0.001104 & 0.42862 & 0.00075 & 0.45055 & 0.001709 & 0.42914 \\
\hline 0.001453 & 0.35779 & 0.002184 & 0.3572 & 0.0019 & 0.35779 & 0.004284 & 0.35779 \\
\hline 0.003536 & 0.28643 & 0.015827 & 0.28578 & 0.00542 & 0.28643 & 0.027098 & 0.28643 \\
\hline 0.033944 & 0.21507 & 0.098007 & 0.21407 & 0.015365 & 0.21507 & 0.076825 & 0.21507 \\
\hline 4360.3 & 0.14371 & 96.147 & 0.15834 & 0.41027 & 0.14371 & 18.60589 & 0.14371 \\
\hline 3625.1 & 0.072357 & 80.825 & 0.15712 & 2.6429 & 0.10919 & 118.5373 & 0.078527 \\
\hline
\end{tabular}

\section{J.2 Aircraft Hinge}

\begin{tabular}{|c|c|c||c||}
\hline \multicolumn{5}{|c|}{ Pareto Frontier Data - Aircraft Hinge } \\
\hline \hline Design Point & Cumulative Damage & $\log ($ Damage) & Mass Fraction \\
\hline \hline Point 0 & $1.50 \mathrm{E}-15$ & -34.1402 & 1 \\
\hline Point1 & $7.13 \mathrm{E}-14$ & -30.2754 & 0.59893 \\
\hline Point2 & $3.3572 \mathrm{E}-13$ & -28.7255 & 0.49873 \\
\hline Point3 & $8.98 \mathrm{E}-12$ & -25.4387 & 0.39988 \\
\hline Point4 & $2.7467 \mathrm{E}-10$ & -22.0177 & 0.29950 \\
\hline Point5 & $1.85 \mathrm{E}-08$ & -17.8067 & 0.19934 \\
\hline
\end{tabular}

\begin{tabular}{||c|c|c|c||}
\hline \multicolumn{4}{|c|}{ Pareto Frontier Data (No Filter) - Aircraft Hinge } \\
\hline \hline Design Point & Cumulative Damage & $\log$ (Damage) & Mass Fraction \\
\hline \hline Point 0 & $1.50 \mathrm{E}-15$ & -34.1402 & 1 \\
\hline
\end{tabular}




\begin{tabular}{||c|c|c|c||}
\hline Point1 & $4.01 \mathrm{E}-14$ & -30.8514 & 0.59129 \\
\hline Point2 & $4.73 \mathrm{E}-13$ & -28.3827 & 0.49234 \\
\hline Point3 & $1.08 \mathrm{E}-11$ & -25.2572 & 0.39683 \\
\hline Point4 & $1.17 \mathrm{E}-09$ & -20.5679 & 0.29378 \\
\hline Point5 & $8.3259 \mathrm{E}-07$ & -14.0002 & 0.19736 \\
\hline
\end{tabular}

\begin{tabular}{|c|c|c|c|c|c|c|}
\hline \multicolumn{7}{|c|}{ Surrogate Model Training Data } \\
\hline ISO & Rotation & Mass (Kg) & Violation (\%) & Compliance & Beams & Design Variables \\
\hline 0.1 & 0 & 0.92093 & 0 & 0.033349 & 9 & 9 \\
\hline 0.432 & 20.225 & 0.78128 & 100 & 0.033931 & 573 & 494 \\
\hline 0.6 & 23 & 0.78086 & 100 & 0.033932 & 549 & 461 \\
\hline 0.75 & 1 & 0.52924 & 100 & 0.042231 & 1649 & 1156 \\
\hline 0.556 & 4.866 & 0.69933 & 100 & 0.03525 & 916 & 715 \\
\hline 1 & 1.632 & 0.67769 & 183.97 & 0.14027 & 4527 & 1898 \\
\hline 0 & 0 & 0.92415 & 0 & $\mathrm{NaN}$ & 0 & 0 \\
\hline 0 & 1 & 0.92415 & 0 & $\mathrm{NaN}$ & 0 & 0 \\
\hline 0 & 2 & 0.92415 & 0 & $\mathrm{NaN}$ & 0 & 0 \\
\hline 0 & 3 & 0.92415 & 0 & $\mathrm{NaN}$ & 0 & 0 \\
\hline 0 & 4 & 0.92415 & 0 & $\mathrm{NaN}$ & 0 & 0 \\
\hline 0 & 5 & 0.92415 & 0 & $\mathrm{NaN}$ & 0 & 0 \\
\hline 0 & 6 & 0.92415 & 0 & $\mathrm{NaN}$ & 0 & 0 \\
\hline 0 & 7 & 0.92415 & 0 & $\mathrm{NaN}$ & 0 & 0 \\
\hline 0 & 8 & 0.92415 & 0 & $\mathrm{NaN}$ & 0 & 0 \\
\hline 0 & 9 & 0.92415 & 0 & $\mathrm{NaN}$ & 0 & 0 \\
\hline 0 & 10 & 0.92415 & 0 & $\mathrm{NaN}$ & 0 & 0 \\
\hline 0 & 11 & 0.92415 & 0 & $\mathrm{NaN}$ & 0 & 0 \\
\hline 0 & 12 & 0.92415 & 0 & $\mathrm{NaN}$ & 0 & 0 \\
\hline 0 & 13 & 0.92415 & 0 & $\mathrm{NaN}$ & 0 & 0 \\
\hline 0 & 14 & 0.92415 & 0 & $\mathrm{NaN}$ & 0 & 0 \\
\hline 0 & 15 & 0.92415 & 0 & $\mathrm{NaN}$ & 0 & 0 \\
\hline 0 & 16 & 0.92415 & 0 & $\mathrm{NaN}$ & 0 & 0 \\
\hline 0 & 17 & 0.92415 & 0 & $\mathrm{NaN}$ & 0 & 0 \\
\hline 0 & 18 & 0.92415 & 0 & $\mathrm{NaN}$ & 0 & 0 \\
\hline 0 & 19 & 0.92415 & 0 & $\mathrm{NaN}$ & 0 & 0 \\
\hline 0 & 20 & 0.92415 & 0 & $\mathrm{NaN}$ & 0 & 0 \\
\hline 0 & 21 & 0.92415 & 0 & $\mathrm{NaN}$ & 0 & 0 \\
\hline 0 & 22 & 0.92415 & 0 & $\mathrm{NaN}$ & 0 & 0 \\
\hline 0 & 23 & 0.92415 & 0 & $\mathrm{NaN}$ & 0 & 0 \\
\hline 0 & 24 & 0.92415 & 0 & $\mathrm{NaN}$ & 0 & 0 \\
\hline
\end{tabular}




\begin{tabular}{|c|c|c|c|c|c|c|}
\hline 0 & 25 & 0.92415 & 0 & $\mathrm{NaN}$ & 0 & 0 \\
\hline 0 & 26 & 0.92415 & 0 & $\mathrm{NaN}$ & 0 & 0 \\
\hline 0 & 27 & 0.92415 & 0 & $\mathrm{NaN}$ & 0 & 0 \\
\hline 0 & 28 & 0.92415 & 0 & $\mathrm{NaN}$ & 0 & 0 \\
\hline 0 & 29 & 0.92415 & 0 & $\mathrm{NaN}$ & 0 & 0 \\
\hline 0 & 30 & 0.92415 & 0 & $\mathrm{NaN}$ & 0 & 0 \\
\hline 0 & 31 & 0.92415 & 0 & $\mathrm{NaN}$ & 0 & 0 \\
\hline 0 & 32 & 0.92415 & 0 & $\mathrm{NaN}$ & 0 & 0 \\
\hline 0 & 33 & 0.92415 & 0 & $\mathrm{NaN}$ & 0 & 0 \\
\hline 0 & 34 & 0.92415 & 0 & $\mathrm{NaN}$ & 0 & 0 \\
\hline 0 & 35 & 0.92415 & 0 & $\mathrm{NaN}$ & 0 & 0 \\
\hline 0 & 36 & 0.92415 & 0 & $\mathrm{NaN}$ & 0 & 0 \\
\hline 0 & 37 & 0.92415 & 0 & $\mathrm{NaN}$ & 0 & 0 \\
\hline 0 & 38 & 0.92415 & 0 & $\mathrm{NaN}$ & 0 & 0 \\
\hline 0 & 39 & 0.92415 & 0 & $\mathrm{NaN}$ & 0 & 0 \\
\hline 0 & 40 & 0.92415 & 0 & $\mathrm{NaN}$ & 0 & 0 \\
\hline 0 & 41 & 0.92415 & 0 & $\mathrm{NaN}$ & 0 & 0 \\
\hline 0 & 42 & 0.92415 & 0 & $\mathrm{NaN}$ & 0 & 0 \\
\hline 0 & 43 & 0.92415 & 0 & $\mathrm{NaN}$ & 0 & 0 \\
\hline 0 & 44 & 0.92415 & 0 & $\mathrm{NaN}$ & 0 & 0 \\
\hline 0 & 45 & 0.92415 & 0 & $\mathrm{NaN}$ & 0 & 0 \\
\hline 0.432 & 40 & 0.81132 & 100 & 0.033836 & 619 & 531 \\
\hline 0.12 & 1.382 & 0.92088 & 0 & 0.033371 & 9 & 9 \\
\hline 0.156 & 5.766 & 0.92071 & 100 & 0.033314 & 11 & 13 \\
\hline 0.209 & 9.795 & 0.92041 & 100 & 0.033348 & 9 & 9 \\
\hline 0.292 & 13.187 & 0.85203 & 100 & 0.033527 & 263 & 247 \\
\hline 0.293 & 17.49 & 0.85167 & 100 & 0.033491 & 306 & 271 \\
\hline 0.132 & 21.446 & 0.9208 & 0 & 0.033329 & 7 & 8 \\
\hline 0.294 & 25.646 & 0.85138 & 100 & 0.033521 & 254 & 230 \\
\hline 0.291 & 29.709 & 0.85241 & 100 & 0.033497 & 286 & 262 \\
\hline 0.197 & 33.755 & 0.92055 & 100 & 0.033331 & 18 & 15 \\
\hline 0.26 & 37.276 & 0.866 & 100 & 0.033357 & 208 & 196 \\
\hline 0.128 & 41.68 & 0.92083 & 0 & 0.033338 & 7 & 8 \\
\hline 0.184 & 45 & 0.92059 & 100 & 0.033349 & 18 & 18 \\
\hline 0.383 & 1.163 & 0.80823 & 100 & 0.033754 & 429 & 370 \\
\hline 0.358 & 5.119 & 0.82059 & 100 & 0.03358 & 363 & 317 \\
\hline 0.392 & 9.498 & 0.80448 & 100 & 0.033748 & 441 & 385 \\
\hline 0.331 & 13.96 & 0.83455 & 100 & 0.033511 & 324 & 285 \\
\hline 0.207 & 17.34 & 0.92053 & 0 & 0.033339 & 22 & 22 \\
\hline 0.37 & 21.585 & 0.81615 & 0 & 0.033648 & 413 & 355 \\
\hline
\end{tabular}




\begin{tabular}{|c|c|c|c|c|c|c|}
\hline 0.387 & 25.224 & 0.80659 & 100 & 0.033778 & 459 & 394 \\
\hline 0.336 & 29.751 & 0.83302 & 0 & 0.033516 & 364 & 323 \\
\hline 0.352 & 33.255 & 0.8239 & 100 & 0.033617 & 424 & 369 \\
\hline 0.349 & 37.506 & 0.82919 & 100 & 0.033622 & 391 & 354 \\
\hline 0.278 & 41.699 & 0.85709 & 100 & 0.033404 & 250 & 239 \\
\hline 0.331 & 45 & 0.83499 & 0 & 0.033505 & 346 & 317 \\
\hline 0.334 & 1.959 & 0.83315 & 100 & 0.033496 & 348 & 302 \\
\hline 0.441 & 5.547 & 0.77814 & 100 & 0.033974 & 568 & 471 \\
\hline 0.306 & 9.139 & 0.84609 & 100 & 0.033529 & 287 & 262 \\
\hline 0.355 & 13.149 & 0.82195 & 100 & 0.033648 & 353 & 312 \\
\hline 0.309 & 17.258 & 0.84503 & 0 & 0.033547 & 332 & 286 \\
\hline 0.319 & 21.841 & 0.84109 & 0 & 0.033551 & 324 & 287 \\
\hline 0.674 & 0.24 & 0.6058 & 100 & 0.038056 & 1358 & 978 \\
\hline 0.517 & 4.417 & 0.72717 & 100 & 0.034662 & 780 & 627 \\
\hline 0.58 & 8.05 & 0.68382 & 100 & 0.03564 & 1028 & 800 \\
\hline 0.552 & 12.903 & 0.70216 & 100 & 0.035173 & 931 & 729 \\
\hline 0.66 & 16.945 & 0.61789 & 100 & 0.037463 & 1352 & 990 \\
\hline 0.586 & 20.491 & 0.67866 & 100 & 0.035735 & 1092 & 848 \\
\hline 0.682 & 24.489 & 0.59699 & 100 & 0.038465 & 1463 & 1053 \\
\hline 0.536 & 28.338 & 0.71377 & 100 & 0.034952 & 933 & 747 \\
\hline 0.553 & 32.9 & 0.70214 & 100 & 0.035207 & 962 & 770 \\
\hline 0.529 & 36.369 & 0.71875 & 100 & 0.034877 & 845 & 677 \\
\hline 0.527 & 40.111 & 0.73905 & 100 & 0.034765 & 861 & 704 \\
\hline 0.674 & 44.78 & 0.60555 & 100 & 0.038144 & 1417 & 1029 \\
\hline 0.816 & 0.39 & 0.45349 & 100 & 0.04993 & 2028 & 1387 \\
\hline 0.3 & 0 & 0.84824 & 100 & 0.033561 & 285 & 253 \\
\hline 0.95 & 14.762 & 0.41112 & 100 & 0.10367 & 3528 & 1983 \\
\hline 0.95 & 26.23 & 0.42278 & 100 & 0.10451 & 3595 & 2050 \\
\hline 0.95 & 45 & 0.45362 & 102.05 & 0.10354 & 3654 & 2058 \\
\hline 0.95 & 10.007 & 0.42854 & 100 & 0.10138 & 3421 & 1933 \\
\hline 0.95 & 14.039 & 0.4372 & 100 & 0.10111 & 3525 & 1975 \\
\hline
\end{tabular}

